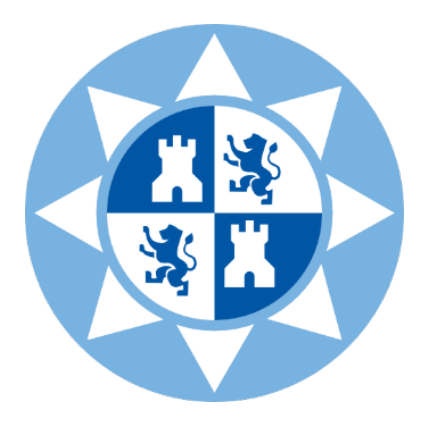

Universidad Politécnica de Cartagena Departamento de Ingeniería Civil

\title{
“Caracterización del problema de intrusión salina de Henry basada en la adimensionalización discriminada avanzada"
}

TESIS DOCTORAL

Manuel Alcaraz Aparicio

Julio, 2016 



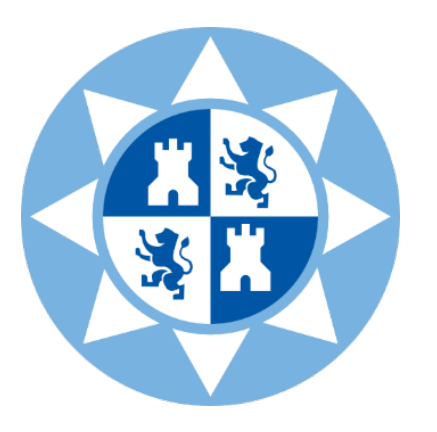

\title{
Universidad Politécnica de Cartagena Departamento de Ingeniería Civil
}

\section{“Caracterización del problema de intrusión salina de Henry basada en la adimensionalización discriminada avanzada”}

\author{
TESIS DOCTORAL
}

Manuel Alcaraz Aparicio

Directores: Dr. Iván Alhama Manteca y Dr. Antonio Soto Meca 

A mi mujer Pilar y a mis hijas Piluca y Mercedes.

A mis padres 



\section{AGRADECIMIENTOS}

Muchas son las personas a las que me gustaría reconocer el apoyo que me han prestado durante estos años para la realización de la tesis, y que me han hecho crecer tanto profesional como personalmente.

En primer lugar, quisiera agradecer a mis directores, Iván Alhama y Antonio Soto, por ser los que me propusieron continuar con sus investigaciones, y los que han sabido guiarme por los caminos que he tenido que recorrer hasta la consecución de esta tesis.

A los miembros del grupo de Simulación por Redes que siempre me han resuelto las dudas que se iban planteando, y en especial, a su Investigador Responsable, Paco Alhama, por transmitirme qué es y cómo se realiza la investigación. A Manuel Cánovas, miembro del grupo, investigando allende los mares, por derrochar y contagiar ilusión y frescura en el trabajo.

Al Catedrático de la Universidad de Granada, el profesor Mario Chica, por brindarme la posibilidad de hacer investigación y acogerme en su grupo, aunque la distancia y mis circunstancias personales no me permitieran lograr el objetivo.

A Emilio Trigueros, compañero de café junto a Mercedes, donde analizamos el devenir de nuestra querida UPCT, compartiendo mucha profesión y donde siempre he recibido el calor necesario para terminar la tesis.

A los miembros del equipo de dirección de la E.T.S. de Ingeniería de Caminos, Canales y Puertos y de Ingeniería de Minas, por su paciencia y comprensión, y por trabajar diariamente en un proyecto de Escuela común.

Para finalizar, agradecer a mi familia por el tiempo que les robo todos los días. A mi mujer y mis hijas, que son el motor de mi vida, y a mis padres, por inculcarme el valor del esfuerzo y del trabajo. 



\section{RESUMEN}

El problema de Henry es ya muy antiguo, pero sin duda, su estudio para el escenario más general no está cerrado pues la literatura científica sigue recogiendo aspectos nuevos sobre el mismo. Desde el primer momento, antes de ser aceptado como problema patrón por la comunidad científica en hidrogeología, Henry caracteriza su problema isótropo siguiendo las técnicas más o menos formales de adimensionalización clásica de las ecuaciones de gobierno, técnicas que conducen a dos parámetros adimensionales (a los que Henry no atribuye significado físico alguna) a los que añade la relación de aspecto sin justificación formal sino sólo por el hecho de ser adimensional. A continuación, asigna valores concretos a estos tres parámetros con criterios prácticos y para salvar los problemas de convergencia de los métodos numéricos al uso en aquellos tiempos. Otros autores posteriores y recientes han corregido estos valores y tratado de asignar significado físico a los (dos) parámetros deducidos de la adimensionalización. En 2014, Kalakan [2014] estudia la caracterización del problema dispersivo de Henry mediante técnicas de análisis dimensional con unos resultados que se reducen a los parámetros de Henry en el problema no dispersivo.

Sin embargo, la consideración de una difusividad anisótropa efectiva (por efectos de la tortuosidad anisótropa de los canales de flujo), no abordada en la literatura sino a través del efecto de dispersión, no hubiera permitido expulsar la relación de aspecto como grupo adimensional independiente. Así, de considerar una difusividad anisótropa, todos los autores que han tratado de caracterizar este problema hubieran derivado un monomio adimensional más, lo cual se demuestra un resultado impreciso en esta memoria. Lo mismo hubiera ocurrido con el problema dispersivo.

El objetivo de esta memoria es la deducción y verificación mediante simulaciones numéricas de los grupos adimensionales discriminados que caracterizan el problema de Henry no dispersivo, pero con anisotropía en la conductividad hidráulica y la difusividad efectiva. Como herramienta para esta caracterización se utiliza la adimensionalización discriminada de las ecuaciones de gobierno, una técnica formal que se ha mostrado muy efectiva en problemas complejos de otros campos de la ingeniería.

Tras explicar los fundamentos de la adimensionalización discriminada, su aplicación al problema anisótropo de Henry conduce a cuatro nuevos grupos adimensionales discriminados (en los que no aparece la relación de aspecto) que se 
demuestra son los verdaderos grupos independientes que controlan los patrones de solución de este problema, es decir, los mapas de isolíneas de concentración y flujo estacionarios. La discriminación, por otro lado, permite asignar un significado físico preciso y un orden de magnitud a los grupos adimensionales derivados de ella. Tras verificar la veracidad de los grupos deducidos, se estudian sus reducciones a escenarios simplificados (incluyendo el problema original de Henry) en los que alguno de los coeficientes dimensionales del problema tiene una influencia despreciable.

Se discuten los valores numéricos propuestos por Henry y el efecto de sus cambios en los patrones de solución del problema original. Se discuten también los patrones resultantes de asignar valor unidad a los grupos discriminados y la influencia de sus cambios (de valores) en los mismos patrones del problema anisótropo. De todo ello, se analiza la idoneidad del problema original de Henry como problema patrón desde el punto de vista de verificación de los códigos de computación en procesos de flujo y transporte de sal.

Finalmente, se estudia la influencia de la longitud del dominio en los patrones y los cambios de estos patrones en los dos grupos discriminados emergentes en problemas en los que la longitud del dominio no influye en la solución (escenarios esbeltos); para estos escenarios, las cuñas de intrusión (difusión) y recirculación se muestran desacopladas, lo que plantea la necesidad de un estudio más profundo del problema: los dos grupos adimensionales deducidos no reproducen la complejidad manifiesta de los patrones de concentración y flujo. Sólo la introducción de nuevas referencias para la coordenada horizontal, relacionadas con las longitudes ocultas de las cuñas de intrusión y recirculación, y la reducción de las ecuaciones de gobierno a las regiones definidas por estas cuñas, podrían conducir a nuevos grupos adimensionales de los que cabe deducir expresiones para el orden de magnitud de las longitudes ocultas. 


\begin{abstract}
Henry`s problem is already ancient but, no doubt, its study for the most general scenario is not close since new aspects on the subject appear each year in the scientific literature. From the first moment, before to be accepted as benchmark by the hydrogeology scientific community, Henry characterizes his original isotropic problem following more or less formal mathematical techniques based on the classical nondimensionalization of governing equations, a tool that leads to two dimensionless parameters (to which he does not assigned any physical meaning) to which the aspect relation or geometric form factor is added by the only fact of being dimensionless. Then, Henry assigns particular values to these three parameters following practical criteria and with the aim of solving the convergence problems that emerge from the numerical methods of those years. Later authors and recent have slightly corrected these values and given physical meaning to the (two) parameters derived from nondimensionalization. In 2014, Kalakan [2014] studies the characterization of dispersive Henry problem by using classical dimensional analysis (pi theorem) with results that reduced to those of Henry for the nondispersive case.
\end{abstract}

However, the assumption of an effective anisotropic diffusivity (caused by the tortuosity effects of the flow channels), not dealing with in the literature but through the dispersive effects, had no allowed to delete the aspect ratio as a dimensionless independent group. Thus, with the hypothesis of anisotropic diffusivity, all authors that have intended to characterize this problem had obtained the aspect ratio monomial as an independent group, a less precise result as it is proved in this memory. The same came be said in relation with the dispersive problem.

The aim of this memory is the derivation and verification, by numerical simulations, of the discriminated dimensionless groups that characterize the nondispersive Henry problem, but assuming anisotropy in the hydraulic conductivity and effective diffusivity. As the tool for this subject it is used the discriminated nondimensionalization of the governing equations, a formal technique that has been proved effective in complex problems in other engineering fields.

After explaining the fundamentals of discriminate nondimensionalization, its application to anisotropic Henry problem provides the four new discriminate dimensionless groups (aspect ratio is not included separately in these numbers) that are 
the really independent true groups that rule the steady-state solution patterns, i.e., the concentration and stream function flow iso-line maps). In addition, discrimination allows to attribute a precise physical meaning and order of magnitude to the dimensionless group derived from its application. After verifying the veracity of the derived groups, their reduction to simplified scenarios (including the original Henry problem) in which some of the physical properties has a negligible influence is investigated.

Numerical values of the dimensionless parameters proposed by Henry and the effect of their changes in the solution patterns of the original problem are discussed. Also, resulting patterns derived from assigning a value of unity to the discriminated groups as well as the influence of their (value) changes in those patterns for the anisotropic problem are investigated. From this, the suitability of the original Henry problem, as benchmark, is also discussed from the point of view of the verification of the standard computational codes in these coupled fluid and salt transport process.

Finally, the influence of the length of the domain as well as the changes in the values of the two parameters that result from the assumption of very large (horizontally extended) domains, in the steady state patterns, are also studied theoretical (nondimensionalization) and numerically. For these scenarios, the intrusion (diffusion) and recirculation wedges are decoupled demonstrating the need of a deeper understanding: the two dimensionless groups derived from nondimensionalization does not reproduce the emergent complexity of the concentration and flow steady patterns. Only the introduction of new references for the horizontal coordinate, related to the 'hidden' lengths of the intrusion and recirculation wedges, as well as the simplification of the governing equations to the subdomains defined by these wedges, could lead to new dimensionless groups from which the order of magnitude of these hidden quantities could be obtained. 


\section{INDICE DE FIGURAS}

Figura Il.1 Geometría del dominio y condiciones de contorno

Figura II.2 Geometría del problema

Figura III.1 Modelo físico del problema de Henry y condiciones de contorno

Figura III.2 Patrones estacionarios de solución del problema de Henry obtenidos mediante FATSIM-A. Isolíneas de concentración (arriba) e isolíneas de función de corriente (abajo)

Figura III.3a Patrones estacionarios de isolíneas de concentración para casos 1 a 3 de la Tabla III.2

Figura III.3b. Patrones estacionarios de isolíneas de concentración para los casos 4 a 6 de la Tabla III.2

Figura III.4a Patrones estacionarios de isolíneas de flujo para los casos 1, 3 y 5 de la Tabla III.2

Figura III.4b Patrones estacionarios de isolíneas de flujo para los casos 2, 4 y 6 de la Tabla III.2

Figura III.5 Isolíneas típicas de concentración (arriba) y función de corriente (abajo) para los seis casos de la Tabla III.2, en escala normalizada, $\mathrm{c}^{\prime}=0.2,0.5$ y $0.8 ; \Psi_{\mathrm{H}}=0$

Figura III.6 Isolíneas de concentración para un patrón global óptimo. Casos pg-1 y pg2. $\left(\pi_{\mathrm{d}, 1}=\pi_{\mathrm{d}, 2}=\pi_{\mathrm{d}, 3}=1\right)$, Tabla 5

Figure III.7 Isolíneas de función de corriente para un patrón global óptimo. Casos pg-1 y pg-2. $\left(\pi_{\mathrm{d}, 1}=\pi_{\mathrm{d}, 2}=\pi_{\mathrm{d}, 3}=1\right)$, Tabla 5

Figura III.8 Patrones globales óptimos de isoconcentración y función de corriente del caso isótropo (problema original de Henry, $\pi_{\mathrm{d}, \text { iso }, 1}=\pi_{\mathrm{d}, \text { iso }, 2}=\pi_{\mathrm{d}, \text { iso }, 3}=1$ ). Tabla III.6

Figura IV.1a Patrones de concentración para los casos 1 a 5 de difusividad despreciable (Casos 1 a 3, Tabla IV.1)

Figura IV.1b Patrones de concentración para los casos 1 a 5 de difusividad despreciable (Casos 4 y 5, Tabla IV.1)

Figura IV. 2a Patrones de función de corriente para los casos 1 a 5 de difusividad despreciable (Casos 1 a 3, Tabla IV.1)

Figura IV. 2b Patrones de función de corriente para los casos 1 a 5 de difusividad despreciable (Casos 4 y 5, Tabla IV.1)

Figura IV.3 Patrones de concentración y función de corriente para el caso 6 de difusividad despreciable (Tabla IV.1)

Figura IV. 4a Patrones de concentración para los casos de difusividad vertical despreciable (Casos 1. 2 y 3, Tabla IV.2)

Figura IV. 4b Patrones de concentración para los casos de difusividad vertical despreciable (Casos 4, 5 y 6, Tabla IV.2) 
Figura IV. 5a Patrones de función de corriente para los casos de difusividad vertical despreciable (Casos 1, 2 y 3, Tabla IV.2)

Figura IV. 5b Patrones de función de corriente para los casos de difusividad vertical despreciable (Casos 4, 5 y 6, Tabla IV.2)

Figura IV.6 Patrones de concentración para los casos de difusividad isótropa (Casos 2 a 5, Tabla IV.3)

Figura IV.7 Patrones de función de corriente para los casos de difusividad isótropa (Casos 2 a 5, Tabla IV.3)

Figura IV.8. Patrones de concentración para los casos de $v_{a m b}$ despreciable, $\operatorname{Lv}_{\mathrm{amb}}<<\mathrm{D}_{\mathrm{x}}$ (Tabla IV.4)

Figura IV.9 Patrones de función de corriente para los casos de vamb despreciable, $\mathrm{LV}_{\mathrm{amb}}<<\mathrm{D}_{\mathrm{x}}$ (Tabla IV.4)

Figura IV.10 Patrones de concentración para los casos de $\Delta \rho$ despreciable (Tabla IV.5)

Figura IV.11 Patrones de función de corriente para los casos de $\Delta \rho$ despreciable (Tabla IV.5)

Figura IV.12 Patrones de concentración y función de corriente para la hipótesis L >>H (dominios isótropos) (Caso 1, Tabla IV.6)

Figura IV.13 Patrones de concentración y función de corriente para la hipótesis L >>H (dominios isótropos) (Caso 2, Tabla IV.6)

Figura IV.14 Patrones de concentración y función de corriente para la hipótesis L >>H (dominios isótropos) (Caso 3, Tabla IV.6)

Figura IV.15 Patrones de concentración y función de corriente para la hipótesis L >>H (dominios anisótropos) (Caso 4, Tabla IV.6)

Figura IV.16 Patrones de concentración y función de corriente para la hipótesis L >>H (dominios anisótropos) (Caso 5, Tabla IV.6)

Figura IV.17 Patrones de concentración y función de corriente para la hipótesis L >>H (dominios anisótropos) (Caso 6, Tabla IV.6)

Figura IV.18 Patrones de concentración y función de corriente para la hipótesis L >>H (dominios anisótropos) (Caso 7, Tabla IV.6)

Figura IV.19 Patrones de concentración y función de corriente para la hipótesis L >>H (dominios anisótropos) (Caso 8, Tabla IV.6)

Figura IV.20 Patrones de concentración y función de corriente (difusividad despreciable)

Figura IV.21 Patrones de concentración y función de corriente (difusividad vertical despreciable)

Figura IV.22 Patrones de concentración y función de corriente (difusividad isótropa)

Figura IV.23 Patrones de concentración y función de corriente (difusividad y conductividad hidráulica isótropas) 
Figura IV.24 Patrones de concentración y función de corriente (velocidad regional despreciable, $\mathrm{Lv}_{\mathrm{amb}}<<\mathrm{D}_{\mathrm{x}}$ )

Figura IV.25 Patrones de concentración y función de corriente (velocidad regional despreciable, $\mathrm{Lv}_{\mathrm{amb}}<<\mathrm{D}_{\mathrm{x}}, \mathrm{k}_{\mathrm{x}}=\mathrm{k}_{\mathrm{y}}$ y $\mathrm{D}_{\mathrm{x}}=\mathrm{D}_{\mathrm{y}}$ )

Figura IV.26 Patrones de concentración y función de corriente (diferencia de densidad despreciable, $\pi_{\mathrm{d}, 2}=\pi_{\mathrm{d}, 3}=\pi_{\mathrm{d}, 4}=1$ )

Figura IV.27 Patrones de concentración y función de corriente (dominios extensos, $\mathrm{L}>>\mathrm{H}$, y medios isótropos, $\pi_{\mathrm{d}, 2}=\pi_{\mathrm{d}, 3}=\pi_{\mathrm{d}, 4}=1$ )

Figura V.1a Patrones de concentración. $\pi_{\mathrm{d}, 1}: 1$, 3, 5.5, 6.5, 7.58, 8.5, 11 y 14 (de arriba abajo), $\pi_{\mathrm{d}, 3}=0.05, \pi_{\mathrm{d}, 2}=\pi_{\mathrm{d}, 4}=0.25$

Figura V.1b Patrones de concentración. $\pi_{\mathrm{d}, 1}:$ 1, 3, 5.5, 6.5, 7.58, 8.5, 11 y 14 (de arriba abajo), $\pi_{\mathrm{d}, 3}=0.05, \pi_{\mathrm{d}, 2}=\pi_{\mathrm{d}, 4}=0.25$

Figura V.2a Patrones de flujo. $\pi_{\mathrm{d}, 1}: \pi_{\mathrm{d}, 1}: 1,3,5.5,6.5,7.58,8.5,11$ y 14 (de arriba abajo), $\pi_{\mathrm{d}, 3}=0.05, \pi_{\mathrm{d}, 2}=\pi_{\mathrm{d}, 4}=0.25$

Figura V.2b Patrones de flujo. $\pi_{\mathrm{d}, 1}: \pi_{\mathrm{d}, 1}: 1,3,5.5,6.5,7.58,8.5,11$ y 14 (de arriba abajo), $\pi_{\mathrm{d}, 3}=0.05, \pi_{\mathrm{d}, 2}=\pi_{\mathrm{d}, 4}=0.25$

Figura V.3a Patrones de concentración. $\pi_{\mathrm{d}, 3}=0.02$, 0.05, 0.1, 0.2, 0.3, 0.5, 1, y 2 (de arriba abajo), $\pi_{\mathrm{d}, 1}=7.58, \pi_{\mathrm{d}, 2}=\pi_{\mathrm{d}, 4}=0.25$

Figura V.3b Patrones de concentración. $\pi_{\mathrm{d}, 3}=0.02,0.05,0.1,0.2,0.3,0.5,1, \mathrm{y} 2$ (de arriba abajo), $\pi_{\mathrm{d}, 1}=7.58, \pi_{\mathrm{d}, 2}=\pi_{\mathrm{d}, 4}=0.25$

Figura V.4a Patrones de flujo. $\pi_{\mathrm{d}, 3}=0.02,0.05,0.1,0.2,0.3,0.5,1$, y 2 (de arriba abajo), $\pi_{\mathrm{d}, 1}=7.58, \pi_{\mathrm{d}, 2}=\pi_{\mathrm{d}, 4}=0.25$

Figura V.4b Patrones de flujo. $\pi_{\mathrm{d}, 3}=0.02,0.05,0.1,0.2,0.3,0.5,1, \mathrm{y} 2$ (de arriba abajo), $\pi_{\mathrm{d}, 1}=7.58, \pi_{\mathrm{d}, 2}=\pi_{\mathrm{d}, 4}=0.25$

Figura V.5a Patrones de concentración. $\pi_{\mathrm{d}, 2}=0.01,0.15,0.2,0.25,0.35,0.5,0.8$, y 1 (de arriba abajo), $\pi_{\mathrm{d}, 1}=7.58, \pi_{\mathrm{d}, 3}=0.05 \mathrm{y} \pi_{\mathrm{d}, 4}=0.25$

Figura V.5b Patrones de concentración. $\pi_{\mathrm{d}, 2}=0.01,0.15,0.2,0.25,0.35,0.5,0.8$, y 1 (de arriba abajo), $\pi_{\mathrm{d}, 1}=7.58, \pi_{\mathrm{d}, 3}=0.05 \mathrm{y} \pi_{\mathrm{d}, 4}=0.25$

Figura V.6a Patrones de flujo. $\pi_{\mathrm{d}, 2}=0.01,0.15,0.2,0.25,0.35,0.5,0.8$, y 1 (de arriba abajo), $\pi_{\mathrm{d}, 1}=7.58, \pi_{\mathrm{d}, 3}=0.05 \mathrm{y} \pi_{\mathrm{d}, 4}=0.25$

Figura V.6b Patrones de flujo. $\pi_{\mathrm{d}, 2}=0.01,0.15,0.2,0.25,0.35,0.5,0.8$, y 1 (de arriba abajo), $\pi_{\mathrm{d}, 1}=7.58, \pi_{\mathrm{d}, 3}=0.05 \mathrm{y} \pi_{\mathrm{d}, 4}=0.25$

Figura V.7a Patrones de concentración. $\pi_{\mathrm{d}, 4}=0.01,0.15,0.2,0.25,0.3,0.5,0.8$ y 2 (de arriba abajo), $\pi_{\mathrm{d}, 1}=7.58, \pi_{\mathrm{d}, 3}=0.05 \mathrm{y} \pi_{\mathrm{d}, 2}=0.25$

Figura V.7b Patrones de concentración. $\pi_{\mathrm{d}, 4}=0.01,0.15,0.2,0.25,0.3,0.5,0.8$ y 2 (de arriba abajo), $\pi_{\mathrm{d}, 1}=7.58, \pi_{\mathrm{d}, 3}=0.05 \mathrm{y} \pi_{\mathrm{d}, 2}=0.25$ 
Figura V.8a Patrones de flujo. $\pi_{\mathrm{d}, 4}=0.01,0.15,0.2,0.25,0.3,0.5,0.8$ y 2 (de arriba abajo), $\pi_{\mathrm{d}, 1}=7.58, \pi_{\mathrm{d}, 3}=0.05 \mathrm{y} \pi_{\mathrm{d}, 2}=0.25$

Figura V.8b Patrones de flujo. $\pi_{\mathrm{d}, 4}=0.01,0.15,0.2,0.25,0.3,0.5,0.8$ y 2 (de arriba abajo), $\pi_{\mathrm{d}, 1}=7.58, \pi_{\mathrm{d}, 3}=0.05$ y $\pi_{\mathrm{d}, 2}=0.25$

Figura V.9a Patrones de concentración. $\pi_{\mathrm{d}, 2}=\pi_{\mathrm{d}, 4}=0.3,0.4,0.5,0.8,1$ y 2 (de arriba abajo), $\pi_{\mathrm{d}, 1}=7.58, \pi_{\mathrm{d}, 3}=0.05$

Figura V.9b Patrones de concentración. $\pi_{\mathrm{d}, 2}=\pi_{\mathrm{d}, 4}=0.3,0.4,0.5,0.8,1$ y 2 (de arriba abajo), $\pi_{\mathrm{d}, 1}=7.58, \pi_{\mathrm{d}, 3}=0.05$

Figura V.10a Patrones de flujo. $\pi_{\mathrm{d}, 2}=\pi_{\mathrm{d}, 4}=0.3,0.4,0.5,0.8,1$ y 2 (de arriba abajo), $\pi_{\mathrm{d}, 1}=7.58, \pi_{\mathrm{d}, 3}=0.05$

Figura V.10b Patrones de flujo. $\pi_{\mathrm{d}, 2}=\pi_{\mathrm{d}, 4}=0.3,0.4,0.5,0.8,1$ y 2 (de arriba abajo), $\pi_{\mathrm{d}, 1}=7.58, \pi_{\mathrm{d}, 3}=0.05$

Figura V.11a Patrones de concentración. $\pi_{\mathrm{d}, 2}=\pi_{\mathrm{d}, 4}=0.3,0.4,0.5,0.8,1$ y 2 (de arriba abajo), $\pi_{\mathrm{d}, 1}=3.79, \pi_{\mathrm{d}, 3}=0.5$

Figura V.11b Patrones de concentración. $\pi_{\mathrm{d}, 2}=\pi_{\mathrm{d}, 4}=0.3,0.4,0.5,0.8,1$ y 2 (de arriba abajo), $\pi_{\mathrm{d}, 1}=3.79, \pi_{\mathrm{d}, 3}=0.5$

Figura V.12a Patrones de flujo. $\pi_{\mathrm{d}, 2}=\pi_{\mathrm{d}, 4}=0.3,0.4,0.5,0.8,1$ y 2 (de arriba abajo), $\pi_{\mathrm{d}, 1}=3.79, \pi_{\mathrm{d}, 3}=0.5$

Figura V.12b Patrones de flujo. $\pi_{\mathrm{d}, 2}=\pi_{\mathrm{d}, 4}=0.3,0.4,0.5,0.8,1$ y 2 (de arriba abajo), $\pi_{\mathrm{d}, 1}=3.79, \pi_{\mathrm{d}, 3}=0.5$

Figura V.13a Patrones de concentración. $\pi_{\mathrm{d}, 1}:$ 0.05, 0,5, 0.8, 1, 3, 5, 10 y 20, $\pi_{\mathrm{d}, 2}=\pi_{\mathrm{d}, 3}$ $=\pi_{\mathrm{d}, 4}=1$

Figura V.13b Patrones de concentración. $\pi_{\mathrm{d}, 1}: 0.05,0,5,0.8,1,3,5,10$ y 20, $\pi_{\mathrm{d}, 2}=\pi_{\mathrm{d}, 3}$ $=\pi_{\mathrm{d}, 4}=1$

Figura V.14a Patrones de flujo. $\pi_{\mathrm{d}, 1}: 0.05,0,5,0.8,1,3,5,10$ y $20, \pi_{\mathrm{d}, 2}=\pi_{\mathrm{d}, 3}=\pi_{\mathrm{d}, 4}=1$

Figura V.14b Patrones de flujo. $\pi_{\mathrm{d}, 1}: 0.05,0,5,0.8,1,3,5,10$ y $20, \pi_{\mathrm{d}, 2}=\pi_{\mathrm{d}, 3}=\pi_{\mathrm{d}, 4}=1$

Figura V.15a Patrones de concentración. $\pi_{\mathrm{d}, 3}=.05,0.1,0,2,0,5,1,3,5$ y 20,

$\pi_{\mathrm{d}, 1}=\pi_{\mathrm{d}, 2}=\pi_{\mathrm{d}, 4}=1$

Figura V.15b Patrones de concentración. $\pi_{\mathrm{d}, 3}: 0.05,0.1,0,2,0,5,1,3,5$ y $20, \pi_{\mathrm{d}, 1}=\pi_{\mathrm{d}, 2}$ $=\pi_{\mathrm{d}, 4}=1$

Figura V.16a Patrones de flujo. $\pi_{\mathrm{d}, 3}: 0.05,0.1,0,2,0,5,1,3,5$ y 20, $\pi_{\mathrm{d}, 1}=\pi_{\mathrm{d}, 2}=\pi_{\mathrm{d}, 4}=1$ 166 Figura V.16b Patrones de flujo. $\pi_{\mathrm{d}, 3}: 0.05,0.1,0,2,0,5,1,3,5$ y 20, $\pi_{\mathrm{d}, 1}=\pi_{\mathrm{d}, 2}=\pi_{\mathrm{d}, 4}=1$ igura V.17a Patrones de concentración. $\pi_{\mathrm{d}, 2}: 0.05,0,5,1,2,5,8,10$ y 20, $\pi_{\mathrm{d}, 1}=\pi_{\mathrm{d}, 3}=$ $\pi_{\mathrm{d}, 4}=1$ 
Figura V.17b Patrones de concentración. $\pi_{\mathrm{d}, 2}: 0.05,0,5,1,2,5,8,10$ y $20, \pi_{\mathrm{d}, 1}=\pi_{\mathrm{d}, 3}=$ $\pi_{\mathrm{d}, 4}=1$

Figura V.18a Patrones de flujo. $\pi_{\mathrm{d}, 2}: 0.05,0,5,1,2,5,8,10$ y 20, $\pi_{\mathrm{d}, 1}=\pi_{\mathrm{d}, 3}=\pi_{\mathrm{d}, 4}=1$

Figura V.18b Patrones de flujo. $\pi_{\mathrm{d}, 2}: 0.05,0,5,1,2,5,8,10$ y 20, $\pi_{\mathrm{d}, 1}=\pi_{\mathrm{d}, 3}=\pi_{\mathrm{d}, 4}=1$

Figura V.19a Patrones de concentración. $\pi_{\mathrm{d}, 4}: 0.05,0,5,1,2,5,8,10$ y 20, $\pi_{\mathrm{d}, 1}=\pi_{\mathrm{d}, 2}=$ $\pi_{\mathrm{d}, 3}=1$

Figura V.19b Patrones de concentración. $\pi_{\mathrm{d}, 4}: 0.05,0,5,1,2,5,8,10$ y 20, $\pi_{\mathrm{d}, 1}=\pi_{\mathrm{d}, 2}=$ $\pi_{\mathrm{d}, 3}=1$

Figura V.20a Patrones de flujo. $\pi_{\mathrm{d}, 4}: 0.05,0,5,1,2,5,8,10$ y 20, $\pi_{\mathrm{d}, 1}=\pi_{\mathrm{d}, 2}=\pi_{\mathrm{d}, 3}=1$

Figura V.20a Patrones de flujo. $\pi_{\mathrm{d}, 4}: 0.05,0,5,1,2,5,8,10$ y 20, $\pi_{\mathrm{d}, 1}=\pi_{\mathrm{d}, 2}=\pi_{\mathrm{d}, 3}=1$

Figura V.21a Patrones de concentración del problema de Henry para L=0.8, 1 y 1.5

Figura V.21b Patrones de concentración del problema de Henry para L= 2, 3 y 4

Figura V.22a Patrones de flujo del problema de Henry para L= 0.8, 1 y 1.5

Figura V.22b Patrones de flujo del problema de Henry para L= 2, 3 y 4

Figura V.23 l(c=0.1), eje vertical, frente a $\pi_{2}$, eje horizontal. El parámetro de las curvas es $\pi_{1}=0.1$ (curva inferior), 0.2, 0.5, 0.6, 0.7, 0.8, 0.9, 1, 2, 3, 4, 5, 6, 8, 10 (curva superior)

Figura V.24 l(c=0.5), eje vertical, frente a $\pi_{2}$, eje horizontal. El parámetro de las curvas es $\pi_{1}=0.1$ (curva inferior), 0.2, 0.5, 0.6, 0.7, 0.8, 0.9, 1, 2, 3, 4, 5, 6, 8, 10 (curva superior)

Figura V.25 l(c=0.1) - l(c=0.5), eje vertical, frente a $\pi_{2}$, eje horizontal. $\pi_{1}$ (parámetro) $=0.1$ (curva inferior), 0.2, 0.5, 0.6, 0.7, 0.8, 0.9, 1, 2, 3, 4, 5, 6, 8, 10 (curva superior)

Figura V.26 Pie de la línea divisoria de flujo, eje vertical, frente a $\pi_{1}$, eje horizontal. $\pi_{2}$ (parámetro) $=0.05$ (curva superior), 0.1, 0.2, 0.3, 0.4, 0.5, 0.6, 0.7, 0.8, 0.9 y 1 (curva inferior)

Figura V.27 Pie de la línea divisoria de flujo, eje vertical, frente a $\pi_{2}$, eje horizontal. $\pi_{1}$ (parámetro) $=0.8$ (curva inferior), 0.9, 1, 2, 3, 4, 5, 6, 8, 10 (curva superior) 
Índice de figuras 


\section{INDICE DE TABLAS}

Tabla III.1 Posibles significados de los grupos discriminados

Tabla III.2 Valores de los parámetros para distintos escenarios

Tabla III.3 Posición relativa horizontal $\left(\mathrm{x}^{\prime}=\mathrm{x} / \mathrm{L}\right)$ de las isolíneas $\mathrm{c}^{\prime}=0.2,0.5$ y 0.8, para $\mathrm{y}^{\prime}=0.5$

Tabla III.4 Parámetros para un patrón global óptimo

Tabla III.5 Valores de los grupos adimensionales clásicos y discriminados para un patrón global óptimo

Tabla III.6 Parámetros para un patrón global óptimo del caso isótropo (Henry original)

Tabla IV.1 Valores de los parámetros para los casos de difusividad despreciable

Tabla IV.2 Valores de los parámetros para los casos de difusividad vertical despreciable

Tabla IV.3 Valores de los parámetros para los casos de difusividad isotrópica

Tabla IV.4 Valores de los parámetros para los casos de velocidad regional despreciable

Tabla IV.5 Valores de los parámetros para casos de diferencia de densidad despreciable

Tabla IV.6 Valores de los parámetros para la simplificación L >>H

Tabla IV.7 Valores de los parámetros para los casos de difusividad despreciable, $\pi_{\mathrm{d}, 2}=$ $\pi_{\mathrm{d}, 2}=1$

Tabla IV.8 Valores de los parámetros para los casos de difusividad vertical despreciable, $\pi_{\mathrm{d}, 2}=\pi_{\mathrm{d}, 2}=\pi_{\mathrm{d}, 3}=1$

Tabla IV.9 Valores de los parámetros para los casos de difusividad isótropa, $\pi_{\mathrm{d}, 2}=\pi_{\mathrm{d}, 2}=\pi_{\mathrm{d}, 3}=\pi_{\mathrm{d}, 4}=1$

Tabla IV.10 Valores de los parámetros para los casos de difusividad y conductividad hidráulica isótropas, $\pi_{\mathrm{d}, 2}=\pi_{\mathrm{d}, 2}=\pi_{\mathrm{d}, 3}=1$

Tabla IV.11 Valores de los parámetros para el caso de velocidad regional despreciable $\left(\mathrm{Lv}_{\mathrm{amb}}<<\mathrm{D}_{\mathrm{x}}\right), \pi_{\mathrm{d}, 2}=\pi_{\mathrm{d}, 2}=\pi_{\mathrm{d}, 3}=1$

Tabla IV.12 Valores de los parámetros para el caso de velocidad regional despreciable $\left(\mathrm{Lv}_{\mathrm{amb}}<<\mathrm{D}_{\mathrm{x}}\right), \mathrm{k}_{\mathrm{x}}=\mathrm{k}_{\mathrm{y}} \mathrm{y} \mathrm{D}_{\mathrm{x}}=\mathrm{D}_{\mathrm{y}} \cdot \pi_{\mathrm{d}, 1}=\pi_{\mathrm{d}, 2}=1$

Tabla IV.13 Valores de los parámetros para el caso de diferencia de densidad despreciable, $\pi_{\mathrm{d}, 2}=\pi_{\mathrm{d}, 3}=\pi_{\mathrm{d}, 4}=1$

Tabla IV.14 Valores de los parámetros para el caso de dominios extensos ( $\mathrm{L}>\mathrm{H})$ y medios isótropos, $\pi_{\mathrm{d}, 1}=\pi_{\mathrm{d}, 3}=1$

Tabla V.0 Grupos discriminados y sus valores para el problema original de Henry

Tabla V.1 Casos para la influencia de un cambio de valor de $\pi_{\mathrm{d}, 1}$ 
Tabla V.2 Casos para la influencia de un cambio de valor de $\pi_{\mathrm{d}, 3}$

Tabla V.3 Casos para la influencia de un cambio de valor de $\pi_{\mathrm{d}, 2}$

Tabla V.4 Casos para la influencia de un cambio de valor de $\pi_{\mathrm{d}, 4}$

Tabla V.5 Casos para la influencia simultánea de os monomios $\pi_{\mathrm{d}, 2}$ y $\pi_{\mathrm{d}, 4}$

Tabla V.6 Casos para la influencia simultánea de los monomios $\pi_{\mathrm{d}, 2} \mathrm{y} \pi_{\mathrm{d}, 4}$ con aumento de la difusión y disminución delas fuerzas de flotación

Tabla V.7 Casos para el estudio de la influencia de $\pi \mathrm{d}, 1$

Tabla V.8 Casos para el estudio de la influencia de $\pi \mathrm{d}, 3$

Tabla V.9 Casos para el estudio de la influencia de $\pi \mathrm{d}, 3$

Tabla V.10 Casos para el estudio de la influencia de $\pi \mathrm{d}, 4$

Tabla V.11 Pies de las isolíneas de concentración c = 0.1 y 0.9 y pie de la línea divisoria para diferentes longitudes del acuífero en el problema de Henry

Tabla V.12 Pie de la isolínea c=0.1 en función de $\pi_{1}$ y $\pi_{2}$

Tabla V.13 Pie de la isolínea c=0.5 en función de $\pi_{1}$ y $\pi_{2}$

Tabla V.13 Pie de la isolínea c=0.5 en función de $\pi_{1}$ y $\pi_{2}$ (continuación)

Tabla V.14 Diferencia de los pies de las isolíneas c=0.1 y 0.5 en función de $\pi_{1}$ y $\pi_{2}$

Tabla V.14 Diferencia de los pies de las isolíneas c=0.1 y 0.5 en función de $\pi_{1}$ y $\pi_{2}$ (continuación) 


\section{INDICE}

CAPÍTULO I. Introducción, objetivos y perspectivas 1

I.1 Introducción y antecedentes 1

I.2 La adimensionalización discriminada. Un salto cualitativo en su aplicación 3

I.3 Objetivos 8

$\begin{array}{ll}\text { I.4 Perspectivas } & 10\end{array}$

$\begin{array}{ll}\text { I.5 Organización de la presente memoria } & 11\end{array}$

\section{CAPITULO II. La adimensionalización discriminada}

II.1 La caracterización de un problema mediante grupos adimensionales 13

II.2 Técnicas para la obtención de grupos adimensionales 17

$\begin{array}{ll}\text { II.2.1 El análisis dimensional } & 17\end{array}$

II.2.1.1 Concepción clásica del análisis dimensional 17

II.2.1.2 El análisis dimensional discriminado 20

II.2.2 La adimensionalización de ecuaciones $\quad 22$

II.2.2.1 El proceso de adimensionalización clásico 22

II.2.2.2 El proceso de adimensionalización discriminada 23

II.2.3 Protocolo de aplicación de la adimensionalización discriminada 31

II.2.4 La definición de problemas patrón a través de los grupos

$\begin{array}{ll}\text { adimensionales discriminados } & 37\end{array}$

\section{CAPÍTULO III. Aplicación de la adimensionalización discriminada al problema de Henry}

III.1 El problema de Henry 39

III.1.1 Antecedentes y discriminación 39

$\begin{array}{ll}\text { III.1.2 Nomenclatura } & 44\end{array}$ 
III.1.3 El modelo físico y matemático del problema de Henry 45

III.1.4 Deducción de los grupos adimensionales discriminados 46

III.1.5 Justificación de los números adimensionales deducidos mediante la discriminación

III.1.6 Uso de los grupos discriminados para una elección óptima del problema patrón

III.2 Conclusiones

\section{CAPÍTULO IV. Simplificación de escenarios}

IV.1 Escenarios simplificados en torno al problema original de Henry

IV.1.1 Primera simplificación. Difusividad despreciable

IV.1.2 Segunda simplificación. Difusividad vertical $\left(\mathrm{D}_{Y}\right)$ despreciable, $\mathrm{D}_{\mathrm{Y}}<<\mathrm{D}_{\mathrm{X}}$

IV.1.3 Tercera simplificación. Difusividad isotrópica, $\mathrm{D}_{\mathrm{Y}}=\mathrm{D}_{\mathrm{X}}$

IV.1.4 Cuarta simplificación. Velocidad Regional despreciable frente a la difusión, $\left(\mathrm{LV}_{\mathrm{amb}}<<\mathrm{D}_{\mathrm{X}}\right)$

IV.1.5 Quinta simplificación. Diferencia de densidades $(\Delta \rho)$ despreciable

IV.1.6 Sexta simplificación. Dominios extensos $(\mathrm{L}>>\mathrm{H})$

IV.2 Escenarios simplificados derivados de problemas con grupos discriminados de orden de magnitud unidad

IV.2.1 Primera simplificación. Difusividad despreciable

IV.2.2 Segunda simplificación. Difusividad vertical ( $\left.D_{Y}\right)$ despreciable,

$$
\mathrm{D}_{\mathrm{Y}}<<\mathrm{D}_{\mathrm{X}}
$$

IV.2.3 Tercera simplificación. Difusividad isotrópica, $\mathrm{D}_{\mathrm{X}}=\mathrm{D}_{\mathrm{Y}}$ 106

IV.2.4 Cuarta simplificación. Difusividad y conductividad hidráulica isotrópicas, $\mathrm{D}_{\mathrm{X}}=\mathrm{D}_{\mathrm{Y}} \mathrm{y} \mathrm{k}_{\mathrm{x}}=\mathrm{k}_{\mathrm{y}}$

IV.2.5 Quinta simplificación. Velocidad Regional despreciable frente a la difusión, ( $\left.\mathrm{LV}_{\mathrm{amb}}<<\mathrm{D}_{\mathrm{X}}\right)$

IV.2.6 Sexta simplificación. Diferencia de densidades $(\Delta \rho)$ despreciable

IV.2.7 Séptima simplificación. Dominios extensos, L >> H y medios Isótropos 


\section{CAPÍTULO V. Influencia de los grupos discriminados en los patrones del problema de Henry.}

V.1 Influencia del valor de los grupos discriminados en los patrones de solución solución del problema original de Henry

V.1.1 Influencia del monomio $\left.\pi_{\mathrm{d}, 1}=\left[(\Delta \rho) \mathrm{gk}_{\mathrm{y}} / \varepsilon \mu \mathrm{v}_{\mathrm{amb}}{ }^{*}\right)\right](\mathrm{L} / \mathrm{H})$

V.1.2 Influencia del monomio $\pi_{\mathrm{d}, 3}=\mathrm{D}_{\mathrm{x}} /\left(\mathrm{V}_{\mathrm{amb}}{ }^{*} \mathrm{~L}\right)$

V.1.3 Influencia del monomio $\pi_{\mathrm{d}, 2}=\left(\mathrm{k}_{\mathrm{x}} \mathrm{H}^{2}\right) /\left(\mathrm{k}_{\mathrm{y}} \mathrm{L}^{2}\right)$

V.1.4 Influencia del monomio $\pi_{\mathrm{d}, 4}=\left(\mathrm{D}_{\mathrm{x}} \mathrm{H}^{2}\right) /\left(\mathrm{D}_{\mathrm{y}} \mathrm{L}^{2}\right)$

V.1.5 Influencia simultánea de los monomios $\pi_{\mathrm{d}, 2}=\left(\mathrm{k}_{\mathrm{x}} \mathrm{H}^{2}\right) /\left(\mathrm{k}_{\mathrm{y}} \mathrm{L}^{2}\right)$

$$
\mathrm{y} \pi_{\mathrm{d}, 4}=\left(\mathrm{D}_{\mathrm{x}} \mathrm{H}^{2}\right) /\left(\mathrm{D}_{\mathrm{y}} \mathrm{L}^{2}\right)
$$

V.1.6 Influencia simultánea de los monomios $\pi_{\mathrm{d}, 2}$ y $\pi_{\mathrm{d}, 4}$ con aumento del efecto difusivo general y disminución del efecto de flotación

V.2 Influencia del valor de los grupos discriminados en los patrones del problema general de Henry (anisótropo e isótropo). Cuñas de intrusión y de recirculación

V.2.1 Valores de los parámetros para el problema anisótropo

V.2.2 Influencia de los monomios, individualmente, en el problema general anisótropo

V.2.2.1 Influencia del monomio $\left.\pi_{\mathrm{d}, 1}=\left[(\Delta \rho) \mathrm{gk}_{\mathrm{y}} / \varepsilon \mu \mathrm{vamb}_{\mathrm{amb}}{ }^{*}\right)\right](\mathrm{L} / \mathrm{H}) \quad 156$

V.2.2.2 Influencia del monomio $\pi_{\mathrm{d}, 3}=\mathrm{D}_{\mathrm{x}} /\left(\mathrm{V}_{\mathrm{amb}}{ }^{*} \mathrm{~L}\right)$

V.2.2.3 Influencia del monomio $\pi_{\mathrm{d}, 2}=\left(\mathrm{k}_{\mathrm{x}} \mathrm{H}^{2}\right) /\left(\mathrm{k}_{\mathrm{y}} \mathrm{L}^{2}\right) \quad 168$

V.2.2.4 Influencia del monomio $\pi_{\mathrm{d}, 4}=\left(\mathrm{D}_{\mathrm{x}} \mathrm{H}^{2}\right) /\left(\mathrm{D}_{\mathrm{y}} \mathrm{L}^{2}\right)$

V.2.3 Evolución de las cuñas en medios isótropos. Influencia de los $\begin{array}{ll}\text { Monomios y del tamaño del dominio } & 179\end{array}$ 

CAPÍTULO I

\section{Introducción, objetivos y perspectivas}

\section{I.1 INTRODUCCIÓN Y ANTECEDENTES}

En el año 2002 empecé a trabajar en el campo de la Teledetección Espacial y en concreto en el tratamiento de imágenes hyperespectrales, con el catedrático D. Mario Chica Olmo de la Universidad de Granada, con la intención de realizar una tesis doctoral bajo su dirección. La distancia entre la UPCT, universidad donde vengo realizando mi labor docente y otras tareas administrativas desde 1998, y la Universidad de Granada fue el principal obstáculo para desarrollar el trabajo continuo y constante, necesario, para llevar a buen término ese objetivo.

Durante algún tiempo mantuve la ilusión, pero otras circunstancias personales y familiares incidieron para tomar conciencia de que una tesis ‘a distancia' constituía un reto excesivamente grande para mí en esos momentos.

En torno al año 2011 el profesor del Departamento de Física F. Alhama, vinculado a la Escuela Técnica Superior de Ingeniería de Caminos, Canales y Puertos y de Ingeniería 
de Minas, me invitó a colaborar en trabajos de investigación y proyectar la realización de una nueva tesis doctoral. Poco tiempo después me propuso continuar con las investigaciones sobre flujo de aguas subterráneas con transporte de soluto, llevadas a cabo por los doctores Iván Alhama y Antonio Soto para la realización de sus respectivas tesis, profesores que, finalmente, asumieron la responsabilidad de la dirección de la presente tesis doctoral. A. Soto desarrolló un programa numérico para simular este tipo de procesos en problemas patrón como los de Henry y Elder mientras que I. Alhama investigó un escenario complejo de intrusión salina en el litoral alicantino de Agua Amarga, donde había instaladas antiguas industrias salineras, con presencia de extracciones para plantas desalinizadoras cercanas. Se trataba de un cambio de tema que me pareció atractivo y novedoso, y la cercanía física era una garantía de trabajo continuado, por lo que acepté el reto.

Tras una dedicación ciertamente irregular por las tareas de implantación y dirección de la Escuela de Ingeniería de Caminos y Minas, he conseguido mi anhelado objetivo merced a la siempre dispuesta ayuda y ánimo de mis directores y del resto de los compañeros del equipo de dirección. Al final, la satisfacción del esfuerzo realizado compensa con creces las enormes dificultades que han supuesto para mí este objetivo.

El presente trabajo supone un paso más en la línea de caracterización adimensional de procesos físicos y de ingeniería del Grupo de Investigación ‘Simulación por redes’ de la UPCT. En la última década, el grupo combina tareas propias de simulación numérica, que han dado lugar a registros de programas sobre diferentes problemas de interés aplicados en docencia e investigación (PROCCA-09 [2005], FATSIM-A [2010], FAHET [2011], EPSNET_10 [2011], OXIPSIS-12 [2013] y CODENS [2013]), con trabajos de caracterización dimensional de escenarios, en los que han aplicado técnicas propias de adimensionalización discriminada con notable éxito (Alhama y Madrid [2007, 2008], Luna y col. [2010], Alhama, I. y col. [2011], Alhama y Madrid [2012], Cánovas y col. [2014, 2015] y Conesa y col. [2016]).

Puede decirse que la orientación actual del grupo va sustituyendo, paulatinamente, el objetivo de la simulación numérica (diseño de modelos en red y su verificación numérica) por el de la caracterización adimensional (estudio de los grupos adimensionales que gobiernan los procesos físicos), usando la primera técnica para comprobar los resultados derivados de la segunda. 
Sin duda, la competencia de un centro universitario para desarrollar tareas de simulación numérica y elaborar códigos específicos, si bien está al alcance de sus investigadores desde el punto de vista del conocimiento requerido, no lo está con mucho al alcance de sus recursos económicos, ni de sus recursos humanos. Estos códigos son más bien objetivo de empresas especializadas, para las que el potencial papel de la Universidad queda relegado al asesoramiento y colaboración en el campo científico específico.

Por otro lado, la caracterización adimensional sí es una tarea específica del investigador universitario puesto que los recursos para su desarrollo y aplicación son el propio y profundo conocimiento de los fenómenos involucrados en el campo particular de trabajo más un conocimiento bien asentado de la teoría dimensional, disciplina que engloba y aglutina campos como la teoría de modelos, el análisis dimensional, la adimensionalización y normalización de ecuaciones, 'scaling', teoría de las funciones homogéneas y otros.

\section{I.2 LA ADIMENSIONAIZACIÓN DISCRIMINADA. UN SALTO CUALITATIVO EN SU APLICACIÓN}

¿Qué se entiende por caracterización adimensional de un proceso físico? El concepto no es nuevo 'sensu stricto', ya que su objetivo está bien definido desde hace décadas, pero la introducción de un protocolo formal para su aplicación, que incluye un conjunto de pasos ordenados y sujetos a reglas precisas, merece el acuerdo sobre un nombre definitivo y sencillo que aluda a este objetivo (con este término, no obstante, ya se han publicado numerosos trabajos en revistas científicas y congresos internacionales, Cánovas [2014], , Alhama I. y col. [2014], Cánovas y col. [2014, 2015, 2016.] y Conesa y col. [2016]).

Históricamente, los investigadores experimentalistas que trabajan con modelos a escala han buscado reducir los numerosos parámetros tanto geométricos como físicos que intervienen en un problema complejo, y que con frecuencia lo convierten en inmanejable como el estudiado en esta memoria, a unas pocas agrupaciones de estos parámetros llamadas grupos o números adimensionales que se comporten como los verdaderos 'parámetros' o monomios que, de forma independiente, rigen o determinan la solución o patrones de solución del problema. 
Unas veces, han sido los propios experimentalistas los que han encontrado estos grupos, a base de un trabajo inagotable de laboratorio y una perspicaz intuición física. Otras, su deducción ha sido fruto de razonamientos teóricos derivados de la aplicación del análisis dimensional, buscando directamente agrupaciones no dimensionales de los parámetros que definen el problema, o la adimensionalización clásica de las ecuaciones de gobierno.

Lo cierto es que el nacimiento de un nuevo grupo o número adimensional dentro de un campo de la ciencia o de la ingeniería ha sido acogido con gozo por la comunidad científica, sin reparar en un detallado estudio de su valor (a veces de orden de magnitud extrañamente superior o inferior a la unidad) y/o de su significado físico (pensamos que un grupo de esta categoría e influencia debería ir invariablemente asociado a un significado físico concreto), hasta el punto de bautizar el grupo con el nombre del investigador. Los grupos o números de Reynolds, Rayleigh, Nusselt, Courant, Boussinesq, Mach, Peclet, Prandtl y otros muchos son un ejemplo de ello.

Un conjunto de grupos adimensionales deducidos con precisión de unas ecuaciones de gobierno homogéneas en términos de las dimensiones de sus términos (sumandos) merced a la invarianza de los mismos frente a la adopción de diferentes sistemas de medida (teoría de las funciones homogéneas, San Juan [1947]), permite representar la solución de un problema expresando la variable incógnita en forma adimensional como una función indeterminada cuyos argumentos son dichos grupos, es el conocido teorema de Pi. Esta es la base de la teoría de modelos, tan fundamental para el ingeniero de cualquier especialidad.

Podemos decir, en suma, que en la búsqueda de estos grupos se han seguido, aparte de la línea estrictamente experimental, dos caminos aparentemente bien diferenciados: i) el análisis dimensional, y ii) la adimensionalización de ecuaciones; Alhama y Madrid [2012] afirman que las bases sobre las que se asienta la teoría que siguen ambos procedimientos es la misma.

Dejamos para el Capítulo II una discusión sobre las analogías y diferencias de estos procedimientos y sobre cómo se han producido los avances particulares de estas técnicas en las últimas décadas, una incursión interesante y (a nuestro modo de ver) necesaria para converger hacia la caracterización adimensional que aglutina los principios 
de ambas y establece un protocolo inequívoco de aplicación en este campo de investigación básica.

En rigor, la caracterización adimensional que aquí aplicamos debería contener el vocablo 'discriminada' porque es una diferencia esencial que, en contraposición con la adimensionalización digamos clásica, proporciona en general (máxime en problemas complejos) el menor número de grupos independientes que rigen o determinan la solución del problema. Finalmente, hemos incluido el término discriminada por dos motivos; en primer lugar, para expresar el concepto (caracterización dimensional) de la forma más concreta y, en segundo lugar, porque la discriminación es necesaria siempre en problemas de cierta complejidad; en éstos, la discriminación conduce a la caracterización más precisa.

Recientemente Kalakan, [2014], mediante técnicas no discriminadas de análisis dimensional que conducen a los mismos grupos adimensionales que la adimensionalización clásica, grupos derivados por Abarca y col. [2007] mediante esta última técnica, ha abordado el problema de Henry objeto de estudio en esta memoria. Estos autores, en ausencia de fenómenos dispersivos, y con permeabilidad anisótropa, obtienen los mismos grupos adimensionales que los derivados de la adimensionalización discriminada merced a la isotropía de la difusividad molecular, cuyo efecto es separar la relación de aspecto (largo/ancho del dominio) como un grupo independiente.

La consideración de una difusividad anisótropa por efectos de la tortuosidad anisótropa de los canales de flujo, no abordada en la literatura sino a través del efecto de dispersión, excepto por Kakinuma y col. [1988], no hubiera permitido expulsar la relación de aspecto como grupo adimensional independiente, sino que éste hubiera quedado vinculado, como veremos, a los cocientes entre los valores de permeabilidades vertical y horizontal, y difusividades vertical y horizontal.

Así, de considerar una difusividad anisótropa, los autores anteriores hubieran derivado un grupo más que con el uso de la adimensionalización discriminada, pues esta última hubiera expulsado la relación de aspecto, integrándola como un factor de corrección de los cocientes mencionados anteriormente.

Por otro lado, para el caso dispersivo, la adimensionalización discriminada tampoco hubiera permitido deducir grupos como el clásico cociente de dispersividades 
(longitudinal/transversal) sino corrigiéndolo de nuevo con la relación de aspecto. Es una comprobación que queda pendiente para trabajos posteriores.

La discriminación impone que las ecuaciones de dimensión de ciertas magnitudes de carácter vectorial como longitudes y velocidades tengan una ecuación dimensional diferente según sea la dirección espacial (es como distinguir entre velocidad en la dirección $\mathrm{x}$, de ecuación dimensional $\mathrm{L}_{\mathrm{x}} \mathrm{T}^{-1}$, y velocidad en la dirección $\mathrm{y}$, de ecuación dimensional $\mathrm{Ly}_{\mathrm{y}} \mathrm{T}^{-1}$ ).

Esta distinción puede extenderse también a magnitudes escalares; por ejemplo, asignando dimensiones temporales diferentes a tiempos asociados a una u otra frecuencia en un problema con dos modos de vibración acoplados, o atribuyendo diferente ecuación dimensional a la energía térmica y a la energía mecánica en problemas termodinámicos en los que no existe transferencia de energía mecánica (viscosa) a térmica. Incluso en un fenómeno 1-D, podrían existir dos longitudes en la misma dirección con diferente ecuación dimensional si están asociados a dos fenómenos físicos diferentes, involucrados en el problema, que discurren con diferente velocidad espacial.

Con todo, queremos decir que la discriminación es un avance más cualitativo que cuantitativo en el proceso de adimensionalización y que sólo su consideración conduce a resultados inequívocamente precisos y fiables.

El profesor F. Alhama, que ha orientado sobre muchos de los controvertidos aspectos y encrucijadas que han surgido a lo largo del trabajo de esta tesis, junto con el profesor Madrid (Alhama y Madrid, [2012]) han dedicado un gran esfuerzo en las dos últimas décadas a los siguientes temas (Madrid y Alhama [2005a, 2005b, 2006, 2008], Luna y col [2010], Alhama I. y col. [2011, 2014, 2015]) y Cánovas y col. [2014, 2015, 2016]:

i. Adimensionalización discriminada.

ii. Significado y orden de magnitud de los grupos adimensionales obtenidos mediante la discriminación.

iii. Definición de subdominios dentro de un problema, asociados con ciertas simplificaciones de las ecuaciones de gobierno de esos dominios.

iv. Deducción del orden de magnitud de ciertas incógnitas llamadas magnitudes ocultas, etc. 
La tesis doctoral de M. Cánovas [2014], que versa sobre la caracterización de escenarios geotérmicos de flujo de fluidos con transporte de calor, puede considerarse un antecedente de la actual en tanto que sentó alguno de los criterios que aquí se han seguido. Cabe mencionar, por otro lado, que el esfuerzo de los profesores F. Alhama y N. Madrid fue continuación de los trabajos del grupo de investigación de ‘Análisis Dimensional’ del Departamento de física Aplicada de la UPCT dirigido por el profesor A. Herranz ya jubilado, a la sazón alumno de Julio Palacios.

El guion de la actual tesis tuvo que ser modificado a lo largo de su realización por la necesidad de una investigación más profunda sobre los fenómenos emergentes, a lo largo del propio desarrollo de la tesis, involucrados en el problema de Henry. En comparación con otros problemas dentro de este campo, como el del lago salado y el domo salino, éste es el problema que más literatura científica ha generado y por tanto el de mayor interés.

La anisotropía del medio poroso en el problema original de Henry da lugar a cuatro grupos adimensionales independientes de los que derivan los patrones resultantes de isolíneas de concentración y flujo. Las pruebas numéricas para verificar la fiabilidad de estos grupos han sido numerosas y han resultado exitosas en todos los casos, aunque quedan temas en los que profundizar.

También, la reducción del problema a diferentes escenarios más simples (entre los que se incluye el escenario isótropo del problema original) ha reducido y redefinido los grupos, pero su validez ha sido también verificada en todos los casos en esta memoria mediante simulaciones numéricas.

El problema original de Henry es especialmente singular ya que el particular valor de cada uno de sus parámetros geométricos e hidrogeológicos conduce a un patrón de concentración y flujo, con una cuña salina común que engloba los fenómenos de:

i) recirculación de agua de mar en el fondo derecho del acuífero, y

ii) difusión de sal en el medio procedente del medio marino.

Cuando se alteran algunos de los parámetros de este problema (y más en escenarios anisótropos), la cuña anterior se desdobla claramente en dos, las cuales, aunque interactuando entre sí, se asocian (por separado) a los fenómenos mencionados anteriormente. Esta emergente complejidad requiere un estudio fenomenológico más 
profundo del problema, estudio que debe abordarse y explicarse a partir de los grupos adimensionales que caracterizan el proceso, y creemos que estos deben ser los grupos deducidos mediante la adimensionalización discriminada.

Por otro lado, los escenarios reales son marcadamente diferentes a los patrones del problema original de Henry, razón por la que muchos autores han recurrido a la inclusión de efectos dispersivos (no abordados en esta memoria) para explicar estas diferencias en lugar del uso de una difusividad anisótropa causada por la tortuosidad de los caminos de flujo entre poros.

Las simulaciones numéricas 2-D se han hecho mediante el programa FATSIM-A (C UPCT, 2010), elaborado por el grupo de investigación y verificado con la simulación de éste y otros problemas patrón (Soto [2007a, 2007b, 2007c]).

\section{I.3 OBJETIVOS}

Pueden sintetizarse en los siguientes puntos:

1. Revisar el estado del arte sobre el problema de Henry en dos aspectos: i) como problema patrón en el campo de la hidrogeología, y ii) en cuanto a la aplicación de técnicas para deducir los grupos adimensionales que determinan sus patrones de solución.

2. En relación con el problema general anisótropo:

2.1 Deducir los grupos adimensionales que rigen la solución de este problema, en escenarios anisótropos de sus propiedades permeabilidad intrínseca y difusión salina, mediante la técnica de adimensionalización discriminada de sus ecuaciones de gobierno.

2.2 Verificar los resultados obtenidos demostrando (mediante simulaciones numéricas) que un cambio en el valor de los parámetros individuales, que mantiene un mismo valor en los grupos adimensionales discriminados, produce los mismos patrones estacionarios finales.

2.3 Verificar que la asignación de valores unidad a los grupos adimensionales en el problema anisótropo produce un patrón de concentración y flujo que cubre la totalidad del dominio de manera regular. Verificar la influencia de cada grupo adimensional (dando valores por encima y por debajo de la unidad a 
dicho grupo) en el patrón de soluciones del problema anisótropo: para ello se modifica el valor de cada grupo reteniendo el del resto de los grupos y observando la evolución de los patrones.

2.4 Investigar la emergencia de la cuña de intrusión al modificar los valores de los grupos adimensionales.

2.5 Obtener los grupos que derivan de escenarios simplificados de este problema y verificar su validez mediante simulaciones numéricas.

3. En relación con el problema isótropo original:

3.1 Reducir los grupos anteriores a los del problema original isótropo como una nueva simplificación del problema general anisótropo, comparando dichos grupos con los deducidos por Henry y otros autores.

3.2 Verificar que la asignación de valores unidad a los grupos adimensionales en el problema isótropo produce igualmente a un patrón de concentración y flujo que cubre la totalidad del dominio de manera regular. Verificar la influencia de cada grupo adimensional (dando valores por encima y por debajo de la unidad a dicho grupo) en el patrón de soluciones del problema isótropo.

3.3 Investigar la emergencia de la cuña de intrusión.

3.4 Investigar las desviaciones de la cuña de intrusión para valores por encima y por debajo de los asignados al problema patrón y observar su influencia en el cambio de los patrones de flujo y concentración.

4. Discutir el mérito del problema de Henry como problema patrón en el campo de la hidrogeología y la intrusión salina, a la luz de los resultados de esta memoria.

5. Investigar y caracterizar las cuñas emergentes de recirculación y flujo. Obtener, hasta donde sea posible, una dependencia de la extensión de estas cuñas con los grupos adimensionales discriminados de los problemas isótropo y anisótropo.

6. Discutir el papel que juega la extensión del dominio en el patrón de solución, tanto en el problema isótropo como en el anisótropo.

7. Establecer las ventajas de la elección del método de adimensionalización discriminada como técnica para la obtención de grupos adimensionales frente a otros métodos clásicos. 


\section{I.4 PERSPECTIVAS}

El problema de Henry no se agota ni con mucho con lo aportado en la presente tesis, aunque a nuestro modo de ver, se trate de un avance cualitativo que profundiza en la comprensión de los complejos fenómenos acoplados que tienen lugar en su seno. Por un lado, la emergencia de dos cuñas bien diferenciadas (de recirculación de agua de mar en el interior del dominio y de difusión o mezcla agua dulce-agua salada entre la cuña de recirculación y la divisoria de aguas) puede ser fruto de nuevas investigaciones para su total caracterización.

La inclusión del fenómeno dispersivo en la ecuación de soluto, puede ser también abordada con la técnica de adimensionalización para deducir los grupos derivados de este nuevo y complejo término en las ecuaciones, imprescindible cuando las velocidades de flujo superan cierto valor. Esta inclusión del efecto dispersivo en la literatura ha sido necesaria para justificar la forma alargada, extendida en exceso, de algunas de las cuñas medidas en acuíferos reales, en comparación con los patrones de Henry.

Una primera incursión en este aspecto, aún no publicada, conduce a que los coeficientes de dispersión longitudinal y transversal, $\alpha_{L}$ y $\alpha_{T}$ respectivamente, tienen una ecuación de dimensiones no homogénea desde la discriminación con lo que su cociente $\alpha_{L} / \alpha_{T}$ no es un número adimensional discriminado hasta que no se corrige con la relación de aspecto (como ocurre con la difusividad y la permeabilidad intrínseca).

En la literatura existente hasta la fecha (Abarca [2006], Abarca y col. [2007] y Kalakan [2014]), en contra de lo anterior, dicho grupo aparece como grupo independiente para establecer el patrón de solución del problema. A luz de las conclusiones de esta memoria cabe asegurar que los resultados derivados en forma clásica (sin discriminación), para el problema de Henry, no pueden conducir, en modo alguno, a los grupos adimensionales más precisos.

El estudio de otros problemas, sobre los que existe igualmente una gran controversia en la literatura en relación con los grupos adimensionales que controlan los patrones de solución, promete ser una perspectiva muy interesante. Un ejemplo de ello es la discusión establecida entre Holzbecher, Simpson y Clement, y Simpson y col. en relación con el problema ‘sedimentary basin’ (Holzbecher [1998] y Simpson y Clement 
[2003, 2004] y). Cabe mencionar en este campo otros problemas de índole similar como el del lago salado, domo salino y Elder salino.

La adimensionalización en problemas termohalinos (aún en sus versiones más simplificadas), aprovechando los trabajos ya elaborados por el grupo en problemas de flujo y transporte de calor en medios porosos, pueden ser motivo también de futuros trabajos, a nuestro modo de ver muy prometedores.

\section{I.5 ORGANIZACIÓN DE LA PRESENTE MEMORIA}

En el Capítulo II se exponen los fundamentos teóricos que sirven de base para el desarrollo de esta memoria, a saber, la técnica de adimensionalización discriminada de ecuaciones y los conceptos involucrados en su aplicación, formación de ecuaciones adimensionales, grupos adimensionales y aplicación del teorema de Pi para deducir los órdenes de magnitud de las incógnitas proporcionadas por esta técnica. Se establecen las diferencias con el análisis dimensional y se relacionan los pasos para una correcta aplicación de esta técnica, diferenciándola con las técnicas clásicas de adimensionalización. También, se hace referencia a la conexión entre el concepto de problema patrón y la caracterización del mismo mediante los grupos discriminados.

El Capítulo III aplica la adimensionalización discriminada al problema clásico (isótropo) de Henry y al mismo escenario con permeabilidad y difusividad (efectiva) anisótropas, deduciendo los grupos adimensionales independientes que caracterizan estos escenarios e interpretando su significado físico en términos de balances de magnitudes. Se verifican estos resultados mediante simulaciones numéricas con FATSIM interpretando la influencia del valor de cada grupo en el patrón de soluciones, tanto mayor cuanto más cercano a la unidad es el valor del grupo, lo que está de acuerdo con las conclusiones del proceso de adimensionalización. Por último, se relaciona el valor de los grupos discriminados con el carácter del problema de Henry como problema patrón para la verificación de códigos de computación. Se recogen al final del capítulo las contribuciones y conclusiones del mismo.

El Capítulo IV se dedica a la simplificación de escenarios estudiando por un lado un conjunto de ellos que derivan del problema original de Henry y, por otro, un conjunto que deriva de problemas con grupos adimensionales del orden de la unidad. Tanto en un 
caso como en otro se asumen las simplificaciones de difusividad, velocidad regional y diferencia de densidades despreciables (separadamente), con algunas variantes, así como el escenario de dominios extensos horizontalmente para determinar hasta qué punto la longitud del acuífero influye en el patrón de soluciones. En todos los casos se verifican los resultados mediante simulaciones numéricas con FATSIM. Las contribuciones y conclusiones se resumen al final del capítulo.

La influencia separada de los grupos discriminados en los patrones del problema original de Henry se estudia en el Capítulo V, así como algunas influencias combinadas de interés, demostrándose que los patrones de solución son coherentes con las desviaciones de los valores de los grupos en torno al valor de Henry. A continuación, se estudia la influencia del valor de los grupos discriminados en los patrones del problema general de Henry (anisótropo e isótropo) que dan lugar a cuñas de intrusión y recirculación desacopladas. Finalmente, se estudia la influencia del tamaño del dominio en los patrones de solución; la eliminación de este dato extendiendo el escenario para eliminar su influencia reduce a dos los monomios del problema, y la simulación de estos escenarios permite explicar coherentemente la evolución de las longitudes ocultas que definen las cuñas. El trabajo de una nueva adimensionalización usando estas longitudes queda planteado pero no resuelto en esta memoria. Una relación detallada de las contribuciones y conclusiones sobre este tema se recoge al final del capítulo.

Finalmente, una síntesis de las contribuciones y conclusiones de esta tesis se recoge separadamente al final de la memoria. 


\section{CAPÍTULO II}

\section{La adimensionalización discriminada}

\section{II.1 LA CARACTERIZACIÓN DE UN PROBLEMA MEDIANTE GRUPOS ADIMENSIONALES}

En principio, caracterizar un problema, desde el punto de vista físico o físicomatemático, es buscar los parámetros que influyen o determinan su solución o patrón de soluciones. Son, naturalmente, los parámetros físicos y geométricos contenidos en su modelo matemático, esto es, en sus ecuaciones de gobierno y en las ecuaciones que definen sus condiciones de contorno e iniciales. Sin embargo, no todos los parámetros que aparecen en estas ecuaciones determinan de forma independiente o separada el patrón (caso de una sola variable dependiente) o patrones (caso de más de una variable dependiente) de solución del problema. Algunos parámetros podrían aparecer directamente agrupados, como ocurre por ejemplo con la permeabilidad intrínseca y la viscosidad en la ecuación de flujo de Darcy, y hasta ser irrelevantes, como podría ocurrir con algunas longitudes del problema asociadas a las condiciones de contorno. 
Un ejemplo paradigmático de longitud superflua, como se denomina en el argot de los estudiosos del Análisis Dimensional (Palacios, [1964]), es la extensión horizontal (L) del problema 2-D de Bénard, que estudia la dinámica de un fluido confinado entre placas de gran longitud (L), separadas una distancia $\mathrm{H}$, bajo un gradiente de temperaturas. El fluido se organiza en celdas de convección que se repiten regularmente a lo largo del dominio con independencia de la extensión de las placas. De nada sirve introducir L en la lista de parámetros relevantes que definen el problema, para deducir de ella los grupos adimensionales, ni de usar esa longitud para definir la variable adimensional asociada a la coordenada horizontal. La inclusión de L induce la introducción del grupo llamado relación de aspecto (largo/ancho del dominio), que en absoluto es relevante en la solución de este problema.

Por otro lado, los parámetros pueden combinarse en las propias ecuaciones mediante operaciones matemáticas sencillas para formar grupos de parámetros más compactos. Pero aclararemos algo sobre el concepto de grupos adimensionales sin entrar en los formalismos que requeriría una teoría axiomática formal que no necesitamos para los objetivos de esta memoria.

La mayor parte de las magnitudes y características físicas tienen dimensiones (en una determinada base dimensional elegida a priori) y éstas se expresan mediante las llamadas ecuaciones de dimensión. En la base de la disciplina mecánica, que incluye los procesos de difusión, en la que se incardinan los problemas de flujo y transporte de esta tesis, existen tres magnitudes fundamentales en función de las cuales pueden expresarse las ecuaciones de dimensión de cualquier magnitud o característica física dentro de esta ciencia. Estas magnitudes son longitud, masa y tiempo; y sus símbolos dimensionales L, M y T, respectivamente. Esta base dimensional se escribe, generalmente, en la forma $[\mathrm{L}, \mathrm{M}, \mathrm{T}]$.

Ejemplos de ecuaciones dimensionales, que denotaremos mediante llaves, de algunas magnitudes físicas son: la velocidad (de unidad $\mathrm{m} / \mathrm{s}$ ), $\{\mathrm{v}\}=\mathrm{LT}^{-1}$; la fuerza $(\mathrm{N})$, $\{\mathrm{f}\}=\mathrm{MLT}^{-2} \mathrm{y}$ la presión $(\mathrm{Pa}),\{\mathrm{p}\}=\mathrm{ML}^{-1} \mathrm{~T}^{-2}$. Las características físicas tienen una ecuación de dimensiones que deriva, a través de su definición, de su expresión en términos de otras magnitudes; por ejemplo la viscosidad de un fluido (derivada de la ecuación de Newton), $\{\mu\}=\mathrm{ML}^{-1} \mathrm{~T}^{-1} \mathrm{y}$ la difusividad de un soluto (derivada de la ecuación de difusión de Fick), $\{\mathrm{D}\}=\mathrm{L}^{2} \mathrm{~T}^{-1}$, etc. La cantidad de soluto se expresa mediante 
la concentración, una magnitud adimensional cuando soluto y disolvente se miden en unidades de masa, por ejemplo. Se trata de los fundamentos de la teoría del análisis dimensional.

Los grupos adimensionales clásicos (todos los conocidos), que denotaremos con la letra griega $\pi$, están formados por agrupaciones de magnitudes y características geométricas y físicas tales que la ecuación dimensional del conjunto es $\left\{\mathrm{L}^{0} \mathrm{M}^{0} \mathrm{~T}^{0}\right\}$. Decimos que $\pi$ no tiene dimensiones, que es un número, grupo o monomio adimensional.

Si a lo anterior añadimos la discriminación, no hay más que distinguir las direcciones espaciales en la base dimensional mediante un símbolo diferenciado. Así, las longitudes en las direcciones $\mathrm{x}, \mathrm{y}$, z tienen por ecuación dimensional $\{\mathrm{x}\}=\mathrm{L}_{\mathrm{x}},\{\mathrm{y}\}=\mathrm{L}_{\mathrm{y}}$, $\mathrm{y}\{\mathrm{z}\}=\mathrm{L}_{\mathrm{z}}$, respectivamente; las velocidades $\left\{\mathrm{v}_{\mathrm{x}}\right\}=\mathrm{L}_{\mathrm{x}} \mathrm{T}^{-1},\left\{\mathrm{v}_{\mathrm{y}}\right\}=\mathrm{L}_{\mathrm{y}} \mathrm{T}^{-1} \mathrm{y}\left\{\mathrm{v}_{\mathrm{z}}\right\}=\mathrm{L}_{\mathrm{z}} \mathrm{T}^{-1}$. Procediendo de igual forma se obtendrían las ecuaciones de dimensión del resto de las magnitudes y parámetros físicos contenidas en las ecuaciones de gobierno.

Por último, decir que algunos parámetros se definen directamente como adimensionales, por ejemplo, la porosidad. Si estos parámetros definen o no, por sí mismos, un grupo adimensional independiente, o si forman parte de otro grupo independiente combinados con otras magnitudes, es una tarea difícil si los grupos se investigan con la técnica del análisis dimensional puesto que, sin más, dichos parámetros constituirían grupos adimensionales independientes (algunos autores como Kalakan [2014] no los consideran como tales sin justificación alguna), pero si se usa la adimensionalización de ecuaciones, esta dificultad se soslaya ya que dichos parámetros quedan agrupados automáticamente, o no, en los grupos adimensionales derivados mediante esta técnica.

Una vez caracterizado el problema mediante un conjunto de grupos adimensionales, los valores de las variables dependientes para una misma posición relativa o adimensional del dominio y un mismo tiempo adimensional no varían si los grupos adimensionales retienen el mismo valor. Esto equivale a decir que los patrones de solución permanecen inalterables (son universales) para los mismos valores numéricos de los grupos adimensionales independientes.

Por otro lado, los grupos, una vez obtenidos, pueden redefinirse mediante operaciones entre ellos (multiplicaciones, divisiones u otras operaciones más 
complicadas), dando lugar a nuevos grupos con significado físico diferente, pero siempre manteniendo su número global. De esta forma, como veremos, puede elegirse su presentación final en la forma en que más convenga.

Cuando menor sea el número de grupos independientes tanto más fácil es presentar las soluciones universales que gobiernan el problema. El caso más sencillo sería el de un problema con una variable dependiente, una independiente y un único grupo adimensional; bastaría un ábaco de curvas que relacionan dichas variables con el grupo adimensional como parámetro. Pero puede imaginarse la información que se genera (prácticamente inmanejable) en problemas transitorios acoplados, con más de un monomio adimensional. El investigador conoce bien la ventaja de conocer estos grupos adimensionales y su uso de la manera más conveniente para reducir al mínimo las costosas pruebas experimentales y/o numéricas que ha de llevar a cabo.

En relación con el significado físico de los números adimensionales, Alhama y Madrid [2012] recogen cómo se interpretan estos números (Reynolds, Froude, Euler, Rossby, Weber, Nusselt, Peclet, Rayleigh..., así hasta veintiséis) en doce textos clásicos de ingeniería, de uso muy extendido, como por ejemplo los de Kreith y Bhon [1997], Potter y Wiggert [1997] y Bejan [2013]. En los citados textos, es frecuente atribuir a muchos de estos números, por ejemplo, el significado de balance de fuerzas. Así, el número de Reynolds se interpreta como el cociente entre fuerzas de inercia y viscosas en Grober y Erk [1967], Potter y Wiggert [1997] y Roshenow y col. [1998], entre otros. El de Rayleigh, de mayor complejidad que el de Reynolds, se define por Kreith y Bhon [1997] y Roshenow y col. [1998] como el producto de los números de Grashof y Prandtl; el primero de ellos interpretado como el cociente entre fuerzas de flotación y viscosas por numerosos autores (Grober y Erk [1967], Kreith y Bhon [1997] y Cengel [2004], por ejemplo), y el segundo interpretado como el cociente entre difusividades de cantidad de movimiento y de calor por otros (Grober y Erk [1967], Thomas [1992], Kreith y Bhon [1997] y Roshenow y col. [1998]). Interpretaciones parecidas se atribuyen a otros tantos grupos.

No obstante, la asignación de un significado físico a los números adimensionales no siempre es concordante entre los autores (Arpaci y Larsen [1984], Gebhart y col. [1988], Kays y Crawford [1993], Ózisik [1993], Holman [1997] y Alhama y Madrid [2012]) y, de hecho, nada se dice de este significado en muchos otros textos de conocido 
prestigio como los de Landau y Lifshitz [1985] y Bejan [1984, 1995, 2004, 2013]. Estos últimos autores, si bien hacen uso de estos números, no reconocen las interpretaciones clásicas mencionadas.

Arpaci, que ha dedicado un esfuerzo enorme a esta tarea, ha atribuido significados muy complejos a muchos de estos números mientras que Bejan les da un significado claro en términos de balances, pero atribuido a subdominios dentro del escenario global del problema. Alhama y Madrid [2012] demuestran que la búsqueda de significados físicos en términos de balances está invariablemente asociada al proceso de adimensionalización. Cuando este proceso se realiza aplicando la discriminación, los resultados en cuanto a la interpretación física son inequívocos y los números adimensionales resultantes tienen, además, un orden de magnitud unidad, en coherencia con un balance de magnitudes físicas del mismo rango numérico de valores.

\section{II.2 TÉCNICAS PARA LA OBTENCIÓN DE GRUPOS ADIMENSIONALES}

Las técnicas clásicas de análisis dimensional y adimensionalización están descritas en el texto Alhama y Madrid [2012], con numerosos ejemplos y aplicaciones en los campos de dinámica de fluidos y transmisión de calor. Las recogemos aquí brevemente y ampliamos la técnica de adimensionalización discriminada con las nuevas particularidades de la misma que han surgido recientemente a raíz, precisamente, de los problemas de flujo y transporte (Cánovas [2014]).

\section{II.2.1 EL ANÁLISIS DIMENSIONAL}

\section{II.2.1.1 Concepción clásica del análisis dimensional}

Aún en la actualidad, básicamente con una orientación hacia la teoría de modelos, son enormes los esfuerzos de algunos especialistas en este tema (Sedov [1993], Sonin [1997], Gibbings [2011], con continuas publicaciones en los últimos años particularmente orientadas hacia la teoría de modelos) por seguir presentando el análisis dimensional clásico como una de las (dos) vías de acceso a la formación de grupos adimensionales. Sin embargo, son muy pocos los artículos científicos que formalmente emplean esta técnica, si no es para corroborar que los grupos derivados de la aplicación de otras técnicas son, en efecto, adimensionales. 
Den o no den resultado las aplicaciones de esta técnica, es difícil desvincular el análisis dimensional de la teoría de modelos merced a la relación inseparable de estos conceptos a lo largo de muchas décadas.

Una revisión histórica de la evolución del análisis dimensional como técnica de obtención de grupos adimensionales puede encontrarse en el texto de Alhama y Madrid [2012]. Estos autores concluyen en que su utilidad en problemas de cierta complejidad es escasa, ambigua y poco precisa; una dura conclusión compartida por otros investigadores de prestigio (Bejan [1984 y 1995]). Sobre este particular este último autor, que crea su propia teoría llamada ‘análisis de escala’ basada en la adimensionalización de ecuaciones, afirma irónicamente en la primera referencia [1984] que el objetivo del análisis dimensional es determinar la dimensión de una determinada magnitud (por ejemplo, cita textualmente, la dimensión del espesor de la capa límite en problemas de convección térmica, llamada longitud). En la segunda referencia [1995], más concesivo, menciona que el análisis dimensional se fundamenta en el hecho de que todas las ecuaciones que describen el comportamiento de un sistema físico deben ser dimensionalmente consistentes, y que cuando estas ecuaciones no pueden explicitarse o son demasiado complejas el análisis dimensional puede usarse como una técnica semiempírica para obtener la forma de la solución y para reducir el número de variables que requieren investigación, sugiriendo los grupos adimensionales derivados de ella para la mejor presentación de los resultados.

Muchos textos relevantes, particularmente en el campo de la mecánica de fluidos y transmisión de calor, contienen un capítulo más o menos extenso que habla de análisis dimensional (Ozixik [1993], Incropera y DeWitt [1996] y Potter y Wiggert [1997]), aunque no hagan uso formal del mismo para deducir grupos adimensionales en problemas complejos dentro de sus propios textos. Otros de referencia ineludible en su campo como Landau y Lifshitz [1985] optan por no hacer mención explícita de esta técnica.

Pero, yendo al problema que nos ocupa, el de flujo de fluidos con transporte de soluto (problema de Henry), la mayor parte de la literatura científica admite como grupo adimensional, por ejemplo, la relación de aspecto del dominio (altura/longitud), un grupo adimensional que se obtiene directamente de las variables geométricas del problema una vez se ha justificado que ambas longitudes son, efectivamente, relevantes; aspecto sobre el que no aportan justificación explicativa alguna y que como veremos a lo largo de la 
memoria es crucial. De hecho, es dudoso que L (la longitud horizontal del dominio) sea una magnitud relevante en el problema de Henry, como veremos.

La relación de aspecto es un grupo adimensional que no se deduciría si se tratara de un problema anisótropo, y es precisamente la isotropía del escenario de Henry la que degenera dos de sus grupos adimensionales discriminados reduciéndolos a la relación de aspecto. De hecho, basta que haya isotropía en una de las propiedades físicas (permeabilidad o difusividad) para que tenga lugar esta simplificación. Esta singularidad referente a la isotropía es la que produce resultados correctos en la determinación de los grupos adimensionales mediante el análisis dimensional clásico, atribuyendo a éste una potencialidad de la que carece en términos generales.

En relación con el significado y orden de magnitud de los grupos obtenidos mediante esta técnica, nada puede concluirse formalmente a pesar de que, como ya se ha mencionado, son numerosos los textos que asocian significados físicos a estos grupos clásicos en términos de balances de magnitudes que compiten en el proceso. Adviértase que órdenes de magnitud elevados para los grupos son incompatibles con significados físicos de balance, pues si las magnitudes efectivamente compiten entre sí, sus órdenes de magnitud deben ser los mismos.

Kalakan [2014] defiende su tesis doctoral sobre la base teórica del análisis dimensional clásico obteniendo, en consecuencia, resultados muy pobres formados por un elevado número de grupos adimensionales. Lo mismo ocurre con Abarca [2007] quién aplica la adimensionalización clásica para deducir los grupos. Más tarde haremos una revisión de estos recientes trabajos que, por otro lado, introducen el fenómeno necesario de la dispersión para justificar las desviaciones entre los patrones reales y los derivados del escenario clásico del problema original de Henry.

En conclusión, la vía de usar el análisis dimensional clásico como técnica para la obtención de grupos adimensionales exige, por un lado, ser un profundo conocedor de los fenómenos físicos involucrados en el proceso para definir de forma precisa la lista relevante de variables sin conocer el modelo matemático, e incluso conociéndolo. La inclusión de una variable superflua o la exclusión de una relevante, conduce invariablemente a resultados incorrectos o poco precisos. 
Por otro lado, el pequeño número de dimensiones de la base dimensional da lugar a la emergencia de un número de monomios generalmente grande e inmanejable, poco útil para describir el problema o para representar sus patrones de solución. Todo ello acentuado en problemas definidos por dominios anisótropos en los que directamente se forman grupos adimensionales formados por los cocientes del parámetro anisótropo (relación entre sus valores para cada dirección), grupos que como demostraremos nunca juegan un papel independiente como tales sino agrupados con factores de forma geométricos (y viceversa) que, a su vez, también se introducen sin justificación formal como grupos adimensionales independientes con esta técnica del análisis dimensional clásico.

\section{II.2.1.2 El análisis dimensional discriminado}

La discriminación en análisis dimensional, siempre referida al tema de las direcciones espaciales, es un concepto antiguo. Su primera aparición se atribuye a Willians [1892] pero es Palacios [1955, 1964], décadas más tarde, quien como complemento a su tratado axiomático del análisis dimensional, presenta la discriminación como una herramienta útil que produce resultados más precisos en muchos problemas.

En su texto de 1955 justifica el uso de esta acepción, afirmando que deriva del hecho de poder medir en distintas unidades según la dirección espacial, mediante su aplicación a numerosos problemas de dinámica de fluidos y transferencia de calor. Desde 1964 hasta 2000, sólo un reducido grupo de investigadores liderados por A. Herranz de la UPCT (Herranz y Arenas [2003, 2005]), ha profundizado con relativo éxito en la discriminación. Con posterioridad a esas fechas sí aparecen algunos trabajos relacionados con el análisis dimensional discriminado, Hristov y col. [2004], Hristov [2007], Zimparov y Petkov [2009], Capobianchi y Aziz [2012], y los trabajos de Alhama y Madrid [2007, 2008] y Madrid y Alhama [2005a, 2005b, 2006 y 2008].

Para Palacios y sus seguidores, insistimos, el concepto de discriminación se justifica en la posibilidad de medir con distintas unidades en las diferentes direcciones espaciales; una base pobre pero de la que se hubiera esperado construir con más rapidez una teoría discriminada más general. Resulta curioso, sin embargo, que la divulgación en idioma inglés de texto de Palacios fuera escasa o prácticamente nula. Amstrong [1965] hace un comentario muy favorable del libro y en relación con la discriminación menciona: 
'In the work of mechanics comes another most interesting notion: that of dealing with the three (or two) spatial dimensions as if they were of different dimensions...'.

También Sonin [2001], el principal autor sobre el que funda su investigación la tesis de Kalakan [2014], hace referencia al libro de Palacios pero, incomprensiblemente, sin citar la discriminación. Sonin, haciendo sólo referencia a la normalización de ecuaciones, finalmente afirma: 'Dimensional analysis is, however, the only option in problems where the equations and boundary conditions are not completely articulated, and always useful because it is simple to apply and quick to give insight.'

Aunque es raro en la época actual que los resultados de una investigación no estén al alcance de toda la comunidad científica, podríamos concluir que en el tema de la discriminación ha sido así. No hay otra forma de explicar el escaso avance en las últimas décadas de esta técnica, que complementa y mejora cualitativamente los resultados del análisis dimensional clásico. Recientemente, sí han sido mostrados a la comunidad científica trabajos relacionados con esta aplicación (ya citados anteriormente).

En cuanto a la conclusión sobre el uso de esta herramienta para la obtención de grupos adimensionales cabe mencionar, como en el análisis dimensional clásico, la exigencia de conocer en profundidad los fenómenos físicos involucrados en el problema, en ausencia de un conocimiento de las ecuaciones de gobierno. La lista de variables relevantes es mayor pero este inconveniente se compensa con el aumento de la multiplicidad de la base dimensional que incluye tres nuevas dimensiones para la longitud en lugar de una, dando lugar globalmente a un menor y más manejable número de grupos adimensionales independientes que además, ahora sí, tienen un claro significado físico en términos de balances de magnitudes y son de orden de magnitud unidad. Las relaciones de aspecto o factores de forma geométricos, así como los cocientes de un mismo parámetro en problemas anisótropos son expulsados como grupos adimensionales independientes mediante esta técnica.

También, la posibilidad añadida de discriminar otras magnitudes como la masa (para separar fenómenos de inercia de fenómenos de simple contabilidad de materia), la energía (para separar la transmisión de calor y de la de energía mecánica por efectos viscosos), o el tiempo (para distinguir procesos estacionarios de transitorios, o para distinguir diferentes frecuencias concurrentes en un mismo proceso), incrementan 
asimismo la multiplicidad de la base dimensional desacoplando magnitudes y anulando grupos derivados de la adimensionalización clásica. La posible discriminación de estas magnitudes echa por tierra, de alguna forma, el argumento de 'la posibilidad de medir con diferentes unidades las longitudes asociadas a cada dirección espacial.

Pero la principal razón que justifica el casi uso nulo de esta vía, en las últimas décadas, para la obtención de grupos adimensionales, es sin duda el desconocimiento de esta herramienta teórica. Esta suerte de ‘mala suerte’ hace ahora muy difícil, tras muchas décadas de investigación, la sustitución de viejos grupos adimensionales (Reynolds, Nusselt, Rayleigh...), aunque no todos (por ejemplo, el número de Prandtl es un número discriminado), por otros nuevos mejor definidos. Los revisores de los trabajos científicos en revistas de ingeniería son demasiado reacios a estos cambios $\mathrm{y}$, sin embargo, es frecuente encontrar correlaciones entre grupos adimensionales clásicos (soluciones de problemas) en las que éstos aparecen multiplicados por cocientes de longitudes; ¡ni más ni menos los grupos que definen los números discriminados!

\section{II.2.2 LA ADIMENSIONALIZACIÓN DE ECUACIONES}

\section{II.2.2.1 El proceso de adimensionalización clásico}

Es el proceso de adimensionalización corrientemente aplicado en la mayor parte de los textos y en la literatura científica. Casi toda la comunidad científica asume que:

i) los grupos o números adimensionales son de cualquier valor y orden de magnitud,

ii) dichos grupos, en general, carecen de significado físico concreto, y

iii) se derivan mediante una técnica ya establecida que elige una referencia común para definir las variables adimensionales espaciales.

Así es lógico asociar la existencia de grupos adimensionales con una especie de suerte que permite simplificar el estudio de muchos problemas. Es la ortodoxia bien establecida de una técnica que ha dado numerosos frutos en dominios 1-D y 2-D, durante décadas, con mejor o menor acierto en los últimos años, y que en consecuencia no hay que tocar.

Pero la adimensionalización debería conducir a ecuaciones normalizadas y éstas a las soluciones universales del problema. Sin embargo, ni las ecuaciones derivadas con este proceso están normalizadas ni los grupos que derivan de ellas son, en general, los 
argumentos que determinan unos patrones universales. El protocolo que deduce los grupos adimensionales en un problema complejo, partiendo del conocimiento de las ecuaciones de gobierno y condiciones de contorno, se resume en lo siguiente:

i) Establecer magnitudes de referencia para cada una de las magnitudes dependientes e independientes del problema, fijando una única referencia para cada magnitud de la misma dimensión física. La magnitud elegida como referencia debe ser un dato explícito en el enunciado del problema.

ii) Definir las variables adimensionales, dependientes e independientes, refiriendo las originales a la magnitud de referencia.

iii) Sustituir las variables dimensionales originales por las nuevas en las ecuaciones de gobierno. Con ello, cada término de la ecuación tiene un coeficiente que, eventualmente, aglutina parámetros, características físicas y referencias.

iv) Dividir cada término de la ecuación por el coeficiente que más convenga para dejar uno de los términos con un coeficiente unidad. Los grupos adimensionales son los coeficientes de los otros términos de la ecuación, más los que se introducen directamente, como las relaciones de aspecto.

En efecto, la ecuación obtenida es adimensional y los coeficientes grupos adimensionales, pero las variables adimensionales no están normalizadas en general (su valor numérico no se restringe al intervalo $[0,1]$ ) y la ecuación, en consecuencia, tampoco es universal. Es curioso que, en dominios finitos 2-D, ningún investigador se haya aventurado a seleccionar distintas referencias a las dos variables espaciales (a pesar de que estaban definidas explícitamente en el problema) con objeto de que ambas quedaran normalizadas.

En conclusión, en cuanto a esta vía de obtención de grupos adimensionales, podemos afirmar que es una técnica que conduce a los mismos resultados que el análisis dimensional; resultados poco precisos en problemas complejos que producen números de orden de magnitud arbitrario y sin significado físico claro.

\section{II.2.2.2 El proceso de adimensionalización discriminada}

Ilustremos la introducción a este proceso con dos ejemplos sencillos, pero suficientemente ilustrativos de muchas de las observaciones y deducciones $\mathbf{o}$ 
conclusiones, que permite la discriminación, a los que aplicaremos las tres técnicas anteriores.

El primer ejemplo se refiere a un dominio rectangular 2-D, constituido por un medio poroso de permeabilidad intrínseca $\left(\mathrm{k}_{\mathrm{x}}, \mathrm{k}_{\mathrm{y}}\right)$ sometido a las condiciones de contorno mostradas en la Figura II.1. Estamos interesados en identificar los grupos adimensionales que caracterizan los patrones estacionarios de flujo y presión.

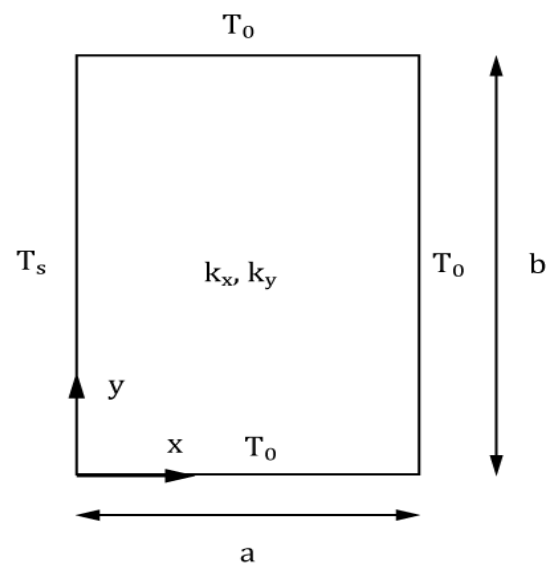

Figura II.1 Geometría del dominio y condiciones de contorno

Para el análisis dimensional clásico las magnitudes relevantes son $\left[\mathrm{k}_{\mathrm{x}}, \mathrm{k}_{\mathrm{y}}, \mathrm{a}, \mathrm{b}\right.$, $\Delta \mathrm{p}=\mathrm{p}_{\mathrm{e}}-\mathrm{p}_{\mathrm{s}}$, siendo las ecuaciones de dimensión de las mismas en la base dimensional clásica [M,L,T] las siguientes: $\left\{\mathrm{k}_{\mathrm{x}}\right\}=\left[\mathrm{k}_{\mathrm{y}}\right]=\mathrm{L}^{2},\{\mathrm{a}\}=\{\mathrm{b}\}=\mathrm{L}$ y $\{\Delta \mathrm{p}\}=\mathrm{ML}^{-1} \mathrm{~T}^{-1}$. La expulsión de la magnitud $\Delta$ p para formar grupos adimensionales es inmediata, y las demás magnitudes forman los grupos, teóricamente independientes,

$$
\pi_{\mathrm{adc}, 1}=\frac{\mathrm{k}_{\mathrm{x}}}{\mathrm{k}_{\mathrm{y}}} \quad \pi_{\mathrm{adc}, 2}=\frac{\mathrm{a}}{\mathrm{b}}
$$

En el análisis dimensional discriminado la base dimensional se incrementa en una nueva magnitud, [M, $\left.\mathrm{L}_{x}, \mathrm{~L}_{y}, \mathrm{~T}\right]$, las ecuaciones dimensionales de las magnitudes relevantes son ahora $\left\{\mathrm{k}_{\mathrm{x}}\right\}=\mathrm{Lx}_{\mathrm{x}}{ }^{2},\left\{\mathrm{k}_{\mathrm{y}}\right\}=\mathrm{Ly}_{\mathrm{y}}{ }^{2},\{\mathrm{a}\}=\mathrm{L}_{\mathrm{x}} \mathrm{y}\{\mathrm{b}\}=\mathrm{L}_{\mathrm{y}}$ (Cánovas, [2015]), y el único grupo posible debe combinar las cuatro magnitudes (por aplicación del teorema de Pi) en la forma

$$
\pi_{\mathrm{add}, 1}=\left(\frac{\mathrm{k}_{\mathrm{x}}}{\mathrm{a}^{2}}\right)\left(\frac{\mathrm{b}^{2}}{\mathrm{k}_{y}}\right)
$$


Para la adimensionalización clásica utilizaremos como ecuación de gobierno la de Laplace, combinación de la ecuación de flujo de Darcy con la de conservación de la masa del fluido,

$$
\mathrm{k}_{\mathrm{x}}\left(\frac{\partial^{2} \mathrm{p}}{\partial \mathrm{x}^{2}}\right)+\mathrm{k}_{\mathrm{y}}\left(\frac{\partial^{2} \mathrm{p}}{\partial \mathrm{y}^{2}}\right)=0
$$

Adoptando como referencias las magnitudes a y $\Delta$ p, que definen las variables adimensionales $x^{\prime}=x / a, y^{\prime}=y / a, p^{\prime}=p / \Delta p$, la ecuación anterior se reduce a su forma adimensional

$$
\frac{\mathrm{k}_{\mathrm{x}}}{\mathrm{a}^{2}} \Delta \mathrm{p}\left(\frac{\partial^{2} \mathrm{p} \prime}{\partial \mathrm{x}^{\prime 2}}\right)+\frac{\mathrm{k}_{\mathrm{y}}}{\mathrm{a}^{2}} \Delta \mathrm{p}\left(\frac{\partial^{2} \mathrm{p}}{\partial \mathrm{y}^{\prime 2}}\right)=0
$$

que se simplifica a

$$
\frac{\mathrm{k}_{\mathrm{x}}}{\mathrm{k}_{\mathrm{y}}}\left(\frac{\partial^{2} \mathrm{p} \prime}{\partial \mathrm{x}^{\prime 2}}\right)+\frac{\partial^{2} \mathrm{p} \prime}{\partial \mathrm{y}^{\prime 2}}=0
$$

Si a esta solución añadimos la relación de aspecto, los grupos adimensionales derivados de la adimensionalización discriminada coinciden con los obtenidos mediante el análisis dimensional clásico, $\mathrm{k}_{\mathrm{x}} / \mathrm{k}_{\mathrm{y}}$ y a/b. De ahí el uso alternativo de estas técnicas en la literatura.

Finalmente, la adimensionalización discriminada fija referencias distintas y coherentes para las variables espaciales, a para la variable x y b para la variable y. Usando estas referencias, las variables adimensionales quedan definidas en la forma $\mathrm{x}^{\prime}=\mathrm{x} / \mathrm{a}$, e $\mathrm{y}^{\prime}$ = y/b. Introduciéndolas en la ecuación de gobierno se obtiene su forma adimensional discriminada

$$
\frac{\mathrm{k}_{\mathrm{x}}}{\mathrm{a}^{2}} \Delta \mathrm{p}\left(\frac{\partial^{2} \mathrm{p} \prime}{\partial \mathrm{x}^{\prime 2}}\right)+\frac{\mathrm{k}_{\mathrm{y}}}{\mathrm{b}^{2}} \Delta \mathrm{p}\left(\frac{\partial^{2} \mathrm{p} \prime}{\partial \mathrm{y}^{\prime 2}}\right)=0
$$

o bien

$$
\frac{\mathrm{k}_{\mathrm{x}}}{\mathrm{k}_{\mathrm{y}}} \frac{\mathrm{b}^{2}}{\mathrm{a}^{2}}\left(\frac{\partial^{2} \mathrm{p} \prime}{\partial \mathrm{x}^{\prime 2}}\right)+\frac{\partial^{2} \mathrm{p} \prime}{\partial \mathrm{y}^{\prime 2}}=0
$$

Ecuación que proporciona un único grupo

$$
\pi=\frac{\mathrm{k}_{\mathrm{x}}}{\mathrm{k}_{\mathrm{y}}} \frac{\mathrm{b}^{2}}{\mathrm{a}^{2}}
$$


Un resultado idéntico al derivado por el análisis dimensional discriminado, pero aquí partiendo de las ecuaciones de gobierno, lo que añade cierta garantía a la deducción. En estas técnicas la relación de aspecto no juega un papel independiente, al fin y al cabo no se puede obtener de las ecuaciones de gobierno ni de las condiciones de contorno en las que reside toda la información del problema. Y el resultado es el doble de preciso (un solo monomio) que el procedente de las técnicas clásicas (dos monomios).

La solución analítica o numérica del problema demuestra que, en efecto, sólo hay un monomio independiente que caracterice los patrones estacionarios de presión y flujo, el obtenido mediante la discriminación. Estos patrones, cuando se representan a escala normalizada de todas las variables, son idénticos cuando el grupo adimensional tiene un mismo valor con independencia de los valores particulares de los parámetros que lo forman. Adviértase que tal monomio puede considerarse una corrección del grupo clásico $\mathrm{k}_{\mathrm{x}} / \mathrm{k}_{\mathrm{y}}$, producto de éste por la relación de aspecto del dominio; una agrupación que, como veremos en los problemas de esta memoria, es común a los grupos adimensionales similares que proporciona la discriminación.

La referencia a soluciones universales, asociadas a patrones universales, en relación con los grupos adimensionales discriminados que rigen la solución de un problema, aplicado al anterior ejemplo, quiere decir lo siguiente: el valor de cualquier variable adimensional como la presión en un punto, la velocidad, el caudal global que entre y sale del dominio... dependen de este grupo y, eventualmente, de las posiciones adimensionales en las que se pide la información. Así, la presión en un punto (x,y) de dominio vendrá dada por

$$
\mathrm{p}_{(x, y)}^{\prime}=\psi_{p}\left(\frac{x}{a}, \frac{y}{b}, \frac{\mathrm{k}_{\mathrm{x}}}{\mathrm{k}_{\mathrm{y}}} \frac{\mathrm{b}^{2}}{\mathrm{a}^{2}}\right)
$$

con $\mathrm{p}_{(\mathrm{x}, \mathrm{y})}$ habitualmente definida en la forma $\mathrm{p}_{(\mathrm{x}, \mathrm{y})}=\left(\mathrm{p}^{\prime}(\mathrm{x}, \mathrm{y})-\mathrm{p}_{\mathrm{s}}\right) /(\Delta \mathrm{p})$, y $\psi_{\mathrm{p}}$ una función arbitraria (desconocida) de los argumentos indicados. De la misma forma, las componentes de la velocidad vienen dadas por las expresiones

$$
v_{x}^{\prime}=\psi_{v x}\left(\frac{x}{a}, \frac{y}{b}, \frac{k_{x}}{k_{y}} \frac{b^{2}}{a^{2}}\right), \quad v_{y}^{\prime}=\psi_{v y}\left(\frac{x}{a}, \frac{y}{b}, \frac{k_{x}}{k_{y}} \frac{b^{2}}{a^{2}}\right),
$$

donde $\mathrm{v}^{\prime}{ }_{\mathrm{x}}=\mathrm{v}_{\mathrm{x}} /\left(\mathrm{k}_{\mathrm{x}} / \mathrm{a}\right)$ y $\mathrm{v}_{\mathrm{y}}^{\prime}=\mathrm{v}_{\mathrm{y}} /\left(\mathrm{k}_{\mathrm{y}} / \mathrm{b}\right), \mathrm{y} \quad \psi_{\mathrm{vx}}$ y $\psi_{\mathrm{vy}}$ funciones desconocida de sus argumentos. Por último, el caudal adimensional que circula por el dominio no es una 
variable local por lo que su forma adimensional sólo depende del monomio derivado de la discriminación,

$$
\mathrm{Q}^{\prime}=\psi_{\mathrm{Q}}\left(\frac{\mathrm{k}_{\mathrm{x}}}{\mathrm{k}_{\mathrm{y}}} \frac{\mathrm{b}^{2}}{\mathrm{a}^{2}}\right)
$$

Las funciones desconocidas, en todos los casos, sólo pueden determinarse mediante simulaciones numéricas o experimentales, pero adviértase que bastarían unas pocas simulaciones para obtener un número igual de puntos de la curva $\mathrm{Q}^{\prime}=$ $\psi_{\mathrm{Q}}\left(\mathrm{k}_{\mathrm{x}} \mathrm{b}^{2} / \mathrm{k}_{\mathrm{y}} \mathrm{a}^{2}\right)$; una información de valiosa importancia proporcionada por la adimensionalización discriminada.

No acabaremos el ejemplo sin comentar algo sobre el orden de magnitud del monomio emergente de la discriminación (2.2), que deriva de la ecuación de gobierno adimensional (2.1). En dicha ecuación se balancean los sumandos $\frac{\mathrm{k}_{\mathrm{x}}}{\mathrm{a}^{2}}\left(\frac{\partial^{2} \mathrm{p} \prime}{\partial \mathrm{x}^{\prime 2}}\right)$ y $\frac{\mathrm{k}_{\mathrm{y}}}{\mathrm{b}^{2}}\left(\frac{\partial^{2} \mathrm{p}^{\prime}}{\partial \mathrm{y}^{\prime 2}}\right)$, por lo que ambos se asumen comparables entre sí. Pero los paréntesis son derivadas segundas de magnitudes cuyos valores están confinados en el intervalo [0,1]. Podemos aproximar, en promedio, dichos cambios a la unidad (afirmación que en problemas lineales e incluso en problema con no linealidades suaves es válida), con lo que el balance de la ecuación tiene lugar entre los coeficientes $\mathrm{k}_{\mathrm{x}} / \mathrm{a}^{2} \mathrm{y} \mathrm{k}_{\mathrm{y}} / \mathrm{b}^{2}$. Esto supone que su valor debe ser del mismo orden y su cociente, que define el orden de magnitud del monomio, del orden de la unidad.

Un valor elevado del monomio (de varias unidades) supone que la importancia numérica del primer término de la ecuación frente al segundo es las mismas unidades mayor, y ello con independencia de qué parámetro particular dentro del monomio cause el aumento de su valor. Por el contrario, un valor bajo del monomio da importancia al primer término frente al segundo.

Supóngase un dominio muy ancho $(b<<a)$ con permeabilidades anisótropas de valor similar. El monomio es de valor muy inferior a la unidad y el flujo de entrada del fluido se dirige a las fronteras superior e inferior en una región cercana al borde de entrada, y a partir de una cierta distancia a dicho borde los flujos horizontales y verticales se hacen despreciables y el resto del dominio sea cual sea su extensión (a) no causa ningún efecto en el patrón. Mas esta observación no nos puede llevar a la conclusión de eliminar el parámetro (a) del problema o su término asociado (el primero) de la ecuación de 
gobierno (2.1). Por otro lado, los cambios que hemos aproximado a la unidad de los cocientes $\left(\frac{\partial^{2} \mathrm{p} \prime}{\partial \mathrm{x}^{\prime 2}}\right)$ y $\left(\frac{\partial^{2} \mathrm{p} \prime}{\partial \mathrm{y}^{\prime 2}}\right)$ no son ahora válidos en todo el dominio. Pero la ecuación (2.1) sigue siendo válida en todo el dominio. Así que, ¿cómo procedemos cuando un grupo adimensional toma valores superiores o inferiores al orden de magnitud unidad?

La discriminación, tras una detallada inspección del problema, elimina la longitud superflua del problema y la sustituye por otra de la misma dimensión discriminada, con carácter de incógnita, que se usará para definir la variable adimensional y'. Si llamamos $\mathrm{l}_{\mathrm{a}}^{*}$ esta longitud, la ecuación (2.1) tiene la forma

$$
\frac{\mathrm{k}_{\mathrm{x}}}{\mathrm{l}_{\mathrm{a}}^{* 2}} \Delta \mathrm{p}\left(\frac{\partial^{2} \mathrm{p} \prime}{\partial \mathrm{x}^{\prime 2}}\right)+\frac{\mathrm{k}_{\mathrm{y}}}{\mathrm{b}^{2}} \Delta \mathrm{p}\left(\frac{\partial^{2} \mathrm{p} \prime}{\partial \mathrm{y}^{\prime 2}}\right)=0
$$

y el grupo adimensional resultante es ahora

$$
\pi=\frac{\mathrm{k}_{\mathrm{x}}}{\mathrm{k}_{\mathrm{y}}} \frac{\mathrm{b}^{2}}{\mathrm{l}_{a}^{* 2}}
$$

La hipótesis de asumir los cambios $\left(\frac{\partial^{2} \mathrm{p}^{\prime}}{\partial \mathrm{x}^{\prime 2}}\right)$ y $\left(\frac{\partial^{2} \mathrm{p}}{\partial \mathrm{y}^{\prime 2}}\right)$ de orden de la unidad es equivalente ahora a asumir que la región en donde el flujo es sensible está definida por el dominio $0 \leq \mathrm{x} \leq \mathrm{l}^{*}$ a (equivalente a $0 \leq \mathrm{x}^{\prime} \leq 1$ ), $0 \leq \mathrm{y} \leq \mathrm{b}$ (equivalente a $0 \leq \mathrm{y}^{\prime} \leq 1$ ), que ahora denominamos sub-dominio. La asignación de un orden de magnitud unidad al grupo anterior proporciona el orden de magnitud de la incógnita $\mathrm{l}_{\mathrm{a}}^{*}$,

$$
\frac{\mathrm{k}_{\mathrm{x}}}{\mathrm{k}_{\mathrm{y}}} \frac{\mathrm{b}^{2}}{\mathrm{l}_{\mathrm{a}}^{* 2}} \sim 1 \quad \Rightarrow \quad \mathrm{l}_{\mathrm{a}}^{*} \sim\left(\frac{\mathrm{k}_{\mathrm{x}} \mathrm{b}^{2}}{\mathrm{k}_{\mathrm{y}}}\right)^{1 / 2}
$$

El segundo ejemplo es un problema transitorio. Se refiere igualmente a un dominio rectangular 2-D, Figura II.2, constituido por una placa de material conductor de calor, de conductividad térmica anisótropa $\left(\mathrm{k}_{\mathrm{x}}, \mathrm{k}_{\mathrm{y}}\right)$ y calor específico $\rho c_{\mathrm{e}}$, sometido a las siguientes condiciones de temperatura $(\theta)$ en el contorno:

Frontera izquierda (condición isoterma): $\quad \theta_{(\mathrm{x}=0,0 \leq \mathrm{y} \leq \mathrm{b}, \mathrm{t} \geq 0)}=\theta_{\mathrm{o}}$

Resto de las fronteras (condición adiabática):

$$
(\partial \theta / \partial x)_{(x=a, 0 \leq y \leq b, t \geq 0)}=(\partial \theta / \partial y)_{(0 \leq x \leq a, y=0, t \geq 0)}=(\partial \theta / \partial y)_{(0 \leq x \leq a, y=b, t \geq 0)}=0
$$

Condición inicial:

$$
\theta_{(0 \leq x \leq a, 0 \leq y \leq b, t=0)}=0
$$


Estamos interesados en identificar los grupos adimensionales discriminados que rigen la solución transitoria del problema.

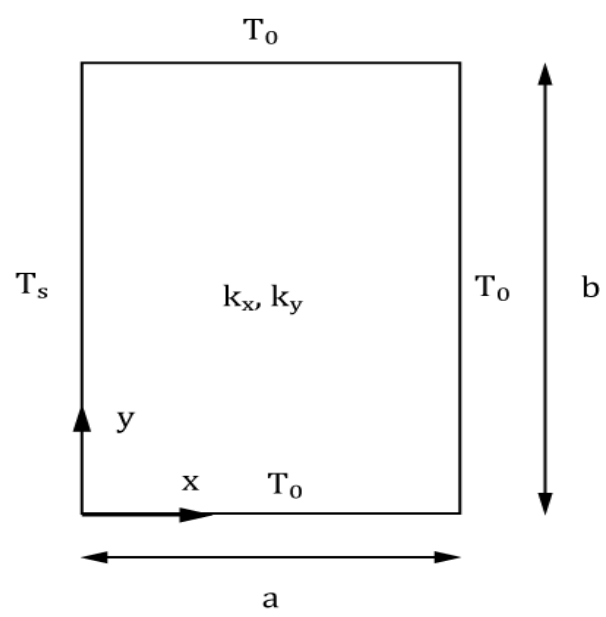

Figura II.2 Geometría del problema

La ecuación de gobierno, introduciendo el parámetro difusividad térmica, $\alpha=\mathrm{k} /\left(\rho c_{\mathrm{e}}\right)$, tiene la forma

$$
\alpha_{\mathrm{x}}\left(\frac{\partial^{2} \theta}{\partial \mathrm{x}^{2}}\right)+\alpha_{\mathrm{y}}\left(\frac{\partial^{2} \theta}{\partial \mathrm{y}^{2}}\right)=\frac{\partial \theta}{\partial \mathrm{t}}
$$

Para el análisis dimensional clásico las magnitudes relevantes son $\left[\alpha_{\mathrm{x}}, \alpha_{\mathrm{y}}, \mathrm{a}, \mathrm{b}\right.$, $\theta_{0}$ ], siendo las ecuaciones de dimensión de las mismas en la base dimensional clásica de la termodinámica [M,L,T, $\theta$ ] las siguientes: $\left\{\alpha_{\mathrm{x}}\right\}=\left\{\alpha_{\mathrm{y}}\right\}=\mathrm{L}^{2} \mathrm{~T}^{-1},\{\mathrm{a}\}=\{\mathrm{b}\}=\mathrm{L},\left\{\mathrm{t}^{*}\right\}=\mathrm{T}$ $\mathrm{y}\{\theta\}=\theta$. $\mathrm{t}^{*}$ representa el tiempo característico de duración del proceso transitorio, una magnitud desconocida o incógnita. La temperatura no puede formar monomio alguno mientras que las otras magnitudes dan lugar a tres monomios independientes:

$$
\pi_{\mathrm{adc}, 1}=\frac{\alpha_{\mathrm{x}}}{\alpha_{\mathrm{y}}} \quad \pi_{\mathrm{adc}, 2}=\frac{\mathrm{a}}{\mathrm{b}} \quad \pi_{\mathrm{adc}, 1}=\frac{\mathrm{t}^{*} \alpha_{\mathrm{x}}}{\mathrm{a}^{2}}
$$

En el análisis dimensional discriminado, con base dimensional $\left[M, L_{x}, L_{y}, T, \theta\right]$, las ecuaciones dimensionales de las magnitudes relevantes son ahora $\left\{\alpha_{\mathrm{x}}\right\}=\mathrm{L}_{\mathrm{x}}{ }^{2} \mathrm{~T}^{-1},\left\{\alpha_{\mathrm{y}}\right\}=$ $\mathrm{L}_{\mathrm{y}}{ }^{2} \mathrm{~T}^{-1},\{\mathrm{a}\}=\mathrm{L}_{\mathrm{x}} \mathrm{y}\{\mathrm{b}\}=\mathrm{L}_{\mathrm{y}}, \mathrm{y}$ los grupos emergentes son dos

$$
\pi_{\mathrm{add}, 1}=\left(\frac{\alpha_{\mathrm{x}}}{\mathrm{a}^{2}}\right)\left(\frac{\mathrm{b}^{2}}{\alpha_{\mathrm{y}}}\right), \quad \pi_{\mathrm{add}, 2}=\left(\frac{\mathrm{t}^{*} \alpha_{\mathrm{x}}}{\mathrm{a}^{2}}\right)
$$


En cuanto a la adimensionalización clásica, adoptando las referencias a, $\theta_{\mathrm{o}} \mathrm{y} \mathrm{t}^{*}$, que definen las variables adimensionales $\mathrm{x}^{\prime}=\mathrm{x} / \mathrm{a}, \mathrm{y}^{\prime}=\mathrm{y} / \mathrm{a}, \theta^{\prime}=\theta / \theta_{0} \mathrm{y} \mathrm{t}^{\prime}=\mathrm{t} / \mathrm{t}^{*}$, la ecuación (2.3) se reduce a su forma adimensional

$$
\frac{\alpha_{\mathrm{x}} \theta_{\mathrm{o}}}{\mathrm{a}^{2}}\left(\frac{\partial^{2} \theta^{\prime}}{\partial \mathrm{x}^{\prime 2}}\right)+\frac{\alpha_{\mathrm{y}} \theta_{\mathrm{o}}}{\mathrm{a}^{2}}\left(\frac{\partial^{2} \theta^{\prime}}{\partial \mathrm{x}^{\prime 2}}\right)=\frac{\theta_{\mathrm{o}} \partial \theta^{\prime}}{\mathrm{t}^{*} \partial \mathrm{t}^{\prime}}
$$

que se simplifica a

$$
\frac{\alpha_{\mathrm{x}}}{\alpha_{\mathrm{y}}}\left(\frac{\partial^{2} \theta^{\prime}}{\partial \mathrm{x}^{\prime 2}}\right)+\frac{\partial^{2} \theta^{\prime}}{\partial \mathrm{y}^{\prime 2}}=\frac{\mathrm{a}^{2}}{\mathrm{t}^{*} \alpha_{\mathrm{y}}} \frac{\partial \theta^{\prime}}{\partial \mathrm{t}^{\prime}}
$$

Añadiendo la relación de aspecto, los grupos adimensionales que resultan de esta adimensionalización coinciden con los del análisis dimensional clásico, a saber, $\alpha_{x} / \alpha_{y}$, $t^{*} \alpha_{y} / a^{2}$ y a/b.

Finalmente, la adimensionalización discriminada elige las referencias a, b y t ${ }^{*}$ que definen las variables adimensionales $\mathrm{x}^{\prime}=\mathrm{x} / \mathrm{a}, \mathrm{y}^{\prime}=\mathrm{y} / \mathrm{b}, \mathrm{t}^{\prime}=\mathrm{t} / \mathrm{t}^{*}$, $\mathrm{y}$ la ecuación adimensional discriminada

$$
\frac{b^{2}}{a^{2}} \frac{\alpha_{\mathrm{x}}}{\alpha_{\mathrm{y}}}\left(\frac{\partial^{2} \theta^{\prime}}{\partial \mathrm{x}^{\prime 2}}\right)+\frac{\partial^{2} \theta^{\prime}}{\partial \mathrm{y}^{\prime 2}}=\frac{\mathrm{b}^{2}}{\mathrm{t}^{*} \alpha_{\mathrm{y}}} \frac{\partial \theta^{\prime}}{\partial \mathrm{t}^{\prime}}
$$

Los grupos resultantes se simplifican a dos, idénticos a los del análisis dimensional discriminado:

$$
\pi_{1}=\frac{\alpha_{x}}{\alpha_{y}} \frac{b^{2}}{a^{2}}
$$

La solución para el tiempo característico consiste en igualar su forma adimensional (precisamente la expresión del segundo monomio) a una función del único monomio que no contiene incógnitas

$$
\pi_{2}=\psi\left(\pi_{1}\right)
$$

o bien

$$
\mathrm{t}^{*}=\frac{\mathrm{a}^{2}}{\alpha_{\mathrm{x}}} \psi\left(\frac{\alpha_{\mathrm{x}}}{\alpha_{\mathrm{y}}} \frac{\mathrm{b}^{2}}{\mathrm{a}^{2}}\right)
$$


Otras soluciones, como la temperatura en cualquier instante y en cualquier posición, viene dada por su forma adimensional en función de los monomios x/a, y/b, $\mathrm{t} /\left(\mathrm{a}^{2} / \alpha_{\mathrm{x}}\right)$ y $\left(\alpha_{\mathrm{x}} \mathrm{b}^{2} /\left(\alpha_{\mathrm{y}} \mathrm{a}^{2}\right)\right.$ :

$$
\frac{\theta}{\theta_{0}}=\psi\left(\frac{\mathrm{x}}{\mathrm{a}}, \frac{\mathrm{y}}{\mathrm{b}}, \frac{\mathrm{t} \alpha_{\mathrm{x}}}{\mathrm{a}^{2}}, \frac{\alpha_{\mathrm{x}}}{\alpha_{\mathrm{y}}} \frac{\mathrm{b}^{2}}{\mathrm{a}^{2}}\right)
$$

Los recursos aplicados a estos problemas pueden considerarse que derivan del concepto de discriminación que abarca más de lo que supone sencillamente atribuir diferente ecuación de dimensiones a las magnitudes del espacio de carácter vectorial. Los propios monomios que derivan de esta técnica de adimensionalización, una vez obtenidos, nos pueden informar de la buena o mala elección de las magnitudes de referencia con las que han sido obtenidos.

¿Qué ocurre cuando el problema está definido por varios monomios?, ¿puede introducirse más de una incógnita en el proceso de adimensionalización?, ¿cómo se obtiene la solución en este caso?... Sin perjuicio de las reglas para aplicar el protocolo de adimensionalización que estableceremos más adelante, cada problema de suficiente complejidad debe ser estudiado en detalle y sus resultados verificados mediante experimentación o simulación numérica para aceptarlos o redefinir el planteamiento de su determinación para llegar a los grupos precisos. Es una línea de investigación reciente y, por tanto, abierta a previsibles mejoras en su aplicación.

\section{II.2.3 PROTOCOLO DE APLICACIÓN DE LA ADIMENSIONALIZACIÓN DISCRIMINADA}

Como se ha mencionado, convertir una ecuación de gobierno o condición de contorno en una ecuación adimensional no es más que sustituir las variables dimensionales (dependientes e independientes) por sus correspondientes nuevas variables, adimensionales, mediante un adecuado cambio de variable. La naturaleza de las variables puede ser cualquiera - coordenadas espaciales, velocidades, tiempo, temperatura... - y el proceso de adimensionalización ha de aplicarse a todas y cada una de las ecuaciones que constituyen el modelo matemático completo del sistema (ecuaciones constitutivas, de conservación, de momento, de energía...). La relación entre cada variable dimensional y su correspondiente adimensional se establece mediante la 
selección de magnitudes de referencia. Esta relación, en esencia la definición de la variable adimensional, tiene la siguiente forma general

$$
\xi^{\prime}=\frac{\xi}{\xi_{\text {ref }}}
$$

donde $\xi^{\prime}$ es la nueva variable adimensional, $\xi$ la variable dimensional original y $\xi_{\text {ref }}$ un valor de referencia arbitrario con la misma dimensión que $\xi$, la misma ecuación de dimensión discriminada. Sustituidas todas las variables dimensionales por sus correspondientes adimensionales, la ecuación de gobierno y las ecuaciones asociadas con las condiciones de contorno se convierten en ecuaciones adimensionales. Todas juntas constituyen el modelo matemático adimensional discriminado.

El aspecto general de cada uno de los términos de estas ecuaciones es el de un producto de dos factores: uno que contiene las nuevas variables adimensionales y/o sus derivadas, o ambas cosas, y otro formado por una agrupación de características físicas asociadas a los fenómenos involucrados en el problema - y/o parámetros.

La primera cuestión es: ¿Cómo elegir la magnitud de referencia, $\xi_{\text {ref }}$ ? ¿Debe o no debe ser, necesariamente, una magnitud cuyo valor se explicita en el enunciado del problema, como habitualmente se adopta en los procesos no discriminados? En la adimensionalización discriminada esta elección sólo exige que la ecuación de dimensiones discriminada de $\xi_{\text {ref }}$ y $\xi$ sea la misma. Así, la referencia para la coordenada x ha de ser una longitud característica en esa misma dirección, y lo mismo para la otra coordenada y para las velocidades $\mathrm{v}_{\mathrm{x}} \mathrm{y} \mathrm{v}_{\mathrm{y}}$ en problemas 2-D.

Si no existen referencias explícitas en el enunciado del problema se elegirán, en principio, referencias arbitrarias u 'ocultas' cuyo significado y orden de magnitud se deducirán una vez obtenidos los grupos adimensionales. Ésta es una diferencia esencial con los métodos clásicos introducida por Alhama y Madrid [2012] para la búsqueda de grupos adimensionales, tanto en el análisis dimensional (aunque en éste ya se discriminaban las magnitudes, constates físicas y demás parámetros con significado físico) como en la adimensionalización de ecuaciones.

Como se verá inmediatamente, si queremos dar un significado físico preciso a los grupos adimensionales que buscamos y, por ende, atribuirles un orden de magnitud unidad, debemos asociar un rango de variación unidad a las nuevas variables 
adimensionales. En dominios 2-D finitos, de geometría $\mathrm{a}_{\mathrm{x}}\left(\right.$ horizontal) $\times \mathrm{a}_{\mathrm{y}}($ vertical), si el proceso físico se extiende a todo el dominio, lo lógico en principio es adoptar las referencias $\mathrm{a}_{\mathrm{x}} \mathrm{y} \mathrm{a}_{\mathrm{y}}$ para adimensionalizar las variables $\mathrm{x}$ e y, respectivamente. En cuanto a velocidades, es frecuente que exista al menos una velocidad característica (asociada a un flujo regional o a una ecuación constitutiva como la de Darcy); si es así, la ecuación de conservación nos permitirá construir la otra velocidad de referencia mientras que si existen dos velocidades características en el problema, la ecuación de conservación nos permitirá deducir directamente uno de los grupos adimensionales buscados: la relación entre velocidades características corregida por la relación de aspecto.

En relación con el tiempo, se elegirá uno de los muchos tiempos característicos que se pueden formar con las variables y características físicas del problema. Dependiendo de la referencia elegida se interpretarán físicamente los balances de los grupos adimensionales obtenidos.

Con todo, el rango de variación de las variables adimensionales se restringe al intervalo de valores [0-1] haciendo que, en promedio, sus cambios y derivadas sean de orden de magnitud unidad (esto es cierto siempre que la no linealidad del problema no sobrepase un grado severo). Con esta hipótesis, los factores de cada término de la ecuación formados por las variables adimensionales y sus cambios pueden simplificarse numéricamente, quedando una ecuación asociada a los coeficientes de estos factores más los coeficientes de los términos que no contienen variables; coeficientes que, obviamente, han de tener el mismo orden de magnitud, así como su suma o diferencia. Los grupos adimensionales son las relaciones entre estos coeficientes, y el número máximo de ellos en una ecuación es como máximo el número de términos de la ecuación menos uno. El hecho de que los coeficientes citados sean del mismo orden de magnitud obliga a que los grupos discriminados sean todos de orden de magnitud unidad.

Existen tantos grupos adimensionales diferentes (no independientes necesariamente) como pares de relaciones entre coeficientes en cada ecuación. Pero cada una de las diferentes ecuaciones, e incluso una misma ecuación, puede contener grupos adimensionales comunes. Para seguir un protocolo formal en la obtención del mínimo número de estos grupos que determina la solución del problema, puede seleccionarse un coeficiente y comparar los restantes con él. Una vez obtenidos los grupos, pueden establecerse nuevos conjuntos de grupos independientes mediante operaciones 
matemáticas simples (multiplicaciones o divisiones), u otras más complejas, entre los primeros.

Todas las consecuencias anteriores se basan en las hipótesis asumidas para la selección de las magnitudes de referencia. La complejidad del problema, sin embargo, puede dificultar el formalismo de la adimensionalización discriminada y producir grupos adimensionales discriminados de orden de magnitud no unidad. Piénsese, por ejemplo, en un problema en el que, en determinadas sub-regiones del dominio preponderan unos procesos físicos frente a otros cuyo efecto podría despreciarse (como se ha visto en los ejemplos citados anteriormente). En estos casos no es válido elegir las longitudes totales del dominio $\left(\mathrm{a}_{\mathrm{x}} \mathrm{y} \mathrm{b}_{\mathrm{x}}\right)$ para obtener los grupos buscados, sino que es preciso reducir las ecuaciones (simplificando su aplicación) a estas sub-regiones, para poder atribuir a los grupos adimensionales resultantes las propiedades de orden de magnitud unidad y significado físico de un balance entre magnitudes dentro del subdominio.

En general, ya lo hemos comentado, poco se profundiza en la literatura científica sobre estos temas de carácter teórico, aunque fundamental desde nuestro punto de vista, aun cuando disponemos de herramientas numéricas tan potentes que nos proporcionan una solución fiable de casi todos los problemas. Es un tema que consideramos abierto a la investigación, aunque nos constan las dificultades para publicar resultados de este tipo en las revistas internacionales al uso. A fin de cuentas, la adimensionalización discriminada conduce a grupos que, en general, no son los clásicos cuyo uso está profundamente introducido y aceptado en la comunidad científica.

En escenarios isótropos, los nuevos números discriminados son intercambiables con los clásicos aunque no coinciden ni en su significado ni en su valor, pero en escenarios anisótropos son absolutamente diferentes. Por fortuna, muchos de estos complejos escenarios anisótropos (como el que nos ocupa en esta memoria) han sido poco estudiados, debido a que la solución contiene un número elevado de monomios que la hace poco manejable. Siendo así, es más fácil introducir los nuevos grupos adimensionales discriminados.

Una vez obtenido el conjunto de los n grupos adimensionales independientes de un problema $\left(\pi_{1}, \pi_{2}, \pi_{3} \ldots \pi_{\mathrm{n}}\right)$, la solución es una función del tipo

$$
\pi_{1}=\mathrm{f}_{1}\left(\pi_{2}, \pi_{3}, \pi_{4,}, \ldots \pi_{\mathrm{n}}\right)
$$


o, alternativamente, de los tipos

$$
\begin{aligned}
& \pi_{2}=\mathrm{f}_{1}\left(\pi_{1}, \pi_{3}, \pi_{4}, \ldots \pi_{\mathrm{n}}\right), \\
& \pi_{3}=\mathrm{f}_{1}\left(\pi_{1}, \pi_{2}, \pi_{4}, \ldots \pi_{\mathrm{n}}\right) \ldots,
\end{aligned}
$$

donde $\mathrm{f}_{1}, \mathrm{f}_{2} \ldots$ son funciones arbitrarias ( $\mathrm{y}$ desconocidas) de los argumentos indicados. La posibilidad de elección del conjunto de grupos independientes como la de elección de cualquiera de las anteriores soluciones permite que la magnitud incógnita del problema aparezca en un solo grupo adimensional, lo que a su vez lleva a expresar directamente el orden de magnitud de dicha incógnita en función de los parámetros de su grupo y del resto de los grupos que no contienen incógnitas.

Afortunadamente, no son excesivos los grupos adimensionales de un problema fundamental por complejo que sea (como el tratado en esta memoria, donde el número máximo de grupos para casos estacionarios es cuatro), de forma que las ecuaciones anteriores son muy útiles. Por ejemplo: si existe un solo monomio queda explicitado el orden de magnitud de la incógnita que contiene, sin la existencia de una función indeterminada. Si existen dos, este orden de magnitud está modulado por una función arbitraria del otro monomio; función que, por otro lado, tiene un rango de valores de orden de magnitud unidad, una información sin duda interesante para el investigador. Además, los valores de la función arbitraria podrían determinarse mediante un conjunto limitado de pruebas experimentales o numéricas. Con tres o más monomios la información, obviamente, no es tan útil pues requiere la elaboración de ábacos.

La aparición de más de una incógnita es un nuevo reto, pero los avances en el campo de adimensionalización de ecuaciones ordinarias acopladas (Conesa [2016] y Conesa y col. [2016]) permiten orientar los pasos para obtener resultados útiles. En general, si no se conocen otras relaciones entre las incógnitas (relaciones deducidas de ecuaciones diferentes a las ecuaciones de gobierno), los procesos de adimensionalización que conducen a la solución de cada una de ellas suelen referirse a distintas ecuaciones de gobierno o a ecuaciones de gobierno con diferentes términos, lo que conduce a la existencia de distintos grupos para cada incógnita. Estos grupos permiten obtener el orden de magnitud de la incógnita por el procedimiento y expresión explicado anteriormente. 
Los pasos para aplicar el protocolo de adimensionalización discriminada a un problema, que deberá aplicarse tras un minucioso estudio y una reflexión profunda sobre los fenómenos involucrados en el mismo, pueden resumirse en:

i) Seleccionar de las magnitudes de referencia (tantas como variables existan en las ecuaciones de gobierno y condiciones de contorno del problema). Estas magnitudes, nunca superfluas, pueden estar explicitadas en el enunciado del problema o ser magnitudes ocultas pero con un claro significado físico.

ii) Definir las variables adimensionales a partir de las referencias anteriores.

iii) Formar las ecuaciones de gobierno adimensionales sustituyendo las viejas variables dimensionales por las nuevas adimensionales definidas en ii).

iv) Establecer los coeficientes dimensionales de cada término de las ecuaciones adimensionales.

v) Formar los grupos adimensionales independientes (tantos en cada ecuación como número de términos menos uno) a partir de los coeficientes anteriores, como cocientes entre pares de los coeficientes listados en iv).

vi) Añadir los factores de forma o relaciones de aspecto (cocientes de longitudes en una misma dirección espacial), si existen. En general, estas relaciones derivan de las ecuaciones asociadas a las condiciones de contorno.

vii) Re-definir los grupos, si conviene, por operaciones simples entre ellos, bien para elegir significados físicos concretos bien para que las incógnitas de interés estén convenientemente ubicadas en los grupos (una en cada grupo).

viii) Establecer las correlaciones entre grupos y las expresiones de las magnitudes incógnita.

ix) Explicitar el orden de magnitud de las incógnitas buscadas.

x) Los patrones de solución del problema sólo dependen de los grupos adimensionales que no contienen incógnitas, aunque, eventualmente, las variables o magnitudes de interés (en su forma adimensional) pueden depender también de posiciones y tiempos adimensionales. 
xi) Las soluciones pueden no verificarse. En este caso el procedimiento ha sido mal aplicado y produce errores. Hay que reestudiar el problema comprobando que las ecuaciones de gobierno son correctas y que las referencias han sido bien seleccionadas.

Entre los pasos iii) y iv) se ha asumido que las variables y sus cambios, en promedio, son de orden de magnitud unidad. Pueden darse circunstancias especiales que requieran pasos diferentes a los indicados en el protocolo anterior. Un ejemplo: en los problemas transitorios con solución asintótica la referencia temporal requiere fijar un criterio porcentual; por ejemplo, el tiempo necesario para que la variable dependiente se mueva un buen porcentaje (80 ó 90 \%) de su recorrido total. Otro ejemplo: aunque infrecuente, la existencia de diferentes fenómenos físicos en una misma dirección espacial y que se producen a distinta velocidad puede requerir la selección de dos referencias distintas en una misma dirección espacial y hacer adimensional cada término de las ecuaciones usando las referencias adecuadas. Con seguridad, otros ejemplos están por estudiar.

Finalmente, ya hemos comentado la existencia de subdominios. La reducción del problema a ellos simplifica las ecuaciones de gobierno, exige referencias distintas y, en suma, conduce a nuevos grupos y balances que definen el patrón del subdominio particular. Como en el caso anterior, puede deducirse información interesante de estos subdominios, en particular alguno de sus límites, tras introducir alguna referencia incógnita.

\section{II.2.4 LA DEFINICIÓN DE PROBLEMAS PATRÓN A TRAVÉS DE LOS GRUPOS ADIMENSIONALES DISCRIMINADOS}

La definición de problemas patrón ('benchmarking') es un tema, en principio, ajeno y desconectado de la investigación de los grupos adimensionales, aunque, evidentemente, la búsqueda de estos grupos sea también un objetivo fundamental en estos problemas. Así, por ejemplo, el problema de Henry [1964] trata de definir un escenario 2-D, típico de intrusión salina en acuíferos costeros, a partir de las ecuaciones de flujo y trasporte de soluto, buscando que la cuña de intrusión reproduzca lo más aproximadamente la respuesta de acuíferos bajo unas condiciones de contorno lo más reales posible. 
La existencia de esta cuña, localizada en el borde del acuífero que linda con el mar, define una zona de mezcla cuyo tamaño, hasta cierto punto, es independiente de la extensión del acuífero. Esto implica que los números adimensionales discriminados e independientes que caracterizan este problema patrón no son del orden de magnitud unidad en tanto que los patrones de solución no se extienden por todo el dominio. Algo similar ocurre con otros problemas patrón de flujo con transporte de sal, como el del lago salado ('salt lake') y el del domo salino ('salt dome'), o de flujo con transporte de calor, como el problema de Elder o el de Yusa; en este último se definen varios sub-problemas en función de la velocidad paralela horizontal establecida en la superficie del dominio.

Cuando estos problemas, como es habitual, se utilizan para verificar los métodos numéricos propuestos para su solución, y los investigadores se centran, bien en la comparación de patrones, bien en la comparación de los puntos de intersección de ciertas isolíneas con el borde del dominio, la mejor elección es la de números adimensionales del orden de la unidad, pues esta condición proporciona patrones regularmente distribuidos en todo el dominio en lugar de patrones concentrados en una parte del mismo.

La comparación en el primer caso permite una mayor sensibilidad en la prueba. En el problema de intrusión de Henry, la selección de números adimensionales discriminados de valor unidad extiende la cuña de intrusión a todo el recinto con una distribución muy regular como se verá en posteriores capítulos, y lo mismo ocurre con el patrón de isolíneas de corriente, aunque desaparece la cuña de recirculación de flujo de agua salada desde el mar, que emerge en la región inferior derecha del dominio.

En los problemas patrón estudiados en esta memoria se revisa la idoneidad de los grupos adimensionales discriminados bajo el punto de vista de su referencia para la verificación de los diferentes métodos numéricos propuestos para su simulación. 


\section{CAPÍTULO III}

\section{Aplicación de la adimensionalización discriminada al problema de Henry}

\section{III.1 EL PROBLEMA DE HENRY}

\section{III.1.1 ANTECEDENTES Y DISCRIMINACIÓN}

Aunque el problema de intrusión salina de Henry [1959 y 1964] y Croucher y O’Sullivan [1995]), que trata de los procesos acoplados de flujo y transporte que tienen lugar en la frontera salina de acuíferos costeros, ha sido motivo de gran controversia en las últimas décadas (Segol y col. [1975]), disfruta aún de gran popularidad como problema patrón para verificar los códigos de computación de flujo de aguas subterráneas (medios porosos) controlados por densidad variable (Frind [1982], Voss y Souza [1987], Holzbecher [1998], Simpson y Clement [2003] y Soto y col. [2007b]). Una descripción con gran detalle de lo que el problema de Henry ha supuesto en la historia del estudio del flujo de aguas subterráneas con transporte de sal, en relación con el establecimiento de problemas patrón, puede leerse en los trabajos de Croucher y O’Sullivan [1995] y Simpson y Clement [2004].

Con anterioridad al trabajo de Henry de 1964, la interfaz entre agua dulce y agua salada se tratada como una fina línea bien definida (Hubbert, 1940; Kozeny (1953), 
Cooper [1959], Henry [1959] y Glover [1964]), que proporcionaba una primera aproximación al problema de intrusión y que, por otra parte, reflejaba los resultados de algunos escenarios reales. En otros escenarios, sin embargo, con cuñas de intrusión muy extensas, esta solución se alejada de la realidad.

Henry en su trabajo de 1964 parte de la forma adimensional de las ecuaciones de gobierno de flujo y transporte (ecuación de Darcy con densidad variable más las ecuaciones de continuidad para el agua y el soluto) formuladas en términos de las variables dependientes función de corriente y concentración, en estado estacionario, deduciendo su solución semianalítica y presentando los patrones de estas variables (isolíneas de función de corriente y de concentración).

Henry reduce el problema no-lineal de valor de contorno a un conjunto infinito de ecuaciones algebraicas no lineales asumiendo una expresión para las soluciones (concentración y función de corriente) en series de Fourier y deduciendo los coeficientes de estas series por el método de Garlekin, una vez truncado el conjunto de ecuaciones, por un método iterativo. Para comparar soluciones, el patrón obtenido por Henry se extiende a los casos extremos de ausencia de difusión y de difusión con diferencia de densidad nula. Sus soluciones numéricas muestran como conclusión importante que la dispersión efectiva o difusividad, el flujo regional y el flujo de densidad variable, en su interacción, inducen un flujo de agua salada en la parte inferior del acuífero, procedente de la frontera del mar, y una región de elevado gradiente entre esta zona de recirculación y la zona de flujo de agua dulce continental.

Así, siguiendo un procedimiento de adimensionalización clásico (no discriminado), Henry y más recientemente otros autores como Holzbecher [1998] y Abarca y col. [2007], definen las variables adimensionales que conducen a los grupos o números adimensionales que, de forma independiente, rigen los patrones de solución del problema. Este procedimiento se denomina clásico por estar asociado al hecho de que los valores característicos usados como referencia para adimensionalizar las variables de problema, coordenadas y velocidades, no tienen un 'carácter' inherente diferenciado, direccional o vectorial. De este modo, una misma referencia longitudinal (la profundidad del acuífero) define la forma adimensional tanto de la coordenada x como de la y, como también, una misma referencia de velocidad (la entrada de agua dulce continental en la frontera izquierda de acuífero) define las velocidades adimensionales horizontal y 
vertical. Sustituidas estas nuevas variables en las ecuaciones de gobierno, las ecuaciones adimensionales resultantes deducidas por Henry contienen dos de los tres los parámetros adimensionales del problema isótropo.

Además, Henry asume un valor constante, y por tanto independiente de la dirección y la velocidad, para el coeficiente de dispersión D a lo largo del medio, es lo que llama dispersión efectiva (aunque se trata de un fenómeno de difusión pura). Este autor escribe textualmente: 'This representative value, even though it supposes a broad assumption, retains the essential features of the problem: the circulation of salt water and the reduction of the inland extent of salt water'.

De acuerdo con las conclusiones de Henry los parámetros adimensionales resultantes son tres. Dos de ellos - que denominaremos $\mathrm{a}_{\mathrm{H}}$ у $\mathrm{b}_{\mathrm{H}}$ (ver nomenclatura)-, a los cuales Henry no atribuye significado físico alguno, emergen de forma natural de la adimensionalización de las ecuaciones de flujo (Darcy) y transporte, respectivamente. El tercero, un factor geométrico o relación de aspecto del dominio - $\xi-$, tanto Henry como otros autores que estudian el problema con posterioridad lo introducen directamente (sin más explicación) merced a su propio carácter adimensional, con independencia del proceso de adimensionalización.

En cuanto a los valores de estos parámetros Henry asume un criterio práctico: por un lado, deben ajustarse en la medida de lo posible a los datos de campo, para lo cual el autor sigue las predicciones de Cooper [1959] y los datos experimentales de Kohout [1960]; por otro, deben permitir la convergencia de las soluciones numéricas al uso en aquellas décadas. Los problemas analíticos de truncamiento y convergencia impusieron límites a los valores de la relación de aspecto 'longitud/profundidad del acuífero', del orden de la unidad como máximo, y del parámetro b En relación con estos límites, los parámetros de campo obtenidos por Kohout (1964) eran: $\mathrm{a}_{\mathrm{H}}=0.04, \mathrm{~b}_{\mathrm{H}}=0.1 \div 0.001$, y relación de aspecto $\xi=16$.

Después de algunos resultados computacionales preliminares, Henry elige sin más explicaciones dos pares de valores concretos para estudiar el efecto de los cambios producidos en la región de descarga de agua dulce:

i) $\quad \mathrm{a}_{\mathrm{H}}=0.45, \quad \mathrm{~b}_{\mathrm{H}}=0.1, \quad \xi=2$,

ii) $\quad \mathrm{a}_{\mathrm{H}}=0.263, \quad \mathrm{~b}_{\mathrm{H}}=0.1, \quad \xi=2$. 
Cuatro décadas más tarde, Simpson y Clement [2003, 2004] asignan significado físico a $\mathrm{a}_{\mathrm{H}}$, un parámetro que escriben en la forma $\mathrm{a}_{\mathrm{H}}=\mathrm{Q} /\left[(\mathrm{kgH} / \mu)\left(\rho_{\mathrm{s}}-\rho_{\mathrm{o}}\right)\right]$, diciendo: 'it can be thought as a dimensionless number that reflects a balance of the advective flux, in the numerator, to the density-driven flux in the denominator'. En su artículo, estos autores intentan mejorar el mérito del problema de Henry incrementando el efecto del flujo controlado por densidad variable en relación con el flujo regional causado por la diferencia de presión entre los bordes izquierdos y derecho del acuífero (condición de contorno).

Para Voss y Souza [1987] y Sanz y Voss [2006], el parámetro ан controla la distribución de presiones la cual, a su vez, define la extensión de la región de intrusión tierra adentro. En cuanto al significado físico de $b_{H}$, podemos decir que se trata de una especie de número de Peclet ya que compara los flujos salinos difusivo y advectivo, Abarca [2006].

Autores posteriores a Henry, quienes comprobaron que las soluciones numéricas aportadas por los valores de los parámetros $\mathrm{a}_{\mathrm{H}}$, $\mathrm{b}_{\mathrm{H}} \mathrm{y} \xi$ propuestos por Henry no reflejaban los resultados observados en acuíferos reales, modificaron algo dichos parámetros. Particularmente, disminuyeron $b_{н}$ en la búsqueda de regiones más estrechas de la zona de mezcla en la interfaz agua dulce-agua salada (Voss y Soza [1987] y Bues y Oltean [2000]). Sin embargo, desde el punto de vista del significado físico atribuido por los autores, con $b_{H}=0.1$ (el valor de Henry), el efecto difusivo es del orden del 10\% del efecto advectivo, un valor de por sí comparativamente bajo; si ahora disminuimos aún más el valor de este coeficiente deberíamos casi eliminar el efecto difusivo de las ecuaciones de balance y simplificar las mismas, es decir, bн no debería ejercer influencia apreciable en los patrones estacionarios.

Esta aparente controversia que atrae nuestra atención está asociada a la forma en que se definen las variables adimensionales, particularmente en problemas isótropos. La conclusión es que cuando dichos grupos se obtienen mediante la adimensionalización clásica, un valor numérico unidad de cualquier grupo no significa necesariamente que los efectos físicos que se balancean o pretenden balancearse sean del mismo orden de magnitud (véanse los fundamentos teóricos expuestos en el Capítulo II). En suma, cuando se elige una única referencia para definir las variables coordenadas adimensionales, y el escenario es alargado o esbelto (no de tipo cuadrado), el rango de variación de estas 
coordenadas y sus cambios pueden ser muy diferentes lo que no permite fijar un mismo orden de magnitud a los coeficientes de la ecuación adimensionalizada y, en consecuencia, los grupos adimensionales resultantes no son necesariamente de orden de magnitud unidad. Cabe esperar conclusiones similares si otras variables de carácter vectorial como la velocidad se definen también en forma clásica. Esa controversia, como veremos, se evita con la adimensionalización discriminada.

Como se ha mencionado en el capítulo anterior, el concepto de discriminación involucra la elección de referencias propias (discriminadas) para cada variable o magnitud que precise definirse en forma adimensional. Los grupos adimensionales independientes resultantes, como veremos, pueden expresarse de distinta forma, pero su número no cambia, su orden de magnitud es la unidad y su significado puede interpretarse siempre en términos del balance de magnitudes que interactúan o se contrarrestan en todo o parte del dominio del problema.

En medios isótropos los grupos resultantes de la adimensionalización discriminada pueden reducirse a los de Henry mediante operaciones simples y sus valores numéricos explican tanto las desviaciones de los patrones resultantes como la reducción de su significado físico a balances en sub-regiones del dominio, en lugar de en toda la región cuando los grupos tienen valor unidad. En el caso más general de medios anisótropos emergen nuevos grupos adimensionales no estudiados por otros autores cuyo número es inferior al obtenido mediante la adimensionalización clásica, un resultado fundamental y una aportación importante de esta memoria.

A pesar de su crítica, el problema de Henry ha sido aceptado como caso de prueba patrón para verificar códigos relacionados con flujo de densidad variable desde los años ochenta. Así, ha sido usado por Intera [1979] para SWIFT; por Voss [1984] para SUTRA; por Holzbecher [1991] para la versión temprana de FAST-C(2D); por Yeh y col [1993] para 2DFEMFAT; por Oldenburg y Press [1993] para TOUGH2; por Kolditz [1994] para FEFLOW; por Bastian y col. [1997] para UG y por Soto y col. [2007c] para FATSIM-A.

\section{III.1.2 NOMENCLATURA}

La nomenclatura y unidades de los parámetros físicos y variables del problema de Henry se recogen a continuación: 
$\mathrm{a}_{\mathrm{H}}$ arápetro del problema de Henry, $\mathrm{a}_{\mathrm{H}}=(\operatorname{kg} \Delta \rho \mathrm{L}) /\left(\varepsilon \mu \mathrm{v}_{\mathrm{amb}}{ }^{*} \mathrm{H}\right) \mathrm{b}_{\mathrm{H}}$

parámetro del problema de Henry, $\mathrm{b}_{\mathrm{H}}=\mathrm{D} /\left(\mathrm{v}_{\mathrm{amb}}{ }^{*} \mathrm{H}\right)$

C,Cs concentración de sal en el agua salada y en el agua de mar

D coeficiente de dispersión difusividad (como lo usa Henry) $\left(\mathrm{m}^{2} / \mathrm{s}\right.$ )

g aceleración de la gravedad $\left(\mathrm{m} / \mathrm{s}^{2}\right)$

h altura asociada a la diferencia de presión hidrostática (m)

$\mathrm{H}$ profundidad del acuífero (m)

$\mathrm{k} \quad$ permeabilidad hidráulica $\left(\mathrm{m}^{2}\right)$

L longitud del acuífero (m)

p presión $(\mathrm{Pa})$

q descarga específica $\mathrm{q}=\varepsilon \mathrm{v}(\mathrm{m} / \mathrm{s})$

Q caudal volumétrico entrante de agua dulce $\left(\mathrm{m}^{2} / \mathrm{s}\right)$

$\mathrm{t}$ tiempo (s)

$\mathrm{v} \quad \operatorname{velocidad}(\mathrm{m} / \mathrm{s})$

Vamb flujo regional causado por la diferencia de presión hidrostática (m/s)

$\mathrm{X}, \mathrm{y} \quad$ coordenadas espaciales (m)

$\beta \quad$ coeficiente de aumento de la densidad con la concentración (dimensional)

$\varepsilon \quad$ porosidad (adimensional)

$\xi \quad$ relación de aspecto, $\mathrm{L} / \mathrm{H}$, (adimensional)

$\psi \quad$ función de corriente $\left(\mathrm{m}^{2} / \mathrm{s}\right)$

$\mu \quad$ viscosidad dinámica del agua salada $\left(\mathrm{kg} \mathrm{m}^{-1} \mathrm{~s}^{-1}\right)$

$\pi$ denota monomio, grupo o número adimensional

$\rho_{\mathrm{s}}, \rho_{\mathrm{o}}$ densidades del agua salada y el agua dulce $\left(\mathrm{kg} \mathrm{m}^{-3}\right)$

\section{Subíndices}

amb referido a la velocidad regional

d referencia a un grupo adimensional discriminado

nd referencia a grupo adimensional no discriminado

f referencia al agua dulce

s referencia al agua salada

$\mathrm{x}, \mathrm{y}$ referencia a las direcciones espaciales $\mathrm{x}, \mathrm{y}$

$\mathrm{H} \quad$ asociado al problema de Henry

$1,2,3 \ldots$.. denota distintos grupos en un mismo problema

\section{Superíndices}


* $\quad$ asociado a una magnitud de referencia

, denota magnitud adimensional

\section{III.1.3 EL MODELO FÍSICO Y MATEMÁTICO DEL PROBLEMA DE HENRY}

El modelo físico de este problema se refiere a una sección vertical (2D) de un acuífero homogéneo e isótropo que recibe un flujo constante de agua dulce en su frontera izquierda (flujo continental o regional) mientras que su frontera derecha está en contacto con el mar. Sus fronteras superior e inferior son impermeables al paso de agua y de la sal. En la región derecha del acuífero se forma una región o zona de mezcla en la que el agua salada de mayor densidad procedente de la frontera marina penetra en el acuífero por el fondo mezclándose con el agua dulce hasta alcanzar un estado de equilibrio dinámico que llamaremos estacionario. La Figura III.1 muestra el modelo físico del problema y las condiciones de contorno expresadas en términos de las variables dependientes función de corriente $(\psi)$ y concentración (c), adoptadas por Henry y otros muchos autores.

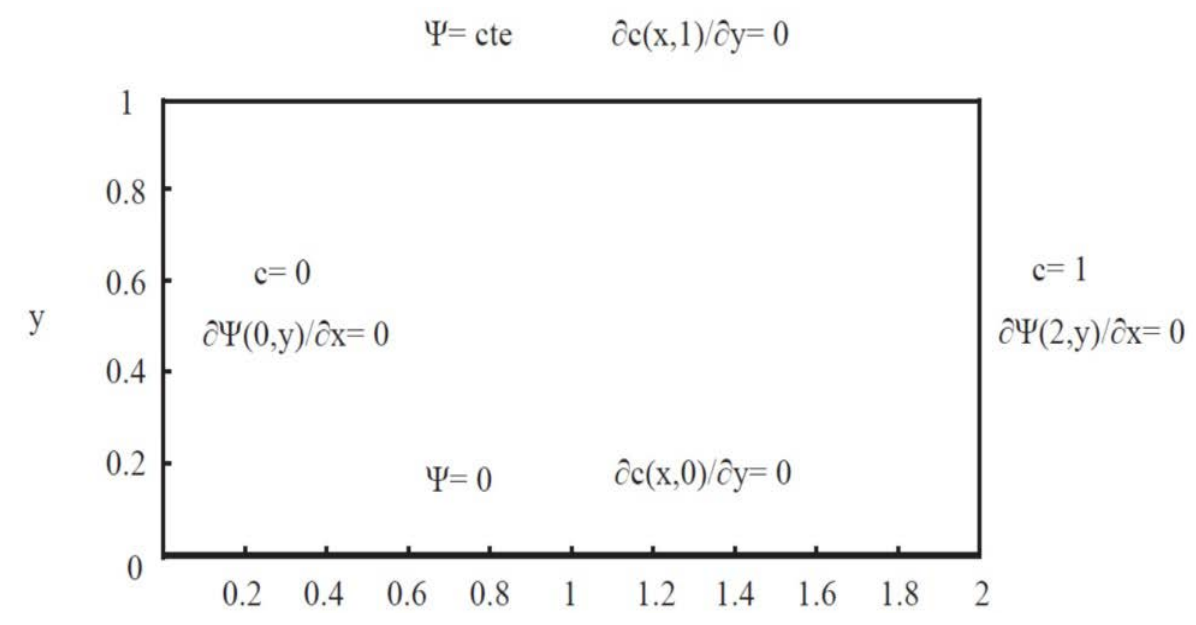

Figura III.1 Modelo físico del problema de Henry y condiciones de contorno

Las ecuaciones de gobierno del problema son las de continuidad (del agua y de la sal), más la ecuación constitutiva de Darcy (ecuación de momento) que relaciona el caudal con el gradiente hidráulico de presión:

$$
\begin{aligned}
& \nabla \mathbf{q}=0 \\
& \frac{\partial \rho}{\partial \mathrm{t}}-\mathbf{v} \cdot \nabla \rho=\nabla \cdot(\mathbf{D} \nabla \rho) \\
& \left(\frac{\mu}{\mathrm{k}}\right) \mathbf{q}+\nabla \mathrm{p}-(\Delta \rho) \mathbf{g}=0
\end{aligned}
$$


$\operatorname{con} \mathrm{q}=\mathrm{v} \varepsilon$.

El cambio de variable de velocidad a función de corriente se realiza mediante las expresiones

$$
\mathrm{q}_{\mathrm{x}}=\frac{\partial \psi}{\partial \mathrm{y}}, \quad \mathrm{q}_{\mathrm{y}}=-\frac{\partial \psi}{\partial \mathrm{x}}
$$

Las ecuaciones asociadas a las condiciones de contorno, mostradas en la figura anterior, se escriben a continuación para referenciarlas en el texto:

$$
\begin{array}{ll}
\text { Frontera inferior: } & \psi=0, \frac{\partial \mathrm{c}(\mathrm{x}, 0)}{\partial \mathrm{y}}=0 \\
\text { Frontera superior: } & \psi=\text { constante }, \frac{\partial \mathrm{c}(\mathrm{x}, 1)}{\partial \mathrm{y}}=0 \\
\text { Frontera izquierda: } & \mathrm{c}=0, \frac{\partial \psi(0, \mathrm{y})}{\partial \mathrm{x}}=0 \\
\text { Frontera derecha: } & \mathrm{c}=1, \frac{\partial \psi(1, \mathrm{y})}{\partial \mathrm{x}}=0
\end{array}
$$

La aproximación de Boussinesq,

$$
\Delta \rho=\rho_{\mathrm{o}} \beta(\Delta c)
$$

que aplica a este problema merced al pequeño cambio existente en las diferencias globales de densidad del agua, (cambio que no afecta a las fuerzas de flotación) permite sustituir la variable densidad por la variable concentración y reducir la ecuación (III.2) a

$$
\frac{\partial \rho}{\partial \mathrm{t}}-\mathbf{v} \cdot \nabla \mathrm{c}=\nabla \cdot(\mathbf{D} \nabla \mathbf{c})
$$

\section{III.1.4 DEDUCCIÓN DE LOS GRUPOS ADIMENSIONALES DISCRIMINADOS}

En relación con esta tarea hemos optado por presentarla siguiendo la misma cadencia temporal con que se ha desarrollado la investigación de esta memoria. Es precisamente esta cadencia la que ha hecho replantearnos críticamente, más de una vez, los resultados obtenidos tras cada proceso de adimensionalización bajo diferentes supuestos, para justificar la coherencia de los mismos en relación con los resultados esperados por la adimensionalización discriminada.

Así, el primer planteamiento fue deducir los grupos discriminados para un escenario anisótropo general y luego reducirlos a casos más simples entre los que está el 
escenario isótropo. Todo ello partiendo de L (la extensión del acuífero) como una variable influyente (no superflua) en el problema. La emergencia de magnitudes ocultas se estudió posteriormente y así se presenta en este trabajo.

El primer paso para la búsqueda de estos grupos es definir las magnitudes de referencia (longitudes y velocidades) que permitan definir las variables adimensionales, coordenadas espaciales y velocidad. En cuanto a las longitudes es inmediato elegir las que definen el dominio geométrico del problema (como hemos mencionado, dejamos para más tarde una discusión sobre la idoneidad o no idoneidad de esta elección)

$$
\mathrm{x}^{*}=\mathrm{L}, \quad \mathrm{y}^{*}=\mathrm{H}
$$

En relación con las velocidades disponemos también de dos referencias de velocidad, una para cada dirección espacial: la velocidad regional (horizontal) causada por el gradiente de presiones en los bordes laterales y la velocidad vertical asociada a los efectos de densidad variable o convección natural, derivada de la ley de Darcy,

$$
\begin{aligned}
& \mathrm{v}_{\mathrm{amb}}^{*}=\frac{\mathrm{q}_{\mathrm{amb}}^{*}}{\varepsilon}=\frac{\mathrm{k}_{\mathrm{x}}}{\mu} \nabla \mathrm{p}_{\mathrm{x}} \\
& \mathrm{v}_{\mathrm{y}}^{*}=\frac{\mathrm{q}_{\mathrm{y}}^{*}}{\varepsilon}=\frac{\mathrm{k}_{\mathrm{y}} \mathrm{g} \Delta \rho}{\varepsilon \mu}
\end{aligned}
$$

Con estas referencias, las variables adimensionales discriminadas se definen en la forma

$$
\begin{array}{ll}
\mathrm{x}^{\prime}=\frac{\mathrm{x}}{\mathrm{L}}, & \mathrm{y}^{\prime}=\frac{\mathrm{y}}{\mathrm{H}}, \\
\mathrm{v}_{\mathrm{x}}^{\prime}=\frac{\mathrm{q}_{\mathrm{x}}^{\prime}}{\varepsilon}=\frac{\mathrm{v}_{\mathrm{x}}}{\mathrm{v}_{\mathrm{amb}}^{*}}, & \mathrm{v}_{\mathrm{y}}^{\prime}=\frac{\mathrm{q}_{\mathrm{y}}^{\prime}}{\varepsilon}=\frac{\mathrm{v}_{\mathrm{y}}}{\mathrm{v}_{\mathrm{y}}^{*}}
\end{array}
$$

La ecuación de continuidad (III.1), aplicada a la región de mezcla cuya extensión es desconocida, pero del orden de magnitud L, puede ser escrita en la forma

$$
\frac{\partial \mathrm{q}_{\mathrm{x}}}{\partial \mathrm{x}}=\frac{\partial \mathrm{q}_{\mathrm{y}}}{\partial \mathrm{y}} \quad \text { o bien } \quad \frac{\partial \mathrm{v}_{\mathrm{x}}}{\partial \mathrm{x}}=\frac{\partial \mathrm{v}_{\mathrm{y}}}{\partial \mathrm{y}}
$$

$\mathrm{y}$, usando las referencias $\mathrm{x}^{*}=\mathrm{L}, \mathrm{y}^{*}=\mathrm{H}, \mathrm{v}_{\mathrm{x}}{ }^{*} \mathrm{y} \mathrm{v}_{\mathrm{y}}{ }^{*}=\left(\mathrm{k}_{\mathrm{y}} \mathrm{g} \Delta \rho\right) /(\varepsilon \mu)$, la forma adimensional de esta ecuación, a saber,

$$
\frac{\mathrm{v}_{\mathrm{x}}^{*}}{\mathrm{~L}}\left(\frac{\partial \mathrm{v}_{\mathrm{x}}^{\prime}}{\partial \mathrm{x}^{\prime}}\right)=\frac{\mathrm{v}_{\mathrm{y}}^{*}}{\mathrm{H}}\left(\frac{\partial \mathrm{v}_{\mathrm{y}}^{\prime}}{\partial \mathrm{y}^{\prime}}\right)
$$


proporciona una nueva referencia horizontal en la región de mezcla $\left(\mathrm{v}_{\mathrm{x}}{ }^{*}\right)$ que procede del grupo adimensional (derivado de la expresión anterior)

$$
\frac{\left(\frac{\mathrm{v}_{\mathrm{x}}^{*}}{\mathrm{~L}}\right)}{\frac{\mathrm{v}_{\mathrm{Y}}^{*}}{\mathrm{H}}}=\left(\frac{\mathrm{v}_{\mathrm{X}}^{*} \mathrm{H}}{\mathrm{v}_{\mathrm{y}}^{*} \mathrm{~L}}\right)
$$

Esta velocidad, que puede ser escrita en la forma

$$
\mathrm{V}_{\mathrm{x}}^{*}=\left(\frac{\mathrm{v}_{\mathrm{y}}^{*} \mathrm{~L}}{\mathrm{H}}\right)=\frac{\mathrm{k}_{\mathrm{y}} \mathrm{g} \Delta \rho \mathrm{L}}{\varepsilon \mu \mathrm{H}}
$$

Se trata de una especie de cociente de velocidades corregido, en el que el factor $\mathrm{L} / \mathrm{H}$ convierte el cociente $\mathrm{v}_{\mathrm{x}}{ }^{*} / \mathrm{v}_{\mathrm{y}}{ }^{*}$ en un grupo adimensional discriminado.

La existencia de dos referencias o velocidades características horizontales diferentes, $\mathrm{v}_{\mathrm{amb}}{ }^{*} \mathrm{y}_{\mathrm{x}}{ }^{*} \mathrm{o}\left(\mathrm{k}_{\mathrm{y}} \mathrm{g} \Delta \rho\right) \mathrm{L} /(\varepsilon \mu \mathrm{H})$, proporciona el primer grupo adimensional discriminado del problema de Henry (no recogido anteriormente en ninguna publicación):

$$
\pi_{\mathrm{d}, 1}=\frac{\mathrm{v}_{\mathrm{x}}^{*}}{\mathrm{v}_{\mathrm{amb}}^{*}}=\frac{\mathrm{k}_{\mathrm{y}} \mathrm{g}(\Delta \rho) \mathrm{L}}{\varepsilon \mu v_{\mathrm{amb}}^{*} \mathrm{H}}=\left(\frac{\mathrm{k}_{\mathrm{y}} \mathrm{g} \Delta \rho}{\varepsilon \mu v_{\mathrm{amb}}^{*}}\right)\left(\frac{\mathrm{L}}{\mathrm{H}}\right)
$$

Volviendo a la definición de velocidad horizontal adimensional, $\mathrm{v}_{\mathrm{x}}^{\prime}$, la existencia de dos referencias $\mathrm{v}_{\mathrm{x}}{ }^{*} \mathrm{y} \mathrm{v}_{\mathrm{amb}}{ }^{*}$, permite asociar dicha velocidad a cualquiera de ellas. A fin de hacer que el máximo valor de $v_{x}^{\prime}$ sea la unidad suele elegirse la referencia de mayor valor numérico.

En la propia región de mezcla, la ecuación de flujo, $\nabla \mathbf{q}=0$, proporciona otro número adimensional discriminado que relaciona las permeabilidades horizontal y vertical. En esta región, los flujos verticales y horizontales están impuestos por gradientes de presión (no por los cambios de densidad puesto que se trata de agua dulce) y podemos escribir

$$
\mathrm{q}_{\mathrm{x}}=\left(\frac{\mathrm{k}_{\mathrm{x}}}{\mu_{\mathrm{x}}}\right)\left(\frac{\partial \mathrm{p}}{\partial \mathrm{x}}\right) \quad \mathrm{y} \quad \mathrm{q}_{\mathrm{y}}=\left(\frac{\mathrm{k}_{\mathrm{y}}}{\mu_{\mathrm{y}}}\right)\left(\frac{\partial \mathrm{p}}{\partial \mathrm{y}}\right)
$$

Asumiendo una viscosidad constante y una referencia arbitraria para el gradiente de presión, $\Delta \mathrm{p}_{\text {o }}$, la adimensionalización de la ecuación de continuidad conduce a la expresión

$$
\left(\frac{\mathrm{k}_{\mathrm{x}} \Delta \mathrm{p}_{\mathrm{o}}}{\mu \mathrm{L}^{2}}\right)\left(\frac{\partial \mathrm{q}_{\mathrm{x}}^{\prime}}{\partial \mathrm{x}^{\prime}}\right)=\left(\frac{\mathrm{k}_{\mathrm{y}} \Delta \mathrm{p}_{\mathrm{o}}}{\mu \mathrm{H}^{2}}\right)\left(\frac{\partial \mathrm{q}_{\mathrm{y}}^{\prime}}{\partial \mathrm{y}}\right)
$$


Esta ecuación proporciona un nuevo número adimensional discriminado, una especie de cociente de permeabilidades corregido, que es independiente del tipo de fuerza que causa el movimiento del fluido

$$
\pi_{\mathrm{d}, 2}=\left(\frac{\mathrm{k}_{\mathrm{x}} \mathrm{H}^{2}}{\mathrm{k}_{\mathrm{y}} \mathrm{L}^{2}}\right)
$$

En relación con el coeficiente de dispersión asumiremos, como lo hace Kakinuma y col. [1988], y a diferencia de Henry y los demás autores, una cualidad anisótropa para este parámetro merced a la tortuosidad de los caminos de flujo de agua, en un intento de generalizar lo más posible el escenario de Henry. Con ello, la adimensionalización de la ecuación de transporte, eliminando la referencia común a todos los términos asociada a la concentración, $\Delta \mathrm{c}_{\mathrm{o}}=\mathrm{c}_{\mathrm{s}}-\mathrm{Cf}_{\mathrm{f}}$ y eligiendo como referencia horizontal para la velocidad $\mathrm{V}_{\mathrm{amb}}$, proporciona el resultado

$$
\left(\frac{1}{\mathrm{t}^{*}}\right)\left(\frac{\partial \mathrm{c}^{\prime}}{\partial \mathrm{t}^{\prime}}\right)=\left(\frac{\mathrm{v}_{\mathrm{amb}}^{*}}{\mathrm{~L}}\right)\left(\frac{\mathrm{v}_{\mathrm{x}}^{\prime} \partial \mathrm{c}^{\prime}}{\partial \mathrm{x}^{\prime}}\right)+\left(\frac{\mathrm{v}_{\mathrm{y}}^{*}}{\mathrm{H}}\right)\left(\frac{\mathrm{v}_{\mathrm{y}}^{\prime} \partial \mathrm{c}^{\prime}}{\partial \mathrm{y}^{\prime}}\right)+\left(\frac{\mathrm{D}_{\mathrm{x}}}{\mathrm{L}^{2}}\right)\left(\frac{\partial^{2} \mathrm{c}^{\prime}}{\partial \mathrm{x}^{\prime 2}}\right)+\left(\frac{\mathrm{D}_{\mathrm{y}}}{\mathrm{H}^{2}}\right)\left(\frac{\partial^{2} \mathrm{c}^{\prime}}{\partial \mathrm{y}^{\prime 2}}\right)
$$

Obviaremos el término referente al tiempo ya que estamos interesados por el momento en la solución estacionaria. Para este escenario, la anterior ecuación reducida a

$$
\left(\frac{\mathrm{v}_{\mathrm{amb}}^{*}}{\mathrm{~L}}\right)\left(\frac{\mathrm{v}_{\mathrm{x}}^{\prime} \partial \mathrm{c}^{\prime}}{\partial \mathrm{x}^{\prime}}\right)+\left(\frac{\mathrm{v}_{\mathrm{y}}^{*}}{\mathrm{H}}\right)\left(\frac{\mathrm{v}_{\mathrm{y}}^{\prime} \partial \mathrm{c}^{\prime}}{\partial \mathrm{y}^{\prime}}\right)+\left(\frac{\mathrm{D}_{\mathrm{x}}}{\mathrm{L}^{2}}\right)\left(\frac{\partial^{2} \mathrm{c}^{\prime}}{\partial \mathrm{x}^{\prime 2}}\right)+\left(\frac{\mathrm{D}_{\mathrm{y}}}{\mathrm{H}^{2}}\right)\left(\frac{\partial^{2} \mathrm{c}^{\prime}}{\partial \mathrm{y}^{\prime 2}}\right)=0
$$

proporciona cuatro coeficientes de la misma ecuación dimensional (la inversa de un tiempo), a saber

$$
\begin{aligned}
& \frac{\mathrm{v}_{\mathrm{amb}}^{*}}{\mathrm{~L}} \\
& \left(\frac{\mathrm{v}_{\mathrm{y}}^{*}}{\mathrm{H}}\right)=\frac{(\Delta \rho) \mathrm{gk}_{y}}{\varepsilon \mu \mathrm{H}} \\
& \frac{\mathrm{D}_{x}}{\mathrm{~L}^{2}} \\
& \frac{\mathrm{D}_{y}}{\mathrm{H}^{2}}
\end{aligned}
$$

Para obtener los grupos adimensionales dividiremos los coeficientes anteriores entre uno de ellos, por ejemplo, el primero. El resultado es

$$
\pi_{\mathrm{d}, 1}=\frac{(\Delta \rho) g k_{\mathrm{y}}}{\varepsilon \mu \mathrm{v}_{\mathrm{amb}}^{*}}\left(\frac{\mathrm{L}}{\mathrm{H}}\right)
$$




$$
\begin{aligned}
& \pi_{d, 3}=\frac{D_{x}}{v_{a m b}^{*}{ }^{L}} \\
& \pi_{d, 3}=\frac{D_{x} L}{v_{a m b}^{*} H^{2}}
\end{aligned}
$$

Adviértase que el primero de estos grupos ya fue derivado anteriormente con lo que son cuatro los grupos adimensionales independientes que controlan la solución del problema estacionario. Los presentamos de la forma más conveniente combinando los grupos $\pi_{\mathrm{d}, 3} \mathrm{y} \pi_{\mathrm{d}, 4}$ en (III.15), para eliminar la velocidad en el último grupo. El resultado es el conjunto de grupos

$$
\begin{aligned}
& \pi_{\mathrm{d}, 1}=\frac{(\Delta \rho) \mathrm{gk}_{\mathrm{y}}}{\varepsilon \mu \mathrm{v}_{\mathrm{amb}}^{*}}\left(\frac{\mathrm{L}}{\mathrm{H}}\right) \\
& \pi_{\mathrm{d}, 2}=\frac{\mathrm{k}_{\mathrm{x}} \mathrm{H}^{2}}{\mathrm{k}_{\mathrm{y}} \mathrm{L}^{2}} \\
& \pi_{\mathrm{d}, 3}=\frac{\mathrm{D}_{\mathrm{x}}}{\mathrm{v}_{\mathrm{amb}}^{*} \mathrm{~L}} \\
& \pi_{\mathrm{d}, 4}=\frac{\mathrm{D}_{\mathrm{x}} \mathrm{H}^{2}}{\mathrm{D}_{\mathrm{y}} \mathrm{L}^{2}}
\end{aligned}
$$

A la vista de estas soluciones es interesante advertir que el uso de la discriminación para adimensionalizar las ecuaciones de gobierno no permite la emergencia en medios anisótropos de grupos adimensionales independientes del tipo de factores de forma ( $\mathrm{L} / \mathrm{H}$, por ejemplo), sino que estos cocientes se unen con los cocientes de los parámetros físicos anisótropos, tales como la permeabilidad y la dispersióndifusión, para formar nuevos grupos adimensionales discriminados. El cociente $\mathrm{L} / \mathrm{H}$, que no puede formarse a partir de los grupos deducidos ni de las condiciones de contorno, actúa como un factor de corrección de los cocientes de estos parámetros físicos anisótropos. Anteriormente ya se ha visto que el cociente L/H también actúa como factor de corrección para el cociente de velocidades en diferentes direcciones, por ejemplo en el grupo $\pi_{\mathrm{d}, 1}$ definido en (III.11).

En relación con su valor, los grupos anteriores (III.16 a III.19) deben ser de orden de magnitud unidad ya que emergen del balance de términos de las ecuaciones de gobierno, habiendo asumido la hipótesis de que en promedio las variables adimensionales y sus cambios son de orden de magnitud unidad. Si se asume una influencia despreciable de uno de los términos de la ecuación de gobierno, no existirá ningún coeficiente asociado 
ni podrá formarse grupo adimensional alguno con los parámetros contenidos en dicho término.

Finalmente, en relación con el significado físico de los grupos discriminados existe una diferencia respecto de los grupos deducidos de forma clásica. Por ejemplo, el grupo $\pi_{\mathrm{d} .1}$ (III.6) escrito en la forma $\left(\mathrm{v}_{\mathrm{y}}{ }^{*} / \mathrm{H}\right) /\left(\mathrm{V}_{\mathrm{amb}}{ }^{*} / \mathrm{L}\right)$, puede interpretarse como el cociente entre dos tiempos: el requerido por el fluido en su movimiento para cubrir la anchura del acuífero (L) animado de la velocidad de referencia horizontal ( $\mathrm{vamb}^{*}$ ) y el requerido para recorrer la profundidad del acuífero $(\mathrm{H})$ con la velocidad de referencia vertical $\left(\mathrm{v}_{\mathrm{y}}{ }^{*}\right)$. Interpretaciones de índole similar pueden ser atribuidas al resto de los grupos. La Tabla III.1 recoge posibles significados físicos para los grupos adimensionales deducidos.

Tabla III.1 Posibles significados de los grupos discriminados

\begin{tabular}{|c|l|l|}
\hline $\begin{array}{c}\text { Grupos } \\
\text { Discriminados }\end{array}$ & \multicolumn{1}{c|}{ Definición } & \multicolumn{1}{c|}{ Significado físico } \\
\hline$\pi_{\mathrm{d}, 1}=\frac{(\Delta \rho) g \mathrm{k}_{\mathrm{y}}}{\varepsilon \mu \mathrm{v}_{\mathrm{amb}}^{*}}\left(\frac{\mathrm{L}}{\mathrm{H}}\right)$ & $\begin{array}{l}\text { Cociente de velocidades } \\
\text { corregido }\end{array}$ & $\begin{array}{l}\text { Tiempo requerido por el fluido en recorrer } \mathrm{L} \\
\text { con velocidad } \mathrm{v}_{\mathrm{amb}}^{*} / \text { ídem para cubrir } \mathrm{H} \text { con } \\
\text { velocidad } \mathrm{v}_{\mathrm{y}}^{*}\end{array}$ \\
\hline$\pi_{\mathrm{d}, 2}=\frac{\mathrm{k}_{\mathrm{x}} \mathrm{H}^{2}}{\mathrm{k}_{\mathrm{y}} \mathrm{L}^{2}}$ & $\begin{array}{l}\text { Cociente de } \\
\text { permeabilidades } \\
\text { corregido }\end{array}$ & $\begin{array}{l}\text { Tiempo requerido por el fluido en recorrer } \mathrm{L} \\
\text { con permeabilidad } \mathrm{k}_{\mathrm{x}} / \text { /ídem para cubrir } \mathrm{H} \\
\text { con permeabilidad } \mathrm{k}_{\mathrm{y}} \text { (para un mismo } \\
\text { gradiente de presión en ambos casos) }\end{array}$ \\
\hline$\pi_{\mathrm{d}, 3}=\frac{\mathrm{D}_{\mathrm{x}}}{\mathrm{v}_{\mathrm{amb}}^{*} \mathrm{~L}}=\frac{\mathrm{D}_{\mathrm{x}} \mathrm{L}}{\mathrm{v}_{\mathrm{amb}}^{*} \mathrm{~L}^{2}}$ & $\begin{array}{l}\text { Cociente de dispersión y } \\
\text { arrastre (horizontales) }\end{array}$ & $\begin{array}{l}\text { Tiempo requerido por el fluido en recorrer } \mathrm{L} \\
\text { con velocidad } \mathrm{v}_{\text {amb }}{ }^{*} / \text { ídem (debido a los } \\
\text { efectos de difusión) }\end{array}$ \\
\hline$\pi_{\mathrm{d}, 4}=\frac{\mathrm{D}_{\mathrm{x}} \mathrm{H}^{2}}{\mathrm{D}_{\mathrm{y}} \mathrm{L}^{2}}$ & $\begin{array}{l}\text { Cociente de difusividades } \\
\text { corregido }\end{array}$ & $\begin{array}{l}\text { Tiempo requerido por el fluido en recorrer } \\
\text { H/ídem para cubrir } \mathrm{L} \text { (debido en ambos } \\
\text { casos a los efectos de difusión) }\end{array}$ \\
\hline
\end{tabular}

La adimensionalización clásica sólo puede interpretar los monomios que de ella derivan en términos de balance de magnitudes cuando los escenarios son isótropos y, quizás, ni siquiera en esos casos. Por un lado, un factor de forma derivado de un cociente corregido de permeabilidades o difusividades no es balance de ninguna magnitud que interactúe en el dominio del problema; por otro, un cociente de velocidades vertical/horizontal, tampoco es ningún balance, su valor puede ser de cualquier orden sin que ello suponga que dicho cociente influye decisivamente en los patrones de la solución. 
Si Vhorizontal $/ \mathrm{V}_{\text {vertical }}>>1$, el monomio puede influir o no en el problema en función del cociente $\mathrm{L} / \mathrm{H}$ : si éste es pequeño $(\mathrm{L}<<\mathrm{H})$ las partículas de fluido recorren el camino vertical en un tiempo despreciable comparado con el tiempo que emplean esas mismas partículas en recorrer el camino horizontal y, en consecuencia, el monomio $\mathrm{V}_{\text {horizontal }} / \mathrm{V}_{\text {vertical }}$ no juega papel alguno en el patrón de solución; si por el contrario L $>>\mathrm{H}$, estos tiempos pueden ser (o no) comparables y el monomio determina (o no) el patrón de solución del problema.

\section{III.1.5 JUSTIFICACIÓN DE LOS NÚMEROS ADIMENSIONALES DEDUCIDOS MEDIANTE LA DISCRIMINACIÓN}

La tabla III.2 muestra diferentes escenarios del este problema, elegidos para verificar la influencia de los grupos adimensionales discriminados en el patrón de soluciones del mismo. Para el escenario 'caso 1', los valores de los parámetros, marcados con el subíndice $\mathrm{H}$, corresponden a los adoptados por Henry:

$\begin{array}{ll}\mathrm{L}_{\mathrm{H}}=2 \mathrm{~m}, & \text { Longitud } \\ \mathrm{H}_{\mathrm{H}}=1 \mathrm{~m}, & \text { Anchura } \\ \mathrm{k}_{\mathrm{H}}=1.0204 \mathrm{E}-9 \mathrm{~m}^{2}, & \text { Permeabilidad } \\ \mathrm{D}_{\mathrm{H}}=1.8857 \mathrm{E}-5 \mathrm{~m}^{2} / \mathrm{s}, & \text { Difusividad } \\ \mu=1 \mathrm{E}-3 \mathrm{~kg} \mathrm{~m}^{-1} \mathrm{~s}^{-1}, & \text { Viscosidad } \\ \varepsilon_{\mathrm{H}}=0.35, & \text { Porosidad } \\ \Delta \rho_{\mathrm{H}}=25 \mathrm{~kg} / \mathrm{m}^{3}, & \text { Incremento de densidad } \\ \mathrm{V}_{\mathrm{amb}, \mathrm{H}}=1.8857 \mathrm{E}-4 \mathrm{~m} / \mathrm{s}, & \text { Flujo regional }\end{array}$

Los valores de la función de corriente, definida en la ecuación (III.4), son los siguientes:

Base del acuífero: $\Psi_{\mathrm{H}}=0 \mathrm{~m}^{2} / \mathrm{s}$

Frontera superior: $\Psi_{\mathrm{H}}=6.6 \mathrm{E}-5 \mathrm{~m}^{2} / \mathrm{s}$

Estos valores cambian en los casos en que cambia la velocidad $\mathrm{v} a m b^{*}$ o cuando cambia H, para poder ajustar el valor de los grupos. En los bordes laterales, la condición es $\mathrm{v}_{\mathrm{y}}=0$ se traduce para la función de corriente en

Fronteras verticales: $\partial \Psi_{\mathrm{H}} / \partial \mathrm{x}=0 \mathrm{~m}^{2} / \mathrm{s}$

Finalmente, la recarga vale $\mathrm{q}_{\mathrm{H}}=\mathrm{v}_{\mathrm{amb}, \mathrm{H}} * \varepsilon_{\mathrm{H}}$ 
Tabla III.2 Valores de los parámetros para distintos escenarios

\begin{tabular}{|c|c|c|c|c|c|c|c|c|c|c|}
\hline Caso & $\mathbf{L}$ & $\mathbf{H}$ & $\mathbf{k}_{\mathrm{x}}$ & $\mathbf{k}_{\mathbf{y}}$ & $\mathbf{D}_{\mathbf{x}}$ & $\mathbf{D}_{\mathbf{y}}$ & $\mathbf{v}_{\mathrm{amb}}{ }^{*}$ & Dr & $\epsilon_{H}$ & $\Psi_{\mathrm{H}}$ \\
\hline \multirow[t]{2}{*}{1} & $\mathbf{L}_{\mathbf{H}}$ & $2 \mathbf{H}_{\mathrm{H}}$ & $\mathbf{k}_{\mathbf{H}}$ & $4 \mathbf{k}_{\mathrm{H}}$ & $\mathbf{D}_{\mathbf{H}}$ & $4 D_{H}$ & $\mathbf{v a m b , H}{ }^{*}$ & $(1 / 2) \mathrm{Dr}_{\mathrm{H}}$ & $\epsilon_{\mathbf{H}}$ & $2 \Psi_{H}$ \\
\hline & 2 & 2 & $1,0204 \mathrm{E}-09$ & 4,0816E-09 & 1,8857E-05 & 7,5428E-05 & 1,8857E-04 & 12,5 & 0,35 & 1,32E-04 \\
\hline \multirow[t]{2}{*}{2} & $2 \mathbf{L}_{\mathrm{H}}$ & $\mathbf{H}_{\mathbf{H}}$ & $4 k_{H}$ & $\mathbf{k}_{\mathrm{H}}$ & $2 \mathbf{D}_{\mathrm{H}}$ & $(1 / 2) D_{H}$ & $\mathbf{V}_{\mathrm{amb}, \mathrm{H}}{ }^{*}$ & $(1 / 2) D r_{H}$ & $\epsilon_{\mathbf{H}}$ & $\Psi_{\mathrm{H}}$ \\
\hline & 4 & 1 & 4,0816E-09 & $1,0204 \mathrm{E}-09$ & 3,7714E-05 & $9,4285 \mathrm{E}-06$ & 1,8857E-04 & 12,5 & 0,35 & $6,60 \mathrm{E}-05$ \\
\hline \multirow[t]{2}{*}{3} & $2 \mathbf{L}_{\mathrm{H}}$ & $\mathbf{H}_{\mathbf{H}}$ & $4 k_{H}$ & $\mathbf{k}_{\mathrm{H}}$ & $4 D_{H}$ & $\mathbf{D}_{\mathrm{H}}$ & $2 \mathbf{v}_{\mathrm{amb}, \mathrm{H}}{ }^{*}$ & $\mathrm{Dr}_{\mathrm{H}}$ & $\epsilon_{\mathbf{H}}$ & $2 \Psi_{H}$ \\
\hline & 4 & 1 & 4,0816E-09 & $1,0204 \mathrm{E}-09$ & 7,5428E-05 & $1,8857 \mathrm{E}-05$ & 3,7714E-04 & 25 & 0,35 & 1,32E-04 \\
\hline \multirow[t]{2}{*}{4} & $\mathbf{L}_{\mathbf{H}}$ & $2 \mathbf{H}_{H}$ & $(1 / 4) k_{H}$ & $\mathbf{k}_{\mathrm{H}}$ & $(\mathbf{1} / 4) D_{H}$ & $\mathbf{D}_{\mathrm{H}}$ & $(1 / 4) \mathbf{V}_{\mathrm{amb}, \mathrm{H}}{ }^{*}$ & $(1 / 2) D r_{H}$ & $\epsilon_{H}$ & $(1 / 2) \Psi_{H}$ \\
\hline & 2 & 2 & 2,5510E-10 & $1,0204 \mathrm{E}-09$ & 4,7143E-06 & 1,8857E-05 & 4,7143E-05 & 12,5 & 0,35 & 3,30E-05 \\
\hline \multirow[t]{2}{*}{5} & $2 \mathbf{L}_{\mathbf{H}}$ & $1.5 \mathrm{H}_{\mathrm{H}}$ & $(2 / 3)^{2} k_{H}$ & $(\mathbf{1} / 4) \mathbf{k}_{\mathrm{H}}$ & $(8 / 9) D_{H}$ & $(1 / 2) D_{H}$ & $(4 / 9) \mathbf{v}_{a m b, H}{ }^{*}$ & (4/3)Dr & $\epsilon_{H}$ & $(2 / 3) \Psi_{H}$ \\
\hline & 4 & 1,5 & $4,5351 \mathrm{E}-10$ & $2,5510 \mathrm{E}-10$ & $1,6762 \mathrm{E}-05$ & $9,4285 \mathrm{E}-06$ & 8,3809E-05 & 33,33333 & 0,35 & 4,40E-05 \\
\hline \multirow[t]{2}{*}{6} & $\mathbf{L}_{\mathbf{H}}$ & $\mathbf{H}_{\mathbf{H}}$ & $\mathbf{k}_{\mathbf{H}}$ & $\mathbf{k}_{\mathrm{H}}$ & $\mathbf{D}_{\mathrm{H}}$ & $\mathbf{D}_{\mathrm{H}}$ & $\mathbf{V}_{\mathrm{amb}, \mathrm{H}}{ }^{*}$ & $(1 / 2) D r_{H}$ & $(1 / 2) \epsilon_{H}$ & $(1 / 2) \Psi_{H}$ \\
\hline & 2 & 1 & $1,0204 \mathrm{E}-09$ & $1,0204 \mathrm{E}-09$ & 1,8857E-05 & 1,8857E-05 & 1,8857E-04 & 12,5 & 0,175 & 3,30E-05 \\
\hline
\end{tabular}

Para los casos 2 a 6 los parámetros de los sucesivos escenarios se han cambiado ordenadamente de forma que los valores numéricos de los grupos adimensionales discriminados permanecen inalterables. Estos valores son:

$$
\pi_{\mathrm{d}, 1}=7.5834 \quad \pi_{\mathrm{d}, 2}=0.25 \quad \pi_{\mathrm{d}, 3}=0.05 \quad \pi_{\mathrm{d}, 4}=0.25
$$

Los cambios de valor de entre parámetros introducidos en los casos 1 a 6 son significativos a fin de elegir un conjunto representativo de escenarios. Las desviaciones de valores pueden dar lugar a un alejamiento de la aproximación de Boussinesq pero esta prueba no depende de que dicha aproximación se cumpla o no; es decir, para los mismos valores de los parámetros adimensionales, los patrones de solución deben ser idénticos de acuerdo con los resultados de la teoría de funciones homogéneas (San Juan [1947]). 
Las simulaciones en FATSIM-A para un mallado de 40 (celdas horizontales) $\times 20$ (celdas verticales) se recogen en las siguientes figuras. La Figura III.2 muestra en primer lugar los patrones de isoconcentración e isofunción de corriente del problema de Henry (Caso 1). Para asegurar que el estado estacionario ha sido alcanzado en todos los casos se ha modificado convenientemente la ventana de simulación de tiempos.
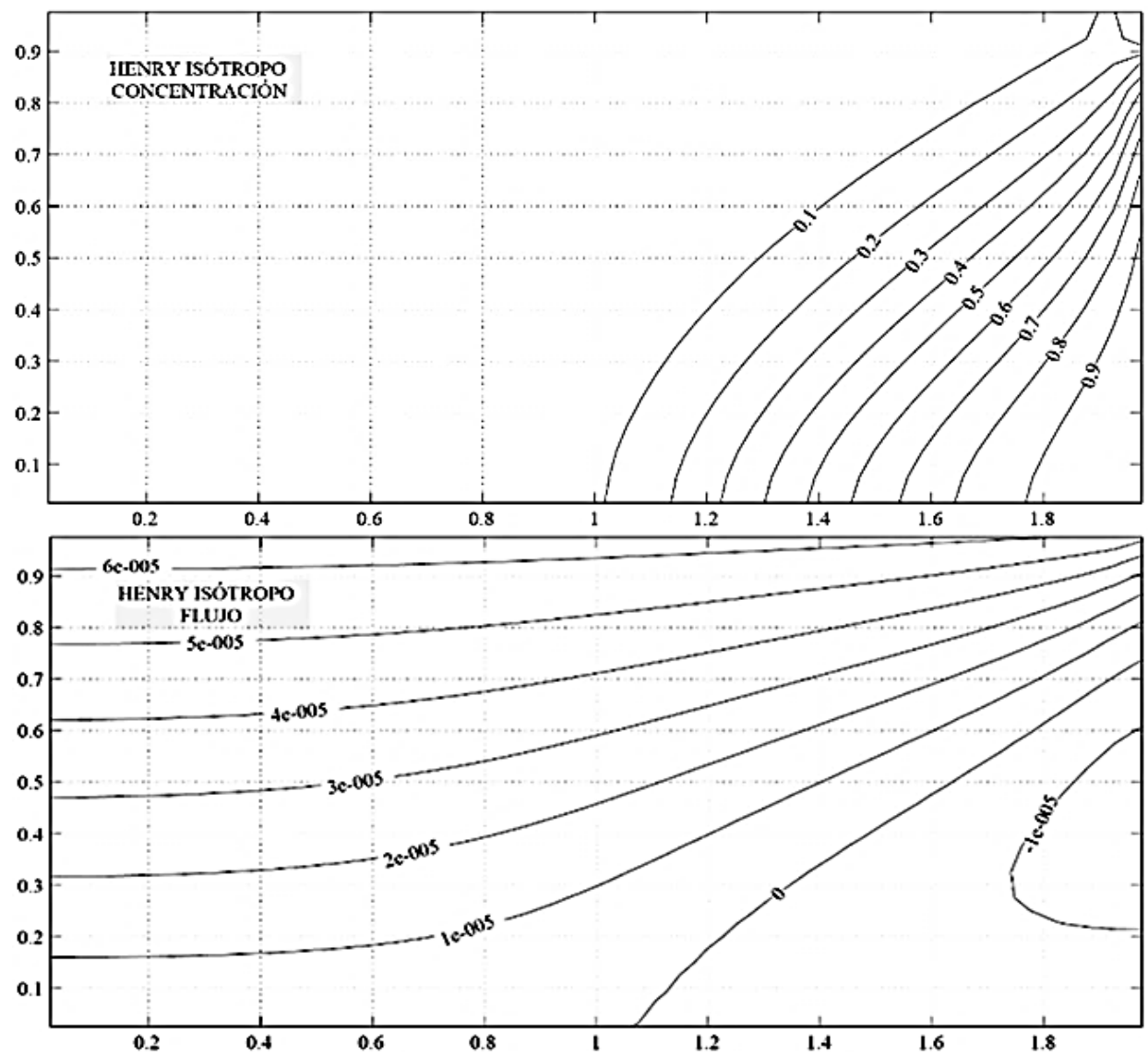

Figura III.2 Patrones estacionarios de solución del problema de Henry obtenidos mediante FATSIM-A. Isolíneas de concentración (arriba) e isolíneas de función de corriente (abajo)

Las Figuras III.3a-b y III.4a-b muestran estos patrones para los seis escenarios de la Tabla III.2. Puede apreciarse que los resultados son prácticamente idénticos, demostrando que los grupos emergentes de la adimensionalización discriminada gobiernan realmente la solución del problema de manera independiente. Adviértase que los ejes del escenario han sido escalados convenientemente (cuando cambia el valor de la longitud o la altura del acuífero) para una mejor comparación a escala de los patrones estacionarios. 
Capítulo III. Aplicación de la adimensionalización discriminada al problema de Henry
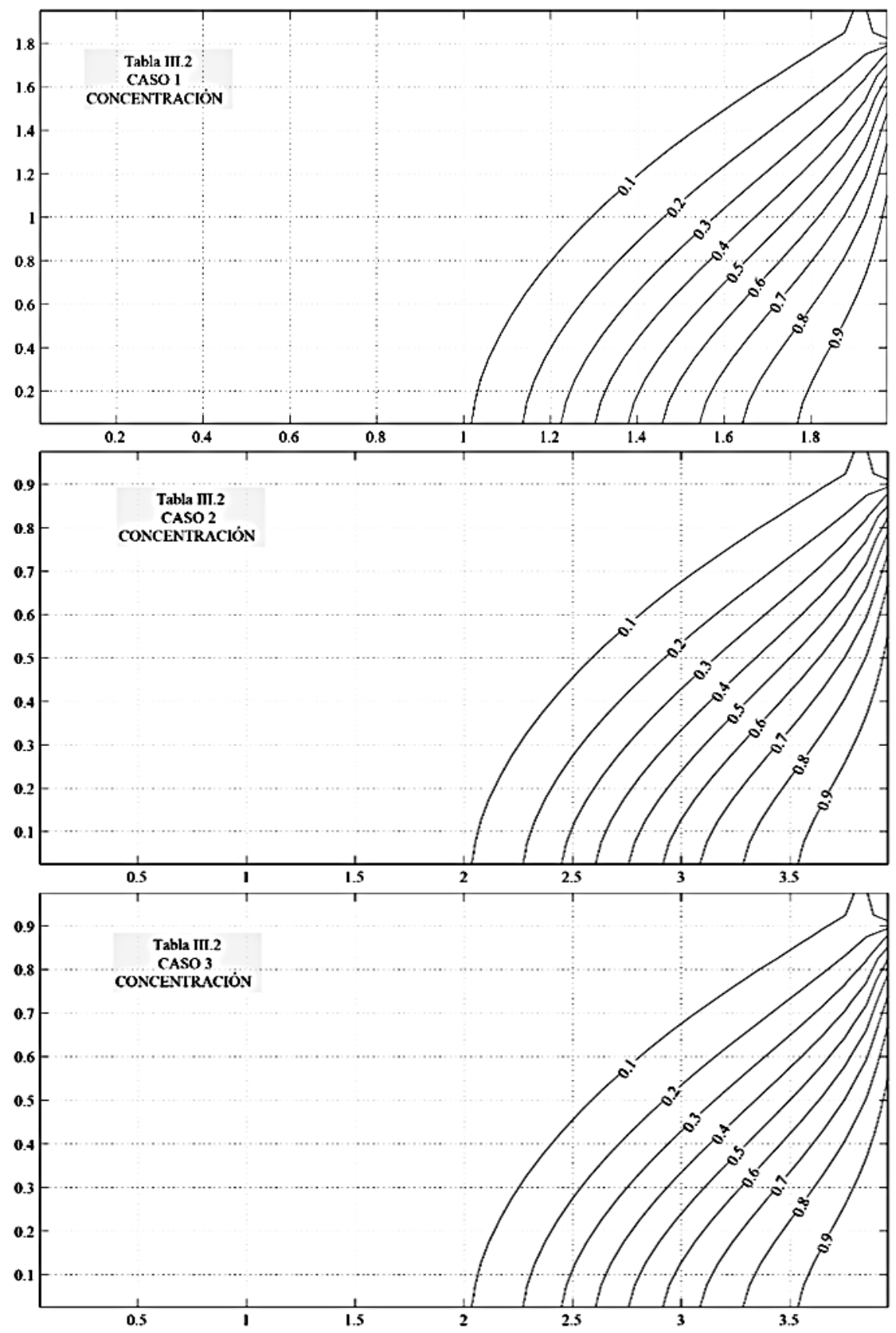

Figura III.3a Patrones estacionarios de isolíneas de concentración para casos 1 a 3 de la Tabla III.2 
Capítulo III. Aplicación de la adimensionalización discriminada al problema de Henry
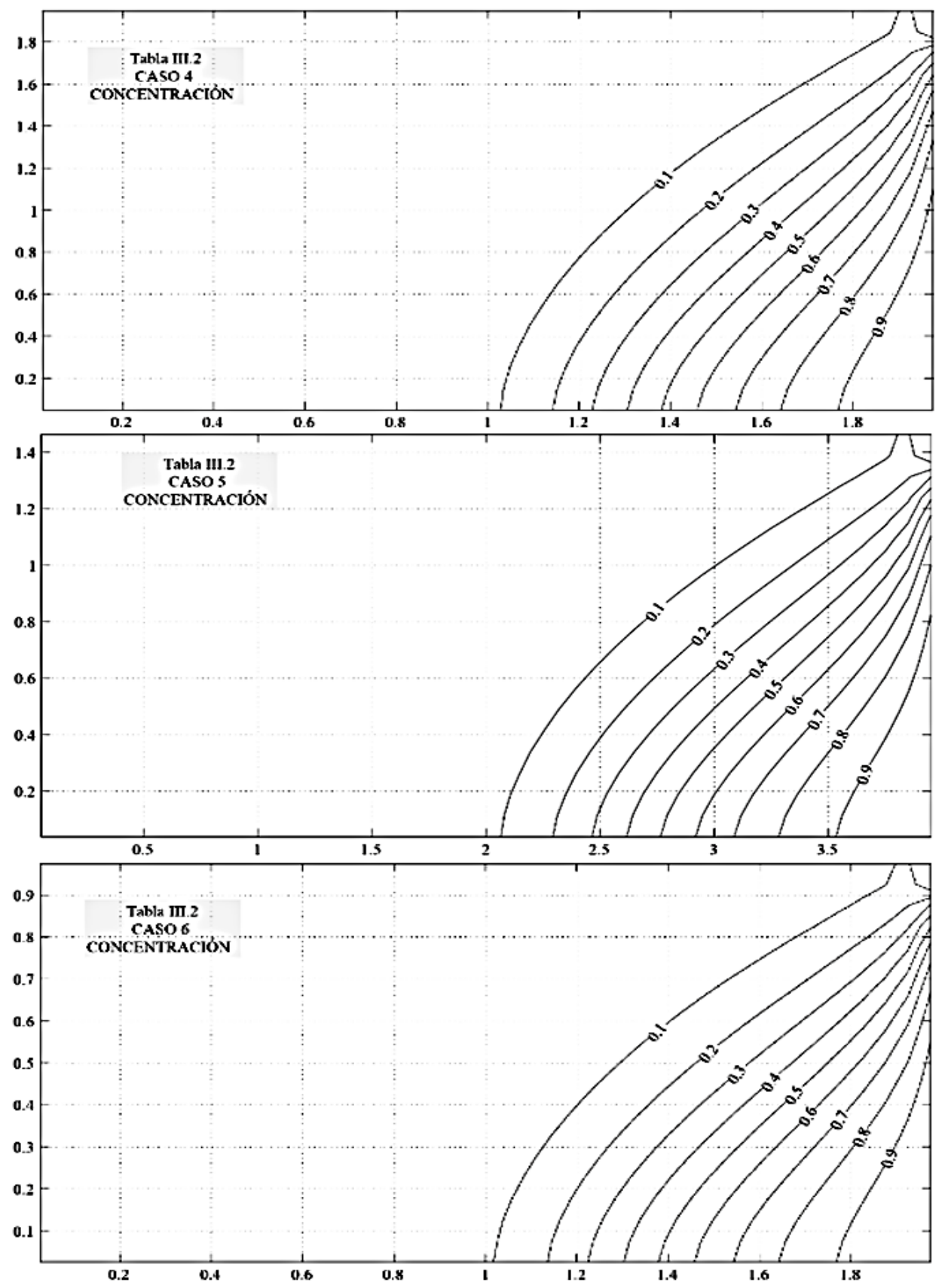

Figura III.3b. Patrones estacionarios de isolíneas de concentración para los casos 4 a 6 de la Tabla III.2 
Capítulo III. Aplicación de la adimensionalización discriminada al problema de Henry
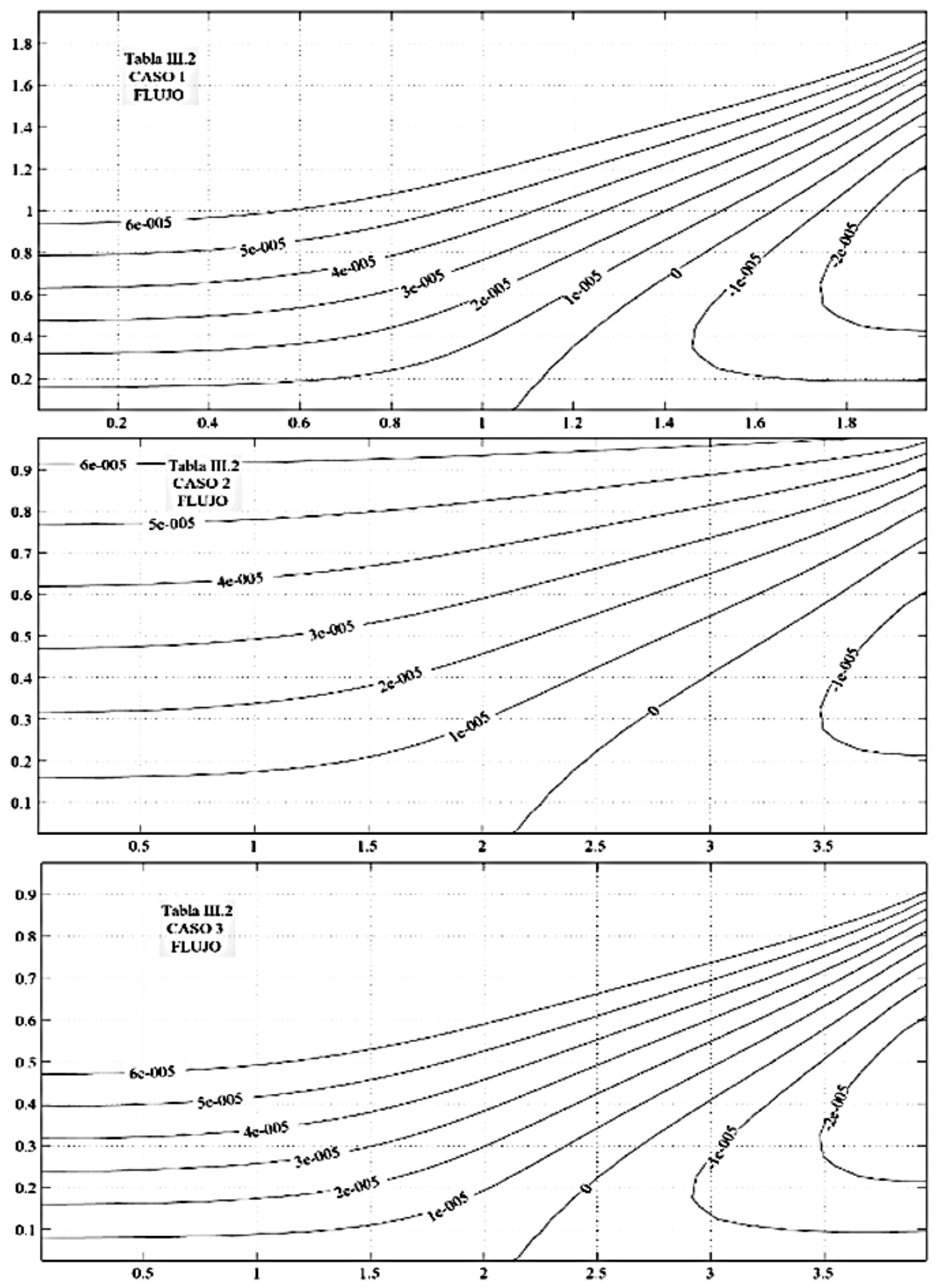

Figura III.4a Patrones estacionarios de isolíneas de flujo para los casos 1, 3 y 5 de la Tabla III.2 

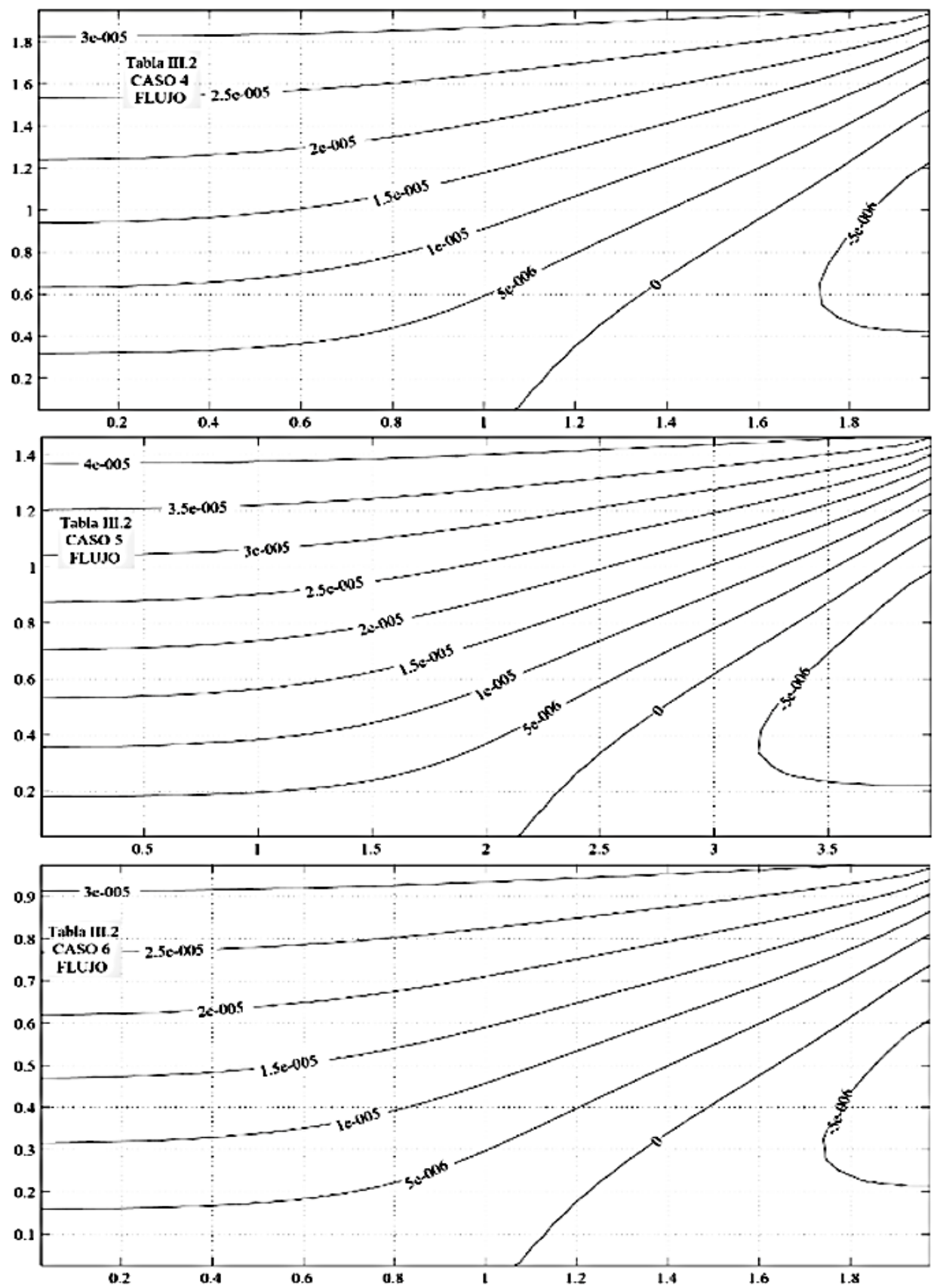

Figura III.4b Patrones estacionarios de isolíneas de flujo para los casos 2, 4 y 6 de la Tabla III.2

Para mejor ilustración, la Figura III.5 muestra, mediante ejes normalizados, tres líneas típicas de concentración y la de función de corriente constante correspondiente a la divisoria, $\mathrm{c}^{\prime}=0.2,0.5$ y $0.8, \mathrm{y} \Psi_{\mathrm{H}}=0$, respectivamente, para los seis casos. De nuevo se trata de soluciones idénticas para una misma posición relativa ( $x^{\prime}$ e y’), como esperábamos. Idénticos resultados se obtienen para las otras líneas de corriente una vez normalizado su valor (el de $\Psi_{\mathrm{H}}=0$ no es preciso normalizarlo obviamente). 


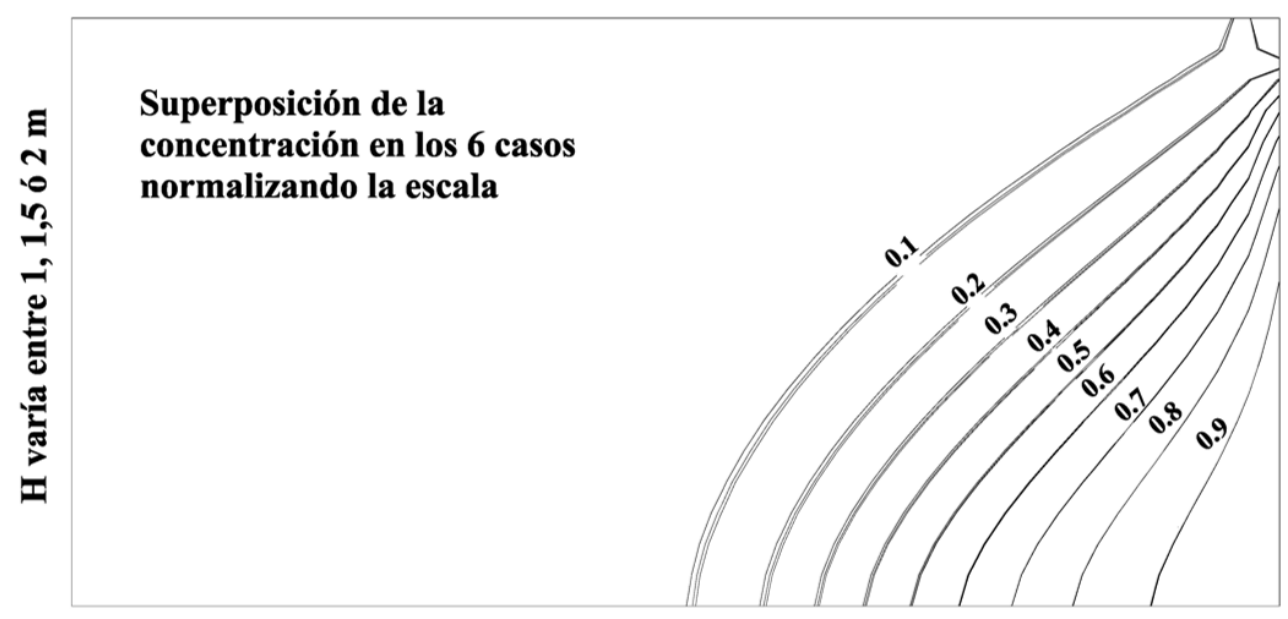

\section{L varía entre 2 ó 4 metros}

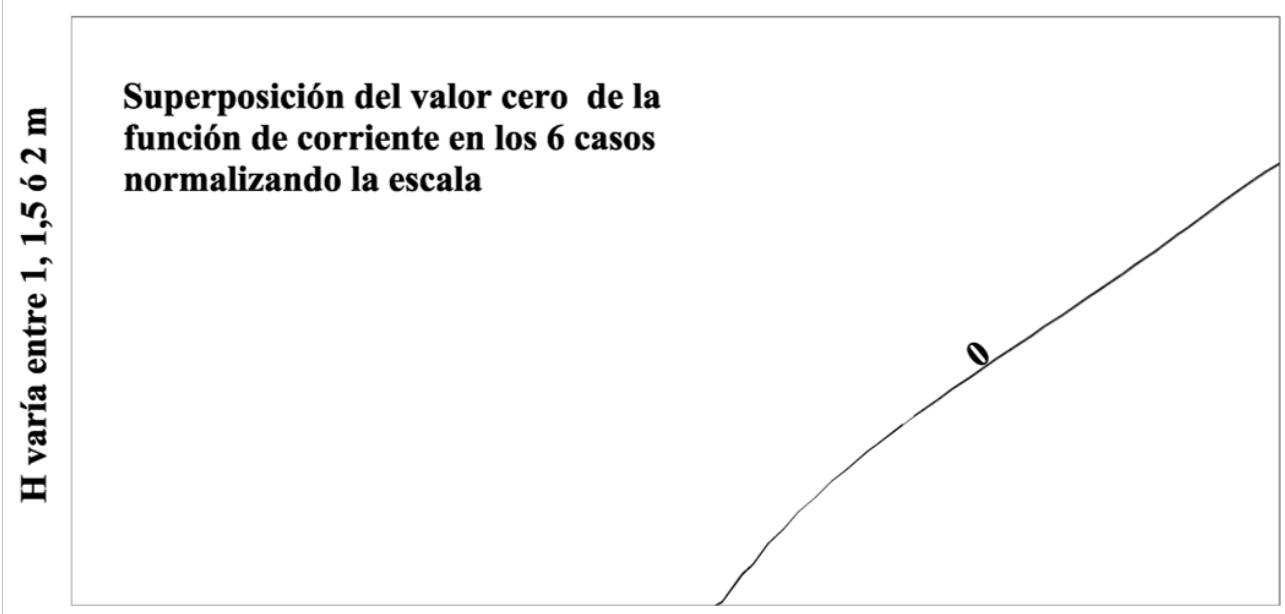

L varía entre 2 ó 4 metros

Figura III.5 Isolíneas típicas de concentración (arriba) y función de corriente (abajo) para los seis casos de la Tabla III.2, en escala normalizada, $c^{\prime}=0.2,0.5$ y $0.8 ; \Psi_{\mathrm{H}}=0$

Para apreciar mejor los resultados, la Tabla III.3 recoge los valores numéricos de la posición vertical relativa $\left(x^{\prime}=x / L\right)$ de tres isolíneas típicas de concentración para una posición horizontal relativa común de valor $\mathrm{y}=\mathrm{H} / 2$ (o y’=0.5).

El error relativo máximo, del orden del 1\%, es atribuido, sin duda, al pequeño tamaño de la malla. Con todo, se vuelve a verificar que los grupos resultantes de proceso de adimensionalización discriminada son los que controlan de manera independiente los patrones de solución del problema de Henry anisótropo. 
Tabla III.3 Posición relativa horizontal $\left(\mathrm{x}^{\prime}=\mathrm{x} / \mathrm{L}\right)$ de las isolíneas $\mathrm{c}^{\prime}=0.2,0.5$ y 0.8, para y’=0.5

\begin{tabular}{|c|c|c|c|c|c|c|c|}
\hline Caso & $\mathbf{1}$ & $\mathbf{2}$ & $\mathbf{3}$ & $\mathbf{4}$ & $\mathbf{5}$ & $\mathbf{6}$ & Henry \\
\hline $\mathrm{c}=0.2$ & 0.725 & 0.730 & 0.729 & 0.732 & 0.734 & 0.730 & 0.730 \\
\hline $\mathrm{c}=0.5$ & 0.874 & 0.875 & 0.874 & 0.875 & 0.878 & 0.873 & 0.870 \\
\hline $\mathrm{c}=0.8$ & 0.961 & 0.960 & 0.960 & 0.963 & 0.962 & 0.962 & 0.960 \\
\hline
\end{tabular}

\section{III.1.6 USO DE LOS GRUPOS DISCRIMINADOS PARA UNA ELECCIÓN ÓPTIMA DEL PROBLEMA PATRÓN}

Como se ha mencionado y demostrado para el problema de Henry, los grupos adimensionales provenientes de la adimensionalización discriminada pueden interpretarse físicamente en términos de balance de magnitudes que interactúan en el dominio del problema, razón por la cual su orden de magnitud es la unidad. Ésta es una propiedad de la que, en general, carece la mayor parte de los grupos adimensionales clásicos usados en ingeniería.

La cuestión que ahora nos planteamos es: ¿qué valores deberían atribuirse a estos grupos discriminados para una óptima elección del problema patrón que tratan de definir?

Para responder a esta pregunta es necesario conectar el escenario buscado como problema patrón, seleccionado bajo argumentos puramente de interés fenomenológico o físico, con los balances globales o regionales que emergen de dicho escenario, balances que implican la existencia de sus correspondientes grupos adimensionales discriminados (también globales o regionales).

La razón esencial por la que el problema de Henry se ha convertido en problema patrón (Pinder y Cooper, [1970]; Segol, Pinder y Gray, [1975]; Frind, [1982]; Huyakorn, Andersen, Mercer y White, [1987]; Voss y Souza, [1987], Segol, [1994], Croucher y O’Sulivan [1995] y Simpson y Clement [2003]) es, seguramente, la de que es el único problema que proporciona una solución semianalítica a condiciones de contorno que reflejan el fenómeno de intrusión salina, Abarca, [2006]. Las discrepancias surgieron sólo en la forma en que diferentes autores llegaron a resultados controvertidos, algunos de los cuales fueron recogidos y discutidos por Croucher y O’Sulivan [1995] y Bues y Oltean [2000]. 
Por ejemplo, Simpson y Clement [2003] detectaron que la distribución de concentraciones del problema desacoplado presentaba un patrón similar al encontrado en el problema acoplado merced a la acusada condición impuesta en la frontera de intrusión, lo que debería convertir el problema de Henry en irrelevante desde el punto de vista de su elección como problema patrón. Con todo, la comunidad científica hidrogeológica sencillamente aceptó el calificativo de patrón con algunas críticas obviamente razonables en relación con el valor de sus parámetros (los de Henry), pero sin entrar en el significado y valor meritorio de estos números como guías para definir el problema ya que, en efecto, el escenario proporcionaba con bastante aproximación los patrones esperados.

El primer paso quizá hubiera debido ser el de definir correctamente unos grupos adimensionales globales, es decir referidos a todo el dominio. Un valor cercano a la unidad para estos grupos implica un patrón regularmente distribuido (en la totalidad del dominio) y la desviación del orden de magnitud (por encima o por debajo de la unidad) de uno o más grupos debería alterar tanto cualitativa como cuantitativamente el patrón global, cada uno con una tendencia particular y diferenciada de los otros. Esto permitiría estudiar y comprobar mediante simulaciones numéricas precisas la influencia de cada grupo en el patrón de soluciones, es decir, la influencia que representa en el patrón el desbalance de magnitudes impuesto por cada grupo.

En problemas complejos y conjugados como el de Henry, la desviación en el orden de magnitud unidad para uno o más grupos transforma sensiblemente el patrón de soluciones produciendo nuevas subregiones dentro del dominio global en las que emergen nuevos grupos adimensionales locales que representan nuevos balances.

Bien, con todo podemos buscar un escenario que, para unos valores particulares de los grupos adimensionales generales (de orden de magnitud apropiado), reproduzca la solución de los escenarios reales que tratamos de reproducir (el problema patrón) y, tras un nuevo estudio más profundo, los grupos adimensionales locales que emergen de los mismos (estos sí, de orden de magnitud unidad). La definición de estos últimos nuevos números podría ser más útil para la verificación de códigos de computación, un objetivo esencial asociado a la búsqueda de problemas patrones, ya que mientras una variación de los grupos globales no induciría cambios apreciables en los patrones (como ocurre de hecho en el problema de Henry), un pequeño cambio en los grupos locales sí que induciría 
cambios apreciables en las soluciones que debería ser recogidos por los códigos a verificar.

En esta línea de razonamiento vamos a buscar, en primer lugar, los patrones que emergen de la asignación de valores unidad a los grupos deducidos con la adimensionalización discriminada. Así, a partir de sus valores para el caso de Henry

$$
\pi_{\mathrm{d}, 1}=7.5834, \pi_{\mathrm{d}, 2}=0.25, \pi_{\mathrm{d}, 3}=0.05 \mathrm{y} \pi_{\mathrm{d}, 4}=0.25
$$

deberíamos incrementar Vamb $^{*}$ para contrarrestar la fuerte influencia de la condición de contorno de la frontera marina, e incrementar al mismo tiempo (y en mayor medida) la difusión, a través del parámetro D, para contrarrestar el empuje del flujo regional. Esto disminuiría $\pi_{\mathrm{d}, 1}$ y aumentaría $\pi_{\mathrm{d}, 3}$ hasta valores unidad. Los valores de $\mathrm{L}$ y $\mathrm{H}$ se corrigen adecuadamente para equilibrar los cocientes de permeabilidades y difusividades y así asignar, también, un valor unidad a los grupos $\pi_{\mathrm{d}, 2} \mathrm{y} \pi_{\mathrm{d}, 4}$.

Si nos referimos al caso isótropo, de una comparación entre los parámetros discriminados y los de Henry, encontramos que la mejor opción para obtener un patrón global es también disminuir $\pi_{\mathrm{d}, 1}$ e incrementar $\pi_{\mathrm{d}, 2}$ y $\pi_{\mathrm{d}, 3}$, lo que se podría obtener incrementando $\mathrm{Vamb}^{*}$, disminuyendo $\mathrm{D}$ y equilibrando el cociente de permeabilidades disminuyendo sensiblemente la longitud del acuífero, L.

Simpson y Clement [2003] recomendaron reducir $\mathrm{a}_{\mathrm{H}}$ para mejorar la sensibilidad del problema de Henry a fin de usarlo como prueba para verificar los códigos de flujo impulsado por densidad variable, mientras que Abarca [2006] apunta que dicha mejora puede también conseguirse disminuyendo $b_{H}$. Ambas recomendaciones están de acuerdo con las conclusiones derivadas de un análisis de los grupos ya obtenidos mediante la adimensionalización discriminada.

Se estudian dos casos, que estimamos son suficientes para verificar los resultados de lo que llamaremos un patrón global óptimo, con los parámetros mostrados en la Tabla III.4. En ambos, $\pi_{\mathrm{d}, 1}=\pi_{\mathrm{d}, 2}=\pi_{\mathrm{d}, 3}=\pi_{\mathrm{d}, 4}=1$, mientras que los grupos clásicos ( $\mathrm{a}_{\mathrm{H}}, \mathrm{b}_{\mathrm{H}} \mathrm{y} \mathrm{H} / \mathrm{L}$ ) tienen valores diferentes a la unidad, Tabla III.5. Como el valor de ан $_{\mathrm{H}}$ depende de la elección de la permeabilidad $k_{\mathrm{x}}$ o $\mathrm{k}_{\mathrm{y}}$ en su definición, y el de $b_{\mathrm{H}}$, a su vez, depende de la elección de la difusividad $\mathrm{D}_{\mathrm{x}}$ o $\mathrm{D}_{\mathrm{y}}$, la Tabla III.5 muestra también los valores de los grupos clásicos para cada elección. 
Las simulaciones de estos casos de patrón óptimo global, isolíneas de concentración salina y flujo, se muestran en las Figuras III.6 y III.7, respectivamente. Come esperábamos, se trata de patrones normalizados idénticos (salvo escala) que demuestran el equilibrio entre las magnitudes físicas que interactúan en el dominio global. Se acentúa que la verificación de un código se hace más precisa en tanto que las isolíneas cruzan el dominio completo y las lecturas y comparación de los valores locales de función de corriente y concentración pueden compararse con mayor precisión (además, con las escalas normalizadas las intersecciones de las líneas con las fronteras concurren en las mismas posiciones relativas), demostrando que la investigación de grupos adimensionales discriminados emerge como una ayuda substancial en la definición o selección de problemas patrón.

Tabla III.4 Parámetros para un patrón global óptimo

\begin{tabular}{|c|c|c|c|c|c|c|c|c|c|}
\hline Parámetro & $\mathbf{L}$ & $\mathbf{H}$ & $\mathbf{k}_{\mathbf{x}}$ & $\mathbf{k}_{\mathbf{y}}$ & $\mathbf{D}_{\mathbf{x}}$ & $\mathbf{D}_{\mathbf{y}}$ & $\mathbf{v a m b}^{*}$ & $\Delta \rho$ & $\Psi$ \\
\hline Henry & $\mathrm{L}_{\mathrm{H}}$ & $\mathrm{H}_{\mathrm{H}}$ & $\mathrm{k}_{\mathrm{H}}$ & $\mathrm{k}_{\mathrm{H}}$ & $\mathrm{D}_{\mathrm{H}}$ & $\mathrm{D}_{\mathrm{H}}$ & $\mathrm{v}_{\mathrm{amb}, \mathrm{H}}{ }^{*}$ & $\Delta \rho_{\mathrm{H}}$ & $\Psi_{\mathrm{H}}$ \\
\hline $\begin{array}{c}\text { Patrón global } \\
\text { óptimo } \\
\text { (Caso 1-pg) }\end{array}$ & $\mathrm{L}_{\mathrm{H}}$ & $\mathrm{H}_{\mathrm{H}}$ & $\mathrm{k}_{\mathrm{H}}(1 / 4) \mathrm{k}_{\mathrm{H}}$ & $4 \mathrm{D}_{\mathrm{H}}$ & $\mathrm{D}_{\mathrm{H}}$ & $(1 / 5) \mathrm{v}_{\mathrm{amb}, \mathrm{H}}{ }^{*}$ & $0.106 \Delta \rho_{\mathrm{H}}$ & $(1 / 5) \Psi_{\mathrm{H}}$ \\
\hline $\begin{array}{c}\text { Patrón global } \\
\text { óptimo } \\
\begin{array}{c}\text { (Caso 2-pg) } \\
\hline\end{array}\end{array}$ & $2^{1 / 2} \mathrm{~L}_{\mathrm{H}}$ & $\mathrm{H}_{\mathrm{H}}$ & $8 \mathrm{k}_{\mathrm{H}}$ & $\mathrm{k}_{\mathrm{H}}$ & $4\left(2^{1 / 2}\right) \mathrm{D}_{\mathrm{H}}$ & $2^{-1 / 2} \mathrm{D}_{\mathrm{H}}$ & $(1 / 5) \mathrm{v}_{\mathrm{amb}, \mathrm{H}}{ }^{*}$ & $0.019 \Delta \rho_{\mathrm{H}}$ & $(1 / 5) \Psi_{\mathrm{H}}$ \\
\hline
\end{tabular}

Tabla III.5 Valores de los grupos adimensionales clásicos y discriminados para un patrón global óptimo

\begin{tabular}{|c|c|c|c|c|c|c|c|c|}
\hline \multirow[b]{2}{*}{ Caso } & \multicolumn{4}{|c|}{ Grupos discriminados } & \multicolumn{4}{|c|}{ Grupos clásicos } \\
\hline & $\pi_{\delta, 1}$ & $\pi_{\delta, 2}$ & $\pi_{\delta, 3}$ & $\pi \delta, 4$ & aH & $\mathbf{b}_{\mathbf{H}}$ & $\begin{array}{c}\mathbf{k}_{\mathrm{x}} / \mathbf{k}_{\mathrm{y}} \\
\mathbf{D}_{\mathrm{x}} / \mathbf{D}_{\mathrm{y}}\end{array}$ & $\mathbf{H} / \mathbf{L}$ \\
\hline Henry & 7.58 & 0.25 & 0.05 & 0.25 & 3.79 & $0.1(0.0397)^{*}$ & 1 & 0.5 \\
\hline $\begin{array}{l}\text { Patrón global óptimo } \\
\text { (Caso1 1-pg) }\end{array}$ & 1 & 1 & 1 & 1 & $\begin{array}{l}1.99 \text { (usando } \mathrm{k}_{\mathrm{x}} \text { ) } \\
0.50 \text { (usando } \mathrm{k}_{\mathrm{y}} \text { ) }\end{array}$ & $\begin{array}{c}0.5 \text { (usando } \mathrm{D}_{\mathrm{x}} \text { ) } \\
0.125 \text { (usando } \mathrm{D}_{\mathrm{y}} \text { ) }\end{array}$ & 4 & 0.5 \\
\hline $\begin{array}{l}\text { Patrón global óptimo } \\
\text { (Caso 2-pg) }\end{array}$ & 1 & 1 & 1 & 1 & $\begin{array}{l}2.85 \text { (usando } \mathrm{k}_{\mathrm{x}} \text { ) } \\
0.36 \text { (usando } \mathrm{k}_{\mathrm{y}} \text { ) }\end{array}$ & $\begin{array}{l}2.83 \text { (usando } \mathrm{D}_{\mathrm{x}} \text { ) } \\
0.35 \text { (usando } \mathrm{D}_{\mathrm{y}} \text { ) }\end{array}$ & 8 & 0.354 \\
\hline
\end{tabular}


Capítulo III. Aplicación de la adimensionalización discriminada al problema de Henry
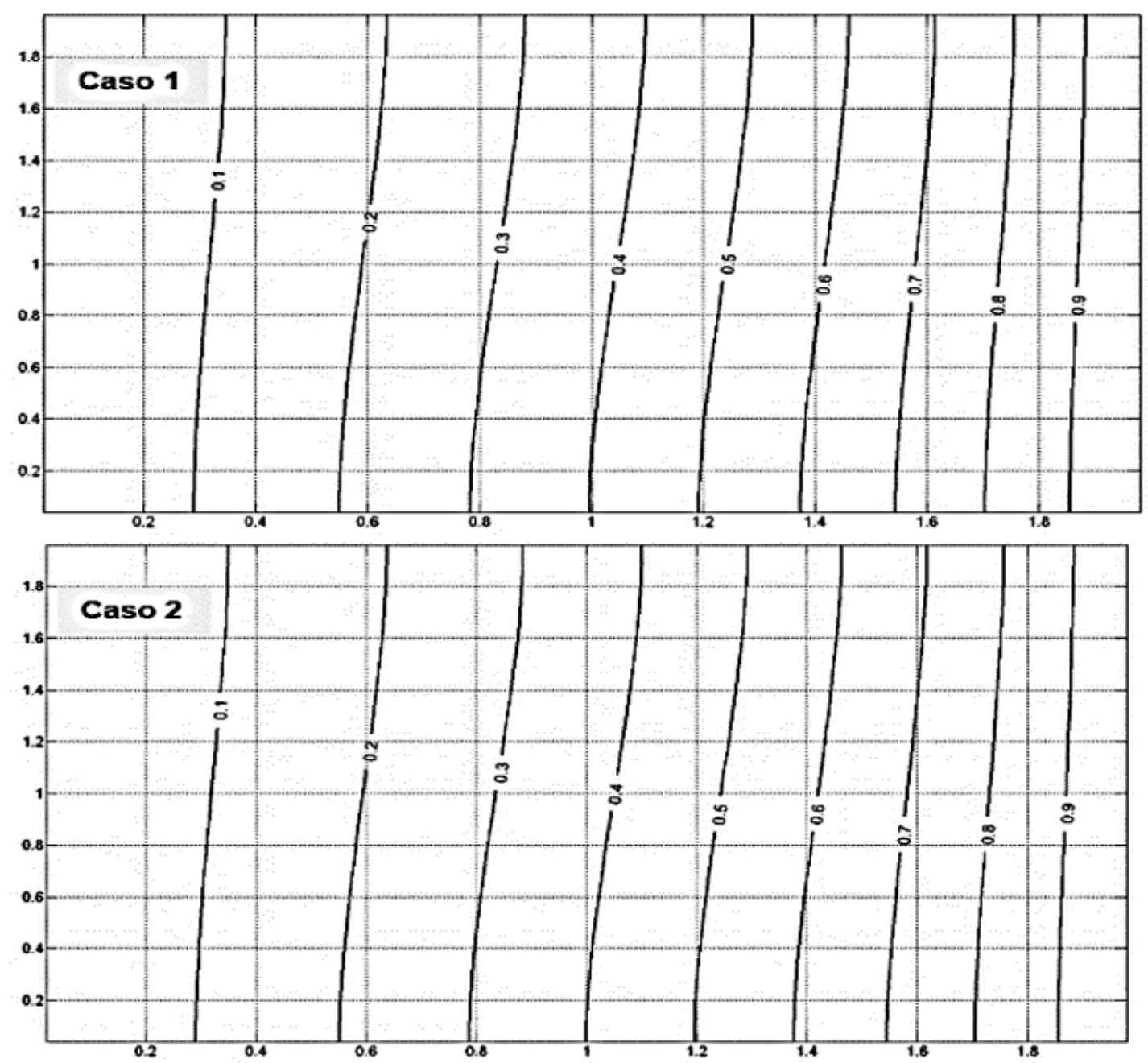

Figura III.6 Isolíneas de concentración para un patrón global óptimo. Casos pg-1 y pg-2. $\left(\pi_{\mathrm{d}, 1}=\pi_{\mathrm{d}, 2}=\pi_{\mathrm{d}, 3}=1\right)$, Tabla 5 

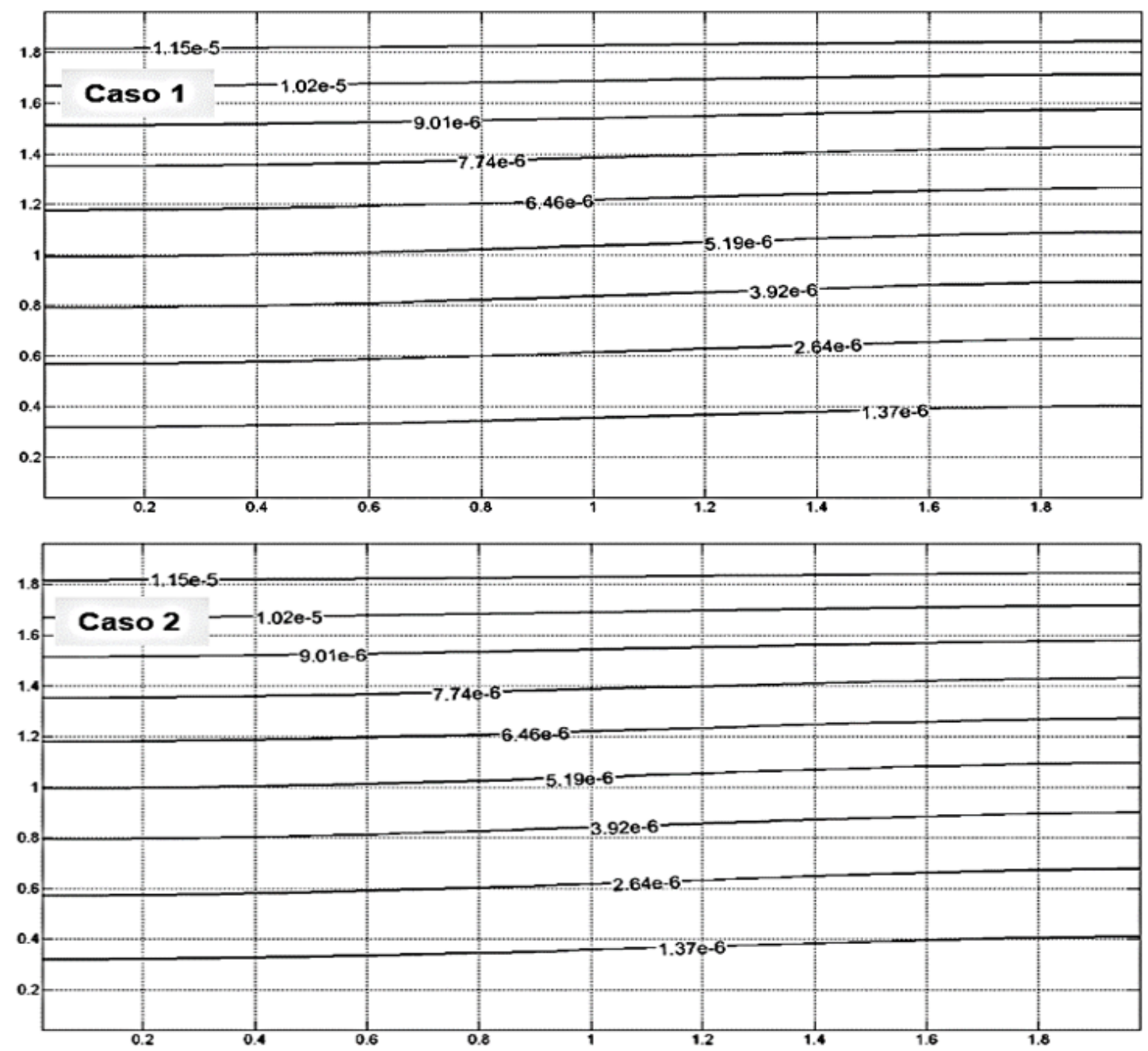

Figure III.7 Isolíneas de función de corriente para un patrón global óptimo. Casos pg-1 y pg-2.

$$
\left(\pi_{\mathrm{d}, 1}=\pi_{\mathrm{d}, 2}=\pi_{\mathrm{d}, 3}=1\right) \text {, Tabla } 5
$$

Para el caso isótropo, problema original de Henry con $k_{x}=k_{y}$ y $D_{x}=D_{y}$, los cuatro grupos discriminados (III.16 a III.19) se reducen a tres que pueden escribirse en la forma

$$
\begin{aligned}
& \pi_{\mathrm{nd},(\mathrm{isót} t r o p o) 1}=\frac{(\Delta \rho) \mathrm{gk}}{\varepsilon \mu \mathrm{v}_{\mathrm{amb}}^{*}} \\
& \pi_{\mathrm{nd},(\mathrm{isót} t r o p o) 2}=\frac{\mathrm{H}}{\mathrm{L}} \\
& \pi_{\mathrm{nd},(\text { isótropo)3 }}=\frac{\mathrm{D}}{v_{a m b}^{*} \mathrm{~L}}
\end{aligned}
$$

Los valores de los parámetros mostrados en la Tabla III.6, que corresponden a un valor unidad de los grupos no discriminados anteriores $\left(\pi_{\mathrm{nd} \text {,(isótropo) } 1}=\pi_{\mathrm{nd} \text {,(isótropo)2 }}=\right.$ $\pi_{\text {nd,(isótropo)3 }}=1$ ), definen un patrón global unidad cuya simulación se muestra en la Figura III.8. Se trata de un patrón idéntico (salvo escala) al de los casos no-isótropos anteriores 
(Figuras III.6 y 7). Es interesante (aunque esperado) advertir que los valores $\mathrm{a}_{\mathrm{H}}=\mathrm{b}_{\mathrm{H}}=\mathrm{H} / \mathrm{L}=1$ corresponden a un patrón óptimo cuyas isolíneas cubren todo el dominio demostrando que los grupos usados por Henry definen correctamente el caso isótropo.

Tabla III.6 Parámetros para un patrón global óptimo del caso isótropo (Henry original)

\begin{tabular}{|c|c|c|c|c|c|c|c|c|c|}
\hline Caso & $\mathbf{L}$ & $\mathbf{H}$ & $\mathbf{k}_{\mathbf{x}}$ & $\mathbf{k}_{\mathbf{y}}$ & $\mathbf{D}_{\mathbf{x}}$ & $\mathbf{D}_{\mathbf{y}}$ & $\mathbf{V a m b}^{*}$ & $\Delta \boldsymbol{\rho}$ & $\Psi$ \\
\hline $\begin{array}{c}\text { Patrón global óptimo } \\
\text { (caso isótropo) }\end{array}$ & $\mathrm{L}_{\mathrm{H}}$ & $2 \mathrm{H}_{\mathrm{H}}$ & $0.4 \mathrm{k}_{\mathrm{H}}$ & $0.4 \mathrm{k}_{\mathrm{H}}$ & $4 \mathrm{D}_{\mathrm{H}}$ & $4 \mathrm{D}_{\mathrm{H}}$ & $(1 / 5) \mathrm{V}_{\mathrm{amb}, \mathrm{H}}{ }^{*}$ & $0.131 \Delta \rho_{\mathrm{H}}$ & $0.4 \Psi_{\mathrm{H}}$ \\
\hline
\end{tabular}
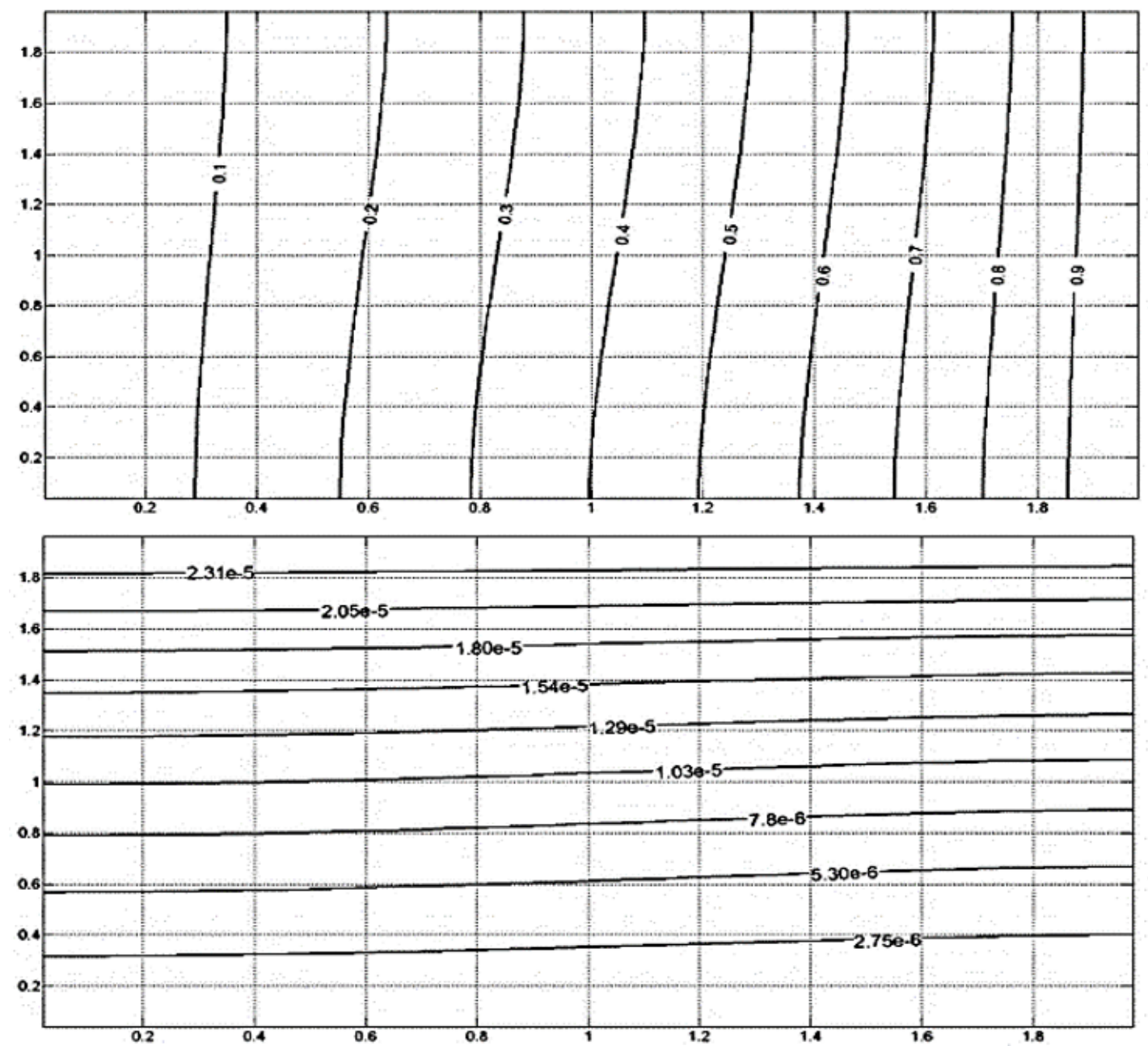

Figura III.8 Patrones globales óptimos de isoconcentración y función de corriente del caso isótropo (problema original de Henry, $\pi_{\mathrm{d}, \mathrm{iso}, 1}=\pi_{\mathrm{d}, \mathrm{iso}, 2}=\pi_{\mathrm{d}, \mathrm{iso}, 3}=1$ ). Tabla III.6 


\section{III.2 CONCLUSIONES}

De lo estudiado en este capítulo pueden establecerse las siguientes contribuciones y conclusiones:

i) Mediante la adimensionalización discriminada se deducen los cuatro grupos adimensionales (discriminados) del problema general de Henry, no dispersivo, para un medio anisótropo en las propiedades permeabilidad y difusividad efectiva.

ii) Se reducen los anteriores números al caso isótropo, demostrándose que los tres grupos resultantes son intercambiables (y por tanto equivalentes) a los obtenidos mediante análisis dimensional por Henry [1964], y a los obtenidos mediante la adimensionalización clásica por Abarca [2006]; también son equivalentes a los obtenidos por Abarca para medios anisótropos en la permeabilidad e isótropos en la difusividad molecular.

iv) Se justifica la expulsión del factor de forma o relación de aspecto $(\mathrm{L} / \mathrm{H})$ en escenarios anisótropos bajo el uso de la adimensionalización discriminada, una conclusión que no se deduciría con el uso de otras técnicas de determinación de los grupos adimensionales.

v) Mediante simulaciones numéricas con el código FATSIM, se verifica la influencia e independencia de los grupos deducidos en los patrones de solución del problema de Henry anisótropo e isótropo, para un conjunto de casos en el que se mantiene el valor numérico de los grupos y se cambia sensiblemente los valores de cualquiera de los parámetros.

vi) Se demuestra que para valores unidad de los grupos deducidos, tanto en el caso isótropo como en el anisótropo, el patrón de flujo y concentración se distribuye de manera regular cubriendo la totalidad del dominio.

vii) Se discute la idoneidad del carácter patrón del problema en base al valor numérico de los grupos discriminados deducidos.

viii) Se interpretan los grupos deducidos en términos de balance de las magnitudes físicas que interactúan en el problema, justificándose al mismo tiempo su orden de magnitud unidad cuando el patrón cubre todo el dominio de manera regular. 
ix) De lo anterior, queda justificada también la distribución no regular del patrón de flujo y concentración para los valores de los grupos adoptados por Henry, así como la previsible menor influencia de un cambio de estos valores en el patrón de Henry, frente a la mayor influencia de un cambio de valores de dichos grupos alrededor de su valor unidad. 


\section{CAPÍTULO IV}

\section{Simplificación de escenarios}

\section{IV.1 ESCENARIOS SIMPLIFICADOS EN TORNO AL PROBLEMA ORIGINAL DE HENRY}

La reducción del problema de Henry a escenarios más simples merced a que los valores de las características físicas induzcan efectos despreciables, debe realizarse eliminando de las ecuaciones de gobierno aquellos términos asociados a tales efectos y deduciendo los nuevos grupos asociados a estos escenarios mediante la aplicación de la adimensionalización discriminada a las ecuaciones simplificadas.

Alternativamente, aunque para ello es necesario proceder con más cuidado, pueden derivarse los grupos asociados a los escenarios simplificados mediante la manipulación directa de los grupos generales y aplicando a los mismos las hipótesis simplificadoras, un procedimiento más rápido que será el que adoptaremos.

En lo que sigue se plantea una serie de escenarios que simplifica el estudio del caso más general del problema de Henry anisótropo (con difusividad efectiva, pero no dispersividad) para valores de los parámetros cercanos a los adoptados por Henry. En cada simplificación se adopta como caso de referencia (que llamaremos caso 1) un 
escenario anisótropo de Henry, es decir, con parámetros iguales a los de Henry excepto en aquellos relacionados con la simplificación.

\section{IV.1.1 PRIMERA SIMPLIFICACIÓN. DIFUSIVIDAD DESPRECIABLE}

Esta hipótesis supone eliminar los términos relacionados con la difusividad en la ecuación del soluto (III.13), términos que contienen los parámetros $\mathrm{D}_{\mathrm{x}} \mathrm{y} \mathrm{D}_{\mathrm{y}}$, lo que reduce sus coeficientes a dos,

$$
\mathrm{vamb}^{*} / \mathrm{L} \mathrm{y} \mathrm{v}{ }_{\mathrm{y}}^{*} / \mathrm{H}=(\Delta \rho) \mathrm{gk}_{\mathrm{y}} /(\varepsilon \mu \mathrm{H}),
$$

y lós parámetros adimensionales a uno, el cociente de velocidades características corregido,

$$
\pi_{\mathrm{d}, 1}=\left[(\Delta \rho) \mathrm{gk}_{\mathrm{y}} /\left(\varepsilon \mu \mathrm{V}_{\mathrm{amb}}\right)\right](\mathrm{L} / \mathrm{H}) .
$$

La ecuación del momento y los grupos adimensionales que resultan de ella son los mismos que en el caso general. Así, tan sólo dos monomios adimensionales diferentes emergen de las ecuaciones de gobierno en este escenario:

$$
\pi_{\mathrm{d}, 1}=\frac{(\Delta \rho) \mathrm{gk}_{\mathrm{y}}}{\varepsilon \mu \mathrm{v}_{\mathrm{amb}}^{*}}\left(\frac{\mathrm{L}}{\mathrm{H}}\right) \quad \pi_{\mathrm{d}, 2}=\frac{\mathrm{k}_{\mathrm{x}} \mathrm{H}^{2}}{\mathrm{k}_{\mathrm{y}} \mathrm{L}^{2}}
$$

que también pueden escribirse en la forma (cuyo interés se apreciará más tarde)

$$
\pi_{\mathrm{d}, 1}=\frac{(\Delta \rho) \mathrm{g}}{\varepsilon \mu \mathrm{v}_{\mathrm{amb}}^{*}}\left(\mathrm{k}_{\mathrm{x}} \mathrm{k}_{\mathrm{y}}\right)^{1 / 2} \quad \pi_{\mathrm{d}, 2}=\frac{\mathrm{k}_{\mathrm{x}} \mathrm{H}^{2}}{\mathrm{k}_{\mathrm{y}} \mathrm{L}^{2}}
$$

De nuevo, la discriminación acopla características físicas relacionadas con las direcciones espaciales, con las longitudes de referencia del problema (asimismo asociadas a las direcciones espaciales), no permitiendo que cocientes tales como H/L, $\mathrm{k}_{\mathrm{x}} / \mathrm{k}_{\mathrm{y}} \mathrm{O} \mathrm{v}_{\mathrm{y}}{ }^{*} / \mathrm{V}_{\mathrm{amb}}$ se constituyan en grupos adimensionales independientes (como se propone con frecuencia con los dos primeros).

La Tabla IV.1 muestra los valores de los parámetros de seis casos correspondientes a esta simplificación. El caso 1 corresponde a un escenario de Henry que se ha elegido isótropo en relación con la conductividad hidráulica (pero podría haberse elegido valores anisótropos para este parámetro que hubieran conducido a las mismas conclusiones). Dado que la difusividad interviene en los parámetros $\pi_{\mathrm{d}, 3}=$ $\mathrm{D}_{\mathrm{x}} /\left(\mathrm{vamb}^{*} \mathrm{~L}\right)$ y $\pi_{\mathrm{d}, 4}=\mathrm{D}_{\mathrm{x}} \mathrm{H}^{2} /\left(\mathrm{D}_{\mathrm{y}} \mathrm{L}^{2}\right)$, será suficiente con asumir un valor $\mathrm{D}_{\mathrm{x}}=\mathrm{D}_{\mathrm{y}} \approx 0.01\left(\mathrm{v}_{\mathrm{amb}, \mathrm{H}} \mathrm{L}\right)$, que para los parámetros del problema de Henry resulta 


$$
\mathrm{D}_{\mathrm{x}}=\mathrm{D}_{\mathrm{y}} \approx 0.01\left(\mathrm{Lv}_{\mathrm{amb}}\right)=0.01(2 \times 1.8857 \mathrm{E}-4)=3.7714 \mathrm{E}-6
$$

Tabla IV.1 Valores de los parámetros para los casos de difusividad despreciable

\begin{tabular}{|c|c|c|c|c|c|c|c|c|c|c|c|}
\hline Caso & $\Delta \rho$ & g & $\mathbf{k}_{\mathbf{x}}$ & $\mathbf{k y}_{\mathbf{y}}$ & $\varepsilon$ & $\mu$ & Vamb & $\mathbf{L}$ & $\mathbf{H}$ & $\mathbf{D}_{\mathbf{x}}, \mathbf{D}_{\mathbf{y}}$ & $\Psi$ \\
\hline \multirow[t]{2}{*}{1} & $\Delta \rho_{\mathrm{H}}$ & $\mathbf{g}_{\mathbf{H}}$ & $\mathbf{k}_{\mathbf{x}, \mathbf{H}}$ & $\mathbf{k}_{\mathbf{y}, \mathbf{H}}$ & $\varepsilon_{\mathrm{H}}$ & $\mu_{\mathrm{H}}$ & Vamb,H & $\mathbf{L}_{\mathbf{H}}$ & $\mathbf{H}_{\mathbf{H}}$ & 3,77E-06 & $\Psi_{\mathrm{H}}$ \\
\hline & 25 & 9,81 & $1,0204 \mathrm{E}-09$ & $1,0204 \mathrm{E}-09$ & 0,35 & $1,0000 \mathrm{E}-03$ & $1,8857 \mathrm{E}-04$ & 2 & 1 & 3,77E-06 & $6,60 \mathrm{E}-05$ \\
\hline \multirow[t]{2}{*}{2} & $\Delta \rho_{\mathrm{H}}$ & $\mathbf{g}_{\mathbf{H}}$ & $2 \mathbf{k}_{\mathbf{x}, \mathrm{H}}$ & $2 \mathbf{k}_{\mathbf{y}, \mathrm{H}}$ & $\varepsilon_{\mathrm{H}}$ & $\mu_{\mathrm{H}}$ & 2 Vamb,H & $\mathbf{L H}_{\mathbf{H}}$ & $\mathbf{H}_{\mathbf{H}}$ & 7,54E-06 & $2 \Psi_{H}$ \\
\hline & 25 & 9,81 & $2,0408 \mathrm{E}-09$ & $2,0408 \mathrm{E}-09$ & 0,35 & $1,0000 \mathrm{E}-03$ & $3,7714 \mathrm{E}-04$ & 2 & 1 & 7,54E-06 & $1,32 \mathrm{E}-04$ \\
\hline \multirow[t]{2}{*}{3} & $\Delta \rho_{\mathrm{H}}$ & $\mathbf{g}_{\mathbf{H}}$ & $2 \mathbf{k}_{\mathrm{x}, \mathrm{H}}$ & $2 \mathbf{k}_{\mathbf{y}, \mathbf{H}}$ & $\varepsilon_{\mathrm{H}}$ & $2 \mu_{H}$ & Vamb,H & $\mathbf{L}_{\mathbf{H}}$ & $\mathbf{H}_{\mathbf{H}}$ & 3,77E-06 & $\Psi_{\mathrm{H}}$ \\
\hline & 25 & 9,81 & $2,0408 \mathrm{E}-09$ & $2,0408 \mathrm{E}-09$ & 0,35 & $2,0000 \mathrm{E}-03$ & $1,8857 \mathrm{E}-04$ & 2 & 1 & 3,77E-06 & $6,60 \mathrm{E}-05$ \\
\hline \multirow[t]{2}{*}{4} & 2 & $\mathbf{g}_{\mathbf{H}}$ & $2 \mathbf{k}_{\mathbf{x}, \mathrm{H}}$ & $2 \mathbf{k}_{\mathbf{y}, \mathbf{H}}$ & $\varepsilon_{\mathrm{H}}$ & $4 \mu_{H}$ & Vamb,H & $\mathbf{L}_{\mathbf{H}}$ & $\mathbf{H}_{\mathbf{H}}$ & 3,77E-06 & $\Psi_{H}$ \\
\hline & 50 & 9,81 & $2,0408 \mathrm{E}-09$ & $2,0408 \mathrm{E}-09$ & 0,35 & $4,0000 \mathrm{E}-03$ & $1,8857 \mathrm{E}-04$ & 2 & 1 & 3,77E-06 & $6,60 \mathrm{E}-05$ \\
\hline \multirow[t]{2}{*}{5} & $\Delta \rho_{\mathrm{H}}$ & $\mathbf{g H}_{\mathbf{H}}$ & $\mathbf{k}_{\mathbf{x}, \mathbf{H}}$ & $\mathbf{k}_{\mathbf{y}, \mathbf{H}}$ & $\varepsilon_{\mathrm{H}}$ & $2 \mu_{\mathrm{H}}$ & 1/2 Vamb,H & $\mathbf{L H}_{\mathbf{H}}$ & $\mathbf{H}_{\mathbf{H}}$ & 1,88E-06 & $0.5 \Psi_{\mathrm{H}}$ \\
\hline & 25 & 9,81 & $1,0204 \mathrm{E}-09$ & $1,0204 \mathrm{E}-09$ & 0,35 & $2,0000 \mathrm{E}-03$ & $9,4285 \mathrm{E}-05$ & 2 & 1 & 1,88E-06 & $3,30 \mathrm{E}-05$ \\
\hline \multirow[t]{2}{*}{6} & $\Delta \rho_{\mathrm{H}}$ & $\mathbf{g}_{\mathbf{H}}$ & $\mathbf{k}_{\mathbf{x}, \mathbf{H}}$ & $\mathbf{k}_{\mathbf{y}, \mathbf{H}}$ & $\varepsilon_{\mathrm{H}}$ & $2 \mu_{H}$ & $1 / 2$ Vamb,H & $\mathbf{L}_{\mathbf{H}}$ & $\mathbf{H}_{\mathbf{H}}$ & 3,77E-06 & $0.5 \Psi_{\mathrm{H}}$ \\
\hline & 25 & 9,81 & $1,0204 \mathrm{E}-09$ & $1,0204 \mathrm{E}-09$ & 0,35 & $2,0000 \mathrm{E}-03$ & $9,4285 \mathrm{E}-05$ & 2 & 1 & 3,77E-06 & $3,30 \mathrm{E}-05$ \\
\hline
\end{tabular}

Por otro lado, como la difusividad de Henry tiene un valor de 1.8857E-5 (cinco veces la adoptada en esta hipótesis), el problema de Henry puede considerarse ciertamente cercano a esta simplificación, y como consecuencia de ello, no se esperan patrones muy diferentes a los de dicho problema.

El bajo valor de $\pi_{\mathrm{d}, 1}$ hace que prepondere el flujo o velocidad regional frente al flujo de Darcy, lo que empuja el patrón hacia la región izquierda del dominio. Adviértase que el monomio que se suprime, $\mathrm{D} /\left(\mathrm{v}_{\mathrm{amb}}{ }^{*} \mathrm{~L}\right)$, tiene un valor de 0.05 , tan solo 5 veces mayor que el adoptado en esta hipótesis. En los casos de la tabla en los que cambia la velocidad regional, se ha corregido convenientemente la difusividad para seguir cumpliendo la condición $\mathrm{D}_{\mathrm{x}}=\mathrm{D}_{\mathrm{y}} \approx 0.01(\operatorname{Lvamb})$.

Las Figuras IV.1a-b y IV.2a-b muestran el patrón de concentración y flujo, respectivamente, de los cinco primeros casos de la Tabla (se trata de patrones idénticos salvo en el valor de la función de corriente que no se ha normalizado). En efecto, este resultado es coherente ya que los monomios deducidos de esta simplificación retienen el mismo valor, a saber, 


$$
\pi_{\mathrm{d}, 1}=\frac{(\Delta \rho) \mathrm{gk}_{\mathrm{y}}}{\varepsilon \mu v_{\mathrm{amb}}^{*}}\left(\frac{\mathrm{L}}{\mathrm{H}}\right)=7,58, \quad \pi_{\mathrm{d}, 2}=\frac{\mathrm{k}_{\mathrm{x}} \mathrm{H}^{2}}{\mathrm{k}_{\mathrm{y}} \mathrm{L}^{2}}=0,25
$$
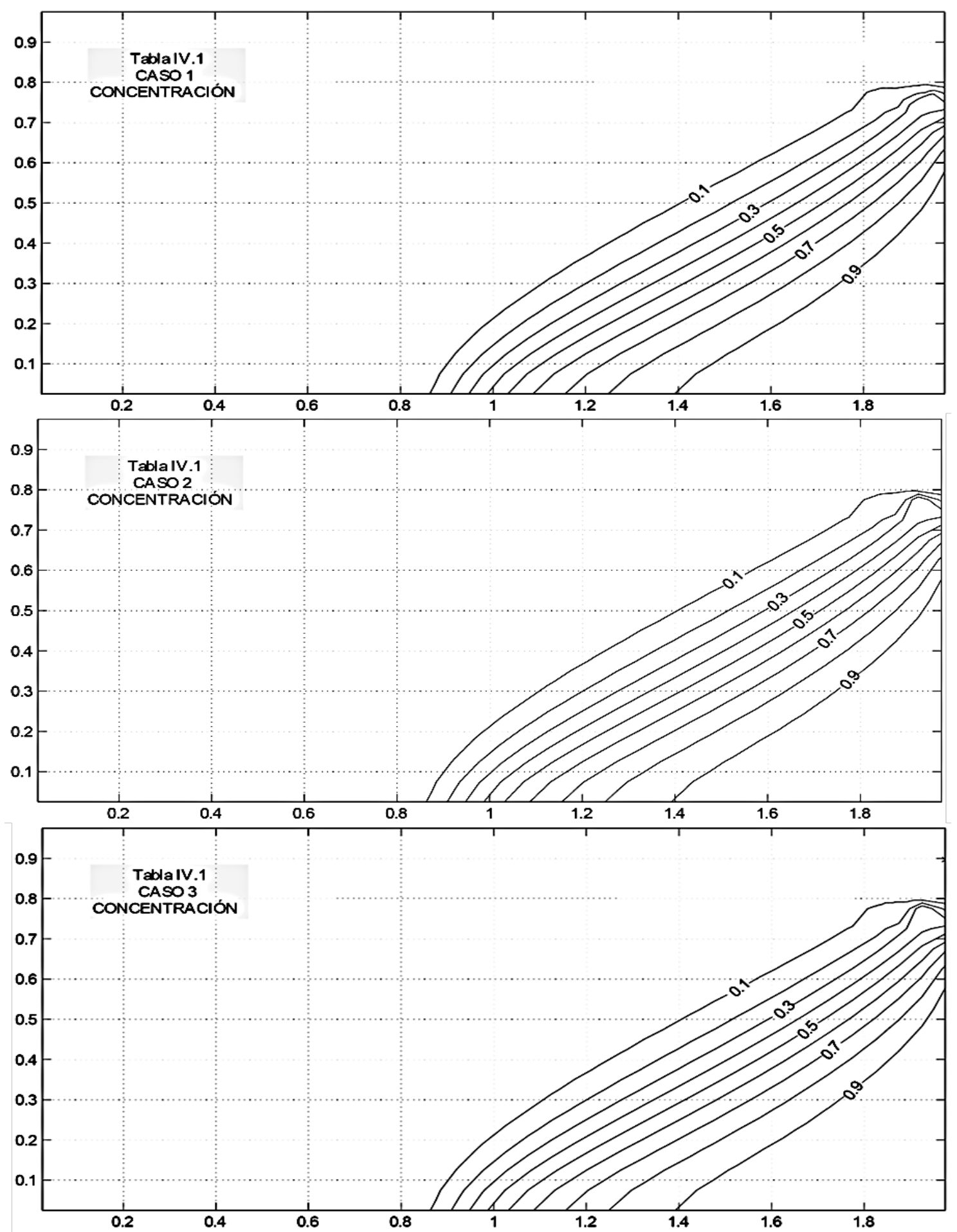

Figura IV.1a Patrones de concentración para los casos 1 a 5 de difusividad despreciable (Casos 1 a 3, Tabla IV.1) 

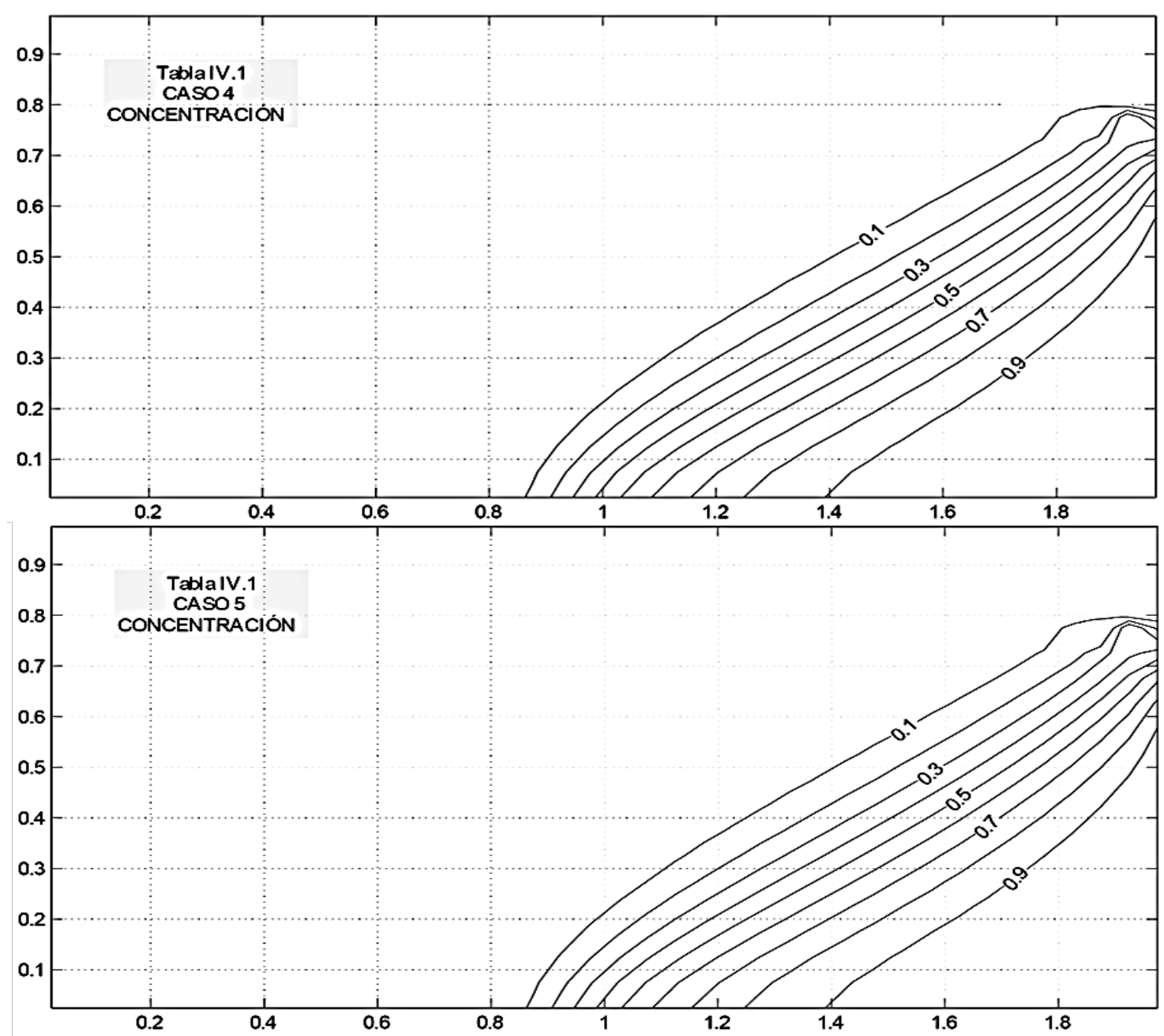

Figura IV.1b Patrones de concentración para los casos 1 a 5 de difusividad despreciable (Casos 4 y 5, Tabla IV.1)

Téngase en cuenta que en los casos en los que cambia la velocidad regional, debe modificarse bien el valor de la función de corriente en la frontera superior bien la altura del dominio, o ambos datos a la vez, por lo que las variables función de corriente y coordenada vertical deben normalizarse para poder comparar los patrones de solución. Así, en los casos 2 y 5, los límites superiores de la función de corrientes se extienden a los valores 7.54E-6 y 1.88E-6, respectivamente.

En el caso 6, donde el límite superior de la función de corriente (para reducir la velocidad regional a la mitad) es 1.88E-6, el valor de la difusividad no se ha corregido para mantener los valores de los monomios, con lo que

$$
\pi_{\mathrm{d}, 1}=\frac{(\Delta \rho) \mathrm{gk}_{\mathrm{y}}}{\varepsilon \mu \mathrm{v}_{\mathrm{amb}}^{*}}\left(\frac{\mathrm{L}}{\mathrm{H}}\right)=7,58 \quad \pi_{\mathrm{d}, 2}=\frac{\mathrm{k}_{\mathrm{x}} \mathrm{H}^{2}}{\mathrm{k}_{\mathrm{y}} \mathrm{L}^{2}}=0,25
$$


resultando un patrón ligeramente diferente (ya que, si bien la difusividad es despreciable, no tiene el mismo valor en los dos casos), Figura IV.3.
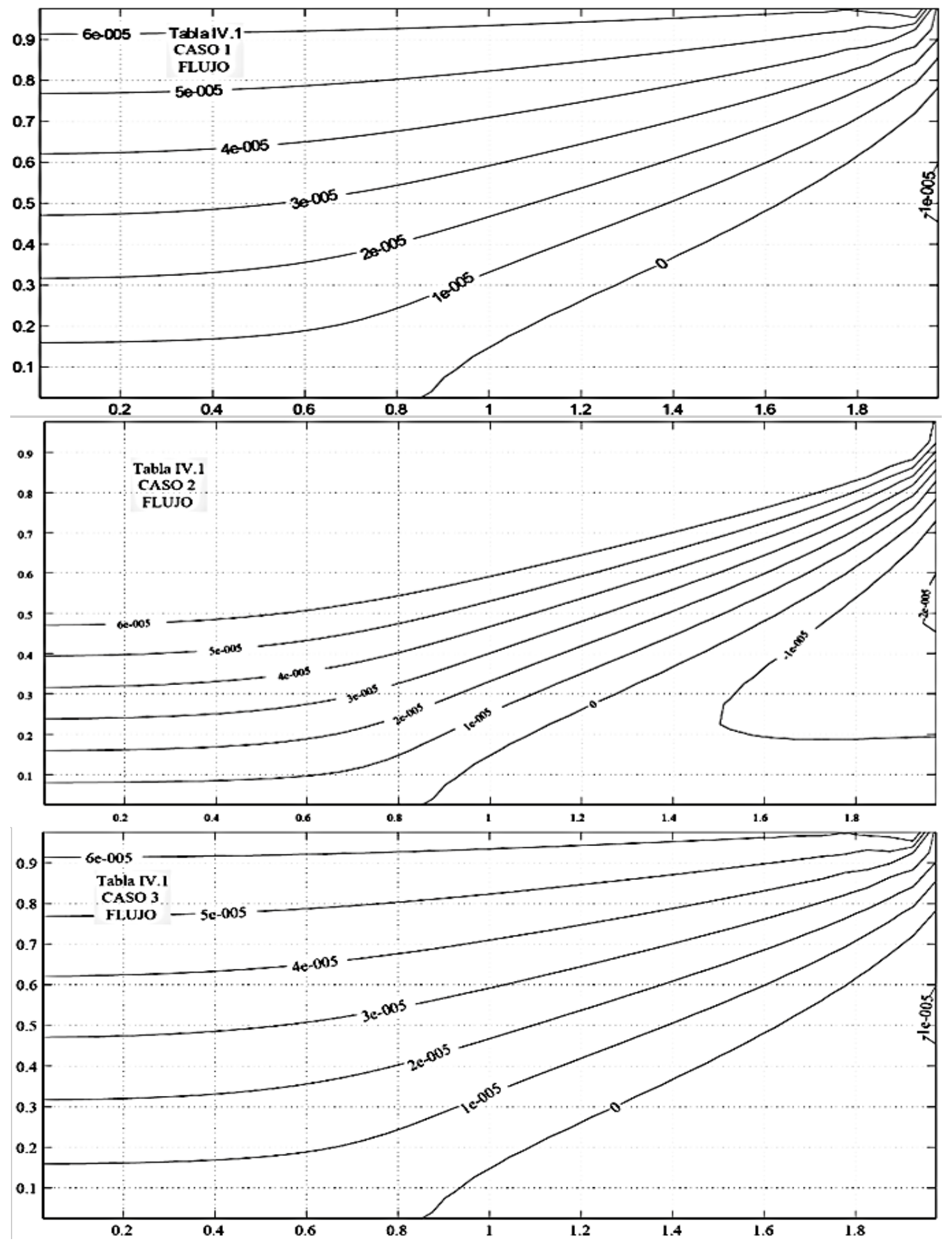

Figura IV. 2a Patrones de función de corriente para los casos 1 a 5 de difusividad despreciable (Casos 1 a 3, Tabla IV.1) 
El efecto de un cambio de difusividad en los patrones de concentración puede parecer contradictorio a primera vista, al comparar con los resultados del problema de Henry original, dado que la concentración (isolíneas 0.1 y 0.5, por ejemplo) penetra más hacia el continente para todos los casos de la tabla, siendo la difusividad de los mismos mucho menor que la de problema patrón de Henry. No es ésta, sin embargo, la comparación que debe hacerse ya que el alcance de la zona inundada por la contaminación salina no depende sólo de la difusividad, sino que este parámetro lo que determina es la anchura de la zona de mayor gradiente de sal, por ejemplo, la anchura que separa las líneas de concentración límites 0.1 y 0.9.

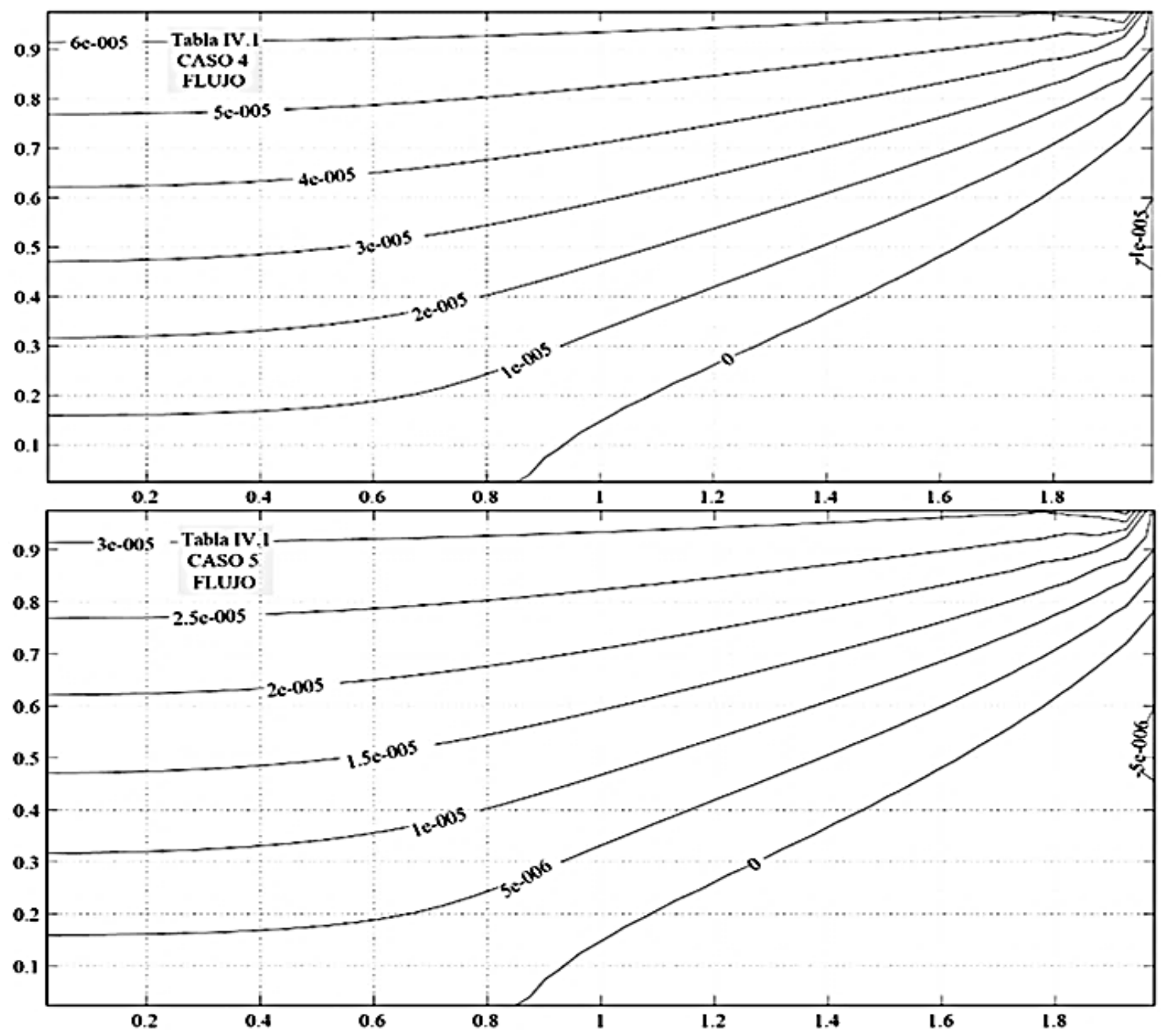

Figura IV. 2b Patrones de función de corriente para los casos 1 a 5 de difusividad despreciable (Casos 4 y 5, Tabla IV.1) 
La recirculación inunda la región inferior-derecha del acuífero con agua de concentración alta, y el elevado gradiente entre el agua de recirculación y el agua dulce crea una zona difusa cuyo espesor depende directamente de la difusividad.

Adviértase que para los casos 1 a 5, la región de salinidad por encima del 90\% es bastante superior a la que existe en el problema original.

Por otro lado, en el caso 6, de difusividad también despreciable, pero de valor doble que el del caso 5, que mantiene iguales el valor del resto de los parámetros (y de los grupos adimensionales resultantes de esta simplificación), la anchura de la región parcialmente contaminada (entre isolíneas 0.1 y 0.9 ) es sensiblemente mayor como cabía esperar de lo mencionado anteriormente.
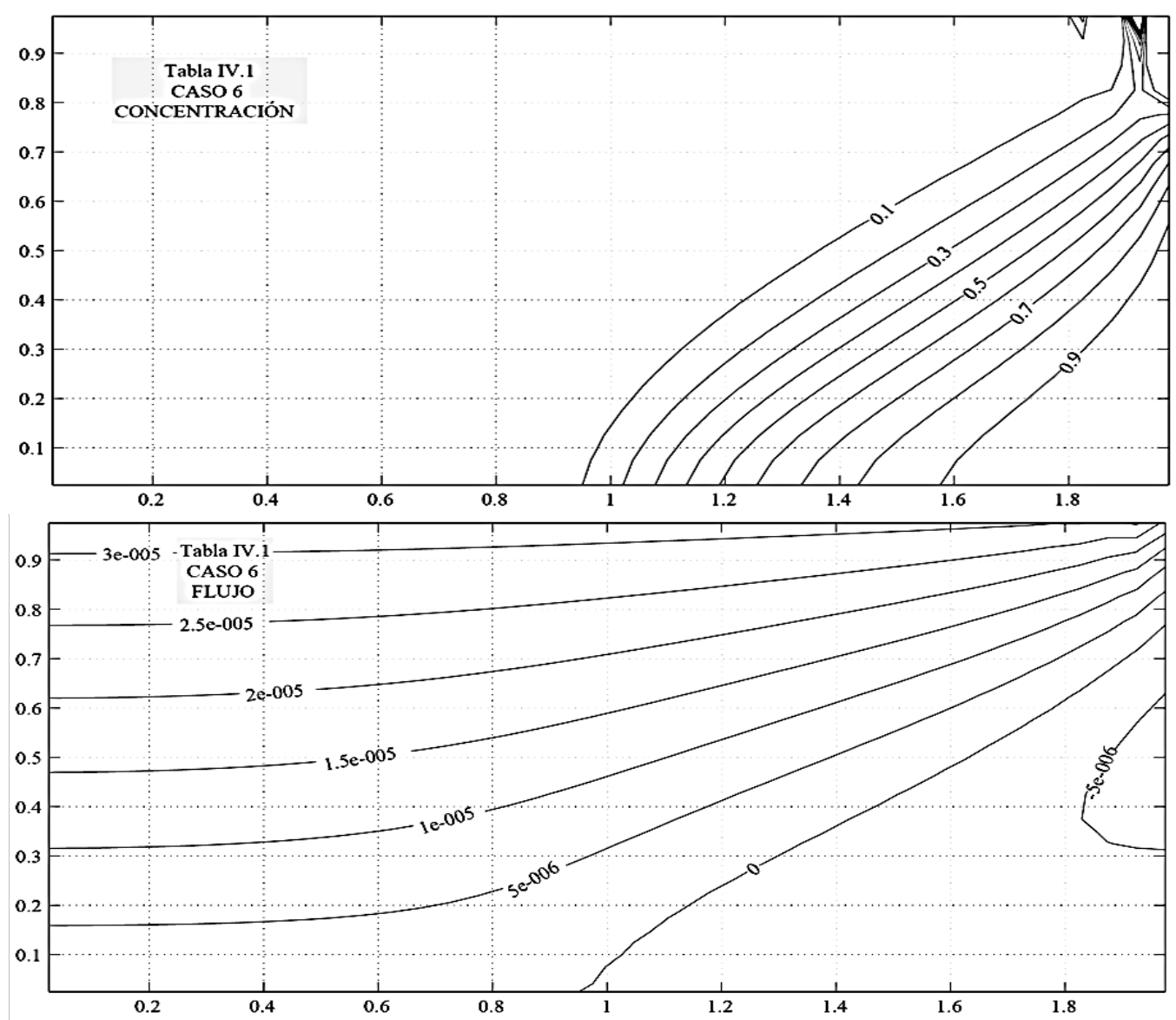

Figura IV.3 Patrones de concentración y función de corriente para el caso 6 de difusividad despreciable (Tabla IV.1) 


\section{IV.1.2 SEGUNDA SIMPLIFICACIÓN. DIFUSIVIDAD VERTICAL (DY) \\ DESPRECIABLE, $\mathrm{D}_{\mathrm{Y}}<<\mathrm{D}_{\mathrm{X}}$}

Esta hipótesis, para la que se adopta una relación entre difusividades de la forma $\mathrm{D}_{\mathrm{y}}=0.01 \mathrm{D}_{\mathrm{x}}$, conduce a tres coeficientes en la ecuación de transporte,

$$
\begin{aligned}
& \mathrm{V}_{\mathrm{amb}}{ }^{*} / \mathrm{L}, \\
& \mathrm{v}_{\mathrm{y}}{ }^{*} / \mathrm{H}=(\Delta \rho) \mathrm{gk}_{\mathrm{y}} /(\varepsilon \mu \mathrm{H}) \\
& \mathrm{D}_{\mathrm{x}} / \mathrm{L}^{2},
\end{aligned}
$$

que dan lugar a dos grupos adimensionales

$$
\begin{aligned}
& \pi_{\mathrm{d}, 1}=\frac{(\Delta \rho) \mathrm{gk}_{\mathrm{y}}}{\varepsilon \mu \mathrm{v}_{\mathrm{amb}}^{*}}\left(\frac{\mathrm{L}}{\mathrm{H}}\right) \\
& \pi_{\mathrm{d}, 3}=\frac{\mathrm{D}_{\mathrm{x}}}{\mathrm{v}_{\mathrm{amb}}^{*} \mathrm{~L}}
\end{aligned}
$$

Éstos, junto con los derivados de la ecuación del momento, proporcionan en total tres grupos adimensionales para gobernar los patrones de solución

$$
\begin{aligned}
& \pi_{\mathrm{d}, 1}=\frac{(\Delta \rho) \mathrm{gk}_{\mathrm{y}}}{\varepsilon \mu \mathrm{v}_{\mathrm{amb}}^{*}}\left(\frac{\mathrm{L}}{\mathrm{H}}\right) \\
& \pi_{\mathrm{d}, 2}=\frac{\mathrm{k}_{\mathrm{x}} \mathrm{H}^{2}}{\mathrm{k}_{\mathrm{y}} \mathrm{L}^{2}} \\
& \pi_{\mathrm{d}, 3}=\frac{\mathrm{D}_{\mathrm{x}}}{\mathrm{v}_{\mathrm{amb}}^{*} \mathrm{~L}}
\end{aligned}
$$

Una vez más, la relación de aspecto no juega un papel separado, sino que se une a los cocientes de velocidad y permeabilidad para formar los dos primeros monomios, corrigiendo estos cocientes.

La Tabla IV.2 muestra los valores de los parámetros de cinco casos típicos de esta simplificación. Como en la primera, el caso 1 corresponde a un escenario de Henry que se ha elegido isótropo en relación con la conductividad hidráulica. Dado que no queremos alterar el dominio geométrico del problema $(\mathrm{L}=2, \mathrm{H}=1)$, mantenemos la conductividad isótropa a fin de conservar en todos los casos un mismo valor para el monomio $\left(\mathrm{k}_{\mathrm{x}} \mathrm{H}^{2}\right) /\left(\mathrm{k}_{\mathrm{y}} \mathrm{L}^{2}\right)$.

Las Figuras IV.4a-b y IV.5a-b muestran el patrón de concentración y de flujo, respectivamente, de los casos de la tabla anterior; como esperábamos, se trata de nuevo 
de patrones idénticos. Para todos, los monomios deducidos retienen obviamente el mismo valor

$$
\begin{aligned}
& \pi_{\mathrm{d}, 1}=\frac{(\Delta \rho) \mathrm{gk}_{\mathrm{y}}}{\varepsilon \mu \mathrm{v}_{\mathrm{amb}}^{*}}\left(\frac{\mathrm{L}}{\mathrm{H}}\right)=7,58 \\
& \pi_{\mathrm{d}, 2}=\frac{\mathrm{k}_{\mathrm{x}} \mathrm{H}^{2}}{\mathrm{k}_{\mathrm{y}} \mathrm{L}^{2}}=0,25 \\
& \pi_{\mathrm{d}, 3}=\frac{\mathrm{D}_{\mathrm{x}}}{\mathrm{v}_{\mathrm{amb}}^{*} \mathrm{~L}}=0,05
\end{aligned}
$$

\begin{tabular}{|c|c|c|c|c|c|c|c|c|c|c|c|c|}
\hline Caso & $\Delta \rho$ & g & $\mathbf{k}_{\mathbf{x}}$ & $\mathbf{k}_{\mathbf{y}}$ & $\varepsilon$ & $\mu$ & $\mathbf{V}_{\mathrm{amb}}$ & $\mathbf{L}$ & $\mathbf{H}$ & $\mathbf{D}_{\mathbf{x}}$ & Dy & $\Psi$ \\
\hline \multirow[t]{2}{*}{1} & $\Delta \rho_{H}$ & $\mathbf{g}_{\mathbf{H}}$ & $\mathbf{k}_{\mathbf{x}, \mathbf{H}}$ & $\mathbf{k}_{\mathbf{y}, \mathbf{H}}$ & $\varepsilon_{H}$ & $\mu_{H}$ & $\mathbf{V}_{\mathbf{a m b}, \mathbf{H}}$ & $\mathbf{L}_{\mathbf{H}}$ & $\mathbf{H}_{\mathbf{H}}$ & $\mathbf{D}_{\mathrm{x}, \mathrm{H}}$ & $\mathbf{0 , 0 1 D _ { x , H }}$ & $\Psi_{\mathrm{H}}$ \\
\hline & 25 & 9,81 & 1,0204E-09 & 1,0204E-09 & 0,35 & 1,0000E-03 & 1,8857E-04 & 2 & 1 & 1,8857E-05 & 1,8857E-07 & $6,60 \mathrm{E}-05$ \\
\hline \multirow[t]{2}{*}{2} & $\Delta \rho_{\mathrm{H}}$ & $\mathbf{g}_{\mathbf{H}}$ & $2 \mathbf{k}_{\mathrm{x}, \mathrm{H}}$ & $2 \mathbf{k}_{\mathbf{y}, \mathbf{H}}$ & $\varepsilon_{H}$ & $2 \mu_{\mathrm{H}}$ & $\mathbf{V}_{\mathrm{amb}, \mathrm{H}}$ & $\mathbf{L}_{\mathbf{H}}$ & $\mathbf{H}_{\mathbf{H}}$ & $\mathbf{D}_{\mathrm{x}, \mathrm{H}}$ & $\mathbf{0 , 0 1 D _ { x , H }}$ & $\Psi_{H}$ \\
\hline & 25 & 9,81 & $2,0408 \mathrm{E}-09$ & $2,0408 \mathrm{E}-09$ & 0,35 & $2,0000 \mathrm{E}-03$ & 1,8857E-04 & 2 & 1 & 1,8857E-05 & 1,8857E-07 & $6,60 \mathrm{E}-05$ \\
\hline \multirow[t]{2}{*}{3} & $\Delta \rho_{H}$ & $\mathbf{g}_{\mathbf{H}}$ & $2 \mathbf{k}_{\mathrm{x}, \mathrm{H}}$ & $2 k_{y, H}$ & $\varepsilon_{\mathrm{H}}$ & $\mu_{H}$ & $2 v_{a m b, H}$ & $\mathbf{L}_{\mathbf{H}}$ & $\mathbf{H}_{\mathbf{H}}$ & $\mathbf{D}_{\mathrm{x}, \mathrm{H}}$ & $\mathbf{0 , 0 1 D _ { x , H }}$ & $2 \Psi_{H}$ \\
\hline & 25 & 9,81 & $2,0408 \mathrm{E}-09$ & $2,0408 \mathrm{E}-09$ & 0,35 & $1,0000 \mathrm{E}-03$ & $3,7714 \mathrm{E}-04$ & 2 & 1 & $3,7714 \mathrm{E}-05$ & $3,7714 \mathrm{E}-07$ & $1,32 \mathrm{E}-04$ \\
\hline \multirow[t]{2}{*}{ 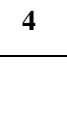 } & 2 & $\mathbf{g}_{\mathrm{H}}$ & $\mathbf{k}_{\mathbf{x}, \mathrm{H}}$ & $\mathbf{k}_{\mathbf{y}, \mathbf{H}}$ & $\varepsilon_{H}$ & $2 \mu_{\mathrm{H}}$ & $\mathbf{V}_{\mathrm{amb}, \mathrm{H}}$ & $\mathbf{L}_{\mathbf{H}}$ & $\mathbf{H}_{\mathbf{H}}$ & $\mathbf{D}_{\mathrm{x}, \mathrm{H}}$ & $\mathbf{0 , 0 1 D _ { x , H }}$ & $\Psi_{\mathrm{H}}$ \\
\hline & 50 & 9,81 & $1,0204 \mathrm{E}-09$ & $1,0204 \mathrm{E}-09$ & 0,35 & $2,0000 \mathrm{E}-03$ & 1,8857E-04 & 2 & 1 & 1,8857E-05 & 1,8857E-07 & $6,60 \mathrm{E}-05$ \\
\hline \multirow[t]{2}{*}{5} & 2 & $\mathbf{g}_{\mathbf{H}}$ & $2 \mathbf{k}_{\mathrm{x}, \mathrm{H}}$ & $2 k_{y, H}$ & $\varepsilon_{H}$ & $4 \mu_{H}$ & $\mathbf{V}_{\text {amb,H }}$ & $\mathbf{L}_{\mathbf{H}}$ & $\mathbf{H}_{\mathbf{H}}$ & $\mathbf{D}_{\mathbf{x}, \mathrm{H}}$ & $\mathbf{0 , 0 1 D _ { x , H }}$ & $\Psi_{\mathrm{H}}$ \\
\hline & 50 & 9,81 & $2,0408 \mathrm{E}-09$ & $2,0408 \mathrm{E}-09$ & 0,35 & $4,0000 E-03$ & 1,8857E-04 & 2 & 1 & 1,8857E-05 & 1,8857E-07 & $6,60 \mathrm{E}-05$ \\
\hline \multirow[t]{2}{*}{6} & $\Delta \rho_{H}$ & $\mathbf{g}_{\mathbf{H}}$ & $\mathbf{k}_{\mathbf{x}, \mathbf{H}}$ & $\mathbf{k}_{\mathbf{y}, \mathbf{H}}$ & $\varepsilon_{\mathrm{H}}$ & $2 \mu_{H}$ & $(1 / 2) \mathbf{v}_{a m b, H}$ & $\mathbf{L}_{\mathbf{H}}$ & $\mathbf{H}_{\mathbf{H}}$ & $(1 / 2) D_{x, H}$ & $\mathbf{0 , 0 1 D _ { x , H }}$ & $(1 / 2) \Psi_{H}$ \\
\hline & 25 & 9,81 & $1,0204 \mathrm{E}-09$ & 1,0204E-09 & 0,35 & $2,0000 \mathrm{E}-03$ & $9,4285 \mathrm{E}-05$ & 2 & 1 & $9,4285 \mathrm{E}-06$ & $9,4285 \mathrm{E}-08$ & $3,30 \mathrm{E}-05$ \\
\hline
\end{tabular}

Tabla IV.2 Valores de los parámetros para los casos de difusividad vertical despreciable

Caben comentarios similares en cierto modo a los expuestos en la primera simplificación. Al no ser despreciable la difusividad horizontal, la extensión horizontal de la zona del acuífero parcialmente contaminada se extiende en relación con la primera simplificación. Es inmediato observar esta extensión de la región en la base del acuífero que se incremente aproximadamente de 0.56 a $0.8 \mathrm{~m}$.

Adviértase, sin embargo, que la región ocupada por la recirculación de agua de mar (línea divisoria en los patrones de función de corriente) define la misma extensión en la base del acuífero en concordancia con la constancia del valor de los grupos adimensionales que no contienen la difusividad. 

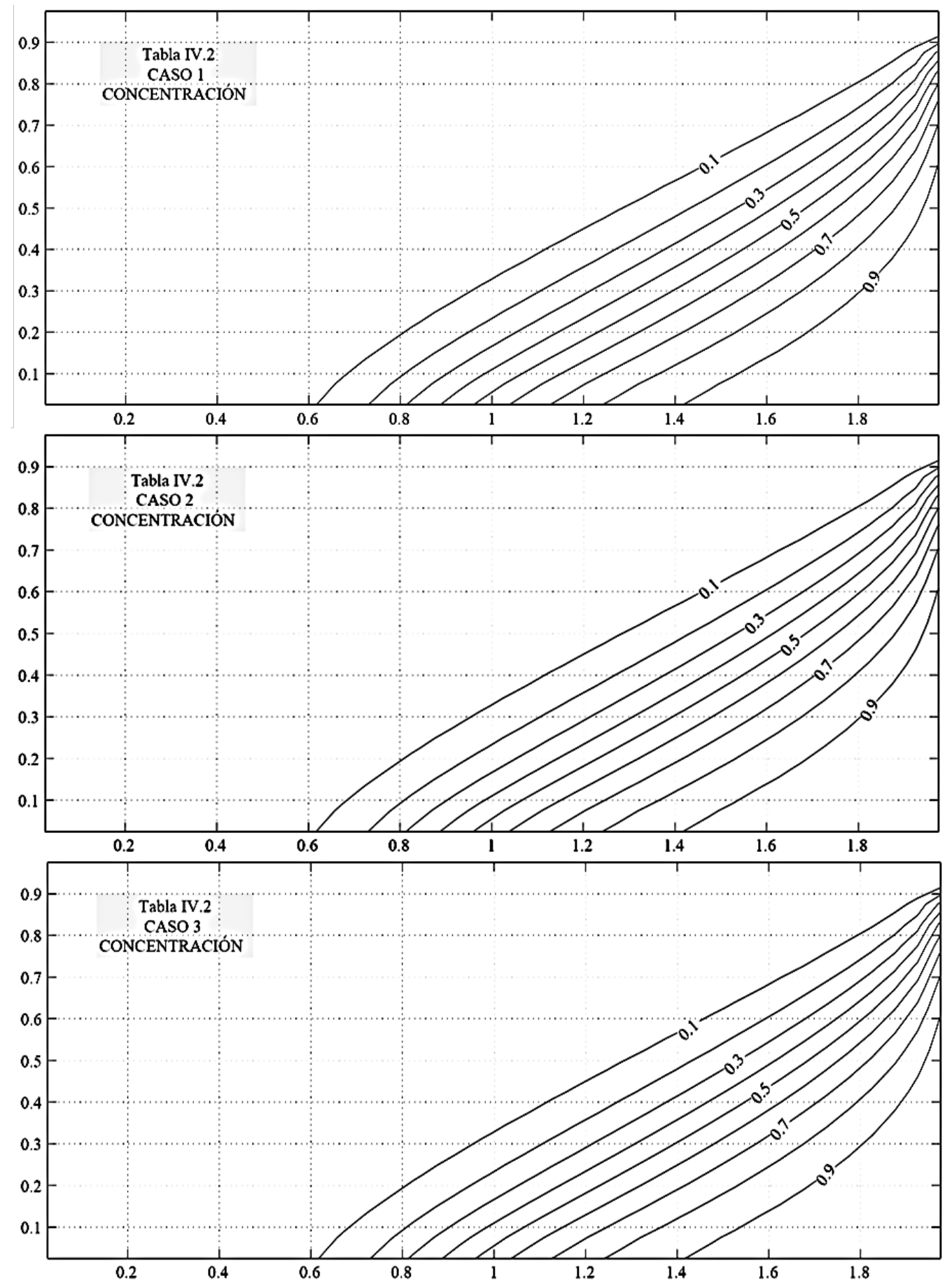

Figura IV. 4a Patrones de concentración para los casos de difusividad vertical despreciable (Casos 1.2 y 3, Tabla IV.2) 

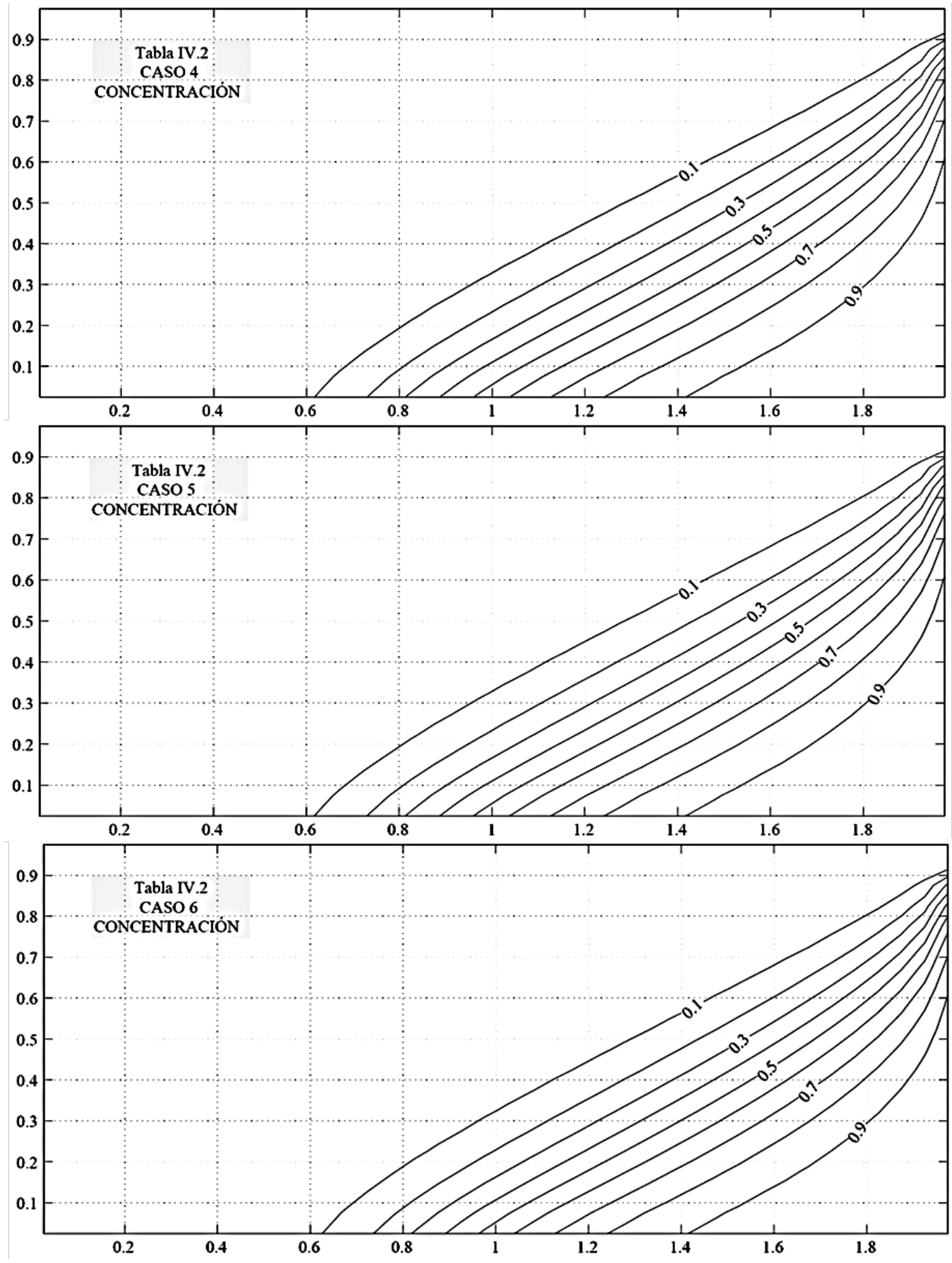

Figura IV. 4b Patrones de concentración para los casos de difusividad vertical despreciable (Casos 4, 5 y 6, Tabla IV.2) 

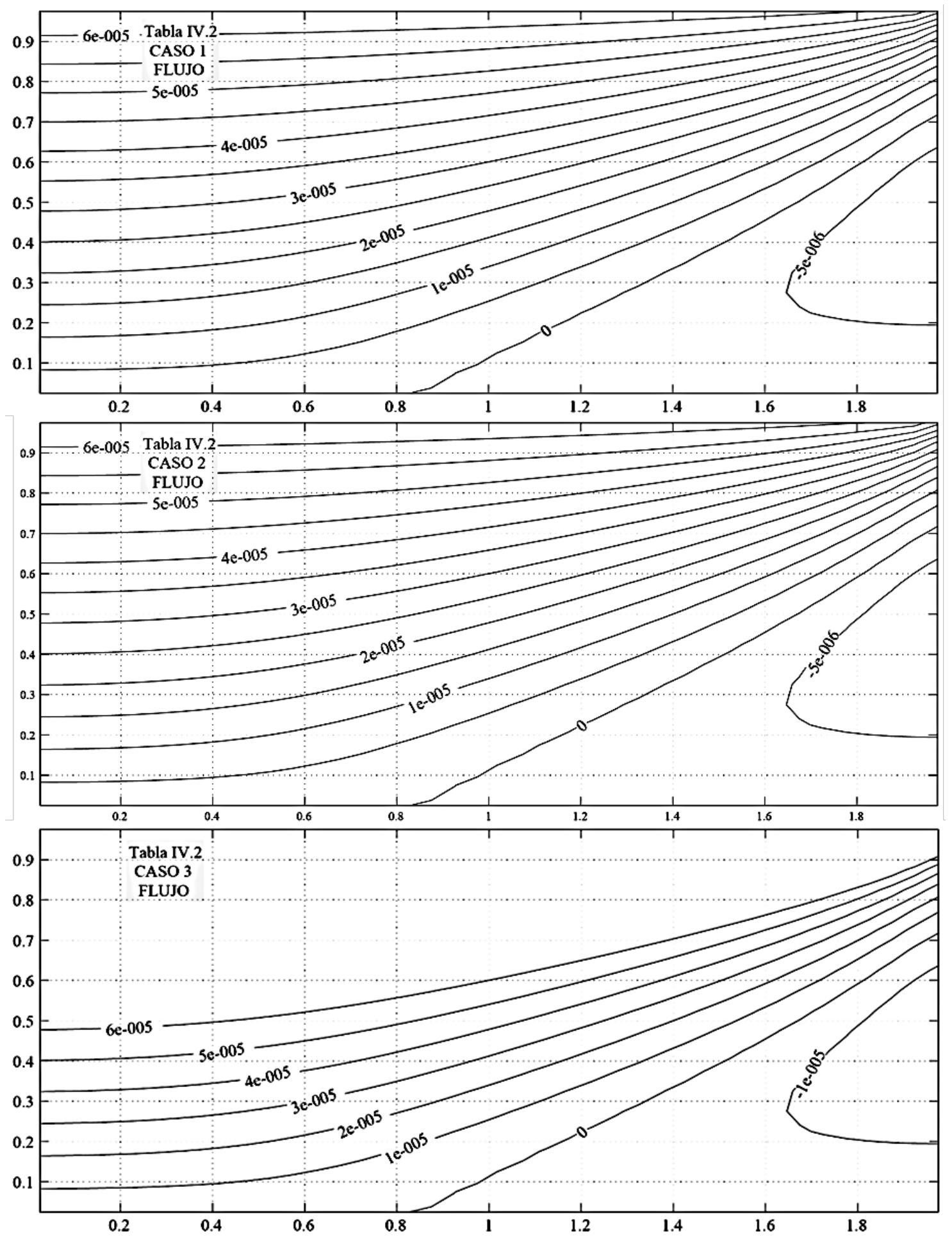

Figura IV. 5a Patrones de función de corriente para los casos de difusividad vertical despreciable (Casos 1, 2 y 3, Tabla IV.2) 
Capítulo IV. Simplificación de escenarios
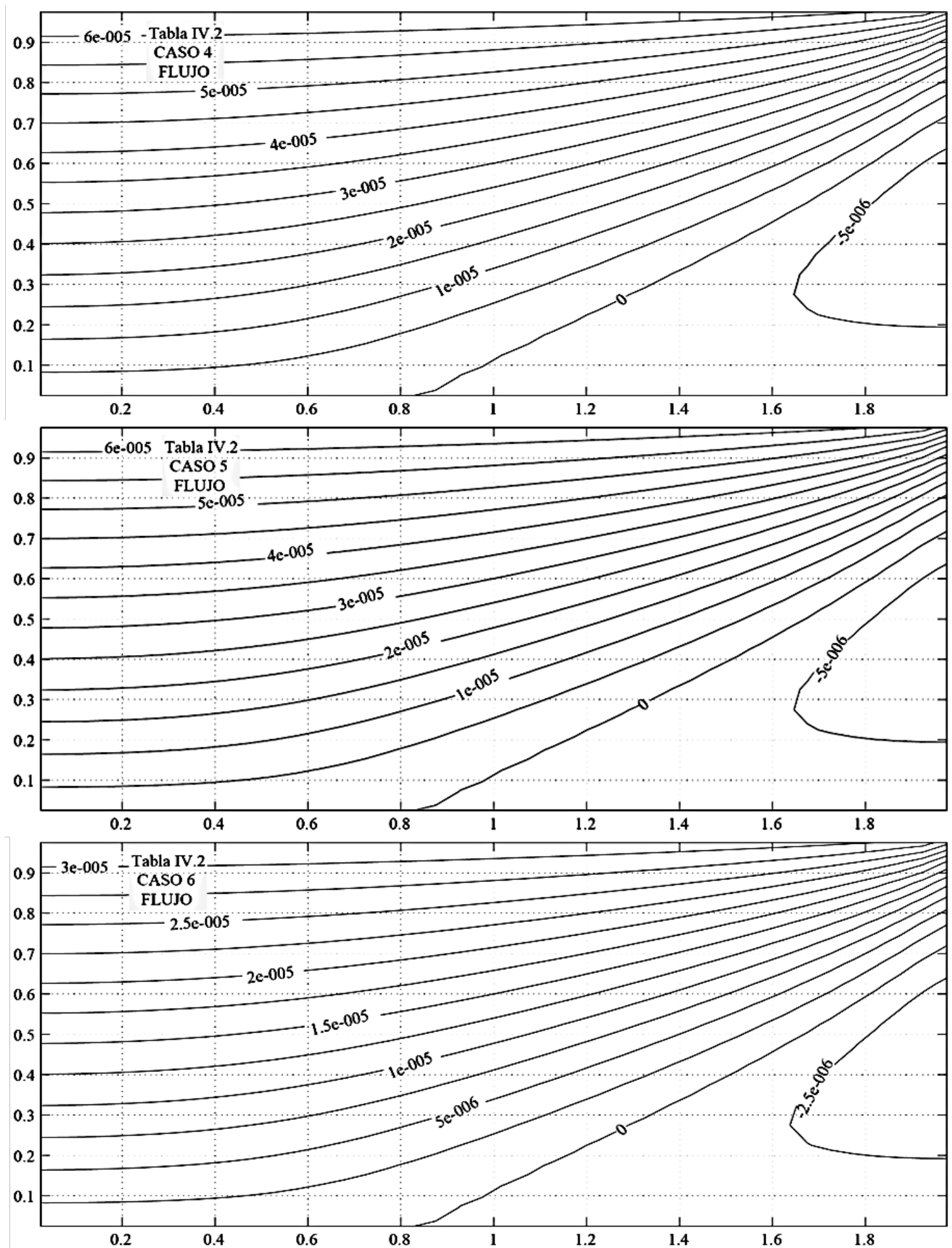

Figura IV. 5b Patrones de función de corriente para los casos de difusividad vertical despreciable (Casos 4, 5 y 6, Tabla IV.2) 


\section{IV.1.3 TERCERA SIMPLIFICACIÓN. DIFUSIVIDAD ISOTRÓPICA, $D_{Y}=D_{x}$}

Para esta hipótesis, de los cuatro coeficientes de la ecuación de soluto emergen tres grupos adimensionales que pueden ser escritos en la forma siguiente (adoptando Vamb ${ }^{*}$ como la velocidad de referencia horizontal),

$$
\begin{aligned}
& \pi_{\mathrm{d}, 1}=\frac{(\Delta \rho) \mathrm{gk}_{\mathrm{y}}}{\varepsilon \mu \mathrm{v}_{\mathrm{amb}}^{*}}\left(\frac{\mathrm{L}}{\mathrm{H}}\right) \\
& \pi_{\mathrm{d}, 3}=\frac{\mathrm{D}}{\mathrm{v}_{\mathrm{amb}}^{*} \mathrm{~L}} \\
& \pi_{\mathrm{d}, 4}=\frac{\mathrm{H}^{2}}{\mathrm{~L}^{2}}
\end{aligned}
$$

Así, el número total de grupos independientes son los anteriores, más uno nuevo procedente de la ecuación del momento (como en el caso general). Los escribiremos en la forma

$$
\begin{aligned}
& \pi_{\mathrm{d}, 1}=\frac{(\Delta \rho) \mathrm{gk}_{\mathrm{y}}}{\varepsilon \mu \mathrm{v}_{\mathrm{amb}}^{*}}\left(\frac{\mathrm{L}}{\mathrm{H}}\right) \\
& \pi_{\mathrm{d}, 2}=\frac{\mathrm{k}_{\mathrm{x}} \mathrm{H}^{2}}{\mathrm{k}_{\mathrm{y}} \mathrm{L}^{2}} \\
& \pi_{\mathrm{d}, 3}=\frac{\mathrm{D}}{\mathrm{v}_{\mathrm{amb}}^{*} \mathrm{~L}} \\
& \pi_{\mathrm{d}, 4}=\frac{\mathrm{H}^{2}}{\mathrm{~L}^{2}}
\end{aligned}
$$

La isotropía de la difusividad, D, sin embargo, revela ahora el factor de forma H/L como un grupo independiente. Mediante manipulaciones matemáticas sencillas, los grupos anteriores pueden expresarse en su forma más simple (propuesta también por Abarca [2006]):

$$
\pi_{\mathrm{d}, 1}=\frac{(\Delta \rho) \mathrm{gk}_{\mathrm{y}}}{\varepsilon \mu \mathrm{v}_{\mathrm{amb}}^{*}} \quad \pi_{\mathrm{d}, 2}=\frac{\mathrm{k}_{\mathrm{x}}}{\mathrm{k}_{\mathrm{y}}} \quad \pi_{\mathrm{d}, 3}=\frac{\mathrm{D}}{\mathrm{v}_{\mathrm{amb}}^{*}{ }^{\mathrm{H}}} \quad \pi_{\mathrm{d}, 4}=\frac{\mathrm{H}}{\mathrm{L}}
$$

Adviértase, sin embargo, que el grupo $\mathrm{D} /\left(\mathrm{Vamb}^{*} \mathrm{H}\right)$ pierde su significado físico lógico asignado en el caso general ya que, ahora, escrito en la forma $\left(\mathrm{H} / \mathrm{v}_{\mathrm{amb}}{ }^{*}\right) /\left(\mathrm{H}^{2} / \mathrm{D}\right)$, es la razón entre el tiempo requerido por el fluido para recorrer la distancia $\mathrm{H}$ animado de la velocidad $\mathrm{Vamb}^{*}$ (arrastre) y el tiempo para recorrer la misma distancia por efecto de la difusión. La Tabla IV.3 muestra los parámetros de cinco casos típicos de esta simplificación, con el caso 1 como escenario de Henry. 
Las Figuras IV.6 y IV.7 muestran los patrones de concentración y flujo, respectivamente, de los casos de la tabla anterior. Para todos, los monomios deducidos retienen un mismo valor

$\pi_{\mathrm{d}, 1}=\frac{(\Delta \rho) \mathrm{gk}_{\mathrm{y}}}{\varepsilon \mu \mathrm{v}_{\mathrm{amb}}^{*}}=3,79 \quad \pi_{\mathrm{d}, 2}=\frac{\mathrm{k}_{\mathrm{x}}}{\mathrm{k}_{\mathrm{y}}}=1 \quad \pi_{\mathrm{d}, 3}=\frac{\mathrm{D}}{\mathrm{v}_{\mathrm{amb}}^{*} \mathrm{H}}=0,05 \quad \pi_{\mathrm{d}, 4}=\frac{\mathrm{H}}{\mathrm{L}}=0,5$

por lo que los patrones resultantes son idénticos como muestra la simulación.

Tabla IV.3 Valores de los parámetros para los casos de difusividad isotrópica

\begin{tabular}{|c|c|c|c|c|c|c|c|c|c|c|c|}
\hline Caso & $\Delta \rho$ & g & $\mathbf{k}_{\mathrm{x}}$ & $\mathbf{k}_{\mathbf{y}}$ & $\varepsilon$ & $\mu$ & $\mathbf{v}_{\mathrm{amb}}$ & $\mathbf{L}$ & $\mathbf{H}$ & $\mathbf{D}_{\mathrm{x}}=\mathrm{D}_{\mathrm{y}}$ & $\Psi$ \\
\hline \multirow[t]{2}{*}{1} & $\Delta \rho_{\mathrm{H}}$ & $\mathbf{g}_{\mathrm{H}}$ & $\mathbf{k}_{\mathrm{x}, \mathrm{H}}$ & $\mathbf{k}_{\mathbf{y}, \mathrm{H}}$ & $\varepsilon_{\mathrm{H}}$ & $\mu_{\mathrm{H}}$ & $\mathbf{V}_{\mathbf{a m b}, \mathbf{H}}$ & $\mathbf{L}_{\mathbf{H}}$ & $\mathbf{H}_{\mathbf{H}}$ & $\mathbf{D}_{\mathrm{H}}$ & $\Psi_{\mathrm{H}}$ \\
\hline & 25 & 9,81 & 1,0204E-09 & $1,0204 \mathrm{E}-09$ & 0,35 & $1,0000 \mathrm{E}-03$ & 1,8857E-04 & 2 & 1 & 1,8857E-05 & 6,60E-05 \\
\hline \multirow[t]{2}{*}{2} & $2 \Delta \rho_{\mathrm{H}}$ & $\mathbf{g}_{\mathrm{H}}$ & $\mathbf{k}_{\mathrm{x}, \mathrm{H}}$ & $\mathbf{k}_{\mathrm{y}, \mathrm{H}}$ & $\varepsilon_{\mathrm{H}}$ & $\mu_{\mathrm{H}}$ & $2 v_{a m b, H}$ & $\mathbf{L}_{\mathbf{H}}$ & $\mathbf{H}_{\mathbf{H}}$ & $2 D_{H}$ & $2 \Psi_{\mathrm{H}}$ \\
\hline & 50 & 9,81 & 1,0204E-09 & 1,0204E-09 & 0,35 & $1,0000 \mathrm{E}-03$ & 3,7714E-04 & 2 & 1 & 3,7714E-05 & $1,32 \mathrm{E}-04$ \\
\hline \multirow[t]{2}{*}{3} & $\Delta \rho_{\mathrm{H}}$ & $\mathbf{g}_{\mathrm{H}}$ & $2 \mathbf{k}_{\mathrm{x}, \mathrm{H}}$ & $2 \mathrm{k}_{\mathrm{y}, \mathrm{H}}$ & $\varepsilon_{\mathrm{H}}$ & $2 \mu_{\mathrm{H}}$ & $\mathbf{V}_{\mathbf{a m b}, \mathbf{H}}$ & $\mathbf{L}_{\mathbf{H}}$ & $\mathbf{H}_{\mathbf{H}}$ & $\mathbf{D}_{\mathrm{H}}$ & $\Psi_{\mathrm{H}}$ \\
\hline & 25 & 9,81 & 2,0408E-09 & 2,0408E-09 & 0,35 & $2,0000 \mathrm{E}-03$ & 1,8857E-04 & 2 & 1 & 1,8857E-05 & $6,60 \mathrm{E}-05$ \\
\hline \multirow[t]{2}{*}{4} & $4 \Delta \rho_{\mathrm{H}}$ & $\mathbf{g}_{\mathrm{H}}$ & $\mathbf{k}_{\mathrm{x}, \mathrm{H}}$ & $\mathbf{k}_{\mathbf{y}, \mathbf{H}}$ & $\varepsilon_{\mathrm{H}}$ & $4 \mu_{H}$ & $\mathbf{V}_{\mathbf{a m b}, \mathbf{H}}$ & $\mathbf{L}_{\mathbf{H}}$ & $\mathbf{H}_{\mathbf{H}}$ & $\mathbf{D}_{\mathrm{H}}$ & $\Psi_{\mathrm{H}}$ \\
\hline & 100 & 9,81 & $1,0204 \mathrm{E}-09$ & $1,0204 \mathrm{E}-09$ & 0,35 & $4,0000 \mathrm{E}-03$ & 1,8857E-04 & 2 & 1 & 1,8857E-05 & $6,60 \mathrm{E}-05$ \\
\hline \multirow[t]{2}{*}{5} & $\Delta \rho_{\mathrm{H}}$ & $\mathbf{g}_{\mathrm{H}}$ & $2 k_{x, H}$ & $2 \mathrm{k}_{\mathrm{y}, \mathrm{H}}$ & $\varepsilon_{\mathrm{H}}$ & $(1 / 2) \mu_{H}$ & $4 \mathrm{v}_{\mathrm{amb}, \mathrm{H}}$ & $\mathbf{L}_{\mathbf{H}}$ & $\mathbf{H}_{\mathbf{H}}$ & $4 D_{H}$ & $4 \Psi_{\mathrm{H}}$ \\
\hline & 25 & 9,81 & 2,0408E-09 & $2,0408 \mathrm{E}-09$ & 0,35 & $5,0000 \mathrm{E}-04$ & 7,5428E-04 & 2 & 1 & 7,5428E-05 & 2,64E-04 \\
\hline
\end{tabular}

Se observa que el patrón de concentraciones es el mismo en todos los casos tanto cualitativa como cuantitativamente. El patrón de flujo es cuantitativamente diferente merced al cambio de flujo regional de un caso a otro, manteniendo siempre la línea divisoria en la misma posición. Estos resultados son coherentes en tanto que se ha mantenido la permeabilidad como parámetro isótropo, forzado por la isotropía en la difusividad, para mantener el valor de los monomios. Esto hace que los grupos anteriores se simplifiquen realmente a:

$$
\pi_{\mathrm{d}, 1}=\frac{(\Delta \rho) \mathrm{gk}}{\varepsilon \mu \mathrm{v}_{\mathrm{amb}}^{*}}\left(\frac{\mathrm{L}}{\mathrm{H}}\right) \quad \pi_{\mathrm{d}, 3}=\frac{\mathrm{D}}{\mathrm{v}_{\mathrm{amb}}^{*} \mathrm{~L}} \quad \pi_{\mathrm{d}, 4}=\frac{\mathrm{H}^{2}}{\mathrm{~L}^{2}}
$$

que se reducen, mediante manipulaciones matemáticas sencillas, a los siguientes

$$
\pi_{\mathrm{d}, 1}=\frac{(\Delta \rho) \mathrm{gk}}{\varepsilon \mu \mathrm{v}_{\mathrm{amb}}^{*}} \quad \pi_{\mathrm{d}, 3}=\frac{\mathrm{D}}{\mathrm{v}_{\mathrm{amb}}^{*} \mathrm{H}} \quad \pi_{\mathrm{d}, 4}=\frac{\mathrm{H}}{\mathrm{L}}
$$

Se trata del caso de Henry, cuya verificación ya se ha realizado en el Capítulo III (Tabla III.2 y figuras subsiguientes). 
Capítulo IV. Simplificación de escenarios
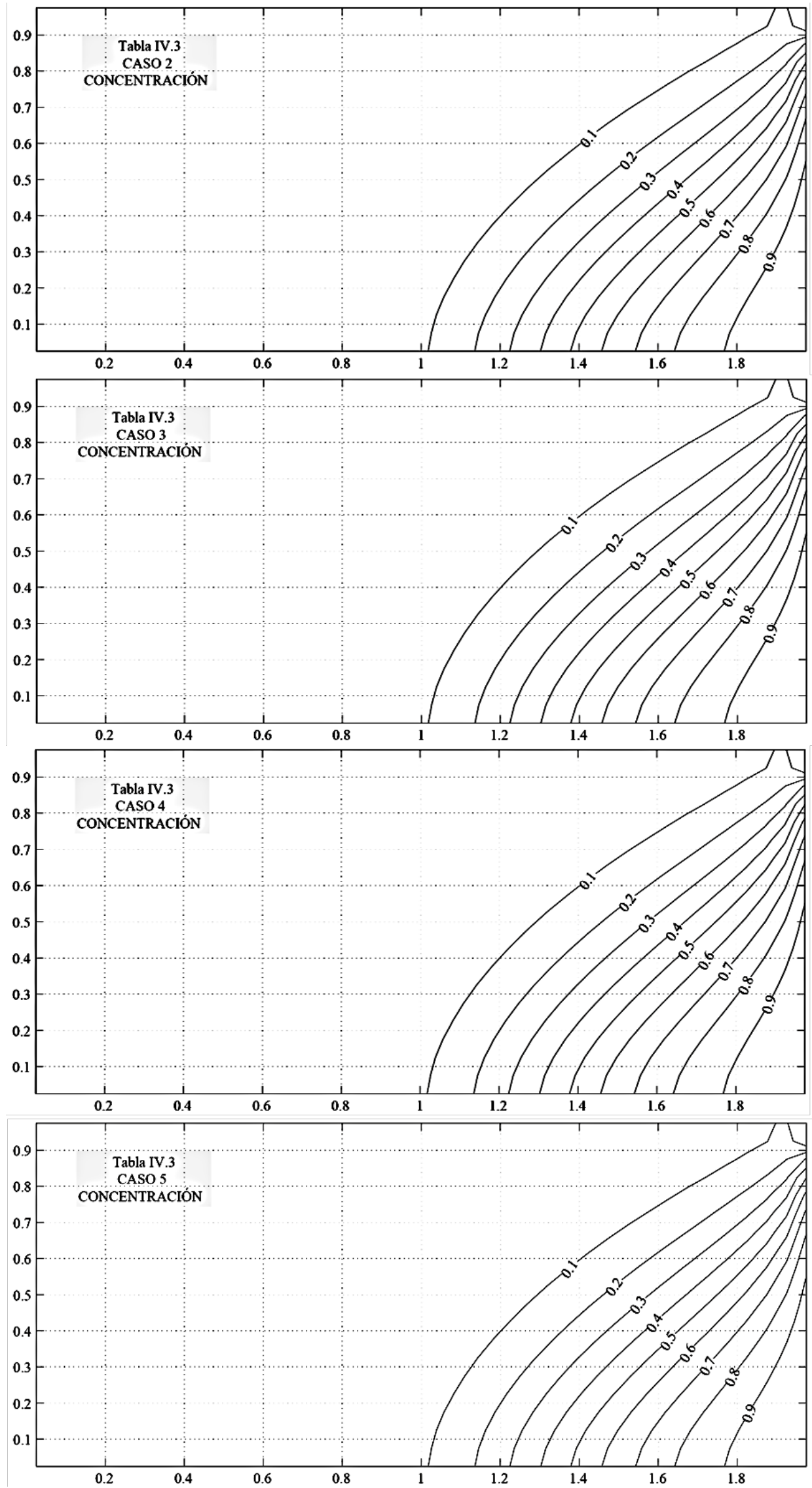

Figura IV.6 Patrones de concentración para los casos de difusividad isótropa (Casos 2 a 5, Tabla IV.3) 
Capítulo IV. Simplificación de escenarios
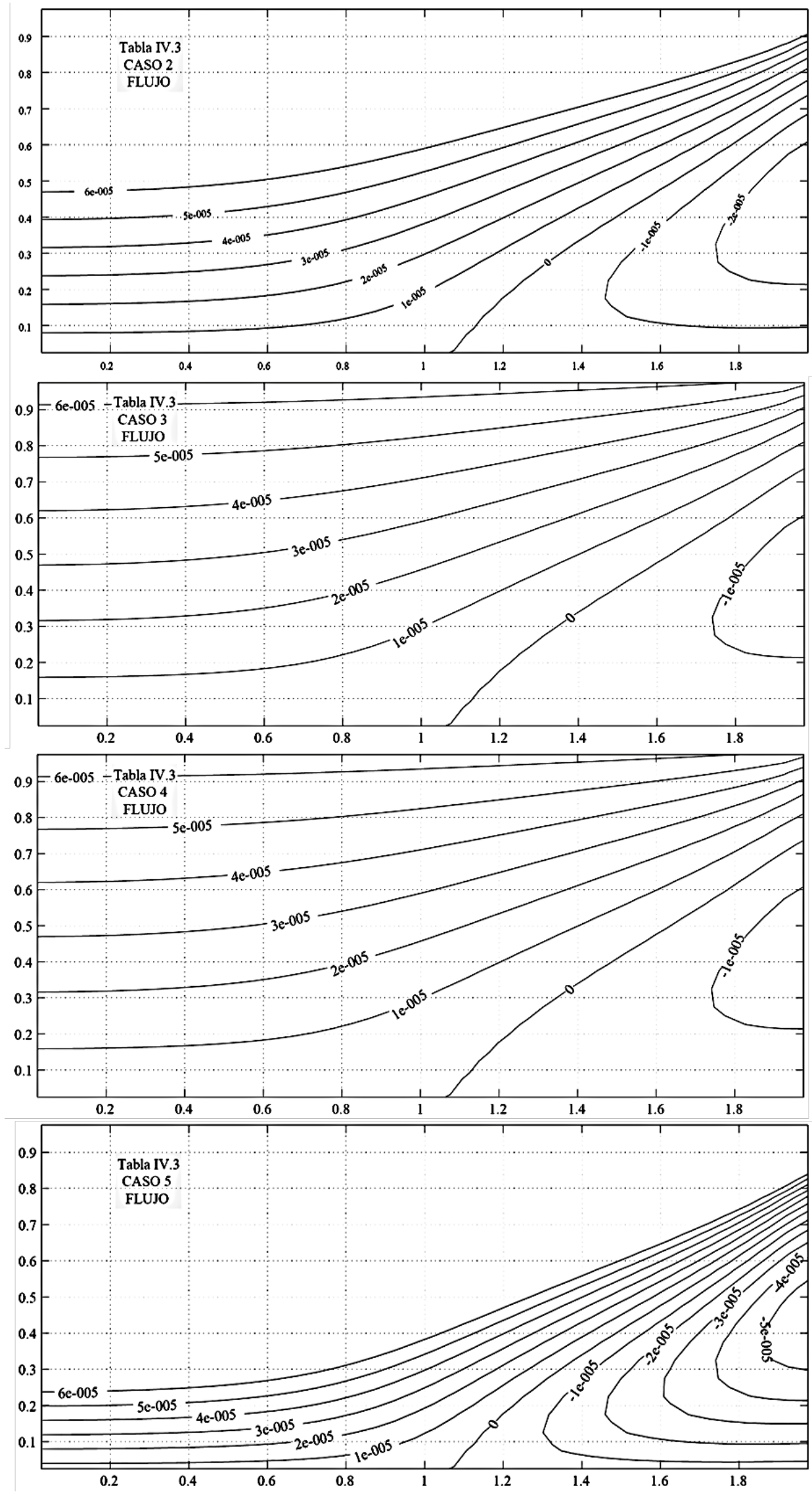

Figura IV.7 Patrones de función de corriente para los casos de difusividad isótropa (Casos 2 a 5, Tabla IV.3) 


\section{IV.1.4 CUARTA SIMPLIFICACIÓN. VELOCIDAD REGIONAL DESPRECIABLE FRENTE A LA DIFUSIÓN (LVamb $<<$ Dx)}

Si queremos mantener bajo esta simplificación la influencia de una difusividad anisótropa en los patrones, y a la vista de la forma de los monomios del problema general, asumiremos una aproximación del tipo $\mathrm{LV}_{\mathrm{amb}}<<\mathrm{D}_{\mathrm{x}}$. Esta hipótesis permite retener el monomio $\pi_{\mathrm{d}, 4}=\mathrm{D}_{\mathrm{x}} \mathrm{H}^{2} /\left(\mathrm{D}_{\mathrm{y}} \mathrm{L}^{2}\right)$. Partiremos de los cuatro monomios del caso general

$$
\begin{aligned}
\pi_{\mathrm{d}, 1} & =\frac{(\Delta \rho) g \mathrm{k}_{\mathrm{y}}}{\varepsilon \mu \mathrm{v}_{\mathrm{amb}}^{*}}\left(\frac{\mathrm{L}}{\mathrm{H}}\right) \\
\pi_{\mathrm{d}, 2} & =\frac{\mathrm{k}_{\mathrm{x}} \mathrm{H}^{2}}{\mathrm{k}_{\mathrm{y}} \mathrm{L}^{2}} \\
\pi_{\mathrm{d}, 3} & =\frac{\mathrm{D}_{\mathrm{x}}}{\mathrm{v}_{\mathrm{amb}}^{*} \mathrm{~L}} \\
\pi_{\mathrm{d}, 4} & =\frac{\mathrm{D}_{\mathrm{x}} \mathrm{H}^{2}}{\mathrm{D}_{\mathrm{y}} \mathrm{L}^{2}}
\end{aligned}
$$

y eliminaremos la influencia de $\mathrm{vamb}_{\mathrm{amb}}$ combinando los monomios $\pi_{\mathrm{d}, 1} \mathrm{y} \pi_{\mathrm{d}, 3}$. Los grupos independientes se reducen a tres, a saber,

$$
\begin{aligned}
& \pi_{\mathrm{d}, 1}=\frac{(\Delta \rho) \mathrm{gk}_{\mathrm{y}}}{\varepsilon \mu \mathrm{D}_{x}}\left(\frac{\mathrm{L}^{2}}{\mathrm{H}}\right) \\
& \pi_{\mathrm{d}, 2}=\frac{\mathrm{k}_{\mathrm{x}} \mathrm{H}^{2}}{\mathrm{k}_{\mathrm{y}} \mathrm{L}^{2}} \\
& \pi_{\mathrm{d}, 4}=\frac{\mathrm{D}_{\mathrm{x}} \mathrm{H}^{2}}{\mathrm{D}_{\mathrm{y}} \mathrm{L}^{2}}
\end{aligned}
$$

Razonando de otra forma, la única velocidad horizontal característica tras asumir $\mathrm{V}_{\mathrm{amb}}$ despreciable, deriva de la ecuación de continuidad: $\mathrm{v}_{\mathrm{x}}{ }^{*}=\left(\mathrm{k}_{\mathrm{y}} \mathrm{g} \Delta \rho \mathrm{L}\right) /(\varepsilon \mu \mathrm{H})$. Sustituyendo esta referencia en la ecuación de continuidad del soluto se obtienen los coeficientes

$$
\mathrm{v}_{\mathrm{x}}^{*} / \mathrm{L}, \quad \mathrm{v}_{\mathrm{y}}{ }^{*} / \mathrm{H}=(\Delta \rho) \mathrm{gk}_{\mathrm{y}} /(\varepsilon \mu \mathrm{H}), \quad \mathrm{D}_{\mathrm{x}} / \mathrm{L}^{2} \quad \mathrm{y} \quad \mathrm{D}_{\mathrm{y}} / \mathrm{H}^{2}
$$

de los que los dos primeros son iguales merced a la ecuación de continuidad del fluido. Estos coeficientes dan lugar a dos monomios independientes

$$
\pi_{\mathrm{d}, 1}=\frac{\mathrm{D}_{\mathrm{x}} \mathrm{H} \varepsilon \mu}{\mathrm{gk}_{\mathrm{y}} \Delta \rho \mathrm{L}^{2}} \quad \pi_{\mathrm{d}, 4}=\frac{\mathrm{D}_{\mathrm{y}} \mathrm{H} \varepsilon \mu}{\mathrm{gk}_{\mathrm{y}} \Delta \rho \mathrm{H}}=\frac{\mathrm{D}_{\mathrm{x}} \mathrm{H}^{2}}{\mathrm{D}_{\mathrm{y}} \mathrm{L}^{2}}
$$

Con todo, se llega a un total de tres monomios independientes. Si además $\mathrm{k}_{\mathrm{x}}=\mathrm{k}_{\mathrm{y}}=\mathrm{k}$ y $\mathrm{D}_{\mathrm{x}}=\mathrm{D}_{\mathrm{y}}=\mathrm{D}$, los grupos resultantes se reducen a dos:

$$
\pi_{\mathrm{d}, 1}=\frac{\mathrm{DH} \varepsilon \mu}{\mathrm{gk} \Delta \rho \mathrm{L}^{2}} \quad \pi_{\mathrm{d}, 4}=\left(\frac{\mathrm{H}}{\mathrm{L}}\right)
$$

o bien, simplificando, a los monomios 
$\pi_{\mathrm{d}, 1}=\frac{\mathrm{D} \varepsilon \mu}{\mathrm{gk} \Delta \rho \mathrm{L}} \quad \pi_{\mathrm{d}, 4}=\left(\frac{\mathrm{H}}{\mathrm{L}}\right)$

La Tabla IV.4 selecciona tres casos en los que se fija el valor numérico de los grupos anteriores

$$
\pi_{\mathrm{d}, 1}=\frac{(\Delta \rho) \mathrm{gk}_{\mathrm{y}}}{\varepsilon \mu \mathrm{D}_{\mathrm{x}}}\left(\frac{\mathrm{L}^{2}}{\mathrm{H}}\right)=151,67 \quad \pi_{\mathrm{d}, 2}=\frac{\mathrm{k}_{\mathrm{x}} \mathrm{H}^{2}}{\mathrm{k}_{\mathrm{y}} \mathrm{L}^{2}}=0,25 \quad \pi_{\mathrm{d}, 4}=\frac{\mathrm{D}_{\mathrm{x}} \mathrm{H}^{2}}{\mathrm{D}_{\mathrm{y}} \mathrm{L}^{2}}=0,25
$$

La hipótesis se asegura haciendo que en los tres casos se cumpla $\mathrm{V}_{\mathrm{amb}}=$ 0.05( $\left.\mathrm{D}_{\mathrm{X}} / \mathrm{L}\right)$. Esto determina que en el caso 1 (problema cuyos parámetros coinciden con los de Henry, excepto la velocidad regional) resulte una velocidad de valor vamb (Caso 1) = $0.05\left(\mathrm{D}_{\mathrm{x}, \mathrm{H}} / \mathrm{L}\right)=(0.05 \times 1.8857 \mathrm{E}-5) / 2=4.6 \mathrm{E}-7=0.0025 \mathrm{v}_{\mathrm{amb}, \mathrm{H}}$, justo la mitad de la velocidad de Henry. Esto significa que el propio problema de Henry puede considerarse un caso cercano a esta hipótesis, es decir, sus patrones no pueden ser muy diferentes como se comprobará a continuación. Para los casos 2 y 3 de la Tabla, es inmediato deducir los valores de la velocidad regional a partir de la condición $v_{a m b}=0.01\left(D_{x} / L\right)$. Estos valores son: $\mathrm{V}_{\mathrm{amb}}$ (caso 2) $=0.15 \times \mathrm{V}_{\mathrm{amb}, \mathrm{H}} \mathrm{y} \mathrm{V}_{\mathrm{amb}}$ (caso 3) $=0.5 \times \mathrm{V}_{\mathrm{amb}, \mathrm{H}}$.

Tabla IV.4 Valores de los parámetros para los casos de velocidad regional despreciable

\begin{tabular}{|c|c|c|c|c|c|c|c|c|c|c|c|c|}
\hline Caso & $\Delta \rho$ & g & $\mathbf{k}_{\mathrm{x}}$ & $\mathbf{k}_{\mathbf{y}}$ & $\varepsilon$ & $\mu$ & $\mathbf{V}_{\mathrm{amb}}$ & $\mathbf{L}$ & $\mathbf{H}$ & $\mathbf{D}_{\mathrm{x}}$ & $\mathbf{D}_{\mathbf{y}}$ & $\Psi$ \\
\hline \multirow[t]{2}{*}{1} & $\Delta \rho_{\mathrm{H}}$ & $\mathbf{g}_{\mathrm{H}}$ & $\mathbf{k}_{\mathrm{x}, \mathrm{H}}$ & $\mathbf{k}_{\mathrm{y}, \mathrm{H}}$ & $\varepsilon_{\mathrm{H}}$ & $\mu_{\mathrm{H}}$ & $0.0025 v_{\mathrm{amb}, \mathrm{H}}$ & $\mathbf{L}_{\mathbf{H}}$ & $\mathbf{H}_{\mathbf{H}}$ & $\mathbf{D}_{\mathrm{x}, \mathrm{H}}$ & $\mathbf{D}_{\mathbf{y}, \mathbf{H}}$ & $0.0025 \Psi_{H}$ \\
\hline & 25 & 9,81 & 1,0204E-09 & 1,0204E-09 & 0,35 & $1,0 \mathrm{E}-03$ & 4,7143E-07 & 2 & 1 & 1,8857E-05 & 1,8857E-05 & $1,65 \mathrm{E}-07$ \\
\hline \multirow[t]{2}{*}{2} & $3 \Delta \rho_{\mathrm{H}}$ & $\mathbf{g}_{\mathrm{H}}$ & $2 k_{x, H}$ & $2 k_{y, H}$ & $\varepsilon_{\mathrm{H}}$ & $2 \mu_{\mathrm{H}}$ & 0.0075vamb, & $\mathbf{L}_{\mathbf{H}}$ & $\mathbf{H}_{\mathbf{H}}$ & $3 \mathbf{D}_{\mathbf{x}, \mathrm{H}}$ & $3 \mathbf{D}_{\mathbf{y}, \mathrm{H}}$ & $0.0075 \Psi_{H}$ \\
\hline & 75 & 9,81 & 2,0408E-09 & 2,0408E-09 & 0,35 & $2,0 \mathrm{E}-03$ & 1,4143E-06 & 2 & 1 & 5,6571E-05 & 5,6571E-05 & 4,95E-07 \\
\hline \multirow[t]{2}{*}{3} & $4 \Delta \rho_{\mathrm{H}}$ & $\mathbf{g}_{\mathrm{H}}$ & $\mathbf{k}_{\mathrm{x}, \mathrm{H}}$ & $k_{y, H}$ & $\varepsilon_{\mathrm{H}}$ & $4 \mu_{\mathrm{H}}$ & $0.0025 v_{a m b, H}$ & $\mathbf{L}_{\mathbf{H}}$ & $\mathbf{H}_{\mathbf{H}}$ & $\mathbf{D}_{\mathbf{x}, \mathrm{H}}$ & $\mathbf{D}_{\mathbf{x}, \mathrm{H}}$ & $0.0025 \Psi_{H}$ \\
\hline & 100 & 9,81 & 1,0204E-09 & 1,0204E-09 & 0,35 & $4,0 \mathrm{E}-03$ & 4,7143E-07 & 2 & 1 & 1,8857E-05 & 1,8857E-05 & 1,65E-07 \\
\hline
\end{tabular}

Las Figuras IV.8 y IV.9 muestran las simulaciones (patrones de concentración y flujo, respectivamente) de los casos de la tabla anterior. Como esperábamos se trata de patrones idénticos. Los patrones de salinidad, derivados de la fuerte influencia de la difusividad frente al flujo horizontal, se han extendido no sólo cubriendo todo el acuífero sino creando un gradiente alto en la región inferior izquierda del dominio demostrando con ello que la longitud del acuífero influye decisivamente en el patrón. En cuanto a los patrones de flujo se ha creado una evidente distorsión en relación con el problema patrón, haciendo emerger dos regiones de recirculación de agua en los bordes 
laterales del acuífero, mientras que en su frontera inferior aparece un flujo invertido de agua salada desde el mar hacia el continente.
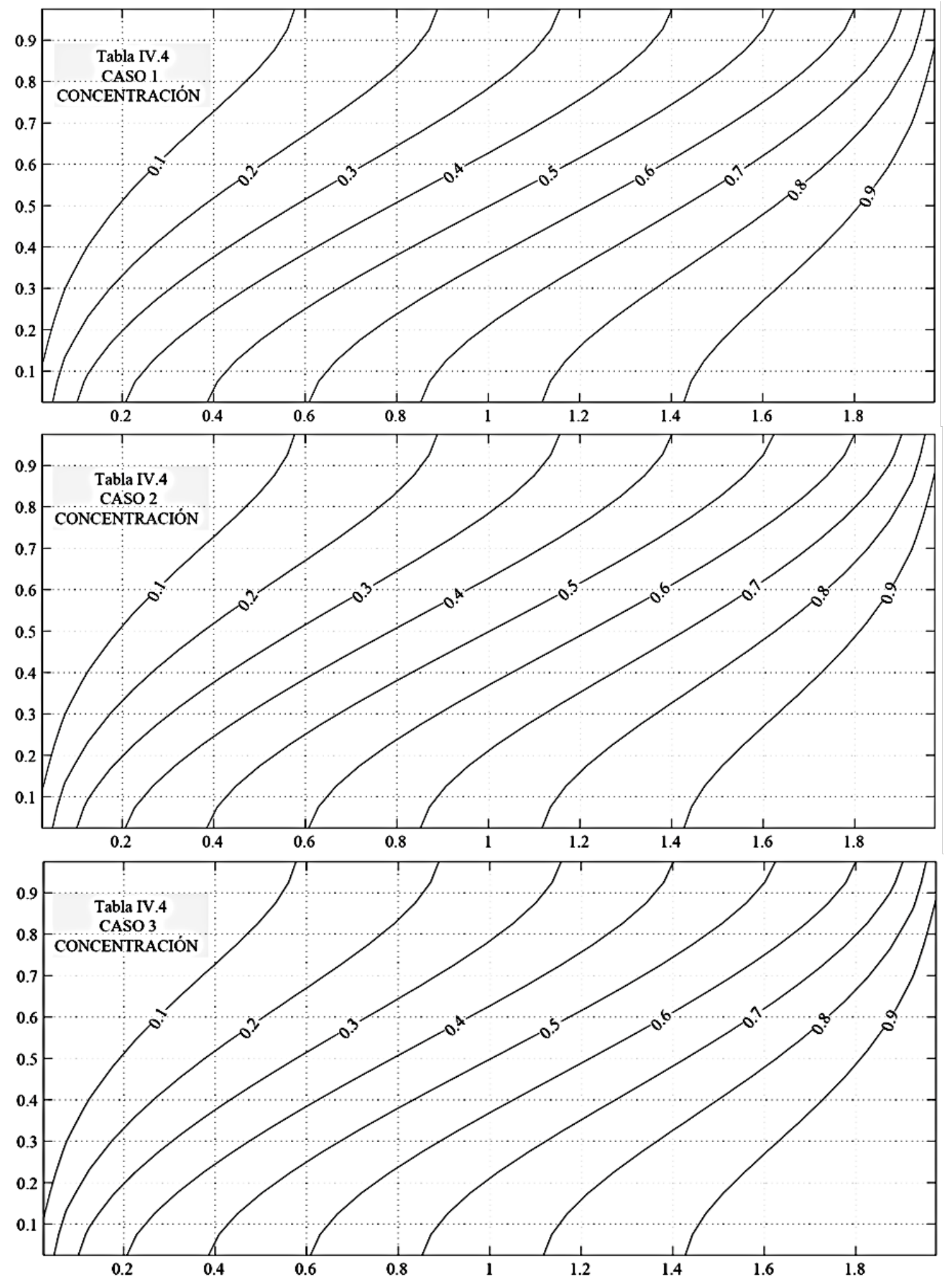

Figura IV.8. Patrones de concentración para los casos de $v_{a m b}$ despreciable, $L v_{a m b}<<D_{x}$ (Tabla IV.4) 

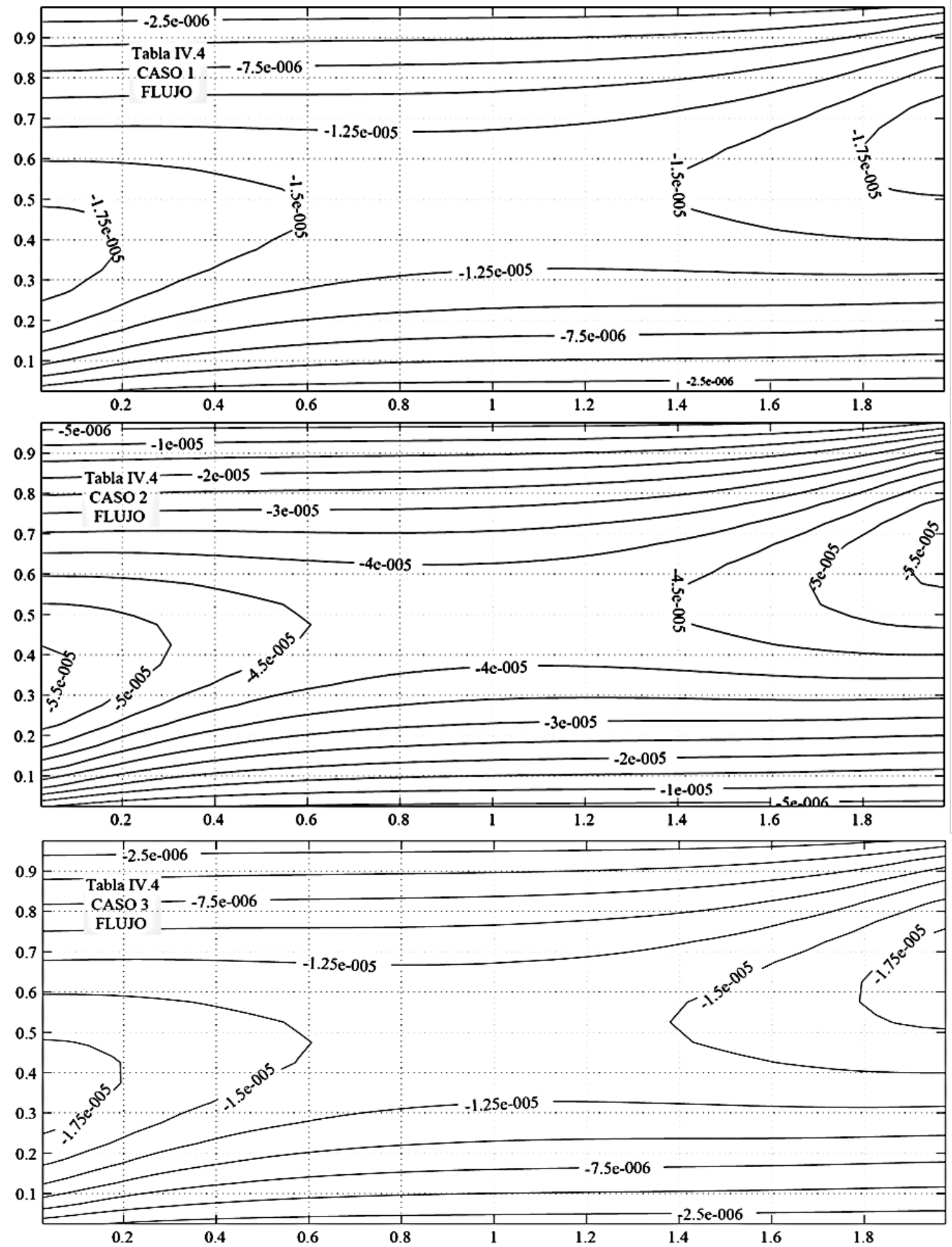

Figura IV.9 Patrones de función de corriente para los casos de $v_{a m b}$ despreciable, $\operatorname{Lv}_{\mathrm{amb}}<<\mathrm{D}_{\mathrm{x}}$ (Tabla IV.4) 


\section{IV.1.5 QUINTA SIMPLIFICACIÓN. DIFERENCIA DE DENSIDADES $(\Delta \rho)$ DESPRECIABLE}

Dado que la diferencia de densidades sólo interviene en el monomio $\pi_{\mathrm{d}, 1}=$ $\left[(\Delta \rho) \mathrm{gk}_{\mathrm{y}} /\left(\varepsilon \mu \mathrm{vamb}^{*}\right)\right](\mathrm{L} / \mathrm{H})$, su valor de 7,58 indica una preponderancia del parámetro $\Delta \rho$ frente al resto del monomio; así, en el problema de Henry esta simplificación no aplica sino al contrario.

Para imponer esta simplificación adoptaremos una reducción del 1\% en su valor actual, es decir, $\Delta \rho=0.25$. Eliminando el monomio anterior, el proceso queda regido por los monomios

$$
\begin{aligned}
\pi_{d, 2} & =\frac{k_{x} H^{2}}{k_{y} L^{2}} \\
\pi_{d, 3} & =\frac{D_{x}}{v_{a m b}^{*} L} \\
\pi_{d, 4} & =\frac{D_{x} H^{2}}{D_{y} L^{2}}
\end{aligned}
$$

Un razonamiento alternativo se fundamenta en asumir directamente que un cambio de densidad despreciable (como una gravedad despreciable o una viscosidad elevada) inducen velocidades verticales $\left(\mathrm{v}_{\mathrm{y}}{ }^{*}\right)$ pequeñas. Suprimiendo $\mathrm{v}_{\mathrm{y}}{ }^{*}$ en la ecuación del soluto, los tres coeficientes resultantes dan lugar a dos grupos adimensionales independientes:

$$
\left(D_{x} / L^{2}\right) /\left(v^{*}{ }^{*} b / L\right) \text { y } D_{y} L /\left(v^{*}{ }_{a m b} H^{2}\right), \quad \text { o } \quad D_{x} H^{2} /\left(D_{y} L^{2}\right) .
$$

Éstos, junto con el grupo proveniente de la ecuación del momento conducen a los tres grupos independientes obtenidos con el razonamiento anterior. La relación de aspecto no se obtiene como grupo independiente a menos que se trate de un medio isótropo en cuyo caso los grupos adimensionales se reducen a dos:

$$
\pi_{\mathrm{d}, 2}=\frac{\mathrm{H}}{\mathrm{L}} \quad \pi_{\mathrm{d}, 3}=\frac{\mathrm{D}}{\mathrm{v}_{\mathrm{amb}}^{*} \mathrm{~L}}
$$

La comprobación se lleva a cabo simulando los casos de la Tabla III.5, para los que los monomios anteriores toman valores fijos:

$$
\pi_{\mathrm{d}, 2}=\frac{\mathrm{k}_{\mathrm{x}} \mathrm{H}^{2}}{\mathrm{k}_{\mathrm{y}} \mathrm{L}^{2}}=0,25 \quad \pi_{\mathrm{d}, 3}=\frac{\mathrm{D}_{\mathrm{x}}}{\mathrm{v}_{\mathrm{amb}}^{*} \mathrm{~L}}=0,05 \quad \pi_{\mathrm{d}, 4}=\frac{\mathrm{D}_{\mathrm{x}} \mathrm{H}^{2}}{\mathrm{D}_{\mathrm{y}} \mathrm{L}^{2}}=0,25
$$


Con objeto de mantener el mismo valor para el monomio $\pi_{\mathrm{d}, 1}=$ $\left[(\Delta \rho) g k_{\mathrm{y}} /\left(\varepsilon \mu \mathrm{vamb}^{*}\right)\right](\mathrm{L} / \mathrm{H})$, a pesar de su despreciable influencia, el valor de $\Delta \rho$ se corrige convenientemente en los tres casos.

Tabla IV.5 Valores de los parámetros para casos de diferencia de densidad despreciable

\begin{tabular}{|c|c|c|c|c|c|c|c|c|c|c|c|c|}
\hline Caso & $\Delta \rho$ & g & $\mathbf{k}_{\mathrm{x}}$ & $\mathbf{k}_{\mathbf{y}}$ & $\varepsilon$ & $\mu$ & $\mathbf{V}_{\mathrm{amb}}$ & $\mathbf{L}$ & $\mathbf{H}$ & $\mathbf{D}_{\mathrm{x}}$ & $\mathbf{D}_{\mathbf{y}}$ & $\Psi$ \\
\hline \multirow[t]{2}{*}{1} & $0.01 \Delta \rho_{\mathrm{H}}$ & $\mathbf{g}_{\mathrm{H}}$ & $\mathbf{k}_{\mathrm{x}, \mathrm{H}}$ & $\mathbf{k}_{\mathbf{y}, \mathrm{H}}$ & $\varepsilon_{\mathrm{H}}$ & $\mu_{\mathrm{H}}$ & $\mathbf{V}_{\text {amb,H }}$ & $\mathbf{L}_{\mathbf{H}}$ & $\mathbf{H}_{\mathbf{H}}$ & $\mathbf{D}_{\mathrm{x}, \mathrm{H}}$ & $\mathbf{D}_{\mathbf{y}, \mathbf{H}}$ & $\Psi_{\mathrm{H}}$ \\
\hline & 0,25 & 9,81 & $1,0204 \mathrm{E}-09$ & 1,0204E-09 & 0,35 & $1,0 \mathrm{E}-03$ & 1,8857E-04 & 2 & 1 & 1,8857E-05 & 1,8857E-05 & $6,60 \mathrm{E}-05$ \\
\hline \multirow[t]{2}{*}{2} & $0.005 \Delta \rho_{\mathrm{H}}$ & $\mathbf{g}_{\mathrm{H}}$ & $2 k_{x, H}$ & $2 \mathrm{k}_{\mathrm{y}, \mathrm{H}}$ & $\varepsilon_{\mathrm{H}}$ & $\mu_{\mathrm{H}}$ & $\mathbf{V}_{\mathbf{a m b}, \mathbf{H}}$ & $\mathbf{L}_{\mathbf{H}}$ & $\mathbf{H}_{\mathbf{H}}$ & $\mathbf{D}_{\mathbf{x}, \mathbf{H}}$ & $\mathbf{D}_{\mathbf{y}, \mathbf{H}}$ & $\Psi_{\mathrm{H}}$ \\
\hline & 0,125 & 9,81 & 2,0408E-09 & 2,0408E-09 & 0,35 & $1,0 \mathrm{E}-03$ & 1,8857E-04 & 2 & 1 & 1,8857E-05 & 1,8857E-05 & 6,60E-05 \\
\hline \multirow[t]{2}{*}{3} & $0.02 \Delta \rho_{\mathrm{H}}$ & $\mathbf{g}_{\mathbf{H}}$ & $\mathbf{k}_{\mathrm{x}, \mathrm{H}}$ & $\mathbf{k}_{\mathrm{y}, \mathrm{H}}$ & $\varepsilon_{\mathrm{H}}$ & $\mu_{\mathrm{H}}$ & $2 v_{a m b, H}$ & $\mathbf{L}_{\mathbf{H}}$ & $\mathbf{H}_{\mathbf{H}}$ & $2 D_{x, H}$ & $2 D_{y, H}$ & $2 \Psi_{H}$ \\
\hline & 0,5 & 9,81 & $1,0204 \mathrm{E}-09$ & $1,0204 \mathrm{E}-09$ & 0,35 & $1,0 \mathrm{E}-03$ & 3,7714E-04 & 2 & 1 & 3,7714E-05 & 3,7714E-05 & 1,32E-04 \\
\hline \multirow[t]{2}{*}{4} & $0.02 \Delta \rho_{\mathrm{H}}$ & $\mathbf{g}_{\mathbf{H}}$ & $(1 / 2) k_{x, H}$ & $(1 / 2) k_{y, H}$ & $\varepsilon_{\mathrm{H}}$ & $\mu_{\mathrm{H}}$ & $2 v_{a m b, H}$ & $\mathbf{L}_{\mathbf{H}}$ & $\mathbf{H}_{\mathbf{H}}$ & $2 D_{x, H}$ & $2 D_{y, H}$ & $2 \Psi_{H}$ \\
\hline & 0,5 & 9,81 & $5,1020 \mathrm{E}-10$ & $5,1020 \mathrm{E}-10$ & 0,35 & $1,0 \mathrm{E}-03$ & 3,7714E-04 & 2 & 1 & 3,7714E-05 & 3,7714E-05 & 1,32E-04 \\
\hline
\end{tabular}

Las Figuras IV.10 y IV.11 muestran las simulaciones de los cuatro casos de la tabla anterior. Los patrones son idénticos, lo que demuestra que los números adimensionales deducidos gobiernan la solución.

El efecto de densidad despreciable apenas modifica la verticalidad de las isolíneas de salinidad que por otro lado se concentran junto al borde del mar con la gran influencia del flujo regional frente a la difusividad (monomio $\pi_{\mathrm{d}, 3}$ ). Finalmente, el patrón de isolíneas de corriente está influenciado por el valor del flujo regional que se dobla de los casos 1 y 2 a los casos 3 y 4, merced a la duplicación del valor máximo de la función de corriente, $\Psi$.

Con todo, las cinco simplificaciones mostradas hasta aquí se han querido referir fundamentalmente a la influencia de los cambios de algunos parámetros físicos concretos del problema, contenidos en los monomios resultantes de la simplificación, en el patrón de soluciones asumiendo como parámetros geométricos influyentes las dimensiones del dominio, L y H. En la siguiente simplificación se profundiza en la influencia específica de estos parámetros con el objeto de discernir hasta qué punto y bajo qué condiciones dichos parámetros son, efectivamente, determinantes o no en la solución del problema de Henry. 
Capítulo IV. Simplificación de escenarios
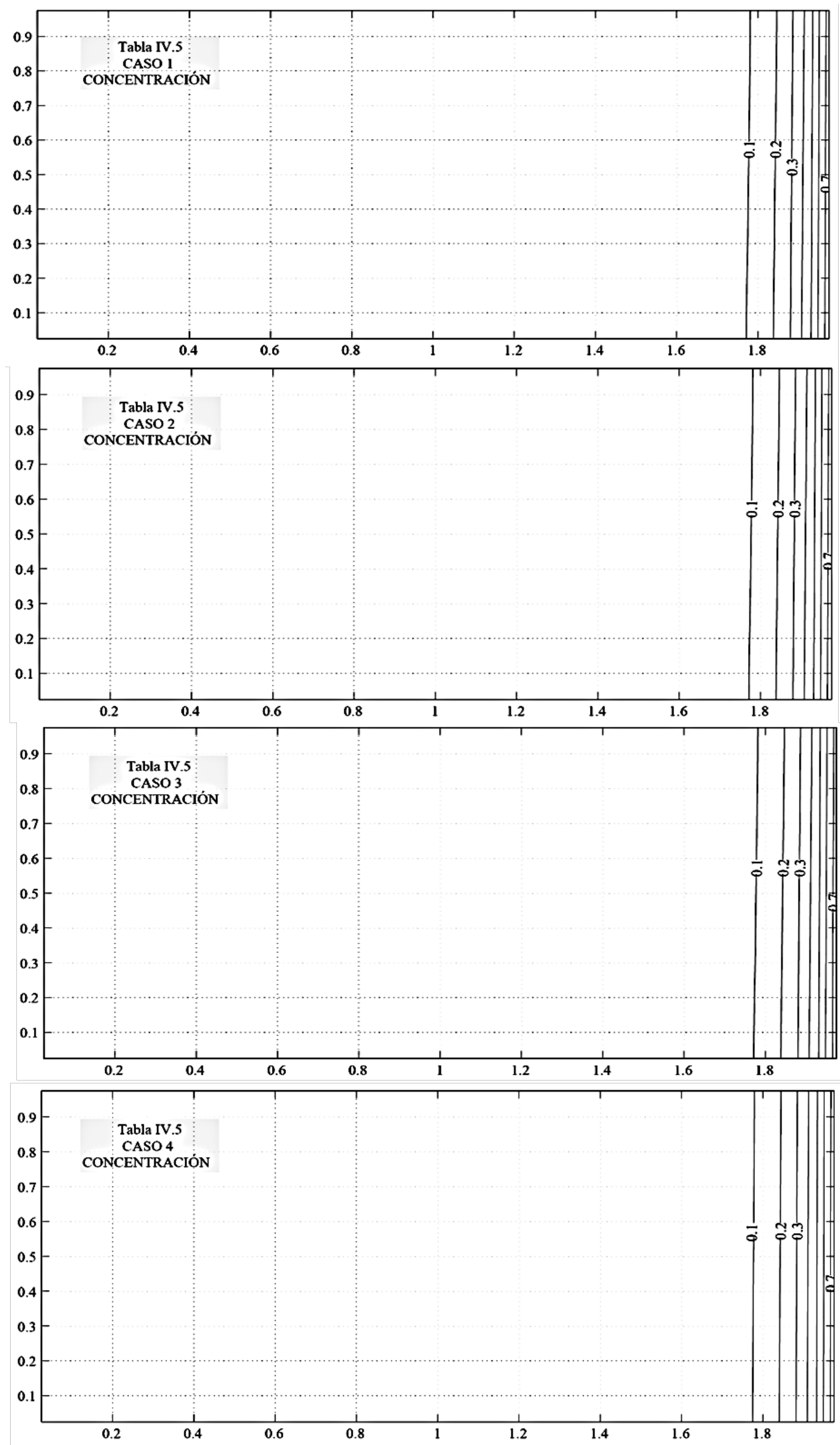

Figura IV.10 Patrones de concentración para los casos de $\Delta \rho$ despreciable (Tabla IV.5) 
Capítulo IV. Simplificación de escenarios
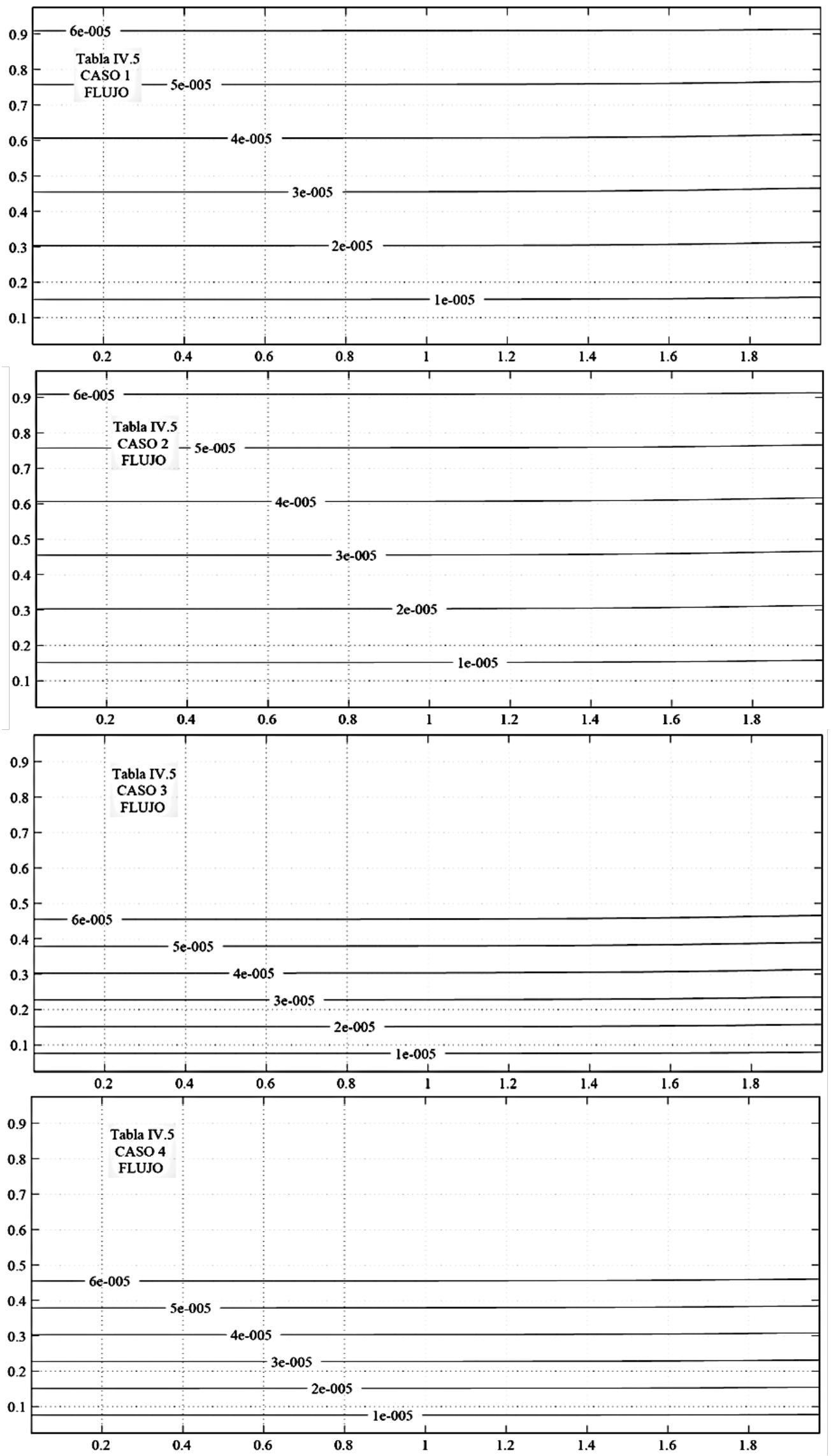

Figura IV.11 Patrones de función de corriente para los casos de $\Delta \rho$ despreciable (Tabla IV.5) 


\section{IV.1.6 SEXTA SIMPLIFICACIÓN. DOMINIOS EXTENSOS (L>>H)}

Aunque más tarde profundizaremos en el tema de los dominios extensos, en los que suprimiremos directamente L como parámetro influyente en los resultados, en esta simplificación seremos menos restrictivos ya que en medios anisótropos los valores de la conductividad hidráulica y la difusividad podrían compensar el efecto de la gran extensión del dominio y hacer que los monomios $\left(k_{x} H^{2}\right) /\left(k_{y} L^{2}\right)$ y $D_{x} H^{2} /\left(D_{y} L^{2}\right)$ influyeran efectivamente en la solución del problema.

Dentro de esta simplificación podemos considerar, pues, dos categorías:

i) Una primera, en la que los medios sean isótropos $\left(\mathrm{k}_{\mathrm{x}}=\mathrm{k}_{\mathrm{y}} \mathrm{y} \mathrm{D}_{\mathrm{x}}=\mathrm{D}_{\mathrm{y}}\right)$ o cuasiisótropos $\left(\mathrm{k}_{\mathrm{x}} \approx \mathrm{k}_{\mathrm{y}} \mathrm{y} \mathrm{D}_{\mathrm{x}} \approx \mathrm{D}_{\mathrm{y}}\right)$, en la que los monomios $\pi_{\mathrm{d}, 2}=\left(\mathrm{k}_{\mathrm{x}} \mathrm{H}^{2}\right) /\left(\mathrm{k}_{\mathrm{y}} \mathrm{L}^{2}\right) \mathrm{y}$ $\pi_{\mathrm{d}, 4}=\mathrm{D}_{\mathrm{x}} \mathrm{H}^{2} /\left(\mathrm{D}_{\mathrm{y}} \mathrm{L}^{2}\right)$ pueden eliminarse directamente del problema por simple inspección física. Esta categoría, a su vez, podría separarse en dos asumiendo isotropía en sólo una de las propiedades (esta separación la dejaremos de momento).

ii) Una segunda, en la que la anisotropía de los parámetros conductividad hidráulica y difusividad compensaran el efecto de la gran extensión del acuífero haciendo que los monomios $\pi_{\mathrm{d}, 2}=\left(\mathrm{k}_{\mathrm{x}} \mathrm{H}^{2}\right) /\left(\mathrm{k}_{\mathrm{y}} \mathrm{L}^{2}\right)$ y $\pi_{\mathrm{d}, 4}=$ $\mathrm{D}_{\mathrm{x}} \mathrm{H}^{2} /\left(\mathrm{D}_{\mathrm{y}} \mathrm{L}^{2}\right)$ tengan que ser considerados en el estudio.

Deduciremos los grupos influyentes en cada categoría partiendo de los cuatro grupos que caracterizan el caso más general, aunque el camino más formal sería el de partir de las ecuaciones de gobierno simplificadas y deducir los monomios procediendo a su adimensionalización discriminada.

Para la primera categoría, como hemos dicho, $\mathrm{H} / \mathrm{L}$ es despreciable y las hipótesis $\mathrm{k}_{\mathrm{x}} \approx \mathrm{k}_{\mathrm{y}}$ y $\mathrm{D}_{\mathrm{x}} \approx \mathrm{D}_{\mathrm{y}}$ eliminan los monomios $\pi_{\mathrm{d}, 2}=\left(\mathrm{k}_{\mathrm{x}} \mathrm{H}^{2}\right) /\left(\mathrm{k}_{\mathrm{y}} \mathrm{L}^{2}\right)$ y $\pi_{\mathrm{d}, 4}=\mathrm{D}_{\mathrm{x}} \mathrm{H}^{2} /\left(\mathrm{D}_{\mathrm{y}} \mathrm{L}^{2}\right)$, reduciendo así los grupos independientes a dos:

$$
\begin{aligned}
& \pi_{\mathrm{d}, 1}=\frac{(\Delta \rho) \mathrm{gk}}{\varepsilon \mu \mathrm{v}_{\mathrm{amb}}^{*}}\left(\frac{\mathrm{L}}{\mathrm{H}}\right) \\
& \pi_{\mathrm{d}, 3}=\frac{\mathrm{D}}{\mathrm{v}_{\mathrm{amb}}^{*} \mathrm{~L}}
\end{aligned}
$$

¡Sorprendente! La longitud de acuífero se retiene como un parámetro influyente en el patrón de soluciones de esta categoría (¡esto no hubiera ocurrido si partimos de las 
ecuaciones simplificadas en las que no hubiera aparecido L!). Combinando estos monomios para que L sólo aparezca en uno de ellos, los podemos presentar alternativamente en la forma

$$
\begin{aligned}
& \pi_{\mathrm{d}, 1}=\frac{(\Delta \rho) \mathrm{gkD}}{\varepsilon \mu \mathrm{v}_{\mathrm{amb}}^{* 2} \mathrm{H}} \\
& \pi_{\mathrm{d}, 3}=\frac{\mathrm{D}}{\mathrm{v}_{\mathrm{amb}}^{*} \mathrm{~L}}
\end{aligned}
$$

El resultado no es de recibo. Si asumimos que L es grande en comparación con H (dentro de esta categoría), este parámetro (L) no puede aparecer en los monomios resultantes. ¡Algo está mal! Un aumento de L en forma alguna puede cambiar el patrón bajo el supuesto L>>H y la solución nos está diciendo que sí.

La inspección física del problema apoya estos resultados: por ejemplo, duplicar L en estas condiciones sólo hace alargar el recorrido horizontal del agua en la región de entrada anterior a la zona de mezcla (región cuya extensión aumenta en el mismo valor que lo hace la longitud del acuífero al ser duplicada); lo único que debería cambiar (y obviamente cambia) en el problema sería las isolíneas de presión a lo largo del dominio, variable que sacamos de las ecuaciones de gobierno.

Para deducir los grupos independientes bajo la hipótesis $\mathrm{L}>>\mathrm{H}$, no podemos partir de unos grupos cuya obtención no ha asumido la misma hipótesis; de unos grupos para los que se ha adoptado una referencia L incompatible con la hipótesis inicial. Sólo cabe una interpretación basada en la adimensionalización discriminada más general que discutiremos más tarde. Hay que volver al principio de la adimensionalización y, a la hora de elegir referencias, en lugar de adoptar L (variable superflua) como referencia para la coordenada $\mathrm{x}$, introducir una magnitud oculta $\mathrm{l}^{*}$ asociada a la extensión del dominio en donde tienen lugar los procesos de mezcla.

A tenor de la isotropía del problema, esta magnitud oculta deberá tener el mismo orden de magnitud que $\mathrm{H}$ para que los monomios $\pi_{\mathrm{d}, 2}=\left(\mathrm{k}_{\mathrm{x}} \mathrm{H}^{2}\right) /\left(\mathrm{k}_{\mathrm{y}} \mathrm{L}^{2}\right)$ y $\pi_{\mathrm{d}, 4}=$ $\mathrm{D}_{\mathrm{x}} \mathrm{H}^{2} /\left(\mathrm{D}_{\mathrm{y}} \mathrm{L}^{2}\right)$ se balanceen convenientemente, es decir, orden de magnitud unidad.

En efecto, con $\mathrm{l}^{*} \sim \mathrm{H}$, los otros dos monomios que intervienen en la solución

$$
\pi_{\mathrm{d}, 1}=\frac{(\Delta \rho) \mathrm{gk}}{\varepsilon \mu v_{\mathrm{amb}}^{*}}\left(\frac{\mathrm{l}^{*}}{\mathrm{H}}\right)
$$




$$
\pi_{\mathrm{d}, 3}=\frac{\mathrm{D}}{\mathrm{v}_{\mathrm{amb}}^{*} \mathrm{l}^{*}}
$$

quedarían simplificados a

$$
\begin{aligned}
& \pi_{d, 1}=\frac{(\Delta \rho) g k}{\varepsilon \mu v_{a m b}^{*}} \\
& \pi_{d, 3}=\frac{D}{v_{a m b}^{*} l^{*}}
\end{aligned}
$$

o bien a

$$
\begin{aligned}
& \pi_{\mathrm{d}, 1}=\frac{(\Delta \rho) \mathrm{gk}}{\varepsilon \mu \mathrm{v}_{\mathrm{amb}}^{*}} \\
& \pi_{\mathrm{d}, 3}=\frac{\mathrm{D}}{\mathrm{v}_{\mathrm{amb}}^{*} \mathrm{H}}
\end{aligned}
$$

¡Pero estos son precisamente los parámetros de problema original de Henry que ya hemos comprobado son correctos! De todo lo anterior podemos extraer, por el momento, dos interesantes conclusiones:

i) en el problema original de Henry puede asumirse que $\mathrm{L}>>\mathrm{H}$, lo que equivale a decir que un incremento de L (por ejemplo, de 2 a 2.5) no altera el patrón en el sentido de que basta incrementar las líneas horizontales del patrón de flujo en una longitud igual al aumento de L, (la simulación demuestra que esto es cierto),

ii) la zona de mezcla es del orden de magnitud de la altura del acuífero (una afirmación ya comprobada).

En relación con la segunda categoría (propiedades claramente anisótropas), se mantendrían los cuatro grupos adimensionales originales como monomios influyentes en la solución del problema:

$$
\pi_{\mathrm{d}, 1}=\left[(\Delta \rho) \mathrm{gk}_{\mathrm{y}} /\left(\varepsilon \mu \mathrm{vamb}_{\mathrm{amb}}{ }^{*}\right)\right](\mathrm{L} / \mathrm{H}), \pi_{\mathrm{d}, 2}=\left(\mathrm{k}_{\mathrm{x}} \mathrm{H}^{2}\right) /\left(\mathrm{k}_{\mathrm{y}} \mathrm{L}^{2}\right), \pi_{\mathrm{d}, 3}=\mathrm{D}_{\mathrm{x}} /\left(\mathrm{vamb}^{*} \mathrm{~L}\right) \text { y } \pi_{\mathrm{d}, 4}=\mathrm{D}_{\mathrm{x}} \mathrm{H}^{2} /\left(\mathrm{D}_{\mathrm{y}} \mathrm{L}^{2}\right) .
$$

Comprobaremos que los grupos adimensionales derivados de la primera categoría, $\pi_{\mathrm{d}, 1}=\left[(\mathrm{kg} \Delta \rho) /\left(\varepsilon \mu \mathrm{vamb}^{*}\right](\mathrm{L} / \mathrm{H})\right.$ y $\pi_{\mathrm{d}, 3}=\mathrm{D} /\left(\mathrm{v}^{*}{ }_{\mathrm{amb}} \mathrm{L}\right)$, no son correctos. Se toman para ello tres casos ( 1 a 3 de la Tabla IV.6) en los que L=3H, y k y D isótropas. Vemos que al aumentar $\mathrm{L}$, manteniendo constantes los valores de $\pi_{\mathrm{d}, 1} \mathrm{y} \pi_{\mathrm{d}, 3}$, los patrones de solución (Figuras IV.12 a IV.14) cambian en contra de lo esperado, lo que demuestra que L no debe aparecer en los parámetros que rigen la solución de esta simplificación. 
Capítulo IV. Simplificación de escenarios

Tabla IV.6 Valores de los parámetros para la simplificación L>>H

\begin{tabular}{|c|c|c|c|c|c|c|c|c|c|c|c|c|}
\hline Caso & $\Delta \rho$ & g & $\mathbf{k}_{\mathbf{x}}$ & $\mathbf{k}_{\mathbf{y}}$ & $\varepsilon$ & $\mu$ & Vamb & $\mathbf{L}$ & $\mathbf{H}$ & $\mathbf{D}_{\mathbf{x}}$ & $\mathbf{D}_{\mathbf{y}}$ & $\Psi$ \\
\hline \multirow[t]{2}{*}{1} & $\Delta \rho_{\mathrm{H}}$ & $\mathbf{g}_{\mathbf{H}}$ & $\mathbf{k}_{\mathbf{x}, \mathbf{H}}$ & $\mathbf{k}_{\mathbf{y}, \mathbf{H}}$ & $\varepsilon_{\mathrm{H}}$ & $\mu_{\mathrm{H}}$ & Vamb,H & $1.5 \mathrm{~L}_{\mathrm{H}}$ & $\mathbf{H}_{\mathbf{H}}$ & $\mathbf{D}_{\mathbf{x}, \mathbf{H}}$ & $\mathbf{D}_{\mathbf{y}, \mathrm{H}}$ & $\Psi_{\mathrm{H}}$ \\
\hline & 25 & 9,81 & 1,0204E-09 & $1,0204 \mathrm{E}-09$ & 0,35 & $1 \mathrm{E}-03$ & 1,8857E-04 & 3 & 1 & 1,8857E-05 & 1,8857E-05 & $6,60 \mathrm{E}-05$ \\
\hline \multirow[t]{2}{*}{2} & $\Delta \rho_{\mathrm{H}}$ & $\mathrm{g}_{\mathrm{H}}$ & $\mathbf{k}_{\mathrm{x}, \mathrm{H}}$ & $k_{\mathbf{y}, \mathrm{H}}$ & $\varepsilon_{\mathrm{H}}$ & $\mu_{\mathrm{H}}$ & Vamb,H & $2,25 \mathrm{~L}_{\mathrm{H}}$ & $1.5 \mathrm{H}_{\mathrm{H}}$ & $1.5 D_{x, H}$ & $1.5 D_{y, H}$ & $1.5 \Psi_{H}$ \\
\hline & 25 & 9,81 & 1,0204E-09 & $1,0204 \mathrm{E}-09$ & 0,35 & $1 \mathrm{E}-03$ & $1,8857 \mathrm{E}-04$ & 4,5 & 1,5 & 2,8286E-05 & 2,8286E-05 & $9,90 \mathrm{E}-05$ \\
\hline \multirow[t]{2}{*}{3} & $\Delta \rho_{\mathrm{H}}$ & $\mathbf{g}_{\mathbf{H}}$ & $\mathbf{k}_{\mathrm{x}, \mathrm{H}}$ & $\mathbf{k}_{\mathbf{y}, \mathbf{H}}$ & $\varepsilon_{\mathrm{H}}$ & $0.5 \mu_{\mathrm{H}}$ & $2 \mathbf{v}_{\mathbf{a m b}, \mathbf{H}}$ & $1.5 \mathrm{~L}_{\mathrm{H}}$ & $\mathbf{H}_{\mathbf{H}}$ & $2 D_{x, H}$ & $2 D_{y, H}$ & $2 \Psi_{H}$ \\
\hline & 25 & 9,81 & 1,0204E-09 & $1,0204 \mathrm{E}-09$ & 0,35 & 5E-04 & 3,7714E-04 & 3 & 1 & 3,7714E-05 & 3,7714E-05 & $1,32 \mathrm{E}-04$ \\
\hline \multirow[t]{2}{*}{4} & $0.67 \Delta \rho_{\mathrm{H}}$ & $\mathbf{g}_{\mathbf{H}}$ & $\mathbf{k}_{\mathbf{x}, \mathrm{H}}$ & $\mathbf{k}_{\mathbf{y}, \mathbf{H}}$ & $\varepsilon_{\mathrm{H}}$ & $\mu_{\mathrm{H}}$ & 0.6 Vamb,H 7 & $2,25 \mathrm{~L}_{\mathrm{H}}$ & $1.5 \mathrm{H}_{\mathrm{H}}$ & $\mathbf{D}_{\mathbf{x}, \mathrm{H}}$ & $\mathbf{D}_{\mathbf{y}, \mathbf{H}}$ & $\Psi_{H}$ \\
\hline & 16,67 & 9,81 & 1,0204E-09 & 1,0204E-09 & 0,35 & 1E-03 & $1,2571 \mathrm{E}-04$ & 4,5 & 1,5 & 1,8857E-05 & 1,8857E-05 & $6,60 \mathrm{E}-05$ \\
\hline \multirow[t]{2}{*}{$\begin{array}{c}5 \\
\text { patrón }\end{array}$} & $\Delta \rho_{\mathrm{H}}$ & $\mathbf{g}_{\mathbf{H}}$ & $2 k_{x, H}$ & $\mathbf{k}_{\mathbf{y}, \mathbf{H}}$ & $\varepsilon_{H}$ & $\mu_{\mathrm{H}}$ & Vamb,H & $1.5 \mathrm{LH}_{\mathrm{H}}$ & $\mathbf{H}_{\mathbf{H}}$ & $\mathbf{D}_{\mathbf{x}, \mathrm{H}}$ & $2 D_{y, H}$ & $\Psi_{H}$ \\
\hline & 25 & 9,81 & 2,0408E-09 & $1,0204 \mathrm{E}-09$ & 0,35 & $1 \mathrm{E}-03$ & 1,8857E-04 & 3 & 1 & 1,8857E-05 & 3,7714E-05 & $6,60 \mathrm{E}-05$ \\
\hline \multirow[t]{2}{*}{6} & $\Delta \rho_{\mathrm{H}}$ & $\mathbf{g}_{\mathbf{H}}$ & $4 k_{x, H}$ & $2 \mathbf{k}_{\mathbf{y}, \mathrm{H}}$ & $\varepsilon_{\mathrm{H}}$ & $\mu_{\mathrm{H}}$ & $2 v_{a m b, H}$ & $1.5 \mathrm{~L}_{\mathrm{H}}$ & $\mathbf{H}_{\mathbf{H}}$ & $2 \mathbf{D}_{x, \mathrm{H}}$ & $4 D_{y, H}$ & $2 \Psi_{H}$ \\
\hline & 25 & 9,81 & 4,0816E-09 & 2,0408E-09 & 0,35 & $1 \mathrm{E}-03$ & $3,7714 \mathrm{E}-04$ & 3 & 1 & 3,7714E-05 & 7,5428E-05 & $1,32 \mathrm{E}-04$ \\
\hline \multirow[t]{2}{*}{7} & $\Delta \rho_{\mathrm{H}}$ & $\mathbf{g}_{\mathbf{H}}$ & $4 k_{x, H}$ & $\mathbf{k}_{\mathbf{y}, \mathbf{H}}$ & $\varepsilon_{H}$ & $\mu_{\mathrm{H}}$ & $\sqrt{ } \mathbf{2}$ vamb,H & $\begin{array}{c}1.5 \sqrt{ } 2 \\
L_{H}\end{array}$ & $\mathbf{H}_{\mathbf{H}}$ & $2 \mathbf{D}_{\mathrm{x}, \mathrm{H}}$ & $2 D_{y, H}$ & $\sqrt{ } 2 \Psi_{H}$ \\
\hline & 25 & 9,81 & 4,0816E-09 & $1,0204 \mathrm{E}-09$ & 0,35 & $1 \mathrm{E}-03$ & 2,6668E-04 & 4,2426 & 1 & 3,7714E-05 & 3,7714E-05 & $9,33 \mathrm{E}-05$ \\
\hline \multirow[t]{2}{*}{8} & $\Delta \rho_{\mathrm{H}}$ & $\mathbf{g}_{\mathbf{H}}$ & $2 \mathbf{k}_{\mathrm{x}, \mathrm{H}}$ & $2 \mathbf{k}_{\mathbf{y}, \mathrm{H}}$ & $\varepsilon_{\mathrm{H}}$ & $\sqrt{ } \mathbf{2} \mu_{\mathrm{H}}$ & $\mathbf{V}_{\mathrm{amb}, \mathrm{H}}$ & $1.5 \mathrm{~L}_{\mathrm{H}}$ & $\sqrt{ } \mathbf{2} \mathbf{H}_{\mathbf{H}}$ & $\mathbf{D}_{\mathbf{x}, \mathrm{H}}$ & $4 D_{y, H}$ & $\sqrt{2} \Psi_{H}$ \\
\hline & 25 & 9,81 & 2,0408E-09 & 2,0408E-09 & 0,35 & $1 \mathrm{E}-03$ & $1,8857 \mathrm{E}-04$ & 3 & 1,4142 & 1,8857E-05 & 7,5428E-05 & 9,33E-05 \\
\hline
\end{tabular}
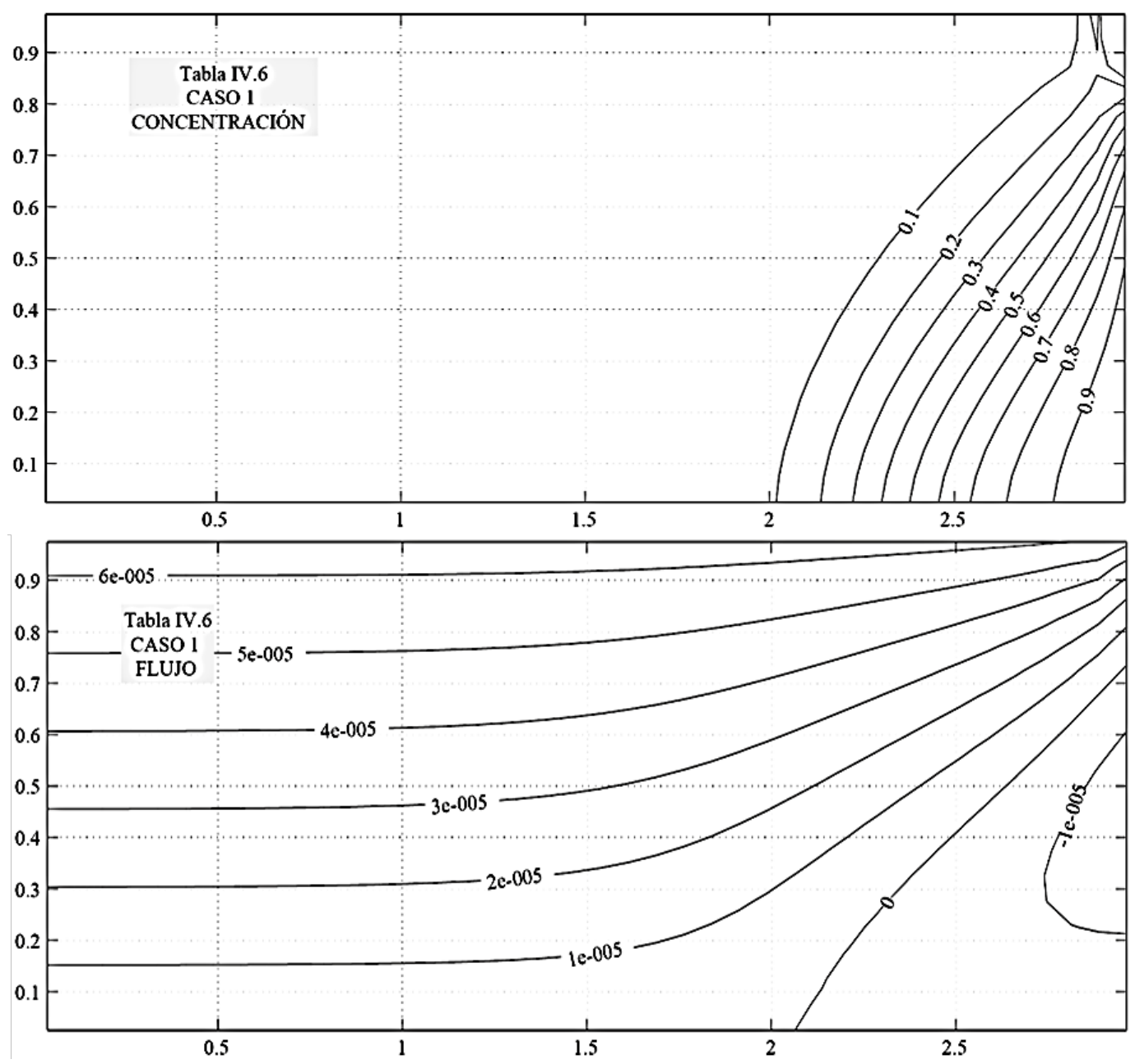

Figura IV.12 Patrones de concentración y función de corriente para la hipótesis L >>H

(dominios isótropos) (Caso 1, Tabla IV.6) 
Capítulo IV. Simplificación de escenarios
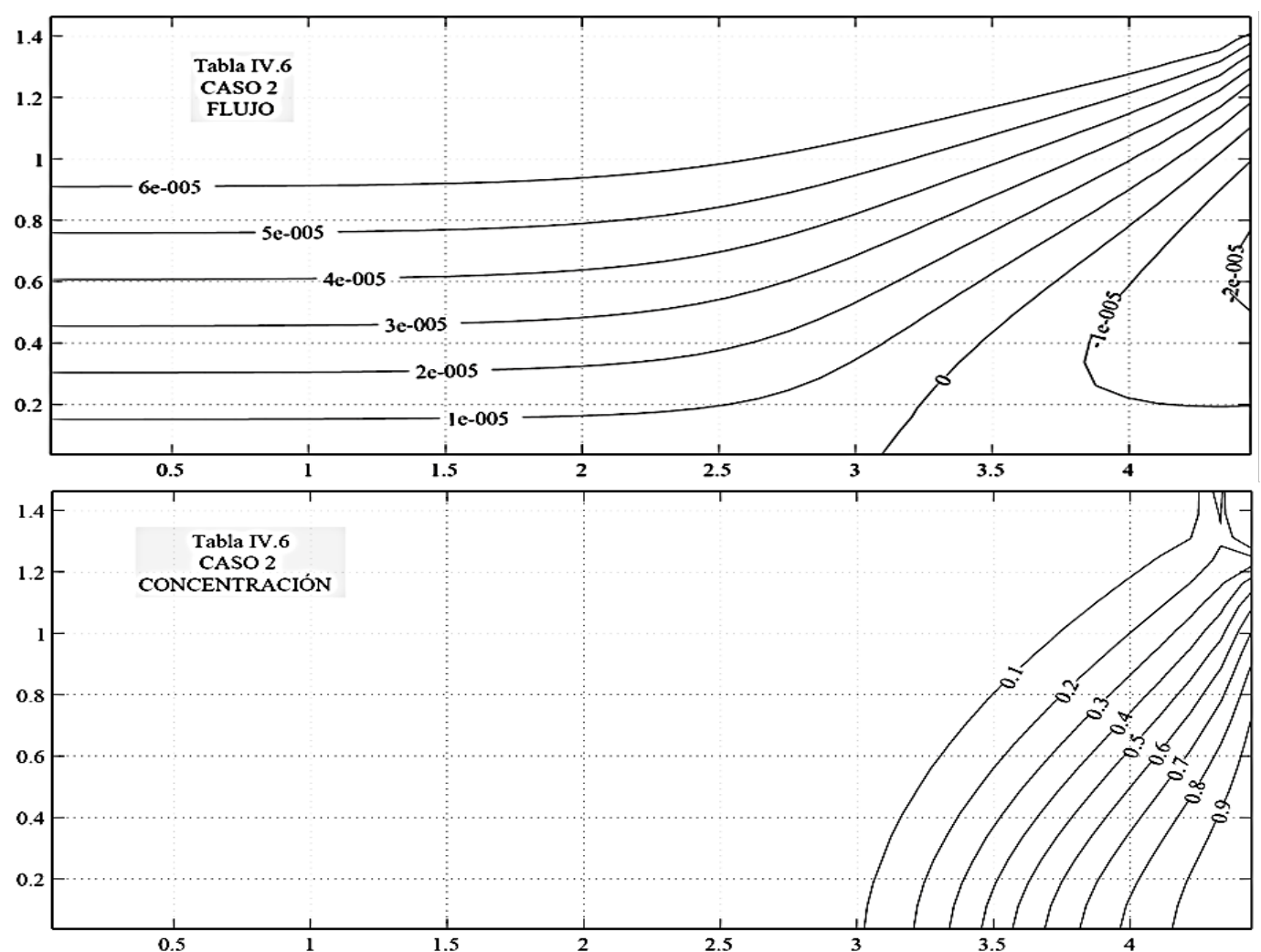

Figura IV.13 Patrones de concentración y función de corriente para la hipótesis L >>H (dominios isótropos) (Caso 2, Tabla IV.6)
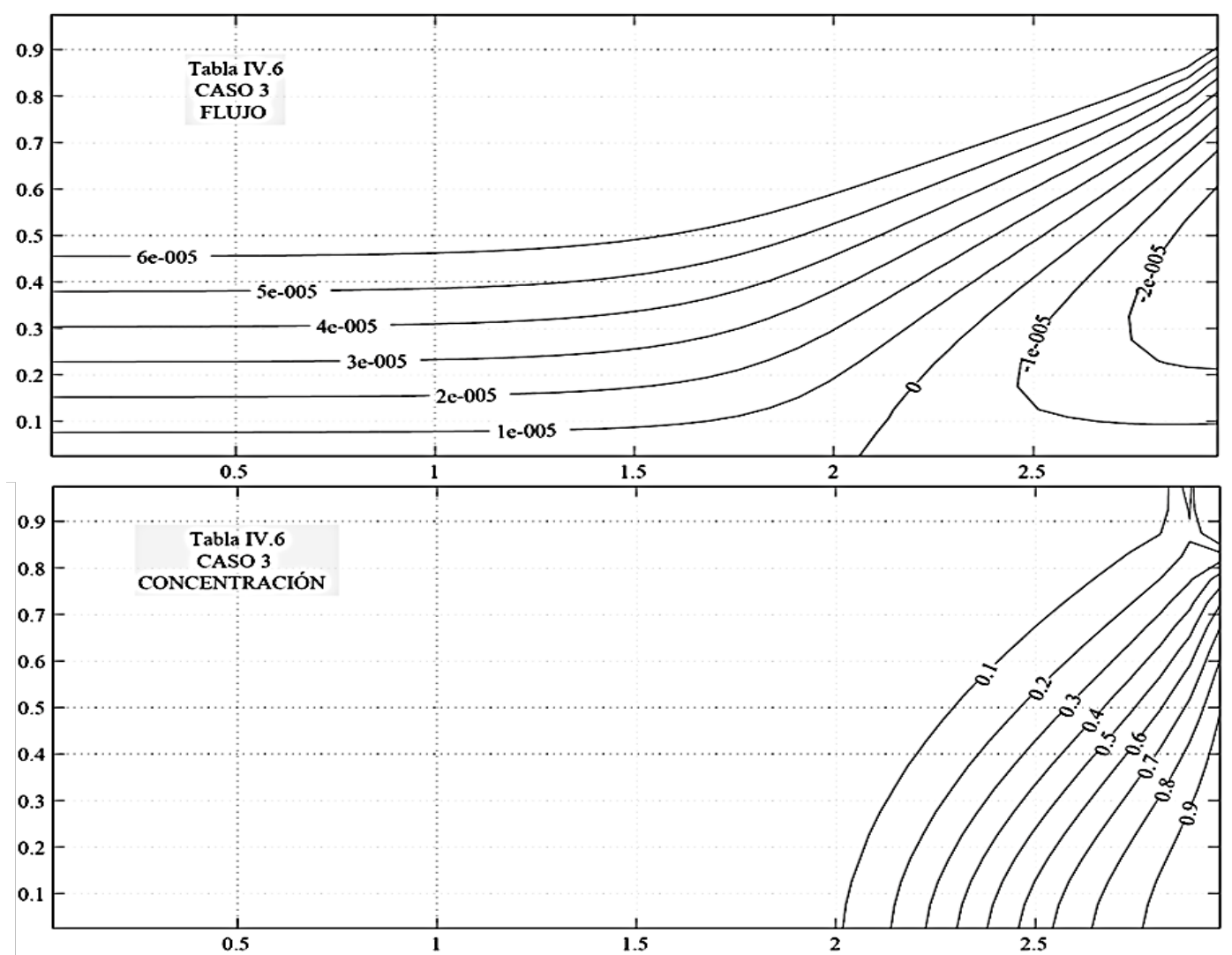

Figura IV.14 Patrones de concentración y función de corriente para la hipótesis L >>H (dominios isótropos) (Caso 3, Tabla IV.6) 
Para la segunda categoría tomaremos en todos los casos $\mathrm{L}=3 \mathrm{H}, \mathrm{k}_{\mathrm{x}}=9 \mathrm{k}_{\mathrm{y}} \mathrm{y}$ $D_{x}=9 D_{y}$, con ello los valores de los monomios $\pi_{d, 2} y \pi_{d, 4}$ no cambian,

$\pi_{\mathrm{d}, 2}=\pi_{\mathrm{d}, 4}=1, \mathrm{y} \pi_{\mathrm{d}, 1}=11,38 \mathrm{y} \pi_{\mathrm{d}, 3}=0,9$.

Se simulan los casos 4 a 8 de la Tabla IV.6. Vemos que, en efecto, al aumentar $\mathrm{L}$, manteniendo también constantes los valores de $\pi_{\mathrm{d}, 1}$ y $\pi_{\mathrm{d}, 3}$ se obtienen diferentes patrones de solución en contra de lo esperado, Figuras IV.15 a IV.19, que lo quiere decir que estos grupos están mal deducidos. Definitivamente, como conclusión, L no forma parte de los parámetros que forman los grupos $\pi_{\mathrm{d}, 1} \mathrm{y} \pi_{\mathrm{d}, 3}$.

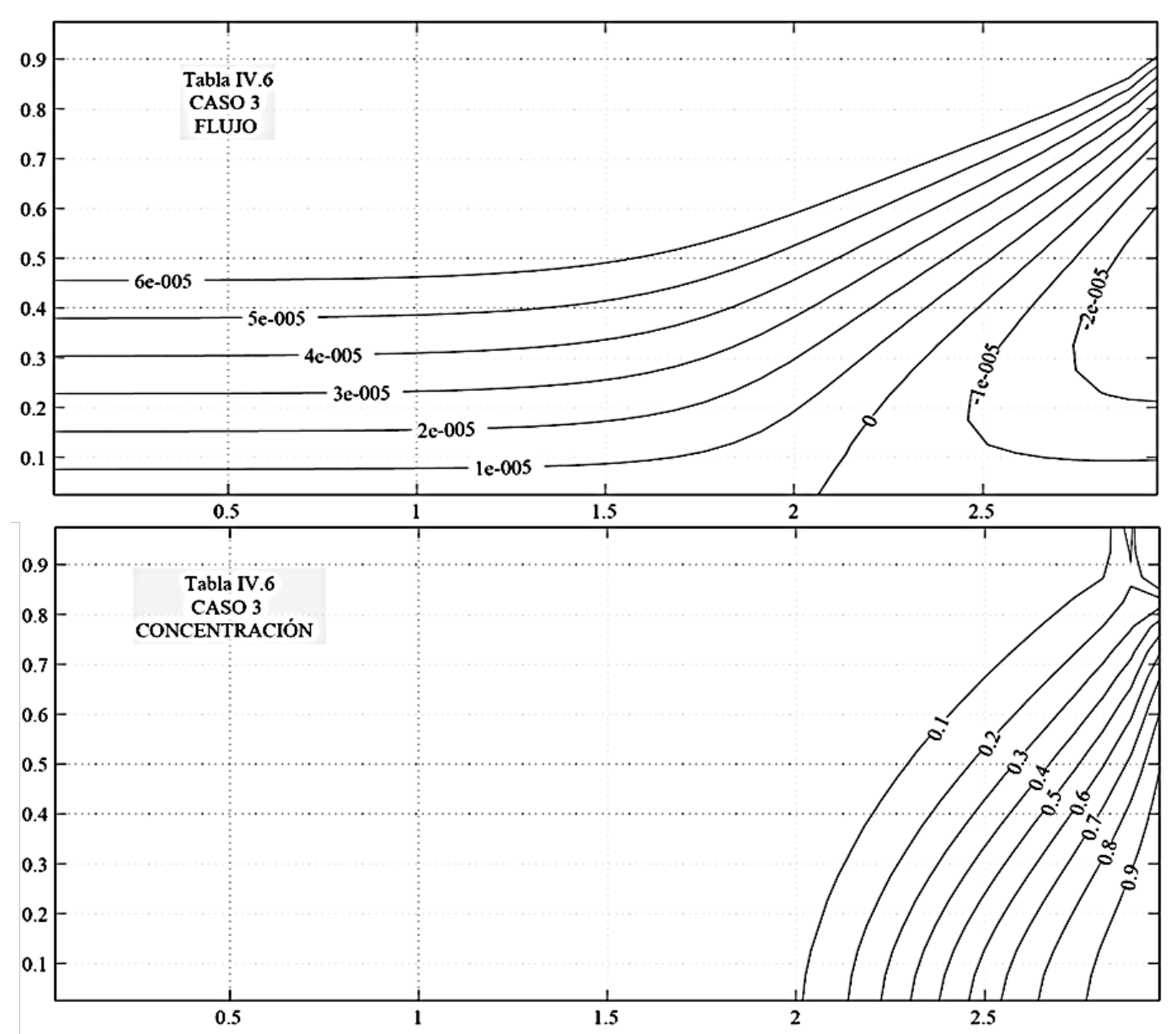

Figura IV.15 Patrones de concentración y función de corriente para la hipótesis L >>H (dominios anisótropos) (Caso 4, Tabla IV.6) 
Capítulo IV. Simplificación de escenarios
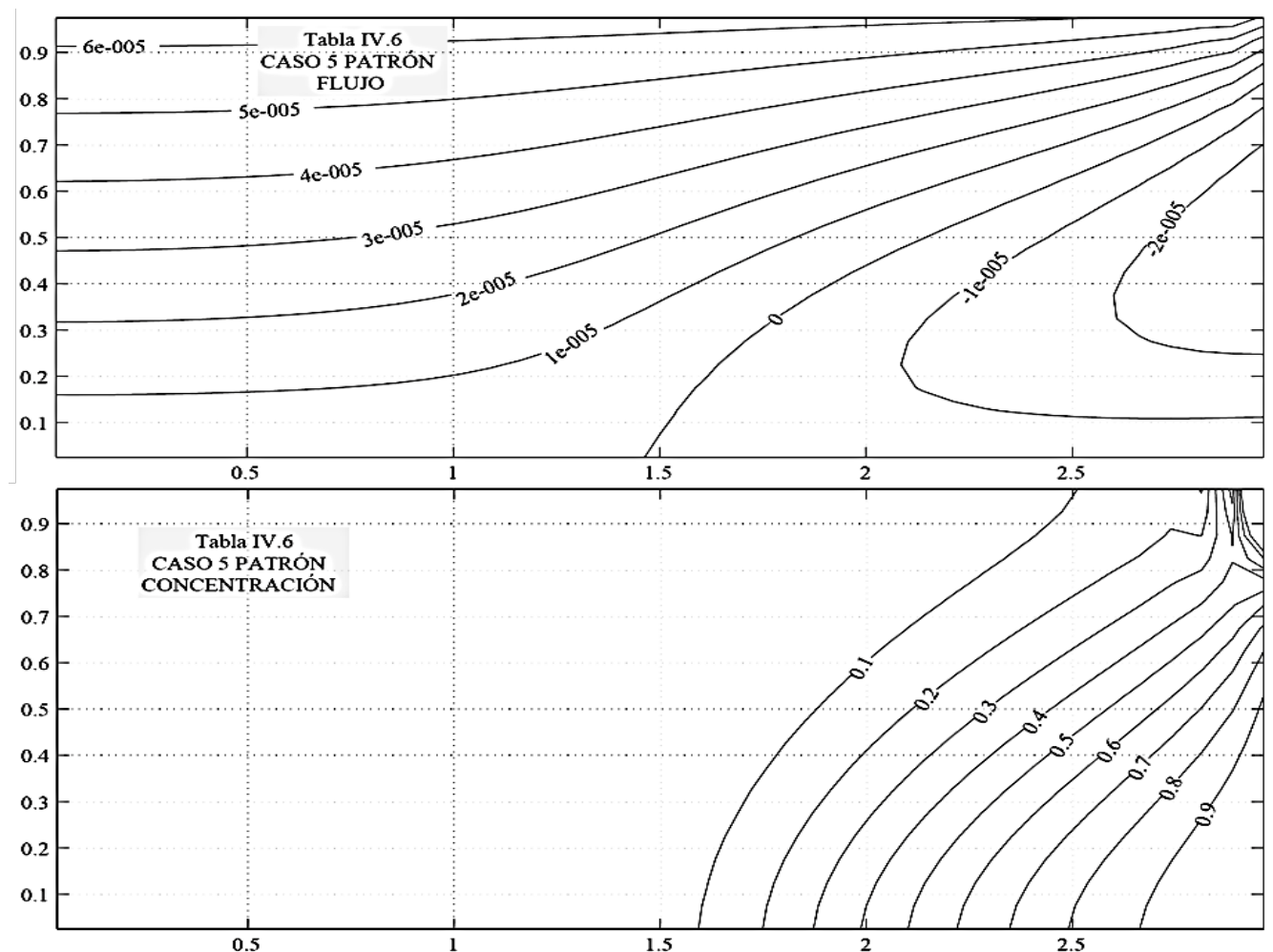

Figura IV.16 Patrones de concentración y función de corriente para la hipótesis L $>>H$ (dominios anisótropos) (Caso 5, Tabla IV.6)
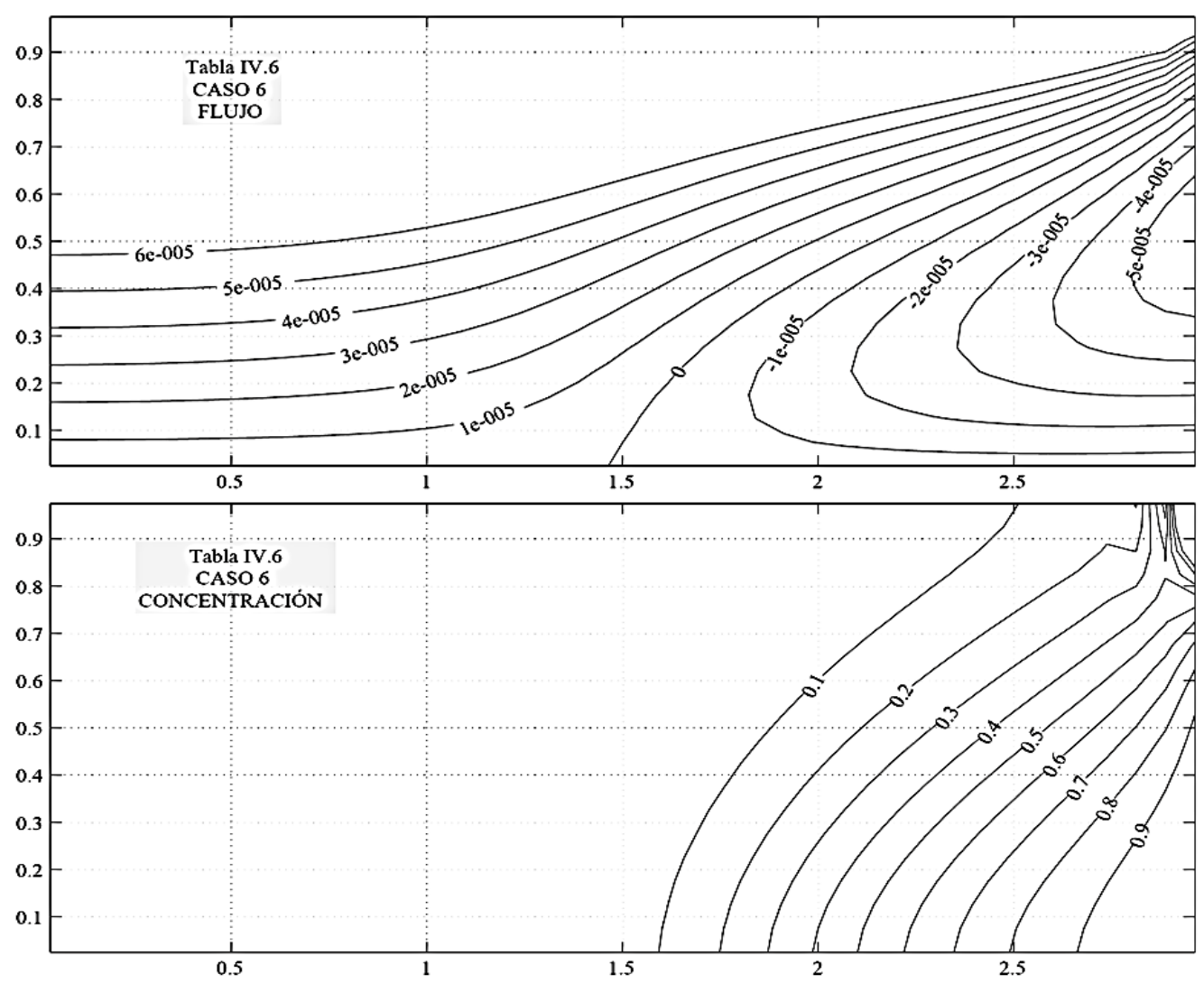

Figura IV.17 Patrones de concentración y función de corriente para la hipótesis L $>>H$ (dominios anisótropos) (Caso 6, Tabla IV.6) 
Capítulo IV. Simplificación de escenarios
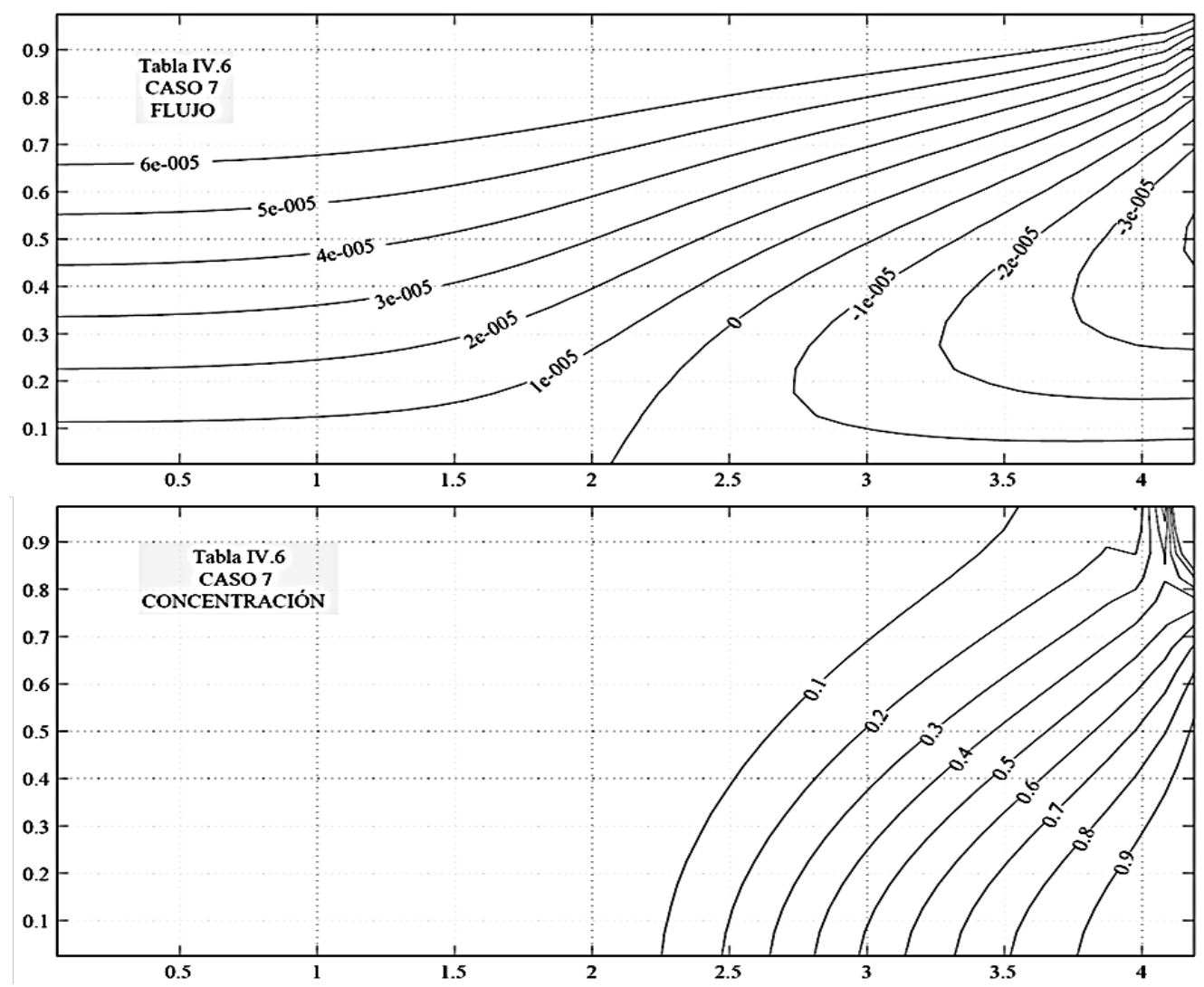

Figura IV.18 Patrones de concentración y función de corriente para la hipótesis L >>H (dominios anisótropos) (Caso 7, Tabla IV.6)
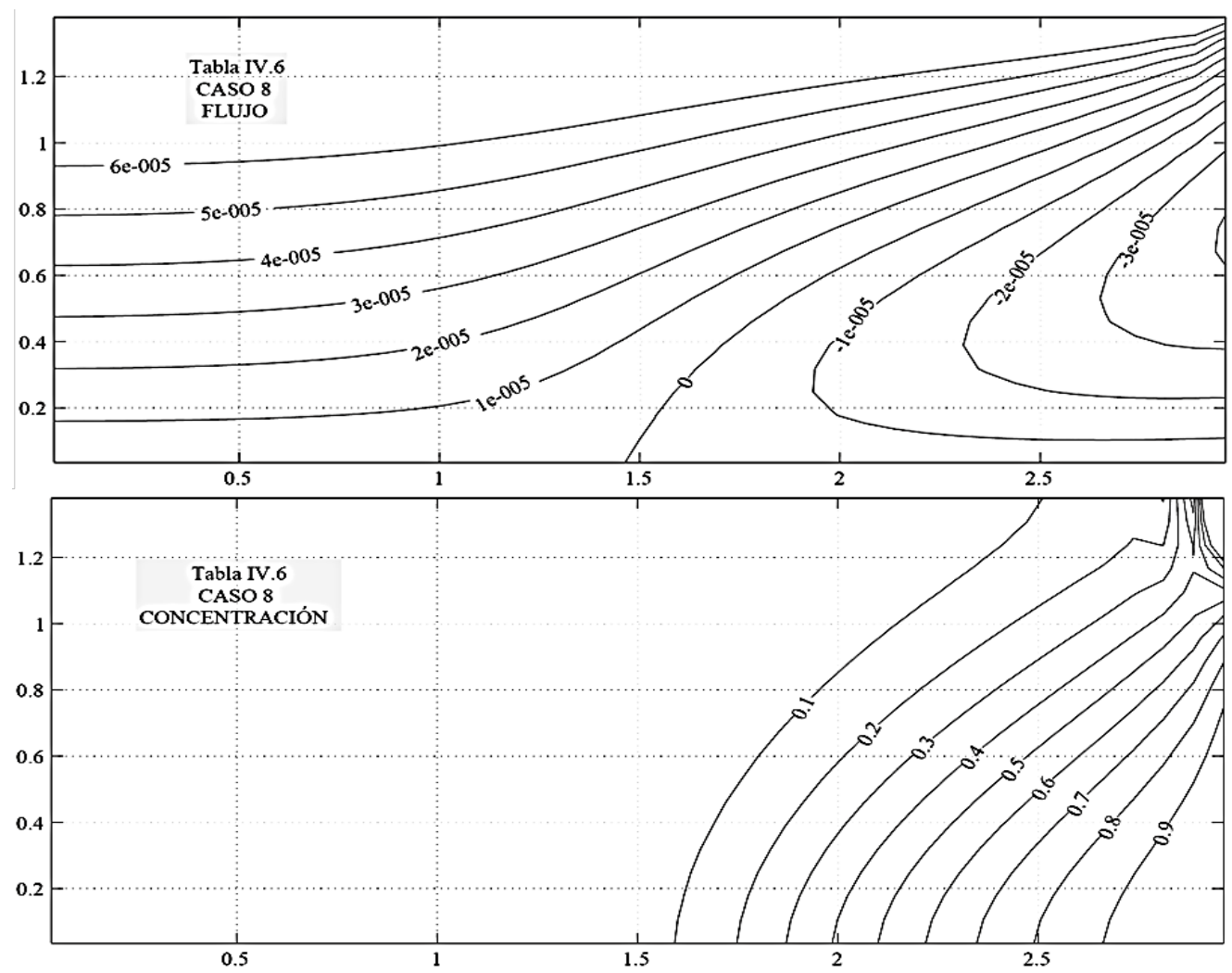

Figura IV.19 Patrones de concentración y función de corriente para la hipótesis L >>H (dominios anisótropos) (Caso 8, Tabla IV.6) 


\section{IV.2 ESCENARIOS SIMPLIFICADOS DERIVADOS DE PROBLEMAS CON GRUPOS DISCRIMINADOS DE ORDEN DE MAGNITUD UNIDAD}

Esta aproximación no tiene mayor interés práctico pero constituye un complemento que ilustra la bondad de los grupos deducidos mediante la adimensionalización discriminada en su simplificación a escenarios más simples. Seguiremos más o menos las simplificaciones adoptadas en el problema de Henry, aunque reduciremos su alcance y la presentación de resultados, las cuales sí son de interés en el campo de la hidrogeología. Hemos optado por hacer una única representación para cada simplificación para no extender el contenido de la memoria, si bien hemos realizado otras simulaciones que extienden los patrones a otros posibles casos de una misma simplificación.

\section{IV.2.1 PRIMERA SIMPLICACIÓN. DIFUSIVIDAD DESPRECIABLE}

Los monomios se reducen a dos

$$
\begin{aligned}
& \pi_{\mathrm{d}, 1}=\frac{(\Delta \rho) \mathrm{gk}_{\mathrm{y}}}{\varepsilon \mu \mathrm{v}_{\mathrm{amb}}^{*}}\left(\frac{\mathrm{L}}{\mathrm{H}}\right) \\
& \pi_{\mathrm{d}, 2}=\frac{\mathrm{k}_{\mathrm{x}} \mathrm{H}^{2}}{\mathrm{k}_{\mathrm{y}} \mathrm{L}^{2}}
\end{aligned}
$$

Se asume $\mathrm{D}_{\mathrm{x}}=\mathrm{D}_{\mathrm{y}} \approx 0.01\left(\mathrm{v}_{\mathrm{amb}, \mathrm{H}} \mathrm{L}\right)$ y H/L fijo. Se toma $\pi_{\mathrm{d}, 2}=1$ jugando con $\mathrm{k}_{\mathrm{x}} \mathrm{y}$ se adopta un valor de $\mu$ que haga $\pi_{\mathrm{d}, 1}=1$. El resto de los parámetros son los de Henry. La Tabla IV.7 muestra los valores de la única simulación cuyos patrones se muestran en la Figura IV.20.

\begin{tabular}{|c|c|c|c|c|c|c|c|c|c|c|}
\hline Caso & $\Delta \rho$ & g & $\mathbf{k}_{\mathrm{x}}$ & $\mathbf{k}_{\mathbf{y}}$ & $\varepsilon$ & $\mu$ & $\mathbf{v}_{\mathrm{amb}}$ & $\mathbf{L}$ & $\mathbf{H}$ & $\mathbf{D}_{\mathbf{x}}, \mathbf{D}_{\mathbf{y}}$ \\
\hline 1 & $\Delta \rho_{\mathrm{H}}$ & $\mathbf{g}_{\mathbf{H}}$ & $\mathbf{k}_{\mathrm{x}, \mathrm{H}}$ & $\mathbf{k}_{\mathrm{y}, \mathrm{H}}$ & $\varepsilon_{\mathrm{H}}$ & $\mu_{\mathrm{H}}$ & $\mathbf{V}_{\mathrm{amb}, \mathrm{H}}$ & $\mathbf{L}_{\mathbf{H}}$ & $\mathbf{H}_{\mathbf{H}}$ & $\mathbf{D}_{\mathrm{x}, \mathrm{H}}$ \\
\hline & 25 & 9,81 & $1,0204 \mathrm{E}-09$ & 4,0816E-09 & 0,35 & 0,007583485 & 1,8857E-04 & 2 & 1 & $3,7714 \mathrm{E}-06$ \\
\hline
\end{tabular}

Tabla IV.7 Valores de los parámetros para los casos de difusividad despreciable, $\pi_{\mathrm{d}, 1}=\pi_{\mathrm{d}, 2}=1$

El desequilibrio de las fuerzas difusivas (despreciables) frente a las restantes (de empuje regional y flotación) se traduce una reducción importante de la cuña de intrusión que queda confinada a la región de recirculación de agua de mar definida por la divisoria de aguas $\Psi=0$. Así sólo existe gradiente de concentración salina en esta región. Esta solución abunda en una crítica a la elección de L y H como magnitudes 
influyentes en los patrones de solución; en su lugar deberían adoptarse las longitudes horizontal y vertical que definen la cuña emergente.
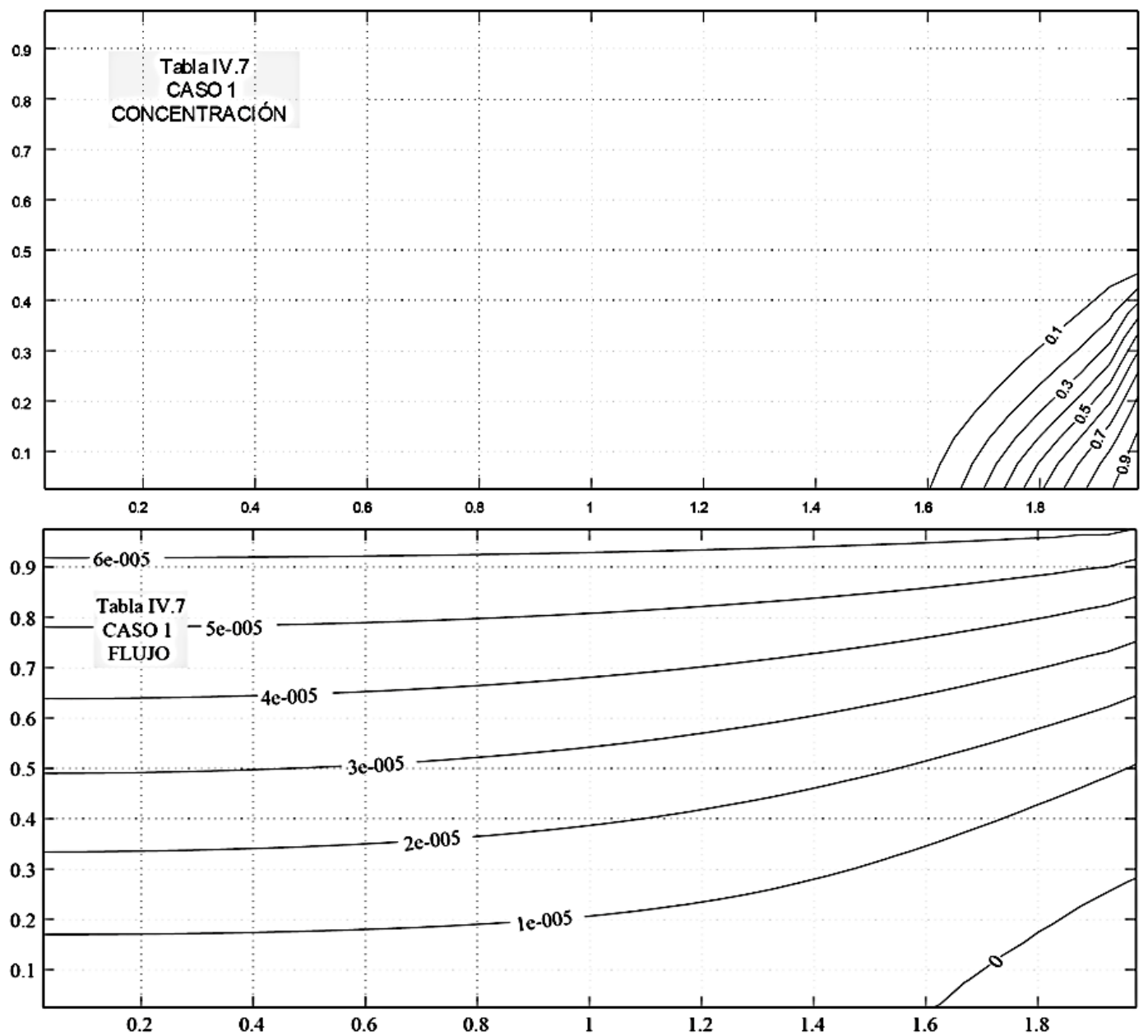

Figura IV.20 Patrones de concentración y función de corriente (difusividad despreciable)

\section{IV.2.2 SEGUNDA SIMPLIFICACIÓN. DIFUSIVIDAD VERTICA(Dy) DESPRECIABLE, $\mathrm{D}_{\mathbf{Y}}<<\mathrm{D}_{\mathbf{X}}$}

Los monomios resultantes son:

$$
\begin{aligned}
& \pi_{\mathrm{d}, 1}=\frac{(\Delta \rho) \mathrm{gk}_{\mathrm{y}}}{\varepsilon \mu \mathrm{v}_{\mathrm{amb}}^{*}}\left(\frac{\mathrm{L}}{\mathrm{H}}\right) \\
& \pi_{\mathrm{d}, 2}=\frac{\mathrm{k}_{\mathrm{x}} \mathrm{H}^{2}}{\mathrm{k}_{\mathrm{y}} \mathrm{L}^{2}} \\
& \pi_{\mathrm{d}, 3}=\frac{\mathrm{D}_{\mathrm{x}}}{\mathrm{v}_{\mathrm{amb}}^{*} \mathrm{~L}}
\end{aligned}
$$

Se asume $D_{\mathrm{x}}=\mathrm{v}_{\mathrm{amb}, \mathrm{H}} \mathrm{L}, \mathrm{D}_{\mathrm{y}}=0.01 \mathrm{D}_{\mathrm{x}} \mathrm{y} \mathrm{H} / \mathrm{L}$ fijo. Se toma $\pi_{\mathrm{d}, 2}=1$ jugando con $\mathrm{k}_{\mathrm{x}}$ y se adopta un valor de $\mu$ que haga $\pi_{\mathrm{d}, 1}=1$. El resto de los parámetros son los de Henry. 
La Tabla IV.8 muestra los valores de la única simulación cuyos patrones de solución se recogen en la Figura IV.21.

Tabla IV.8 Valores de los parámetros para los casos de difusividad vertical despreciable, $\pi_{\mathrm{d}, 1}=\pi_{\mathrm{d}, 2}=\pi_{\mathrm{d}, 3}=1$

\begin{tabular}{|c|c|c|c|c|c|c|c|c|c|c|c|}
\hline Caso & $\Delta \rho$ & $\mathbf{g}$ & $\mathbf{k}_{\mathbf{y}}$ & $\mathbf{k}_{\mathbf{x}}$ & $\boldsymbol{\varepsilon}$ & $\boldsymbol{\mu}$ & $\mathbf{v}_{\mathbf{a m b}}$ & $\mathbf{L}$ & $\mathbf{H}$ & $\mathbf{D}_{\mathbf{x}}$ & $\mathbf{D}_{\mathbf{y}}$ \\
\hline $\mathbf{1}$ & $\Delta \boldsymbol{\rho}_{\mathbf{H}}$ & $\mathbf{g}_{\mathbf{H}}$ & $\mathbf{k}_{\mathbf{y}, \mathbf{H}}$ & $\mathbf{x x} \mathbf{k}_{\mathbf{x}, \mathbf{H}}$ & $\boldsymbol{\varepsilon}_{\mathbf{H}}$ & $\mathbf{x x} \boldsymbol{\mu}_{\mathbf{H}}$ & $\mathbf{v}_{\mathbf{a m b}, \mathbf{H}}$ & $\mathbf{L}_{\mathbf{H}}$ & $\mathbf{H}_{\mathbf{H}}$ & $\mathbf{x x} \mathbf{D}_{\mathbf{x}, \mathbf{H}}$ & $\mathbf{x x} \mathbf{D}_{\mathbf{y}, \mathbf{H}}$ \\
\hline & 25 & 9,81 & $1,0204 \mathrm{E}-09$ & $4,0816 \mathrm{E}-09$ & 0,35 & $7,5835 \mathrm{E}-03$ & $1,8857 \mathrm{E}-04$ & 2 & 1 & $3,7714 \mathrm{E}-04$ & $3,7714 \mathrm{E}-06$ \\
\hline
\end{tabular}

Tanto los patrones de flujo cmo los de concentración, en particular los primeros, no sufren apenas alteración como era de esperar. Tan solo una ligera mayor inclinación de las lineas de concentración del patrón de menor difusividad vertical merced a la menor influencia de este efecto frente al arastre.
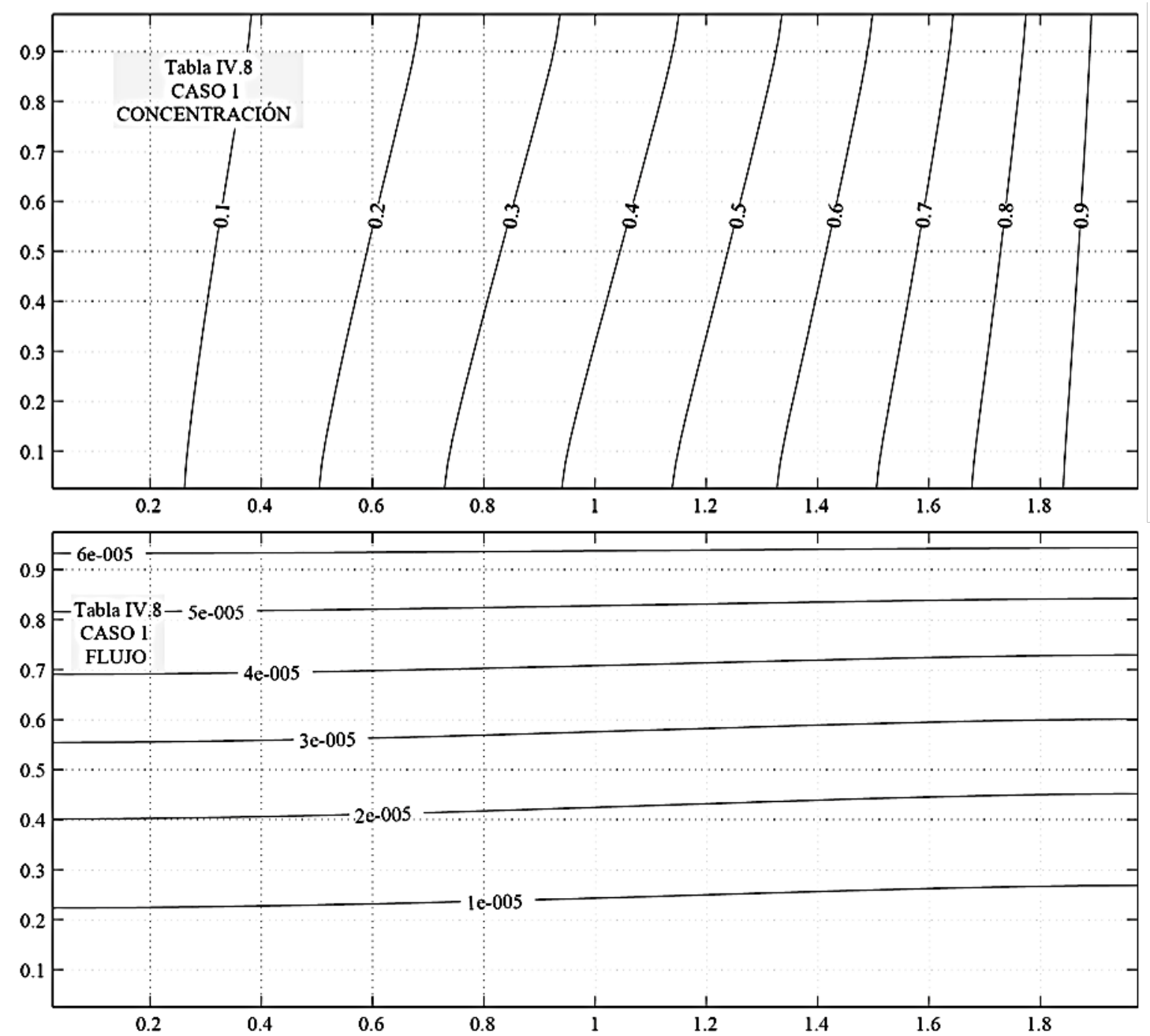

Figura IV.21 Patrones de concentración y función de corriente (difusividad vertical despreciable) 


\section{IV.2.3 TERCERA SIMPLIFICACION. DIFUSIVIDAD ISOTRÓPICA, $\mathrm{DY}_{\mathrm{Y}}=\mathrm{D}_{\mathrm{X}}$}

Los grupos resultantes, escritos en su forma más sencilla, son:

$$
\begin{aligned}
& \pi_{\mathrm{d}, 1}=\frac{(\Delta \rho) \mathrm{gk}_{\mathrm{y}}}{\varepsilon \mu \mathrm{v}_{\mathrm{amb}}^{*}} \\
& \pi_{\mathrm{d}, 2}=\frac{\mathrm{k}_{\mathrm{x}}}{\mathrm{k}_{\mathrm{y}}} \\
& \pi_{\mathrm{d}, 3}=\frac{\mathrm{D}}{\mathrm{v}_{\mathrm{amb}}^{*} \mathrm{~L}} \\
& \pi_{\mathrm{d}, 4}=\frac{\mathrm{H}}{\mathrm{L}}
\end{aligned}
$$

Se adopta la geometría de Henry, una difusividad de valor $\mathrm{D}=\mathrm{vamb, \textrm {H }} \mathrm{L}$ y un valor de $\mu$ que haga $\pi_{\mathrm{d}, 1}=1$. El resto de los parámetros son los de Henry. La Tabla IV.9 muestra los valores de una única simulación cuyos patrones de solución se representan en la Figura IV.22. Las pequeñas diferencias frente al patrón del caso $\pi_{\mathrm{d}, 2}=\pi_{\mathrm{d}, 2}=\pi_{\mathrm{d}, 3}=$ $\pi_{\mathrm{d}, 4}=1$ es el valor del monomio $\pi_{\mathrm{d}, 4}=1 / 2$, apenas influyente en los patrones. El efecto de $\mathrm{D}_{\mathrm{y}}$ es despreciable por el poco valor del gradiente vertical de concentraciones.

En definitiva, como en el caso anterior, las isolíneas de concentración y corriente, verticales y horizontales respectivamente, muestran un patrón coherente con la simplificación, y su distribución regular en todo el dominio es asimismo coherente con el hecho de que los grupos influyentes valgan la unidad.

Tabla IV.9 Valores de los parámetros para los casos de difusividad isótropa,

$$
\pi_{\mathrm{d}, 1}=\pi_{\mathrm{d}, 2}=\pi_{\mathrm{d}, 3}=\pi_{\mathrm{d}, 4}=1
$$

\begin{tabular}{|c|c|c|c|c|c|c|c|c|c|c|}
\hline Caso & $\Delta \boldsymbol{\rho}$ & $\mathbf{g}$ & $\mathbf{k}_{\mathbf{x}}$ & $\mathbf{k}_{\mathbf{y}}$ & $\boldsymbol{\varepsilon}$ & $\boldsymbol{\mu}$ & $\mathbf{v}_{\mathrm{amb}}$ & $\mathbf{L}$ & $\mathbf{H}$ & $\mathbf{D}_{\mathbf{x}}=\mathbf{D}_{\mathbf{y}}$ \\
\hline $\mathbf{1}$ & $\Delta \boldsymbol{\rho}_{\mathrm{H}}$ & $\mathbf{g}_{\mathrm{H}}$ & $\mathbf{k}_{\mathbf{x}, \mathrm{H}}$ & $\mathbf{k}_{\mathbf{y}, \mathbf{H}}$ & $\boldsymbol{\varepsilon}_{\mathrm{H}}$ & $\mathbf{x x} \boldsymbol{\mu}_{\mathbf{H}}$ & $\mathbf{v}_{\mathbf{a m b}, \mathbf{H}}$ & $\mathbf{L}_{\mathbf{H}}$ & $\mathbf{H}_{\mathbf{H}}$ & $\mathbf{D}_{\mathbf{x}, \mathrm{H}}$ \\
\hline & 25 & 9,81 & $1,0204 \mathrm{E}-09$ & $1,0204 \mathrm{E}-09$ & 0,35 & $3,7917 \mathrm{E}-03$ & $1,8857 \mathrm{E}-04$ & 2 & 1 & $3,7714 \mathrm{E}-04$ \\
\hline
\end{tabular}



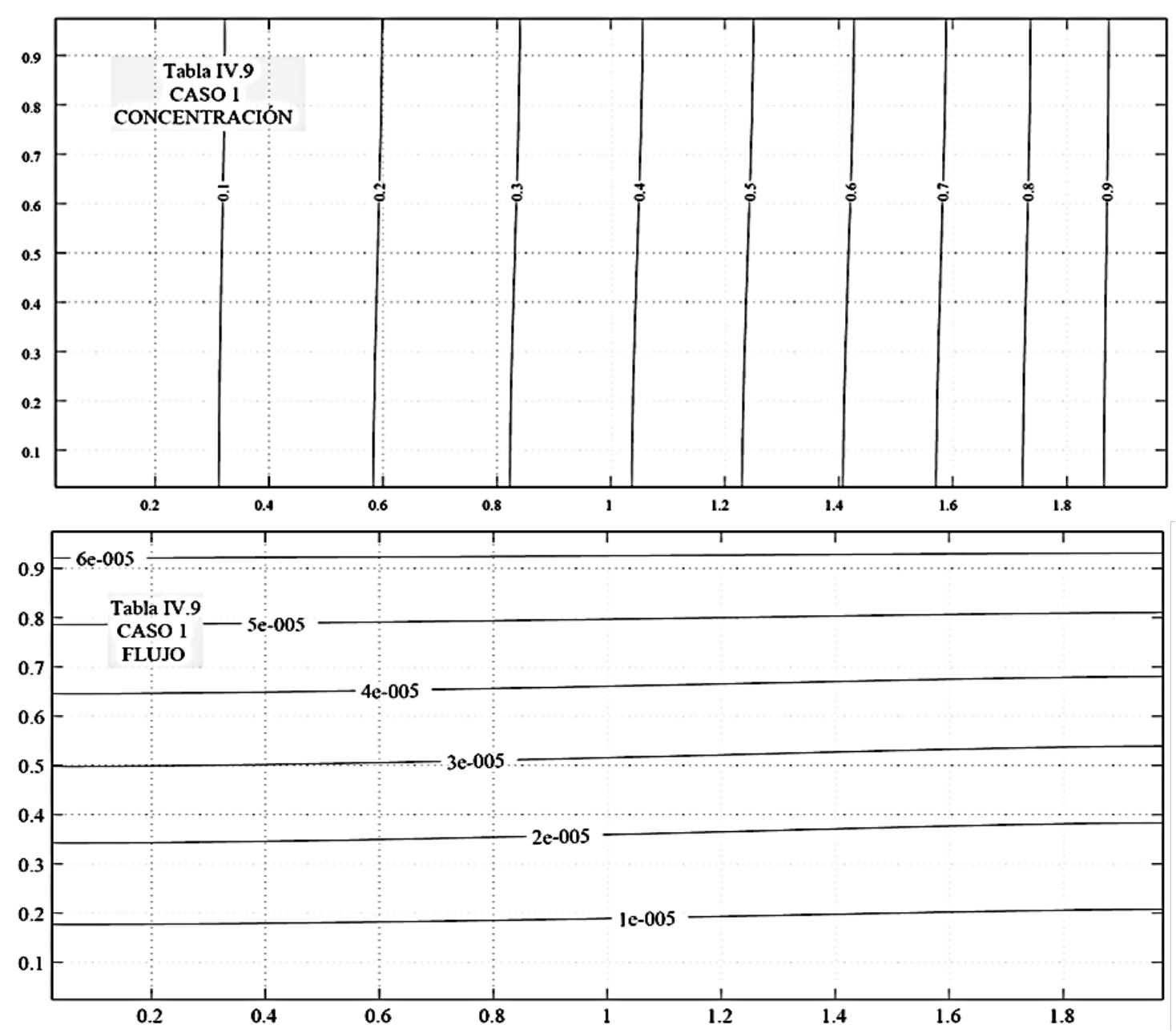

Figura IV.22 Patrones de concentración y función de corriente (difusividad isótropa)

\section{IV.2.4 CUARTA SIMPLIFICACION. DIFUSIVIDAD Y CONDUCTIVIDAD HIDRÁULICA ISOTRÓPICAS, $D_{\mathbf{y}}=D_{x} \mathbf{y} k_{\mathbf{y}}=k_{x}$}

Se obtienen los siguientes grupos independientes:

$$
\begin{aligned}
& \pi_{\mathrm{d}, 1}=\frac{(\Delta \rho) \mathrm{gk}_{\mathrm{y}}}{\varepsilon \mu \mathrm{v}_{\mathrm{amb}}^{*}} \\
& \pi_{\mathrm{d}, 3}=\frac{\mathrm{D}}{\mathrm{v}_{\mathrm{amb}}^{*} \mathrm{~L}} \\
& \pi_{\mathrm{d}, 4}=\frac{\mathrm{H}}{\mathrm{L}}
\end{aligned}
$$

Se adopta la geometría de Henry, una difusividad de valor $\mathrm{D}=\mathrm{vamb, \textrm {H }} \mathrm{\textrm {y }}$ un valor de $\mu$ que haga $\pi_{\mathrm{d}, 1}=1$. El resto de los parámetros son los de Henry. La Tabla IV.10 muestra los valores de una única simulación cuyos patrones de solución se representan 
en la Figura IV.23. Cabe un comentario de índole similar al del caso anterior; las isolíneas de concentración y corriente se distribuyen regularmente en todo el dominio.

Tabla IV.10 Valores de los parámetros para los casos de difusividad y conductividad hidráulica isótropas, $\pi_{\mathrm{d}, 1}=\pi_{\mathrm{d}, 3}=\pi_{\mathrm{d}, 4}=1$

\begin{tabular}{|c|c|c|c|c|c|c|c|c|c|}
\hline Caso & $\Delta \rho$ & $\mathbf{g}$ & $\mathbf{k}_{\mathbf{x}}=\mathbf{k}_{\mathbf{y}}$ & $\boldsymbol{\varepsilon}$ & $\boldsymbol{\mu}$ & $\mathbf{v}_{\mathbf{a m b}}$ & $\mathbf{L}$ & $\mathbf{H}$ & $\mathbf{D}_{\mathbf{x}}=\mathbf{D}_{\mathbf{y}}$ \\
\hline $\mathbf{1}$ & $\Delta \boldsymbol{\rho}_{\mathrm{H}}$ & $\mathbf{g}_{\mathrm{H}}$ & $\mathbf{k}_{\mathbf{x}, \mathrm{H}}$ & $\boldsymbol{\varepsilon}_{\mathbf{H}}$ & $\mu_{\mathbf{H}}$ & $\mathbf{v}_{\mathrm{amb}, \mathbf{H}}$ & $\mathbf{L}_{\mathbf{H}}$ & $\mathbf{H}_{\mathbf{H}}$ & $\mathbf{D}_{\mathbf{x}, \mathbf{H}}$ \\
\hline & 25 & 9,81 & $1,0204 \mathrm{E}-09$ & 0,35 & $3,7917 \mathrm{E}-03$ & $1,8857 \mathrm{E}-04$ & 2 & 1 & $3,7714 \mathrm{E}-04$ \\
\hline
\end{tabular}
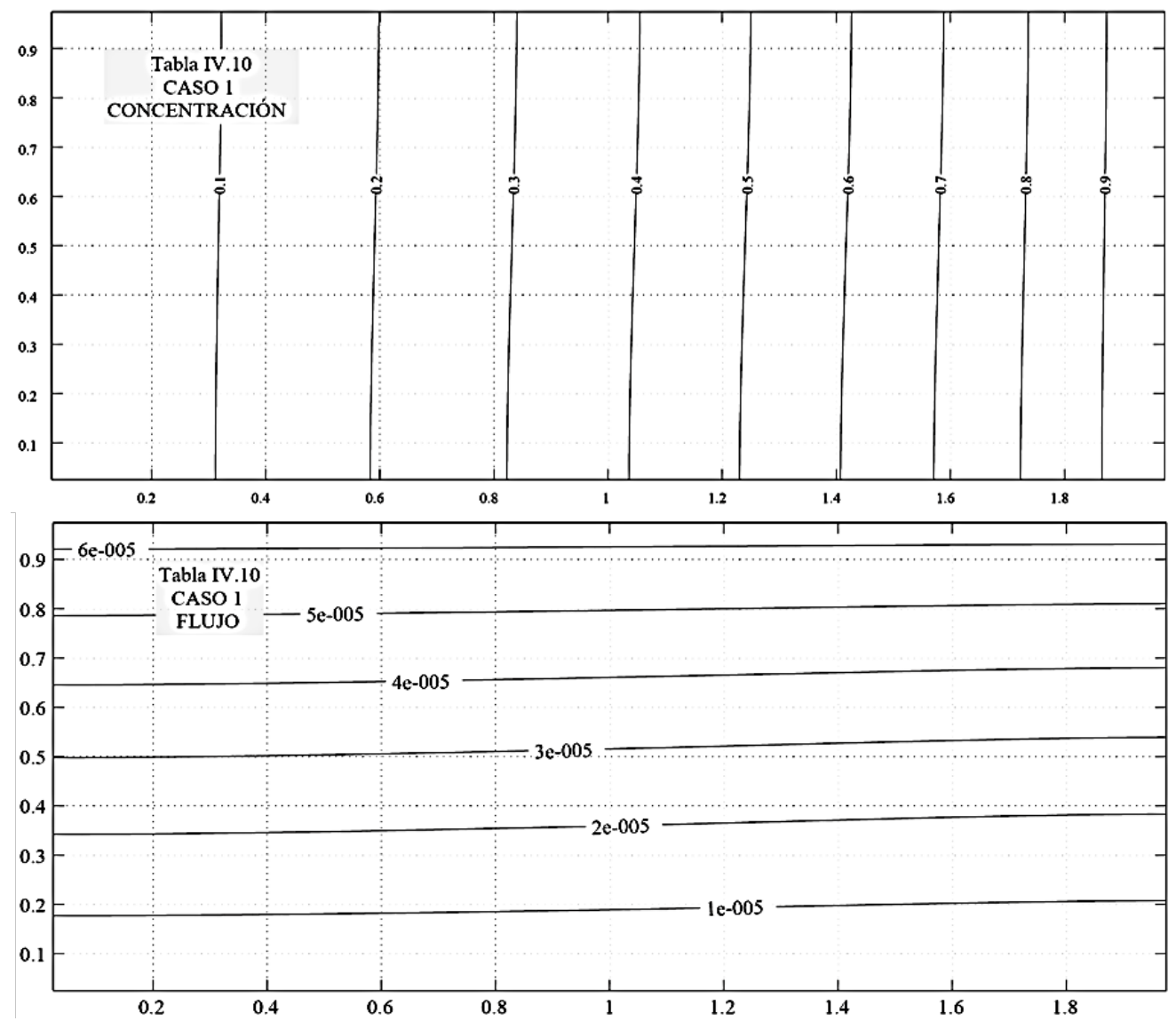

Figura IV.23 Patrones de concentración y función de corriente (difusividad y conductividad hidráulica isótropas)

\section{IV.2.5 QUINTA SIMPLICACIÓN. VELOCIDAD REGIONAL DESPRECIABLE FRENTE A LA DIFUSIÓN (Lvamb $<<$ DX)}

Los grupos independientes se reducen a tres, a saber,

$$
\begin{aligned}
& \pi_{\mathrm{d}, 1}=\frac{(\Delta \rho) \mathrm{gk}_{\mathrm{y}}}{\varepsilon \mu \mathrm{D}_{\mathrm{x}}}\left(\frac{\mathrm{L}^{2}}{\mathrm{H}}\right) \\
& \pi_{\mathrm{d}, 2}=\frac{\mathrm{k}_{\mathrm{x}} \mathrm{H}^{2}}{\mathrm{k}_{\mathrm{y}} \mathrm{L}^{2}}
\end{aligned}
$$




$$
\pi_{\mathrm{d}, 4}=\frac{\mathrm{D}_{\mathrm{x}} \mathrm{H}^{2}}{\mathrm{D}_{\mathrm{y}} \mathrm{L}^{2}}
$$

Se asume para la velocidad regional el valor $\mathrm{v}_{\mathrm{amb}}=0.01\left(\mathrm{D}_{\mathrm{x}} / \mathrm{L}\right)$, la geometría de Henry, un valor de $\mathrm{k}_{\mathrm{x}}$ que cumpla $\pi_{\mathrm{d}, 2}=1$, un valor de $\mathrm{D}_{\mathrm{y}}$ que cumpla $\pi_{\mathrm{d}, 4}=1 \mathrm{y}$ un valor de $\mu$ que haga $\pi_{\mathrm{d}, 1}=1$. El resto de los parámetros son los de Henry. La Tabla IV.11 muestra los valores de un único caso cuya simulación se representa en la Figura IV.24.

De nuevo, el aspecto del patrón retiene una misma forma de distribución regular de isolíneas de concentración y flujo por el valor unidad de los monomios influyentes.

Tabla IV.11 Valores de los parámetros para el caso de velocidad regional despreciable

$$
\left(\operatorname{Lv}_{\mathrm{amb}}<<\mathrm{D}_{\mathrm{x}}\right), \pi_{\mathrm{d}, 1}=\pi_{\mathrm{d}, 2}=\pi_{\mathrm{d}, 4}=1
$$

\begin{tabular}{|c|c|c|c|c|c|c|c|c|c|c|c|c|}
\hline Caso & $\Delta \boldsymbol{\rho}$ & $\mathbf{g}$ & $\mathbf{k}_{\mathbf{x}, \mathbf{H}}$ & $\mathbf{k}_{\mathbf{y}, \mathbf{H}}$ & $\boldsymbol{\varepsilon}$ & $\boldsymbol{\mu}$ & $\mathbf{V a m b}$ & $\mathbf{L}$ & $\mathbf{H}$ & $\mathbf{D}_{\mathbf{x}}$ & $\mathbf{D}_{\mathbf{y}}$ & $\Psi$ \\
\hline $\mathbf{1}$ & $\Delta \boldsymbol{\rho}_{\mathbf{H}}$ & $\mathbf{g}_{\mathbf{H}}$ & $4 \mathbf{k}_{\mathbf{x}, \mathbf{H}}$ & $\mathbf{k}_{\mathbf{y}, \mathbf{H}}$ & $\boldsymbol{\varepsilon}_{\mathbf{H}}$ & $\mathbf{x x} \boldsymbol{\mu}_{\mathbf{H}}$ & $\mathbf{0 . 0 1}\left(\mathbf{D}_{\mathbf{x}} / \mathbf{L}\right)$ & $\mathbf{L}_{\mathbf{H}}$ & $\mathbf{H}_{\mathbf{H}}$ & $4 \mathbf{D}_{\mathbf{x}, \mathbf{H}}$ & $\mathbf{D}_{\mathbf{y}, \mathbf{H}}$ & $\mathbf{0 . 0 0 2} \Psi_{\mathbf{H}}$ \\
\hline & 25 & 9,81 & $4,0816 \mathrm{E}-09$ & $1,0204 \mathrm{E}-09$ & 0,35 & 0,0379 & $3,7714 \mathrm{E}-07$ & 2 & 1 & $7,5428 \mathrm{E}-05$ & $1,8857 \mathrm{E}-05$ & $1,3200 \mathrm{E}-07$ \\
\hline
\end{tabular}
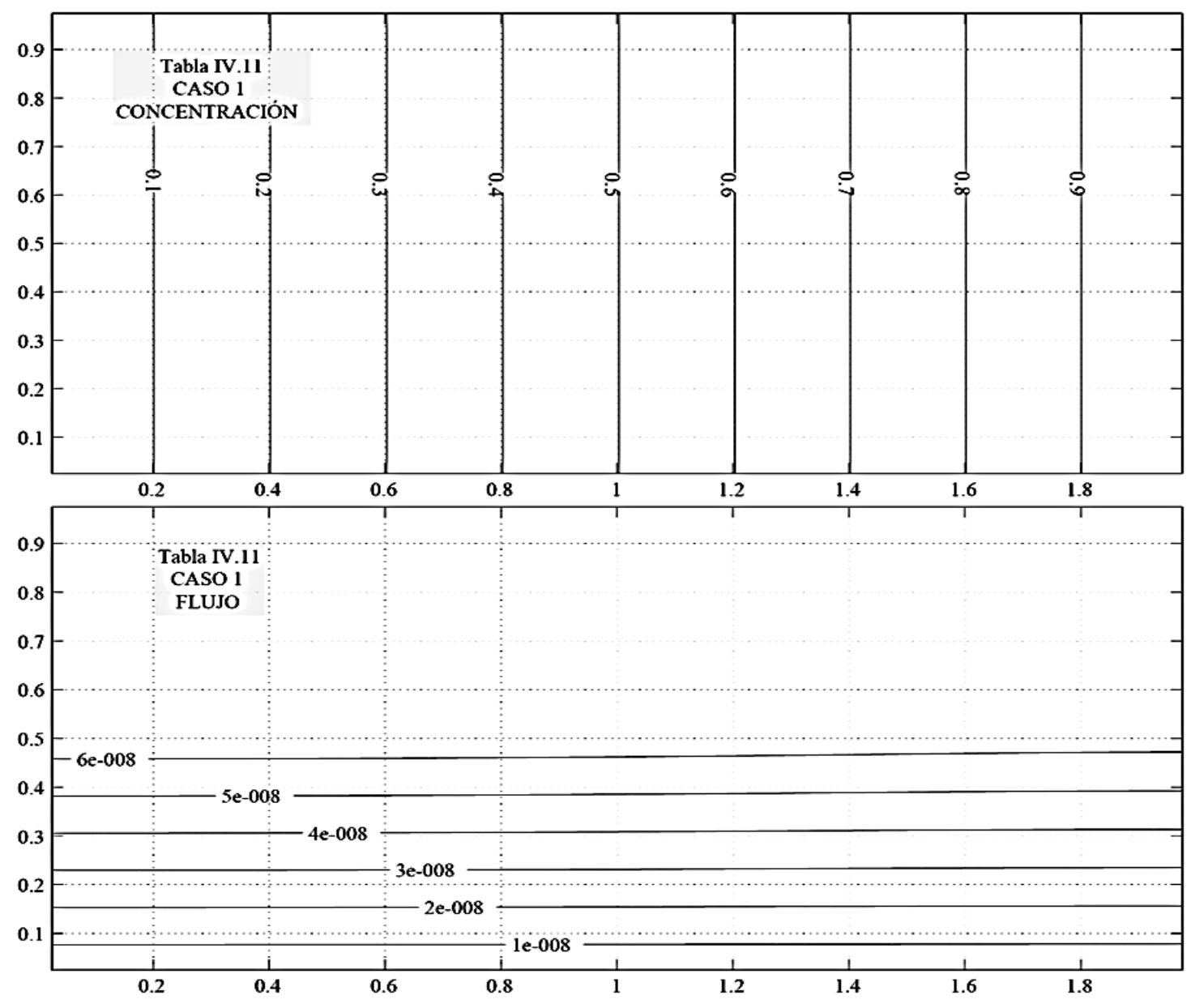

Figura IV.24 Patrones de concentración y función de corriente (velocidad regional despreciable, $\mathrm{LV}_{\mathrm{amb}}<<\mathrm{D}_{\mathrm{x}}$ ) 
$\mathrm{Si}$, además, $\mathrm{k}_{\mathrm{x}}=\mathrm{k}_{\mathrm{y}}$ y $\mathrm{D}_{\mathrm{x}}=\mathrm{D}_{\mathrm{y}}$, los grupos resultantes se reducen a dos:

$$
\pi_{\mathrm{d}, 1}=\frac{(\Delta \rho) \mathrm{gkL}}{\varepsilon \mu \mathrm{D}} \quad \pi_{\mathrm{d}, 2}=\frac{\mathrm{H}}{\mathrm{L}}
$$

Se asume para la velocidad regional el valor $\mathrm{v}_{\mathrm{amb}}=0.01\left(\mathrm{D}_{\mathrm{x}} / \mathrm{L}\right)$, la geometría de Henry, y un valor de $\mu$ que haga $\pi_{\mathrm{d}, 2}=1$. El resto de los parámetros son los de Henry. La Tabla IV.12 muestra los valores del caso simulado cuyo patrón de líneas regularmente espaciadas se presenta en la Figura IV.25.

Tabla IV.12 Valores de los parámetros para el caso de velocidad regional despreciable ( $\mathrm{LV}_{\mathrm{amb}}<<\mathrm{D}_{\mathrm{x}}$ ),

$$
\mathrm{k}_{\mathrm{x}}=\mathrm{k}_{\mathrm{y}} \text { y } \mathrm{D}_{\mathrm{x}}=\mathrm{D}_{\mathrm{y}} \cdot \pi_{\mathrm{d}, 1}=\pi_{\mathrm{d}, 2}=1
$$

\begin{tabular}{|c|c|c|c|c|c|c|c|c|c|c|c|}
\hline Caso & $\Delta \rho$ & g & $\mathbf{k}_{\mathrm{x}}$ & $\mathbf{k}_{\mathbf{y}}$ & $\varepsilon$ & $\mu$ & $\mathbf{V}_{\mathrm{amb}}$ & $\mathbf{L}$ & $\mathbf{H}$ & $\mathbf{D}_{\mathrm{x}}=\mathbf{D}_{\mathbf{Y}}$ & $\Psi$ \\
\hline 1 & $\Delta \rho_{\mathrm{H}}$ & $\mathbf{g}_{\mathrm{H}}$ & $\mathbf{k}_{\mathrm{x}, \mathrm{H}}$ & $\mathbf{k}_{\mathbf{y}, \mathrm{H}}$ & $\varepsilon_{H}$ & $\mathbf{x x} \mu_{\mathrm{H}}$ & $0.01 v_{\mathrm{amb}, \mathrm{H}}$ & $\mathbf{L}_{\mathbf{H}}$ & $\mathbf{H}_{\mathrm{H}}$ & $\mathbf{D}_{\mathbf{x}, \mathrm{H}}$ & $5,00 \mathrm{E}-04 \Psi_{\mathrm{H}}$ \\
\hline & 25 & 9,81 & $1,0204 \mathrm{E}-09$ & $1,0204 \mathrm{E}-09$ & 0,35 & $1,5167 \mathrm{E}+01$ & $9,4285 \mathrm{E}-08$ & 2 & 1 & 1,8857E-05 & 3,300E-08 \\
\hline
\end{tabular}
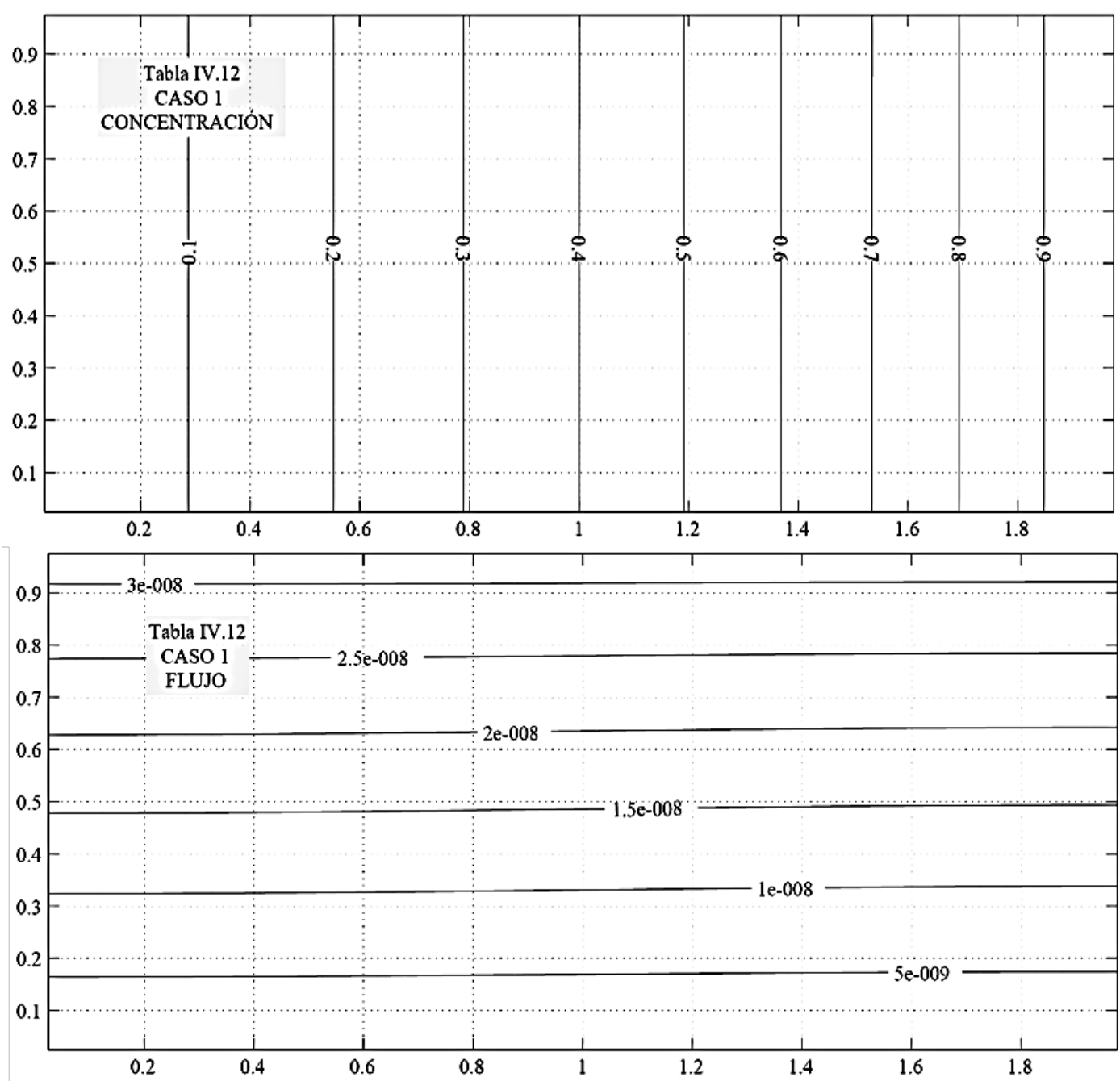

Figura IV.25 Patrones de concentración y función de corriente (velocidad regional despreciable, $\mathrm{Lv}_{\mathrm{amb}}<<\mathrm{D}_{\mathrm{x}}, \mathrm{k}_{\mathrm{x}}=\mathrm{k}_{\mathrm{y}} \mathrm{y} \mathrm{D}_{\mathrm{x}}=\mathrm{D}_{\mathrm{y}}$ ) 


\section{IV.2.6 SEXTA SIMPLIFICACION. DIFERENCIA DE DENSIDADES $(\Delta \rho)$ DESPRECIABLE}

Los monomios independientes del proceso de adimensionalización discriminada son

$$
\pi_{\mathrm{d}, 2}=\frac{\mathrm{k}_{\mathrm{x}} \mathrm{H}^{2}}{\mathrm{k}_{\mathrm{y}} \mathrm{L}^{2}} \quad \pi_{\mathrm{d}, 3}=\frac{\mathrm{D}_{\mathrm{x}}}{\mathrm{v}_{\mathrm{amb}}^{*} \mathrm{~L}} \quad \pi_{\mathrm{d}, 4}=\frac{\mathrm{D}_{\mathrm{x}} \mathrm{H}^{2}}{\mathrm{D}_{\mathrm{y}} \mathrm{L}^{2}}
$$

Se asume el valor $\Delta \rho=0.25$, la geometría de Henry, un valor de $k_{x}$ que cumpla $\pi_{\mathrm{d}, 2}=1$, un valor de $D_{\mathrm{x}}$ que cumpla $\pi_{\mathrm{d}, 3}=1 \mathrm{y}$ un valor de $\mathrm{D}_{\mathrm{y}}$ que cumpla $\pi_{\mathrm{d}, 4}=1$. El resto de los parámetros son los de Henry. La Tabla IV.13 muestra los valores de un único caso cuya simulación, que no cambia en aspecto respecto al de las anteriores simplificaciones, se representa en la Figura IV.26.

Tabla IV.13 Valores de los parámetros para el caso de diferencia de densidad despreciable,

$$
\pi_{\mathrm{d}, 2}=\pi_{\mathrm{d}, 3}=\pi_{\mathrm{d}, 4}=1
$$

\begin{tabular}{|c|c|c|c|c|c|c|c|c|c|c|c|}
\hline Caso & $\Delta \boldsymbol{\rho}$ & $\mathbf{g}$ & $\mathbf{k}_{\mathbf{x}}$ & $\mathbf{k}_{\mathbf{y}}$ & $\boldsymbol{\varepsilon}$ & $\boldsymbol{\mu}$ & $\mathbf{v}_{\mathbf{a m b}}$ & $\mathbf{L}$ & $\mathbf{H}$ & $\mathbf{D}_{\mathbf{x}}$ & $\mathbf{D}_{\mathbf{y}}$ \\
\hline $\mathbf{1}$ & $\mathbf{0 . 0 1} \Delta \boldsymbol{\rho}_{\mathbf{H}}$ & $\mathbf{g}_{\mathbf{H}}$ & $\mathbf{k}_{\mathbf{x}, \mathbf{H}}$ & $\mathbf{k}_{\mathbf{y}, \mathbf{H}}$ & $\boldsymbol{\varepsilon}_{\mathbf{H}}$ & $\boldsymbol{\mu}_{\mathbf{H}}$ & $\mathbf{v}_{\mathbf{a m b}, \mathbf{H}}$ & $\mathbf{L}_{\mathbf{H}}$ & $\mathbf{H}_{\mathbf{H}}$ & $\mathbf{D}_{\mathbf{x}, \mathbf{H}}$ & $\mathbf{D}_{\mathbf{y}, \mathbf{H}}$ \\
\hline & 0,25 & 9,81 & $4,0816 \mathrm{E}-09$ & $1,0204 \mathrm{E}-09$ & 0,35 & $1 \mathrm{E}-03$ & $1,8857 \mathrm{E}-04$ & 2 & 1 & $3,7714 \mathrm{E}-04$ & $9,4285 \mathrm{E}-05$ \\
\hline
\end{tabular}
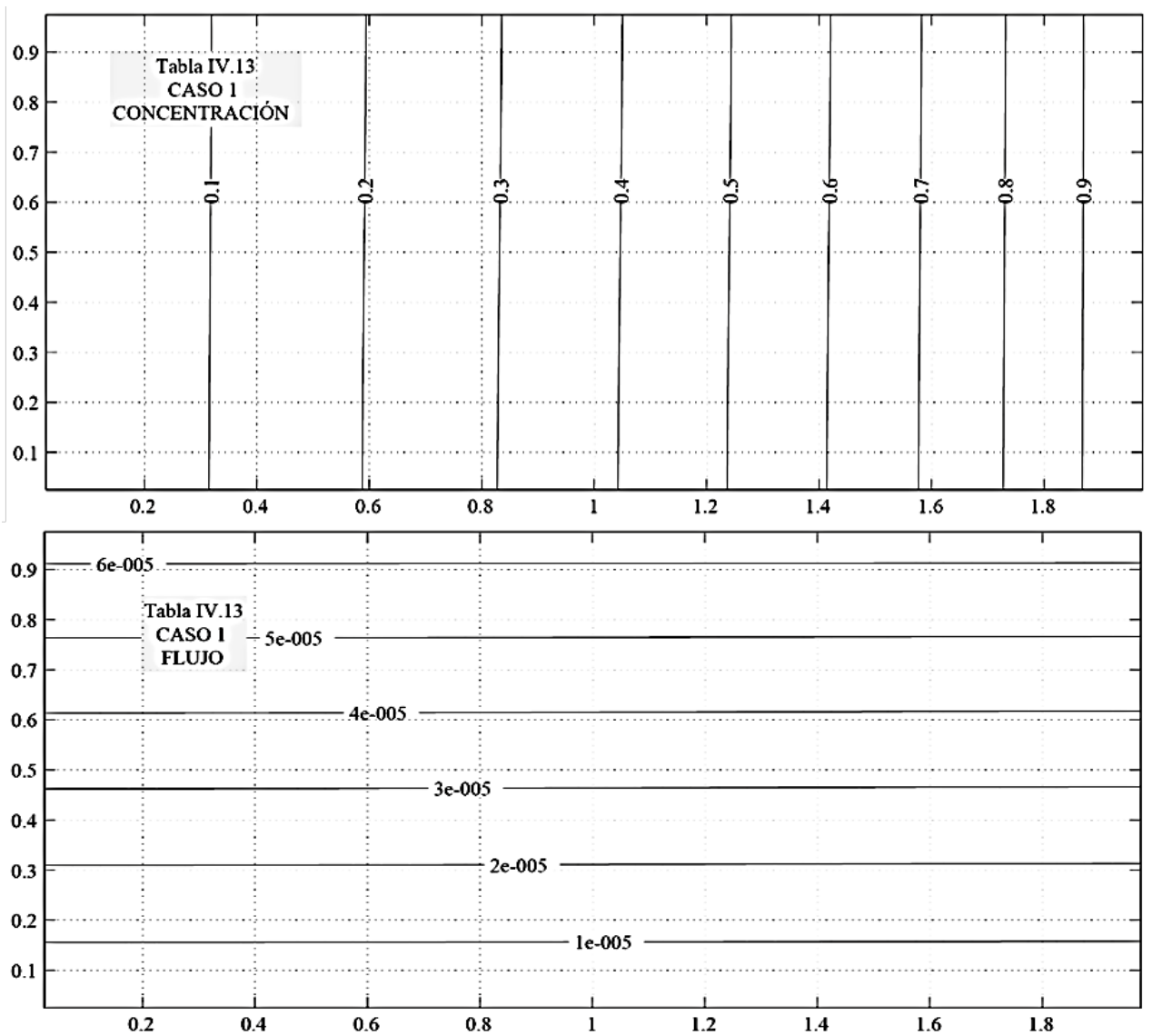

Figura IV.26 Patrones de concentración y función de corriente (diferencia de densidad despreciable, $\pi_{\mathrm{d}, 2}=\pi_{\mathrm{d}, 3}=\pi_{\mathrm{d}, 4}=1$ ) 


\section{IV.2.7 SÉPTIMA SIMPLIFICACION. DOMINIOS EXTENSOS (L>>H) Y MEDIOS ISÓTROPOS}

Los monomios se reducen a dos

$$
\pi_{\mathrm{d}, 1}=\frac{(\Delta \rho) \mathrm{gk}_{\mathrm{y}}}{\varepsilon \mu v_{\mathrm{amb}}^{*}}\left(\frac{\mathrm{L}}{\mathrm{H}}\right) \quad \pi_{\mathrm{d}, 3}=\frac{\mathrm{D}}{\mathrm{v}_{\mathrm{amb}}^{*} \mathrm{~L}}
$$

Se asume el valor $L=6$, un valor de $D$ que cumpla $\pi_{\mathrm{d}, 3}=1$ y un valor de $\mu$ que cumpla $\pi_{\mathrm{d}, 1}=1$. El resto de los parámetros son los de Henry. La Tabla IV.13 muestra los valores de un único caso cuya simulación se representa en la Figura IV.27. De acuerdo con lo esperado, consecuencia del valor unidad de los monomios influyentes, el sensible alargamiento de L no ha afectado al aspecto de distribución regular de líneas en el patrón.

Tabla IV.14 Valores de los parámetros para el caso de dominios extensos ( $\mathrm{L}>>\mathrm{H})$ y medios isótropos,

$$
\pi_{\mathrm{d}, 1}=\pi_{\mathrm{d}, 3}=1
$$

\begin{tabular}{|c|c|c|c|c|c|c|c|c|c|}
\hline Caso & $\Delta \rho$ & $\mathbf{g}$ & $\mathbf{k}_{\mathbf{y}}$ & $\boldsymbol{\varepsilon}$ & $\boldsymbol{\mu}$ & $\mathbf{\mathbf { v a m b } _ { \mathbf { a } }}$ & $\mathbf{L}$ & $\mathbf{H}$ & $\mathbf{D}_{\mathbf{x}}$ \\
\hline $\mathbf{1}$ & $\Delta \boldsymbol{\rho}_{\mathbf{H}}$ & $\mathbf{g}_{\mathbf{H}}$ & $\mathbf{k}_{\mathbf{y}, \mathbf{H}}$ & $\boldsymbol{\varepsilon}_{\mathbf{H}}$ & $\mathbf{x x} \boldsymbol{\mu}_{\mathbf{H}}$ & $\mathbf{V}_{\mathbf{a m b}, \mathbf{H}}$ & $\mathbf{3 L}_{\mathbf{H}}$ & $\mathbf{H}_{\mathbf{H}}$ & $\mathbf{D}_{\mathbf{x}, \mathbf{H}}$ \\
\hline & 25 & 9,81 & $1,0204 \mathrm{E}-09$ & 0,35 & $2,2750 \mathrm{E}-02$ & $1,8857 \mathrm{E}-04$ & 6 & 1 & $1,1314 \mathrm{E}-03$ \\
\hline
\end{tabular}
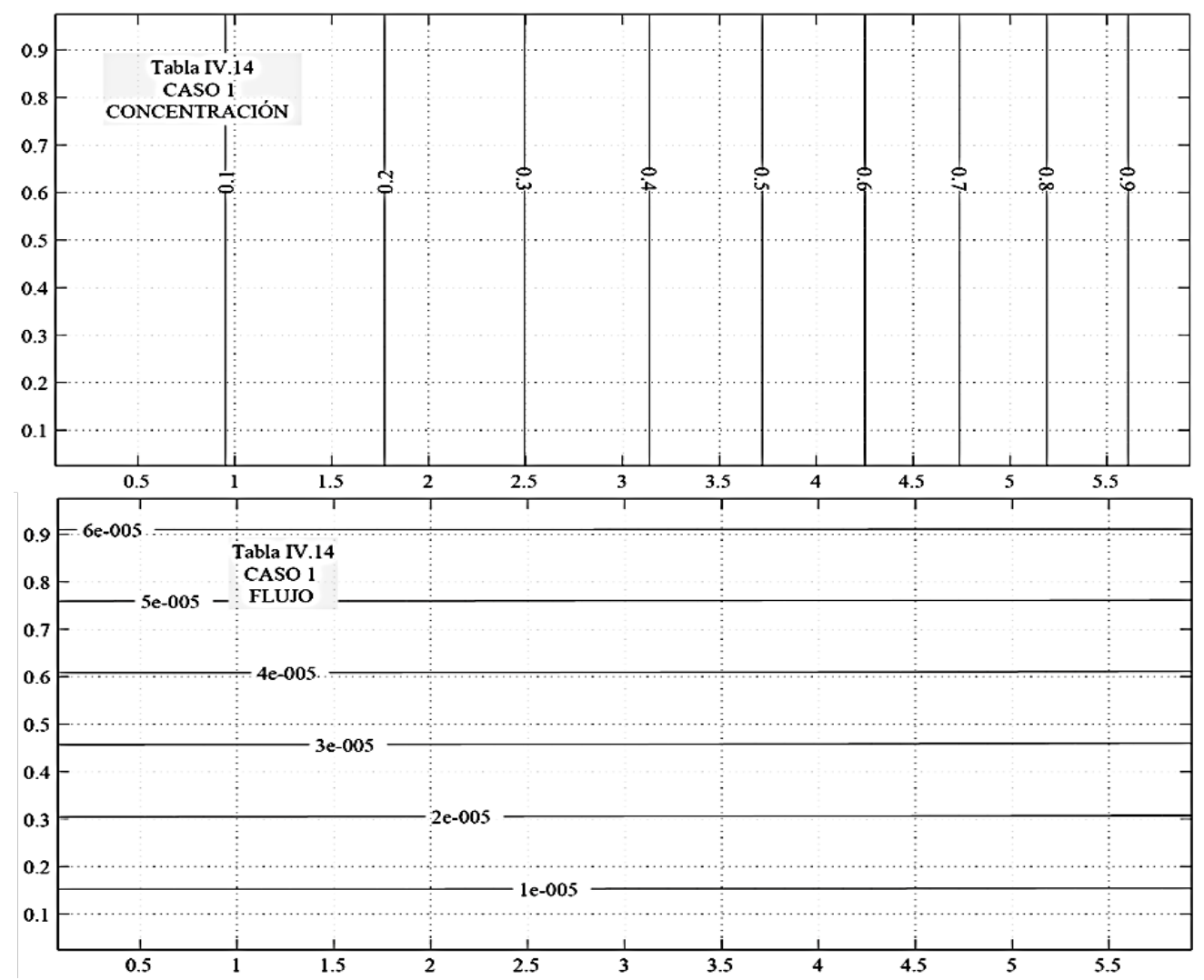

Figura IV.27 Patrones de concentración y función de corriente (dominios extensos, L>>H, y medios isótropos, $\pi_{\mathrm{d}, 2}=\pi_{\mathrm{d}, 3}=\pi_{\mathrm{d}, 4}=1$ ) 


\section{IV.3 CONCLUSIONES}

Se simplifican los escenarios tanto del problema original de Henry, cuyos grupos adimensionales tienen valores diferentes a la unidad, como del problema no isótropo cuyos números adimensionales valen la unidad. En todos los casos se derivan los grupos adimensionales resultantes verificándose los resultados mediante la simulación numérica con FATSIM de un conjunto de casos típicos.

i) Para el problema isótropo original de Henry se consideran seis simplificaciones, las cinco primeras se refieren a la influencia en el patrón de solución de los cambios de algunos parámetros físicos concretos del problema contenidos en los monomios resultantes de la simplificación, asumiendo como parámetros geométricos influyentes (pero de valor constante) las dimensiones del dominio, L y H. En la última simplificación, se investiga la influencia específica de estos parámetros geométricos ( $\mathrm{L}$ y $\mathrm{H}$ ) con el objeto de averiguar hasta qué punto y bajo qué condiciones dichos parámetros son, en efecto, determinantes o no en la solución del problema de Henry. Estas simplificaciones son:

1) Difusividad despreciable.

Los grupos se reducen de tres a dos y se verifica este resultado. La relación de aspecto no emerge como grupo independiente en principio, a menos que se asuma isotropía en la permeabilidad. Los patrones resultantes determinan una zona más estrecha de gradiente de sal. Al mantener el bajo valor del monomio $\pi_{\mathrm{d}, 1}$, prepondera el flujo o velocidad regional frente a la velocidad de Darcy, empujando el patrón hacia la derecha del dominio (como ocurre en el problema de Henry).

2) Difusividad vertical despreciable, $\mathrm{D}_{\mathrm{y}}<<\mathrm{D}_{\mathrm{x}}$.

El número de grupos independientes se mantiene en tres, aunque de nuevo, la relación de aspecto no emerge como grupo independiente. El valor despreciable de la difusividad vertical alarga horizontalmente la interface agua dulce agua salada, pero el efecto es poco perceptible por el ya bajo valor de la difusividad frente a la velocidad regional (monomio $\pi_{\mathrm{d}, 2}$ ) en el problema original. La recirculación de agua de mar (línea divisoria en los patrones de función de corriente) define la misma extensión en la base del acuífero en concordancia con 
la constancia del valor de los grupos adimensionales que no contienen la difusividad.

3) Difusividad isotrópica, $D_{y}=D_{x}$.

Ahora sí aparece la relación de aspecto como grupo independiente. Se mantiene el número de grupos adimensionales en cuatro y se verifica su deducción. El patrón de concentración es idéntico al de Henry, pues aunque se quisiera aumentar el valor de la difusividad para apreciar su efecto en los patrones, habría que compensar los valores de los otros parámetros para mantener el valor de los grupos del problema original, con lo que la velocidad regional seguiría preponderando frente a la difusión y la de Darcy frente a la regional. El patrón de flujo es, pues, cuantitativamente diferente merced al cambio de flujo regional de un caso a otro, manteniendo siempre la línea divisoria en la misma posición.

4) Velocidad regional despreciable frente al efecto de difusión, $L v^{*}{ }_{a m b}<<D_{X}$.

Se verifican los tres monomios resultantes. La acusada disminución de $\mathrm{v}^{*}$ amb frente a su valor en el problema original resulta en un patrón acusadamente diferente ya que la preponderancia de la difusión frente al flujo regional desplaza la cuña salada hacia el continente con gradientes de sal en todo el dominio del acuífero. Por otro lado, la influencia de la velocidad de Darcy induce un enorme flujo de recirculación procedente del mar que alcanza el margen izquierdo del dominio induciendo, por un lado, una nueva recirculación de agua dulce en dicho borde y, por otro, un flujo invertido de agua salada desde el mar hacia el continente en la frontera inferior. Con todo, el patrón de flujo es marcadamente diferente al del problema de Henry original.

5) Diferencia de densidades $(\Delta \rho)$ despreciable.

Los grupos adimensionales se reducen a tres (dos en el caso isótropo). La ausencia de flujo inducido por densidad variable endereza tanto las líneas de flujo (haciéndolas horizontales) como las de concentración (haciéndolas verticales). Por otro lado, la preponderancia del flujo regional frente a la difusión retrae la cuña de intrusión hacia el margen derecho del dominio.

6) Dominios extensos ( $\mathrm{L}>>\mathrm{H})$.

Tanto en escenarios anisótropos como isótropos, si deducimos los grupos por simplificación de los del caso general llegamos a resultados incoherentes (dos grupos para el caso isótropo y cuatro para el anisótropo), pero si procedemos a 
adimensionalizar las ecuaciones bajo esta hipótesis, carecemos de referencia para la coordenada horizontal. Sólo la introducción de una longitud oculta (prevista en la discriminación) permite obtener grupos adimensionales $\mathrm{y}$, al mismo tiempo, deducir el orden de magnitud de dicha longitud oculta. Esto conduce a la importante conclusión de que en el problema de Henry, la extensión L del acuífero es un parámetro poco o nada relevante.

ii) Para escenarios simplificados derivados de problemas con grupos discriminados del orden de la unidad se consideran simplificaciones similares a las del punto i). En este caso y como esperamos, la asignación del valor unidad a los monomios influyentes que resultan de cada caso induce un patrón regular tanto de isolíneas de concentración como de corriente en todo el dominio, patrón que se asemeja mucho cualitativamente en todos los casos.

1) Difusividad despreciable.

El desequilibrio de las fuerzas difusivas (despreciables) frente a las restantes (de empuje regional y de flotación) reduce la cuña de intrusión a la región de recirculación de agua de mar definida por la divisoria. De este patrón se deriva una crítica a la elección de L y H como magnitudes influyentes, que deberían ser sustituidas quizás por las longitudes horizontal y vertical que definen la cuña resultante.

2) Difusividad vertical despreciable, $\mathrm{D}_{\mathrm{y}}<<\mathrm{D}_{\mathrm{x}}$.

Los patrones apenas sufren alteración como era de esperar; sólo una leve mayor inclinación de las lineas de concentración por causa de la disminución de este efecto frente al arastre.

3) Difusividad isotrópica, $D_{y}=D_{x}$.

Como en el caso anterior, los patrones de concentración y corriente (casi perpendiculares entre sí) son coherentes con la simplificación y distribuyen las isolíneas regularmente merced al valor unidad de los monomios influyentes.

4) Difusividad y conductividad hidráulica isotrópicas, $D_{y}=D_{x}, k_{x}=k_{y}$.

El patrón, similar al anterior, tiene una distribución regular de líneas que ocupa todo el dominio por el valor unidad de los grupos adimensionales influyentes.

5) Velocidad regional despreciable frente al efecto de difusión, $\mathrm{Lv}^{*}{ }_{\mathrm{amb}}<<\mathrm{D}_{\mathrm{X}}$. Misma conclusión que en el caso anterior. 
6) Diferencia de densidades $(\Delta \rho)$ despreciable.

Misma conclusión que en los casos anteriores.

7) Dominios extensos $(\mathrm{L}>>\mathrm{H})$ y medios isótropos.

El acusado alargamiento de L no ha afectado a la distribución regular de líneas en todo el dominio por el valor unidad de los grupos. 


\section{CAPITULO V}

\section{Influencia de los grupos discriminados en los patrones del problema de Henry}

\section{V.1 INFLUENCIA DEL VALOR DE LOS GRUPOS DISCRIMINADOS EN LOS PATRONES DE SOLUCIÓN DEL PROBLEMA ORIGINAL DE HENRY}

En este capítulo se investiga la influencia de cada monomio al modificar sus valores en torno al que tienen en el problema original isótropo de Henry, incluyendo en estas modificaciones anisotropías en los valores de la conductividad hidráulica y la difusividad efectiva. Por comodidad, volvemos a escribir la expresión de estos grupos y su valor para el problema original de Henry en la Tabla V.0.

Sus valores para el caso de Henry están, unos claramente por encima de la unidad y otros por debajo de ella, y dado que (como hemos visto en capítulos anteriores) la influencia del valor numérico de estos grupos depende de la desviación respecto de la unidad, es obvio que para desviaciones grandes como las que se dan en el problema de Henry, un cambio de valor en los parámetros deberá ejercer una influencia relativamente pequeña en los patrones estacionarios, tanto más pequeña cuando mayor sea la desviación. 
Capítulo V. Influencia de los grupos discriminados en los patrones del problema de Henry

Merced a que la desviación inicial de todos los parámetros del problema frente a la unidad es grande, los balances que representan dichos monomios están desequilibrados. En particular, el valor elevado del monomio $\pi_{\mathrm{d}, 1}$ induce una preponderancia de la velocidad de Darcy frente a la velocidad regional una vez corregidas ambas por la geometría del dominio; mientras que el reducido valor del monomio $\pi_{\mathrm{d}, 3}$ da cuenta de la despreciable influencia de los efectos difusivos frente a la velocidad regional. Por otro lado, en cuanto a las propiedades isótropas, al no estar corregidas por las dimensiones del dominio, proporcionan valores también relativamente pequeños de los monomios $\pi_{\mathrm{d}, 2} \mathrm{y}$ $\pi_{\mathrm{d}, 4}$. En dominios anisótropos, estos monomios degeneran a la relación de aspecto del acuífero cuya influencia en el patrón, que ya hemos discutido anteriormente, tiende a ser nula al crecer la longitud del acuífero por encima de un cierto valor, manteniendo constante la profundidad H. En dominios anisótropos, la influencia de estos monomios la discutiremos en este apartado.

Tabla V.0 Grupos discriminados y sus valores para el problema original de Henry

\begin{tabular}{|c|c|c|}
\hline Grupo discriminado & Expresión & Valor \\
\hline$\pi_{\mathrm{d}, 1}$ & $\frac{(\Delta \rho) \mathrm{gk}_{\mathrm{y}}}{\varepsilon \mu \mathrm{v}_{\mathrm{amb}}^{*}}\left(\frac{\mathrm{L}}{\mathrm{H}}\right)$ & 7.5834 \\
\hline$\pi_{\mathrm{d}, 2}$ & $\frac{\mathrm{k}_{\mathrm{x}} \mathrm{H}^{2}}{\mathrm{k}_{\mathrm{y}} \mathrm{L}^{2}}$ & 0.25 \\
\hline$\pi_{\mathrm{d}, 3}$ & $\frac{\mathrm{D}_{\mathrm{x}}}{\mathrm{v}_{\mathrm{amb}}^{*} \mathrm{~L}}$ & 0.05 \\
\hline$\pi_{\mathrm{d}, 4}$ & $\frac{\mathrm{D}_{\mathrm{x}} \mathrm{H}^{2}}{\mathrm{D}_{\mathrm{y}} \mathrm{L}^{2}}$ & 0.25 \\
\hline
\end{tabular}

Lo interesante, uno de los objetivos de este capítulo, es investigar valores de estos cuatro monomios discriminados que aproximen los patrones resultantes a casos reales cuyo aspecto es el de cuñas de recirculación más angostas y cuñas de intrusión de menor espesor; esto es, una amplia zona de agua salada de alta concentración qua acuña la región inferior derecha del acuífero, con una estrecha frontera de agua salada de gradiente elevado que la separa la mencionada cuña de la región de agua dulce. Esperamos reproducir estos patrones trabajando con la anisotropía en las propiedades conductividad hidráulica y difusividad efectiva, k y D. 


\section{V.1.1 INFLUENCIA DEL MONOMIO $\pi_{\mathrm{d}, 1}=\left[(\Delta \rho) \mathrm{gk}_{\mathrm{y}} /\left(\varepsilon \mu \mathrm{vamb}^{*}\right)\right](\mathrm{L} / \mathrm{H})$}

Las Figuras V.1a-b y V.2a-b muestran los patrones de concentración y flujo, respectivamente, para el rango de valores de $\pi_{\mathrm{d}, 1}$ en torno a $7.483, \pi_{\mathrm{d}, 1}=1,3,5.5,6.5$, 7.58, 8.5, 11 y 14. Basta para ello modificar el parámetro $\mu$ que sólo aparece en dicho monomio. La Tabla V.1 muestra el conjunto de casos.

El resto de los monomios se mantienen en los valores de Henry: $\pi_{\mathrm{d}, 3}=0.05 \mathrm{y} \pi_{\mathrm{d}, 2}$ $=\pi_{\mathrm{d}, 4}=0.25$. Las desviaciones por encima y por debajo de su valor para el problema de Henry, 7.483, suponen aumentar o disminuir, respectivamente, la influencia (ya de por sí elevada) de la velocidad de Darcy frente a la velocidad regional. Como en todos los casos los efectos difusivos son casi despreciables frente a los efectos de la velocidad regional (merced al bajo valor de $\pi_{\mathrm{d}, 3}$ ). Sin embargo, un aumento de $\pi_{\mathrm{d}, 1}$ por disminución, por ejemplo, de $v_{a m b}$, tiende a anular tanto los efectos del flujo regional frente al de Darcy (de acuerdo con el cambio del monomio $\pi_{\mathrm{d}, 1}$ ) como los difusivos (por el cambio del monomio $\pi_{\mathrm{d}, 3}$ ); del mismo modo, una disminución de $\pi_{\mathrm{d}, 1}$ por aumento de $\mathrm{v}_{\mathrm{amb}}$, tiende a incrementar tanto los efectos del flujo regional como los difusivos.

Tabla V.1 Casos para la influencia de un cambio de valor de $\pi_{\mathrm{d}, 1}$

\begin{tabular}{|c|c|c|c|c|c|}
\hline CASO & $\mu$ & $\pi_{\delta, 1}$ & $\pi_{\delta, 2}$ & $\pi_{\delta, 3}$ & $\boldsymbol{\pi}_{\delta, 4}$ \\
\hline $\mathbf{1}$ & 0,0076 & $\mathbf{1}$ & 0,25 & 0,05 & 0,25 \\
\hline $\mathbf{2}$ & 0,0025 & $\mathbf{3}$ & 0,25 & 0,05 & 0,25 \\
\hline $\mathbf{3}$ & 0,0014 & $\mathbf{5 , 5}$ & 0,25 & 0,05 & 0,25 \\
\hline $\mathbf{4}$ & 0,0012 & $\mathbf{6 , 5}$ & 0,25 & 0,05 & 0,25 \\
\hline $\mathbf{5}$ & $\mathbf{0 , 0 0 1}$ & $\mathbf{7 , 5 8}$ & $\mathbf{0 , 2 5}$ & $\mathbf{0 , 0 5}$ & $\mathbf{0 , 2 5}$ \\
\hline $\mathbf{6}$ & 0,0009 & $\mathbf{8 , 5}$ & 0,25 & 0,05 & 0,25 \\
\hline $\mathbf{7}$ & 0,0007 & $\mathbf{1 1}$ & 0,25 & 0,05 & 0,25 \\
\hline $\mathbf{8}$ & 0,0005 & $\mathbf{1 4}$ & 0,25 & 0,05 & 0,25 \\
\hline
\end{tabular}


Capítulo V. Influencia de los grupos discriminados en los patrones del problema de Henry
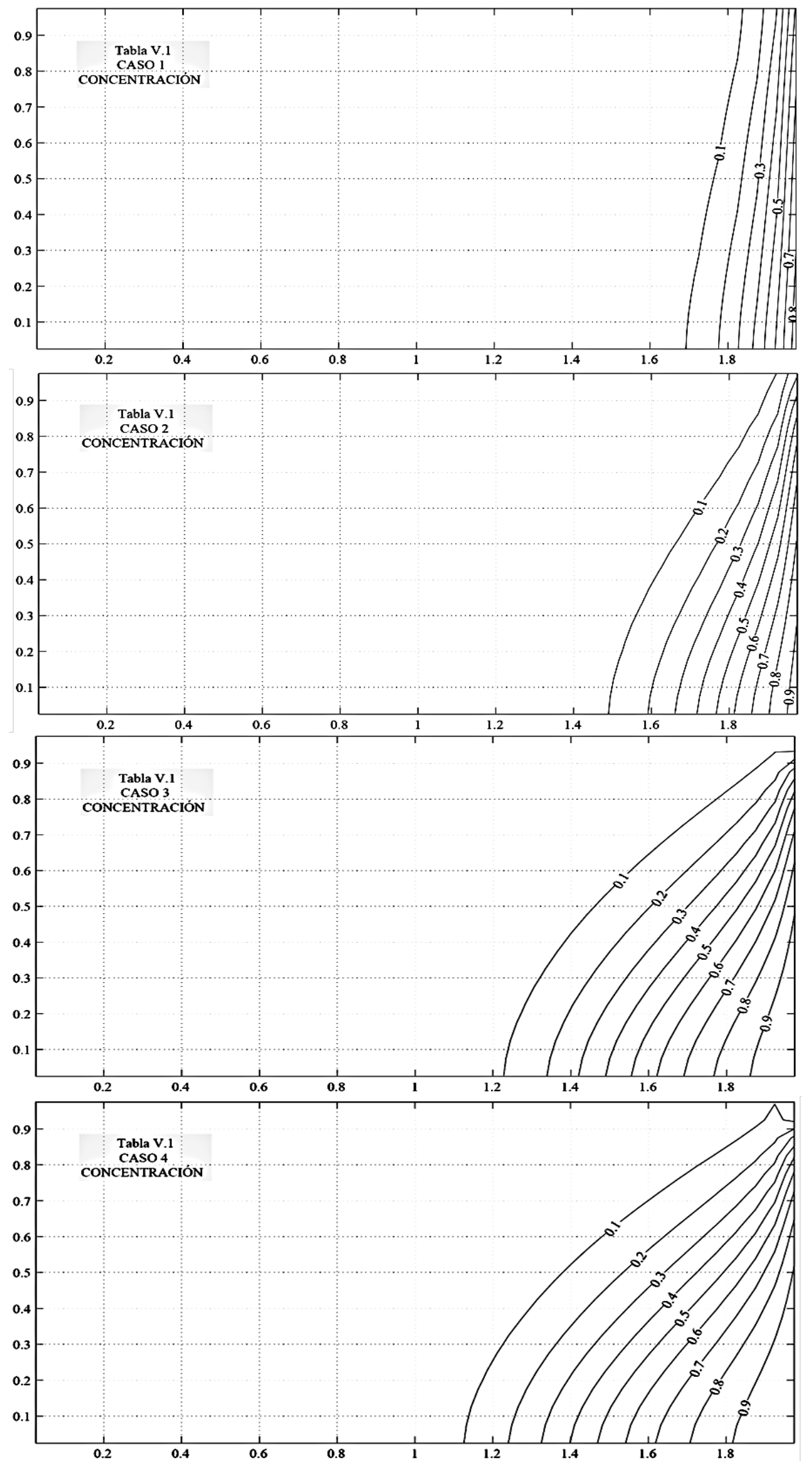

Figura V.1a Patrones de concentración. $\pi_{\mathrm{d}, 1}: 1$, 3, 5.5, 6.5, 7.58, 8.5, 11 y 14 (de arriba abajo), $\pi_{\mathrm{d}, 3}=0.05, \pi_{\mathrm{d}, 2}=\pi_{\mathrm{d}, 4}=0.25$ 
Capítulo V. Influencia de los grupos discriminados en los patrones del problema de Henry
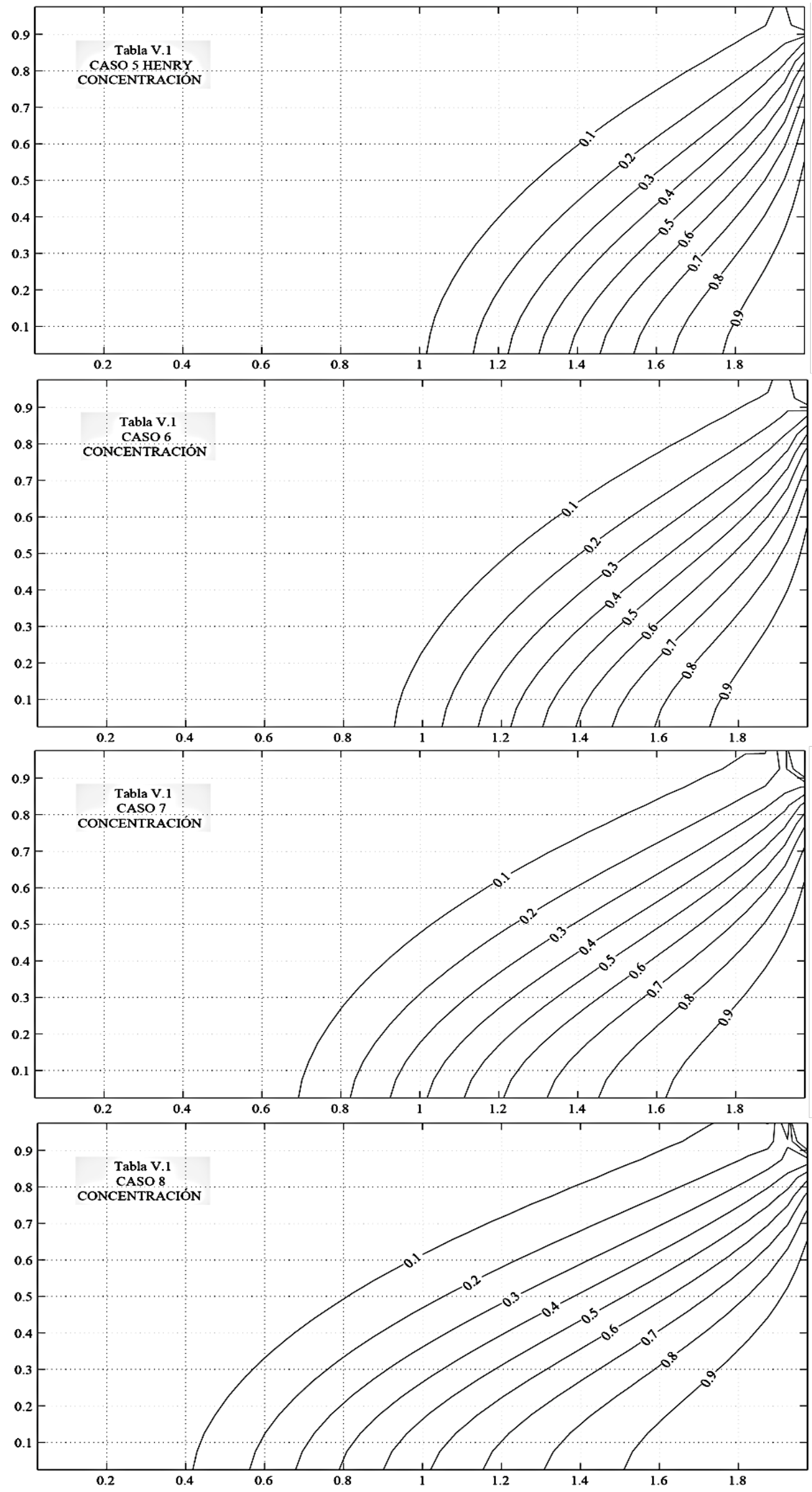

Figura V.1b Patrones de concentración. $\pi_{\mathrm{d}, 1}:$ 1, 3, 5.5, 6.5, 7.58, 8.5, 11 y 14 (de arriba abajo), $\pi_{\mathrm{d}, 3}=0.05, \pi_{\mathrm{d}, 2}=\pi_{\mathrm{d}, 4}=0.25$ 
En referencia a la región de gradiente apreciable de concentración, Figuras V.2ab, una visión global de estas figuras ilustra con claridad la evolución de esta región. Valores de $\pi_{\mathrm{d}, 1}$ pequeños en relación con el problema patrón $\left(\pi_{\mathrm{d}, 1}=7.58\right)$ retraen la cuña hacia el lado del mar, y la estrechan haciéndola más vertical merced a la menor influencia de los efectos del cambio de densidad frente a los debidos al flujo regional. A aumentar paulatinamente el valor de $\pi_{\mathrm{d}, 1}$ a partir de la unidad y hasta llegar al valor de Henry, la cuña se acuesta y se ensancha por la base; sin embargo, la parte superior de ella, presionada por el flujo de agua dulce en su salida hacia el mar, contrae la cuña en torno al borde marino superior.

Por razones similares, para valores de $\pi_{\mathrm{d}, 1}$ superiores al del patrón $\left(\pi_{\mathrm{d}, 1}=7.58\right)$ la cuña continúa ensanchándose en su base hasta cubrir una buena parte del fondo, manteniendo obviamente la misma estructura de contracción en la región de descarga de agua dulce. De acuerdo con las conclusiones de los capítulos anteriores, cuñas muy extensas en su base manifiestan una posible influencia de la extensión del dominio (L) en el patrón por imposición de la condición de salinidad nula en la región de entrada de agua dulce.

Es interesante apuntar, en estas figuras la emergencia de una región acuñada de elevada salinidad en la zona inferior derecha del dominio, cuya extensión crece con $\pi_{\mathrm{d}, 1}$ (flujos regionales despreciables frente a los efecto de flotación). También, hacer notar la forma alargada del patrón de concentraciones para grandes valores de $\pi_{\mathrm{d}, 1}$, un patrón que parece asemejarse a los casos reales.

En cuanto al patrón de flujo, Figuras V.2a-b, se aprecia que la divisoria de aguas, en particular su intersección con la base del dominio, inexistente para valores bajos de $\pi_{\mathrm{d}, 1}$ (muy poca difusión y elevado flujo regional), emerge progresivamente con el aumento de este monomio hasta sobrepasar la posición que ocupa en el problema patrón y llegar a alcanzar la casi totalidad de la base.

Parece existir una casi concordancia entre la extensión de la base de la cuña salina y la intersección de la divisoria con el fondo del dominio, pero también se muestra cierta independencia que merecería ser estudiada entre las áreas de la cuña salina y de la región del dominio a la derecha de la divisoria. También se manifiesta la emergencia de agua de cierta concentración salina más allá (por encima) de la divisoria. 
Capítulo V. Influencia de los grupos discriminados en los patrones del problema de Henry
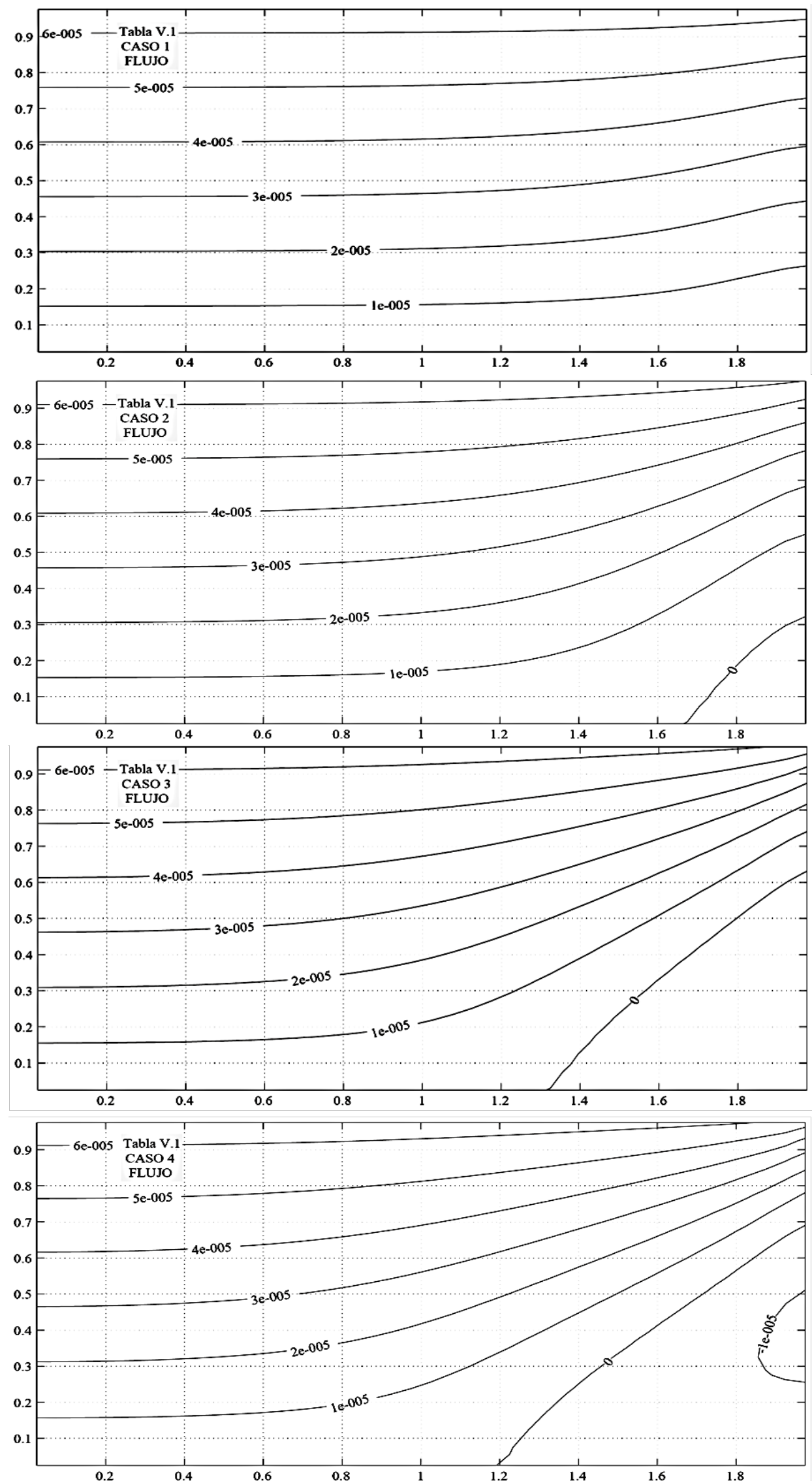

Figura V.2a Patrones de flujo. $\pi_{\mathrm{d}, 1}: \pi_{\mathrm{d}, 1}: 1,3,5.5,6.5,7.58,8.5,11$ y 14 (de arriba abajo), $\pi_{\mathrm{d}, 3}=0.05, \pi_{\mathrm{d}, 2}=\pi_{\mathrm{d}, 4}=0.25$ 
Capítulo V. Influencia de los grupos discriminados en los patrones del problema de Henry
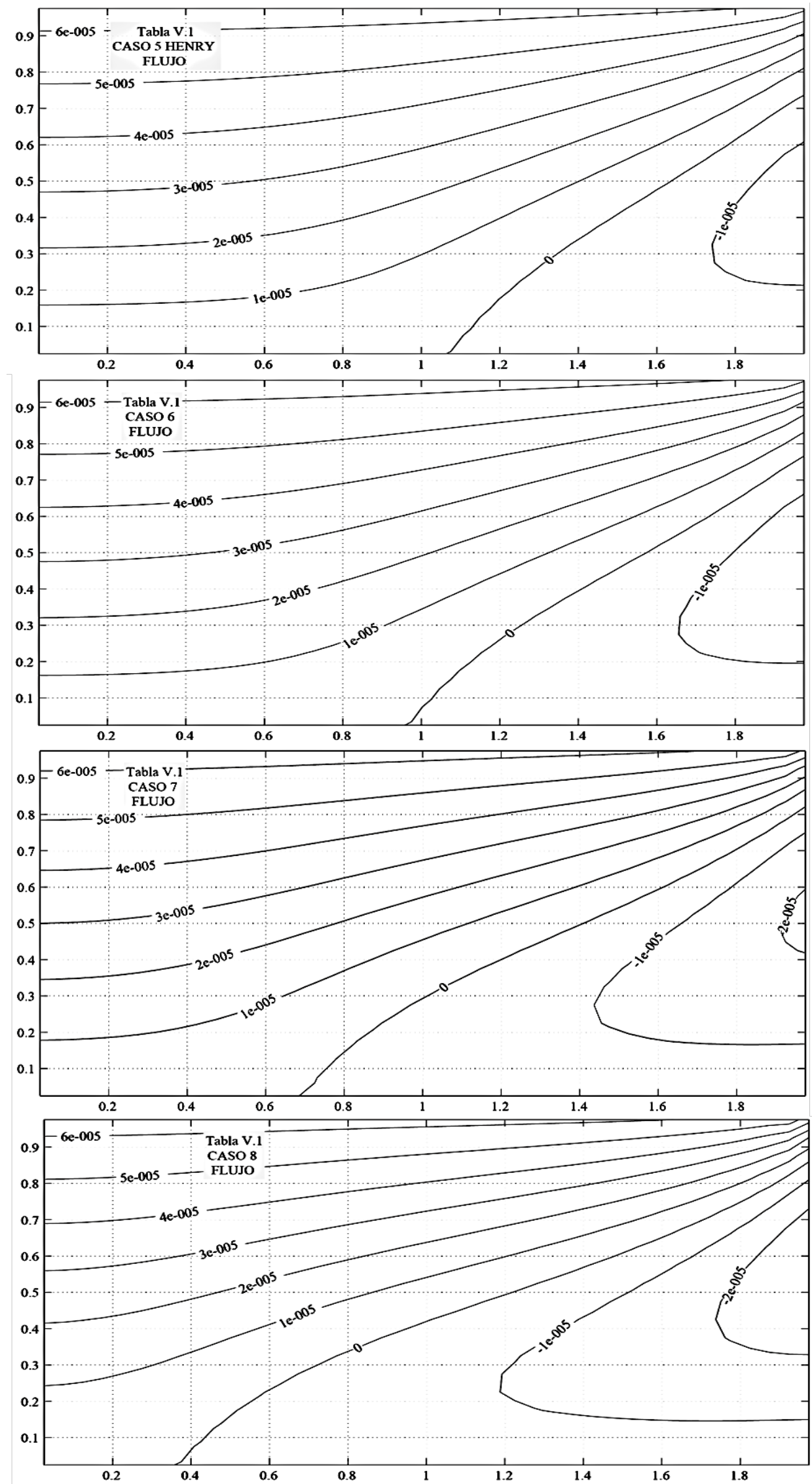

Figura V.2b Patrones de flujo. $\pi_{\mathrm{d}, 1}: \pi_{\mathrm{d}, 1}: 1,3$, 5.5, 6.5, 7.58, 8.5, 11 y 14 (de arriba abajo), $\pi_{\mathrm{d}, 3}=0.05, \pi_{\mathrm{d}, 2}=\pi_{\mathrm{d}, 4}=0.25$ 
En la búsqueda de patrones reales, más parecidos a los que proporcionan valores de $\pi_{\mathrm{d}, 1}$ por encima del problema patrón, Henry debería haber optado por valores superiores de este parámetro. Quizás no lo hizo por la falta de un conocimiento más detallado de estos patrones reales.

\section{V.1.2 INFLUENCIA DEL MONOMIO $\pi_{\mathrm{d}, 3}=\mathrm{D}_{\mathrm{x}} /\left(\mathrm{Vamb}^{*} \mathrm{~L}\right)$}

Un aumento del monomio $\pi_{\mathrm{d}, 3}$ supone incrementar los efectos de difusividad frente a los causados por el flujo regional, que no llegarían a ser comparables hasta valores de este monomio cercanos a la unidad. Por ello, el rango de valores de este monomio se ha confinado al intervalo [0.02, 5]. Valores por debajo de 0.05 (de Henry), hacen aún más despreciables los efectos difusivos. Por otro lado, los efectos de flotación causados por la velocidad de Darcy preponderan siempre sobre los efectos del flujo regional (merced al alto valor de $\pi_{\mathrm{d}, 1}$ ) y sólo son comparables a los difusivos para valores $\pi_{\mathrm{d}, 3}$ cercanos a 7.58 , el valor de $\pi_{\mathrm{d}, 1}$ de Henry. La Tabla V.2 muestra la relación de casos elegidos para estudiar esta influencia, $\pi_{\mathrm{d}, 3}=0.02,0.05,0.1,0.2,0.3,0.5,1, \mathrm{y} 2$. Para cada caso se ha modificado la difusividad, $\mathrm{D}_{\mathrm{x}}$, corrigiendo convenientemente $\mathrm{D}_{\mathrm{y}}$ para mantener constante el monomio $\pi_{\mathrm{d}, 4}$. Los monomios restantes mantienen los valores $\pi_{\mathrm{d}, 1}=7.583, \pi_{\mathrm{d}, 2}=\pi_{\mathrm{d}, 4}=0.25$.

Tabla V.2 Casos para la influencia de un cambio de valor de $\pi_{\mathrm{d}, 3}$

\begin{tabular}{|c|c|c|c|c|c|c|}
\hline Caso & $\mathbf{D}_{\mathbf{x}}$ & $\mathbf{D}_{\mathbf{y}}$ & $\boldsymbol{\pi}_{\delta, \mathbf{3}}$ & $\boldsymbol{\pi}_{\delta, 1}$ & $\boldsymbol{\pi}_{\delta, 2}$ & $\boldsymbol{\pi}_{\delta, 4}$ \\
\hline $\mathbf{1}$ & $7,54 \mathrm{E}-06$ & $7,54 \mathrm{E}-06$ & $\mathbf{0 , 0 2}$ & 7,58 & 0,25 & 0,25 \\
\hline $\mathbf{2}$ & $\mathbf{1 , 8 9 \mathrm { E } - 0 5}$ & $\mathbf{1 , 8 9 \mathrm { E } - 0 5}$ & $\mathbf{0 , 0 5}$ & $\mathbf{7 , 5 8}$ & $\mathbf{0 , 2 5}$ & $\mathbf{0 , 2 5}$ \\
\hline $\mathbf{3}$ & $3,77 \mathrm{E}-05$ & $3,77 \mathrm{E}-05$ & $\mathbf{0 , 1}$ & 7,58 & 0,25 & 0,25 \\
\hline $\mathbf{4}$ & $7,54 \mathrm{E}-05$ & $7,54 \mathrm{E}-05$ & $\mathbf{0 , 2}$ & 7,58 & 0,25 & 0,25 \\
\hline $\mathbf{5}$ & $1,13 \mathrm{E}-04$ & $1,13 \mathrm{E}-04$ & $\mathbf{0 , 3}$ & 7,58 & 0,25 & 0,25 \\
\hline $\mathbf{6}$ & $1,89 \mathrm{E}-04$ & $1,89 \mathrm{E}-04$ & $\mathbf{0 , 5}$ & 7,58 & 0,25 & 0,25 \\
\hline $\mathbf{7}$ & $3,77 \mathrm{E}-04$ & $3,77 \mathrm{E}-04$ & $\mathbf{1}$ & 7,58 & 0,25 & 0,25 \\
\hline $\mathbf{8}$ & $7,54 \mathrm{E}-04$ & $7,54 \mathrm{E}-04$ & $\mathbf{2}$ & 7,58 & 0,25 & 0,25 \\
\hline
\end{tabular}

Las Figuras V.3a-b y V.4a-b muestran los patrones de concentración y flujo, respectivamente, resultantes de la simulación con FATSIM. En ellas se aprecia una evolución regular de ambos patrones con el crecimiento del monomio $\pi_{\mathrm{d}, 3}$. 
Capítulo V. Influencia de los grupos discriminados en los patrones del problema de Henry
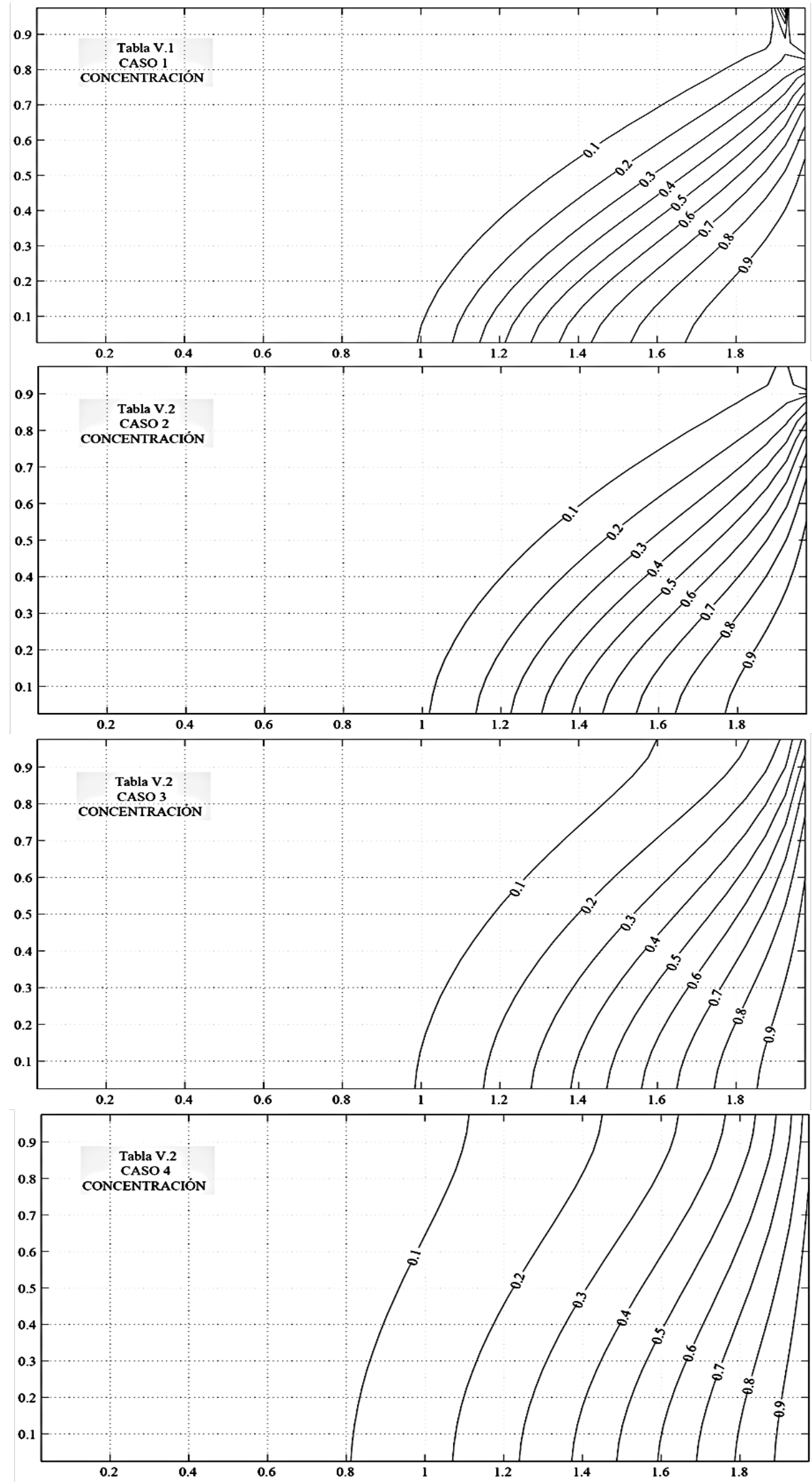

Figura V.3a Patrones de concentración. $\pi_{\mathrm{d}, 3}=0.02,0.05,0.1,0.2,0.3,0.5$, 1, y 2 (de arriba abajo), $\pi_{\mathrm{d}, 1}=7.58, \pi_{\mathrm{d}, 2}=\pi_{\mathrm{d}, 4}=0.25$ 
Capítulo V. Influencia de los grupos discriminados en los patrones del problema de Henry
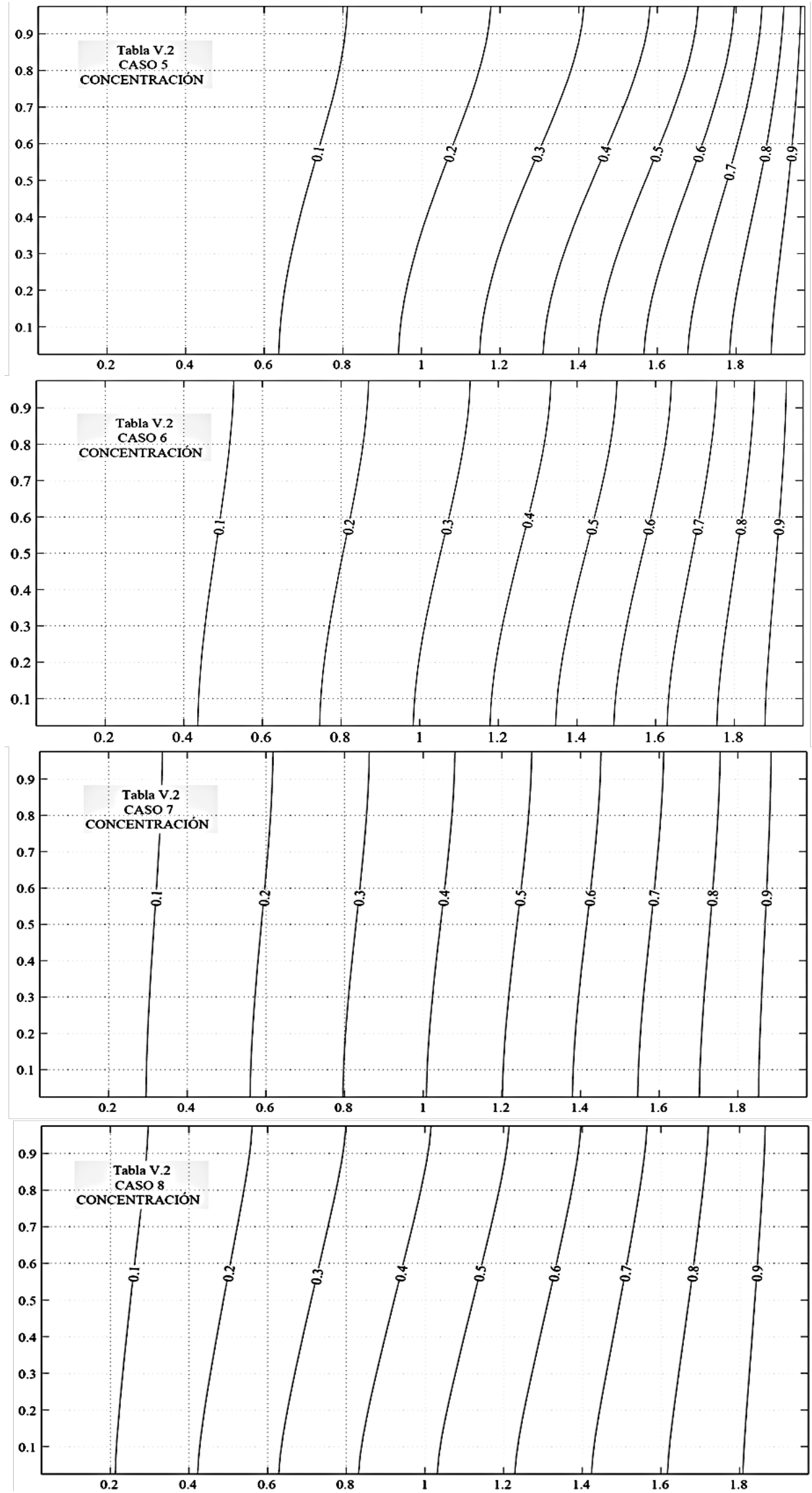

Figura V.3b Patrones de concentración. $\pi_{\mathrm{d}, 3}=0.02,0.05,0.1,0.2,0.3,0.5,1, \mathrm{y} 2$ (de arriba abajo), $\pi_{\mathrm{d}, 1}=7.58, \pi_{\mathrm{d}, 2}=\pi_{\mathrm{d}, 4}=0.25$ 
Capítulo V. Influencia de los grupos discriminados en los patrones del problema de Henry
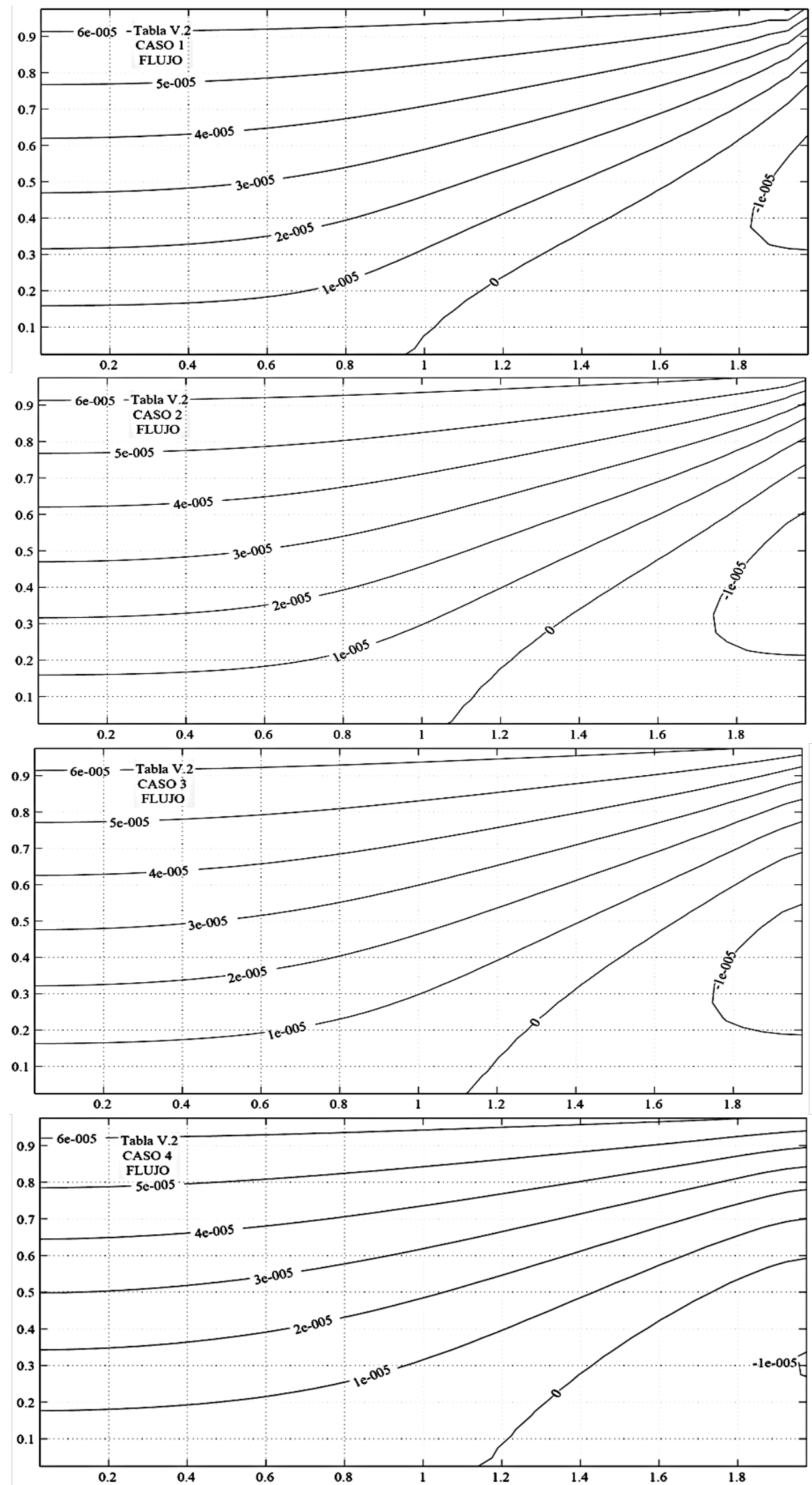

Figura V.4a Patrones de flujo. $\pi_{\mathrm{d}, 3}=0.02,0.05,0.1,0.2,0.3,0.5,1$, y 2 (de arriba abajo), $\pi_{\mathrm{d}, 1}=7.58, \pi_{\mathrm{d}, 2}=\pi_{\mathrm{d}, 4}=0.25$ 
Capítulo V. Influencia de los grupos discriminados en los patrones del problema de Henry
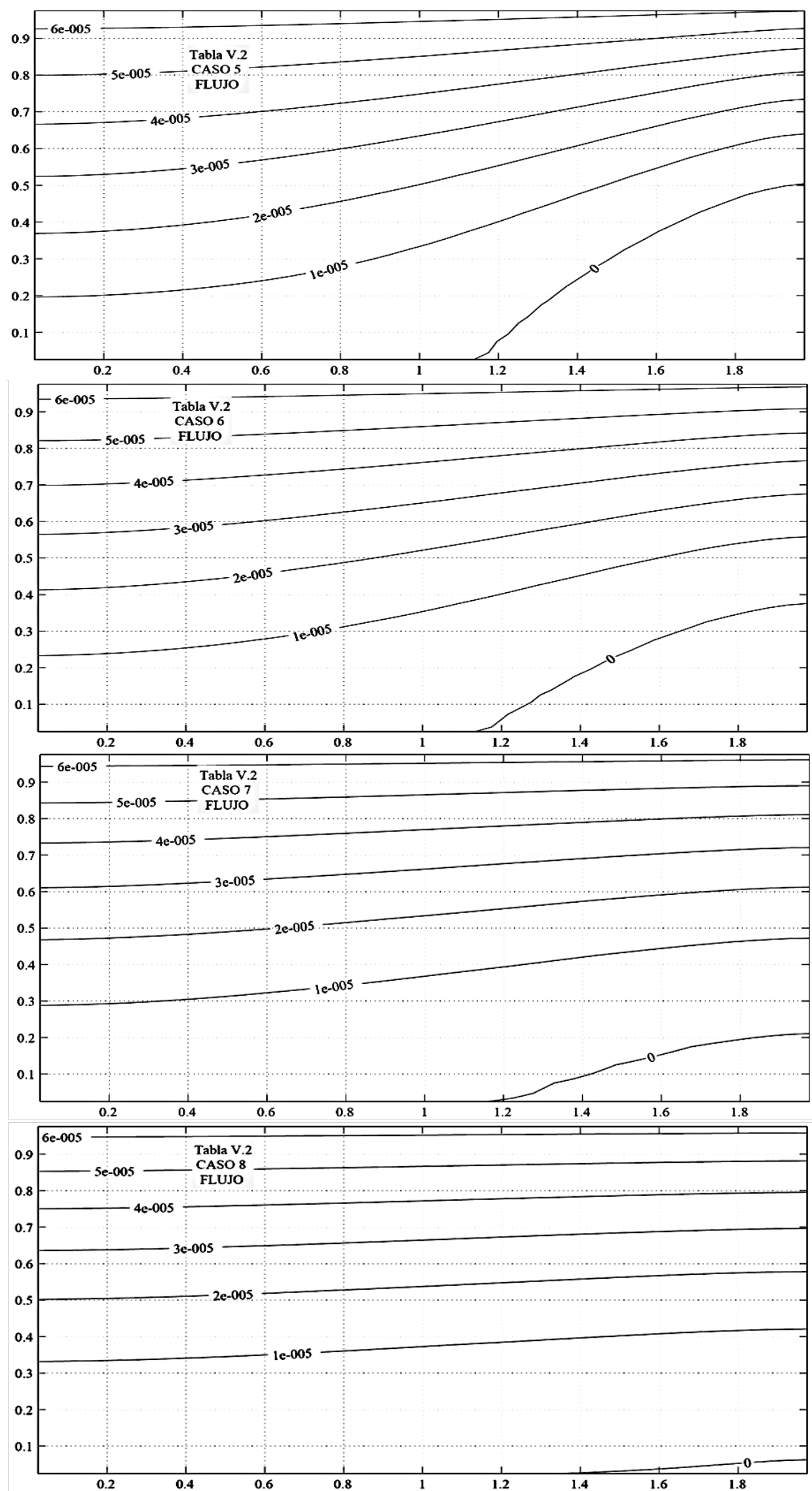

Figura V.4b Patrones de flujo. $\pi_{\mathrm{d}, 3}=0.02,0.05,0.1,0.2,0.3,0.5,1$, y 2 (de arriba abajo), $\pi_{\mathrm{d}, 1}=7.58, \pi_{\mathrm{d}, 2}=\pi_{\mathrm{d}, 4}=0.25$ 
Capítulo V. Influencia de los grupos discriminados en los patrones del problema de Henry

Como se esperaba, el patrón de salinidad, Figura V.3a-b, sufre pocas modificaciones para $\pi_{\mathrm{d}, 3}=0.05$ (el de Henry) y valores inferiores. $\mathrm{Al}$ aumentar $\pi_{\mathrm{d}, 3}$ hasta acercarse a la unidad, los patrones cambian sensiblemente alcanzando la salinidad todo el dominio. Dado que un aumento de $\pi_{\mathrm{d}, 3}$ significa una reducción de la influencia de la velocidad regional frente a la difusión, este cambio lleva aparejado también una mayor influencia del efecto de la flotación frente al empuje regional por lo que se justifica que las líneas de isoconcentración se vuelvan más verticales. Por otro lado, cabe mencionar que valores de $\pi_{\mathrm{d}, 3}$ cercanos a la unidad determinan un patrón en el que aprecia claramente que la longitud del dominio influye decisivamente en dicho patrón merced a la condición de contorno de concentración nula en la frontera izquierda del dominio.

En cuanto al patrón de flujo se puede apuntar dos comentarios importantes. En primer lugar, la existencia de una divisoria de aguas cuya posición apenas sufre cambios para valores pequeños de $\pi_{\mathrm{d}, 3}$, tan solo que su intersección con la frontera marina desciende ligeramente haciendo más angosta la zona de recirculación situada bajo dicha línea. La intersección de la línea divisoria con el fondo del acuífero apenas se modifica para valores bajos de $\pi_{\mathrm{d}, 3}$ pero se retrae hacia el más sensiblemente al acercarse el monomio al valor de la unidad. El mismo comentario cabe en relación con el caudal de recirculación bajo la divisoria, el cual apenas se modifica para valores de $\pi_{\mathrm{d}, 3}$ bastante inferiores a la unidad pero que tiende a cero al acercarse dicho monomio a este valor.

En segundo lugar, y esto puede ser importante desde el punto de vista de un análisis cualitativo del problema, existe un claro desacoplo entre la extensión (medida sobre el fondo del acuífero) de la zona salada resultante del patrón de concentraciones y la longitud que determina la línea divisoria (que marca la zona de recirculación) con la frontera inferior. Mientras que para los valores del problema patrón dicha relación es muy aproximadamente la unidad, al aumentar de $\pi_{\mathrm{d}, 3}$ la relación anterior crece (al principio muy lentamente, hasta valores de $\pi_{\mathrm{d}, 3} \approx 0.5$ ) hasta hacerse muy elevada. El desacoplo emergente entre las longitudes anteriores induce a reflexionar sobre la existencia de nuevos balances entre magnitudes asociadas a los efectos que se contraponen en estas subregiones. Las ecuaciones de gobierno podrían eventualmente simplificarse al ser aplicadas a estas subregiones, y su adimensionalización proporcionaría nuevos grupos que permitirían interpretar correctamente estos balances. Es un tema que dejamos para futuras investigaciones. 


\section{V.1.3 INFLUENCIA DEL MONOMIO $\pi_{\mathrm{d}, 2}=\left(\mathbf{k}_{\mathbf{x}} \mathbf{H}^{2}\right) /\left(\mathbf{k}_{\mathbf{y}} \mathbf{L}^{2}\right)$}

Un aumento (disminución) de este monomio discrimina la permeabilidad hidráulica horizontal frente a la vertical, incrementando (disminuyendo) el efecto de la primera. Sin embargo, al retener el valor de los restantes monomios, los efectos difusivos son despreciables frente a los causados por el flujo regional y estos últimos pequeños frente a los efectos de flotación. La difusividad mantiene su carácter isótropo.

Las Figuras V.5a-b y V.6a-b muestran los patrones de concentración y flujo, respectivamente, de este último caso. El rango de valores de $\pi_{\mathrm{d}, 2}$, en torno a 0.25 , es $\pi_{\mathrm{d}, 2}$ $=0.01,0.15,0.2,0.25,0.35,0.5,0.8, \mathrm{y} 1$, Tabla V.3, conseguido simplemente modificando el parámetro $\mathrm{k}_{\mathrm{x}}$. Los monomios restantes mantienen los valores $\pi_{\mathrm{d}, 1}=7.58$, $\pi_{\mathrm{d}, 3}=0.05$ y $\pi_{\mathrm{d}, 4}=0.25$

Tabla V.3 Casos para la influencia de un cambio de valor de $\pi_{\mathrm{d}, 2}$

\begin{tabular}{|c|c|c|c|c|c|}
\hline Caso & Kx & $\pi_{\delta, 2}$ & $\pi_{\delta, 1}$ & $\pi_{\delta, 3}$ & $\pi_{\delta, 4}$ \\
\hline 1 & $4,0816 \mathrm{E}-11$ & 0,01 & 7,58 & 0,05 & 0,25 \\
\hline 2 & $6,1224 \mathrm{E}-10$ & 0,15 & 7,58 & 0,05 & 0,25 \\
\hline 3 & $8,1632 \mathrm{E}-10$ & 0,2 & 7,58 & 0,05 & 0,25 \\
\hline 4 & $1,0204 \mathrm{E}-09$ & 0,25 & 7,58 & 0,05 & 0,25 \\
\hline 5 & $1,4286 \mathrm{E}-09$ & 0,35 & 7,58 & 0,05 & 0,25 \\
\hline 6 & $2,0408 \mathrm{E}-09$ & 0,5 & 7,58 & 0,05 & 0,25 \\
\hline 7 & $3,2653 \mathrm{E}-09$ & 0,8 & 7,58 & 0,05 & 0,25 \\
\hline 8 & $4,0816 \mathrm{E}-09$ & 1 & 7,58 & 0,05 & 0,25 \\
\hline
\end{tabular}

La influencia de este monomio en el patrón de salinidad es importante tanto cualitativa como cuantitativamente. Su valor, a partir de 0,01 (un valor ya despreciable, poco influyente en los patrones) ensancha y acuesta la región entre el agua casi dulce y el agua casi salada, produciendo patrones que ciertamente reflejan los que se dan en acuíferos reales. Cabe mencionar la influencia de $L$ en los patrones para valores de $\pi_{\mathrm{d}, 2}$ cercanos a la unidad pues las isolíneas de menor valor tocan la frontera inferior del acuífero en la cual se ha impuesto la condición de concentración nula que impide que dichas líneas intersecten la mencionada frontera. 
Capítulo V. Influencia de los grupos discriminados en los patrones del problema de Henry
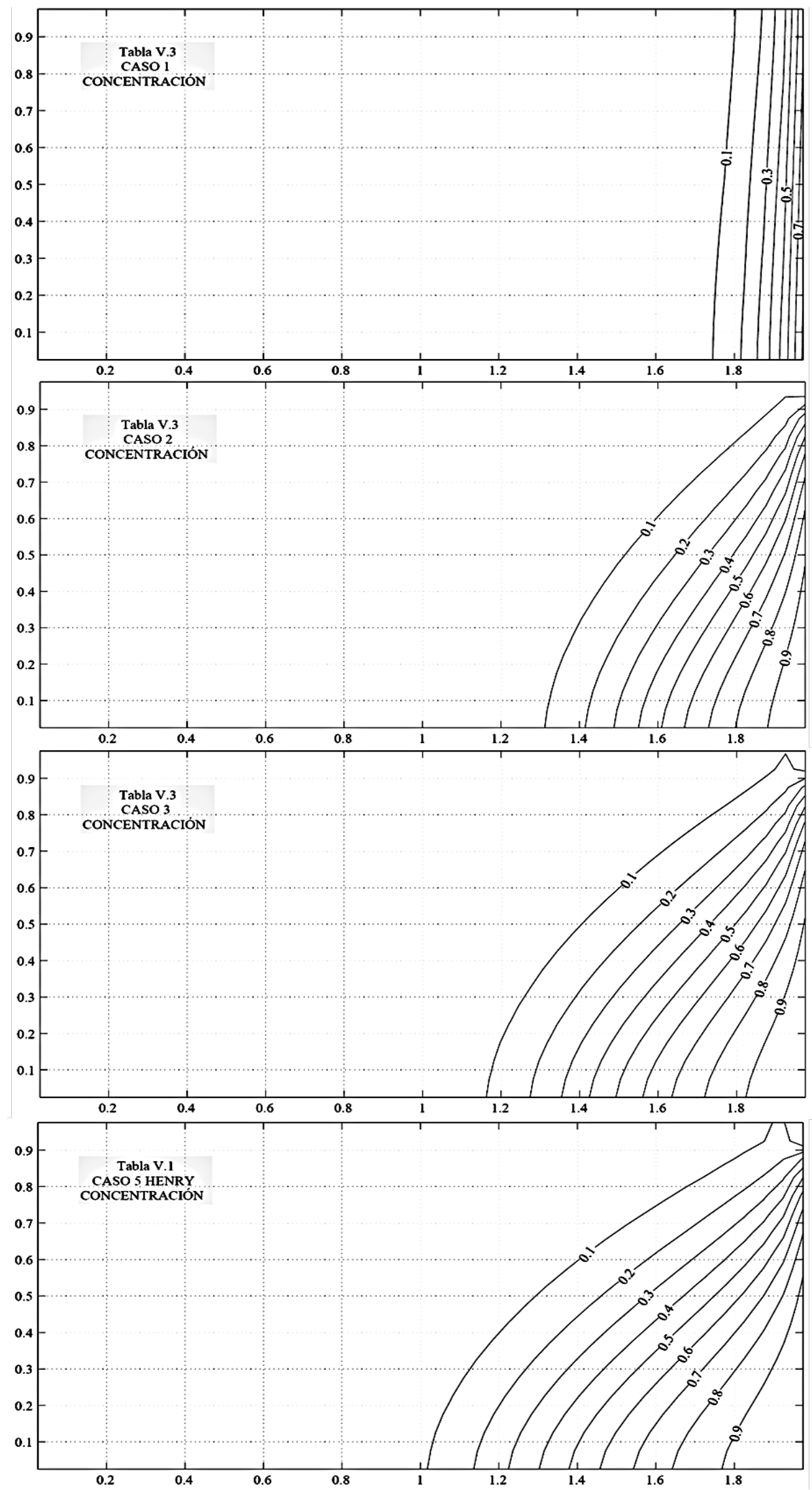

Figura V.5a Patrones de concentración. $\pi_{\mathrm{d}, 2}=0.01,0.15,0.2,0.25,0.35,0.5,0.8$, y 1 (de arriba abajo), $\pi_{\mathrm{d}, 1}=7.58, \pi_{\mathrm{d}, 3}=0.05$ y $\pi_{\mathrm{d}, 4}=0.25$ 
Capítulo V. Influencia de los grupos discriminados en los patrones del problema de Henry
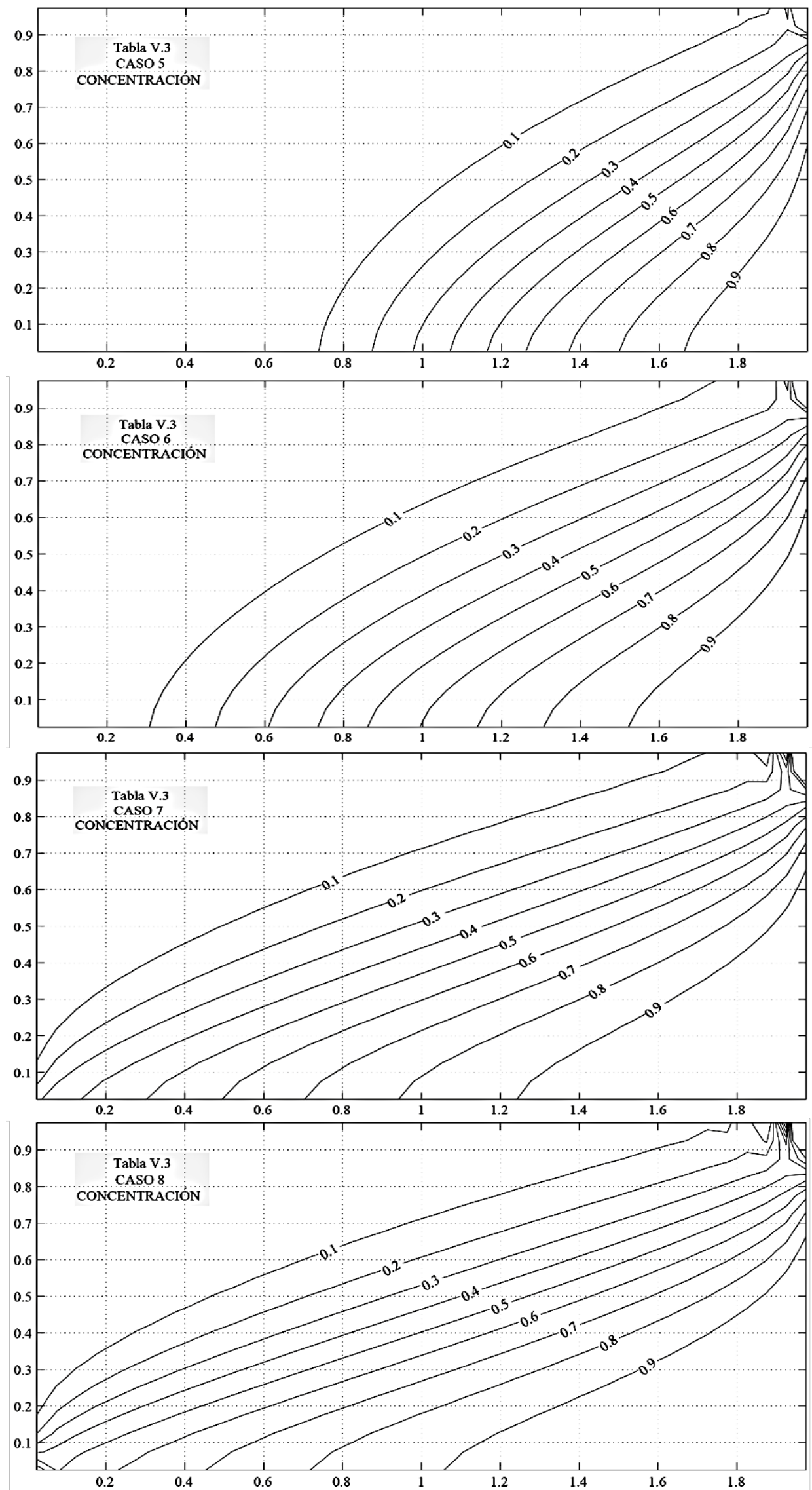

Figura V.5b Patrones de concentración. $\pi_{\mathrm{d}, 2}=0.01,0.15,0.2,0.25,0.35,0.5,0.8$, y 1 (de arriba abajo), $\pi_{\mathrm{d}, 1}=7.58, \pi_{\mathrm{d}, 3}=0.05 \mathrm{y} \pi_{\mathrm{d}, 4}=0.25$ 
Capítulo V. Influencia de los grupos discriminados en los patrones del problema de Henry
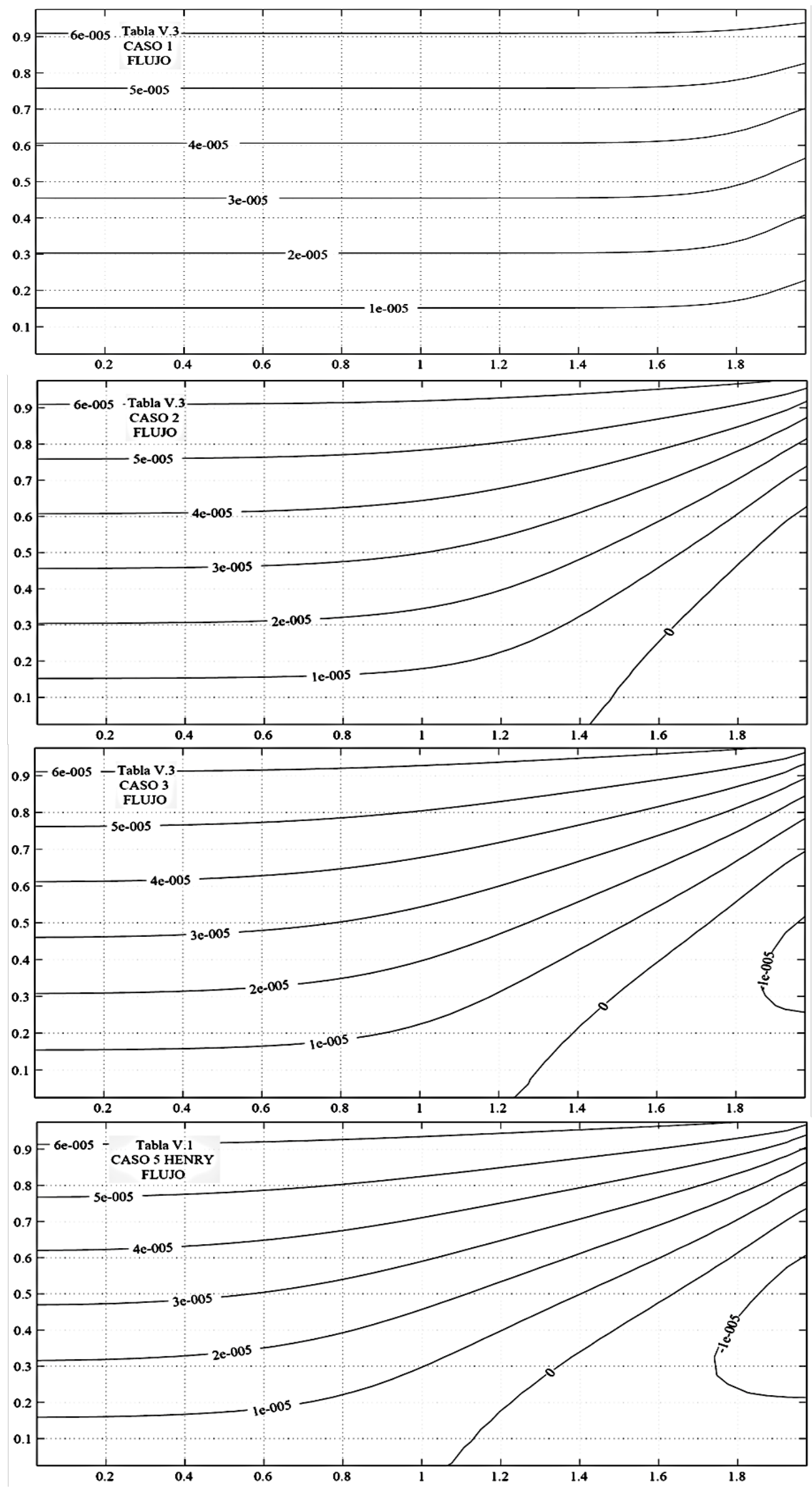

Figura V.6a Patrones de flujo. $\pi_{\mathrm{d}, 2}=0.01,0.15,0.2,0.25,0.35,0.5,0.8, \mathrm{y} 1$ (de arriba abajo), $\pi_{\mathrm{d}, 1}=7.58, \pi_{\mathrm{d}, 3}=0.05 \mathrm{y} \pi_{\mathrm{d}, 4}=0.25$ 
Capítulo V. Influencia de los grupos discriminados en los patrones del problema de Henry
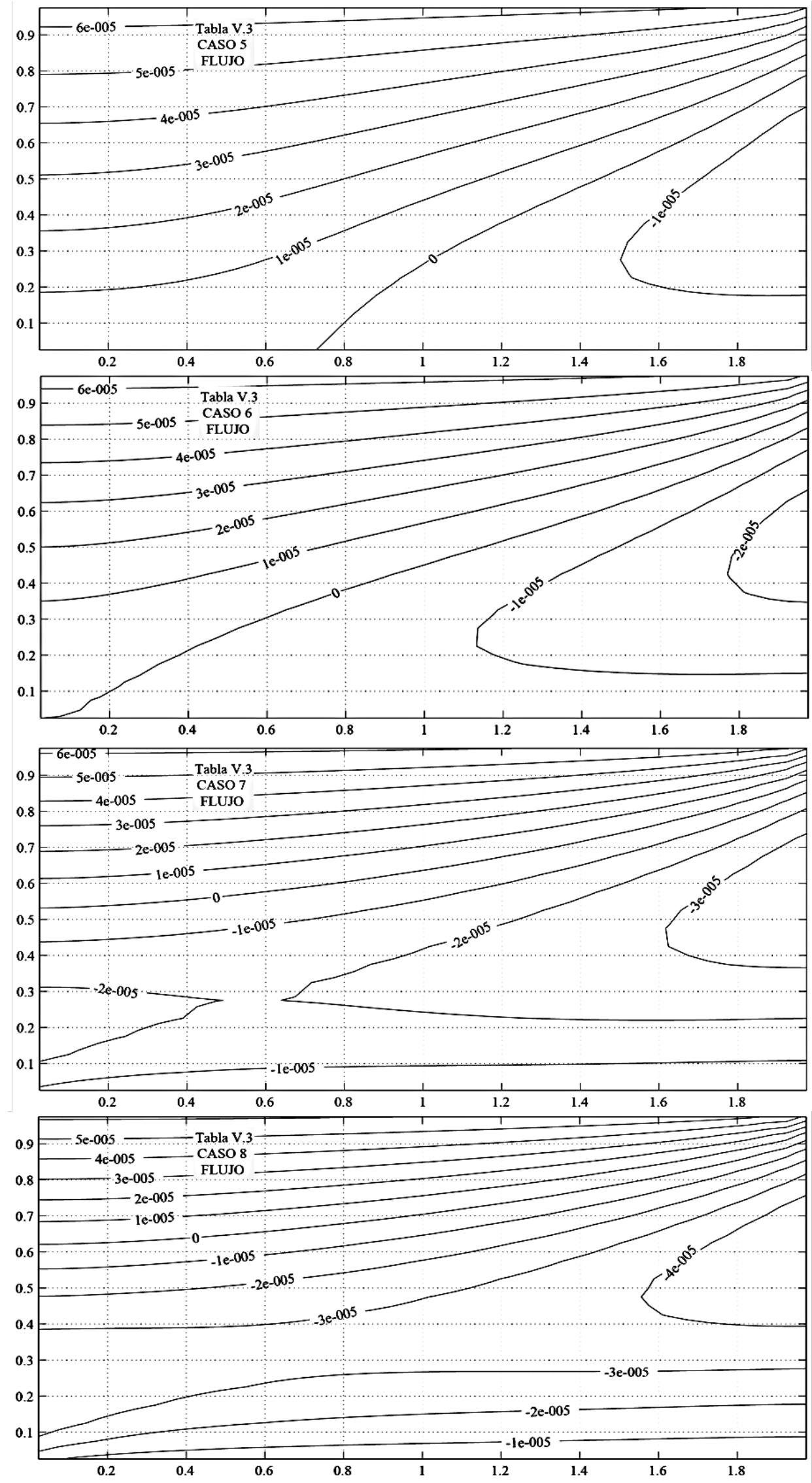

Figura V.6b Patrones de flujo. $\pi_{\mathrm{d}, 2}=0.01,0.15,0.2,0.25,0.35,0.5,0.8, \mathrm{y} 1$ (de arriba abajo), $\pi_{\mathrm{d}, 1}=7.58, \pi_{\mathrm{d}, 3}=0.05 \mathrm{y} \pi_{\mathrm{d}, 4}=0.25$ 
Por otro lado, la emergencia de un subdominio saturado de agua de máxima salinidad dentro del acuífero, en su borde inferior derecho, puede explicarse merced al aumento de $\mathrm{k}_{\mathrm{x}}$ frente a $\mathrm{k}_{\mathrm{y}}$. De este modo, el agua que penetra en el acuífero procedente del mar por su frontera inferior está saturada de sal, y no baja la concentración hasta que alcanza zonas menos saladas en donde emergen fenómenos de flotación que cambian su salinidad.

El patrón de flujo sigue una solución que puede decirse esperada para valores de $\pi_{\mathrm{d}, 2}$ bajos, definiendo una divisoria que prácticamente coincide con el borde de la frontera agua dulce-agua salada. Pero para valores cercanos a la unidad, quizás debido a la influencia de la longitud del dominio (L), la línea divisoria penetra la frontera continental y da lugar a recirculaciones de agua dulce y agua salada en las dos fronteras verticales, zona continental y zona marina, respectivamente. Bajo estas recirculaciones existe una región de circulación inversa de agua, del mar hacia el continente, poco real.

\section{V.1.4 INFLUENCIA DEL MONOMIO $\pi_{\mathrm{d}, 4}=\left(\mathrm{D}_{\mathrm{x}} \mathrm{H}^{2}\right) /\left(\mathrm{D}_{\mathrm{y}} \mathrm{L}^{2}\right)$}

Un aumento de este monomio discrimina la difusividad efectiva horizontal frente a la vertical, incrementando el efecto de la primera. Sin embargo, al retener el valor de los restantes monomios, los efectos difusivos son despreciables frente a los causados por el flujo regional y estos últimos pequeños frente a los efectos de flotación, todo ello debido a los valores particulares de los otros monomios, que son los de Henry. La conductividad hidráulica mantiene su carácter isótropo (lo que aleja la influencia estudiada, en cierto modo, de escenarios reales). Las Figuras V.7a-b y V.8a-b muestran los patrones de concentración y flujo, respectivamente, para el rango de valores de $\pi_{\mathrm{d}, 3}$ en torno a $0.25, \pi_{\mathrm{d}, 4}=0.01,0.15,0.2,0.25,0.3,0.5,0.8$ y 2 , conseguido simplemente modificando el parámetro $D_{\mathrm{y}}$. Los monomios restantes mantienen los valores $\pi_{\mathrm{d}, 1}=7.58$, $\pi_{\mathrm{d}, 3}=0.05 \mathrm{y} \pi_{\mathrm{d}, 2}=0.25$. La Tabla V.4 muestra el conjunto de casos.

Los patrones de salinidad, Figuras V.7a-b, dan lugar a dos comentarios. Por un lado, el aumento de $\pi_{\mathrm{d}, 4}$, partiendo de su valor más bajo, produce cuñas de difusión cuya base se expande paulatina, pero no acusadamente, sobre la frontera inferior. Es, sin duda, una consecuencia del aumento de $\mathrm{D}_{\mathrm{x}}$ frente a $\mathrm{D}_{\mathrm{y}}$. Por otro, el aumento también en extensión, medida sobre la base del acuífero, de una región completamente salada lindante con el mar, también consecuencia del aumento de $\mathrm{D}_{\mathrm{x}}$ frente a $\mathrm{D}_{\mathrm{y}}$. 
Tabla V.4 Casos para la influencia de un cambio de valor de $\pi_{\mathrm{d}, 4}$

\begin{tabular}{|c|c|c|c|c|c|}
\hline Caso & $D_{y}$ & $\pi_{\delta, 4}$ & $\pi_{\delta, 1}$ & $\pi_{\delta, 2}$ & $\pi_{\delta, 3}$ \\
\hline 1 & $4,7143 \mathrm{E}-04$ & 0,01 & 7,58 & 0,25 & 0,05 \\
\hline 2 & $3,1428 \mathrm{E}-05$ & 0,15 & 7,58 & 0,25 & 0,05 \\
\hline 3 & $2,3571 \mathrm{E}-05$ & 0,2 & 7,58 & 0,25 & 0,05 \\
\hline 4 & 1,8857E-05 & 0,25 & 7,58 & 0,25 & 0,05 \\
\hline 5 & $1,5714 \mathrm{E}-05$ & 0,3 & 7,58 & 0,25 & 0,05 \\
\hline 6 & $9,4285 \mathrm{E}-06$ & 0,5 & 7,58 & 0,25 & 0,05 \\
\hline 7 & $5,8928 \mathrm{E}-06$ & 0,8 & 7,58 & 0,25 & 0,05 \\
\hline 8 & $2,3571 \mathrm{E}-06$ & 2 & 7,58 & 0,25 & 0,05 \\
\hline
\end{tabular}

Adviértase que mantener constante el resto de los monomios lleva aparejado un aumento de la velocidad regional (para mantener el valor de $\pi_{\mathrm{d}, 3}$ ) y un aumento de las fuerza de flotación (para mantener $\pi_{\mathrm{d}, 1}$ ), sin embargo, el incremento de estos efectos no compensa el de $D_{x}$ frente a $D_{y}$ y las cuñas de salinización se extienden y acuestan sobre el dominio.

En relación con los patrones de flujo, Figuras V.8a-b, puede apuntarse varios comentarios. En primer lugar, el lento pero monótono crecimiento de la extensión de la línea divisoria (medida sobre la base del acuífero y hasta el borde marino). Esta longitud es sólo parecida en extensión a la de la cuña salina (longitud, medida sobre la base del acuífero, desde la línea de isoconcentración 0.1 hasta la frontera del mar, lo que incluye la región de acuífero completamente salada). En segundo lugar, mientras que los patrones de isolíneas situados por encima de la divisoria son prácticamente idénticos, los de isolíneas por debajo de ésta cambian progresivamente (cuantitativamente) de forma que el caudal de recirculación de agua de mar disminuye sensiblemente al incrementarse el monomio $\pi_{\mathrm{d}, 4}$. En particular, para $\pi_{\mathrm{d}, 4}=1$, la línea divisoria ha sufrido el máximo desplazamiento hacia la derecha del dominio mientras que el caudal de recirculación que encierra bajo ella es prácticamente despreciable (véase que no aparece la isolínea de función de corriente 1E-5 en el patrón de $\pi_{\mathrm{d}, 4}=1$, Figura V.8b). 
Capítulo V. Influencia de los grupos discriminados en los patrones del problema de Henry
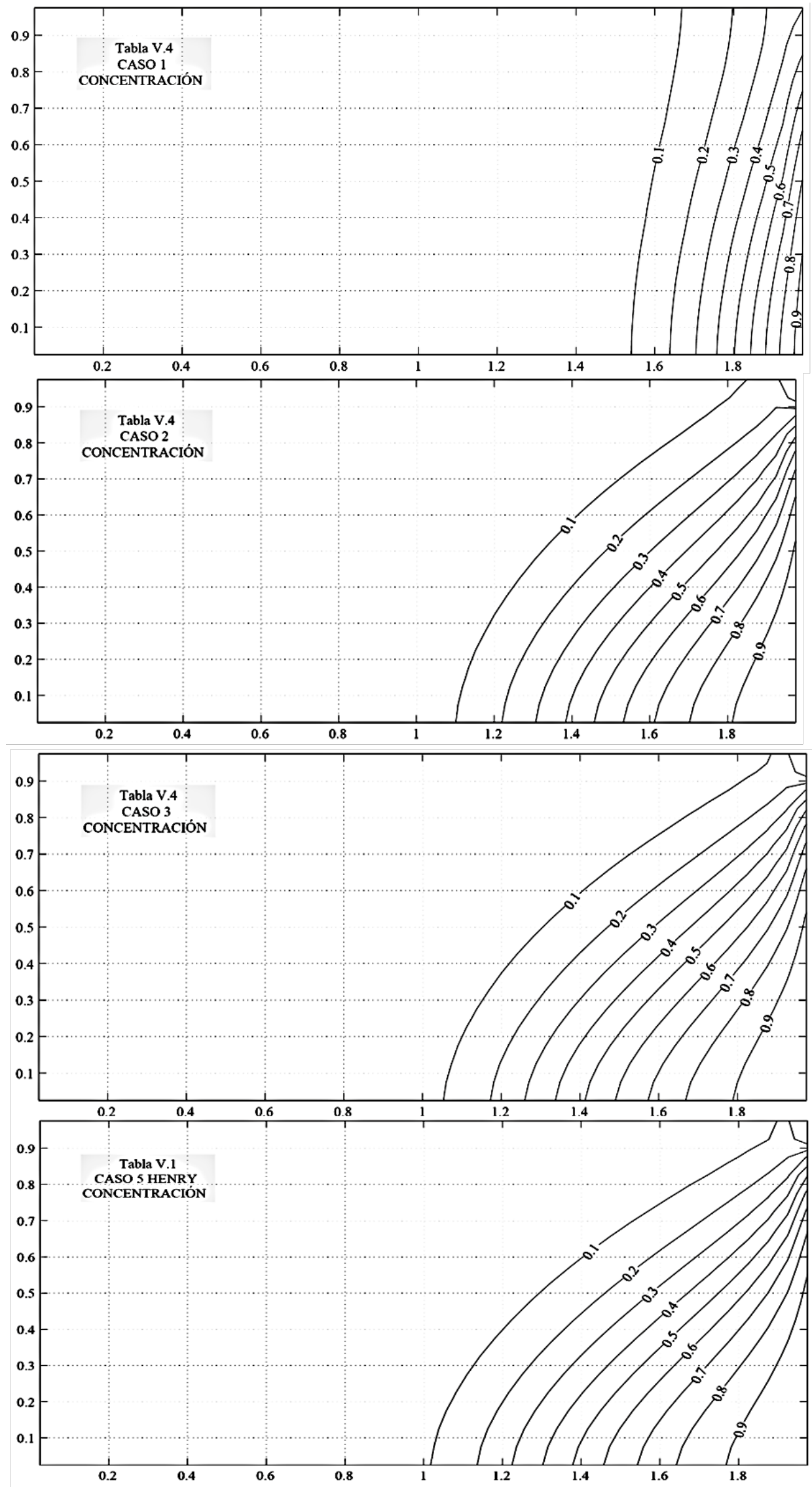

Figura V.7a Patrones de concentración. $\pi_{\mathrm{d}, 4}=0.01,0.15,0.2,0.25,0.3,0.5,0.8$ y 2 (de arriba abajo), $\pi_{\mathrm{d}, 1}=7.58, \pi_{\mathrm{d}, 3}=0.05$ y $\pi_{\mathrm{d}, 2}=0.25$ 
Capítulo V. Influencia de los grupos discriminados en los patrones del problema de Henry
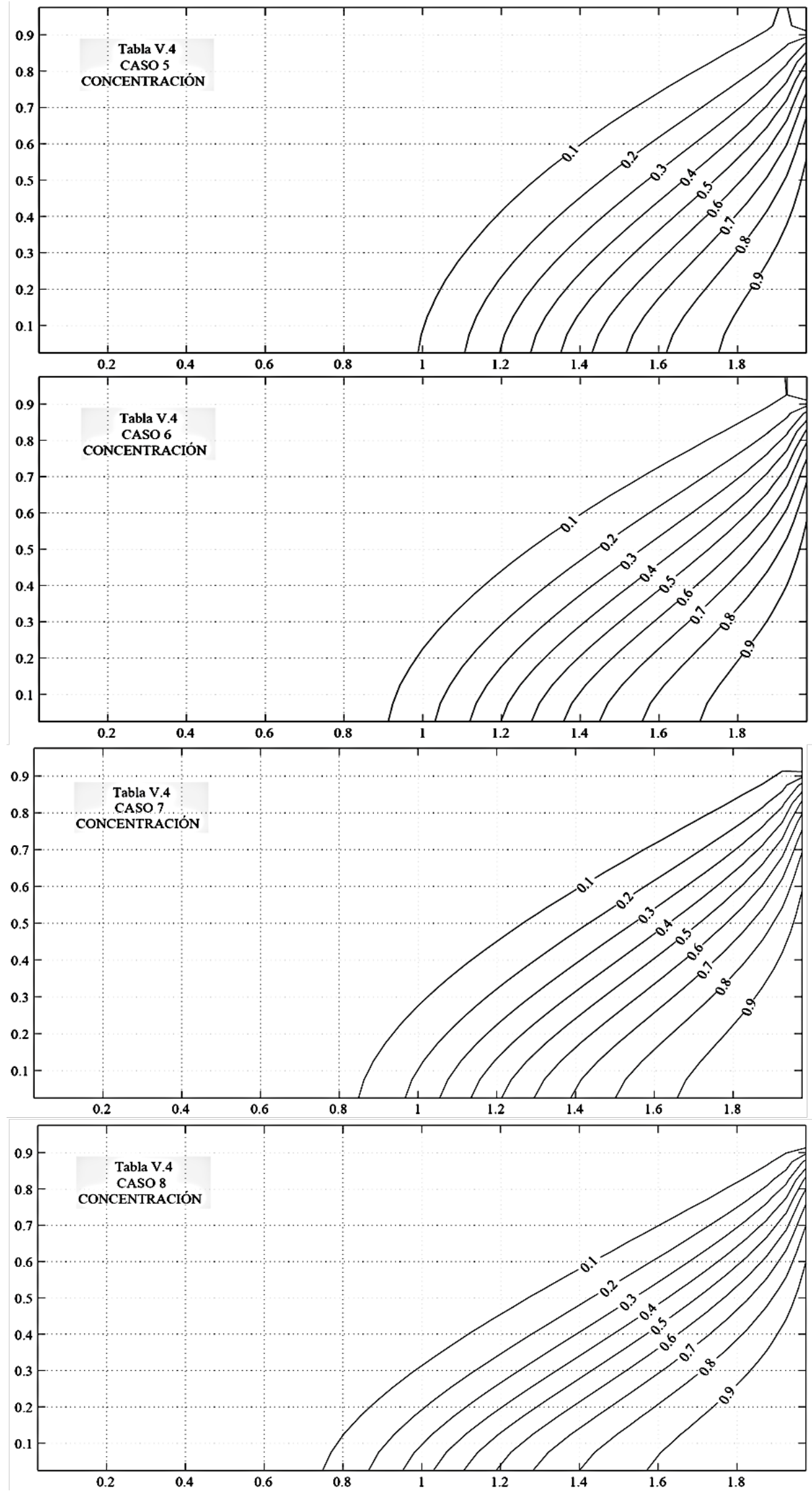

Figura V.7b Patrones de concentración. $\pi_{\mathrm{d}, 4}=0.01,0.15,0.2,0.25,0.3,0.5,0.8$ y 2 (de arriba abajo), $\pi_{\mathrm{d}, 1}=7.58, \pi_{\mathrm{d}, 3}=0.05 \mathrm{y} \pi_{\mathrm{d}, 2}=0.25$ 
Capítulo V. Influencia de los grupos discriminados en los patrones del problema de Henry
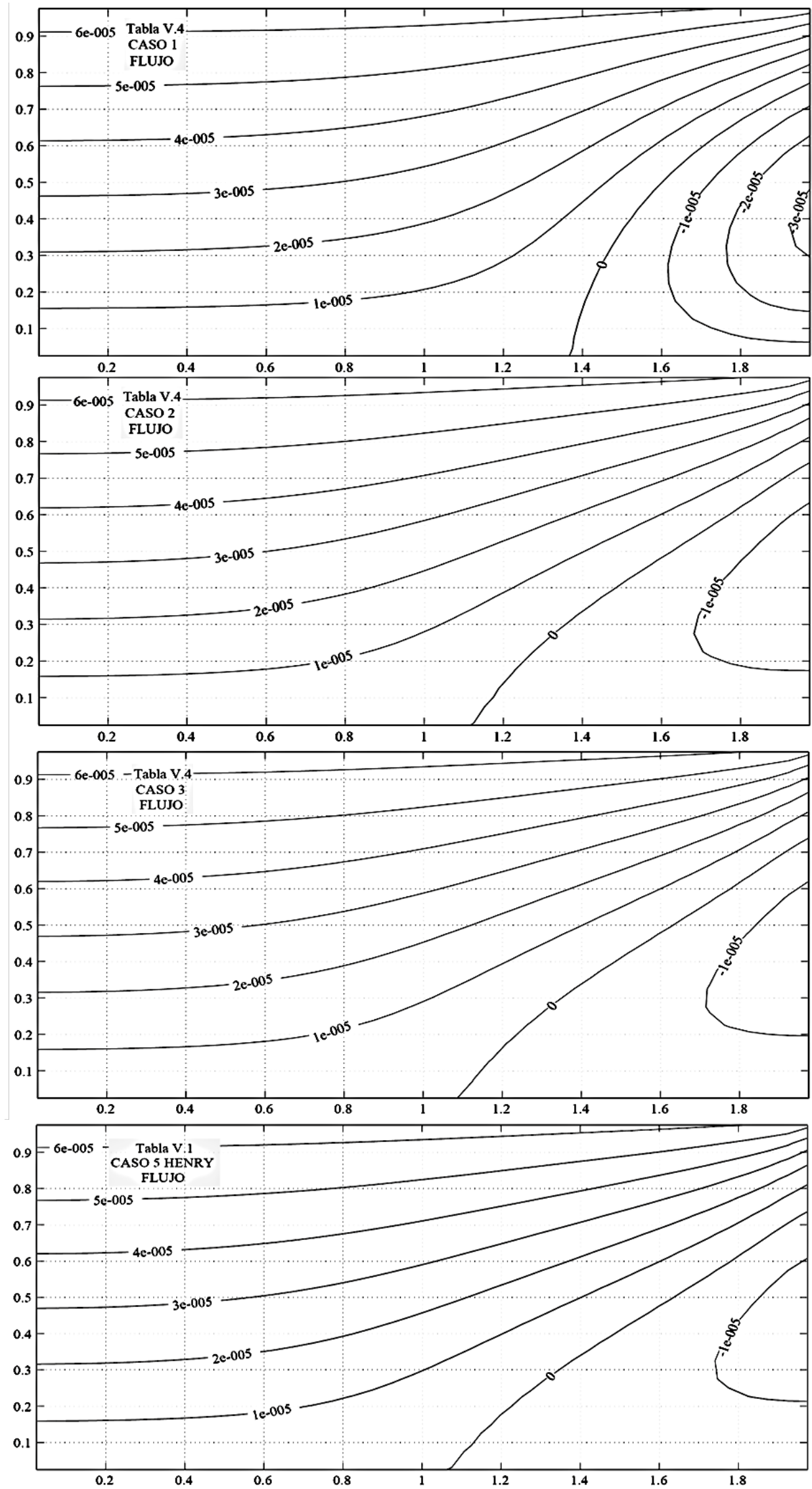

Figura V.8a Patrones de flujo. $\pi_{\mathrm{d}, 4}=0.01,0.15,0.2,0.25,0.3,0.5,0.8$ y 2 (de arriba abajo), $\pi_{\mathrm{d}, 1}=7.58, \pi_{\mathrm{d}, 3}=0.05 \mathrm{y} \pi_{\mathrm{d}, 2}=0.25$ 
Capítulo V. Influencia de los grupos discriminados en los patrones del problema de Henry
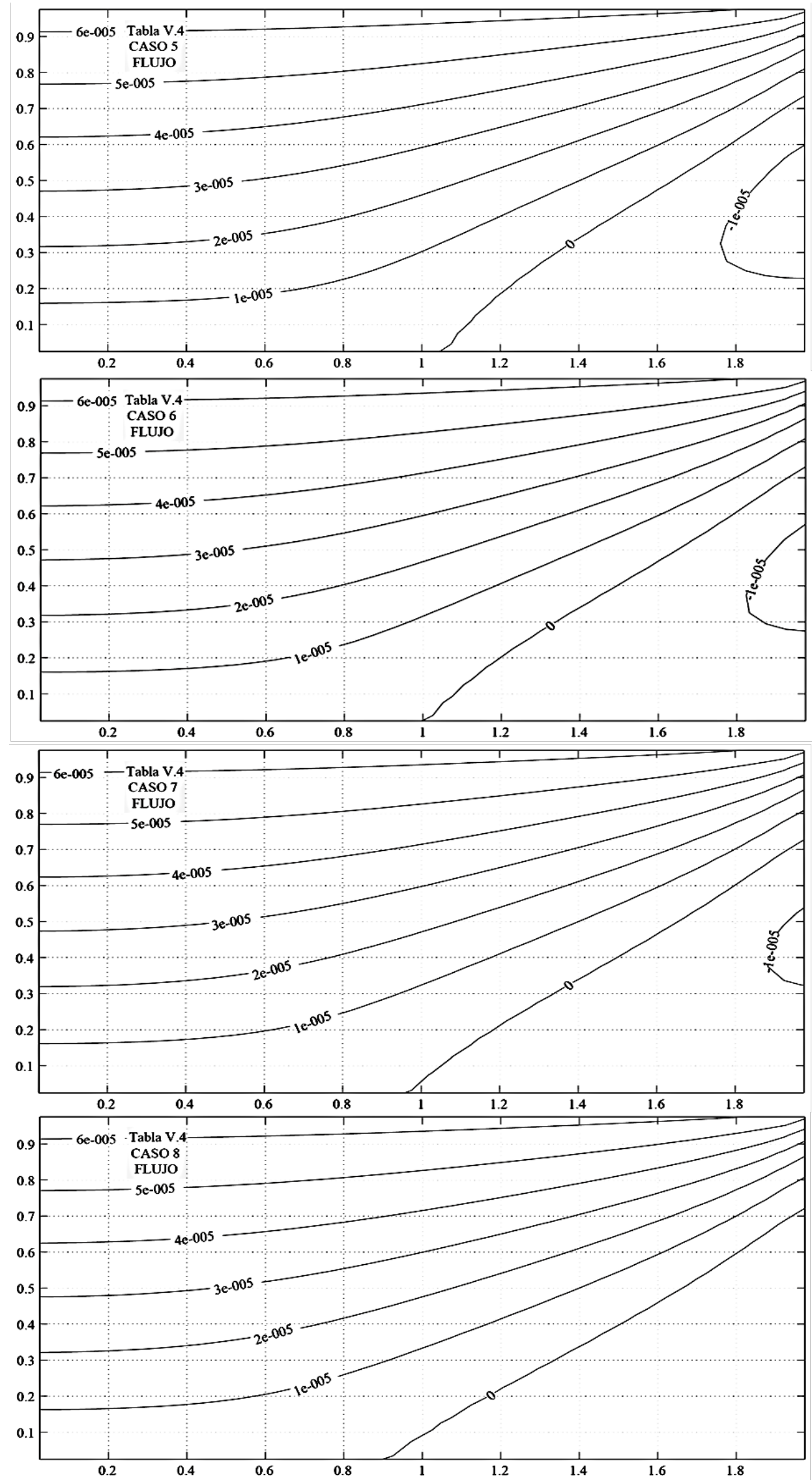

Figura V.8b Patrones de flujo. $\pi_{\mathrm{d}, 4}=0.01,0.15,0.2,0.25,0.3,0.5,0.8$ y 2 (de arriba abajo), $\pi_{\mathrm{d}, 1}=7.58, \pi_{\mathrm{d}, 3}=0.05 \mathrm{y} \pi_{\mathrm{d}, 2}=0.25$ 


\section{V.1.5 INFLUENCIA SIMULTÁNEA DE LOS MONOMIOS $\pi_{\mathrm{d}, 2}=\left(\mathbf{k}_{\mathbf{x}} \mathbf{H}^{2}\right) /\left(\mathbf{k}_{\mathbf{y}} \mathbf{L}^{2}\right) \mathbf{y}$ $\pi_{\mathrm{d}, 4}=\left(D_{\mathrm{x}} \mathbf{H}^{2}\right) /\left(\mathbf{D}_{\mathrm{y}} \mathbf{L}^{2}\right)$}

La influencia simultánea de estos monomios resulta particularmente interesante ya que el carácter anisótropo de la conductividad hidráulica es común en gran parte de los acuíferos reales para los que $\mathrm{k}_{\mathrm{x}}$ suele ser varias veces superior (hasta de un orden de magnitud) a $k_{y}$, lo que asocia un valor de difusividad efectiva $D_{x}$ también varias veces superior a $\mathrm{D}_{\mathrm{y}}$. Todo ello induce a buscar patrones para los que $\pi_{\mathrm{d}, 2} \approx \pi_{\mathrm{d}, 4}$ se acerquen a la unidad, manteniendo en principio el valor de los restantes monomios $\pi_{\mathrm{d}, 1} \mathrm{y} \pi_{\mathrm{d}, 3}$.

Para estudiar estos escenarios no hay más que aumentar el valor de $\mathrm{k}_{\mathrm{x}}$, que provoca un aumento proporcional en $\pi_{\mathrm{d}, 2}$, $\mathrm{y}$ disminuir $\mathrm{D}_{\mathrm{y}}$, que provoca un aumento del monomio $\pi_{\mathrm{d}, 4}$. Para el resto de los parámetros del problema se asumen los valores del problema de Henry. Con todo, los efectos difusivos horizontales son despreciables (los verticales aún más) frente a los efectos asociados al empuje regional, mientras que los efectos de flotación también claramente superiores a los causados por el flujo regional. Balances similares a los existentes en el problema original de Henry.

Las Figuras V.9a-b y V.10a-b muestran los patrones simulados para el rango de valores de $\pi_{\mathrm{d}, 2} \mathrm{y} \pi_{\mathrm{d}, 4}$ en torno a $0.25, \pi_{\mathrm{d}, 2}=\pi_{\mathrm{d}, 4}=0.3,0.4,0.5,0.8,1$ y $2, \pi_{\mathrm{d}, 1}=7.58$ y $\pi_{\mathrm{d}, 3}$ $=0.05$, Tabla V.5

Tabla V.5 Casos para la influencia simultánea de os monomios $\pi_{\mathrm{d}, 2} \mathrm{y} \pi_{\mathrm{d}, 4}$

\begin{tabular}{|c|c|c|c|c|c|c|}
\hline CASO & Kx & Dy & $\pi_{\mathrm{d}, 2}$ & $\pi_{\mathrm{d}, 4}$ & $\pi_{\mathrm{d}, 1}$ & $\pi_{\mathrm{d}, 3}$ \\
\hline $\mathbf{1}$ & $1,22448 \mathrm{E}-09$ & $1,57142 \mathrm{E}-05$ & 0,3 & 0,3 & 7,58 & 0,05 \\
\hline $\mathbf{2}$ & $1,63264 \mathrm{E}-09$ & $1,17856 \mathrm{E}-05$ & 0,4 & 0,4 & 7,58 & 0,05 \\
\hline $\mathbf{3}$ & $2,0408 \mathrm{E}-09$ & $9,4285 \mathrm{E}-06$ & 0,5 & 0,5 & 7,58 & 0,05 \\
\hline $\mathbf{4}$ & $3,26528 \mathrm{E}-09$ & $5,89281 \mathrm{E}-06$ & 0,8 & 0,8 & 7,58 & 0,05 \\
\hline $\mathbf{5}$ & $4,0816 \mathrm{E}-09$ & $4,71425 \mathrm{E}-06$ & 1 & 1 & 7,58 & 0,05 \\
\hline $\mathbf{6}$ & $8,1632 \mathrm{E}-09$ & $2,35713 \mathrm{E}-06$ & 2 & 2 & 7,58 & 0,05 \\
\hline
\end{tabular}

El incremento simultáneo de los monomios $\pi_{\mathrm{d}, 2} \mathrm{y} \pi_{\mathrm{d}, 4}$ desde valores pequeños (0.3) hasta valores del orden de la unidad provoca patrones marcadamente diferentes pero con una evolución que ilustra claramente el efecto de estos cambios. Valores de $\pi_{\mathrm{d}, 2}$ y $\pi_{\mathrm{d}, 4}$ cercanos a la unidad equivalen a acentuar el carácter isótropo de los parámetros permeabilidad y difusividad. En consecuencia, el patrón de concentraciones tiende a extenderse ocupando toda la región del acuífero (patrones semejantes a los casos reales). 
No obstante, la baja difusividad frente a los efectos del flujo regional que causa el bajo valor de $\pi_{\mathrm{d}, 3}(0.05)$, y la de éste en relación con los efectos de flotación que causa el valor relativamente alto de $\pi_{\mathrm{d}, 1}$ (7.58), distorsiona el patrón estrechando progresivamente la región de interface agua dulce-agua salada del acuífero. La región de agua completamente salada se va haciendo mayor definiendo una cuña cuya base alcanza toda la extensión del acuífero. Obviamente, la longitud total del acuífero L es un parámetro definitivamente influyente en estos patrones.

El patrón de flujo es aún más sensible a estos cambios simultáneos de $\pi_{\mathrm{d}, 2}$ y $\pi_{\mathrm{d}, 4}$. La línea divisoria parece coincidir aproximadamente con la frontera agua dulce-agua salada para valores bajos de los monomios. Sin embargo, para un valor de 0.5 esta línea ya alcanza la frontera continental, aunque sólo emerge una recirculación de agua salada por la frontera marina; por encima de 0.5 , la divisoria se desplaza hacia arriba del dominio haciendo emerger una nueva recirculación de agua dulce desde la frontera regional. Es importante apreciar (por los valores de las líneas de corriente) que el caudal de agua dulce del continente al mar, por encima de la línea divisoria, es el impuesto por la condición de contorno continental por lo que el caudal neto bajo dicha línea debe ser cero (el agua entrante del continente al acuífero debe compensarse con el agua saliente (procedente del mar) del acuífero al continente.

El estudio de las direcciones del flujo por debajo de la divisoria nos dice que:

i) Inmediatamente debajo de ésta sigue habiendo un flujo de agua dulce, desde el continente, de valor creciente al aumentar los monomios $\pi_{\mathrm{d}, 2}$ y $\pi_{\mathrm{d}, 4}$ (Figura V.10b); la mayor parte de este flujo llega al mar, el resto recircula volviendo a salir hacia el continente.

ii) En el fondo del acuífero existe un flujo contrario (del mar hacia el continente), que se estrecha al avanzar, y cuyo valor compensa exactamente el caudal de agua entrante por encima del impuesto por la condición de contorno. 
Capítulo V. Influencia de los grupos discriminados en los patrones del problema de Henry
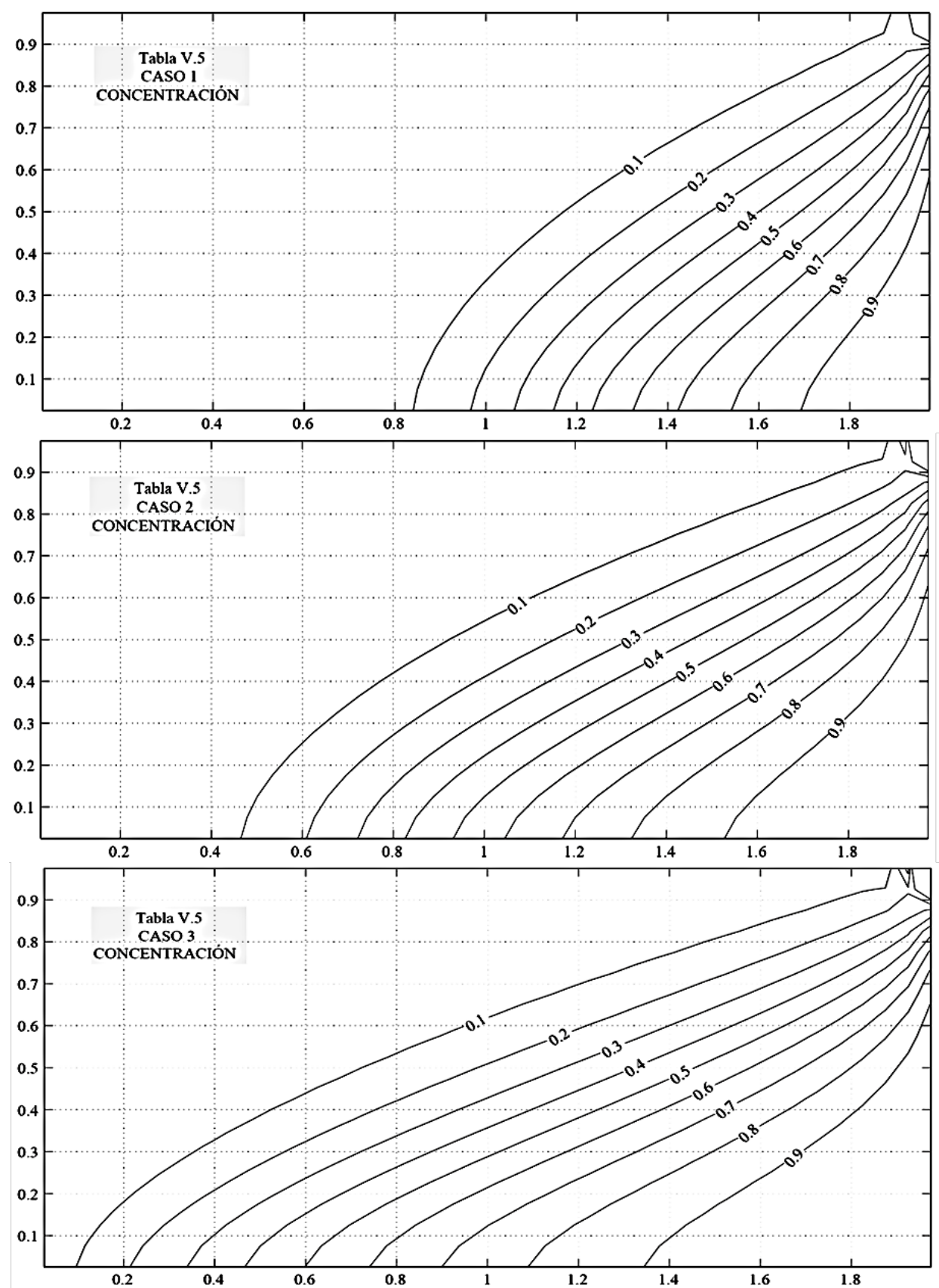

Figura V.9a Patrones de concentración. $\pi_{\mathrm{d}, 2}=\pi_{\mathrm{d}, 4}=0.3,0.4,0.5,0.8,1$ y 2 (de arriba abajo), $\pi_{\mathrm{d}, 1}=7.58, \pi_{\mathrm{d}, 3}=0.05$ 
Capítulo V. Influencia de los grupos discriminados en los patrones del problema de Henry
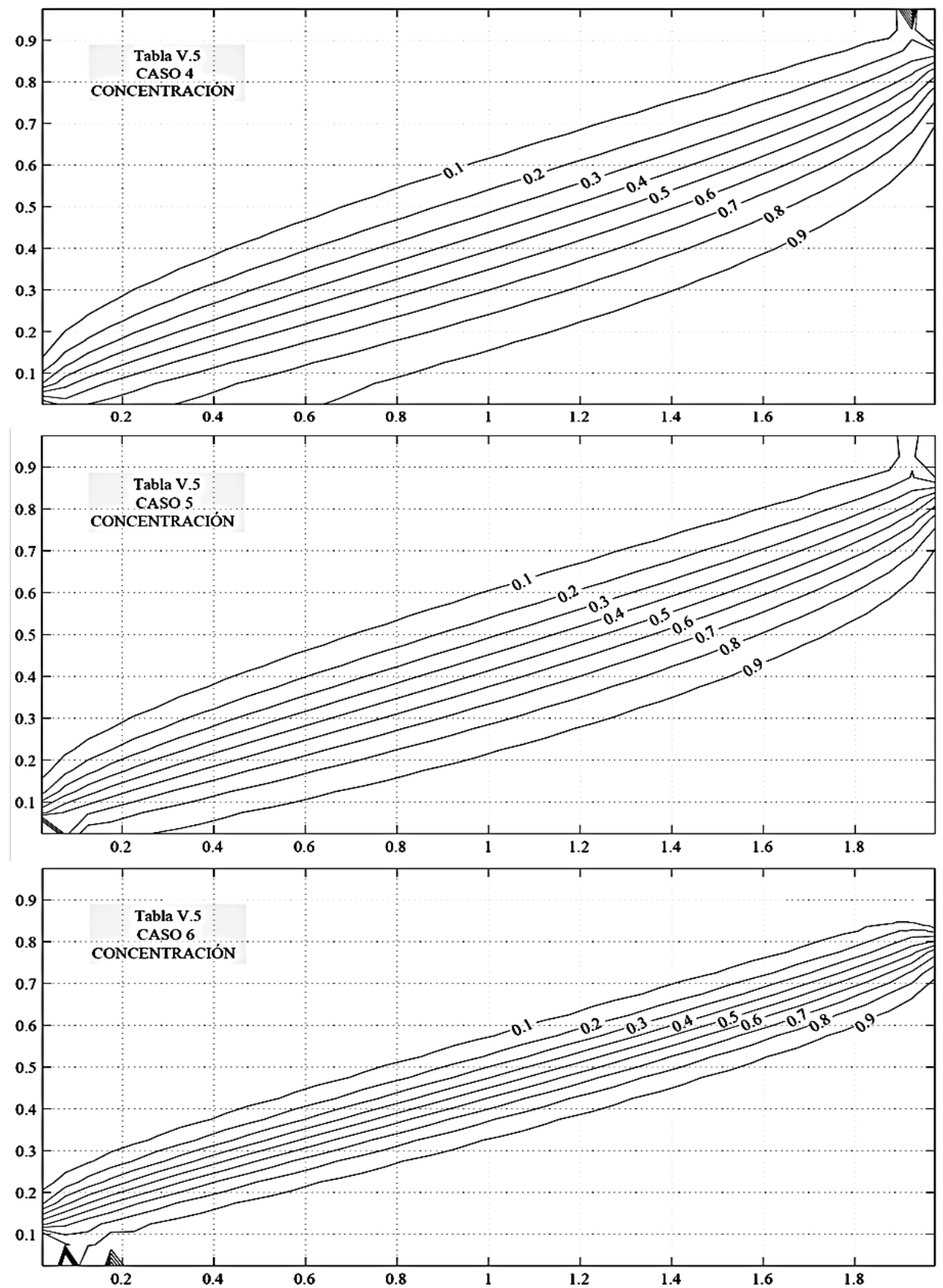

Figura V.9b Patrones de concentración. $\pi_{\mathrm{d}, 2}=\pi_{\mathrm{d}, 4}=0.3,0.4,0.5,0.8,1$ y 2 (de arriba abajo), $\pi_{\mathrm{d}, 1}=7.58, \pi_{\mathrm{d}, 3}=0.05$ 
Capítulo V. Influencia de los grupos discriminados en los patrones del problema de Henry
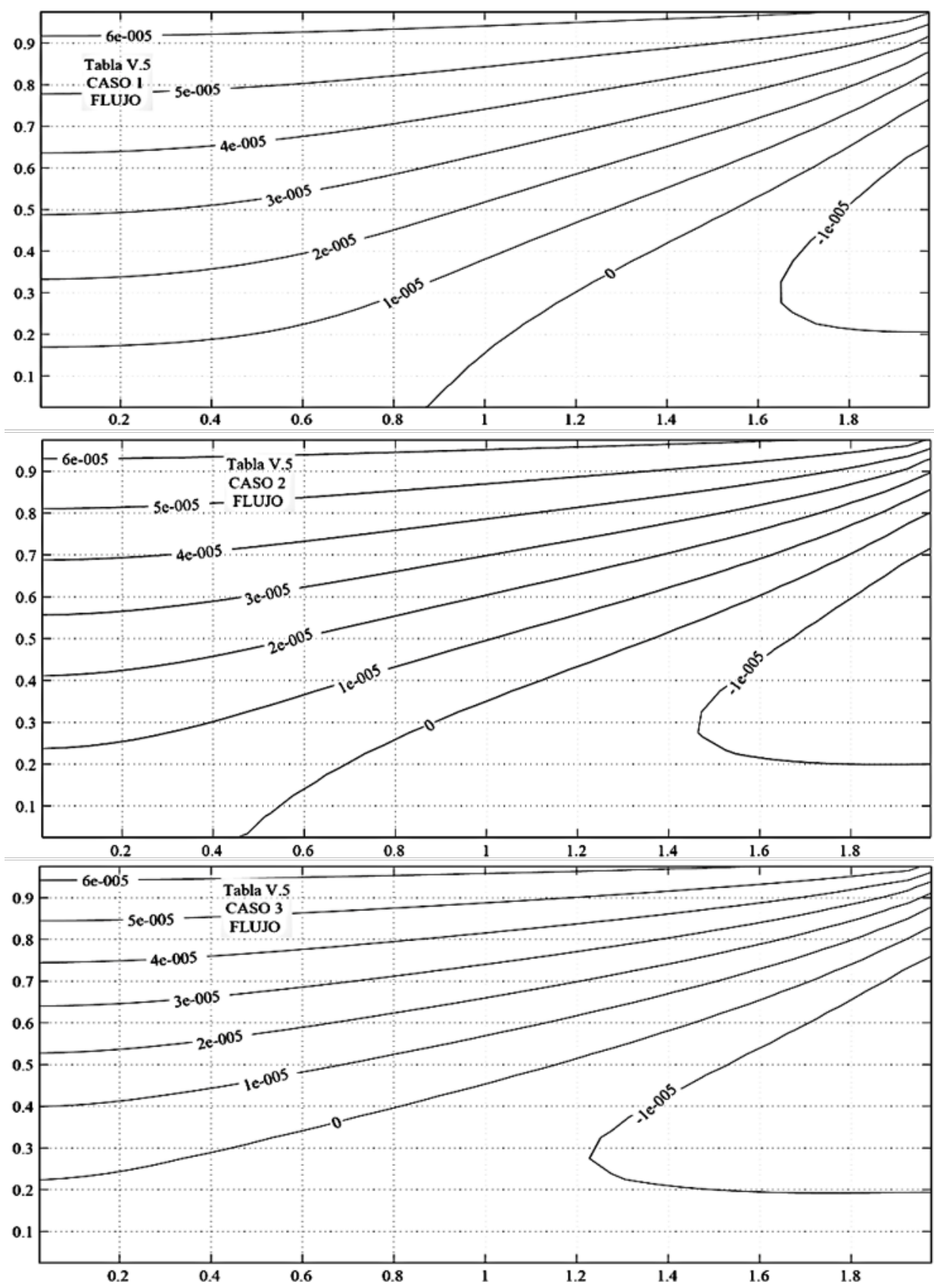

Figura V.10a Patrones de flujo. $\pi_{\mathrm{d}, 2}=\pi_{\mathrm{d}, 4}=0.3,0.4,0.5,0.8,1$ y 2 (de arriba abajo), $\pi_{\mathrm{d}, 1}=7.58, \pi_{\mathrm{d}, 3}=0.05$ 
Capítulo V. Influencia de los grupos discriminados en los patrones del problema de Henry
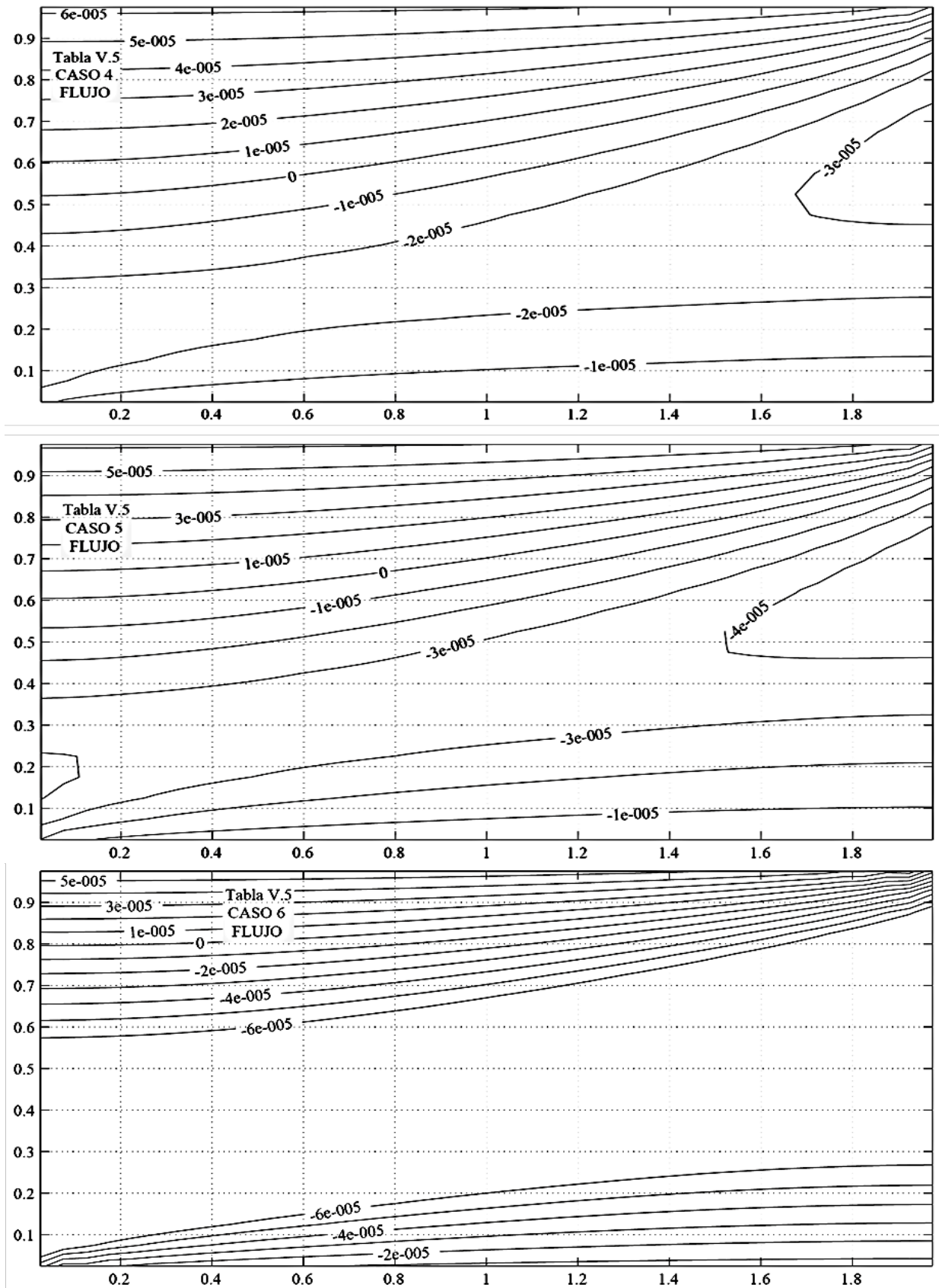

Figura V.10b Patrones de flujo. $\pi_{\mathrm{d}, 2}=\pi_{\mathrm{d}, 4}=0.3,0.4,0.5,0.8,1$ y 2 (de arriba abajo), $\pi_{\mathrm{d}, 1}=7.58, \pi_{\mathrm{d}, 3}=0.05$ 


\section{V.1.6 INFLUENCIA SIMULTÁNEA DE LOS MONOMIOS $\pi_{\mathrm{d}, 2}$ y $\pi_{\mathrm{d}, 4}$, CON AUMENTO DEL EFECTO DIFUSIVO GENERAL Y DISMINUCIÓN DEL EFECTO DE FLOTACIÓN}

Es obvio que esta influencia acerca los patrones al controvertido escenario ideal de problema patrón: unos valores de los monomios que causen una distribución regular de los patrones merced al balance equilibrado de todos los efectos que intervienen en el problema. Se trata de compensar (de acuerdo con lo indicado por muchos autores y ya mencionado en el Capítulo II) la notable influencia en el problema de Henry de los efectos de flotación frente al flujo regional y la despreciable influencia de los efectos difusivos en general frente al mismo flujo, en acuíferos anisótropos con $\mathrm{k}_{\mathrm{x}}>>\mathrm{k}_{\mathrm{y}} \mathrm{y} \mathrm{D}_{\mathrm{x}}>>\mathrm{D}_{\mathrm{y}}$. Así, la disminución relativa de $\pi_{\mathrm{d}, 1}$ puede implementarse, por ejemplo, mediante un aumento de la viscosidad; el aumento de $\pi_{\mathrm{d}, 3}$ con un incremento adecuado de la difusividad efectiva $D_{\mathrm{x}}$ que, a su vez, incrementa $\pi_{\mathrm{d}, 4}$; finalmente, el aumento de $\pi_{\mathrm{d}, 2}$ puede implementarse aumentando $\mathrm{k}_{\mathrm{x}}$.

Con todo, hemos adoptado para los monomios los valores siguientes: $\pi_{\mathrm{d}, 2}=\pi_{\mathrm{d}, 4}=$ $0.3,0.4,0.5,0.8,1$ y $2, \pi_{\mathrm{d}, 1}=3.741$ y $\pi_{\mathrm{d}, 3}=0.5$, los dos últimos la mitad y diez los valores del problema de Henry, respectivamente. La Tabla V.6 muestra los casos propuestos para estudiar esta influencia.

Tabla V.6 Casos para la influencia simultánea de los monomios $\pi_{\mathrm{d}, 2}$ y $\pi_{\mathrm{d}, 4}$ con aumento de la difusión y disminución delas fuerzas de flotación

\begin{tabular}{|c|c|c|c|c|c|c|}
\hline CASO & Kx & Dy & $\pi_{\mathrm{d}, 2}$ & $\pi_{\mathrm{d}, 4}$ & $\pi_{\mathrm{d}, 1}$ & $\pi_{\mathrm{d}, 3}$ \\
\hline $\mathbf{1}$ & $1,22448 \mathrm{E}-09$ & $1,57142 \mathrm{E}-05$ & 0,3 & 0,3 & 3,79 & 0,5 \\
\hline $\mathbf{2}$ & $1,63264 \mathrm{E}-09$ & $1,17856 \mathrm{E}-05$ & 0,4 & 0,4 & 3,79 & 0,5 \\
\hline $\mathbf{3}$ & $2,0408 \mathrm{E}-09$ & $9,4285 \mathrm{E}-06$ & 0,5 & 0,5 & 3,79 & 0,5 \\
\hline $\mathbf{4}$ & $3,26528 \mathrm{E}-09$ & $5,89281 \mathrm{E}-06$ & 0,8 & 0,8 & 3,79 & 0,5 \\
\hline $\mathbf{5}$ & $4,0816 \mathrm{E}-09$ & $4,71425 \mathrm{E}-06$ & 1 & 1 & 3,79 & 0,5 \\
\hline $\mathbf{6}$ & $8,1632 \mathrm{E}-09$ & $2,35713 \mathrm{E}-06$ & 2 & 2 & 3,79 & 0,5 \\
\hline
\end{tabular}

Las Figuras V.11a-b y V.12a-b muestran los resultados de la simulación mediante FATSIM. De acuerdo con lo esperado, los patrones de concentración se muestran casi regularmente distribuidos en todo el dominio; la influencia de $\pi_{\mathrm{d}, 1} \mathrm{y} \pi_{\mathrm{d}, 3}$ sólo empieza a 
Capítulo V. Influencia de los grupos discriminados en los patrones del problema de Henry

ser apreciable cuando las difusividades y permeabilidades horizontales son grandes (parecidas a las verticales) ya que es entones cuando la flotación es importante, por los más altos gradientes verticales de concentración a que dan lugar estos aumentos. La consecuencia es una inclinación de las isolíneas de concentración en todo el dominio, tendiendo a una concentración de las mismas en el borde inferior izquierdo para los valores más altos de $\pi_{\mathrm{d}, 2} \mathrm{y} \pi_{\mathrm{d}, 4}$.

Los patrones de flujo, Figuras 5.12a-b, muestran un claro desacoplo entra las longitudes que definen la línea divisoria y la de menor concentración sobre el fondo del acuífero, particularmente para los valores pequeños de $\pi_{\mathrm{d}, 2} \mathrm{y} \pi_{\mathrm{d}, 4}$. En este rango, la longitud definida por la divisoria pasa de ser nula a llegar hasta la mitad del dominio, sin alterar prácticamente el patrón de líneas por encima de ella. Para valores superiores de estos monomios el patrón reproduce las peculiaridades surgidas en el caso anterior, con la emergencia de i) una zona de flujo de agua dulce por encima de la línea divisoria (en cantidad igual a la impuesta en toda la frontera continental), ii) otra zona por debajo de la línea y junto a ella (también con flujo de agua dulce) que circula en el mismo sentido, iii) sendas zonas de recirculación de agua dulce y agua salada, en el centro de las fronteras laterales izquierda y derecha, respectivamente, y iv) una última región pegada al fondo del acuífero con flujo de agua de mar desde éste hacia el continente. Sin duda, se trata de nuevo de patrones complejos en cuyo estudio podría profundizarse a través de nuevos balances locales definidos por grupos adimensionales aplicables a esos dominios.

Un comentario final en relación con todos los escenarios estudiados en esta sección (V.1). El enfoque fundamental que ha orientado la elaboración de esta tesis doctoral ha sido el de la caracterización del problema de Henry en escenarios anisótropos de sus propiedades; y ello tratando de justificar que tales escenarios son capaces de reproducir los patrones de concentración alargados que emergen en los casos reales.

En nuestra opinión, los grupos deducidos, no sólo se comportan de la manera esperada (controlando los patrones finales) sino que, en efecto, son capaces de reproducir la casi totalidad de escenarios posibles. Además, los escenarios derivados de asignar a algún de los monomios (o a más de uno) un valor alejado de la unidad (por encima o por debajo), puede producir modificaciones en el patrón en las que aparecen subdominios o zonas cuyos balances particulares pueden ser interpretados en términos de nuevos monomios. 
Capítulo V. Influencia de los grupos discriminados en los patrones del problema de Henry
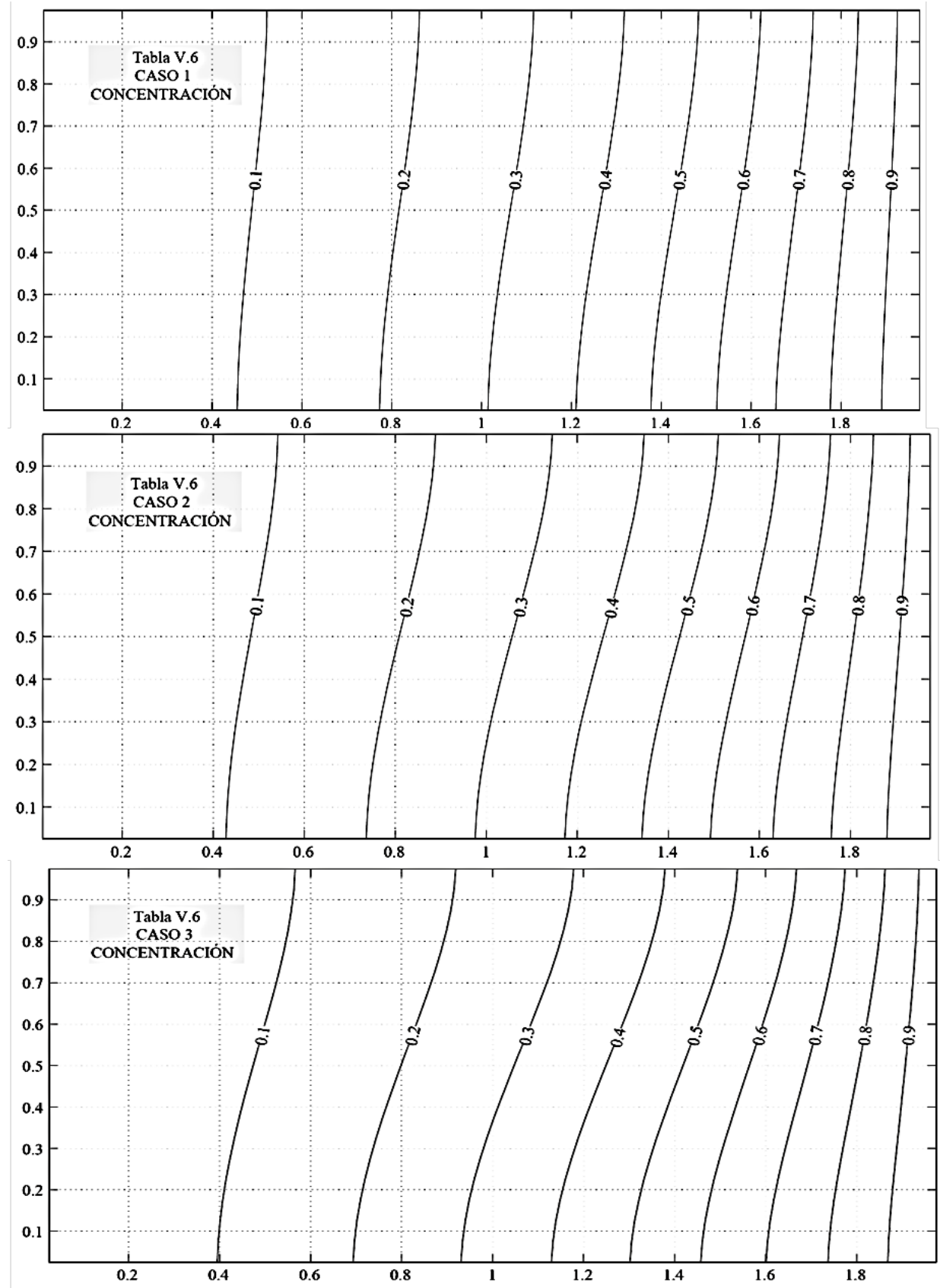

Figura V.11a Patrones de concentración. $\pi_{\mathrm{d}, 2}=\pi_{\mathrm{d}, 4}=0.3,0.4,0.5,0.8,1$ y 2 (de arriba abajo), $\pi_{\mathrm{d}, 1}=3.79, \pi_{\mathrm{d}, 3}=0.5$ 
Capítulo V. Influencia de los grupos discriminados en los patrones del problema de Henry
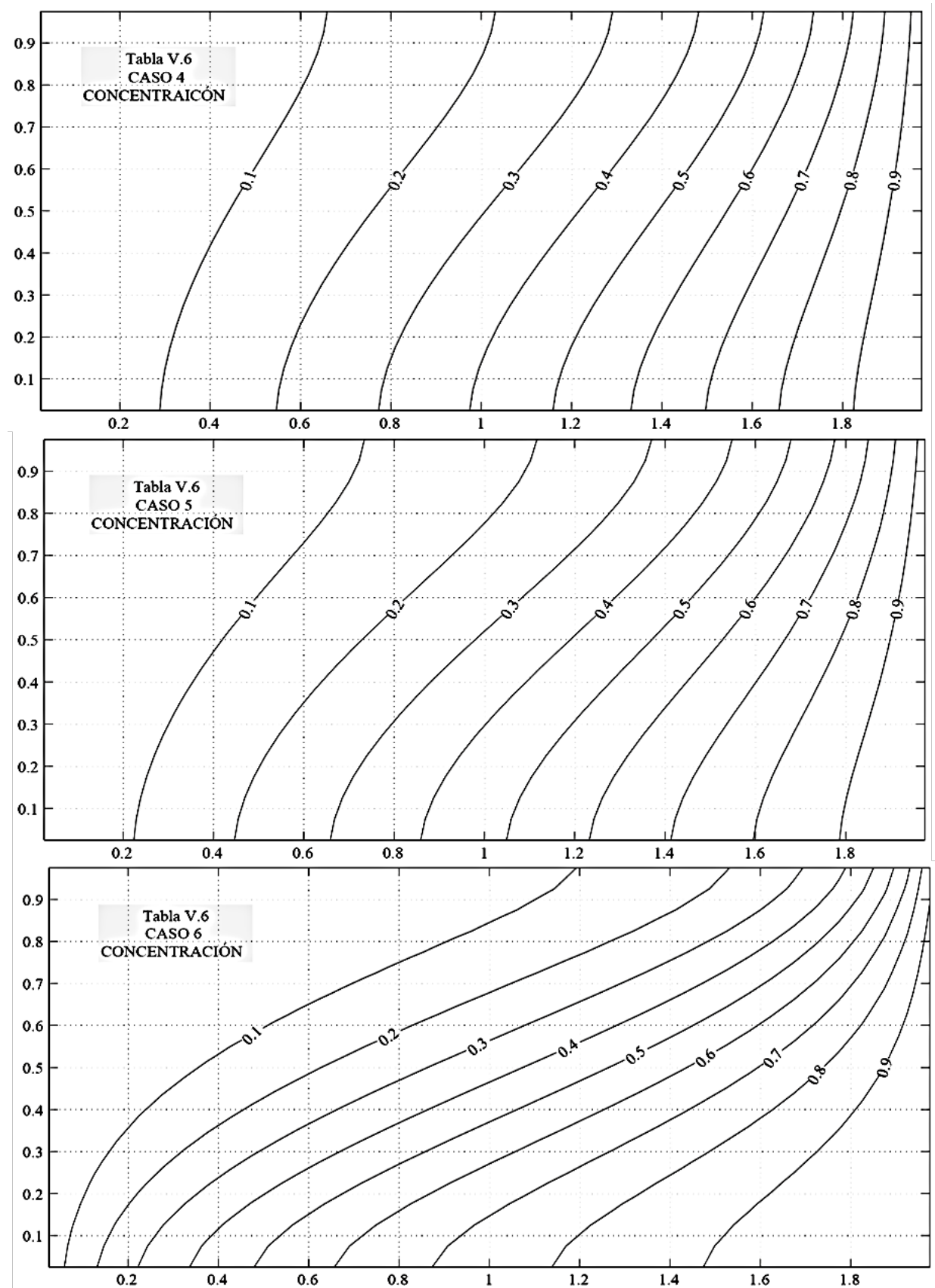

Figura V.11b Patrones de concentración. $\pi_{\mathrm{d}, 2}=\pi_{\mathrm{d}, 4}=0.3,0.4,0.5,0.8,1$ y 2 (de arriba abajo), $\pi_{\mathrm{d}, 1}=3.9, \pi_{\mathrm{d}, 3}=0.5$ 
Capítulo V. Influencia de los grupos discriminados en los patrones del problema de Henry
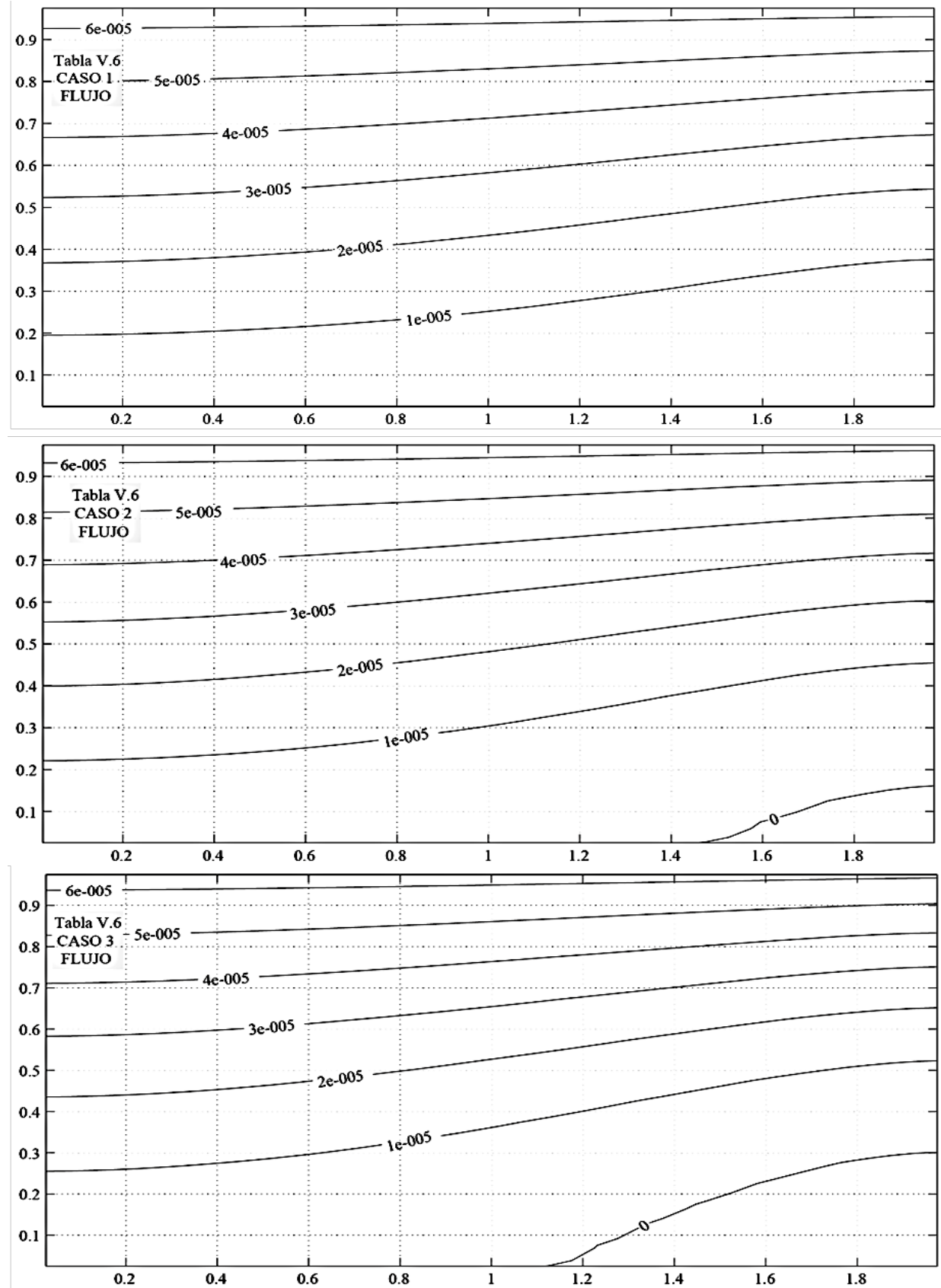

Figura V.12a Patrones de flujo. $\pi_{\mathrm{d}, 2}=\pi_{\mathrm{d}, 4}=0.3,0.4,0.5,0.8,1$ y 2 (de arriba abajo), $\pi_{\mathrm{d}, 1}=3.79, \pi_{\mathrm{d}, 3}=0.5$ 
Capítulo V. Influencia de los grupos discriminados en los patrones del problema de Henry
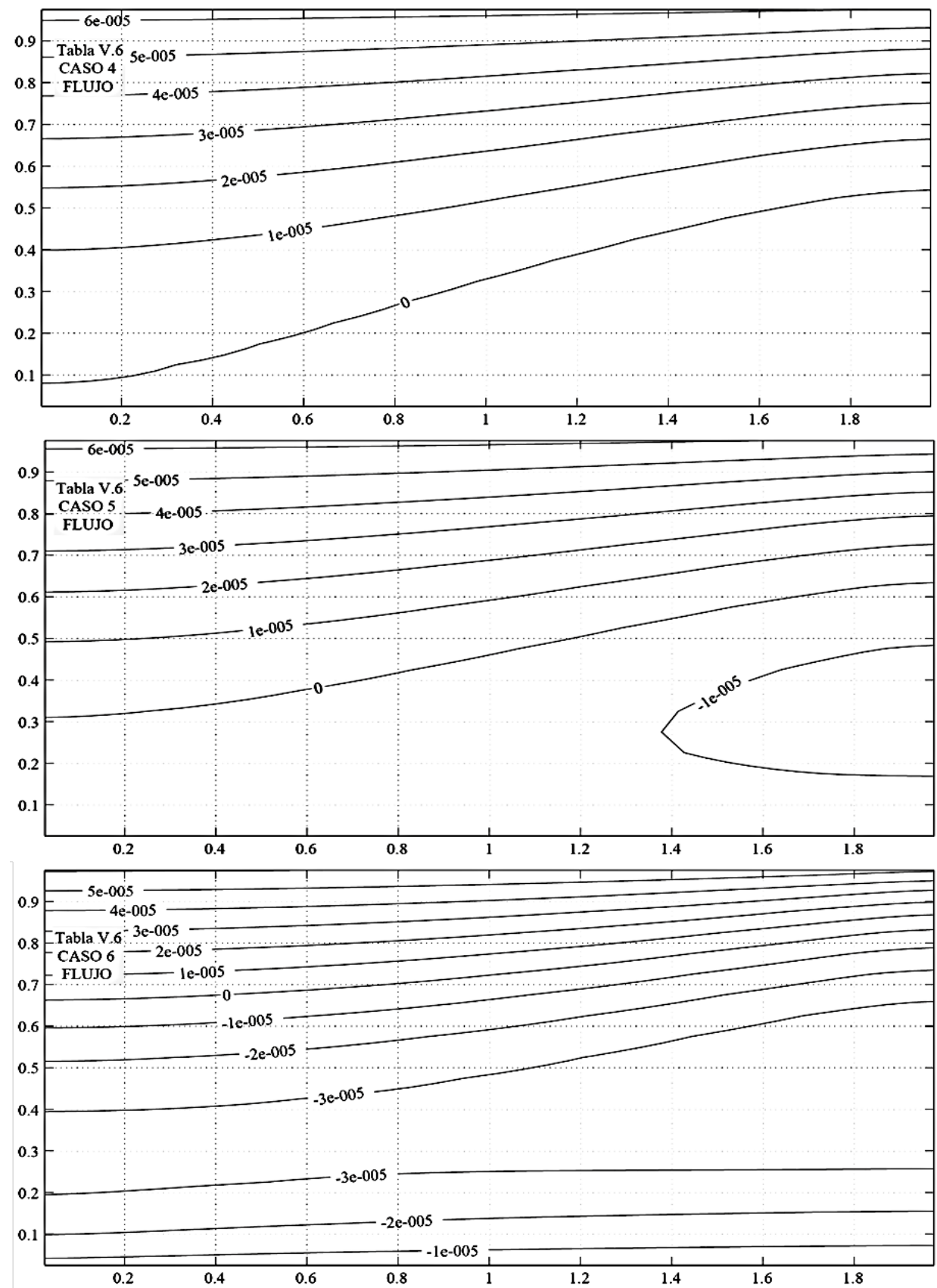

Figura V.12b Patrones de flujo. $\pi_{\mathrm{d}, 2}=\pi_{\mathrm{d}, 4}=0.3,0.4,0.5,0.8,1$ y 2 (de arriba abajo), $\pi_{\mathrm{d}, 1}=3.79, \pi_{\mathrm{d}, 3}=0.5$ 
Capítulo V. Influencia de los grupos discriminados en los patrones del problema de Henry

\section{V.2 INFLUENCIA DEL VALOR DE LOS GRUPOS DISCRIMINADOS EN LOS PATRONES DEL PROBLEMA GENERAL DE HENRY (ANISÓTROPO E ISÓTROPO). CUÑAS DE INTRUSION Y DE RECIRCULACIÓN}

El objetivo de este apartado es más bien académico pues se trata de comprobar la influencia de los cambios de valor de los monomios discriminados en torno a la unidad, valor para el que los balances entre los diferentes efectos que se contraponen en el problema están equilibrados. Un pequeño cambio de valor en torno a la unidad (el doble, el triple, la mitad...) supone desequilibrios apreciables en los balances que, a su vez, determinan cambios de importancia en los patrones de solución.

Recordamos que la ventaja esencial que representa obtener los grupos adimensionales independientes que rigen la solución de un problema es reducir la influencia individual de cada parámetro a la influencia de cada grupo adimensional formado por agrupaciones de estos parámetros. En problemas complejos, la reducción de un número no pequeño de parámetros a un reducido número de grupos es, sin duda, una ventaja para el investigador en todos los órdenes (visión física más completa y aguda de los fenómenos involucrados en el problema, menores tiempos de experimentación o simulación numérica, estudio de la influencia y sensibilidad en la solución de cada uno de los parámetros, etc.).

En la adimensionalización clásica no se asegura que los grupos adimensionales que derivan de ella representen balances de magnitudes que interaccionan en el proceso, lo que no permite asociar la influencia de los grupos con la influencia del valor relativo de las magnitudes que se balancean. Por el contrario, en la adimensionalización discriminada los grupos sí representan balances y la influencia mayor o menor de cada grupo se asocia inmediatamente a la influencia de una magnitud respecto a otra dentro de cada balance, de cada grupo o entre grupos.

La posibilidad, por otro lado, de presentar los grupos independientes de diferente forma, merced a su combinación mediante operaciones matemáticas más o menos sencillas, permite elegir la opción de mayor interés que puede ser diferente según convenga, por ejemplo, por el propio significado físico del grupo o por querer presentar alguno de los parámetros en sólo uno de los grupos (como ocurre cuando se busca el orden de magnitud de alguna incógnita que contiene la ecuación adimensional) 
En este apartado se estudia la influencia separada de los grupos adimensionales discriminados en el patrón de solución del problema de Henry, influencia que, en términos de las magnitudes físicas que se balancean en dichos grupos, nos llevará a una mayor comprensión de los procesos físicos acoplados que tienen lugar. En el límite, cuando un grupo dado deja de influir en el patrón de soluciones, los resultados deben converger con los obtenidos en las simplificaciones estudiadas en el Capítulo IV.

La realización de este estudio permitirá observar que los patrones de solución presentan dos cuñas diferenciadas (ya empezadas a manifestarse en el apartado anterior de este capítulo): una de intrusión salina asociada al patrón de concentración, llamada propiamente cuña de intrusión salina (veremos que puede definirse mejor como la cuña en la que existen gradientes de concentración apreciables, es decir, la extensión de agua con salinidades intermedias), y otra asociada al patrón de flujo llamada cuña de recirculación, definida por la divisoria o línea de valor nulo de la función de corriente.

En el problema de Henry original, merced a los valores particulares de los parámetros, estas dos cuñas tienen la misma extensión. La cuña de recirculación está bien definida y ocupa el remolino de flujo que, procedente de la frontera marina, penetra por el fondo del acuífero y se devuelve al mar en su propia región fronteriza bajo la zona de descarga de agua dulce. Todo el remolino de agua penetrante tiene una concentración prácticamente coincidente con la del agua de mar. Merced a que la extensión de ambas cuñas disminuye al aumentar la cota vertical del acuífero (de abajo arriba), nos referiremos siempre a la medida de éstas sobre la base o fondo del acuífero.

\section{V.2.1 VALORES DE LOS PARÁMETROS PARA EL PROBLEMA ANISÓTROPO}

Para asignar un valor unidad a los grupos discriminados elegimos los siguientes valores para el conjunto de parámetros:

$$
\begin{aligned}
& \mathrm{L}=\mathrm{L}_{\mathrm{H}}=2 \mathrm{~m}, \\
& \mathrm{H}=\mathrm{H}_{\mathrm{H}}=1 \mathrm{~m}, \\
& \varepsilon=\varepsilon_{\mathrm{H}}=0.35 \\
& \mathrm{k}_{\mathrm{y}}=\mathrm{k}_{\mathrm{y}, \mathrm{H}}=1.0204 \mathrm{E}-9 \mathrm{~m}^{2}, \\
& \left.\mathrm{k}_{\mathrm{x}}=4 \mathrm{k}_{\mathrm{x}, \mathrm{H}}=4.0816 \mathrm{E}-9 \mathrm{~m}^{2} \quad \text { (para hacer } \pi_{\mathrm{d}, 2}=1\right) \\
& \mathrm{D}_{\mathrm{y}}=\mathrm{D}_{, \mathrm{H}}=1.8857 \mathrm{E}-5 \mathrm{~m}^{2} / \mathrm{s},
\end{aligned}
$$


Capítulo V. Influencia de los grupos discriminados en los patrones del problema de Henry

$D_{\mathrm{x}}=4 \mathrm{D}_{\mathrm{x}, \mathrm{H}}=7.5428 \mathrm{E}-5 \mathrm{~m}^{2} / \mathrm{s} \quad\left(\right.$ para hacer $\left.\pi_{\mathrm{d}, 4}=1\right)$

$\mathrm{V}_{\mathrm{amb}}=\mathrm{D}_{\mathrm{x}} / \mathrm{L}=3.7714 \mathrm{E}-5 \mathrm{~m} / \mathrm{s} \quad\left(\right.$ para hacer $\left.\pi_{\mathrm{d}, 3}=1\right)$, como $\mathrm{V}_{\mathrm{amb}, \mathrm{H}}=1.8857 \mathrm{E}-4$,

$\mathrm{V}_{\mathrm{amb}}=0.2 \mathrm{v}_{\mathrm{amb}, \mathrm{H}}$

$\mu=\left[(\Delta \rho) \mathrm{gk}_{\mathrm{y}} /\left(\varepsilon \mathrm{Vamb}^{*}\right)\right](\mathrm{L} / \mathrm{H})=0.03792 \mathrm{kgm}^{-1} \mathrm{~s}^{-1}$ (para hacer $\left.\pi_{\mathrm{d}, 1}=1\right), \mu=37.92 \mu_{\mathrm{H}}$

$\Delta \rho=\Delta \rho_{\mathrm{H}}=25 \mathrm{~kg} / \mathrm{m}^{3}$

con lo que

$\pi_{\mathrm{d}, 1}=\pi_{\mathrm{d}, 2}=\pi_{\mathrm{d}, 3}=\pi_{\mathrm{d}, 4}=1$

\section{V.2.2 INFLUENCIA DE LOS MONOMIOS, INDIVIDUALMENTE, EN EL PROBLEMA GENERAL ANISÓTROPO}

Este apartado, que se refiere a escenarios muy alejados del problema de Henry, estudia la influencia de cada monomio en un patrón en el que se balancean por igual las magnitudes del resto de los monomios.

\section{V.2.2.1 Influencia del monomio $\pi_{\mathrm{d}, 1}=\left[(\Delta \rho) \mathrm{gk}_{\mathrm{y}} /\left(\varepsilon \mu \mathrm{Vamb}^{*}\right)\right](\mathrm{L} / \mathrm{H})$}

Asignamos una serie de valores a $\pi_{\mathrm{d}, 1}$ por encima y por debajo de la unidad mientras mantenemos el resto de monomios en valores $\pi_{\mathrm{d}, 2}=\pi_{\mathrm{d}, 3}=\pi_{\mathrm{d}, 4}=1$. Valores de $\pi_{\mathrm{d}, 1}$ superiores a la unidad refuerzan los efectos de las fuerzas de flotación (Darcy) frente a los de arrastre del flujo regional, y al contrario para valores de $\pi_{\mathrm{d}, 1}$ inferiores a la unidad.

Tabla V.7 Casos para el estudio de la influencia de $\pi_{\mathrm{d}, 1}$

\begin{tabular}{|c|c|c|c|c|c|}
\hline CASO & viscosidad & $\boldsymbol{\pi}_{\mathrm{d}, \mathbf{1}}$ & $\boldsymbol{\pi}_{\mathrm{d}, \mathbf{2}}$ & $\boldsymbol{\pi}_{\mathrm{d}, \mathbf{3}}$ & $\boldsymbol{\pi}_{\mathrm{d}, \mathbf{4}}$ \\
\hline $\mathbf{1}$ & 0,75834847 & 0,05 & 1 & 1 & 1 \\
\hline $\mathbf{2}$ & 0,07583485 & 0,5 & 1 & 1 & 1 \\
\hline $\mathbf{3}$ & 0,04739678 & 0,8 & 1 & 1 & 1 \\
\hline $\mathbf{4}$ & $\mathbf{0 , 0 3 7 9 1 7 4 2}$ & $\mathbf{1}$ & $\mathbf{1}$ & $\mathbf{1}$ & $\mathbf{1}$ \\
\hline $\mathbf{5}$ & 0,01263914 & 3 & 1 & 1 & 1 \\
\hline $\mathbf{6}$ & 0,00758348 & 5 & 1 & 1 & 1 \\
\hline $\mathbf{7}$ & 0,00379174 & 10 & 1 & 1 & 1 \\
\hline $\mathbf{8}$ & 0,00189587 & 20 & 1 & 1 & 1 \\
\hline
\end{tabular}

Las Figuras V.13a-b y V.14a-b muestran los patrones de concentración y función de corriente para un significativo rango de valores de $\pi_{\mathrm{d}, 1}=0.05,0,5,0.8,1,3,5,10$ y 20 , Tabla V.7. El cambio de valor de $\pi_{\mathrm{d}, 1}$ se impone modificando cualquier parámetro que no aparezca en los restantes monomios, por ejemplo $\Delta \rho$ o $\mu$. 
Capítulo V. Influencia de los grupos discriminados en los patrones del problema de Henry

El patrón de salinidad, Figura V.13a-b, distribuye las isolíneas de concentración regularmente en todo el dominio con un espaciado casi regular cuando los efectos de flotación son despreciables frente a los de empuje regional $\left(\pi_{\mathrm{d}, 1}<1\right)$. Al equilibrarse estos efectos, $\pi_{\mathrm{d}, 1} \approx 1$, las isolíneas sufren una leve inclinación fruto de la combinación de los movimientos verticales de las partículas (causados por la flotación) y los horizontales (causados por el empuje regional). Prácticamente todas las isolíneas tienen una inclinación similar. Al aumentar $\pi_{\mathrm{d}, 1}$ por encima de la unidad, los efectos de flotación preponderan y las inclinaciones de las isolíneas son más acusadas.

En cuanto al patrón de flujo, Figura V.14a-b, las líneas de corriente son prácticamente horizontales y están igualmente espaciadas cuando las fuerzas de flotación son despreciables frente las de arrastre del flujo regional, $\pi_{\mathrm{d}, 1}<1$.

El valor $\pi_{\mathrm{d}, 3}=1$ equilibra los efectos difusivos y los de empuje regional y ésta es, sin duda, la mejor justificación para esta distribución regular de isolíneas. Al crecer el monomio se manifiestan los efectos de flujo de densidad variable inclinando las isolíneas de corriente ligeramente de forma que el agua dulce entrante flote sobre el agua salada y tienda a salir por la parte superior de la frontera marina. De nuevo, dado que las fuerzas difusivas y las de flujo regional siguen estando equilibradas, $\pi_{\mathrm{d}, 3}=1$, el espaciamiento entre isolíneas sigue siendo aproximadamente regular.

La divisoria (isolínea $\Psi=0$ ), que no emerge hasta valores de $\pi_{\mathrm{d}, 1}$ ligeramente superiores a la unidad, está prácticamente desacoplada de cualquier referencia del patrón de flujo para $\pi_{\mathrm{d}, 1} \approx 1$ y valores inferiores de este monomio; pero para valores ligeramente superiores emerge con fuerza y en un estrecho rango de valores de $\pi_{\mathrm{d}, 1}$ su intersección con la base cubre todo el dominio.

Puede apreciarse que para $\pi_{\mathrm{d}, 1} \approx 3$, que no significa aún fuerzas regionales despreciables frente a las de flotación, la divisoria ha penetrado en la frontera regional generando un pequeño flujo salino de recirculación. Al seguir aumentando $\pi_{\mathrm{d}, 3}$, la divisoria se desplaza hacia arriba, el flujo de recirculación de agua marina se incrementa, y emergen una nueva recirculación en la frontera regional y un canal de agua salada en el fondo desde el mar hacia el continente. 
Capítulo V. Influencia de los grupos discriminados en los patrones del problema de Henry
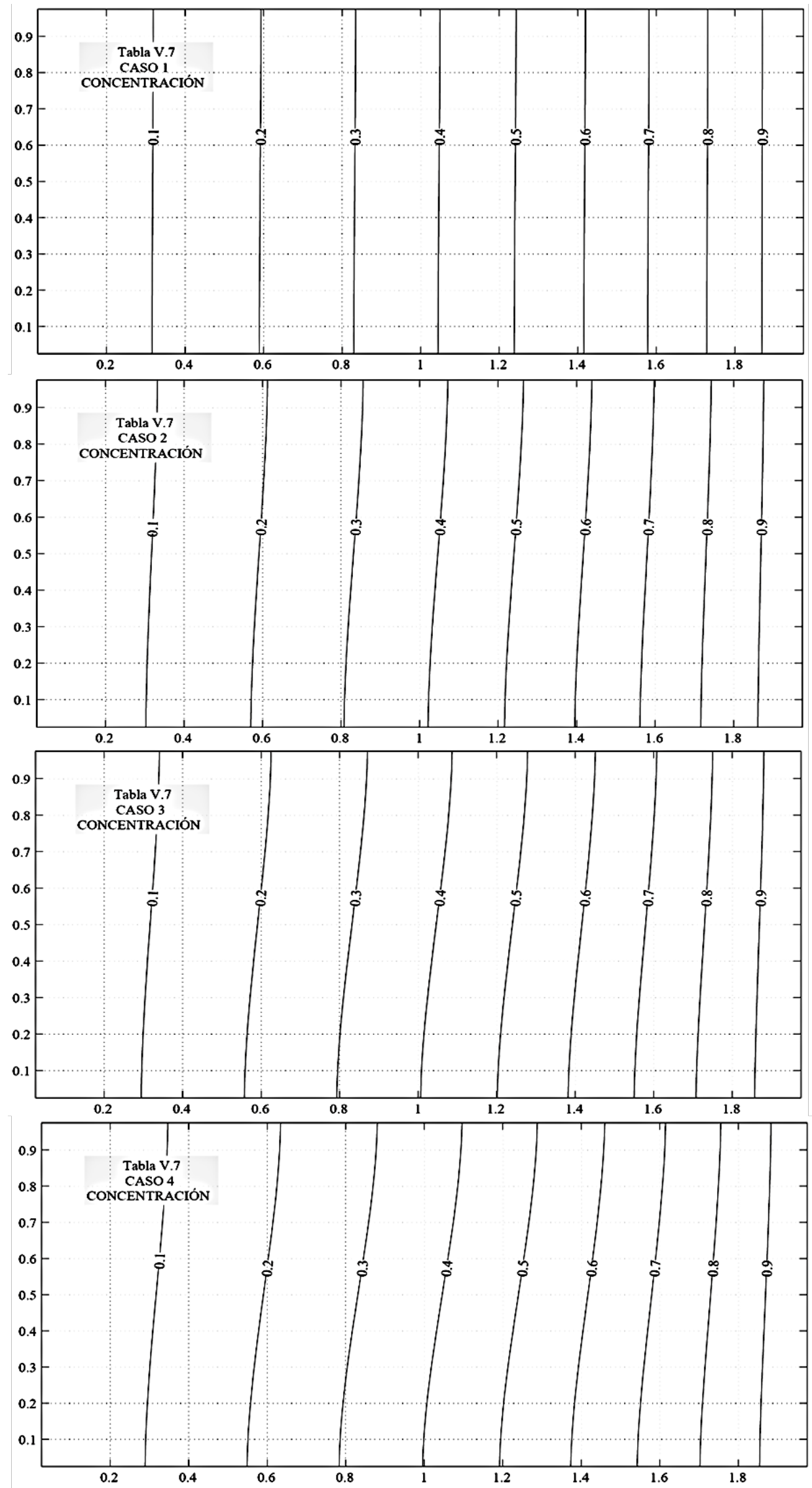

Figura V.13a Patrones de concentración. $\pi_{\mathrm{d}, 1}: 0.05,0,5,0.8,1,3,5,10$ y $20, \pi_{\mathrm{d}, 2}=\pi_{\mathrm{d}, 3}=\pi_{\mathrm{d}, 4}=1$ 
Capítulo V. Influencia de los grupos discriminados en los patrones del problema de Henry
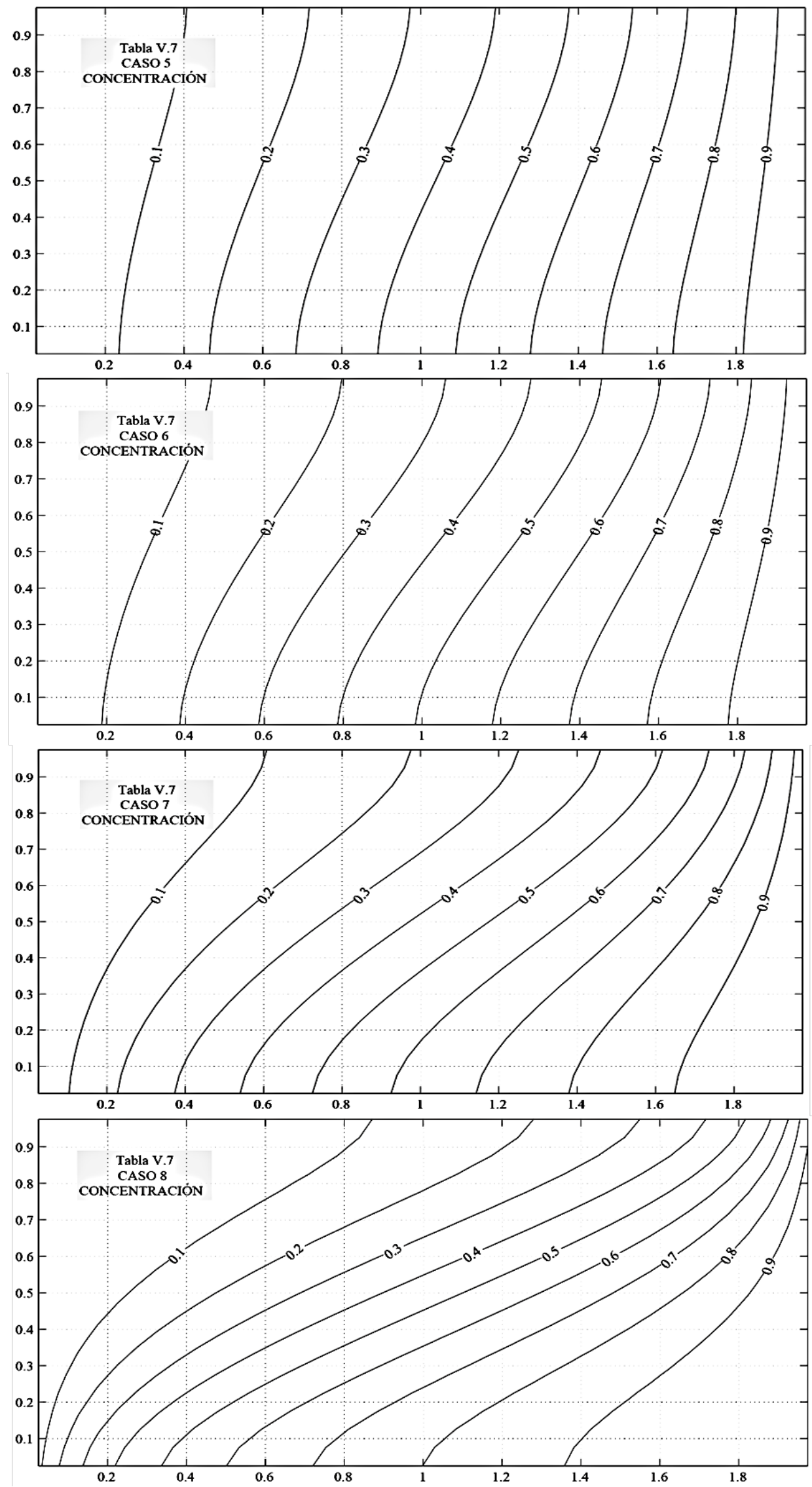

Figura V.13b Patrones de concentración. $\pi_{\mathrm{d}, 1}: 0.05,0,5,0.8,1,3,5,10$ y $20, \pi_{\mathrm{d}, 2}=\pi_{\mathrm{d}, 3}=\pi_{\mathrm{d}, 4}=1$ 
Capítulo V. Influencia de los grupos discriminados en los patrones del problema de Henry
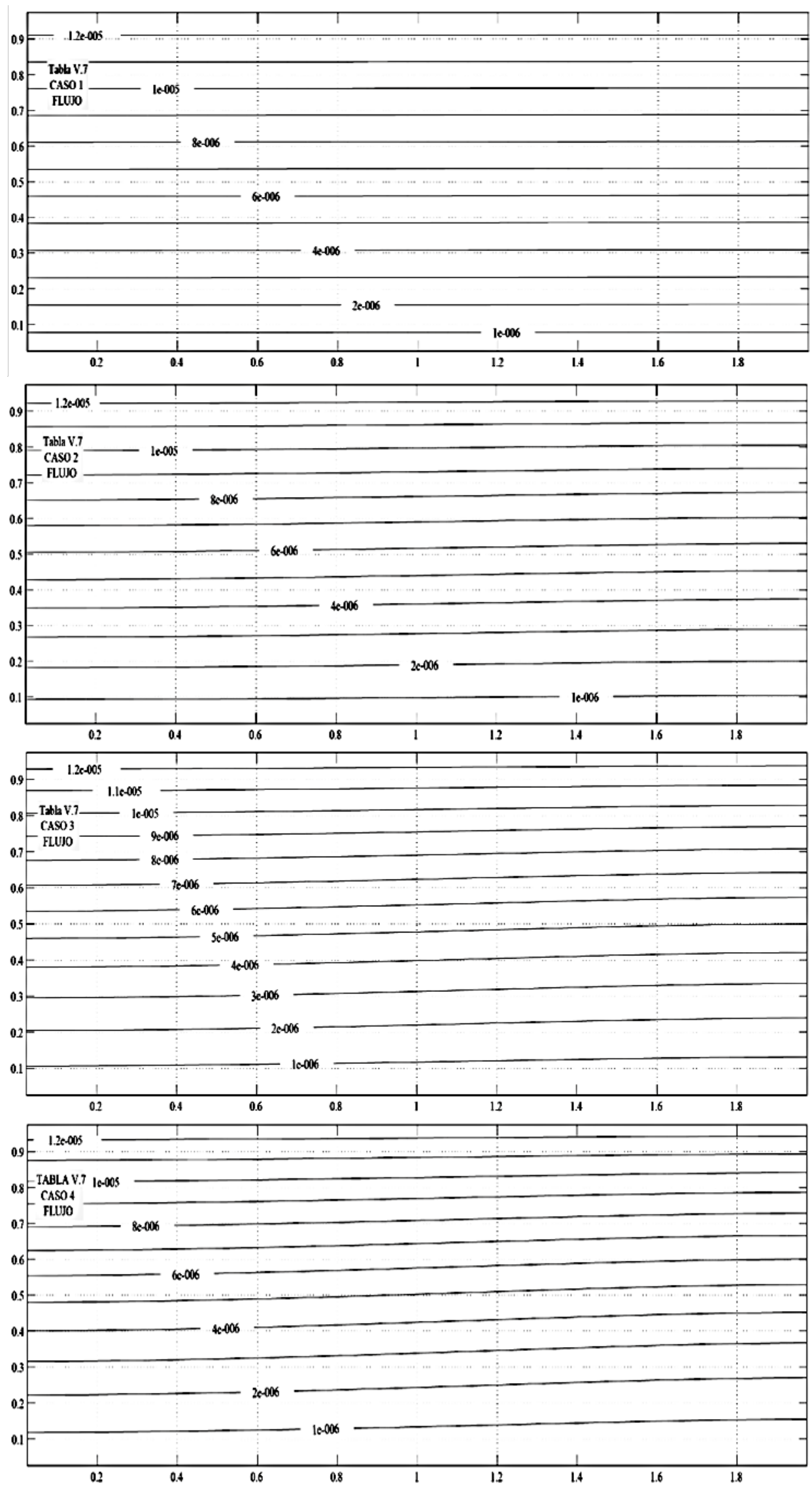

Figura V.14a Patrones de flujo. $\pi_{\mathrm{d}, 1}: 0.05,0,5,0.8,1,3,5,10$ y 20, $\pi_{\mathrm{d}, 2}=\pi_{\mathrm{d}, 3}=\pi_{\mathrm{d}, 4}=1$ 
Capítulo V. Influencia de los grupos discriminados en los patrones del problema de Henry
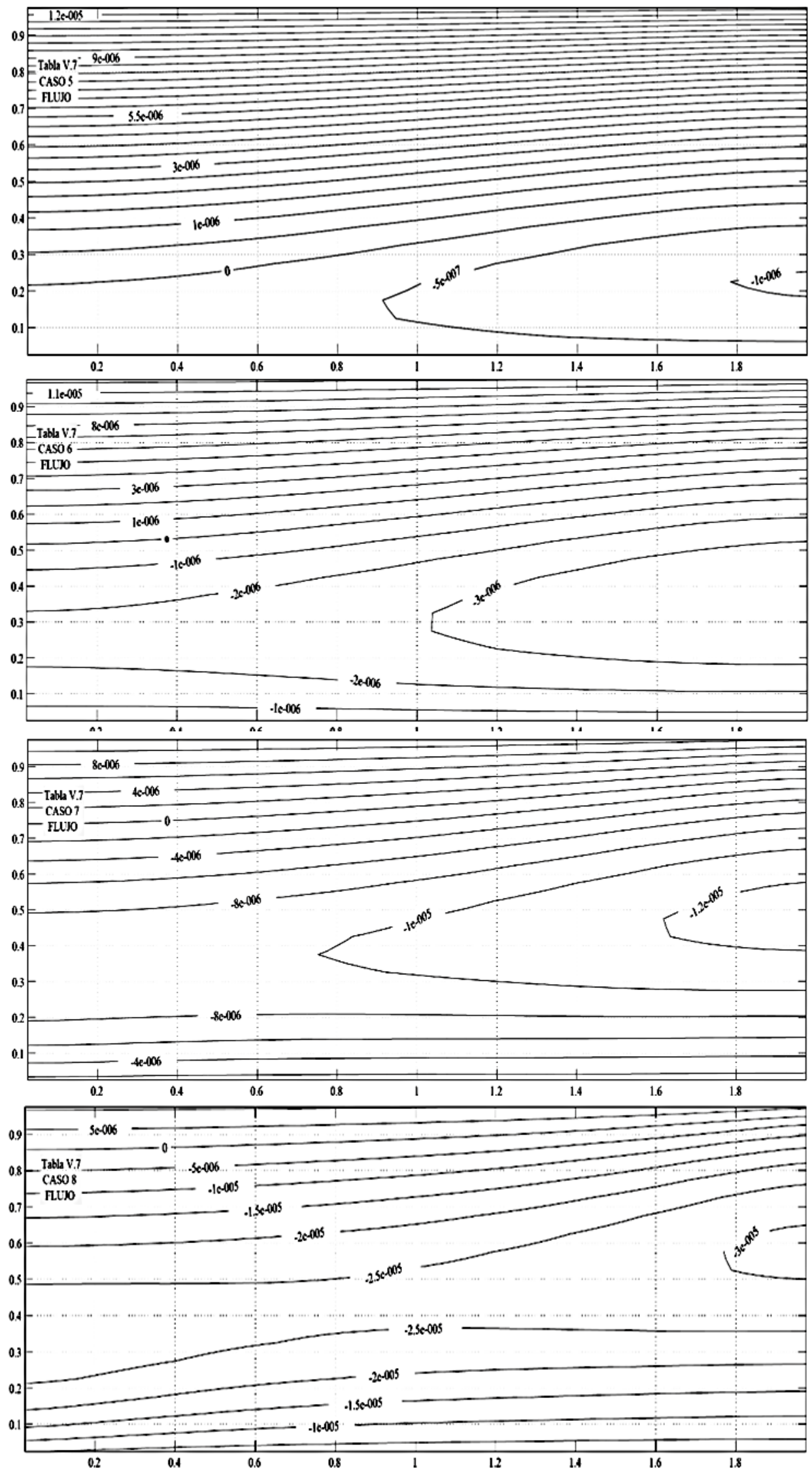

Figura V.14b Patrones de flujo. $\pi_{\mathrm{d}, 1}: 0.05,0,5,0.8,1,3,5,10$ y 20, $\pi_{\mathrm{d}, 2}=\pi_{\mathrm{d}, 3}=\pi_{\mathrm{d}, 4}=1$ 


\section{V.2.2.2 Influencia del monomio $\pi_{\mathrm{d}, 3}=\mathbf{D}_{\mathbf{x}} /\left(\operatorname{Vamb}^{*} \mathbf{L}\right)$}

Ya sabemos que este monomio compara efectos de difusividad horizontal y flujo regional. Para valores de $\pi_{\mathrm{d}, 3}$ por encima de la unidad prepondera el efecto de transporte difusivo mientras que para valores por debajo este efecto es despreciable frente al efecto del arrastre horizontal.

Para modificar el valor de $\pi_{\mathrm{d}, 3}$ en un rango similar al del caso anterior, podemos optar por modificar $\mathrm{D}_{\mathrm{x}}$ y corregir $\mathrm{D}_{\mathrm{y}}$ para mantener el valor $\pi_{\mathrm{d}, 4}=1$, o por modificar $\mathrm{vamb}_{\mathrm{a}}$ y corregir $\mu$ para mantener el valor $\pi_{\mathrm{d}, 1}=1$.

La Tabla V.8 muestra el listado de casos simulados que cubren el rango $\pi_{\mathrm{d}, 3}=$ 0.05, 0.1, 0,2, 0,5, 1, 3, 5 y 20. Las Figuras V.15a-b y V.16a-b muestran los patrones de concentración y función de corriente, respectivamente, resultantes de la simulación con FATSIM.

Tabla V.8 Casos para el estudio de la influencia de $\pi_{\mathrm{d}, 3}$

\begin{tabular}{|c|c|c|c|c|c|}
\hline Caso & $\mathbf{D}_{\mathbf{x}}$ & $\boldsymbol{\pi}_{\mathrm{d}, \mathbf{3}}$ & $\boldsymbol{\pi}_{\mathrm{d}, \mathbf{1}}$ & $\boldsymbol{\pi}_{\mathrm{d}, \mathbf{2}}$ & $\boldsymbol{\pi}_{\mathrm{d}, \mathbf{4}}$ \\
\hline $\mathbf{1}$ & $3,7714 \mathrm{E}-06$ & 0,05 & 1 & 1 & 1 \\
\hline $\mathbf{2}$ & $7,5428 \mathrm{E}-06$ & 0,1 & 1 & 1 & 1 \\
\hline $\mathbf{3}$ & $1,5086 \mathrm{E}-05$ & 0,2 & 1 & 1 & 1 \\
\hline $\mathbf{4}$ & $3,7714 \mathrm{E}-05$ & 0,5 & 1 & 1 & 1 \\
\hline 5 & $7,5428 \mathrm{E}-05$ & 1 & 1 & 1 & 1 \\
\hline $\mathbf{6}$ & 0,00022628 & 3 & 1 & 1 & 1 \\
\hline $\mathbf{7}$ & 0,00037714 & 5 & 1 & 1 & 1 \\
\hline $\mathbf{8}$ & 0,00150856 & 20 & 1 & 1 & 1 \\
\hline
\end{tabular}

La concentración, para pequeños valores de $\pi_{\mathrm{d}, 3}$ frente a la unidad, produce como era de esperar un patrón cuyas líneas se concentran en el borde marino merced a la baja difusividad frente al empuje regional. Pero las líneas quedan inclinadas por el balance equilibrado entre la flotación y el arrastre.

Al crecer $\pi_{\mathrm{d}, 3}$ y acercarse a la unidad, el patrón se ensancha manteniendo su inclinación, hasta que sus isolíneas cubren casi todo el dominio. Finalmente, para valores de $\pi_{\mathrm{d}, 3}$ superiores a la unidad el efecto preponderante de la difusividad frente a todo (los efectos de arrastre y los de flotación, que continúan siendo equilibrados), produce una 
Capítulo V. Influencia de los grupos discriminados en los patrones del problema de Henry

distribución muy regular con isolíneas igualmente distanciadas, verticales, que cubren la totalidad del acuífero.

El patrón de líneas de corriente, Figura V.16a-b, para valores muy pequeños de $\pi_{\mathrm{d}, 3}$, las fuerzas de arrastre y flotación, equilibradas, permiten que cierta cantidad de agua salada entre en el acuífero procedente del mar. Los flujos se mezclan en dicha zona dando lugar a una débil recirculación de agua salada que entra (por la parte inferior de la frontera) y sale (por la superior) bajo la línea divisoria. Con todo, el agua dulce entrante del continente es impulsada hacia arriba por el mencionado flujo salado de recirculación inclinando ligeramente las líneas de corriente conforme se acercan a la frontera del mar.

La región de recirculación marcada por la divisoria disminuye al aumentar el efecto difusivo y desaparece antes de que se equilibren las fuerzas difusivas y de arrastre continental.

Para valores crecientes de $\pi_{\mathrm{d}, 3}$, por encima de la unidad, merced que la salinización ocasionada por la difusión, que se extiende hacia el interior del acuífero desde el mar, y a que las fuerzas continentales de arrastre y las de flotación (equilibradas entre sí) pueden considerarse despreciables frente a la difusión, el patrón de corriente está formado por líneas horizontales regularmente distribuidas que cubren todo el dominio.

Vuelve a llamar la atención, y en este caso de manera más acusada, el desacoplo entre la extensión horizontal de la línea divisoria (medida desde su pie hasta la frontera del mar) y la definida por el patrón de concentraciones (extensión de la región parcialmente salada medida en el fondo del acuífero), que en el problema patrón eran casi idénticas.

El estudio de las variaciones de $\pi_{\mathrm{d}, 3}$ reporta una solución que no sólo desacopla estas longitudes sino que muestra unas tendencias 'aparentemente’ inesperadas: ¡la recirculación de agua salada en la región cercana al mar bajo la línea divisoria, disminuye al aumentar la salinidad global del acuífero! Una consecuencia más de la complejidad del problema merced al acoplamiento de sus ecuaciones de gobierno en el caso más general en el que todos los balances son del mismo orden de magnitud y por tanto ningún término de las mismas puede despreciarse para simplificar el problema. 
Capítulo V. Influencia de los grupos discriminados en los patrones del problema de Henry
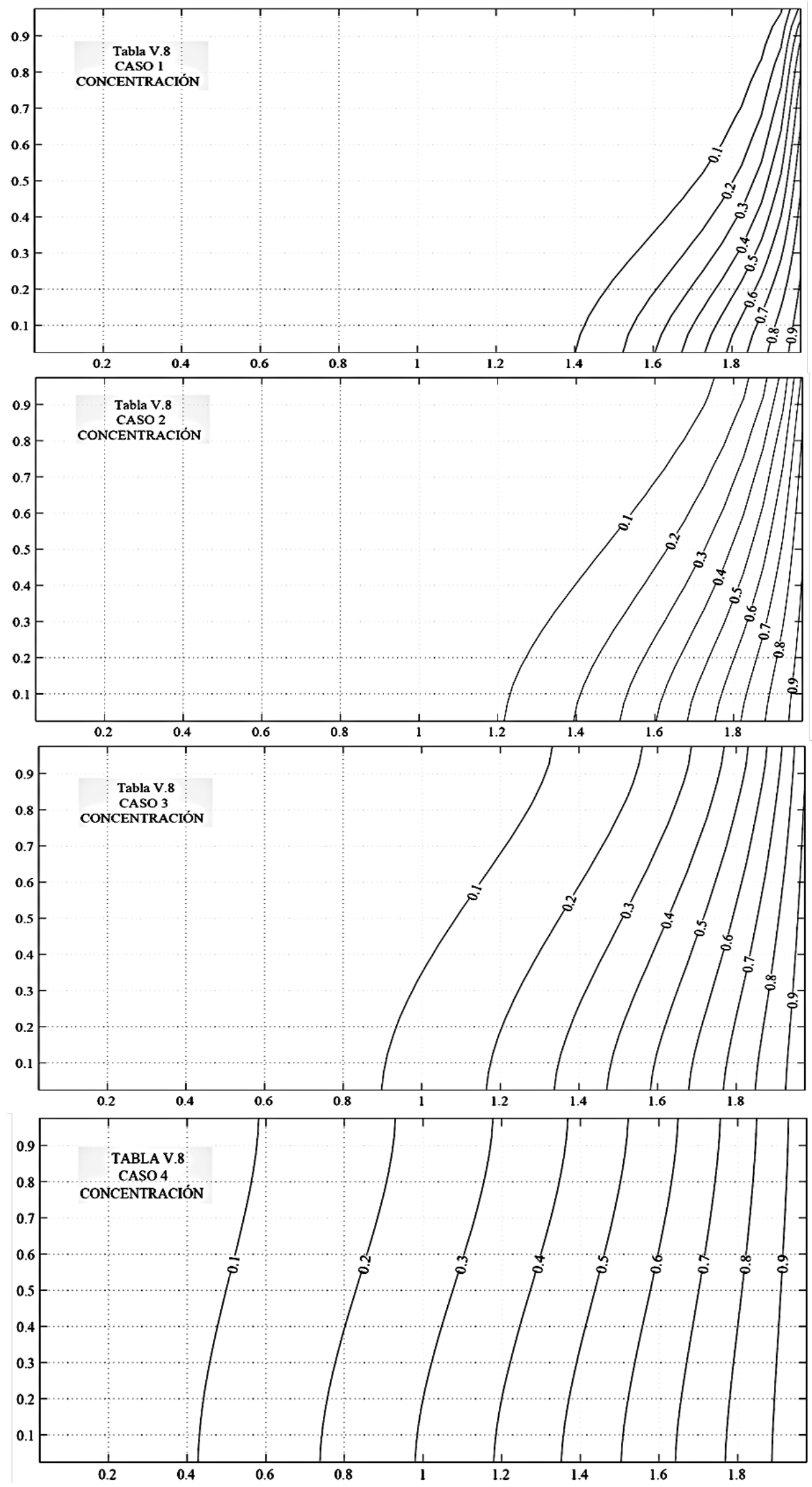

Figura V.15a Patrones de concentración. $\pi_{\mathrm{d}, 3}=.05,0.1,0,2,0,5,1,3,5$ y 20,

$$
\pi_{\mathrm{d}, 1}=\pi_{\mathrm{d}, 2}=\pi_{\mathrm{d}, 4}=1
$$


Capítulo V. Influencia de los grupos discriminados en los patrones del problema de Henry
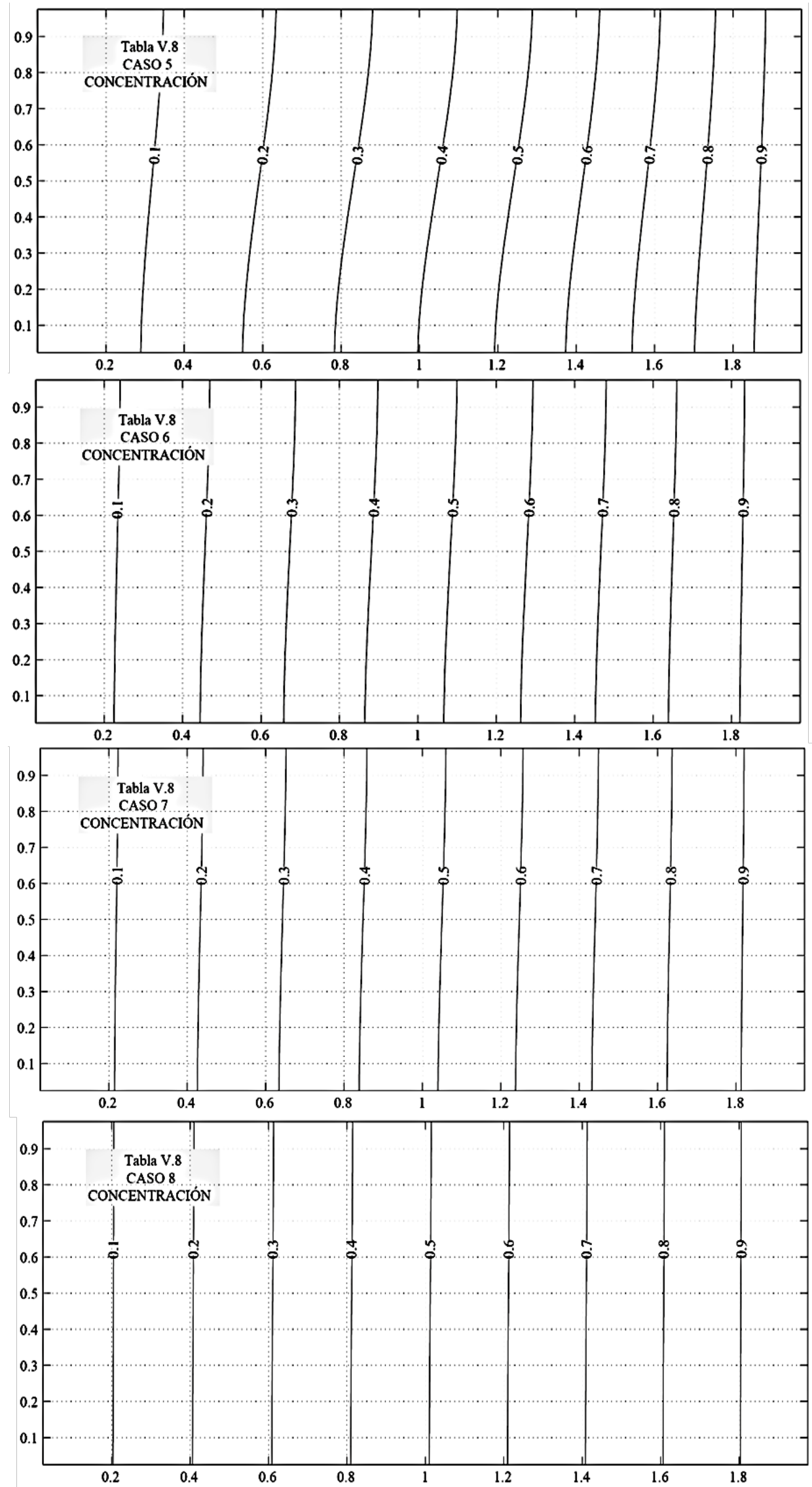

Figura V.15b Patrones de concentración. $\pi_{\mathrm{d}, 3}$ : 0.05, 0.1, 0,2, 0,5, 1, 3, 5 y 20, $\pi_{\mathrm{d}, 1}=\pi_{\mathrm{d}, 2}=\pi_{\mathrm{d}, 4}=1$ 
Capítulo V. Influencia de los grupos discriminados en los patrones del problema de Henry
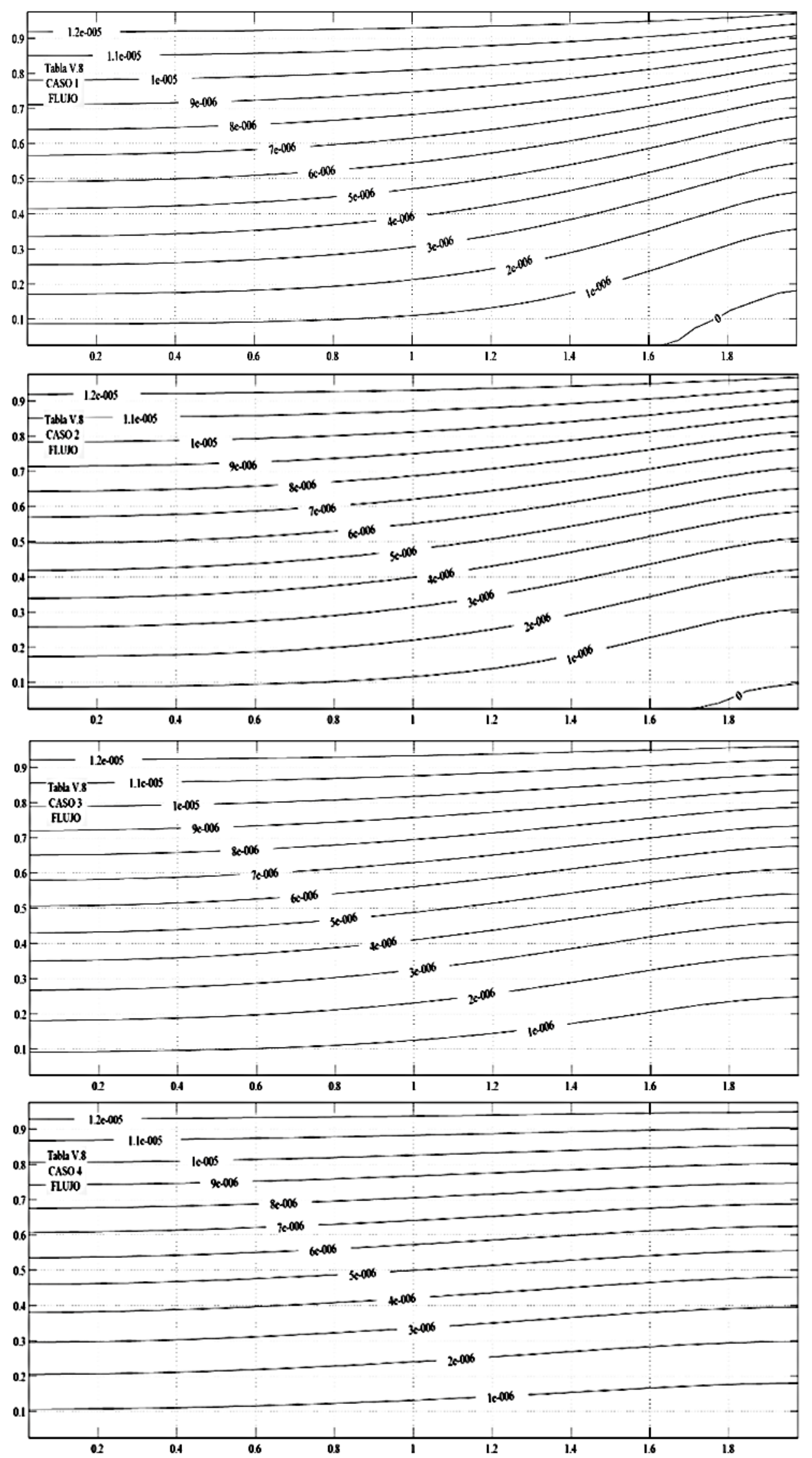

Figura V.16a Patrones de flujo. $\pi_{\mathrm{d}, 3}: 0.05,0.1,0,2,0,5,1,3,5$ y 20, $\pi_{\mathrm{d}, 1}=\pi_{\mathrm{d}, 2}=\pi_{\mathrm{d}, 4}=1$ 
Capítulo V. Influencia de los grupos discriminados en los patrones del problema de Henry
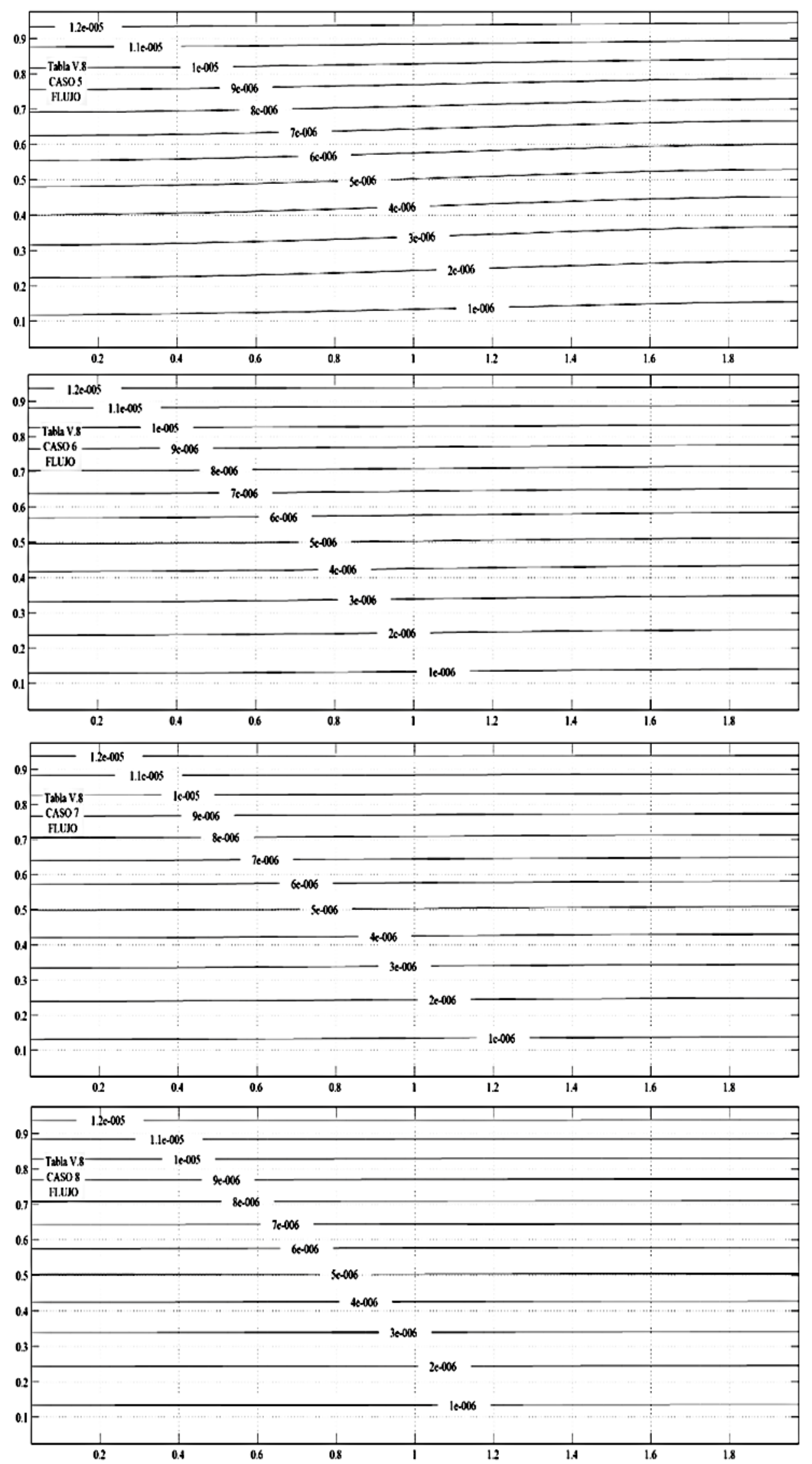

Figura V.16b Patrones de flujo. $\pi_{\mathrm{d}, 3}: 0.05,0.1,0,2,0,5,1,3,5$ y 20, $\pi_{\mathrm{d}, 1}=\pi_{\mathrm{d}, 2}=\pi_{\mathrm{d}, 4}=1$ 


\section{V.2.2.3 Influencia del monomio $\pi_{\mathrm{d}, 2}=\left(\mathbf{k}_{\mathrm{x}} \mathbf{H}^{2}\right) /\left(\mathbf{k}_{\mathbf{y}} \mathbf{L}^{2}\right)$}

Este monomio recoge el efecto de la anisotropía en la conductividad hidráulica corregida por las dimensiones del acuífero. Para valores por encima de la unidad prepondera la conductividad hidráulica horizontal frente a la vertical y viceversa. Para cambiar su valor basta con modificar $\mathrm{k}_{\mathrm{x}}$, un parámetro que sólo interviene en este grupo. Los casos elegidos para la simulación cubren el rango de valores $\pi_{\mathrm{d}, 2}: 0.05,0,5,1,2,5$, 8, 10 y 20, con $\pi_{\mathrm{d}, 1}=\pi_{\mathrm{d}, 3}=\pi_{\mathrm{d}, 4}=1$, Tabla V.9.

Las Figuras V.17a-b y V.18a-b muestran los patrones de concentración y función de corriente resultantes.

Tabla V.9 Casos para el estudio de la influencia de $\pi_{\mathrm{d}, 3}$

\begin{tabular}{|c|c|c|c|c|c|}
\hline Caso & $\mathbf{K}_{\mathbf{x}}$ & $\boldsymbol{\pi}_{\mathrm{d}, \mathbf{2}}$ & $\boldsymbol{\pi}_{\mathrm{d}, \mathbf{1}}$ & $\boldsymbol{\pi}_{\mathrm{d}, \mathbf{3}}$ & $\boldsymbol{\pi}_{\mathrm{d}, \mathbf{4}}$ \\
\hline $\mathbf{1}$ & $2,0408 \mathrm{E}-10$ & 0,05 & 1 & 1 & 1 \\
\hline $\mathbf{2}$ & $2,0408 \mathrm{E}-09$ & 0,5 & 1 & 1 & 1 \\
\hline $\mathbf{3}$ & $3,2653 \mathrm{E}-09$ & 0,8 & 1 & 1 & 1 \\
\hline $\mathbf{4}$ & $\mathbf{4 , 0 8 1 6 \mathrm { E } - 0 9}$ & $\mathbf{1}$ & $\mathbf{1}$ & $\mathbf{1}$ & $\mathbf{1}$ \\
\hline $\mathbf{5}$ & $1,2245 \mathrm{E}-08$ & 3 & 1 & 1 & 1 \\
\hline $\mathbf{6}$ & $2,0408 \mathrm{E}-08$ & 5 & 1 & 1 & 1 \\
\hline $\mathbf{7}$ & $4,0816 \mathrm{E}-08$ & 10 & 1 & 1 & 1 \\
\hline $\mathbf{8}$ & $8,1632 \mathrm{E}-08$ & 20 & 1 & 1 & 1 \\
\hline
\end{tabular}

Valores pequeños de la permeabilidad horizontal $\left(\pi_{\mathrm{d}, 2}<1\right)$, con fuerzas de flotación y de arrastre equilibradas, aumentan en primer lugar, globalmente, las presiones del acuífero merced al establecimiento de un flujo regional constante en el contorno. Por otro lado, el equilibrio de la difusividad y el arrastre de agua dulce hacen que el acuífero se salinice casi completamente y de forma regular, con isolíneas de concentración prácticamente verticales. Este patrón se mantiene para valores creciente de $\pi_{\mathrm{d}, 2}$ hasta que el efecto las permeabilidades anisótropas (corregidas por la longitud) se equilibran $\left(\pi_{\mathrm{d}, 2}\right.$ cercano a la unidad). Las líneas de concentración se inclinan por los efectos de flotación y arrastre (que ahora sí se manifiestan) y se produce el fenómeno de recirculación en la frontera salina con la emergencia de una divisoria rápidamente creciente. Finalmente, valores de $\pi_{\mathrm{d}, 2}$ superiores a la unidad, con mayor influencia de la permeabilidad horizontal frente a la vertical, permiten la entrada de agua procedente de la frontera marina por el fondo del acuífero, Esta recirculación de agua salada empuja el agua dulce hacia arriba y distorsiona al patrón de concentraciones que inclina en mayor grado sus isolíneas sin dejar 
Capítulo V. Influencia de los grupos discriminados en los patrones del problema de Henry

de cubrir la totalidad del dominio. Sin duda, la longitud L es determinante en los patrones ya que el agua salada entrante procedente del mar no puede mantener su salinidad en el recorrido hacia el continente pues se lo impide la condición de frontera de agua dulce en la frontera continental. El resultado es una concentración de líneas en el fondo de dicha frontera (algo similar a lo que ocurre en la zona de descarga).

En cuanto al patrón de flujo, Figura V.18a-b, la preponderancia de la permeabilidad hidráulica vertical frente a la horizontal $\left(\pi_{\mathrm{d}, 2}<1\right)$, con equilibrio de efectos difusivos y de arrastre $\left(\pi_{\mathrm{d}, 1}=1\right)$, produce un patrón de isolíneas casi horizontales sin posibilidad de entrada de agua salada al acuífero procedente del mar. La sal, sin embargo, como hemos mencionado anteriormente, se difunde y contamina la totalidad del acuífero. No existe divisoria para valores del monomio inferiores a la unidad, $\pi_{\mathrm{d}, 2}<1$ ), lo que manifiesta una clara independencia de su emergencia con el hecho de que una parte mayor o menor del acuífero se haya contaminado de sal. Para $\pi_{\mathrm{d}, 2}=1$, todos los balances están equilibrados y los patrones ya se conocen: líneas de concentración y flujo regularmente distribuidas en todo el escenario.

Al seguir creciendo $\pi_{\mathrm{d}, 2}$ por encima de la unidad y mantener el equilibrio entre efectos de flotación y arrastre, por un lado, y entre difusión y arrastre, por otro, el aumento de la conductividad hidráulica horizontal frente a la vertical (naturalmente compensada por la geometría) permite la entrada de agua salada procedente del mar por el borde inferior de su frontera. Esta entrada distorsiona las líneas de corriente confinando el flujo de agua dulce en la región superior. Al seguir incrementándose $\pi_{\mathrm{d}, 2}$ el patrón se desdobla en tres regiones: i) la de flujo de agua dulce continental, confinado por encima de la divisoria, casi horizontal, desplazada hacia la frontera superior del dominio, ii) una de circulación de agua de mar procedente de éste que se dirige horizontalmente a la zona continental por el fondo del acuífero, y iii) una región entre las anteriores que transporta agua, también horizontalmente, desde el continente hacia el mar. En las tres regiones anteriores el agua cambia paulatinamente su salinidad a lo largo del trayecto. Por otro lado, el flujo de agua neta tanto en la frontera izquierda como en la derecha es el impuesto por la condición de contorno, Vamb. Sin duda, se trata quizás de un escenario algo irreal merced a la elección de una longitud L demasiado pequeña para escenarios con estos valores de los parámetros. 
Capítulo V. Influencia de los grupos discriminados en los patrones del problema de Henry
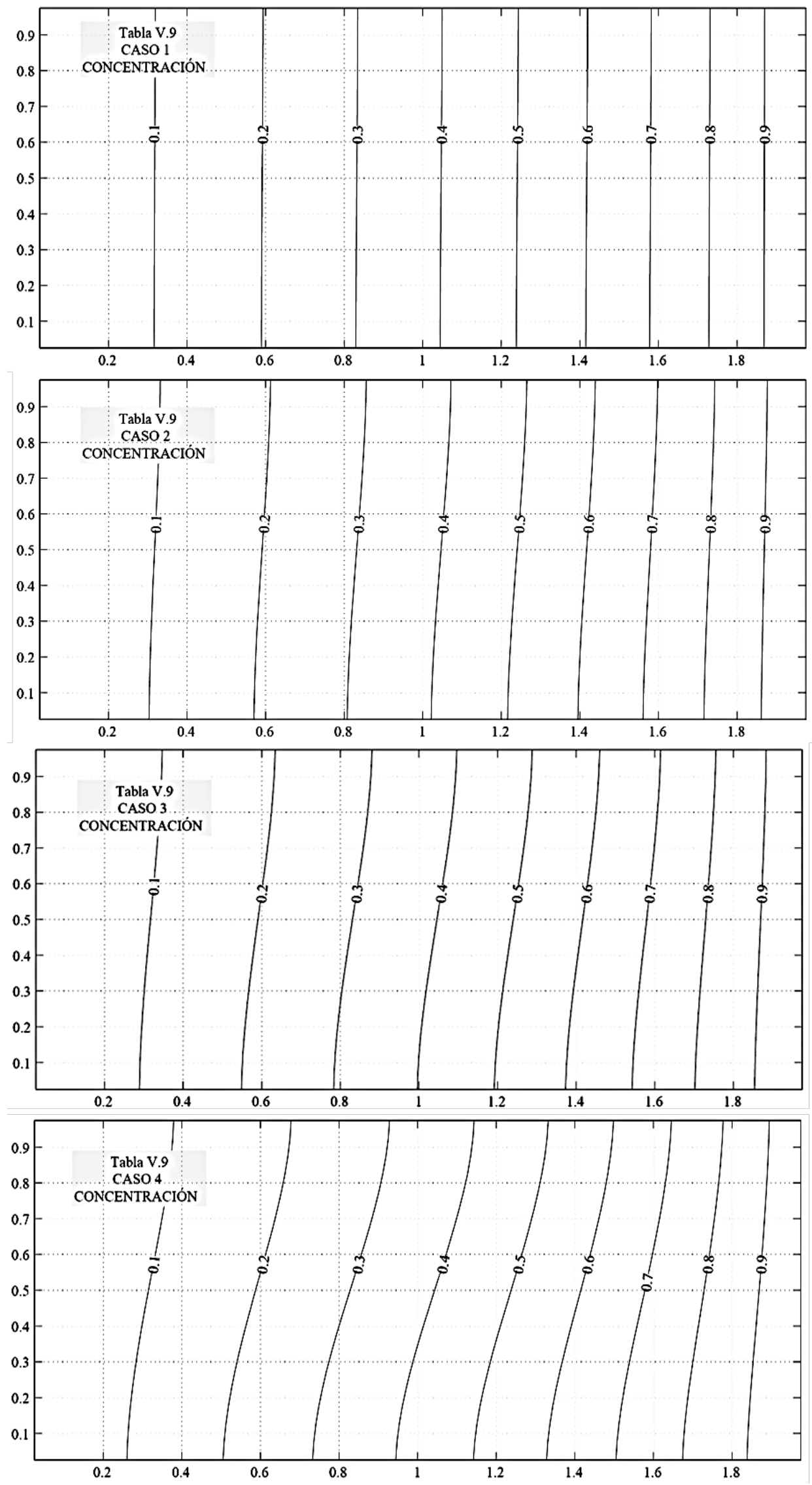

Figura V.17a Patrones de concentración. $\pi_{\mathrm{d}, 2}: 0.05,0,5,1,2,5,8,10$ y $20, \pi_{\mathrm{d}, 1}=\pi_{\mathrm{d}, 3}=\pi_{\mathrm{d}, 4}=1$ 
Capítulo V. Influencia de los grupos discriminados en los patrones del problema de Henry
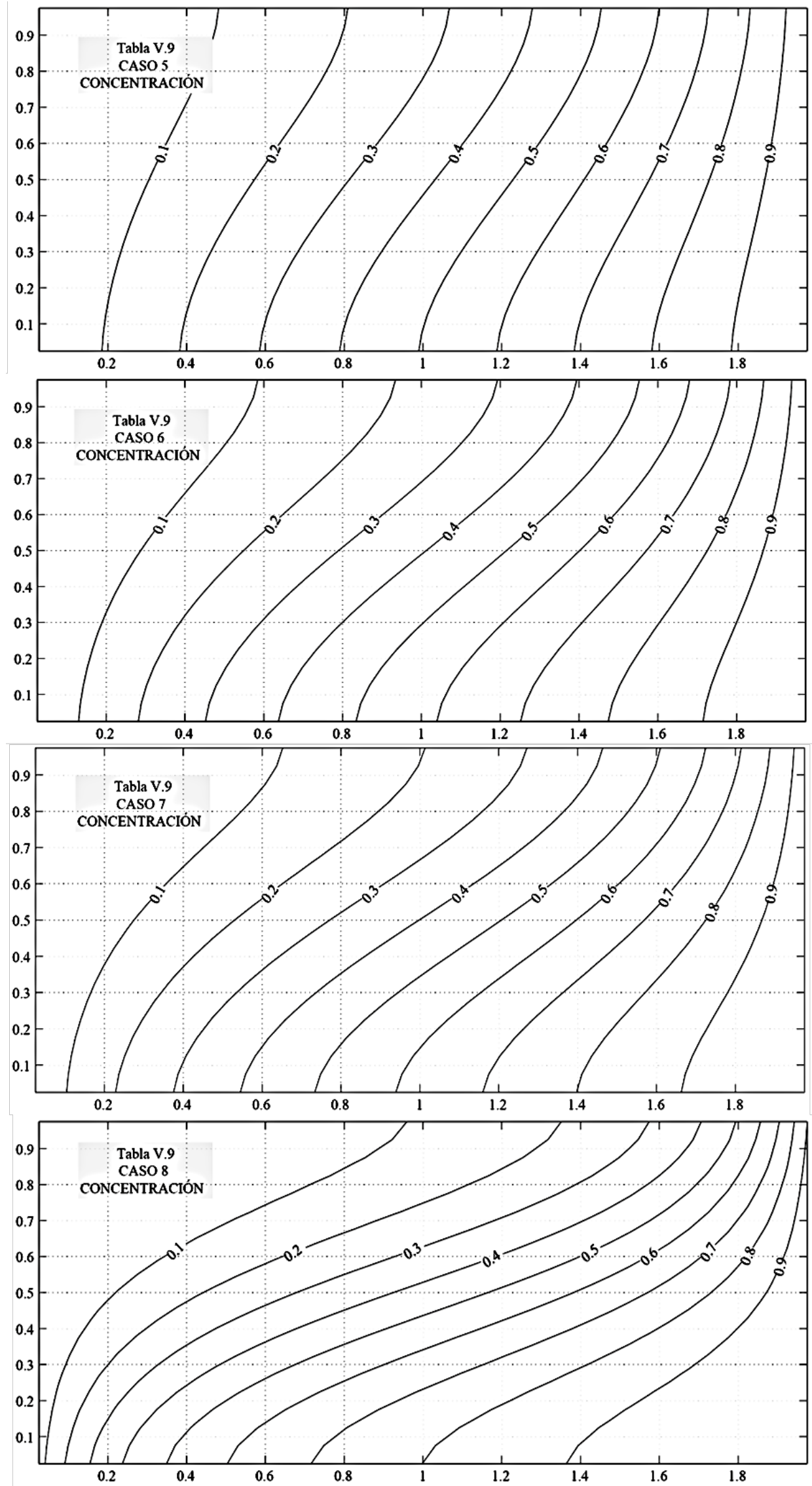

Figura V.17b Patrones de concentración. $\pi_{\mathrm{d}, 2}$ : 0.05, 0,5, 1, 2, 5, 8, 10 y 20, $\pi_{\mathrm{d}, 1}=\pi_{\mathrm{d}, 3}=\pi_{\mathrm{d}, 4}=1$ 
Capítulo V. Influencia de los grupos discriminados en los patrones del problema de Henry
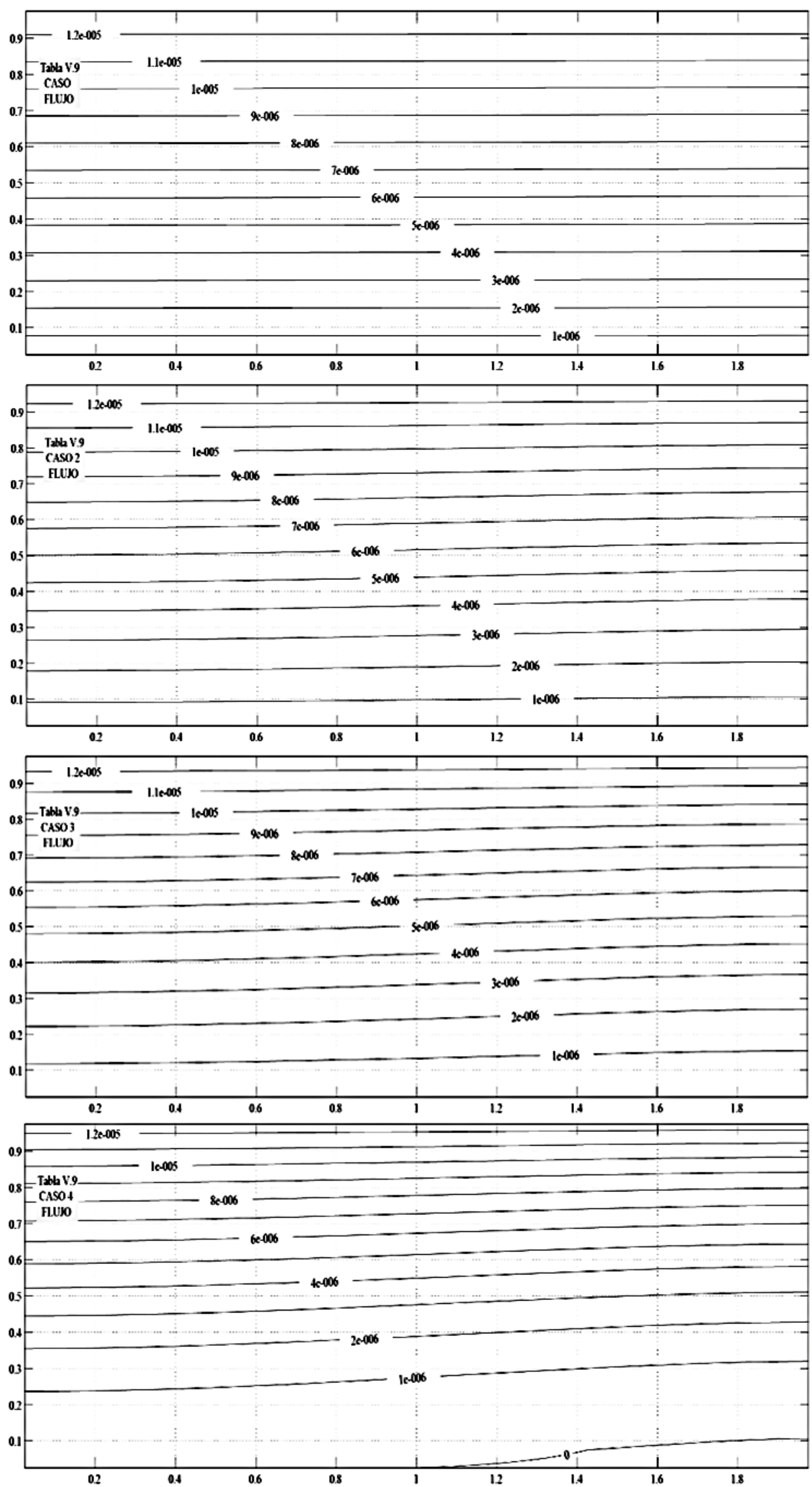

Figura V.18a Patrones de flujo. $\pi_{\mathrm{d}, 2}: 0.05,0,5,1,2,5,8,10$ y 20, $\pi_{\mathrm{d}, 1}=\pi_{\mathrm{d}, 3}=\pi_{\mathrm{d}, 4}=1$ 
Capítulo V. Influencia de los grupos discriminados en los patrones del problema de Henry
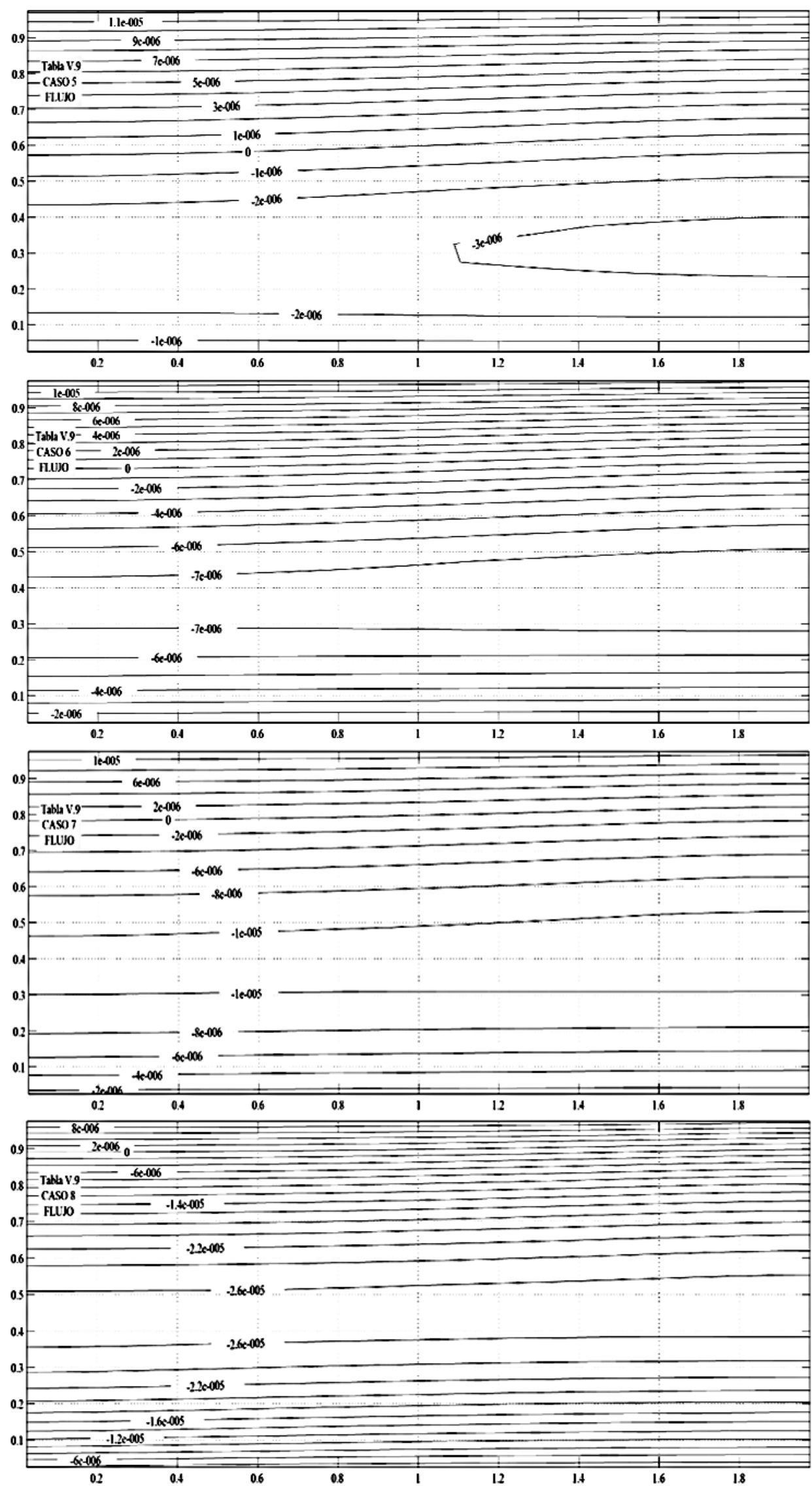

Figura V.18b Patrones de flujo. $\pi_{\mathrm{d}, 2}: 0.05,0,5,1,2,5,8,10$ y 20, $\pi_{\mathrm{d}, 1}=\pi_{\mathrm{d}, 3}=\pi_{\mathrm{d}, 4}=1$ 


\section{V.2.2.4 Influencia del monomio $\pi_{\mathrm{d}, 4}=\left(\mathrm{D}_{\mathrm{x}} \mathrm{H}^{2}\right) /\left(\mathrm{D}_{\mathrm{y}} \mathrm{L}^{2}\right)$}

Este monomio recoge el efecto de la anisotropía en la difusividad efectiva corregida por las dimensiones del acuífero. Para valores por encima de la unidad prepondera la difusividad horizontal frente a la vertical y viceversa. Para cambiar su valor basta con modificar $\mathrm{D}_{\mathrm{y}}$, un parámetro que sólo interviene en este grupo. Los casos estudiados, mostrados en la Tabla V.10, cubren el rango de valores $\pi_{\mathrm{d}, 4}: 0.05,0,5,1,2,5$, 8, 10 y 20, $\pi_{\mathrm{d}, 1}=\pi_{\mathrm{d}, 2}=\pi_{\mathrm{d}, 3}=1$. Son casos relativamente irreales, aunque de interés académico, por la falta de correlación entre la permeabilidad y la difusividad efectiva anisótropas.

Las Figuras V.19a-b y V.20a-b muestran los patrones de concentración y función de corriente resultantes de la simulación.

Tabla V.10 Casos para el estudio de la influencia de $\pi_{\mathrm{d}, 4}$

\begin{tabular}{|c|c|c|c|c|c|}
\hline Caso & $\mathbf{D}_{\mathrm{y}}$ & $\boldsymbol{\pi}_{\mathrm{d}, \mathbf{4}}$ & $\boldsymbol{\pi}_{\mathrm{d}, \mathbf{1}}$ & $\boldsymbol{\pi}_{\mathrm{d}, \mathbf{2}}$ & $\boldsymbol{\pi}_{\mathrm{d}, \mathbf{3}}$ \\
\hline $\mathbf{1}$ & 0,00037714 & 0,05 & 1 & 1 & 1 \\
\hline $\mathbf{2}$ & $3,7714 \mathrm{E}-05$ & 0,5 & 1 & 1 & 1 \\
\hline $\mathbf{3}$ & $\mathbf{1 , 8 8 5 7 E - 0 5}$ & $\mathbf{1}$ & $\mathbf{1}$ & $\mathbf{1}$ & $\mathbf{1}$ \\
\hline $\mathbf{4}$ & $9,4285 \mathrm{E}-06$ & 2 & 1 & 1 & 1 \\
\hline $\mathbf{5}$ & $3,7714 \mathrm{E}-06$ & 5 & 1 & 1 & 1 \\
\hline $\mathbf{6}$ & $2,3571 \mathrm{E}-06$ & $\mathbf{8}$ & 1 & 1 & 1 \\
\hline $\mathbf{7}$ & $1,8857 \mathrm{E}-06$ & 10 & 1 & 1 & 1 \\
\hline $\mathbf{8}$ & $9,4285 \mathrm{E}-07$ & 20 & 1 & 1 & 1 \\
\hline
\end{tabular}

El equilibrio de todas las fuerzas, de arrastre continental, de flotación y de difusión horizontal (resultante del valor unidad de los monomios $\pi_{\mathrm{d}, 1}=\pi_{\mathrm{d}, 2}=\pi_{\mathrm{d}, 3}$ ) conducen a un patrón muy regular para todo el rango de valores de $\pi_{\mathrm{d}, 4}$. Los cambios en la anisotropía de la difusividad sólo modifican ligeramente la inclinación de las líneas de concentración; para valores muy bajos de este monomio (que implican tanto una velocidad regional, $\pi_{\mathrm{d}, 3}$, como un arrastre, $\pi_{\mathrm{d}, 1}$, despreciables) las líneas se presentan casi verticales consecuencia de la elevada difusión vertical. El patrón es el de un problema de difusión pura horizontal, a pesar del despreciable valor de $D_{x}$ frente a $D_{y}$, donde $L$ no altera cualitativamente los patrones. Valores de $\pi_{\mathrm{d}, 4}$ elevados (efectos de $\mathrm{D}_{\mathrm{y}}$ despreciables frente a todas las fuerzas) emergen gradientes verticales de concentración aumentando la inclinación de las isolíneas. Finalmente, el patrón de concentraciones permanece prácticamente idéntico. 
Capítulo V. Influencia de los grupos discriminados en los patrones del problema de Henry
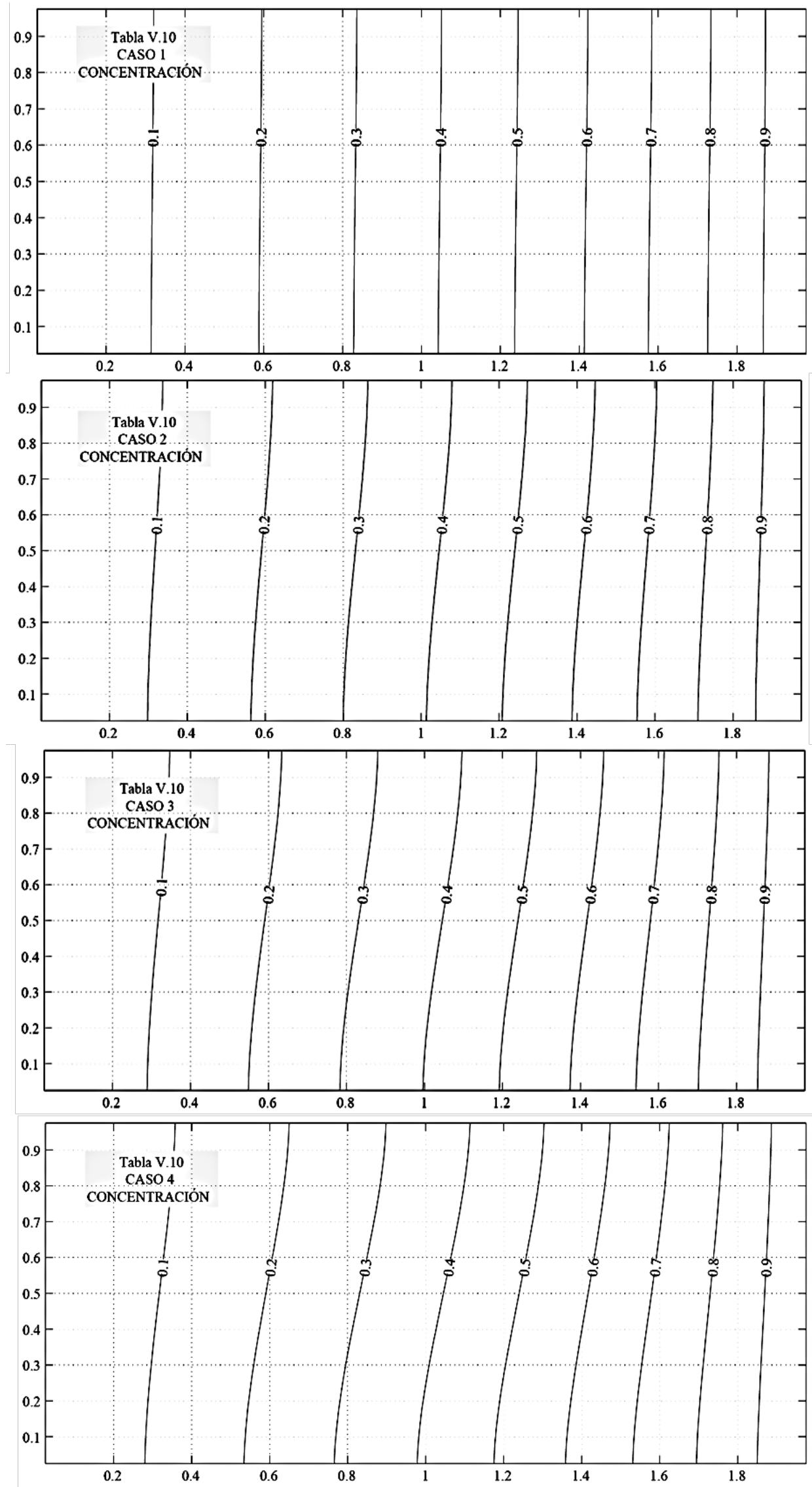

Figura V.19a Patrones de concentración. $\pi_{\mathrm{d}, 4}:$ 0.05, 0,5, 1, 2, 5, 8, 10 y 20, $\pi_{\mathrm{d}, 1}=\pi_{\mathrm{d}, 2}=\pi_{\mathrm{d}, 3}=1$ 
Capítulo V. Influencia de los grupos discriminados en los patrones del problema de Henry
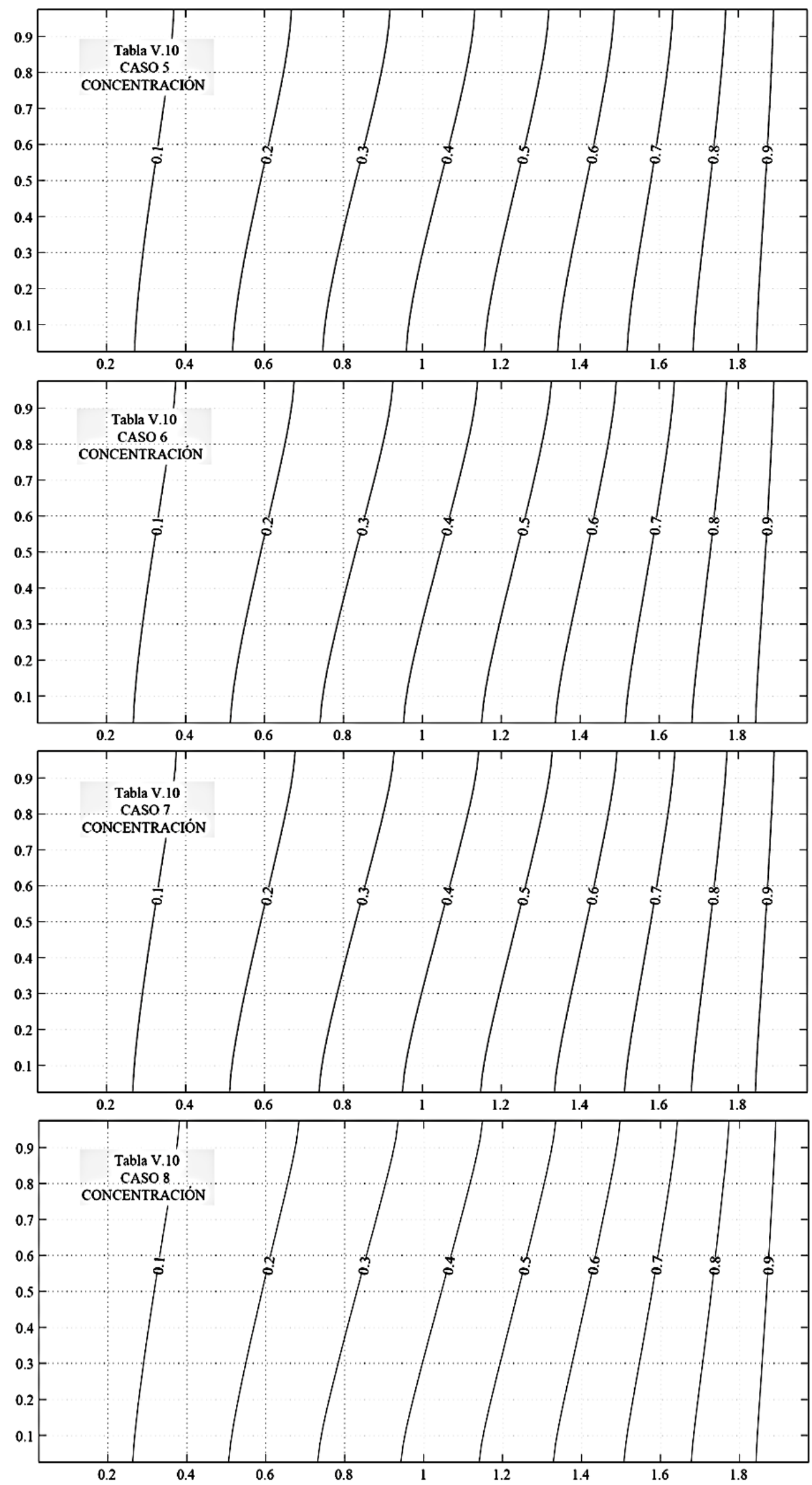

Figura V.19b Patrones de concentración. $\pi_{\mathrm{d}, 4}:$ 0.05, 0,5, 1, 2, 5, 8, 10 y 20, $\pi_{\mathrm{d}, 1}=\pi_{\mathrm{d}, 2}=\pi_{\mathrm{d}, 3}=1$ 
Capítulo V. Influencia de los grupos discriminados en los patrones del problema de Henry
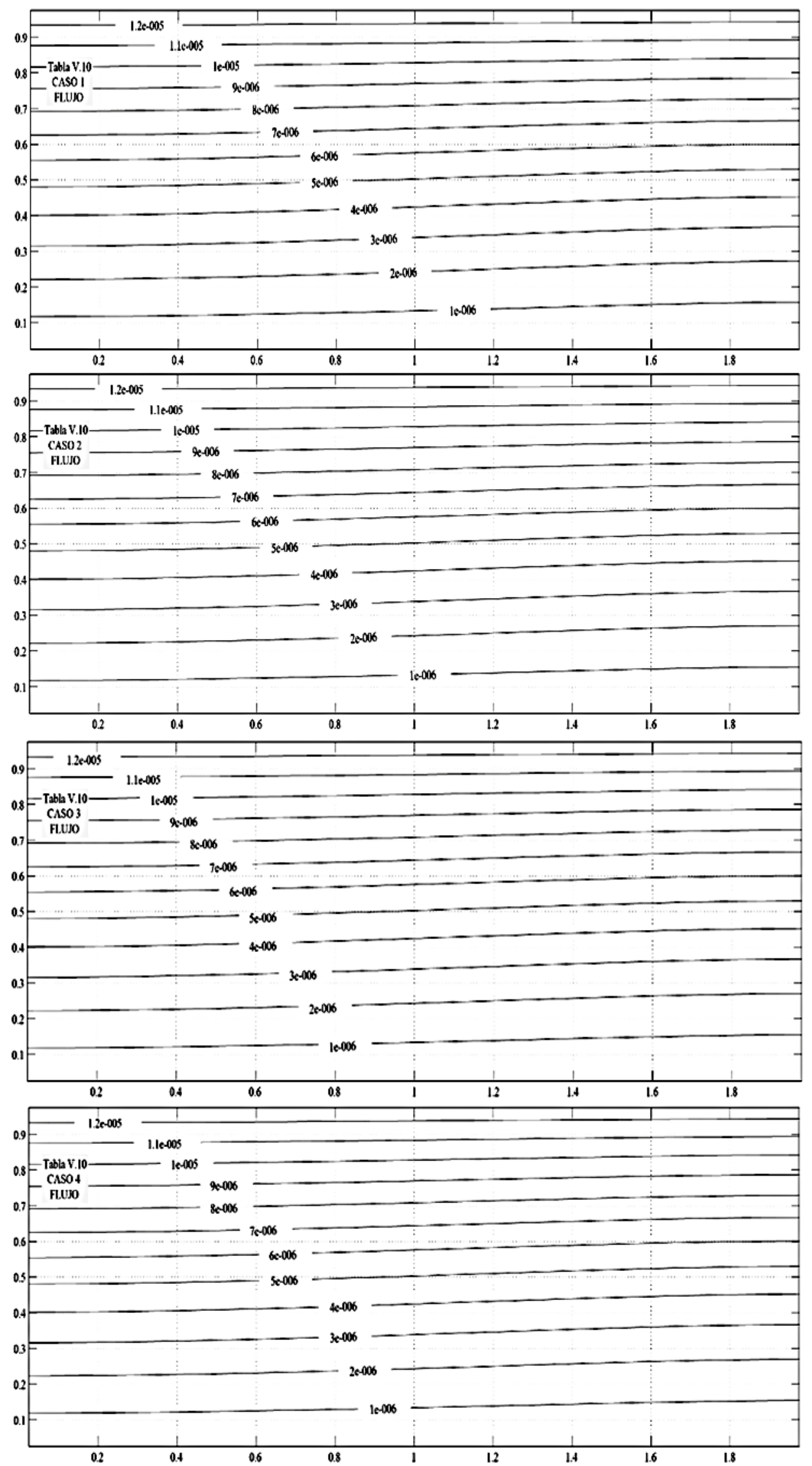

Figura V.20a Patrones de flujo. $\pi_{\mathrm{d}, 4}: 0.05,0,5,1,2,5,8,10$ y 20, $\pi_{\mathrm{d}, 1}=\pi_{\mathrm{d}, 2}=\pi_{\mathrm{d}, 3}=1$ 
Capítulo V. Influencia de los grupos discriminados en los patrones del problema de Henry
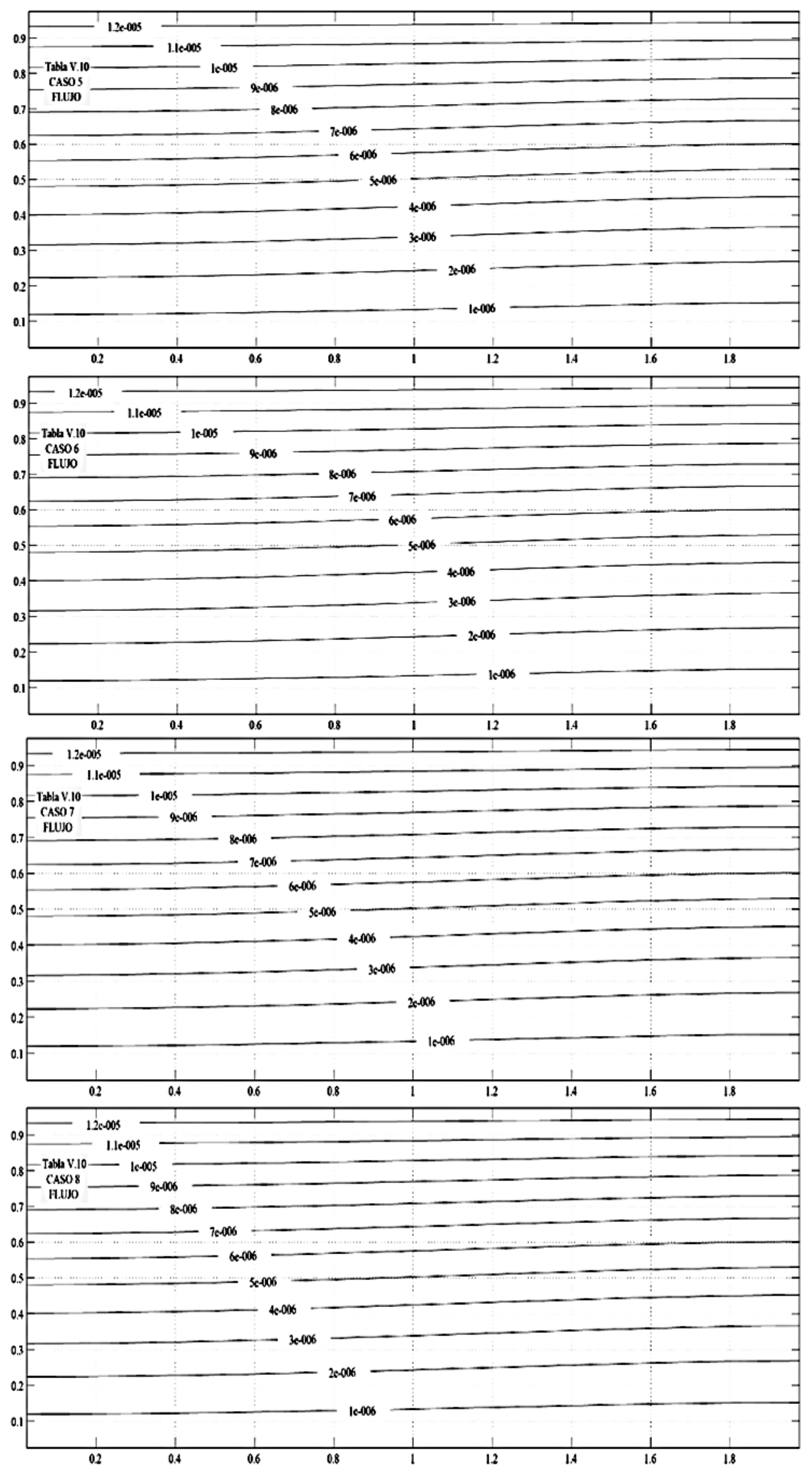

Figura V.20a Patrones de flujo. $\pi_{\mathrm{d}, 4}: 0.05,0,5,1$, 2, 5, 8, 10 y 20, $\pi_{\mathrm{d}, 1}=\pi_{\mathrm{d}, 2}=\pi_{\mathrm{d}, 3}=1$ 


\section{V.2.3 EVOLUCIÓN DE LAS CUÑAS EN MEDIOS ISÓTROPOS. INFLUENCIA DE LOS MONOMIOS Y DEL TAMAÑO DEL DOMINIO}

Estudiamos los tamaños de las cuñas de recirculación y difusión en escenarios isótropos de Henry, resultado de la influencia de los grupos adimensionales, bajo las siguientes hipótesis: i) profundidad constante del acuífero, ii) longitud del acuífero suficientemente grande como para asegurar que no influye en las posiciones de las cuñas.

A la vista de los resultados anteriores, de los conocimientos adquiridos y de las incompletas interpretaciones de ciertos aspectos que emergen para determinados valores de los grupos adimensionales, volvemos a replantearnos una cuestión trascendental en relación con las expresiones de estos grupos (discriminados o no): ¿qué papel juega realmente la longitud el acuífero en los patrones de solución del problema? Lo inmediato es, sencillamente, observar estos patrones en el problema original, variando este parámetro. Los patrones resultantes se muestran en las Figuras V.21a-b y V.22a-b. Para mejor apreciar los mismos se ha construido la Tabla V.11 en la que se dan las longitudes de los pies de las isolíneas c=0.1 y 0.9 así como la del pie de la divisoria de aguas, todos medidos desde el extremo inferior derecho del dominio. Puede apreciarse que valores de $\mathrm{L} \geq 1.5$, no alteran prácticamente nada estos pies, lo que indica que L no determina la forma de estos patrones por encima de este valor. Sí lo hace por debajo del mismo, principalmente en el pie de la línea divisoria que llega a tocar la frontera derecha induciendo una nueva recirculación de agua dulce en dicha frontera para $\mathrm{L}=0.8 \mathrm{y}$ haciendo que algunas de las líneas de corriente procedentes de la frontera salina lleguen al borde izquierdo y penetren en la región continental (escenarios no reales). Por otro lado, la línea de concentración menor $(c=0.1$ ) no se desplaza demasiado merced a la condición de contorno de concentración cero en la frontera continental.

Tabla V.11 Pies de las isolíneas de concentración c = 0.1 y 0.9 y pie de la línea divisoria para diferentes longitudes del acuífero en el problema de Henry

\begin{tabular}{|c|c|c|c|c|c|c|}
\hline $\mathbf{L}$ & $\mathbf{0 . 8}$ & $\mathbf{1}$ & $\mathbf{1 . 5}$ & $\mathbf{2}$ & $\mathbf{2 . 5}$ & $\mathbf{4}$ \\
\hline l (c=0.1) & 0.8 & 0.952 & 0.97 & 0.98 & 0.98 & 0.98 \\
\hline l (c=0.9) & 0.35 & 0.25 & 0.24 & 0.24 & 0.24 & 0.24 \\
\hline l (divisoria) & $>>1$ & $>1$ & 1.01 & 0.933 & 0.93 & 0.93 \\
\hline
\end{tabular}

La conclusión hace que nos replanteemos el monomio relación de aspecto (H/L) como un monomio independiente en el problema original de Henry: lo es, en efecto, para valores inferiores a (aproximadamente) 1.5 y no lo es para valores superiores a 1.5. 
Capítulo V. Influencia de los grupos discriminados en los patrones del problema de Henry
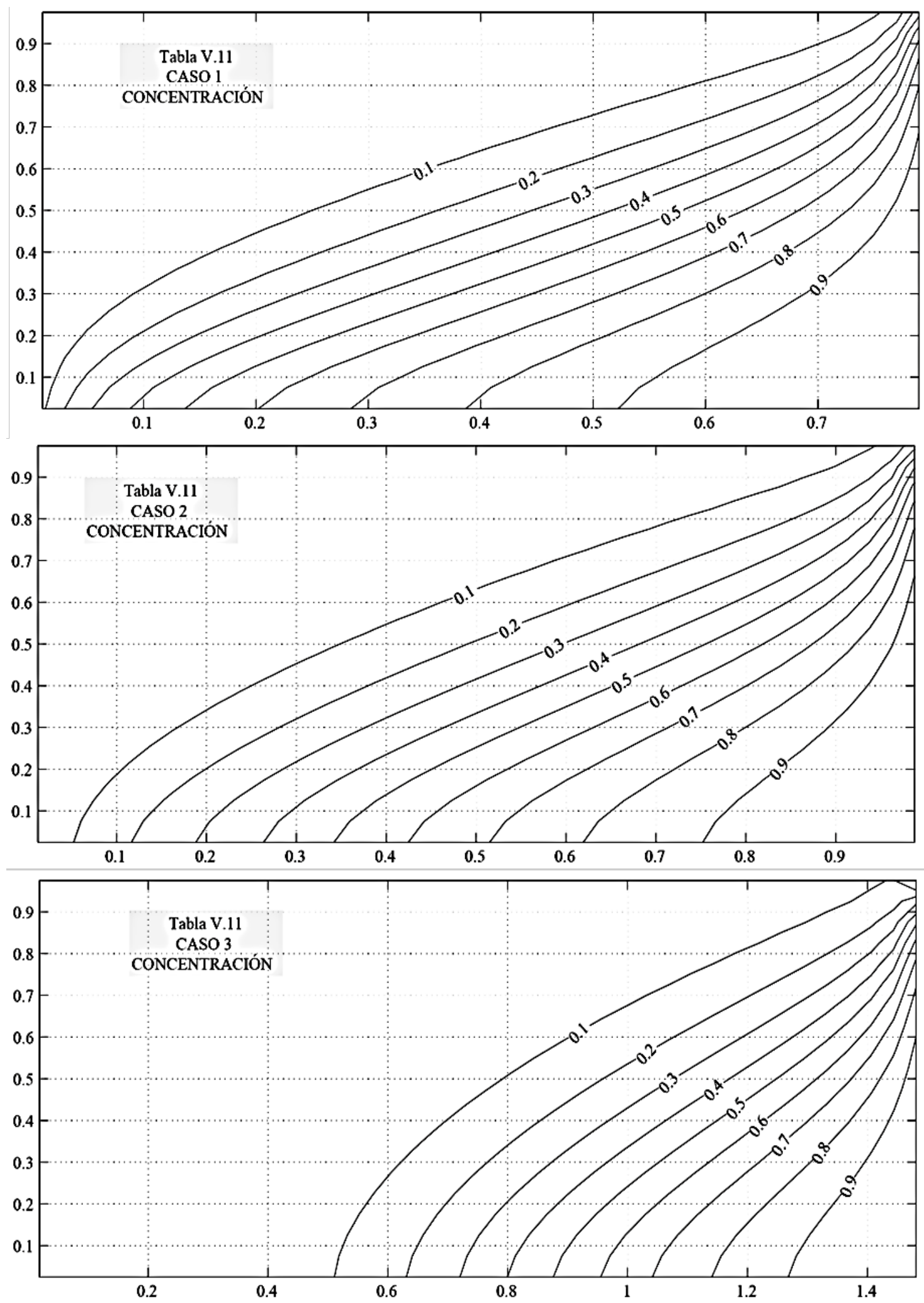

Figura V.21a Patrones de concentración del problema de Henry para L=0.8, 1 y 1.5 
Capítulo V. Influencia de los grupos discriminados en los patrones del problema de Henry
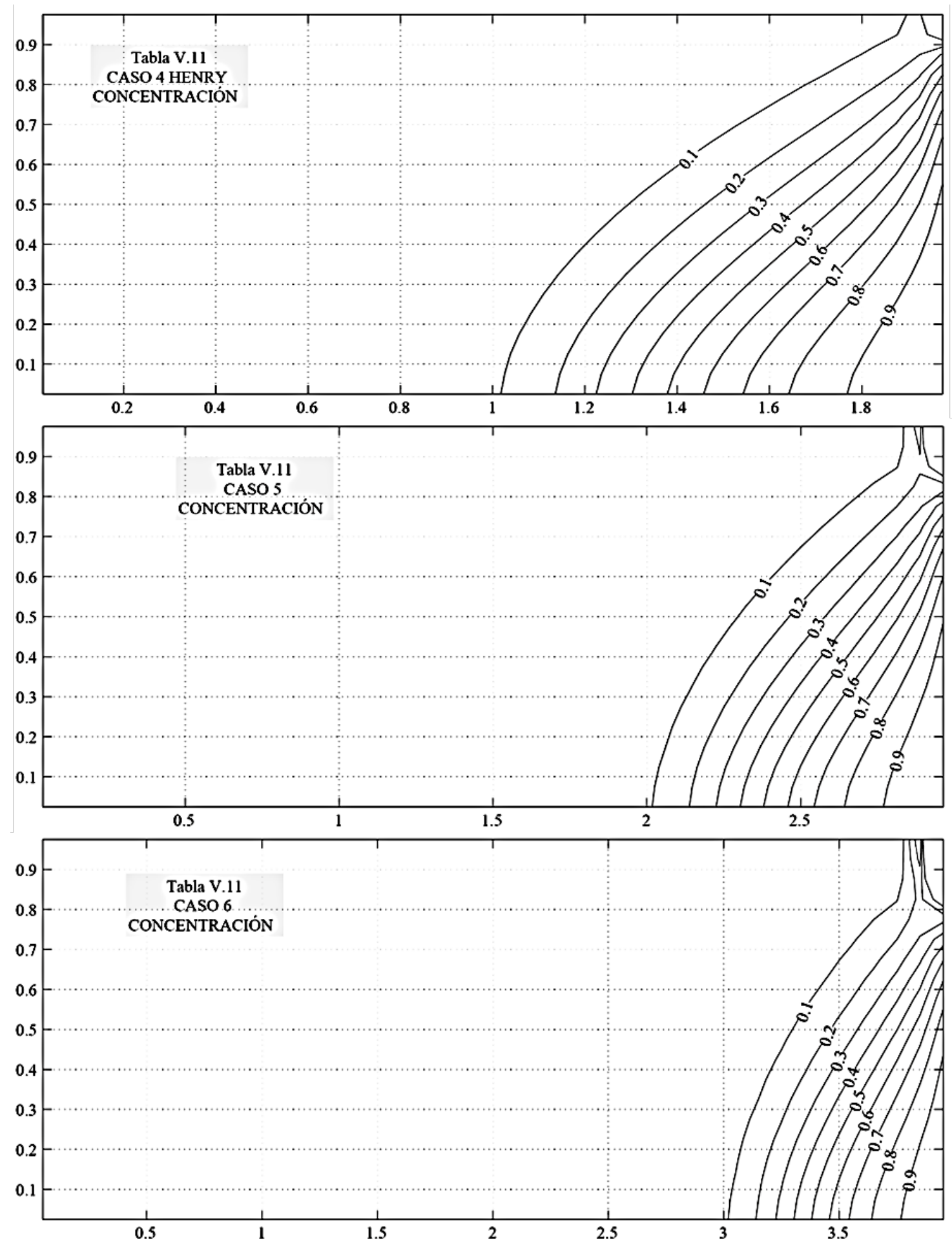

Figura V.21b Patrones de concentración del problema de Henry para L= 2, 3 y 4 
Capítulo V. Influencia de los grupos discriminados en los patrones del problema de Henry
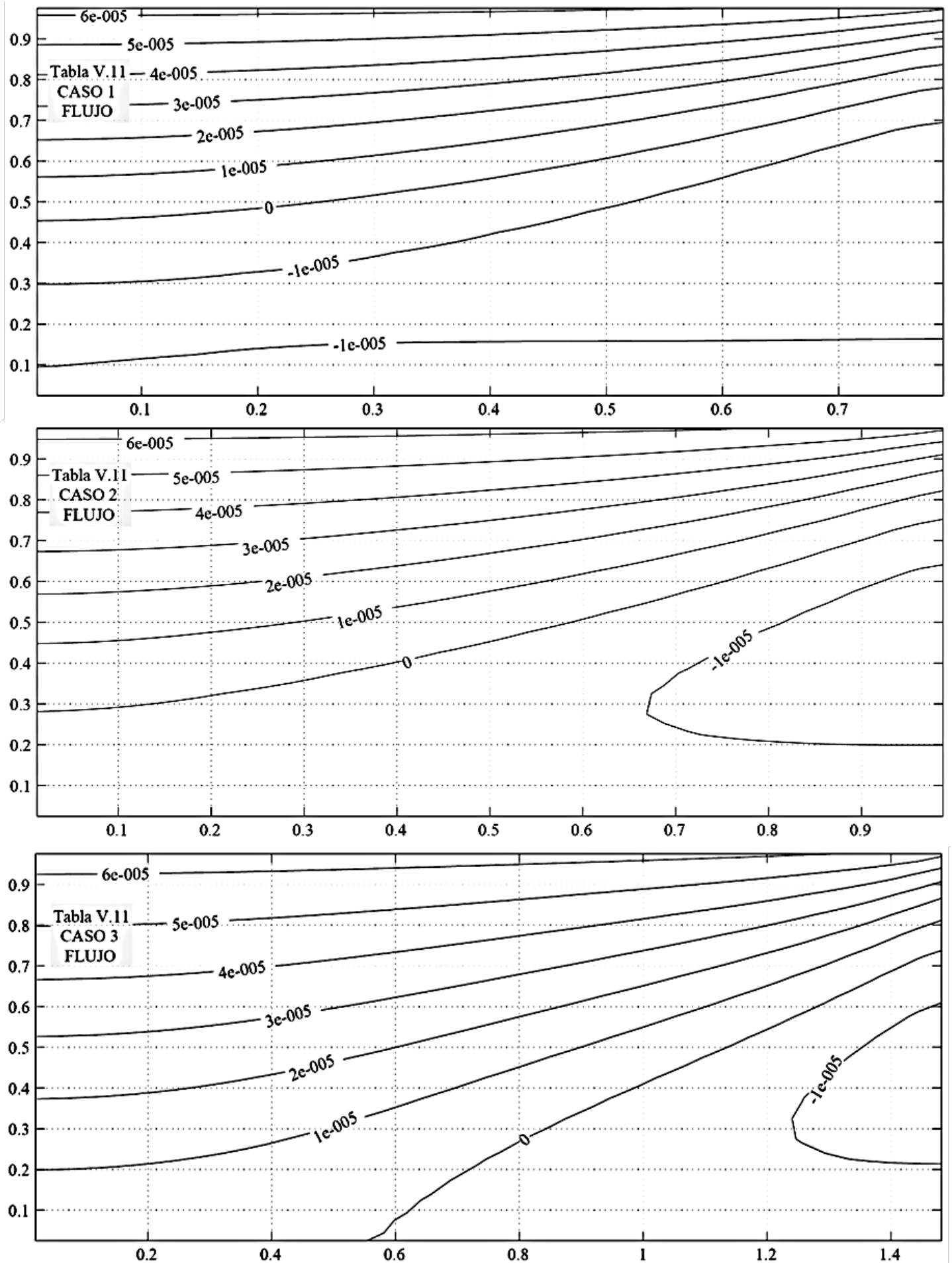

Figura V.22a Patrones de flujo del problema de Henry para L= 0.8, 1 y 1.5 
Capítulo V. Influencia de los grupos discriminados en los patrones del problema de Henry
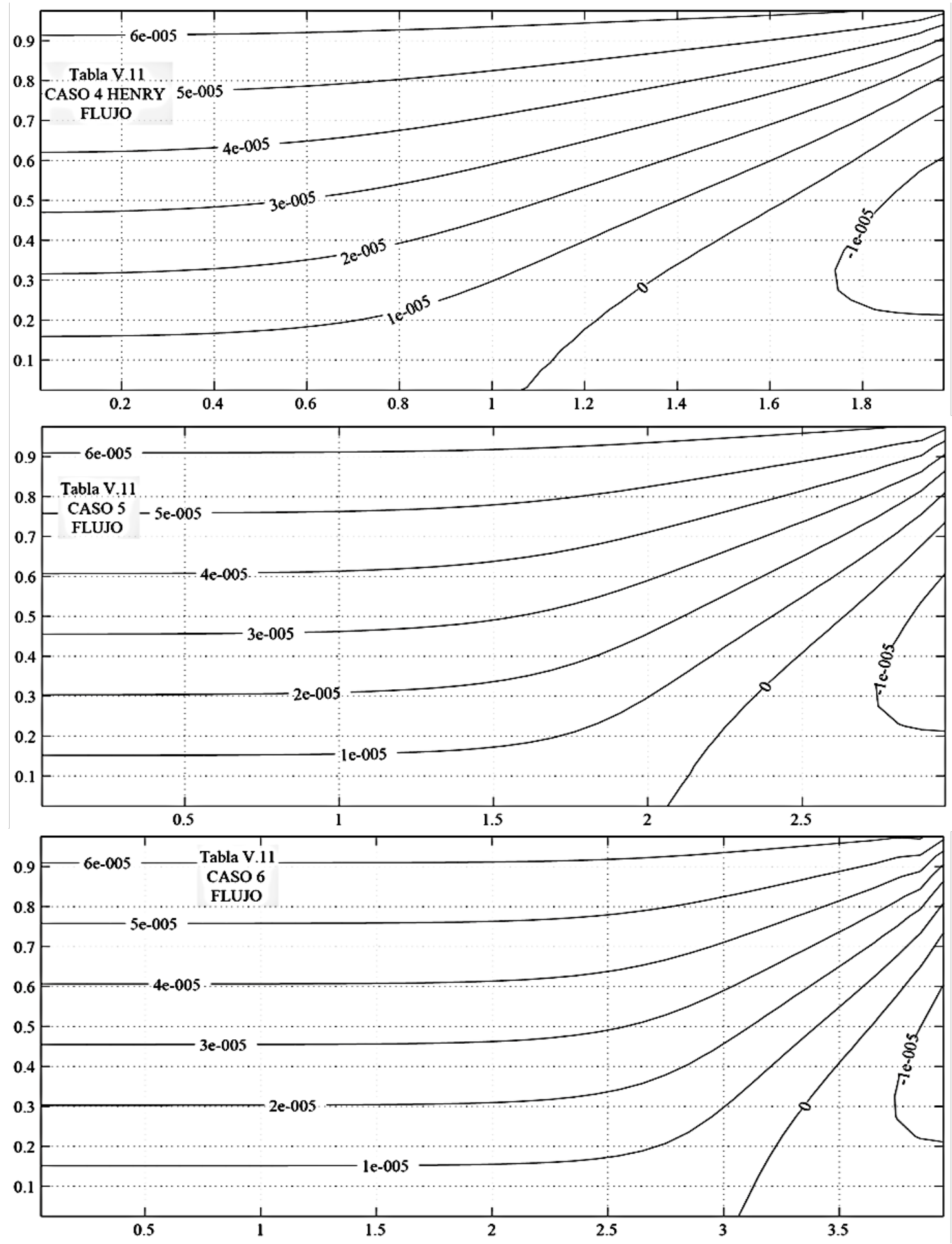

Figura V.22b Patrones de flujo del problema de Henry para L= 2, 3 y 4 
La consideración de un L superfluo en el estudio de las cuñas en el problema isótropo permitirá reducir los tres monomios derivados de la adimensionalización discriminada (equivalentes a los asumidos por Henry y otros autores),

$$
\pi_{1}=\frac{(\Delta \rho) \mathrm{gk}}{\varepsilon \mu v_{\mathrm{amb}}^{*}} \quad \pi_{2}=\frac{\mathrm{D}}{\mathrm{v}_{\mathrm{amb}}^{*} \mathrm{~L}} \quad \pi_{3}=\frac{\mathrm{H}}{\mathrm{L}}
$$

a solo dos, que expresados en términos de $\mathrm{H}$ y no de $\mathrm{L}$ tienen la forma

$$
\pi_{1}=\frac{(\Delta \rho) \mathrm{gk}}{\varepsilon \mu v_{\mathrm{amb}}^{*}} \quad \pi_{2}=\frac{\mathrm{D}}{\mathrm{v}_{\mathrm{amb}}^{*} \mathrm{H}}
$$

y expresar en función de estos grupos los pies de las mencionadas cuñas.

Las Tablas12 a 15 siguientes muestran los resultados de la simulación para un amplio rango de valores de $\pi_{1}$ y $\pi_{2}$ (desde 0.1 a 10 , rango que incluye el caso de Henry original), adoptando para L una longitud suficientemente grande como para una asegurar influencia nula en los resultados y la convergencia numérica de las soluciones. Los parámetros tabulados son los pies de las isolíneas de concentración c=0.1 y 0.5, diferencia entre los pies de estas isolíneas y pie de la divisoria $\Psi=0$.

La forma gráfica ilustrativa de estas dependencias se muestra en los ábacos de las Figuras V.23 a V.27. La dependencia del pie de la isolínea de concentración 0.1 con el monomio $\pi_{2}$, con $\pi_{1}$ como parámetro se muestra en las Figuras V.23 y V.24, la segunda un detalle de la primera para valores de $\pi_{2}<2$, rango para el que este parámetro (aunque de pequeño valor) es muy dependiente de $\pi_{1}$. Este resultado parece coherente si se atiende al significado físico asociado a los monomios.

En efecto, valores elevados de $\pi_{2}$ suponen una preponderancia del efecto difusivo frente al efecto del flujo regional lo que hace penetrar el pie de la concentración salina hacia el continente, relegando la influencia de $\pi_{1}$ a la región del patrón situada en la zona superior al borde inferior. Sin embargo, efectos difusivos poco preponderantes frente el flujo regional $\left(\pi_{2}<1\right)$ dan lugar a pies de pequeño valor que aumentan para fuerzas de flotación grandes $\left(\pi_{1}>>1\right)$, las cuales contrarrestan el citado flujo como se muestra claramente en detalle en la Figura V.24.

Un razonamiento similar cabe aplicar a la línea de concentración 0.5 localizada naturalmente a la derecha de la anterior. Los tamaños de los pies de esta isolínea se 
muestran en las Figuras V.25 y V.26, la segunda un detalle de la primera para valores pequeños de $\pi_{2}$.

Pero lo más interesante es la distancia que separa las líneas anteriores (quizás podría haberse representado la diferencia entre las isolíneas 0.1 y 0.9 , que recogería toda la extensión de la zona de gradiente salino, pero el resultado, cualitativamente, hubiera sido es el mismo como hemos comprobado). Esta distancia, representada en la Figura V.27 frente a $\pi_{2}$ y con $\pi_{1}$ como parámetro, da una idea precisa de la zona de difusión. Su valor es independiente de las fuerzas de flotación $\left(\pi_{1}>1\right)$ para difusividades grandes frente al flujo regional $\left(\pi_{2}>1\right)$, lo que equivale a asumir que los pies de las isolíneas $\mathrm{c}=0.1$ y 0.5 aumentan por igual al crecer $\pi_{1}$ para valores constantes de $\pi_{2}$, una explicación coherente con la dependencia mostrada en las Figuras V.23 y V.25. Por otro lado, para efectos difusivos despreciables frente al flujo regional, las distancias entre isolíneas son pequeñas y apenas dependientes de las fuerzas de flotación (monomio $\pi_{1}$ ).

En relación con el pie de la línea divisoria de flujo, su dependencia con los monomios parece más compleja, aunque coherente. La Figura V.26 muestra que para valores de $\pi_{1}$ mayores que la unidad, que indican preponderancia de las fuerzas del flotación frente a las de flujo regional, la recirculación aumenta su extensión casi linealmente con $\pi_{1}$ para valores de $\pi_{2}$ pequeños y constantes (difusión despreciable frente a flujo regional).

Sin embargo, al aumentar el efecto difusivo, sin llegar a ser comparable con el del flujo regional, la linealidad anterior desaparece parcialmente y cuando los efectos difusivos empiezan a ser comparables con los de flujo, el pie de la divisoria se contrae sensiblemente hasta desaparecer, seguramente debido a que las fuerzas de flotación hacen ascender el fluido de elevada concentración en las inmediaciones de la frontera del mar (nada más penetrar en el acuífero).

Finalmente, fuerzas de flotación pequeñas y difusividades grandes (ambas frente a los efectos del flujo regional), no originan en ningún caso cuña de recirculación, lo que no resulta inmediato de explicar; bajo estas condiciones la sal penetra paulatinamente a lo largo del acuífero con gradientes verticales de flujo pequeños (isolíneas casi verticales) e insuficientes para causar recirculación desde la frontera marina. 
Capítulo V. Influencia de los grupos discriminados en los patrones del problema de Henry

Tabla V.12 Pie de la isolínea c=0.1 en función de $\pi_{1}$ y $\pi_{2}$

\begin{tabular}{|c|c|c|c|c|c|c|c|c|c|c|c|c|c|c|c|c|}
\hline \multirow{2}{*}{$\pi_{1}$} & \multicolumn{8}{|c|}{$\pi_{2}$} & \multirow{2}{*}{$\pi_{2}$} & \multicolumn{7}{|c|}{$\pi_{1}$} \\
\hline & 0,1 & 0,2 & 0,5 & 0,6 & 0,7 & 0,8 & 0,9 & 1 & & 2 & 3 & 4 & 5 & 6 & 8 & 10 \\
\hline 0,05 & 0,13 & 0,14 & 0,1 & 0,11 & 0,13 & 0,16 & 0,18 & 0,31 & 0,05 & 0,59 & 0,83 & 1,07 & 1,31 & 1,54 & 1,75 & 2,27 \\
\hline 0,1 & 0,24 & 0,26 & 0,31 & 0,32 & 0,34 & 0,36 & 0,38 & 0,4 & 0,1 & 0,62 & 0,82 & 1,02 & 1,21 & 1,45 & 1,67 & 2,06 \\
\hline 0,2 & 0,47 & 0,48 & 0,52 & 0,53 & 0,55 & 0,56 & 0,58 & 0,59 & 0,2 & 0,74 & 0,89 & 1,03 & 1,19 & 1,37 & 1,6 & 1,85 \\
\hline 0,3 & 0,69 & 0,7 & 0,73 & 0,74 & 0,76 & 0,74 & 0,76 & 0,77 & 0,3 & 0,89 & 1 & 1,12 & 1,23 & 1,37 & 1,58 & 1,76 \\
\hline 0,4 & 0,9 & 0,91 & 0,95 & 0,95 & 0,96 & 0,93 & 0,96 & 0,97 & 0,4 & 1,05 & 1,12 & 1,24 & 1,33 & 1,42 & 1,6 & 1,78 \\
\hline 0,5 & 1,15 & 1,2 & 1,16 & 1,16 & 1,17 & 1,11 & 1,18 & 1,18 & 0,5 & 1,24 & 1,31 & 1,38 & 1,45 & 1,54 & 1,67 & 1,85 \\
\hline 0,6 & 1,36 & 1,36 & 1,38 & 1,38 & 1,38 & 1,39 & 1,39 & 1,39 & 0,6 & 1,43 & 1,48 & 1,54 & 1,6 & 1,67 & 1,8 & 1,94 \\
\hline 0,7 & 1,59 & 1,59 & 1,59 & 1,59 & 1,6 & 1,6 & 1,61 & 1,59 & 0,7 & 1,63 & 1,67 & 1,71 & 1,76 & 1,81 & 1,93 & 2,05 \\
\hline 0,8 & 1,79 & 1,79 & 1,82 & 1,8 & 1,8 & 1,84 & 1,82 & 1,78 & 0,8 & 1,82 & 1,85 & 1,89 & 1,95 & 1,99 & 2,09 & 2,18 \\
\hline 0,9 & 1,98 & 1,98 & 2,04 & 2,05 & 1,98 & 2 & 2,05 & 1,97 & 0,9 & 2,01 & 2,04 & 2,08 & 2,14 & 2,18 & 2,25 & 2,33 \\
\hline 1 & 2,26 & 2,14 & 2,15 & 2,26 & 2,15 & 2,13 & 2,15 & 2,15 & 1 & 2,19 & 2,22 & 2,27 & 2,33 & 2,37 & 2,43 & 2,5 \\
\hline 2 & 4,3 & 4,2 & 4,19 & 4,28 & 4,19 & 4,19 & 4,2 & 4,21 & 2 & 4,2 & 4,2 & 4,2 & 4,21 & 4,21 & 4,2 & 4,2 \\
\hline 3 & 6,04 & 6 & 6 & 6 & 6 & 6,01 & 6 & 6,01 & 3 & 6,04 & 6 & 6,01 & 6 & 6,02 & 6,01 & 5,8 \\
\hline 4 & 7,58 & 7,33 & 7,58 & 7,57 & 7,56 & 7,57 & 7,47 & 7,55 & 4 & 7,51 & 7,48 & 7,5 & 7,54 & 7,45 & 7,48 & 7,5 \\
\hline 5 & 8,99 & 8,7 & 8,92 & 9,01 & 8,93 & 8,92 & 8,93 & 8,92 & 5 & 9 & 8,92 & 8,8 & 8,93 & 8,91 & 8,92 & 8,92 \\
\hline 6 & 10,3 & 10 & 10,08 & 10,2 & 10,09 & 10,08 & 10,09 & 10,05 & 6 & 10,03 & 10,03 & 10,03 & 10,05 & 10,07 & 10,08 & 10,13 \\
\hline 7 & 11,2 & 11,2 & 11,23 & 11,4 & 11,25 & 11,24 & 11,24 & 11,39 & 7 & 11,39 & 11,2 & 11,2 & 11,19 & 11,23 & 11,25 & 11,3 \\
\hline 8 & 12,61 & 12,39 & 12,39 & 12,59 & 12,41 & 12,4 & 12,4 & 12,39 & 8 & 12,39 & 12,57 & 12,41 & 12,38 & 12,39 & 12,41 & 12,55 \\
\hline 9 & 13,5 & 13,46 & 13,47 & 13,5 & 13,47 & 13,46 & 13,48 & 13,59 & 9 & 13,23 & 13,23 & 13,4 & 13,61 & 13,46 & 13,47 & 13,49 \\
\hline 10 & 14,55 & 14,52 & 14,54 & 14,57 & 14,53 & 14,51 & 14,55 & 14,54 & 10 & 14,52 & 14,56 & 14,54 & 14,51 & 14,53 & 14,53 & 14,57 \\
\hline
\end{tabular}


Capítulo V. Influencia de los grupos discriminados en los patrones del problema de Henry

Tabla V.13 Pie de la isolínea c=0.5 en función de $\pi_{1}$ y $\pi_{2}$

\begin{tabular}{|c|c|c|c|c|c|c|c|c|}
\hline \multirow[t]{2}{*}{$\pi_{2}$} & \multicolumn{8}{|c|}{$\pi_{1}$} \\
\hline & 0,1 & 0,2 & 0,5 & 0,6 & 0,7 & $\mathbf{0 , 8}$ & 0,9 & 1 \\
\hline 0,05 & 0,03 & 0,04 & 0,07 & 0,08 & 0,085 & 0,09 & 0,11 & 0,13 \\
\hline 0,1 & 0,07 & 0,08 & 0,11 & 0,12 & 0,13 & 0,14 & 0,15 & 0,16 \\
\hline 0,2 & 0,15 & 0,15 & 0,17 & 0,18 & 0,19 & 0,2 & 0,21 & 0,22 \\
\hline 0,3 & 0,21 & 0,21 & 0,24 & 0,24 & 0,25 & 0,26 & 0,26 & 0,27 \\
\hline 0,4 & 0,28 & 0,28 & 0,31 & 0,31 & 0,31 & 0,31 & 0,32 & 0,33 \\
\hline 0,5 & 0,35 & 0,35 & 0,37 & 0,37 & 0,37 & 0,37 & 0,38 & 0,39 \\
\hline 0,6 & 0,41 & 0,41 & 0,43 & 0,43 & 0,43 & 0,44 & 0,44 & 0,45 \\
\hline $\mathbf{0 , 7}$ & 0,48 & 0,48 & 0,5 & 0,5 & 0,5 & 0,5 & 0,5 & 0,51 \\
\hline 0,8 & 0,55 & 0,55 & 0,56 & 0,56 & 0,56 & 0,55 & 0,57 & 0,57 \\
\hline 0,9 & 0,62 & 0,62 & 0,63 & 0,63 & 0,63 & 0,62 & 0,63 & 0,63 \\
\hline 1 & 0,68 & 0,68 & 0,68 & 0,68 & 0,69 & 0,69 & 0,69 & 0,69 \\
\hline 2 & 1,32 & 1,32 & 1,32 & 1,34 & 1,32 & 1,33 & 1,33 & 1,33 \\
\hline 3 & 1,92 & 1,92 & 1,91 & 1,93 & 1,93 & 1,94 & 1,93 & 1,94 \\
\hline 4 & 2,45 & 2,44 & 2,46 & 2,45 & 2,45 & 2,46 & 2,44 & 2,45 \\
\hline 5 & 2,97 & 2,91 & 2,96 & 2,98 & 2,97 & 2,96 & 2,95 & 2,95 \\
\hline 6 & 3,45 & 3,37 & 3,4 & 3,42 & 3,41 & 3,41 & 3,4 & 3,43 \\
\hline 7 & 3,83 & 3,82 & 3,85 & 3,86 & 3,85 & 3,86 & 3,84 & 3,8 \\
\hline 8 & 4,29 & 4,28 & 4,29 & 4,3 & 4,29 & 4,31 & 4,29 & 4,3 \\
\hline 9 & 4,7 & 4,69 & 4,71 & 4,7 & 4,7 & 4,7 & 4,71 & 4,73 \\
\hline 10 & 5,12 & 5,09 & 5,12 & 5,1 & 5,1 & 5,09 & 5,12 & 5,1 \\
\hline
\end{tabular}


Capítulo V. Influencia de los grupos discriminados en los patrones del problema de Henry

Tabla V.13 Pie de la isolínea c=0.5 en función de $\pi_{1}$ y $\pi_{2}$ (continuación)

\begin{tabular}{|c|c|c|c|c|c|c|c|}
\hline \multirow[t]{2}{*}{$\pi_{2}$} & \multicolumn{7}{|c|}{$\pi_{1}$} \\
\hline & 2 & 3 & 4 & 5 & 6 & 8 & 10 \\
\hline 0,05 & 0,36 & 0,59 & 0,8 & 1 & 1,19 & 1,56 & 1,77 \\
\hline 0,1 & 0,32 & 0,49 & 0,65 & 0,81 & 0,95 & 1,23 & 1,46 \\
\hline 0,2 & 0,32 & 0,43 & 0,54 & 0,67 & 0,88 & 0,97 & 1,15 \\
\hline 0,3 & 0,35 & 0,43 & 0,52 & 0,61 & 0,81 & 0,92 & 1,06 \\
\hline 0,4 & 0,39 & 0,45 & 0,52 & 0,58 & 0,74 & 0,88 & 0,97 \\
\hline 0,5 & 0,44 & 0,49 & 0,55 & 0,61 & 0,67 & 0,83 & 0,88 \\
\hline 0,6 & 0,49 & 0,53 & 0,58 & 0,62 & 0,68 & 0,78 & 0,87 \\
\hline 0,7 & 0,54 & 0,58 & 0,63 & 0,66 & 0,7 & 0,79 & 0,87 \\
\hline 0,8 & 0,595 & 0,63 & 0,67 & 0,7 & 0,74 & 0,81 & 0,88 \\
\hline 0,9 & 0,65 & 0,68 & 0,72 & 0,74 & 0,78 & 0,83 & 0,9 \\
\hline 1 & 0,71 & 0,74 & 0,76 & 0,79 & 0,82 & 0,87 & 0,93 \\
\hline 2 & 1,33 & 1,34 & 1,35 & 1,34 & 1,36 & 1,39 & 1,4 \\
\hline 3 & 1,93 & 1,93 & 1,93 & 1,93 & 1,94 & 1,94 & 1,95 \\
\hline 4 & 2,46 & 2,45 & 2,47 & 2,48 & 2,45 & 2,46 & 2,46 \\
\hline 5 & 2,96 & 2,96 & 2,92 & 2,96 & 2,96 & 2,95 & 2,98 \\
\hline 6 & 3,42 & 3,42 & 3,43 & 3,45 & 3,4 & 3,4 & 3,41 \\
\hline 7 & 3,88 & 3,84 & 3,84 & 3,89 & 3,84 & 3,86 & 3,85 \\
\hline 8 & 4,3 & 4,29 & 4,3 & 4,31 & 4,28 & 4,31 & 4,28 \\
\hline 9 & 4,7 & 4,69 & 4,69 & 4,73 & 4,69 & 4,71 & 4,65 \\
\hline 10 & 5,05 & 5,1 & 5,04 & 5,06 & 5,1 & 5,1 & 5,02 \\
\hline
\end{tabular}


Capítulo V. Influencia de los grupos discriminados en los patrones del problema de Henry

Tabla V.14 Diferencia de los pies de las isolíneas c=0.1 y 0.5 en función de $\pi_{1}$ y $\pi_{2}$

\begin{tabular}{|c|c|c|c|c|c|c|c|c|}
\hline \multirow[t]{2}{*}{$\pi_{2}$} & \multicolumn{8}{|c|}{$\pi_{1}$} \\
\hline & 0,1 & 0,2 & 0,5 & 0,6 & 0,7 & 0,8 & 0,9 & 1 \\
\hline 0,05 & 0,1 & 0,1 & 0,03 & 0,03 & 0,045 & 0,07 & 0,07 & 0,18 \\
\hline 0,1 & 0,17 & 0,18 & 0,2 & 0,2 & 0,21 & 0,22 & 0,23 & 0,24 \\
\hline 0,2 & 0,32 & 0,33 & 0,35 & 0,35 & 0,36 & 0,36 & 0,37 & 0,37 \\
\hline 0,3 & 0,48 & 0,49 & 0,49 & 0,5 & 0,51 & 0,48 & 0,5 & 0,5 \\
\hline 0,4 & 0,62 & 0,63 & 0,64 & 0,64 & 0,65 & 0,62 & 0,64 & 0,64 \\
\hline 0,5 & 0,8 & 0,85 & 0,79 & 0,79 & 0,8 & 0,74 & 0,8 & 0,79 \\
\hline 0,6 & 0,95 & 0,95 & 0,95 & 0,95 & 0,95 & 0,95 & 0,95 & 0,94 \\
\hline 0,7 & 1,11 & 1,11 & 1,09 & 1,09 & 1,1 & 1,1 & 1,11 & 1,08 \\
\hline 0,8 & 1,24 & 1,24 & 1,26 & 1,24 & 1,24 & 1,29 & 1,25 & 1,21 \\
\hline 0,9 & 1,36 & 1,36 & 1,41 & 1,42 & 1,35 & 1,38 & 1,42 & 1,34 \\
\hline 1 & 1,58 & 1,46 & 1,47 & 1,58 & 1,46 & 1,44 & 1,46 & 1,46 \\
\hline 2 & 2,98 & 2,88 & 2,87 & 2,94 & 2,87 & 2,86 & 2,87 & 2,88 \\
\hline 3 & 4,12 & 4,08 & 4,09 & 4,07 & 4,07 & 4,07 & 4,07 & 4,07 \\
\hline 4 & 5,13 & 4,89 & 5,12 & 5,12 & 5,11 & 5,11 & 5,03 & 5,1 \\
\hline 5 & 6,02 & 5,79 & 5,96 & 6,03 & 5,96 & 5,96 & 5,98 & 5,97 \\
\hline 6 & 6,85 & 6,63 & 6,68 & 6,78 & 6,68 & 6,67 & 6,69 & 6,62 \\
\hline 7 & 7,37 & 7,38 & 7,38 & 7,54 & 7,4 & 7,38 & 7,4 & 7,59 \\
\hline 8 & 8,32 & 8,11 & 8,1 & 8,29 & 8,12 & 8,09 & 8,11 & 8,09 \\
\hline 9 & 8,8 & 8,77 & 8,76 & 8,8 & 8,77 & 8,76 & 8,77 & 8,86 \\
\hline 10 & 9,43 & 9,43 & 9,42 & 9,47 & 9,43 & 9,42 & 9,43 & 9,44 \\
\hline
\end{tabular}


Capítulo V. Influencia de los grupos discriminados en los patrones del problema de Henry

Tabla V.14 Diferencia de los pies de las isolíneas c=0.1 y 0.5 en función de $\pi_{1}$ y $\pi_{2}$ (continuación)

\begin{tabular}{|c|c|c|c|c|c|c|c|c|}
\hline \multirow{2}{*}{$\boldsymbol{\pi}_{\mathbf{2}}$} & \multicolumn{7}{|c|}{$\boldsymbol{\pi}_{\mathbf{1}}$} \\
\cline { 2 - 10 } & $\mathbf{1}$ & $\mathbf{2}$ & $\mathbf{3}$ & $\mathbf{4}$ & $\mathbf{5}$ & $\mathbf{6}$ & $\mathbf{8}$ & $\mathbf{1 0}$ \\
\hline $\mathbf{0 , 0 5}$ & 0,18 & 0,23 & 0,24 & 0,27 & 0,31 & 0,35 & 0,19 & 0,5 \\
\hline $\mathbf{0 , 1}$ & 0,24 & 0,3 & 0,33 & 0,37 & 0,4 & 0,5 & 0,44 & 0,6 \\
\hline $\mathbf{0 , 2}$ & 0,37 & 0,42 & 0,46 & 0,49 & 0,52 & 0,49 & 0,63 & 0,7 \\
\hline $\mathbf{0 , 3}$ & 0,5 & 0,54 & 0,57 & 0,6 & 0,62 & 0,56 & 0,66 & 0,7 \\
\hline $\mathbf{0 , 4}$ & 0,64 & 0,66 & 0,67 & 0,72 & 0,75 & 0,68 & 0,72 & 0,81 \\
\hline $\mathbf{0 , 5}$ & 0,79 & 0,8 & 0,82 & 0,83 & 0,84 & 0,87 & 0,84 & 0,97 \\
\hline $\mathbf{0 , 6}$ & 0,94 & 0,94 & 0,95 & 0,96 & 0,98 & 0,99 & 1,02 & 1,07 \\
\hline $\mathbf{0 , 7}$ & 1,08 & 1,09 & 1,09 & 1,08 & 1,1 & 1,11 & 1,14 & 1,18 \\
\hline $\mathbf{0 , 8}$ & 1,21 & 1,225 & 1,22 & 1,22 & 1,25 & 1,25 & 1,28 & 1,3 \\
\hline $\mathbf{0 , 9}$ & 1,34 & 1,36 & 1,36 & 1,36 & 1,4 & 1,4 & 1,42 & 1,43 \\
\hline $\mathbf{1}$ & 1,46 & 1,48 & 1,48 & 1,51 & 1,54 & 1,55 & 1,56 & 1,57 \\
\hline $\mathbf{2}$ & 2,88 & 2,87 & 2,86 & 2,85 & 2,87 & 2,85 & 2,81 & 2,8 \\
\hline $\mathbf{3}$ & 4,07 & 4,11 & 4,07 & 4,08 & 4,07 & 4,08 & 4,07 & 3,85 \\
\hline $\mathbf{4}$ & 5,1 & 5,05 & 5,03 & 5,03 & 5,06 & 5 & 5,02 & 5,04 \\
\hline $\mathbf{5}$ & 5,97 & 6,04 & 5,96 & 5,88 & 5,97 & 5,95 & 5,97 & 5,94 \\
\hline $\mathbf{6}$ & 6,62 & 6,61 & 6,61 & 6,6 & 6,6 & 6,67 & 6,68 & 6,72 \\
\hline $\mathbf{7}$ & 7,59 & 7,51 & 7,36 & 7,36 & 7,3 & 7,39 & 7,39 & 7,45 \\
\hline $\mathbf{8}$ & 8,09 & 8,09 & 8,28 & 8,11 & 8,07 & 8,11 & 8,1 & 8,27 \\
\hline $\mathbf{9}$ & 8,86 & 8,53 & 8,54 & 8,71 & 8,88 & 8,77 & 8,76 & 8,84 \\
\hline & 9,44 & 9,47 & 9,46 & 9,5 & 9,45 & 9,43 & 9,43 & 9,55 \\
\hline & & & & & & & & \\
\hline & & & & & & & & \\
\hline
\end{tabular}


Capítulo V. Influencia de los grupos discriminados en los patrones del problema de Henry

Tabla V.14 Pie de la divisoria de flujo $\Psi=0$ en función de $\pi_{1}$ y $\pi_{2}$

\begin{tabular}{|c|c|c|c|c|c|c|c|c|c|c|}
\hline \multirow[t]{2}{*}{$\pi_{2}$} & \multicolumn{10}{|c|}{$\pi_{1}$} \\
\hline & 0,8 & 0,9 & 1 & 2 & 3 & 4 & 5 & 6 & 8 & 10 \\
\hline 0,05 & 0 & 0,1 & 0,18 & 0,53 & 0,8 & 1,07 & 1,32 & 1,57 & 2,07 & 2,42 \\
\hline $\mathbf{0 , 1}$ & 0 & 0,07 & 0,15 & 0,48 & 0,75 & 0,98 & 1,19 & 1,47 & 1,8 & 2,18 \\
\hline 0,2 & 0 & 0 & 0 & 0,45 & 0,71 & 0,91 & 1,11 & 1,37 & 1,66 & 1,98 \\
\hline 0,3 & 0 & 0 & 0 & 0,42 & 0,69 & 0,89 & 1,08 & 1,3 & 1,6 & 1,89 \\
\hline 0,4 & 0 & 0 & 0 & 0,36 & 0,68 & 0,88 & 1,07 & 1,25 & 1,58 & 1,84 \\
\hline 0,5 & 0 & 0 & 0 & 0 & 0,65 & 0,87 & 1,07 & 1,23 & 1,55 & 1,79 \\
\hline 0,6 & 0 & 0 & 0 & 0 & 0,61 & 0,86 & 1,06 & 1,24 & 1,54 & 1,79 \\
\hline $\mathbf{0 , 7}$ & 0 & 0 & 0 & 0 & 0,57 & 0,85 & 1,06 & 1,24 & 1,54 & 1,81 \\
\hline 0,8 & 0 & 0 & 0 & 0 & 0,51 & 0,82 & 1,04 & 1,25 & 1,56 & 1,84 \\
\hline 0,9 & 0 & 0 & 0 & 0 & 0,44 & 0,79 & 1,03 & 1,24 & 1,56 & 1,85 \\
\hline 1 & 0 & 0 & 0 & 0 & 0,35 & 0,75 & 1,03 & 1,23 & 1,58 & 1,86 \\
\hline 2 & 0 & 0 & 0 & 0 & 0 & 0 & 0,7 & 0,88 & 1,5 & 1,94 \\
\hline 3 & 0 & 0 & 0 & 0 & 0 & 0 & 0 & 0 & 1,02 & 1,7 \\
\hline 4 & 0 & 0 & 0 & 0 & 0 & 0 & 0 & 0 & 0 & 1,24 \\
\hline 5 & 0 & 0 & 0 & 0 & 0 & 0 & 0 & 0 & 0 & 0,6 \\
\hline 6 & 0 & 0 & 0 & 0 & 0 & 0 & 0 & 0 & 0 & 0 \\
\hline 7 & 0 & 0 & 0 & 0 & 0 & 0 & 0 & 0 & 0 & 0 \\
\hline 8 & 0 & 0 & 0 & 0 & 0 & 0 & 0 & 0 & 0 & 0 \\
\hline 9 & 0 & 0 & 0 & 0 & 0 & 0 & 0 & 0 & 0 & 0 \\
\hline 10 & 0 & 0 & 0 & 0 & 0 & 0 & 0 & 0 & 0 & 0 \\
\hline
\end{tabular}


Capítulo V. Influencia de los grupos discriminados en los patrones del problema de Henry

$$
I^{*}(\mathrm{c}=0.1)
$$

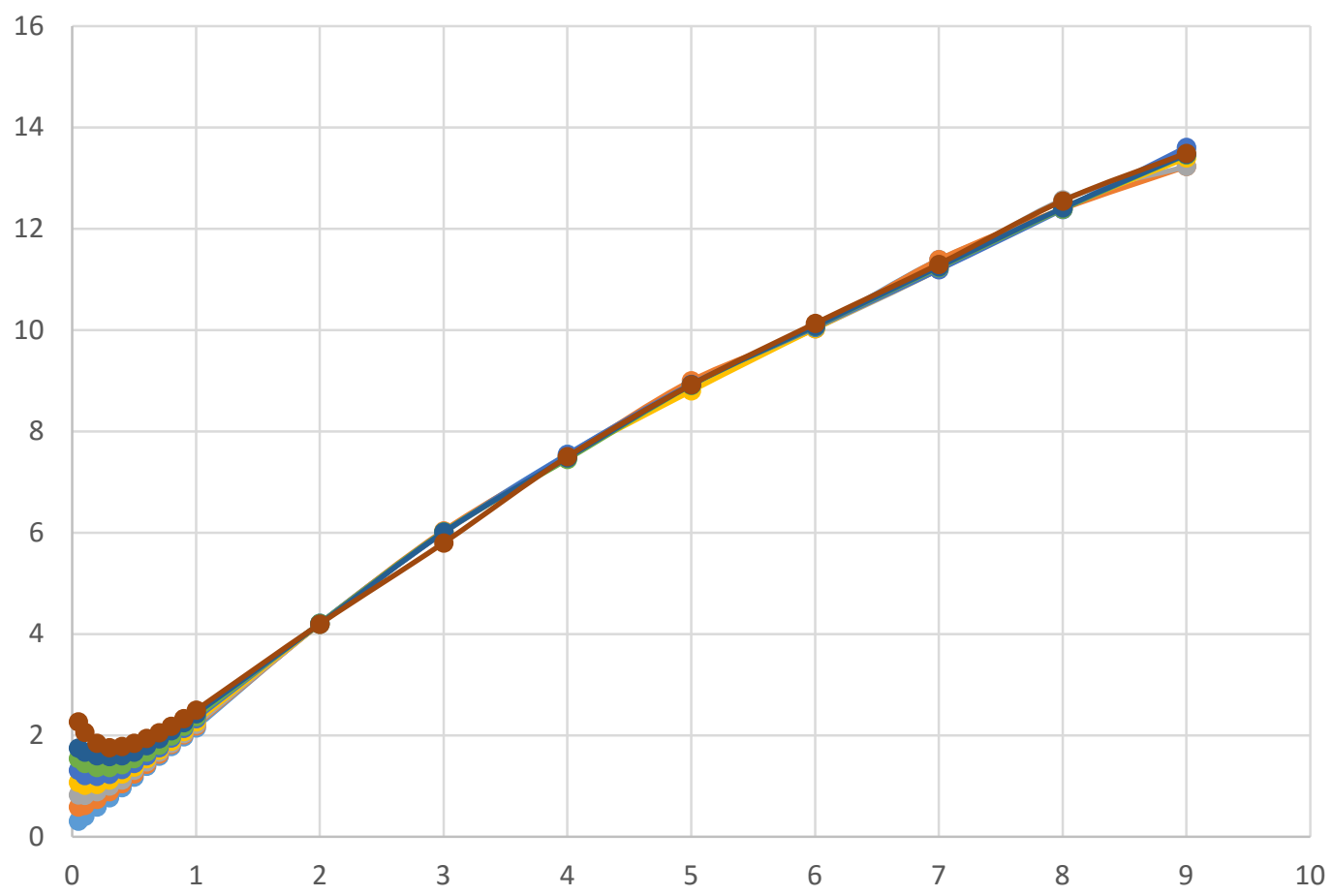

$I^{*}(\mathrm{c}=0.1)$

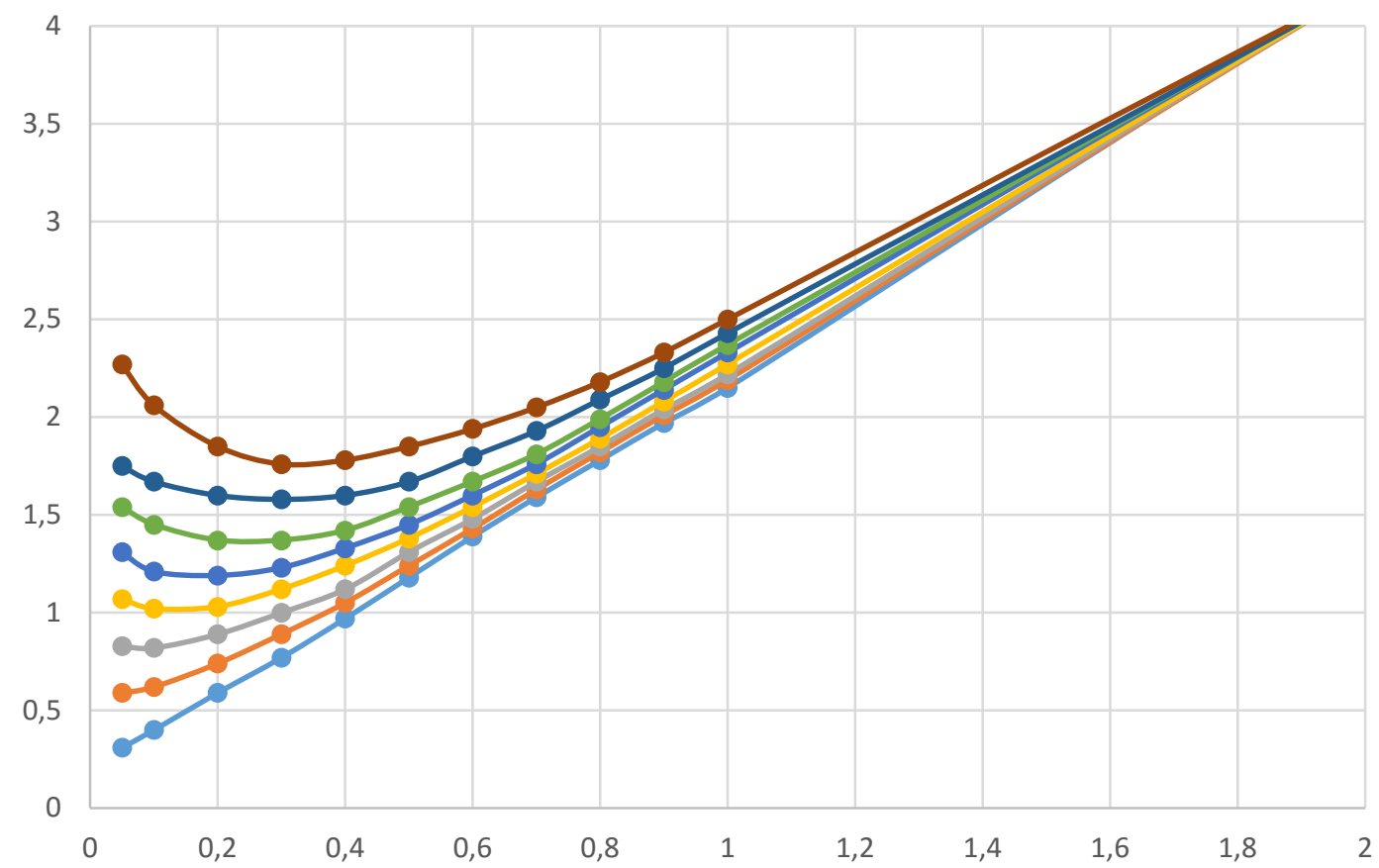

Figura V.23 l(c=0.1), eje vertical, frente a $\pi_{2}$, eje horizontal. El parámetro de las curvas es $\pi_{1}=0.1$ (curva inferior), 0.2, 0.5, 0.6, 0.7, 0.8, 0.9, 1, 2, 3, 4, 5, 6, 8, 10 (curva superior) 
Capítulo V. Influencia de los grupos discriminados en los patrones del problema de Henry

I* $(c=0.5)$

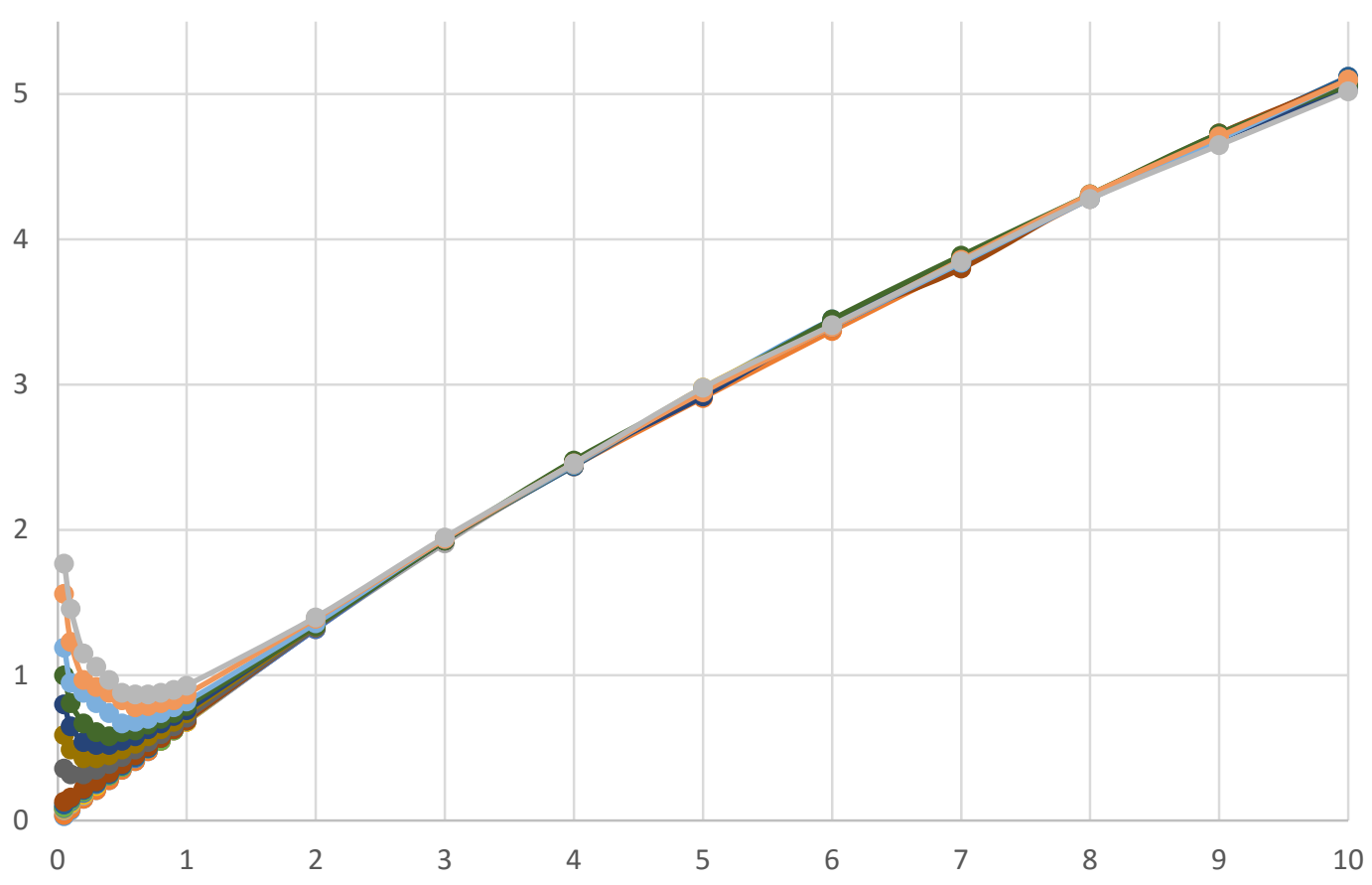

$I^{*}(c=0.5)$

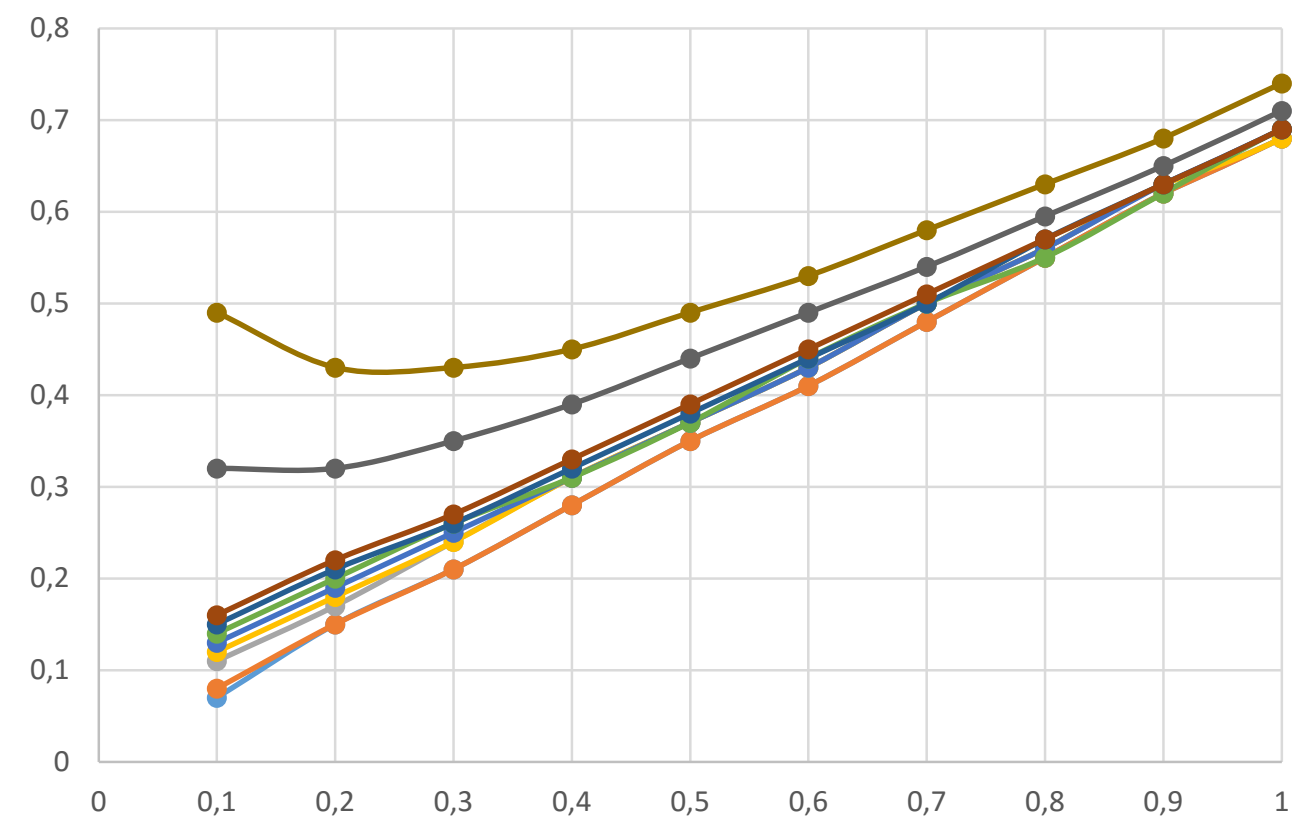

Figura V.24 l(c=0.5), eje vertical, frente a $\pi_{2}$, eje horizontal. El parámetro de las curvas es $\pi_{1}=0.1$ (curva inferior), 0.2, 0.5, 0.6, 0.7, 0.8, 0.9, 1, 2, 3, 4, 5, 6, 8, 10 (curva superior) 
Capítulo V. Influencia de los grupos discriminados en los patrones del problema de Henry

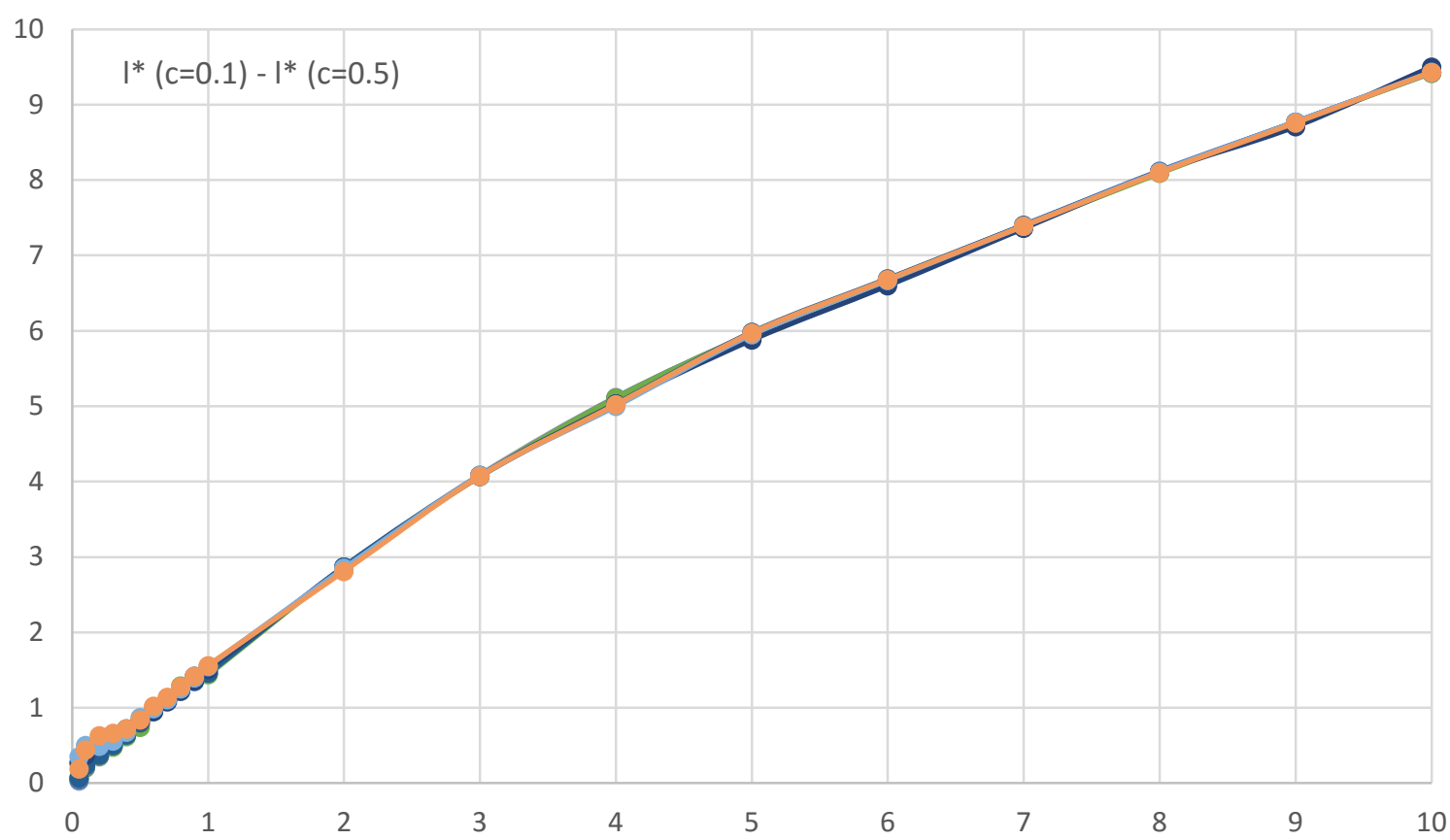

Figura V.25 l(c=0.1) - l(c=0.5), eje vertical, frente a $\pi_{2}$, eje horizontal. $\pi_{1}$ (parámetro) $=0.1$ (curva inferior), 0.2, 0.5, 0.6, 0.7, 0.8, 0.9, 1, 2, 3, 4, 5, 6, 8, 10 (curva superior)

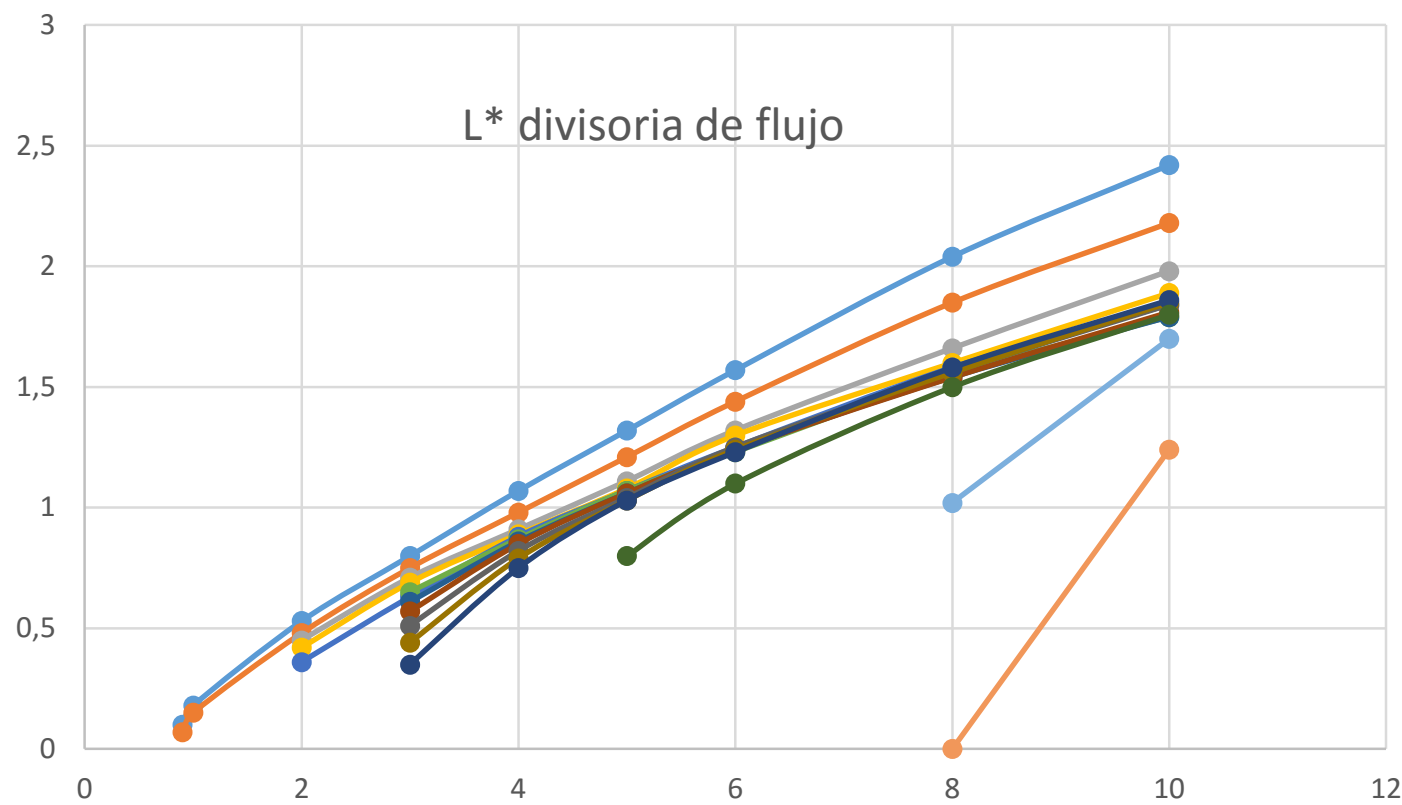

Figura V.26 Pie de la línea divisoria de flujo, eje vertical, frente a $\pi_{1}$, eje horizontal. $\pi_{2}$ (parámetro) $=0.05$ (curva superior), $0.1,0.2,0.3,0.4,0.5,0.6,0.7,0.8,0.9$ y 1 (curva inferior) 
La Figura V.27 es una representación alternativa de la dependencia de la línea divisoria con los monomios, donde vuelve a manifestarse claramente la no linealidad de esta dependencia. Si seguimos una cualquiera de las líneas de esta gráfica, para las que el efecto de la flotación es superior al del flujo regional $\left(\pi_{1}>1\right)$, podemos advertir el siguiente comportamiento: i) un efecto difusivo pequeño frente al flujo regional $\left(\pi_{2}<1\right)$ causa unos pies de recirculación elevados merced a que las fuerzas de flotación hacen ascender el flujo de agua de mar relajando la presión y permitiendo mayor entrada de agua desde el mar; ii) al aumentar el efecto difusivo las isolíneas de concentración se hacen más verticales comprimiendo la cuña de recirculación y haciendo disminuir su pie hasta su desaparición; para un efecto difusivo intermedio los anteriores comportamientos se equilibran y el pie de la línea divisoria se mantiene constante (zona recta de las curvas).

Por último, valores de $\pi_{1}$ claramente inferiores a la unidad no causan recirculación (pie de la línea divisoria de longitud nula).

De la complejidad que emerge en el problema cuando los monomios de los que dependen las variables estudiadas son sólo dos, cabe deducir que si añadimos la anisotropía en la permeablidad hidraúlica, lo que supone la emergencia de un nuevo monomio, podemos encontrarnos con unos resultados demasiado complejos de explicar que fueron parcialmente abordados con anterioridad. Además, ¿¿cuál sería la expresión de este nuevo monomio discriminado si ahora no disponemos de una longitud de refrencia horizontal para construirlo por ser L un parámetro superfluo?, y ¿qué decir si, además, introducimos anisotropía en la difusividad efectiva (coherente con la anisotropía en la permeabilidad)?

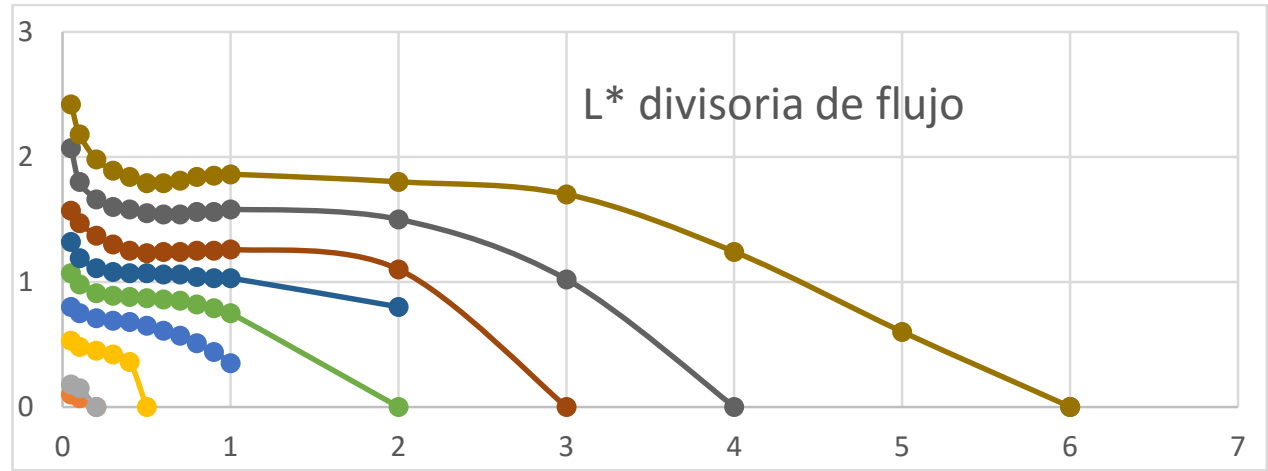

Figura V.27 Pie de la línea divisoria de flujo, eje vertical, frente a $\pi_{2}$, eje horizontal. $\pi_{1}$ (parámetro) $=0.8$ (curva inferior), $0.9,1,2,3,4,5,6,8,10$ (curva superior) 
Capítulo V. Influencia de los grupos discriminados en los patrones del problema de Henry

Dejamos estas preguntas en el aire con la intención de abordarlas con profundidad en un futuro próximo mediante la adimensionalización, restringida a los subdominios del problema, basada en los siguientes razonamientos. Los patrones de concentración y flujo obtenidos en la sección anterior para el problema original de Henry manifiestan claramente la existencia de regiones diferenciadas. En relación con el primero cabe distinguir tres regiones: Una de agua dulce con la forma de un trapecio rectangular inverso y acuñado, cuya base mayor (L) es prácticamente la frontera superior del acuífero y cuya base menor es aproximadamente L/2. El lado oblicuo de dicho trapecio está curvado y puede definirse por una isolínea de pequeña concentración (por ejemplo, c=0.1). ¿Cómo influye la longitud total del acuífero (L) en los límites inferiores de esta primera región si se mantienen constantes los valores de los restantes parámetros del problema, incluido H? Ya hemos verificado que un aumento de L se traduce en un aumento de la extensión de la zona de entrada de flujo horizontal procedente del continente (agua dulce), manteniendo inalterable la extensión de la zona de mezcla, mientras que una disminución de L sí que cambia el patrón de ambas zonas.

La segunda región dentro del patrón de concentración es la definida por el agua de concentración máxima, digamos de concentración por encima de 0.9. En esta región, que emerge en la zona inferior derecha del acuífero, tiene forma de cuña, cuando existe (según los valores de algunos de los grupos adimensionales) y el agua recircula en sentido contrario a las agujas del reloj procedente y con destino al mar. Definimos esta región por la longitud de la base de la cuña. Por último, la tercera región está situada entre las anteriores y tiene salinidades intermedias (por ejemplo $0.1<\mathrm{c}<0.9$ ); la definiremos por su espesor en la base del acuífero. Su forma es variable y depende del valor de los grupos adimensionales.

En relación con el patrón de flujo, en el caso más complejo podemos distinguir dos regiones. La primera definida por agua dulce y agua parcialmente salada (para ciertos valores de los grupos adimensionales esta región ocupa todo el acuífero) formada por líneas de flujo horizontal que procedentes de la frontera continental se curvan a la derecha del dominio dirigiéndose hacia la parte superior de la frontera marina por donde descargan. El agua aumenta su concentración paulatinamente al entrar en la zona curvada, zona en la que se producen los fenómenos de flotación. La base de esta región coincide aproximadamente con la base de la región de agua dulce del patrón de concentraciones 
Capítulo V. Influencia de los grupos discriminados en los patrones del problema de Henry

en el problema original de Henry mientras que en general, depende del valor de los grupos adimensionales. La segunda región en el patrón de flujo, cuando existe, tiene forma acuñada, es de agua muy salada y está separada de la primera por una divisoria de aguas; sus líneas, en general, salen de la parte inferior de la frontera marina y se curvan dirigiéndose a la región inmediatamente superior de dicha frontera. Se define por un pie que coincide, como hemos dicho, con el de la región de agua completamente salada del patrón de concentraciones. T

Todo lo anterior permite la definición de varias longitudes ocultas. En relación con el patrón de flujo definiremos:

$l_{\mathrm{f}}^{*} \quad$ longitud de la cuña de recirculación. Distancia desde el extremo inferior derecho del borde marino hasta la intersección de la línea divisoria $(\psi=0)$ con el fondo del acuífero.

mientras que para el patrón de concentraciones definiremos:

$l_{\mathrm{c}=0.1}{ }^{*}$ : distancia desde el extremo inferior derecho del acuífero a la intersección de la isolínea c=0.1 con la frontera inferior,

$\mathrm{l}_{\mathrm{c}=0.5}{ }^{*}$; ídem a la intersección de la isolínea c $=0.5$,

$\mathrm{l}_{\mathrm{c}=0.9}{ }^{*}$ : ídem a la intersección de la isolínea c $=0.9$,

$\mathrm{l}_{\mathrm{d}}{ }^{*}=\mathrm{l}_{\mathrm{c}=0.1}{ }^{*}-\mathrm{l}_{\mathrm{c}=0.9}{ }^{*}$ : extensión de la cuña difusa o región de agua parcialmente salada, medida sobre la base del acuífero.

Por otro lado, hemos visto también en la sección anterior que los patrones, a menos que sus cuñas se encuentren suficientemente alejadas de la frontera izquierda, están influenciados por la posición relativamente cercana $(L=2 \mathrm{~m})$ de dicha frontera al borde marino; esto es, que los patrones están influenciados por la relativamente pequeña longitud del acuífero. Así, en relación con el patrón de concentraciones, la condición de concentración nula en dicha frontera impide la extensión natural de las isolíneas de menor concentración hacia la izquierda del dominio, isolíneas que penetrarían en el continente en ausencia de dicha condición.

En relación con el patrón de flujo su influencia parece menor ya que las cuñas de recirculación son de menor extensión (excepto, como se ha visto en la memoria, para ciertos valores de los monomios). Adviértase que las condiciones de función de corriente impuestas en la frontera continental y sus valores extremos en las cotas superior e inferior, 
$\mathrm{v}_{\mathrm{y}}=-(\partial \psi / \partial \mathrm{x})=0$ y $\psi_{(\mathrm{x}=0, \mathrm{y}=\mathrm{H})}-\psi_{(\mathrm{x}=0, \mathrm{y}=0)}=\mathrm{v}_{\mathrm{amb}}$, respectivamente, permitirían que el agua saliera y entrara por dicha frontera horizontalmente (componente vertical de velocidad nula), con la única condición de que el caudal total (entrada menos salida) sea de vamb $\mathrm{m}^{3} / \mathrm{s}$, el definido por los valores impuestos a $\psi$ en dicha frontera. Efectivamente, para determinados valores de los grupos discriminados el agua de recirculación llega hasta la frontera izquierda y sale por ella en dirección hacia el continente, compensando el caudal total de entrada con una velocidad mayor en la región superior de esta frontera.

Es difícil imaginar que en los acuíferos reales la extensión del dominio (L) influya en los patrones de solución. Parece más lógico suponer que la extensión de los acuíferos reales es lo suficientemente grande como para no afectar a los patrones de intrusión: L es finita, pero de valor suficiente como para que su influencia sea irrelevante en el problema. Pero entonces dispondríamos de una sola referencia $(\mathrm{H})$ para adimensionalizar las coordenadas espaciales y, en consecuencia, sólo podríamos adimensionalizar las ecuaciones de gobierno siguiendo un procedimiento clásico (no discriminado). Sin embargo, sabemos que la técnica de adimensionalización clásica ha conducido a resultados imprecisos y pobres, aparte de no proporcionar solución a determinadas incógnitas introducidas como referencias. La conclusión es que debemos utilizar las longitudes propuestas $\mathrm{l}_{\mathrm{f}}{ }^{*} \mathrm{y} \mathrm{l}_{\mathrm{d}}{ }^{*}$ para definir la variable adimensional independiente $\mathrm{x}$ en cada ecuación (o simplificación de la misma al subdominio correspondiente) y proceder a la búsqueda de los nuevos grupos adimensionales que, a su vez, proporcionarían el orden de magnitud de las incógnitas introducidas $\mathrm{l}_{\mathrm{f}}{ }^{*} \mathrm{y} \mathrm{l}_{\mathrm{d}}{ }^{*}$. No es una tarea fácil.

En la sección de fundamentos teóricos (Capítulo II) ya se ha dado pistas y establecido el procedimiento de adimensionalización a seguir en estos casos, fundado en una comprensión profunda de los fenómenos físicos involucrados en el problema. Allí mencionamos que cuando no existe una referencia adecuada en el enunciado del problema se establece una referencia desconocida (oculta) o incógnita, cuyo orden de magnitud será obtenido tras la aplicación del propio proceso de adimensionalización. 


\section{V.3 CONCLUSIONES}

i. En relación con el problema isótropo original cabe concluir que los grupos deducidos quedan verificados con las simulaciones ejecutadas y son capaces de reproducir la casi totalidad de potenciales escenarios.

1. Para el monomio $\pi_{\mathrm{d}, 1}=\left[(\Delta \rho) g \mathrm{k}_{\mathrm{y}} /\left(\varepsilon \mu \mathrm{vamb}^{*}\right)\right](\mathrm{L} / \mathrm{H})$, las desviaciones por encima $\mathrm{y}$ por debajo de su valor en el problema original (7.483, que supone una influencia apreciable de la velocidad de Darcy frente a la velocidad regional), manteniendo constantes los valores de los otros monomios, $\pi_{\mathrm{d}, 3}=0.05$ y $\pi_{\mathrm{d}, 2}=\pi_{\mathrm{d}, 4}=0.25$, producen resultados coherentes. Valores decrecientes de $\pi_{\mathrm{d}, 1}$ (partiendo de 14 hasta 1) retraen y estrechan en su base la cuña hacia el lado del mar, progresivamente, lo que se justifica por la creciente menor influencia de los efectos del cambio de densidad frente al flujo regional. El patrón de concentraciones para el valor más alto, $\pi_{\mathrm{d}, 1}=14$, produce una cuña muy angosta e inclinada (como en los casos reales) cuya base se extiende prácticamente a todo el acuífero, manifestando sin duda una posible influencia de la extensión del dominio (L) en el patrón, por imposición de la condición de agua dulce en la frontera continental. Se aprecia la emergencia de una región acuñada de elevada salinidad en la zona inferior derecha del dominio, cuya extensión crece con $\pi_{\mathrm{d}, 1}$ (flujos regionales despreciables frente a los de flotación).

En cuanto al patrón de flujo, la intersección con la base del dominio de la divisoria de aguas, inexistente para valores bajos de $\pi_{\mathrm{d}, 1}$, emerge progresivamente con el aumento de este monomio hasta alcanzar buena parte de la base. Aunque existe una fuerte conexión entre la base de la cuña salina y la intersección de la divisoria con el fondo del dominio, no se mantienen esta concordancia entre las áreas de la cuña salina y de la región del dominio a la derecha de la divisoria. Además, emerge agua parcialmente salada a la izquierda de la divisoria.

2. Para $\pi_{\mathrm{d}, 3}$ los patrones se muestran igualmente coherentes. El patrón de salinidad sufre pocas modificaciones (aunque se ensancha en la zona superior) hasta que $\pi_{\mathrm{d}, 3}$ se acerca a la unidad en donde la sal cubre todo el dominio y se manifiesta la 
Capítulo V. Influencia de los grupos discriminados en los patrones del problema de Henry

influencia de L; y como un aumento de $\pi_{\mathrm{d}, 3}$ por reducción de $\mathrm{V}_{\mathrm{amb}}$ lleva aparejado una mayor influencia de la flotación, las líneas de isoconcentración se vuelvan más verticales.

La intersección de la divisoria de aguas con la base se contrae ligeramente con el aumento de $\pi_{\mathrm{d}, 3}$ haciendo más angosta la zona de recirculación situada bajo la línea y, en consecuencia, disminuyendo el caudal de recirculación que tiende a cero para $\pi_{\mathrm{d}, 3}>1$. Es muy importante advertir el claro desacoplo entre la extensión (medida sobre el fondo del acuífero) de la zona salada resultante del patrón de concentraciones y la longitud que determina la línea divisoria (que marca la zona de recirculación) con la frontera inferior. Este desacoplo induce a reflexionar en el futuro sobre la existencia de nuevos balances (nuevos grupos adimensionales) entre magnitudes asociadas a los efectos que se contraponen, a partir de las ecuaciones simplificadas en estas subregiones.

3. Los cambios de $\pi_{\mathrm{d}, 2}$ afectan a la permeabilidad, un parámetro muy influyente cualitativa y cuantitativamente en los patrones; sin embargo, al retener el valor de los otros monomios, los efectos difusivos son despreciables frente a los causados por el flujo regional y estos últimos pequeños frente a los efectos de flotación. Su valor, a partir de 0,01 ensancha y acuesta la región de mezcla hasta extenderse a todo lo largo del acuífero (con evidente influencia de L), reproduciendo patrones que ciertamente reflejan los de los acuíferos reales. La emergencia de un subdominio saturado, creciente, en la región inferior derecha puede explicarse merced al aumento de $\mathrm{k}_{\mathrm{x}}$ frente a $\mathrm{k}_{\mathrm{y}}$ : el agua saturada de sal que penetra en el acuífero desde el mar no baja la concentración hasta que alcanza zonas menos saturadas sensibles a la flotación.

En cuanto al patrón de flujo, valores pequeños de $\pi_{\mathrm{d}, 2}$ definen una divisoria cuya base casi coincide con la de la frontera agua dulce-agua salada. Pero para $\pi_{\mathrm{d}, 2} \approx 1$, quizás por la influencia de L, la línea divisoria penetra en el continente dando lugar a recirculaciones de agua en las dos fronteras verticales y a una recirculación inversa desde el mar hacia el continente, poco real. 
Capítulo V. Influencia de los grupos discriminados en los patrones del problema de Henry

4. Para $\pi_{\mathrm{d}, 4}=\left(\mathrm{D}_{\mathrm{x}} \mathrm{H}^{2}\right) /\left(\mathrm{D}_{\mathrm{y}} \mathrm{L}^{2}\right)$ un aumento de su valor manteniendo los otros monomios (efectos difusivos despreciables frente a los del flujo regional y estos últimos pequeños frente a los de flotación) produce, por un lado, cuñas de difusión cuya base se expande paulatina, pero no acusadamente, sobre la frontera inferior, y por otro, la emergencia progresiva de una región completamente salada lindante con el mar.

En cuanto al patrón de flujo, se advierte un desplazamiento lento hacia el continente, similar al de la cuña salina (medido hasta la frontera marina). También, las isolíneas de flujo bajo la divisoria manifiestan que el caudal de recirculación de agua de mar disminuye sensiblemente al incrementar el monomio $\pi_{\mathrm{d}, 4}$ hasta hacerse despreciable para $\pi_{\mathrm{d}, 4}=1$.

5. La influencia simultánea de los monomios $\pi_{\mathrm{d}, 2}=\left(\mathrm{k}_{\mathrm{x}} \mathrm{H}^{2}\right) /\left(\mathrm{k}_{\mathrm{y}} \mathrm{L}^{2}\right)$ y $\pi_{\mathrm{d}, 4}=$ $\left(D_{\mathrm{x}} \mathrm{H}^{2}\right) /\left(\mathrm{D}_{\mathrm{y}} \mathrm{L}^{2}\right)$, que estudia el efecto de la anisotropía en $\mathrm{k}$ y (merced a ésta) $\mathrm{D}$ es estudiada para valores que se acerquen a los de los casos reales. Valores de $\pi_{\mathrm{d}, 2} \mathrm{y}$ $\pi_{\mathrm{d}, 4}$ cercanos a la unidad extienden el patrón de concentraciones a todo el acuífero (denotando la influencia de L), reproduciendo casos reales, al estrechar la región de interface agua dulce-agua salada. Esto se debe a la baja difusividad frente a los efectos del flujo regional y más aún frente a los efectos de flotación por los valores de $\pi_{\mathrm{d}, 3}(0.05)$ y $\pi_{\mathrm{d}, 1}(7.58)$.

El patrón de flujo es muy sensible. Para valores bajos de los monomios, la línea divisoria marca aproximadamente la frontera agua dulce-agua salada hasta valores de 0.5; por encima de este valor la divisoria se desplaza hacia arriba dando origen a sendas recirculaciones en las dos fronteras verticales en las que deben cumplirse los balances entre los caudales de entrada y salida de agua impuesto s monomios emerge un flujo inverso del mar hacia el continente que parece muy improbable en casos reales.

6. Por último, se reproducen los patrones que muestran la influencia simultánea de los monomios $\pi_{\mathrm{d}, 2}$ y $\pi_{\mathrm{d}, 4}$ (para $\mathrm{k}_{\mathrm{x}}>>\mathrm{k}_{\mathrm{y}}$ y $\mathrm{D}_{\mathrm{x}}>>\mathrm{D}_{\mathrm{y}}$ ) con aumento del efecto difusivo general y disminución del efecto de flotación, el escenario 'ideal’ de problema 
Capítulo V. Influencia de los grupos discriminados en los patrones del problema de Henry

patrón, tratando de compensar la notable influencia en el problema de Henry de los efectos de flotación frente al flujo regional y la despreciable influencia de los efectos difusivos en general frente al mismo flujo. De acuerdo con lo esperado, la influencia de $\pi_{\mathrm{d}, 1} \mathrm{y} \pi_{\mathrm{d}, 3}$ empieza a ser apreciable (alejando las isolíneas de una distribución regular) cuando las difusividades y permeabilidades horizontales son grandes (parecidas a las verticales), aumentando los efectos de flotación. Las isolíneas de concentración se inclinan progresivamente tendiendo a concentrarse en las esquinas para los valores más altos de $\pi_{\mathrm{d}, 2} \mathrm{y} \pi_{\mathrm{d}, 4}$.

Para los valores pequeños de $\pi_{\mathrm{d}, 2} \mathrm{y} \pi_{\mathrm{d}, 4}$, el flujo muestra un fuerte desacoplo con la concentración en relación con las longitudes que sobre la base del acuífero definen la línea divisoria (que pasa de no existir a llegar a la mitad del acuífero) y la de menor concentración. Para valores superiores de los monomios el patrón se vuelve complejo con la emergencia de subdominios asociados a zonas de recirculación y circulación de flujos de agua dulce y salada que requieren un estudio más detallado.

ii. En relación con la influencia de los grupos discriminados (con valor en torno a la unidad), en los patrones del problema general de Henry (anisótropo e isótropo) se comprueba que pequeñas desviaciones suponen desequilibrios apreciables en los balances que, a su vez, determinan cambios de importancia en los patrones con la emergencia de cuñas de intrusión y de recirculación desacopladas.

1. La influencia de $\pi_{\mathrm{d}, 1}=\left[(\Delta \rho) \mathrm{gk}_{\mathrm{y}} /\left(\varepsilon \mu \mathrm{V}_{\mathrm{amb}}{ }^{*}\right)\right](\mathrm{L} / \mathrm{H})$ en el patrón de salinidad para valores $\pi_{\mathrm{d}, 1}<1$ es pequeña; las isolíneas de concentración quedan espaciadas casi regularmente en todo el dominio, mientras que las líneas de flujo son prácticamente horizontales y están igualmente espaciadas (flotación despreciable). Para $\pi_{\mathrm{d}, 1} \approx 1$ las isolíneas de concentración sufren una leve inclinación que se incrementa progresivamente para $\pi_{\mathrm{d}, 1}>1$, fruto de la combinación de los efectos de flotación y empuje horizontal; las de corriente se inclinan ligeramente para que el agua dulce flote sobre el agua salada y salga por arriba en la frontera marina. 
Capítulo V. Influencia de los grupos discriminados en los patrones del problema de Henry

La divisoria no emerge hasta valores de $\pi_{\mathrm{d}, 1}$ por encima de la unidad y está prácticamente desacoplada de cualquier referencia del patrón de flujo para $\pi_{\mathrm{d}, 1}<1$, emergiendo con fuerza para valores $\pi_{\mathrm{d}, 1} \approx 1 \mathrm{y}$ cubriendo toda la base para valores ligeramente superiores a la unidad. Para $\pi_{\mathrm{d}, 1} \approx 3$, la divisoria alcanza la frontera continental y sigue desplazándose hacia arriba generando flujos de recirculación en ambas fronteras y, posteriormente, un canal inverso de agua desde el mar hacia el continente.

2. En cuanto a la influencia de $\pi_{\mathrm{d}, 3}=\mathrm{D}_{\mathrm{x}} /\left(\right.$ Vamb $\left.^{*} \mathrm{~L}\right)$, valores de este monomio inferiores a la unidad dan lugar a un patrón de salinidad que concentra sus líneas (inclinadas por el balance equilibrado entre la flotación y el arrastre) junto al borde marino, merced a la baja difusividad frente al empuje regional. Al acercarse éste a la unidad, el patrón se ensancha manteniendo su inclinación, hasta cubrir todo el dominio en coherencia con lo esperado. Por último, para $\pi_{\mathrm{d}, 3}>1$ (difusividad preponderante frente a todos los efectos), se produce una distribución muy regular con isolíneas igualmente distanciadas, verticales, que cubren la totalidad del acuífero.

En cuanto al patrón de flujo, para $\pi_{\mathrm{d}, 3}<1$, el equilibrio arrastre-flotación da lugar a una pequeña recirculación en la frontera marina (que desvía las líneas de corriente continentales hacia arriba) que desaparece al crecer $\pi_{\mathrm{d}, 3}$. Al acercarse $\pi_{\mathrm{d}, 3}$ a la unidad, la recirculación desaparece antes de que se equilibren las fuerzas difusivas y de arrastre continental. Para $\pi_{\mathrm{d}, 3}>1$, con fuerzas de arrastre y flotación equilibradas entre sí pero despreciables frente a la difusión, las líneas de corriente, horizontales, cubren regularmente todo el dominio.

Llama la atención que el desacoplo entre la extensión horizontal de la línea divisoria y la definida por el patrón de concentraciones, que en el problema original de Henry eran casi idénticas, se hayan desacoplado más que en el caso anterior. El monomio $\pi_{\mathrm{d}, 3}$ reporta una información aparentemente inesperada pero sólo explicable por la complejidad del problema acoplado, cuando todos los efectos son influyentes: la recirculación de agua salada en la región cercana al mar bajo la línea divisoria, disminuye al aumentar la salinidad global del acuífero. 
Capítulo V. Influencia de los grupos discriminados en los patrones del problema de Henry

3. Los escenarios en los que cambia el monomio $\pi_{\mathrm{d}, 2}=\left(\mathrm{k}_{\mathrm{x}} \mathrm{H}^{2}\right) /\left(\mathrm{k}_{\mathrm{y}} \mathrm{L}^{2}\right)$ parecen algo irreales a la vista de los patrones resultantes ya que la longitud del acuífero L es muy determinante, en particular para valores de $\pi_{\mathrm{d}, 2}>1$; sólo un aumento sensible de L permitiría que el pie de la divisoria se encontrara en la base del acuífero.

Así, valores pequeños de $\pi_{\mathrm{d}, 2}<1$, con fuerzas de flotación y de arrastre equilibradas, aumentan las presiones en el acuífero por la disminución de su permeabilidad a flujo constante y causan unos patrones de concentración con distribución regular en todo el acuífero. Este patrón se mantiene hasta que $\pi_{\mathrm{d}, 2} \approx 1$, valor para el que las líneas se inclinan por la emergencia de los efectos de flotación y arrastre. El patrón de flujo, casi horizontal y regular para $\pi_{\mathrm{d}, 2}<1$, manifiesta una recirculación en la frontera marina para $\pi_{\mathrm{d}, 2} \approx 1$, con una divisoria que se desplaza rápidamente al seguir creciendo $\pi_{\mathrm{d}, 2}$ por encima de la unidad. Valores de $\pi_{\mathrm{d}, 2 \approx 3} \mathrm{y}$ superiores crean tres regiones superpuestas de flujo alternativo (continente-mar, mar-continente), casi horizontales.

4. La influencia del monomio $\pi_{\mathrm{d}, 4}=\mathrm{D}_{\mathrm{x}} \mathrm{H}^{2} /\left(\mathrm{D}_{\mathrm{y}} \mathrm{L}^{2}\right)$ es singular en tanto que al estar equilibradas en todo momento las fuerzas de arrastre, flotación y difusividad (por los valores unidad de $\left.\pi_{\mathrm{d}, 1} \mathrm{y} \pi_{\mathrm{d}, 3}\right)$ los patrones de flujo permanecen inalterables $\mathrm{y}$ regularmente distribuidos en todo el dominio. Los cambios de $\pi_{\mathrm{d}, 4}$ tan solo afectan a la inclinación de las isolíneas de concentración. Para $\pi_{\mathrm{d}, 4}<1$ la fuerte influencia de la difusión vertical frente al resto de las fuerzas elimina sus gradientes, presentando unas líneas verticales, mientras que para $\pi_{\mathrm{d}, 4}>1$ ocurre lo contrario y las líneas se inclinan ligeramente.

iii. En relación con la influencia de la geometría del dominio en la evolución de las cuñas buscando el papel real que juega dicha geometría en el patrón de soluciones, se concluye que valores de $\mathrm{L} \geq 1.5$, no alteran prácticamente nada los pies de las cuñas verificando que L no determina la forma de estos patrones por encima de este valor, ni por tanto la relación de aspecto H/L. Ello permite simplificar los monomios del problema a dos (con H en lugar de L como referencia longitudinal): $\pi_{1}=\frac{(\Delta \rho) \mathrm{gk}}{\varepsilon \mu v_{\mathrm{amb}}^{*}}$ $\mathrm{y} \pi_{2}=\frac{\mathrm{D}}{\mathrm{vamb}_{\mathrm{amb}}^{*}}$. 
La simulación de escenarios (con L suficientemente grande) para un amplio conjunto de valores de estos parámetros proporciona resultados coherentes cuyas no linealidades son solo explicables por los balances que se establece en los subdominios, las longitudes de los cuales deberían ser función de los grupos adimensionales emergentes en los mismos y derivados (tarea que no se aborda en la tesis) de las ecuaciones adimensionales correspondientes. Estas longitudes son: $\mathrm{l}_{\mathrm{f}}{ }^{*}$, longitud de la cuña de recirculación (distancia desde el extremo inferior derecho del borde marino hasta la intersección de la línea divisoria con el fondo del acuífero), y $\mathrm{l}_{\mathrm{c}=0.1}{ }^{*}-\mathrm{l}_{\mathrm{c}=0.9}{ }^{*}$, extensión de la cuña difusa o región de agua parcialmente salada, medida sobre la base del acuífero (donde $\mathrm{l}_{\mathrm{c}=0.1}{ }^{*} \mathrm{y} \mathrm{l}_{\mathrm{c}=0.9}{ }^{*}$ son, respectivamente, las distancias desde el extremo inferior derecho del acuífero a la intersección de las isolíneas c=0.1 y C=0.9 con la frontera inferior. 
Capítulo V. Influencia de los grupos discriminados en los patrones del problema de Henry 


\section{CONTRIBUCIONES Y CONCLUSIONES FINALES}

Aunque al final de los Capítulos III, IV y V que constituyen el núcleo de investigación de esta memoria se han recogido pormenorizadamente las contribuciones y conclusiones específicas de los diferentes aspectos estudiados en la misma, se describe aquí, de forma agrupada y a modo de síntesis global una relación de las contribuciones y conclusiones más importantes.

\section{PRIMERA. En relación con la aplicación de la técnica de adimensionalización} discriminada al problema de Henry.

I.1 Se ha aplicado con éxito la técnica de adimensionalización discriminada para deducir los cuatro grupos adimensionales (discriminados) del problema general de Henry con difusividad efectiva, no dispersivo, para un medio anisótropo en las propiedades permeabilidad y difusividad efectiva.

I.2 Se reducen los anteriores números al caso isótropo, demostrando que los tres grupos resultantes son equivalentes a los obtenidos mediante análisis dimensional por Henry [1964], y mediante la adimensionalización clásica por Abarca [2006]; también son equivalentes a los obtenidos por Abarca para medios anisótropos en la permeabilidad e isótropos en la difusividad molecular.

I.3 La discriminación justifica la expulsión del factor de forma o relación de aspecto $(\mathrm{L} / \mathrm{H})$ como grupo adimensional en escenarios anisótropos, una conclusión coherente con los resultados, no deducida mediante técnicas clásicas de análisis dimensional o adimensionalización.

I.4 Los resultados anteriores se verifican mediante simulaciones numéricas con el código FATSIM para demostrar la independencia y completitud (unicidad) de los grupos deducidos. La verificación consiste en simular diferentes escenarios para los que el valor de los grupos adimensionales no cambia, pero sí lo hace y de forma sensible el valor particular de dos o más de los diferentes parámetros hidrogeológicos y geométricos. En todas las pruebas sin excepción se obtienen los mismos patrones de isolíneas de concentración y flujo para los mismos valores numéricos de los grupos adimensionales. 
I.5 Para valores unidad de los grupos deducidos, tanto en el caso isótropo como en el anisótropo, el patrón de flujo y concentración se distribuye de manera regular cubriendo la totalidad del dominio como cabía esperar. En este sentido:

I.5.1 Se discute la idoneidad del carácter patrón del problema en base al valor numérico de los grupos discriminados deducidos.

I.5.2 Se interpretan los grupos deducidos en términos de balance de magnitudes físicas que interactúan en el problema, justificándose al mismo tiempo su orden de magnitud unidad cuando el patrón cubre todo el dominio.

I.5.3 Se justificada asimismo la distribución no regular del patrón de flujo y concentración para los valores de los grupos adoptados por Henry.

I.5.4 Se justifica la influencia relativa de un cambio en el valor de los grupos para el problema de Henry y para el caso de grupos adimensionales de valor unidad (influencia mayor en el segundo caso que en el primero).

\section{SEGUNDA. En relación con escenarios tipo Henry que admiten simplificaciones por influencia despreciable de alguno de los parámetros del problema.}

Se simplifican los escenarios tanto del problema original de Henry, cuyos grupos adimensionales tienen valores diferentes a la unidad, como del problema no isótropo con números adimensionales de valor unidad. En todos los casos se derivan y verifican los grupos adimensionales resultantes.

II.1 Para el problema isótropo original de Henry se consideran seis simplificaciones, las cinco primeras se refieren a la influencia en el patrón de solución de los cambios de algunos parámetros físicos concretos del problema, contenidos en los monomios resultantes de la simplificación, asumiendo como parámetros geométricos influyentes (pero de valor constante) las dimensiones del dominio, L y H. En la última simplificación, se investiga la influencia específica de estos parámetros geométricos ( $\mathrm{L}$ y H) con el objeto de averiguar hasta qué punto y bajo qué condiciones dichos parámetros son, en efecto, determinantes o no en la solución del problema original. Estas simplificaciones son: 
i) Difusividad despreciable. Los grupos se reducen de tres a dos y la relación de aspecto no emerge como grupo independiente en principio, a menos que se asuma isotropía en la permeabilidad.

ii) Difusividad vertical despreciable, $\mathrm{D}_{\mathrm{y}}<<\mathrm{D}_{\mathrm{x}}$. El número de grupos independientes se mantiene en tres, aunque de nuevo, la relación de aspecto no emerge como grupo independiente. La recirculación de agua de mar (línea divisoria en los patrones de función de corriente) define la misma extensión en la base del acuífero en concordancia con la constancia del valor de los grupos adimensionales que no contienen la difusividad.

iii) Difusividad isotrópica, $\mathrm{D}_{\mathrm{y}}=\mathrm{D}_{\mathrm{x}}$. Ahora sí aparece la relación de aspecto como grupo independiente. Se mantiene el número de grupos adimensionales en cuatro y se verifica su deducción.

iv) Velocidad regional despreciable frente al efecto de difusión, $\mathrm{Lv}^{*}{ }_{\mathrm{amb}}<<\mathrm{D}$. Se verifican los tres monomios resultantes. La acusada disminución de $\mathbf{v}^{*}$ amb frente a su valor en el problema original resulta en un patrón acusadamente diferente ya que la preponderancia de la difusión frente al flujo regional desplaza la cuña salada hacia el continente con gradientes de sal en todo el dominio del acuífero. El patrón de flujo es marcadamente diferente al del problema de Henry original.

v) Diferencia de densidades $(\Delta \rho)$ despreciable. Los grupos adimensionales se reducen a tres (dos en el caso isótropo).

vi) Dominios extensos $(\mathrm{L}>>\mathrm{H})$. Tanto en escenarios anisótropos como isótropos, si deducimos los grupos por simplificación de los del caso general llegamos a resultados incoherentes (dos grupos para el caso isótropo y cuatro para el anisótropo). Se concluye que la extensión L del acuífero es un parámetro poco o nada relevante.

II.2 Para escenarios simplificados derivados de problemas con grupos discriminados del orden de la unidad se consideran simplificaciones similares a las anteriores. En este caso y como esperamos, la asignación del valor unidad a los monomios influyentes que resultan de cada caso induce (en coherencia) un patrón regular tanto de isolíneas de concentración como de corriente en todo el dominio, patrón 
que se asemeja mucho cualitativamente en todos los casos. Se simulan los siguientes casos:

i) Caso de difusividad despreciable. Del patrón resultante se deriva una crítica a la elección de L y H como magnitudes influyentes.

ii) Caso de difusividad vertical despreciable. Los patrones, en coherencia con lo esperado, apenas sufren alteración.

iii) Caso de difusividad isotrópica, $\mathrm{D}_{\mathrm{y}}=\mathrm{D}_{\mathrm{x}}$.

iv) Caso de difusividad y conductividad hidráulica isotrópicas, $\mathrm{D}_{\mathrm{y}}=\mathrm{D}_{\mathrm{x}}, \mathrm{k}_{\mathrm{x}}=\mathrm{k}_{\mathrm{y}}$.

v) Caso de velocidad regional despreciable, $\left(\mathrm{Lv}_{\mathrm{amb}}<<\mathrm{D}_{\mathrm{x}}\right)$.

vi) Caso de diferencia de densidad despreciable.

vii) Caso de dominios extensos $(\mathrm{L}>>\mathrm{H})$ y medios isótropos. El acusado alargamiento de L no ha afectado a la distribución regular de líneas en todo el dominio por el valor unidad de los grupos.

\section{TERCERA. En relación con las desviaciones de los monomios respecto a sus valores en el problema original de Henry.}

Cabe concluir que las simulaciones ejecutadas son coherentes y capaces de reproducir la casi totalidad de potenciales escenarios.

III.1 Para el monomio $\pi_{\mathrm{d}, 1}=\left[(\Delta \rho) g k_{\mathrm{y}} /\left(\varepsilon \mu \mathrm{vamb}^{*}\right)\right](\mathrm{L} / \mathrm{H})$. Valores decrecientes de $\pi_{\mathrm{d}, 1}$ retraen y estrechan en su base la cuña hacia el lado del mar, con la emergencia de una región acuñada de elevada salinidad en la zona inferior derecha del dominio. L influye en el resultado. En el patrón de flujo, la intersección con la base del dominio de la divisoria de aguas, inexistente para valores bajos de $\pi_{\mathrm{d}, 1}$, emerge progresivamente con el aumento de este monomio hasta alcanzar buena parte de la base.

III.2 Para el monomio $\pi_{\mathrm{d}, 3}=\mathrm{D}_{\mathrm{x}} /\left(\mathrm{V}_{\mathrm{amb}}{ }^{*} \mathrm{~L}\right)$. El patrón de salinidad sufre pocas modificaciones hasta que $\pi_{\mathrm{d}, 3}$ se acerca a la unidad en donde la sal cubre todo el dominio. Se manifiesta la influencia de L. La intersección de la divisoria de aguas con la base se contrae ligeramente con el aumento de $\pi_{\mathrm{d}, 3}$. Es importante advertir el claro desacoplo (que induce a reflexionar sobre la existencia de nuevos balances) entre la extensión de la zona salada resultante del patrón de 
concentraciones y la longitud que determina la línea divisoria con la frontera inferior.

III.3 Para el monomio $\pi_{\mathrm{d}, 2}=\left(\mathrm{k}_{\mathrm{x}} \mathrm{H}^{2}\right) /\left(\mathrm{k}_{\mathrm{y}} \mathrm{L}^{2}\right)$. Su valor, a partir de 0,01 ensancha $\mathrm{y}$ acuesta la región de mezcla hasta extenderse a todo lo largo del acuífero (con evidente influencia de L), reproduciendo patrones que ciertamente reflejan los de los acuíferos reales. En cuanto al patrón de flujo, para $\pi_{\mathrm{d}, 2} \approx 1$, la línea divisoria penetra en el continente dando lugar a recirculaciones de agua en las dos fronteras y a una recirculación inversa desde el mar hacia el continente.

III.4. Para el monomio $\pi_{\mathrm{d}, 4}=\left(\mathrm{D}_{\mathrm{x}} \mathrm{H}^{2}\right) /\left(\mathrm{D}_{\mathrm{y}} \mathrm{L}^{2}\right)$. Emergen una cuña de difusión cuya base se expande paulatinamente sobre la frontera inferior y una región completamente salada lindante con el mar. El caudal de recirculación de agua de mar disminuye sensiblemente al incrementar $\pi_{\mathrm{d}, 4}$ hasta hacerse despreciable para $\pi_{\mathrm{d}, 4} \approx 1$.

III.5 Para la influencia simultánea de $\pi_{\mathrm{d}, 2}=\left(\mathrm{k}_{\mathrm{x}} \mathrm{H}^{2}\right) /\left(\mathrm{k}_{\mathrm{y}} \mathrm{L}^{2}\right)$ y $\pi_{\mathrm{d}, 4}=\left(\mathrm{D}_{\mathrm{x}} \mathrm{H}^{2}\right) /\left(\mathrm{D}_{\mathrm{y}} \mathrm{L}^{2}\right)$. Valores de $\pi_{\mathrm{d}, 2} \mathrm{y} \pi_{\mathrm{d}, 4}$ cercanos a la unidad extienden el patrón de concentraciones a todo el acuífero, denotando la influencia de L merced a la baja difusividad frente a los efectos del flujo regional y de la flotación. El patrón de flujo es muy sensible. La divisoria marca la frontera agua dulce-agua salada hasta valores $\pi_{\mathrm{d}, 2}=0.5$; por encima de este valor la divisoria se desplaza hacia arriba dando origen a sendas recirculaciones en las dos fronteras y, más tarde, a un flujo inverso del mar hacia el continente.

III.6 Para la influencia simultánea de $\pi_{\mathrm{d}, 2}$ y $\pi_{\mathrm{d}, 4}$, con aumento del efecto difusivo general y disminución del efecto de flotación. La influencia de $\pi_{\mathrm{d}, 1} \mathrm{y} \pi_{\mathrm{d}, 3}$ empieza a ser apreciable cuando las difusividades y permeabilidades horizontales son grandes. Las isolíneas de sal tienden a concentrarse en las esquinas para los valores más altos de $\pi_{\mathrm{d}, 2} \mathrm{y} \pi_{\mathrm{d}, 4}$. Por otro lado, para valores pequeños de $\pi_{\mathrm{d}, 2} \mathrm{y} \pi_{\mathrm{d}, 4}$ el flujo muestra un fuerte desacoplo con la concentración en relación con las longitudes que sobre la base del acuífero definen la línea divisoria y la de menor concentración. Para valores superiores el patrón se vuelve más complejo. 


\section{CUARTA. En relación con la influencia de los grupos discriminados en los patrones del problema general de Henry (anisótropo e isótropo).}

Las simulaciones ejecutadas son coherentes y se comprueba que pequeñas desviaciones de los monomios (que suponen desequilibrios apreciables en los balances) determinan cambios de importancia en los patrones con la emergencia de cuñas de intrusión y de recirculación desacopladas.

IV.1 Influencia de $\pi_{\mathrm{d}, 1}=\left[(\Delta \rho) g \mathrm{k}_{\mathrm{y}} /\left(\varepsilon \mu \mathrm{v}_{\mathrm{amb}}{ }^{*}\right)\right](\mathrm{L} / \mathrm{H})$. Para $\pi_{\mathrm{d}, 1}<1$, las isolíneas de concentración y flujo están igualmente espaciadas en coherencia con una flotación despreciable. La divisoria no emerge hasta valores de $\pi_{\mathrm{d}, 1}$ por encima de la unidad y está prácticamente desacoplada de cualquier referencia del patrón de flujo para $\pi_{\mathrm{d}, 1}<1$. Para $\pi_{\mathrm{d}, 1}$ creciente, la divisoria alcanza la frontera continental y se desplaza hacia arriba generando flujos de recirculación y, posteriormente, un canal inverso de agua desde el mar hacia el continente.

IV.2 Influencia de $\pi_{\mathrm{d}, 3}=\mathrm{D}_{\mathrm{x}} /\left(\mathrm{V}_{\mathrm{amb}}{ }^{*} \mathrm{~L}\right)$. Las isolíneas de concentración se expanden a partir de un patrón concentrado en el borde marino para $\pi_{\mathrm{d}, 3}<1$, hasta cubrir todo el dominio para valores crecentes de este monomio. También, para $\pi_{\mathrm{d}, 3}<1$, el equilibrio arrastre-flotación da lugar a una pequeña recirculación en la frontera que desaparece al crecer el monomio. Para $\pi_{\mathrm{d}, 3}>1$, con fuerzas de arrastre $y$ flotación equilibradas entre sí pero despreciables frente a la difusión, las líneas de corriente, horizontales, cubren regularmente todo el dominio. Se observa un desacoplo entre la extensión horizontal de la línea divisoria y la definida por el patrón de concentraciones. Sólo la complejidad de este problema conjugado explica que la recirculación de agua salada en la región cercana al mar, bajo la línea divisoria, disminuye al aumentar la salinidad global del acuífero.

IV.3 Influencia de $\pi_{\mathrm{d}, 2}=\left(\mathrm{k}_{\mathrm{x}} \mathrm{H}^{2}\right) /\left(\mathrm{k}_{\mathrm{y}} \mathrm{L}^{2}\right)$. Para $\pi_{\mathrm{d}, 2}>1$, la longitud del acuífero $\mathrm{L}$ es muy determinante en los patrones y sólo un aumento sensible de L permite que el pie de la divisoria se ubique en la base del acuífero. El patrón de flujo, casi horizontal y regular para $\pi_{\mathrm{d}, 2}<1$, manifiesta una recirculación en la frontera marina para $\pi_{\mathrm{d}, 2} \approx 1$ con una divisoria que se desplaza rápidamente al seguir creciendo $\pi_{\mathrm{d}, 2}$ por encima de la unidad. Para $\pi_{\mathrm{d}, 2} \approx 3$ ya emergen tres regiones de flujo alternativo (continente-mar, mar-continente), casi horizontales. 
IV.4 Influencia de $\pi_{\mathrm{d}, 4}=\mathrm{D}_{\mathrm{x}} \mathrm{H}^{2} /\left(\mathrm{D}_{\mathrm{y}} \mathrm{L}^{2}\right)$. Los patrones de flujo permanecen inalterables $\mathrm{y}$ regularmente distribuidos en todo el dominio. Para $\pi_{\mathrm{d}, 4}<1$, la fuerte influencia de la difusión vertical frente al resto de las fuerzas elimina los gradientes verticales de concentración presentando isolíneas verticales, mientras que para $\pi_{\mathrm{d}, 4}>1$ ocurre lo contrario y las líneas se inclinan ligeramente.

\section{QUINTA. En relación con la influencia de la geometría del dominio en la evolución de las cuñas.}

Se concluye que valores de $\mathrm{L} \geq 1.5$, no alteran prácticamente nada los pies de las cuñas por lo que ni L ni la relación de aspecto determinan los patrones en este rango. Así, los monomios del problema se reducen a dos; $\pi_{1}=\frac{(\Delta \rho) g k}{\varepsilon \mu v_{\mathrm{amb}}^{*}}$ y $\pi_{2}=\frac{\mathrm{D}}{\mathrm{v}_{\mathrm{amb}}^{*} \mathrm{H}}$, con $\mathrm{H}$ como única referencia longitudinal para derivar las ecuaciones adimensionales.

Se investigan los patrones para valores de L no influyentes en los mismos con resultados coherentes, pero sólo explicables con los nuevos monomios que resultan de la simplificación de las ecuaciones de gobierno a los subdominios que emergen en el problema, subdominios obviamente definidos por las cuñas (desacopladas) de intrusión (difusión) y recirculación emergentes. Las longitudes que definen estas cuñas deberían asumirse como las nuevas referencias para adimensionalizar las ecuaciones en estos subdominios. Una tarea para continuar este estudio. 


\section{Contribuciones y conclusiones finales}




\section{REFERENCIAS BIBLIOGRÁFICAS}

Abarca, E. (2006). Seawater intrusion in complex geological environments. Tesis doctoral. Universidad Politécnica de Cataluña.

Abarca, E., J. Carrera, X. Sánchez-Vila, y M. Dentz (2007), Anisotropic dispersive Henry Problem, Advances in Water Resources, 30 (4), 913-926.

Alhama, F. y Madrid, C.N. (2012). Discriminated dimensional analysis in fluid mechanics and heat transfer. Ed. Reverté. Barcelona.

Alhama, F. y Madrid, C.N. (2007). Discriminated dimensional analysis versus classical dimensional analysis and aplications to heat transfer and fluid dynamics. Chinese Journal of Chemical Engineering, 15 (5), 626-631.

Alhama, F. y Madrid, C.N. (2008). Discriminate dimensional analysis ans scale analysis applied to forced and free convection: a comparison. Erotherm-08. Eindhoven.

Alhama, I. Alhama, F. y Soto A. (2011). FATSIM-A: An educational tool base on electrical analogy and the code PSpice to simulate fluid flow and solute transport processes. Computer Application in Engineeering Education, 16, 72-82.

Alhama, I., Canovas, M. y Alhama, F. (2014). On the Nondimensionalization Process in Complex Problems: Application to Natural Convection in Anisotropic Porous Media. Mathematical Problems in Engineering, Volume 2014. Article ID 796781, 13p.

Alhama, I., Canovas, M., Alhama, F. (2015). Simulation of fluid flow and heat transport coupled processes using FAHET software. Journal of Porous Media, 18 (5), 537-546.

Alhama, I., Soto Meca, A., Alhama, F. (2011). Simulation of flow and solute coupled 2$\mathrm{D}$ problems with velocity-dependent dispersion coefficient based on the network method. Hydrological Processes, 26(24), 3725-3735.

Amstrong, H.L.(1965). Dimensional analysis. American Journal of Physics. 33, 513

Arpaci, V.S. y Larsen, P.S. (1984). Convection heat transfer. Prentice-Hall, New Jersey.

Bastian, P., Birken, K., Lang, S., Johannsen, K., Neu_, N., Rentz-Reichert y H., Wieners, C., (1997). UG - A flexible software toolbox for solving partial diferential equations. Computing and Visualization in Science, 1(1), 27-40.

Bejan, A. (1984). Convection heat transfer. John Wiley \& Sons, New York.

Bejan, A. (1995). Convection heat transfer, (2ª ed). John Wiley \& Sons, New York.

Bejan, A. (2004). Convection heat transfer, (3 $3^{\mathrm{a} e d) . ~ J o h n ~ W i l e y ~ \& ~ S o n s, ~ N e w ~ Y o r k . ~}$

Bejan, A. (2013). Convection heat transfer, (4 ed). John Wiley \& Sons, New York.

Bues, M., y C. Oltean (2000), Numerical simulations for saltwater intrusion by the mixed hybrid finite element method and discontinuous finite element method, Transport Porous Med., 40(2), 171-200. 
Canovas, M. (2014). Caracterización adimensional y simulación numérica de escenarios patrón (Bénard, Yusa y Elder) de procesos geotérmicos de flujo y transporte. Tesis doctoral, Universidad Politécnica de Cartagena.

Canovas, M., Alhama, I., Trigueros, E. y Alhama, F. (2014). Numerical study of temperatura and streamfunction patterns before full convection in geothermal cells of Bénard type. Proceedings of the $11^{\text {th }}$ World Congress on Computacional Mechanics (WCCM XI) Barcelona, Vol. 4. 3022-3033.

Canovas, M., Alhama, I. y Alhama, F. (2015). Mathematical characterization of Bénardtype geothermal scenarios using discriminated non-dimensionalization of the governing equations. International Journal of Nonlinear Sciences and Numerical Simulation, 16(1), 23-34.

Canovas, M., Alhama, I., Trigueros, E. y Alhama, F. (2016). A review of classical dimensionless numbers for the Yusa problem based on discriminated nondimensionalization of the governing equations. Hidrological process (Accepted article).

Capobianchi, M. y Aziz, A. (2012). A scale analysis for natural convective flows over vertical surfaces. International Journal of Thermal Sciences, 54, 82-88.

Cengel, Y.A. (2004). Heat transfer: A practical approach (2a ed.). McGraw-Hill. New Delhi.

CODENS-13 (2013): Alhama, F., Alhama I., Sánchez J.F., Morales J.L., Programa de resolución de ecuaciones diferenciales ordinarias acopladas, NAR: 08/2014/0056, (C) 2004-2014 UPCT.

Conesa, M. (2016). Sobre la adimensionalización discriminada de ecuaciones y sistemas de ecuaciones diferenciales ordinarias, no lineales y solución numérica mediante el método de redes: aplicación a problemas mecánicos. Tesis doctoral. Universidad Politécnica de Cartagena.

Conesa, M., Sánchez Pérez, J.F., Alhama, I. y Alhama, F. (2016). On the nondimensionalization of coupled, nonlinear ordinary differential equations. Nonlinear Dynamics, 84(1), 91-105.

Cooper, H.H. (1959). A hypothesis concerning the dynamic balance of fresh water and salt water ina a coastal aquifer. Journal of Geophysical Research, 64(4), 461-467.

Croucher, A.E. y O’Sullivan, M.J. (1995). The Henry problem for saltwater intrusion, Water Resources Research, 31 (7), 1809-1814.

EPSNET_10 (2011): Morales J.L., Moreno J.A., Alhama F., Elasticity Problems Simulation Network, NAR: 08/2011/418, (C) 2011 UPCT.

FAHET (2011): Alhama I., Soto Meca A., Cánovas M., Alhama F., Flow and Heat Transport simulator, NAR: 08/2011/419, (C) 2004-2009 UPCT.

FATSIM-A (2010): Alhama I., Soto Meca A., Alhama F., Fluid Flow and Heat Transport simulator, NAR: 08/2010/1110 (DL: MU-1093-2010), (C) 2004-2009 UPCT. 
Frind, E. (1982), Simulation of long-term transient density-dependent transport in groundwater, Advances in Water Resources., 5 (2), 73-88.

Gebhart, B., Jaluria, Y., Mahajan, R.L. y Sammakia, B. (1988). Buoyancy-induced flows and transport. Hemisphere Publishing Cor, Washington.

Gibbings, J.C. (2011) Dimensional Analysis. UK: Springer-Verlag London Limited.

Glover, R.E. (1964). The pattern of Fresh-Water Flow in a Coastal Auifer. Sea water in coastal Aquifers, U.S. Geological Survey Water-Supply Paper-C, 32-35.

Gröber, H y Erk S. (1967). Transmisión de calor. Madrid selecciones científicas.

Henry, H.R. (1959). Salt intrusion into fresh-water aquifers. Journal of Geophysical Research, 64(11), 1911-1919.

Henry, H.R. (1964). Effects of dispersion on salt encroachment in coastal aquifers, in Sea Water in Coastal Aquifers. US Geological Survey Water Supply Paper, 1613-C, 7084.

Herranz, A. y Arenas, A. (2003). Análsis dimensional y sus aplicaciones. IV Geometría Física. Diego Marín, Murcia

Herranz, A. y Arenas, A. (2005). Análsis dimensional y sus aplicaciones. Diego Marín, Murcia.

Holman, J.P. (1997). Heat transfer; (8 ed). McGraw-Hill, New York.

Holzbecher E. (1991). FAST-C(2D) Modellierung von Dichtestromungen im Porosen Medium, Institut fur Wasserbau und Wasserwirtschaft, Technische Universitat Berlin, Mitteilungen Nr. 117, Berlin

Holzbecher, E. (1998). Modelling Density-Driven Flow in Porous Media. Springer, Berlín.

Hristov, J.Y. (2007). Magnetic field assisted fluidization: dimensional analysis addressing the physical basis. China Particuology, 5 (1), 103-110.

Hristov, J.Y., Planas-Cuchi, E., Aranaldos, J. y Casal, J (2004). Accidental burning of a fuel laye ron a waterbed: a scale analysis of the models predicting the pre-bollover time and tests to published data. Intenational Journal of Thermal Sciences, 43(3), 221-239.

Hubbert, M.K. (1940). The theory of ground-water motion. The Journal of Geology, 48(8). $785-944$.

Huyakorn, P., Andersen, F., Mercer, J. y White, H. (1987), Saltwater intrusion in aquifers: Development and testing of a three-dimensional finite element model, Water Resources Research, 2(23), 293-312.

Incropera, F.P. y DeWitt, D.P. (1996). Fundamentals of Heat and Mass Transfer. Wiley, New York. 
Intera (1979). SWIFT Revision of the Documentation for a Model for Calculating Effects of Liquid Waste Disposal in Deep Saline Aquifers, US Geological Society, WRI 79-96, Reston, Virginia.

Kakinuma T., Kishi, Y. y Inouchi, K. (1988) The behavior of groundwater with dispersion in coastal aquifers. Journal of Hydrology; 98(3-4-98), 225-248

Kalakan, Ch. (2014). Investigation of saltwater intrusion based on the Henry problem and a field-scale problem. Ph.D., University of Florida, 424 pages.

Kays, W.M. y Crawford, M. (1993). Convective Heat and Mas Transfer. McGraw-Hill. New York.

Kohout, F. A., Cyclic flow of salt water in the Biscayne aquifer of southeastern F lorida, J. Geophys.R es., 65(7), 2133-2141, 1960

Kohout, F.A., In Sea water in coastal aquifers (Cooper, et., al., 1964) U.S. Geological Survey Water Supply Paper 1613-C

Kolditz, O. (1994). Benchmark for numerical ground water simulations. In: Diersch, H.j. FEFLOW User's Manual. Release 4.20, WASY, BERLIN 5.1-5.129.

Kozeny, J. (1953). Hydaulik. Springer Verlag, Viena.

Kreith, F. y Bohn, M. (1997). Principles of Heat Transfer. PWS Publising Company. 753 pages.

Landau, L.D. y Lifshitz, E.M. (1985). Mecánica de fluidos. Ed. Reverte, Barcelona.

Luna-Abad, J.F., Alhama F. y Campo, A. (2010). Optimization of longitudinal rectangular fins through the concept of relative inverse admittance. Heat Transfer Engineering, 31(5), 395-401.

Madrid, C.N. y Alhama, F. (2005a). Discriminated dimensional analysis of the energy equation: Application to laminar forced convection along a flat plate. International Journal of Thermal Sciences, 44, 333-341.

Madrid, C.N. y Alhama, F. (2005b). A Revision of the Classical Reynolds Numbers for Different Types of Flow Following the Theory of Discriminated Dimensional Analysis. IASME Transactions, 8(2), 1524-1529.

Madrid, C.N. y Alhama, F. (2006). Discrimination: A fundamental and necessary extension of classical dimensional analysis. International Communications in Heat and Mass, 33(3), 287-294

Madrid, C.N. y Alhama, F. (2008) Study of the laminar natural convection problema along an isothermal vertical plate base don dicriminated dimensional analysis. Chemical Engineering Communications, 195(12), 1524-1537.

Oldenburg, C.M., y K. Pruess, (1993) A Two-Dimensional Dispersion Module for the TOUGH2 Simulator, Report LBL-32505, Lawrence Berkeley Laboratory, Berkeley, Calif., 1993. 
OXIPSIS-12 (2013): Sánchez J.F., Alhama F., Moreno, J.A., Programa de simulación de procesos de oxidación, NAR: 08/2013/616, (C) 2004-2013 UPCT.

Ozisik, M.N. (1993). Heat conduction (2a ed.). John Wiley \& Sons. New York. 692 pages.

Palacios, J. (1955). Análisis dimensional. Espasa, Madrid.

Palacios, J. (1964). Dimensional analysis. MacMillan, London.

Pinder, G. F., y H. H. Cooper (1970), A numerical technique for calculating the transient position of the saltwater front. Water Resources Research, 6(3), 875-882.

Potter, M. y Wiggert D. (1997). Mecánica de fluidos. Prentice Hall, London

PROCCA-09 (2005): Alhama F. y Del Cerro F., Programa de conducción de calor, NAR:08/2005/544, (C) 2004-2009 UPCT.

Rohsenow, W.M., Hartnett, J.P. y Cho, Y.I. (1998). Handbook of Heat Transfer ( $\left.3^{a} e d\right)$. McGraw-Hill, New York.

San Juan, R. (1947). Teoría de las magnitudes físicas y sus fundamentos algebraicos. Revista de la Real Academia de Ciencias, Madrid.

Sanz, E. y Voss, C.I. (2006). Inverse modeling for seawater intrusion in coastal aquifers: insights about parameter sensivities, variances, correlations and estimation procedures derived from the Henry problem. Advances in Water Resources 29, 439457.

Sedov, L.I. (1993). Similarity and Dimensional Methods in Mechanics (10 ed.).CRS Press. 496 pages.

Segol, G., Pinder G.F.. y Gray, W.G. (1975). A Galerkin finite element technique for calculating the transient position of the saltwater front, Water Resources Research., 11(2), 343-347.

Segol, G. (1994). Classic Groundwater Simulations Proving and Improving Numerical Models, Prentice-Hall, New Jersy.

Simpson, M.J. y Clement,T.P. (2003). Theoretical analysis of the worthiness of the Henry and Elder problems as benchmarks of density-dependent groundwater flow models. Advances of Water Resources 26 (1), 17-31.

Simpson, M.J. y Clement,T.P. (2004). Improving the worthiness of the Henry problem as a benchmark for density-dependent groundwater flow models. Water Resources Research, 40 (1) W01504.

Sonin, A.A. (1997). The physics basis of dimensional analysis. MIT Cambridge, MA 02139.

Sonin, A.A. (2001). The physics basis of dimensional analysis. Second edition. MIT Cambridge, MA 02139 
Soto, A. (2007a). Simulación numérica de procesos de intrusión salina en acuíferos mediante el método de simulación por redes. Tesis doctoral. Universidad Politécnica de Cartagena.

Soto, A., Alhama, F. y González-Fernández, C.F. (2007b). An efficient model for solving density driven groundwater flow problems base on the network simulation method. Journal of Hidrology, 339 (1-2), 39-53.

Soto, A., Alhama, F. y González-Fernández, C.F. (2007c). Density-driven flow and solute transport problems. A 2-D numerical model based on the network simulation method. Computer Physics Communications, 177 (9), 720-728.

Thomas, L.C., (1992). Heat transfer. Prentice-Hall, New Yersey.

Voss, C.I. (1984). SUTRA. A FE simulation for saturated- unsaturated, Fluid-Density Dependent Ground-water flow with Energy Transport or Chemically-Reactive SingleSpecies Solute Transport. U.S. Geological Survey. Water Resources investigation 844369

Voss, C.I. y Souza W.R. (1987). Variable-density flow and solute transport simulation of regional aquifers containing a narrow freshwater-saltwater transition zone. Water Resources Research, 23(10), 1851-1866.

Williams, W. (1892). On the relation of the dimensions of physical quantities to directions in space. The London, Edinburgh and Dublin Philosophical magazine and Journal of Science, 34(208), 234-271.

Yeh, G.T., Cheng, J.R. y Cheng, H.P (1993). 2DFEMFAT: User's Manual of a 2Dimensional Finite Element Model of Flow and Transport through SaturatedUnsaturated Media in Course Notes on Modeling of Flow and contaminants in the Subsoil. Delft: Engineering and Mine Surveying.

Zimparov, V.D. y Petkov, V.M. (2009). Application of discriminated analysis to low Reynolds numbers swirl flows in circular tubes with twisted-tape inserts. Pressure drops correlations. International Review of Chemical Engineering, 1 (4), 346-356. 